\title{
Childhood overweight : the influence of parenting on children's energy balance-related behavior.
}

Citation for published version (APA):

Sleddens, E. F. C. (2013). Childhood overweight : the influence of parenting on children's energy balancerelated behavior. [Doctoral Thesis, Maastricht University]. Uitgeverij BOXPress. https://doi.org/10.26481/dis.20130405es

Document status and date:

Published: 01/01/2013

DOI:

$10.26481 /$ dis.20130405es

Document Version:

Publisher's PDF, also known as Version of record

\section{Please check the document version of this publication:}

- A submitted manuscript is the version of the article upon submission and before peer-review. There can be important differences between the submitted version and the official published version of record.

People interested in the research are advised to contact the author for the final version of the publication, or visit the DOI to the publisher's website.

- The final author version and the galley proof are versions of the publication after peer review.

- The final published version features the final layout of the paper including the volume, issue and page numbers.

Link to publication

\footnotetext{
General rights rights.

- You may freely distribute the URL identifying the publication in the public portal. please follow below link for the End User Agreement:

www.umlib.nl/taverne-license

Take down policy

If you believe that this document breaches copyright please contact us at:

repository@maastrichtuniversity.nl

providing details and we will investigate your claim.
}

Copyright and moral rights for the publications made accessible in the public portal are retained by the authors and/or other copyright owners and it is a condition of accessing publications that users recognise and abide by the legal requirements associated with these

- Users may download and print one copy of any publication from the public portal for the purpose of private study or research.

- You may not further distribute the material or use it for any profit-making activity or commercial gain

If the publication is distributed under the terms of Article $25 \mathrm{fa}$ of the Dutch Copyright Act, indicated by the "Taverne" license above, 


\title{
CHILDHOOD OVERWEIGHT
}

The influence of parenting on children's energy balance-related behavior

\author{
E.F.C. Sleddens
}




\section{Colophon}

Cover layout: Proefschriftmaken.nl / Uitgeverij BOXPress

Cover illustration: www.fotosearch.nl

Printed by: Proefschriftmaken.nl / Uitgeverij BOXPress

Published by: Uitgeverij BOXPress, 's-Hertogenbosch

ISBN: 978-90-8891-587-1

(C) E.F.C. Sleddens, Maastricht, the Netherlands, 2013

The research presented in this thesis was performed at the Department of Health Promotion at Maastricht University, the Netherlands. The work in this thesis was performed within the NUTRIM School for Nutrition, Toxicology and Metabolism, Maastricht University Medical Center+.

All rights are reserved. No part of this publication may be reproduced or transmitted in any form or by any means, without the written permission from the author or, were appropriate, the publisher of the article. 


\title{
CHILDHOOD OVERWEIGHT
}

\section{The influence of parenting on children's energy balance-related behavior}

\author{
Proefschrift \\ ter verkrijging van de graad van doctor \\ aan de Universiteit Maastricht, \\ op gezag van de Rector Magnificus, \\ Prof. dr. L.L.G. Soete \\ volgens het besluit van het College van Decanen, \\ in het openbaar te verdedigen \\ op vrijdag 5 april 2013 om 10.00 uur \\ door
}

Ester Francisca Catharina Sleddens

Geboren op 9 juni 1985

te Eindhoven, Nederland 


\section{Promotor}

Prof. dr. N.K. De Vries

\section{Copromotores}

Dr. S.P.J. Kremers

Dr. C.T.M.C.N. Thijs

\section{Beoordelingscommissie}

Prof. dr. H. De Vries (voorzitter)

Dr. P. van Assema

Prof. dr. A. Jansen

Em. Prof. dr. R.A. Knibbe

Prof. dr. L. Maes, Universiteit Gent

The research described in this thesis was supported by a grant of the Dutch Heart Foundation (DHF2008B112). Financial support by the Dutch Heart Foundation for the publication of this thesis is gratefully acknowledged. 


\section{Contents}

1. General introduction

\section{PART 1: Parenting reviews}

2. General parenting, childhood overweight and obesity-inducing behaviors: A review

3. Interventions addressing general parenting to prevent or treat childhood obesity

4. Physical activity parenting: A systematic review of existing questionnaires and their associations with child activity levels

\section{PART 2: Measurement}

\section{Child temperament}

5. Validating the Children's Behavior Questionnaire in Dutch children: Psychometric properties and a cross-cultural comparison of factor structures

6. The Children's Behavior Questionnaire very short form: Psychometric properties and validation of a one-item temperament scale

7. Measuring child temperament: Validation of a 3-item temperament scale and 13-item impulsivity scale

\section{Eating and feeding styles}

8. The Children's Eating Behavior Questionnaire: Factorial validity and association with Body Mass Index in Dutch children aged 6 to 7

9. Relationship between parental feeding styles and eating behaviors of Dutch children aged 6 to 7

\section{General parenting}

10. Issues in the measurement of parenting style

11. Development of the Comprehensive General Parenting Questionnaire for parents of 5 to 13 year olds

\section{PART 3: Parenting - child eating behavior relationship}

12. Food parenting practices and child dietary patterns: Prospective relations and the moderating role of general parenting, child temperament, and child eating style

13. General Discussion

References

Summary

Samenvatting

Dankwoord/Acknowledgment

Curriculum Vitea

Publication list 



\section{CHAPTER 1}

General introduction 


\section{The childhood overweight and obesity epidemic}

During the last decades, the prevalence of childhood overweight and obesity has dramatically increased in populations worldwide (De Onis, Blössner, \& Borghi, 2010; Ogden, Carroll, Kit, \& Flegal, 2012; Gupta, Goel, Shah, \& Misra, 2012; Wang \& Lobstein, 2006). In the Netherlands, from 1980 onwards, a steep incline in overweight rates in children has been detected (Schokker, Visscher, Nooyens, Van Baak, \& Seidell, 2006; Schönbeck et al., 2011; Van den Hurk, Van Dommelen, Van Buuren, Verkerk, \& HiraSing, 2007). Schönbeck et al. (2011), using data from the Dutch National Growth Studies in 1980, 1997, and 2009, reported that in 2009, $12.8 \%$ of the Dutch boys and $14.8 \%$ of the Dutch girls aged 2 to 21 years have been classified as overweight and $1.8 \%$ of the boys and $2.2 \%$ of the girls have been classified as obese. Compared to 1980 , this is a two to three fold higher prevalence in overweight and four to six fold increase in obesity.

The complex interplay of environmental, genetic, cultural and evolutionary factors of obesity is widely acknowledged (Heitmann et al., 2012). As our genome has remained largely unchanged for generations, lifestyle changes have caused the prevalence rates of overweight and obesity to rise. Obesity is the result of a chronic imbalance between energy intake (overconsumption of calories) and energy expenditure (low levels of physical activity and high levels of sedentary behavior such as television viewing and computer use) (Pérez-Escamilla et al., 2012; Te Velde et al., 2012). These behaviors are also referred to as 'energy balance-related behaviors' (EBRBs; Kremers, Visscher, Seidell, Van Mechelen, \& Brug, 2005). Both healthy and unhealthy behaviors have been shown to cluster (Boone-Heinonen, Gordon-Larsen, \& Adair, 2008; Gubbels, Kremers, Goldbohm, Stafleu, \& Thijs, 2012; Pearson \& Biddle, 2011) and track into later life (Biddle, Pearson, Ross, \& Braithwaite, 2010; Craigie, Lake, Kelly, Adamson, \& Mathers, 2011; Telama, 2009), thereby decreasing or increasing the likelihood for excessive weight gain to occur. Consequently, also childhood overweight is likely to persist into adulthood (Singh, Mulder, Twisk, Van Mechelen, \& Chinapaw, 2008).

Overweight and obesity have major health implications in childhood, as well as in the long run. The shortterm risks include medical problems such as elevated blood pressure, type 2 diabetes mellitus, abnormal blood lipids (increased LDL, decreased HDL, high triglyceride), sleep apnea, and reduced physical fitness, but also psychological problems such as low levels of quality of life and self-esteem, negative body image, depression and mental distress (Daniels, 2009; Must \& Strauss, 1999). These problems strongly increase the risk of developing cardiovascular diseases later in life, and subsequent adult morbidity and premature mortality (Reilly \& Kelly, 2011). Medical care costs attributable to childhood overweight and obesity are high. In a review by Withrow and Alter (2010) obesity was estimated to account for between $0.7 \%$ and $2.8 \%$ of a country's total healthcare expenditures.

Thus, childhood overweight and obesity is a dominant concern because of its dramatic increase, its persistence into adulthood, its association with a host of negative health outcomes and its burden on healthcare systems. 


\section{The broader context of childhood overweight and obesity}

A complex set of factors influence the development of energy imbalance causing excessive weight gain. The role the environment plays in shaping children's EBRB is pivotal, since children have less autonomy in choosing to perform these behaviors than adults. Environmental correlates of EBRBs include the neighborhood environment (e.g., recreation facilities, neighborhood safety) (De Vet, De Ridder, \& De Wit, 2011; Safron, Cislak, Gaspar, \& Luszczynska, 2011; Van der Horst et al., 2007), school environment (De Vet et al., 2011; Harrison \& Jones, 2012; Safron et al., 2011; Williams, Wyatt, Hurst, \& Williams, 2012), home environment (e.g., parental support, home facilities) (Patrick \& Nicklas, 2005; Van der Horst et al., 2007), interpersonal environment (e.g., peer influence, social norms) (De Vet et al., 2011), and societal/macro environment (e.g, climate, culture, location) (De Vet et al., 2011). These environmental factors are highlighted in so-called ecological models, in which different types and levels of environment are interrelated with factors at the individual level (Sallis \& Owen, 2002). Ecological theories view human development from an interactive contextual perspective. Some conceptual models incorporating different types and levels of environment relevant for child EBRBs adopted this ecological theory approach. These include for instance the 'Ecological model of predictors of childhood overweight' (Birch \& Davison, 2001), the 'Model of the home food environment pertaining to childhood obesity' (Rosenkranz \& Dzewaltowski, 2008), and the 'Environmental Research framework for weight Gain prevention') (EnRG; Kremers, De Bruijn, Visscher, Van Mechelen, De Vries, \& Brug, 2006). These models all have been informative in guiding research efforts towards unraveling the understanding of childhood overweight.

Although it is important to acknowledge the broader context (the influence of familial environment, the school environment, and the community and larger social environments), parents are key players in the development of obesity-inducing behaviors in young children. Parents are the gatekeepers of the home food supply and responsible for providing access to regular physical activity. Despite the increasing interest in the role of parents, the exact mechanisms of their influence remain to be uncovered.

\section{The role of parents and child characteristics}

Parents are utterly important in influencing children's health behaviors and subsequently their weight status. Through the use of parenting practices, defined as content-specific acts of parenting (Darling \& Steinberg, 1993) parents can have an influence on a wide range of health behaviors. In the feeding domain, for instance, previous reviews showed the relationship between parents' use of so-called 'food parenting practices' and their children's dietary intake and weight status (Clark, Goyder, Bissell, Blank, \& Peters, 2007; Faith, Scanlon, Birch, Francis, \& Sherry, 2004; Hurley, Cross, \& Hughes, 2011; Scaglioni, Salvioni, \& Galimberti, 2008; Thompson, 2010; Ventura \& Birch, 2008; Wardle \& Carnell, 2006). Most of the included studies linked highly controlling food parenting practices (e.g., restricting the type and amount of food a child can eat and using food as a reward) to children's dietary intake and weight status, failing to assess other practices such as encouragement of eating healthy foods and guidance. These studies are mainly 
cross-sectional and have yielded contradictory results. The literature on parenting in regard to physical activity and sedentary behaviors is less abundant, but faces the same problems. The majority of these studies fail to assess the larger context of parenting, so-called 'general parenting' styles or dimensions. For instance, Darling and Steinberg (1993) postulated that general parenting modifies the association between parenting practices and adolescent behavior. In more general terms, social ecological theory assumes the operation of higher order moderation processes (Wachs, 1999), implying that parenting and child factors at higher, more distal, levels can alter the impact of factors at a lower level. As such, a factor at a higher level forms the context in which proximal parenting processes operate. The parenting definitions for different levels are given in Box 1 below.

\section{Box 1. Parenting definitions and examples of specific parenting practices}

\section{General parenting}

Reflect parent-child interactions across a wide range of situations, provide the socio-emotional context in which specific parenting practices are processed and internalized by children, and reflect a philosophy of how children should be raised and the goals parents have for their children's development.

\section{Parenting practices}

Goal-directed behaviors or strategies parents use (praise, feedback, reward, punishment, reasoning, limit setting) to influence children's behaviors.

\section{Food parenting}

Limiting intake of snack foods, increasing home availability of fruits and vegetables.

\section{Physical activity parenting}

Encouraging a child to be physically active, using own behavior to encourage a child to be active.

\section{Sedentary (screen media) parenting}

Restricting a child's time engaged in screen media, allowing a child to watch television while eating.

Besides the contextual influence of general parenting, there is also an important influence of child characteristics, in particular temperament and eating style, both directly and in interaction with other ecological levels. Child temperament is a function of biological make-up influenced by interactions with the environment, including interactions with parenting. Previous studies show that general parenting and child temperament interacted in explaining child weight status; children with more difficult temperament and insensitive mothers had higher risks for being overweight or obese $(\mathrm{Wu}$, Dixon, Dalton, Tudiver, \& Liu, 2010; Zeller, Boles, \& Reiter-Purtill, 2008). Other studies found evidence for the moderating influence of child temperament (Gubbels et al., 2009; Kochanska, Aksan, \& Joy, 2007; Van Zeijl et al., 2007) and child eating style (Gubbels et al., 2011) in influencing the parenting - child behavior relationship.

Thus, interventions targeting parenting may benefit from being tailored to the home's emotional climate and the child's temperament and eating style. However, the literature remains inconclusive as to which parenting practices are associated with children's EBRBs and weight status, taking into account the context in which these proximal parenting processes operate. These problems are compounded by poor theoretical development (Kremers, 2010), inconsistent measurement, and relying on findings of cross-sectional studies. 
In Figure 1 the hypothesized model regarding the 'parenting - child weight relationship' is presented. The ultimate goal of this dissertation is to unravel the exact mechanisms of the influence of parenting on EBRBs and subsequent weight development in children. I will take into consideration the influence of additional demographic factors, including parental education level and child age and gender.

Figure 1. Model describing the 'parenting - child weight relationship'

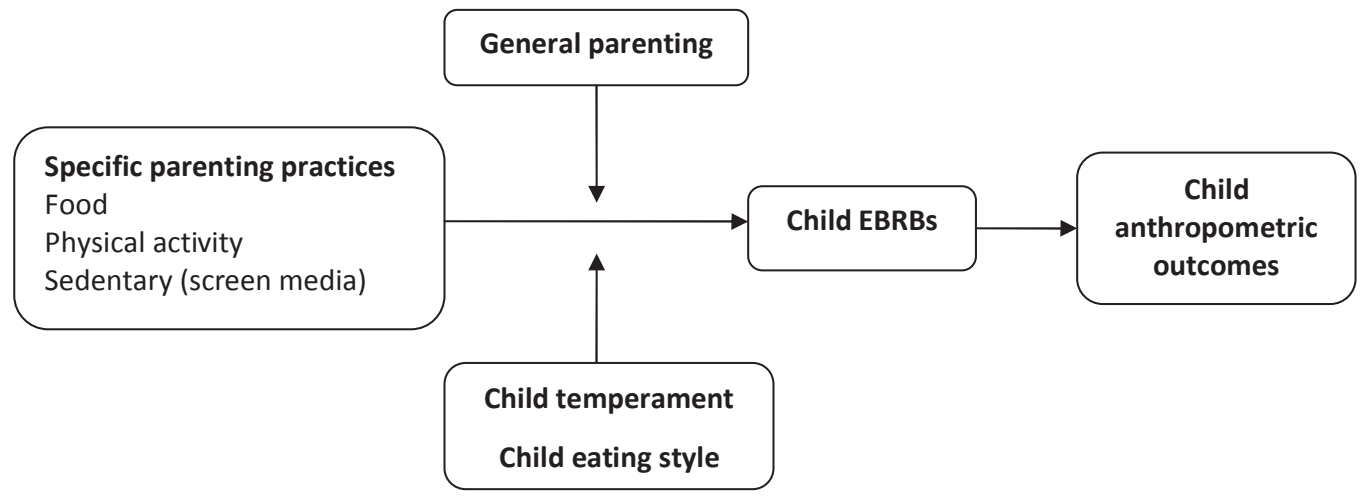

\section{Study objectives}

In this dissertation evidence regarding the influence of parenting on children's EBRBs and weight development is provided, following three steps.

First step, the following three reviews are performed:

- Review 1: Which studies examine relationships between general parenting and child EBRBs and weight status, and what are the findings?

- Review 2: Which intervention programs address general parenting in order to prevent or treat childhood obesity and are they effective?

- Review 3: What questionnaires exist regarding physical activity parenting? Are these measures validated?

Since reviews related to food parenting and sedentary (screen-media) parenting were simultaneously performed among other international research groups, the review presented in this dissertation focuses solely on physical activity parenting.

Second step, measures are developed and/or validated assessing child temperament, child eating styles, feeding styles and general parenting. 
Third step, the contextual role of general parenting and child characteristics (i.e., child temperament and child eating style) are examined, together with the strength of interrelationships between the variables and their contribution to child eating behavior, using the validated instruments in step 2 . These questionnaires were included in the ongoing KOALA Birth Cohort Study (Dutch acronym for 'Child, Parents, and health: Lifestyle and Genetic Constitution').

\section{Outline of the dissertation}

The current dissertation consists of three parts, i.e., parenting-related reviews, development and/or validation of several parent and child-related variables described in the parenting - child weight model, and testing the proposed model in a large longitudinal sample of Dutch children participating in the KOALA Birth Cohort Study. The outline of these parts and the specific chapters are described below.

\section{Parenting reviews}

Chapters 2 to 4 describe findings of systematic reviews about parenting in relation to child EBRBs. Chapter 2 summarizes existing literature about the relationships between general parenting and child EBRBs and weight-related outcomes. Chapter 3 provides an overview of existing interventions addressing general parenting in order to prevent or treat childhood obesity. Chapter 4 reviews existing questionnaires of parenting practices in regard to physical activity, their psychometric performance and the correlation with children's physical activity levels.

\section{Measurement}

Chapters 5 to 11 describe the development and/or validation of child temperament, eating and feeding styles, and general parenting questionnaires. These instruments will be administered to participants of the KOALA Birth Cohort Study to examine the hypothesized parenting model.

\section{Child temperament}

Chapter 5 focuses on validating several forms of the Children's Behavior Questionnaire (195 items and 36 items) using factor analytic procedures to see whether the three-factor temperament structure could be replicated. The global traits of temperament include surgency/extraversion, negative affectivity, and effortful control. Cross-cultural comparisons of temperament structure were also performed. Chapter 6 describes the development and validation of a one-item temperament scale for clinical use (e.g., for use in tailoring interventions such as the 'Kiddio: Food Fight' smart phone application game to help parents of preschool children use effective parenting practices related to eating vegetables; Baranowski et al., 2012), with three vignettes addressing the global temperament traits. The one-item measure was tested against 
the 36-item Children's Behavior Questionnaire in a sample of 3- to 5-year-olds in the United States. Chapter 7 describes the validation of a 3 -item temperament measure and 13-item impulsivity scale. First, the oneitem temperament measure described in chapter 6 was adapted. For each of the three vignettes, parents were asked to select how much it applied to their child. Then, this measure was tested against the 36-item Children's Behavior Questionnaire. Additionally, a child-report 13-item impulsivity questionnaire was tested for its applicability.

\section{Eating and feeding styles}

Chapter 8 describes the translation and validation of the Children's Eating Behavior Questionnaire in a Dutch sample of 6- to 7-year-olds. Factor analyses were performed and relationships between child eating style and BMI were investigated. Chapter 9 describes the translation and validation of the Parental Feeding Style Questionnaire in a Dutch sample of 6- to 7-year-olds. Psychometric evaluations, including factor analyses, were performed. Additionally, associations between parental feeding styles and dietary intake behaviors of both the parent and the child were assessed.

\section{General parenting}

Chapter 10 summarizes discussions about a range of issues regarding the assessment of parenting. These included: 1) general versus domain specific parenting styles and practices; 2) novel approaches to parenting measurement; 3) the role of ethnicity and culture; 4) assessing bidirectional influences; 5) broadening assessments beyond the immediate family; and 6) designing effective interventions. Chapter 11 describes the development and validation of the 'Comprehensive General Parenting Questionnaire'. The questionnaire measures five key aspects of parenting: nurturance, structure, behavioral control, coercive control, and overprotection. The survey was administered to large samples of parents of 5- to 13-year-old children in the Netherlands, Belgium and the United States. Advanced statistical techniques, including Confirmatory Factor Analysis and Item Response Modeling, were used to test the five-factor structure and to reduce the number of items.

\section{Parenting - child eating behavior relationship}

Chapter 12 describes the interplay between food-related parenting practices and child dietary patterns and the role of potential moderating factors (i.e., general parenting, child temperament, and child eating style). The hypothesized model (see Figure 1) is partially tested using longitudinal data from the KOALA Birth Cohort Study. First, the study examines the extent to which food parenting practices predict the development of child eating behavior. Second, the study tests the moderating role of both general parenting and child characteristics (i.e., temperament and eating style) on the relationship between food parenting practices and children's dietary patterns. 


\section{General discussion}

In the final chapter of this dissertation, chapter 13, the findings of the presented studies are integrated and discussed. Implications and recommendations are given for future research and practice, most importantly what considerations new measures must take into account and what approaches to use in the parenting overweight research field. 


\title{
CHAPTER 2
}

General parenting, childhood overweight and obesity-inducing behaviors:

A review

\author{
Ester FC Sleddens \\ Sanne MPL Gerards \\ Carel Thijs \\ Nanne K De Vries \\ Stef PJ Kremers
}

International Journal of Pediatric Obesity, 2011 


\begin{abstract}
Despite emerging efforts to investigate the influence parents have on their children's weight status and related dietary and activity behaviors, reviews regarding the role of general parenting are lacking. We performed a systematic review regarding the relationship between general parenting and these weightrelated outcomes to guide observational research. In total, 36 studies were included. Discrepancies across studies were found, which may be explained by differences in conceptualization of parenting constructs. Overall, however, results suggest that children raised in authoritative homes ate more healthily, were more physically active and had lower Body Mass Index (BMI) levels, compared to children who were raised with other styles (authoritarian, permissive/indulgent, uninvolved/neglectful). Findings of some moderation studies indicate that general parenting has a differential impact on children's weight-related outcomes, depending on child and parental characteristics. These findings underline the importance of acknowledging interactions between general parenting and both child and parent characteristics, as well as behaviorspecific parenting practices.
\end{abstract}




\section{Introduction}

There has been a dramatic increase in prevalence of childhood overweight and obesity over the last few decades (Wang \& Lobstein, 2006). So-called energy balance-related behaviors (Kremers, Visscher, Seidell, Van Mechelen, \& Brug, 2005) contributing to excessive weight gain include the consumption of energydense foods, sugar-sweetened beverages (e.g., Huaidong \& Feskens, 2010; Jebb, 2005) as well as low levels of physical activity and sedentary behaviors (e.g., Janssen et al., 2005; Malina \& Little, 2008). An area of emerging research focuses on the role of parents in the development of obesity-inducing health behaviors of their children. Many of these studies address the influence of parental feeding styles and specific parenting practices regarding food and/or activity (e.g., Brown, Ogden, Vogele, \& Gibson, 2008; Davison, Cutting, \& Birch, 2003; Hughes, Patrick, Power, Fisher, Anderson, \& Nicklas, 2007; Hughes, Power, Fisher, Mueller, \& Nicklas, 2005; Hughes, Shewchuk, Baskin, Nicklas, \& Qu, 2008; Joyce, Zimmer-Gembeck, 2009; Matheson, Robinson, Varady, \& Killen, 2006; Mitchell, Brennan, Hayes, \& Miles, 2009; Musher-Eizenman, De Lauzon-Guillain, Holub, Leporc, \& Charles, 2009; Patrick, Nicklas, Hughes, \& Morales, 2005). Specific parenting practices include, for example, house-rules regarding breakfast consumption, parental control of child snacking and television viewing time. Existing reviews mainly concentrate on these specific types of parental influences affecting children's weight-related health outcomes, (e.g., Faith, Scanlon, Birch, Francis, \& Sherry, 2004; Wardle \& Carnell, 2006). Numerous efforts to unravel the influence of general parenting on children's weight-related behaviors suggest that the causal pathways are likely to be complex (Enten \& Golan, 2008; Golan \& Crow, 2004; Kitzmann, Dalton, \& Buscemi, 2008; Rhee, 2008; Ventura \& Birch, 2008). For instance, the contextual influence of general parenting is assumed to moderate the association between parenting practices and children's health outcomes (Darling \& Steinberg, 1993). The concept of general parenting has been defined as a constellation of attitudes and beliefs that create an emotional climate and determines behavioral expression between parent and child (Darling \& Steinberg, 1993). General parenting in this paper is also referred to as parenting style or dimensions (of parental behavior). In addition to having a potential moderating influence, general parenting may also impact on children's weight status through its influence on various parenting practices with regard to diet and physical (in)activity. Figure 1 depicts the possible mediating and moderating pathways of the influence of parenting on child weight (general parenting - child weight relationship).

The main objective of this review is to synthesize evidence regarding the influence of general parenting on children's diet and activity behaviors, and weight status. To our knowledge, this is the first review to focus exclusively on the influence of general parenting. 
Figure 1. Conceptual model for the relationships between parenting and children's anthropometric-related outcomes

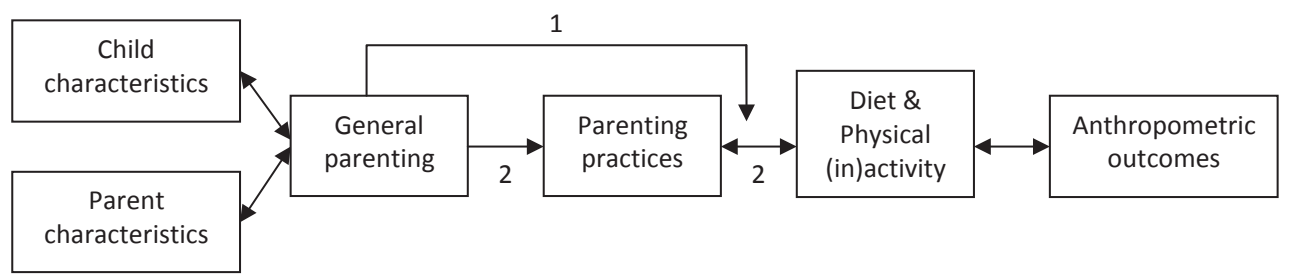

Note: Pathway 1 (moderation): interaction between general parenting and more specific parenting practices in predicting child diet \& physical (in)activity. Pathway 2 (mediation): influence of general parenting on child diet \& physical (in)activity mediated by effects of general parenting on more specific parenting practices. Child and parent characteristics may have an influence on all variables in the model.

\section{Background of parenting typologies}

The commonly used typological approach in parenting research is based on the work of Maccoby and Martin in 1983, who described parenting style as a function of two dimensions of parental behavior: the extent to which parents are (1) responsive to their children's needs (responsiveness) and (2) controlling of their children's behaviors (demandingness). These two dimensions of parenting consistently emerge from factor analytic approaches. 'Responsiveness' has also been referred to as parental warmth (Baumrind, 1967; Becker, 1964; Schaefer, 1959), involvement (Grolnick \& Slowiaczek, 1994), nurturance (Block, 1981), child-centeredness (Pulkkinen, 1982), acceptance (Baumrind, 1967; Rohner, 1986), and caring/empathy (Parker, Tupling, \& Brown, 1979). 'Demandingness', on the other hand, is often related to aspects of control such as behavioral control (Schaefer, 1959) and firm control (Baumrind, 1965), restrictiveness (Becker, 1964), and democracy (Baldwin, 1948). By crossing the dimensions of responsiveness and demandingness, four prototypes of parenting are created (see Table 1) (Maccoby \& Martin, 1983): authoritative (parents who are both responsive and demanding), authoritarian (parents who are less responsive but highly demanding), indulgent or permissive (parents who provide a high level of responsiveness but are less demanding), and neglectful or uninvolved (parents who show relatively low levels of both dimensions).

Table 1: Fourfold typology of parenting based on the two-dimensional classification of Maccoby \& Martin (1983)

\begin{tabular}{lcc} 
& & Responsiveness \\
\cline { 2 - 3 } Demandingness & High & Low \\
\hline High & Authoritative parenting & Authoritarian parenting \\
Low & Indulgent parenting & Neglectful parenting \\
\hline
\end{tabular}




\section{Method}

\section{Search strategy and eligibility criteria}

Comprehensive literature searches were conducted between September 2009 and February 2010 utilizing a range of electronic databases (PubMed, PsycINFO, Scopus) together with lateral searching techniques (reference tracking and author searching). We included studies reporting general parenting and at least one of the following child outcomes: weight status, dietary intake (behaviors), physical (in)activity. To specify, literature searches were performed using at least one of the following parenting-related keywords: parenting (style), (child) rearing, authoritative, authoritarian, permissive, indulgent, or neglectful; weightrelated keywords: physical (in)activity, sedentary behavior, sport(s), television, computer, eating, diet, fruit, vegetable, breakfast, snack(ing), (sugar-sweetened) beverages, (over)weight, obesity, or Body Mass Index (BMI); and age-related keywords: infant, preschool, child, or adolescent. Other inclusion criteria were as follows: all studies should be written in English and published in a scientific peer-reviewed journal or as a dissertation; and the study sample should consist of infants, preschoolers, children or adolescents (here defined as children with an age below 18 years at baseline). No selection criteria with regard to study methodology were formulated. Studies of children with eating disorders were excluded, as well as studies assessing the relationship between general parenting and child eating styles without dietary intake outcomes. We included every eligible study published until February 2010, including e-publications. Using the selected keywords, 2244 papers were identified in Pubmed, PsycINFO and Scopus. Thereafter, all papers were screened on title, leading to 546 eligible hits. Of these, 434 were eliminated based on abstract evaluation. Full-text manuscripts were retrieved for the remaining 112 papers. This resulted in 33 studies which were considered eligible for inclusion, the other 79 papers did not describe on general parenting. Furthermore, we applied reference tracking leading to three additional references. In total, 36 studies were considered eligible for the current review. Manuscripts were mainly excluded because they did not assess general parenting. Furthermore, prevention and intervention studies with regard to childhood overweight were excluded.

Two authors (ES and SG) independently screened all titles and abstracts of the manuscripts identified by the literature search for inclusion in this review. Full text versions of all potential relevant studies were obtained for further evaluation to determine inclusion, with any disagreement being resolved by discussion. In case of doubt, a third author (SK) was consulted. All studies selected for inclusion were scanned for additional references. Following this procedure, 36 publications were included in the review (Mendelson, White, \& Schliecker, 1995; Gable \& Lutz, 2000; Schmitz, Lytle, Philips, Murray, Birnbaum, \& Kubik, 2002; Kremers, Brug, De Vries, \& Engels, 2003; Lytle et al., 2003; Mustillo, Worthman, Erkanli, Keeler, Angold, \& Costello, 2003; Agras, Hammer, McNicholas, \& Kraemer, 2004; Brann \& Skinner, 2005; Chen \& Kennedy, 2004/2005, Chen, Kennedy, Yeh, \& Kools, 2005; Chen, Unnithan, Kennedy, \& Yeh, 2008; Ludrosky, 2005; Kim, 2006, Kim, McIntosh, Anding, Kubena, Reed, \& Moon, 2008; Musher-Eizenman \& Holub, 2006; Rhee, Lumeng, Appugliese, Kaciroti, \& Bradley, 2006; Gibson, Byrne, Davis, Blair, Jacoby, \& Zurbrick, 2007; Hejazi, 2007; Moens, Braet, \& Soetens, 2007; Van der Horst, Kremers, Ferreira, \& Singh, 2007; Wake, Nicholson, Hardy, \& Smith, 2007; West, 2007; Blissett \& Haycraft, 2008; Humenikova \& Gates, 2008; 
Reineke, 2008; Zeller, Boles, \& Reiter-Purtill, 2008; De Bourdeaudhuij, Te Velde, Maes, Pérez-Rodrigo, De Almeida, \& Brug, 2009; Lohaus, Vierhaus, \& Ball, 2009; Pearson, Atkin, Biddle, Gorely, \& Edwardson, 2010; Topham et al., 2010; Vereecken, Legiest, De Bourdeaudhuij, \& Maes, 2009; Berge, Wall, Bauer, \& NeumarkSztainer, 2010a; Berge, Wall, Loth, \& Neumark-Sztainer, 2010b; Hennessy, Hughes, Goldberg, Hyatt, \& Economos, 2010; Olvera \& Power, 2010). Figure 2 depicts the number of all studies published up till 2010 regarding the general parenting - child weight relationship. This figure shows that the number of studies examining this relationship has increased in recent years, from two studies before 2002 to about nine studies published in 2008 and 2009 together.

Figure 2. Number of publications examining the general parenting child weight relationship by year $(n=30)$

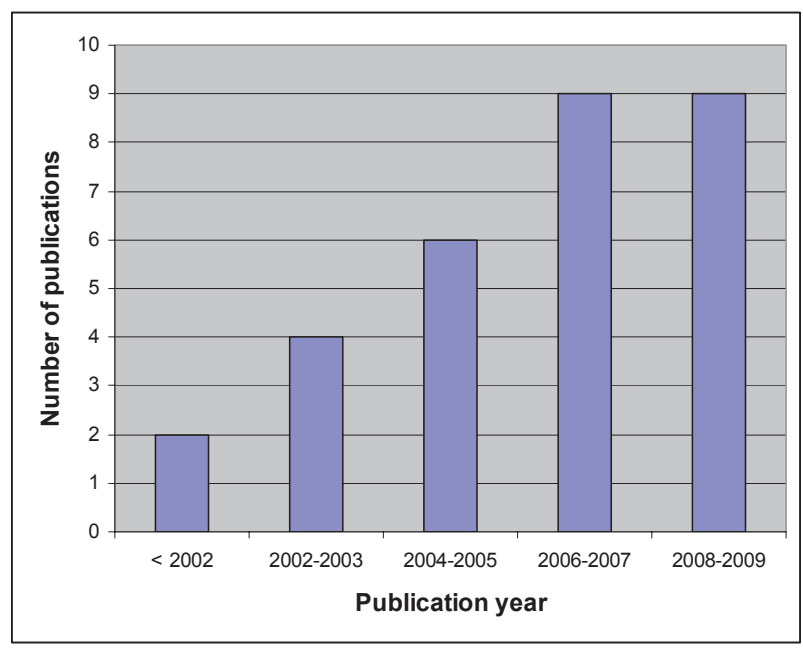

Legend: All studies regarding the general parenting - child weight relationship published in a scientific journal issue (no e-publications) before 2010 are included in this Figure. 


\section{Data extraction}

Data regarding sample characteristics (sample size, child age, gender, ethnicity, location and setting), measurements (overview of instruments assessing general parenting, child dietary and physical (in)activity behavior, child weight status), and study results were abstracted by the first author (ES) and checked by the second author (SG). Instruments measuring independent variables other than parenting styles, such as parenting practices, were only described when interaction was tested with general parenting in predicting children's weight-related outcomes. Studies assessing interaction could be valuable in understanding the complex mechanisms behind the general parenting - child weight relationship (see Figure 1). We report on results of studies with a-priori hypotheses about possible interaction and on results of post-hoc analyses (i.e., interaction patterns that were not specified at the beginning of the study). The results of the reviewed studies are presented in chronological order in the Supplement, available at the end of this chapter (page 40-57). For all studies, both statistically significant results (depicted with closed spheres) and nonsignificant results (depicted with open spheres) are reported to give a complete overview of the associations between all study variables.

\section{Results}

\section{Study characteristics of the included studies}

The sample size of the included studies ranged from 45 to over 4000, representing the absolute number of caregivers and/or children who participated in the study. Different study characteristics in terms of sample size, age, gender, ethnicity and location/setting of the study are depicted in Table 2. Most study populations consisted of North-Americans $(n=23)$, followed by Western Europeans $(n=9)$, Australians $(n=$ $3)$, Asians $(n=3)$, Southern Europeans $(n=2)$ and Eastern Europeans $(n=1)$. Samples from the United States (US) consisted of ethnically diverse populations, including participants with Hispanic, African and/or Asian backgrounds. 


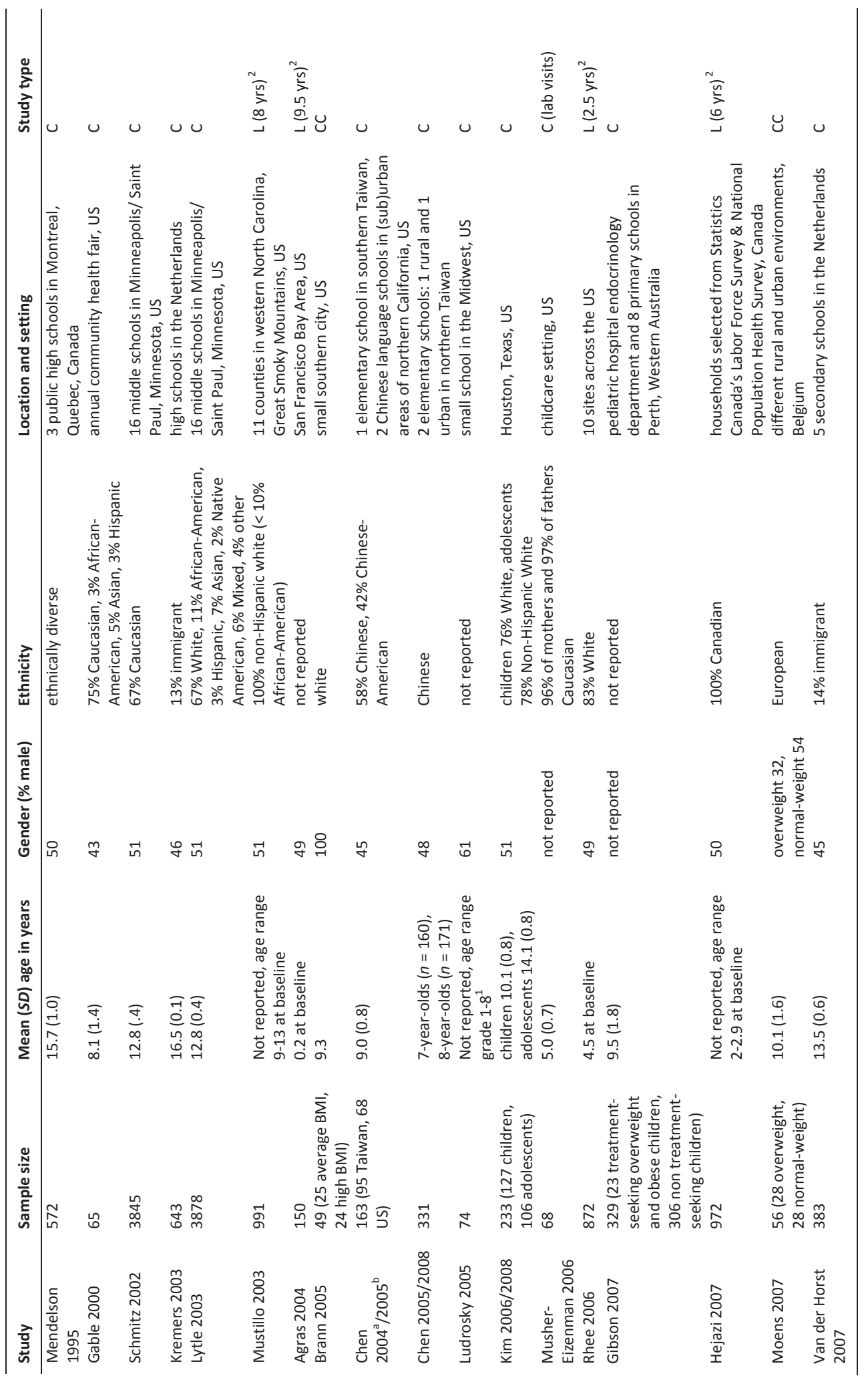




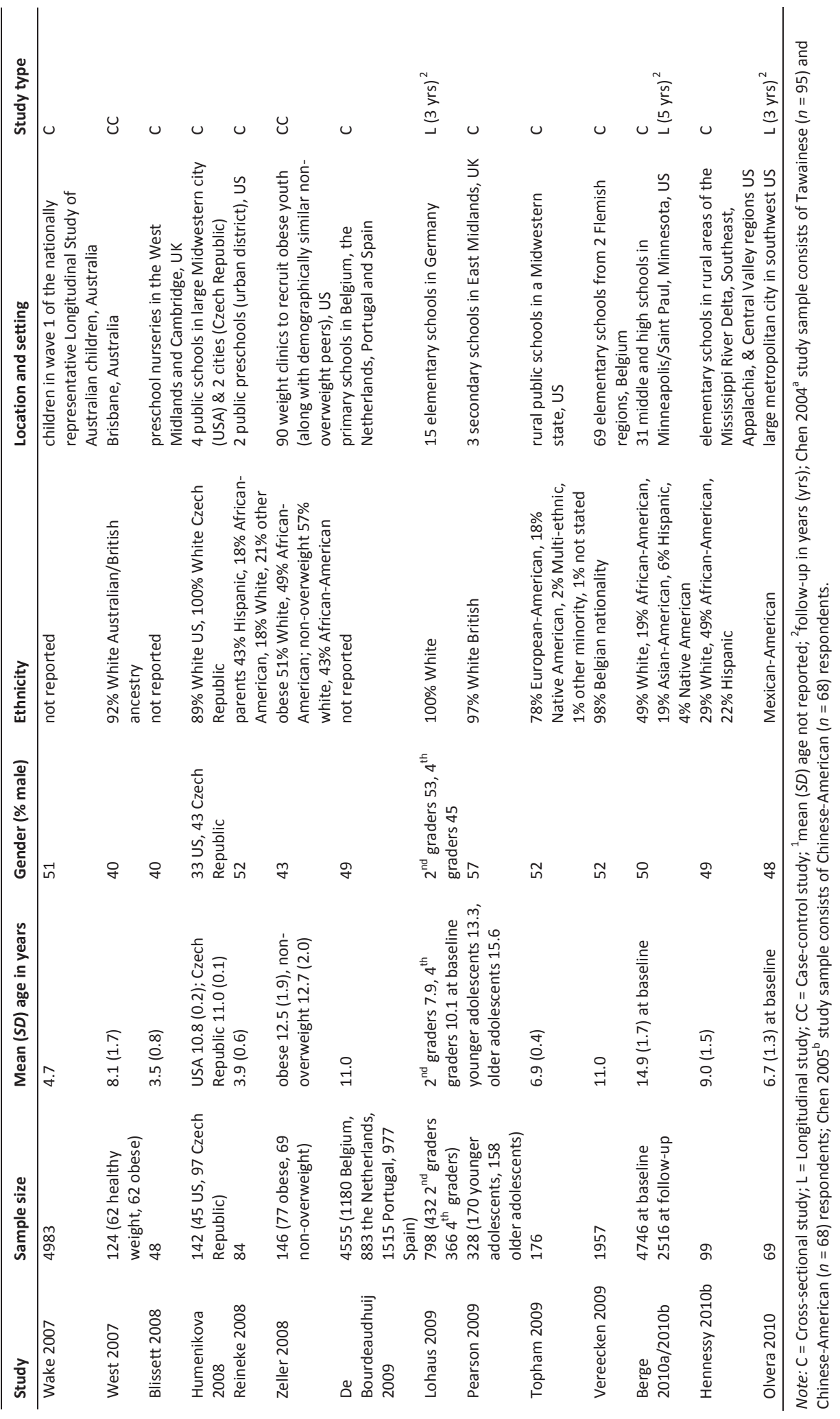




\section{Parenting measures}

In total, 21 different instruments were used to measure parenting dimensions or styles (see Supplement on page 40-57). All of these instruments have proven to be valid and reliable. The parenting tools which are used most often are the 'Child Rearing Practices Report' (Block, 1981) applied in four studies (Chen et al., 2005/2008; Gable \& Lutz, 2000; Ludrosky, 2005), and the 'Parenting Style Instrument' (Den Exter Blokland, Engels, \& Finkenauer, 2001; Lamborn, Mounts, Steinberg, \& Dornbusch, 1991; Steinberg, Elmen, \& Mounts, 1989) also used in four studies (De Bourdeaudhuij et al., 2009; Kremers et al., 2003; Pearson et al., 2010; Van der Horst et al., 2007). The 'Parenting Practices Questionnaire' (Robinson, Mandleco, Olsen, \& Hart, 1995 ) or its short form, the 'Parenting Styles and Dimensions Questionnaire' (Robinson, Mandleco, Olsen, \& Hart, 2001) were administered to participants in three studies $(43,59,66)$. Several parenting instruments were applied in two studies, i.e., the 'Authoritative Parenting Index' (Jackson, Bee-Gates, \& Henriksen, 1994) used by Schmitz et al. (2002) and Lytle et al. (2003); the 'Parental Authority Questionnaire' (Buri, 1991) used by Agras et al. (2004) or its revised version (Reitman, Rhode, Hupp, \& Altobello, 2002) used by Musher-Eizenman and Holub (2006); the 'Parenting Dimension Inventory' (Power, 1993) used by Olvera and Power (2010) and Hennessy et al. (2010); the 'Attitudes Toward Child-Rearing Scales' (Croake \& Hinkle, 1991) used by Chen and Kennedy (2004/2005); and the 'Parenting Scale' (Arnold, O'Leary, Wolff, \& Acker, 1993) used by Gibson et al. (2007) and West (2007). For an overview regarding reporting of general parenting and for a brief description of all parenting instruments used in the included studies, we refer to Table 3 and the Supplement on page 40-57, respectively.

\section{Findings per outcome variable}

The included studies were clustered by outcome variable: dietary behavior ( $n=14)$, physical (in)activity $(n=$ $10)$, and weight status $(n=29)$. Below, we give an overview of the key findings. Further study details are presented in Tables 3, 4 and the Supplement on page 40-57.

\section{Dietary behavior}

Eleven cross-sectional studies (De Bourdeaudhuij et al., 2009; Chen \& Kennedy, 2005; Kim, 2006; Kim et al., 2008; Kremers et al., 2003; Ludrosky, 2005; Lytle et al., 2003; Musher-Eizenman \& Holub, 2006; Pearson et al., 2010; Van der Horst, 2007; Vereecken et al., 2009) and three longitudinal studies (Agras et al., 2004; Berge et al., 2010b; Lohaus et al., 2009) measured the relationship between parenting and children's weight-related dietary behaviors (see Table 4a). 


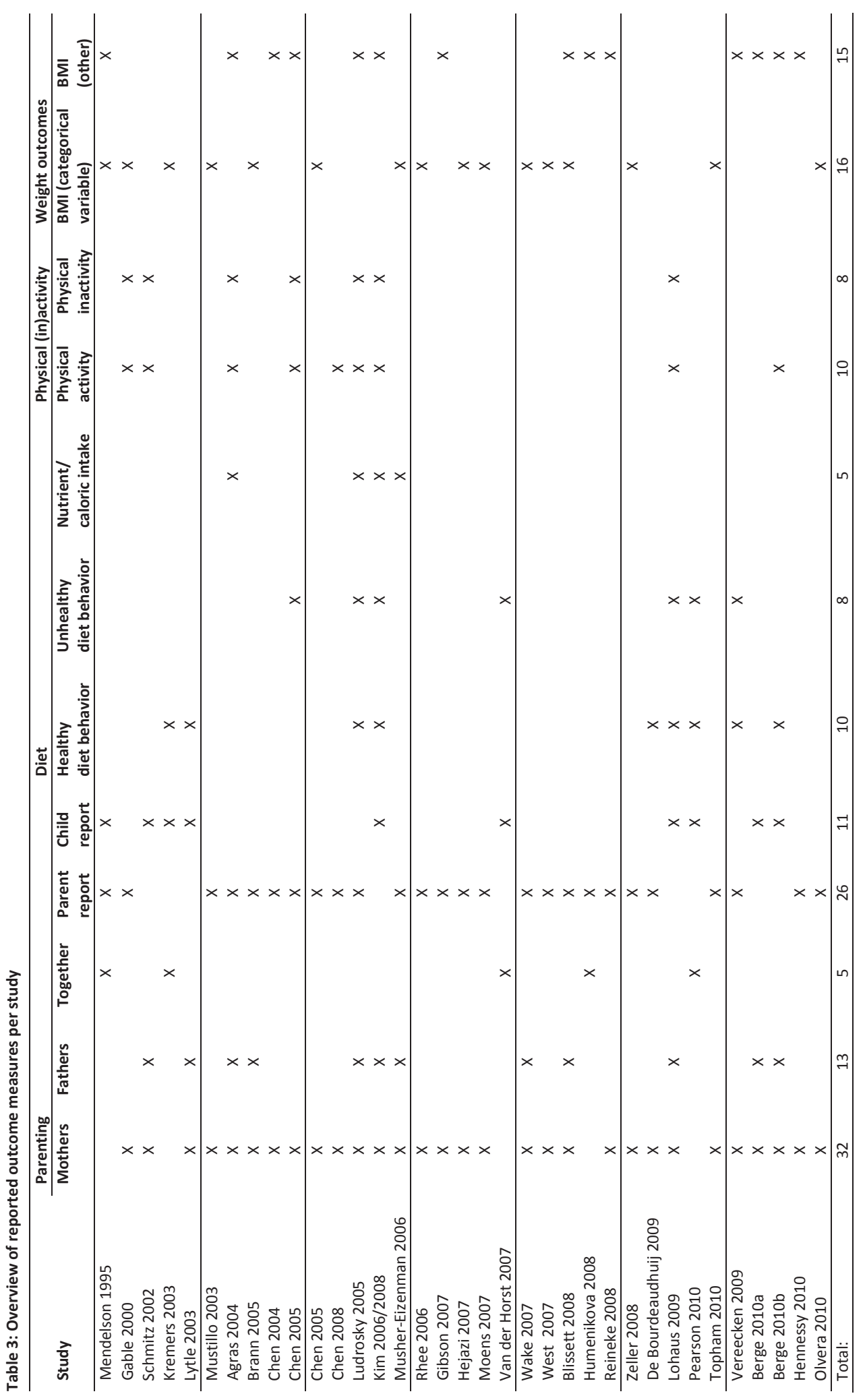




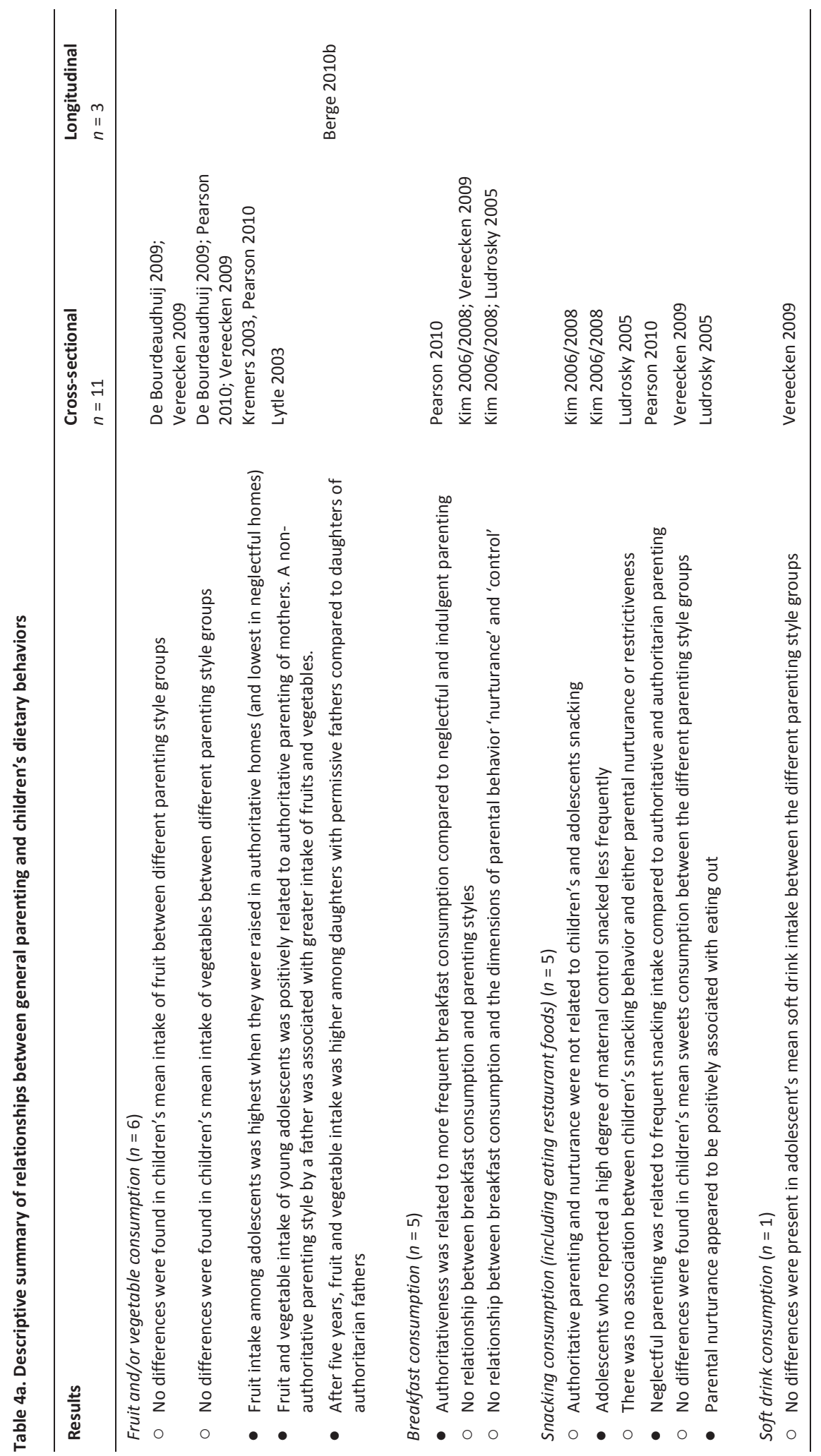




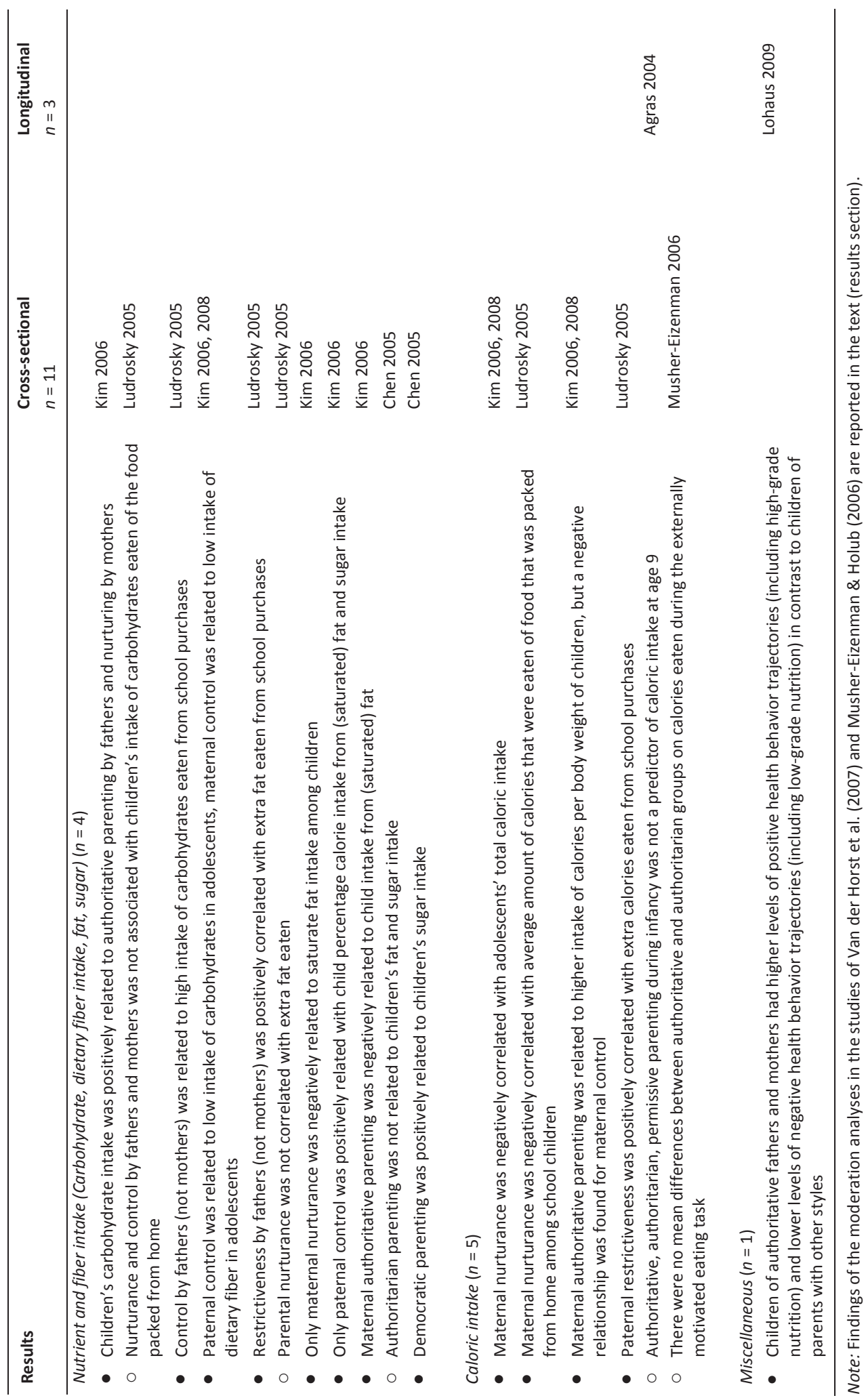


Fruit and/or vegetable intake. In two large scale cross-sectional studies (De Bourdeaudhuij et al., 2009; Vereecken et al. 2009), one including multiple countries (De Bourdeaudhuij et al., 2009), no relationships were found with parenting styles. Other studies found favorable effects of authoritative parenting on fruit intake (Kremers et al., 2003; Lytle et al., 2003, Pearson et al., 2010); in the study of Lytle et al. (2003) this was only true for mothers, and this positive relationship was also present for vegetable intake. Berge et al. (2010b), the only study using a longitudinal design, found different results; daughters of permissive fathers having higher intakes of fruit and vegetables five years later than those of authoritarian fathers.

Breakfast consumption. For the relationship between general parenting and breakfast consumption inconsistent results are reported. Pearson et al. (2010) indicated that authoritative parenting was related to more frequent breakfast consumption compared to neglectful and indulgent parenting. Contrary, other studies found no relationship of breakfast consumption with parenting styles (Kim, 2006; Kim et al., 2008; Vereecken et al., 2009) or the dimensions of parental behavior 'nurturance' and 'control' (Kim, 2006, Kim et al., 2008; Ludrosky, 2005).

Snacking and soft drink intake. Snacking was uncorrelated to most parenting styles and dimensions (Kim, 2006, Kim et al., 2008; Ludrosky, 2005). Only adolescents who reported a high degree of maternal control snacked less frequently (Kim, 2006, Kim et al., 2008). Neglectful parenting was related to frequent snacking compared to authoritative and authoritarian parenting (Pearson et al., 2010). Vereecken et al. (2009), who besides sweets consumption also assessed soft drink consumption, reported that no associations were present between these overweight-inducing behaviors and parenting styles as defined using the four-fold typology.

Van der Horst et al. (2007) executed moderation analyses, examining whether restrictive feeding practices have a different effect on adolescents' sugar-sweetened beverage consumption depending on the parenting style of their caregivers. Results indicated that the parenting dimensions of 'involvement' and 'strictness' modified the associations between restrictive feeding and sugar-sweetened beverage consumption, in a sense that controlling parenting practices had the strongest association with a decreased consumption of these drinks when parents were moderately controlling and highly involved.

Nutrient and fiber intake. Kim (2006) found that children's carbohydrate intake was positively related with authoritative parenting by fathers and nurturance by mothers. Inconsistent findings were found for controlling parenting; this was related either to high (Ludrosky, 2005) or low (Kim, 2006, Kim et al., 2008) intake of carbohydrates or fiber. For fat intake, there was a negative relationship with nurturing and authoritative parenting by mothers, whereas a positive relationship was found with fathers' controlling parenting (Kim, 2006, Kim et al., 2008; Ludrosky, 2005). In a study of Chinese-American children, Chen and Kennedy (2005) indicated that a positive association was found between democratic parenting and sugar intake. 
Caloric intake. In general, caloric intake was negatively correlated with maternal nurturance (Chen et al., 2008; Kim, 2006; Ludrosky, 2005), but positively with parental restrictiveness (Chen et al., 2008). In the longitudinal study of Agras et al. (2004) authoritative, authoritarian or permissive parenting during infancy was not significantly related to caloric intake of children at 9.5 years.

Musher-Eizenman and Holub (2006) conducted moderation analyses to find out whether parenting style would moderate the effects of restrictive feeding practices on children's caloric intake through externally motivated eating. The authors hypothesized that authoritarian parenting is related to high levels of caloric intake among children, whereas authoritative parenting is expected to attenuate the negative effects of restrictive feeding. In this small sample study, an external eating task was performed to assess eating in the absence of hunger and ultimately caloric intake. The results of this study showed that fathers with an authoritative parenting style who applied restrictive feeding practices had a protective effect on their child's caloric intake (i.e., associated with low caloric intake), whereas mothers with a authoritarian parenting style who applied these restrictive feeding practices had a counterproductive effect on caloric intake (i.e., associated with high caloric intake).

Positive and negative health behaviors. Results of a longitudinal study revealed that authoritative fathers and mothers had children with higher levels of positive health behavior trajectories (including high-grade nutrition such as fruit and vegetable consumption) and lower levels of negative health behavior trajectories (including low-grade nutrition) over a three-year period in contrast to children of parents with other styles (Lohaus et al., 2009). However, it should be noted that the positive health-related behavior measure used in this study also incorporated questions regarding physical activity, use of health care and personal hygiene. The negative health-related behavior measure also included statements regarding television viewing, nicotine and alcohol consumption, and risk behavior. 


\section{Physical (in)activity}

Seven cross-sectional studies (Chen \& Kennedy, 2005; Chen et al., 2008; Gable \& Lutz, 2000; Kim, 2006; Kim et al., 2008; Ludrosky, 2005; Schmitz et al., 2002) and three longitudinal studies (Berge et al., 2010b; Agras et al., 2004; Lohaus et al., 2009) examined associations between general parenting and children's physical (in)activity levels (see Table 4b). The cross-sectional studies reported inconsistent results regarding the parenting - physical activity relationship. In the studies assessing parental control no associations were revealed with children's and/or adolescent's physical (in)activity levels (Kim, 2006; Kim et al., 2008; Ludrosky, 2005). In some studies no associations were found between authoritarian parenting (Gable \& Lutz, 2000; Chen \& Kennedy, 2005), non-authoritative parenting (Kim, 2006; Kim et al., 2008), authoritative parenting (Gable \& Lutz, 2000), democratic parenting (Chen \& Kennedy, 2005) and child physical (in)activity. The more positive parenting variables (e.g., nurturance and authoritative parenting) were more often positively associated with activity levels (Kim, 2006; Kim et al., 2008; Ludrosky, 2005).

Two cross-sectional studies found that the relationship between general parenting and child activity was influenced by gender (Chen et al., 2008; Schmitz et al., 2002). However, mixed results were found. Chen et al. (2008) who conducted a study in Taiwan found that physical activity was positively associated with authoritative parenting in 7- and 8-year-old boys, but with authoritarian parenting in girls of the same age. Schmitz et al. (2002) found different results among a large group of young adolescents. Only for female adolescents, maternal authoritativeness was a significant positive predictor of physical activity and a negative predictor of sedentary leisure habits.

Findings of the longitudinal studies indicated that authoritative parenting was a positive predictor of physical activity (Berge et al., 2010b; Lohaus et al., 2009), a negative predictor of sedentary leisure-time activities (leisure-time behaviors which require very little energy, including television viewing) (Lohaus et al., 2009) or a non-significant predictor (Agras et al., 2004) of physical (in)activity (including television viewing and assessment of physical activity via accelerometry) at follow-up. Berge et al. (2010b) showed that only for adolescent sons, authoritative parenting by fathers predicted frequent physical activity at fiveyear follow-up in comparison with sons of neglectful fathers. 

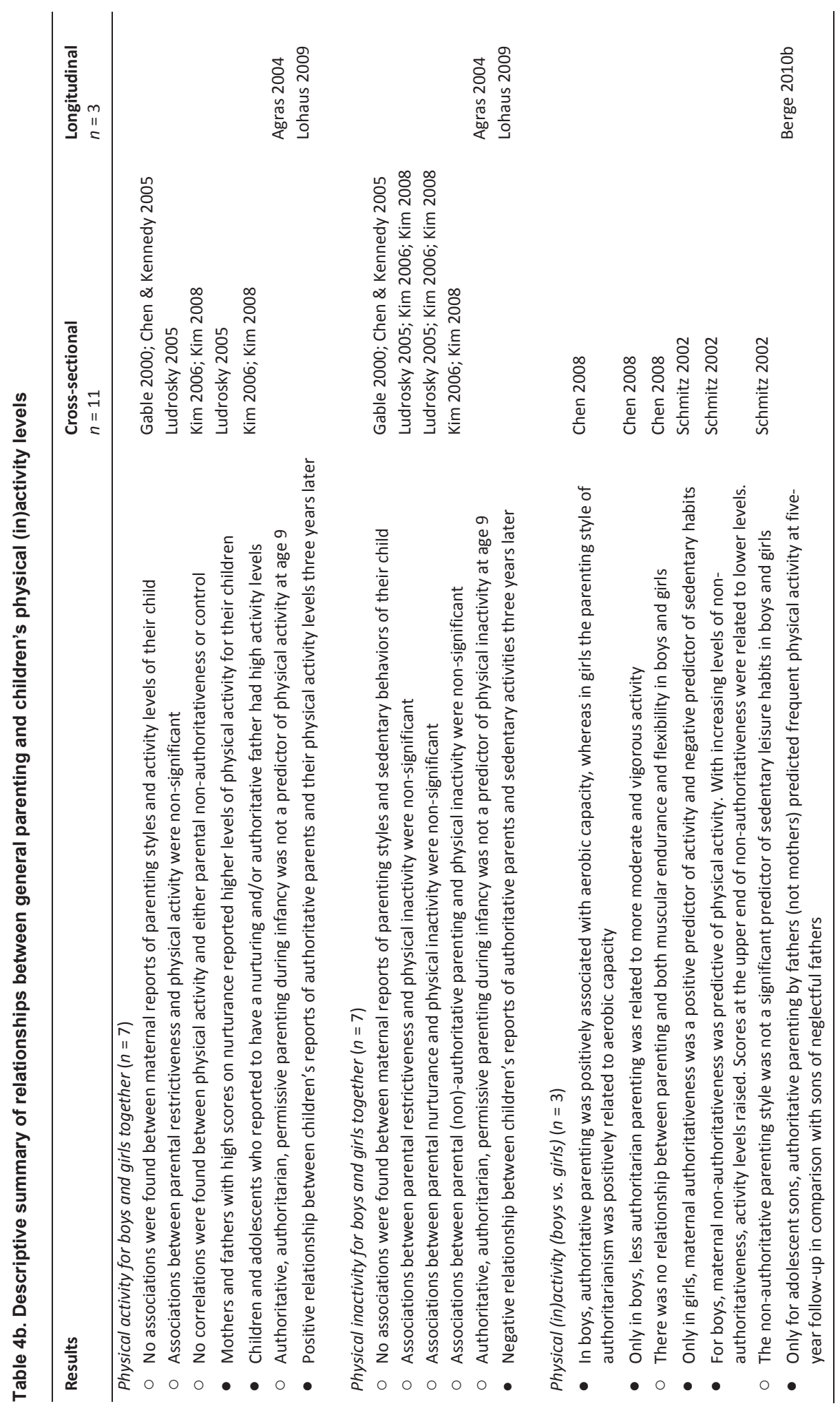


\section{$\underline{\text { Weight status }}$}

In total, 29 studies were identified which examined relationships between general parenting and a weightrelated outcome variable (see Table 4c). The majority of these studies used cross-sectional $(n=19)$ (Berge et al., 2010a; Blissett \& Haycraft, 2008; Chen \& Kennedy, 2004/2005; Chen et al., 2005; Gable \& Lutz, 2000; Gibson et al., 2007; Hennessy et al., 2010b; Humenikova \& Gates, 2008; Kim, 2006; Kim et al., 2008; Musher-Eizenman et al., 2006; Kremers et al., 2003; Ludrosky, 2005; Mendelson et al., 1995; Reineke, 2008; Topham et al., 2010; Vereecken et al., 2009; Wake et al., 2007) or case-control $(n=4)$ (Brann \& Skinner, 2005; Moens et al., 2007; West, 2007; Zeller et al., 2008) rather than a longitudinal design $(n=6)$ (Agras et al., 2004; Berge et al., 2010b; Hejazi, 2007; Mustillo et al., 2003; Olvera \& Power, 2010; Rhee et al., 2006).

Six cross-sectional studies found no significant effects of child weight status group (Blissett \& Haycraft, 2008; Brann \& Skinner, 2005; Chen et al., 2005; Gable \& Lutz, 2000; Mendelson et al., 1995; Moens et al., 2007) on general parenting variables. Some other cross-sectional studies found no mean differences in child BMI between various parenting style groups (Kremers et al., 2003; Musher-Eizenman \& Holub, 2006). However, two studies comparing parenting styles of mothers with obese and normal-weight children did report significant results (West, 2007; Zeller et al., 2008), indicating mothers of obese children scoring higher on 'laxness' and 'overreactivity' (West, 2007) and lower on 'behavioral control' (Zeller et al., 2008).

Many cross-sectional studies reported some non-significant findings regarding associations between particular parenting dimensions or styles and children's BMI (Berge et al., 2010a; Blissett \& Haycraft, 2008; Chen \& Kennedy, 2004/2005; Gibson et al., 2007; Hennessy et al., 2010b; Humenikova \& Gates, 2008; Kim, 2006; Kim et al., 2008; Ludrosky, 2005; Reineke, 2008; Vereecken et al., 2009; Wake et al., 2007). Across studies which found significant relationships, inconsistent findings were reported. Some studies found that authoritative parenting was associated with lower BMI values (Kim, 2006; Ludrosky, 2005; Van der Horst, 2007; Zeller et al., 2008). In contrast, Humenikova and Gates (2008) found that less authoritative parenting was related with lower BMI z-scores in Czech children. Permissive parenting in US children (Humenikova \& Gates, 2008) and democratic parenting in both Taiwanese and Chinese-American children (Chen \& Kennedy, 2004/2005) were positively related to children's BMI z-scores. Other studies found that parenting control of mothers (Kim, 2006; Kim et al., 2008) and fathers (Ludrosky, 2005) (e.g., including forms of authoritarian and psychological control) was positively related to BMI z-score. However, Wake et al. (2007) reported an inverse relationship between paternal control (some aspects of behavioral control) and child BMI.

It seems that relationships which were found depended on characteristics of the outcome variable. In general, no association between general parenting and weight status was found when a categorical variable was used; more often, significant relationships were found when BMI was used as a continuous outcome variable.

Six longitudinal studies were identified assessing whether general parenting predicts weight status at follow-up or weight status development among children (Agras et al., 2004; Berge et al., 2010b; Hejazi, 
2007; Mustillo et al., 2003; Olvera \& Power, 2010; Rhee et al., 2006). Three of these studies found positive effects of authoritative parenting on children's weight status (i.e., authoritative parenting was related with lower weight at follow-up) (Berge et al., 2010b; Olvera \& Power, 2010; Rhee et al., 2006). One study relating parenting styles to child weight status, failed to detect significant effects (Agras et al., 2004). In two studies, children's weight and height were repeatedly measured to define various developmental BMI trajectories (Hejazi, 2007; Mustillo et al., 2003). The aim of these studies was to examine whether there were any differences in parenting dimensions between these defined groups. Only one study reported significant differences (Hejazi, 2007).

A minority of existing (cross-sectional) studies focused on assessing interaction between variables (i.e., parenting styles, parent or child characteristics) in predicting children's weight status (Berge et al., 2010b; Topham et al., 2010; Zeller et al., 2008). Zeller et al. (2008) tested whether parenting interacted with child temperament in predicting child weight status. Findings showed that interaction was present between low maternal warmth and difficult child temperament, indicating that $69 \%$ of obese youth were classified as being high on difficult temperament and low on maternal warmth as compared to $31 \%$ of non-overweight youth (Zeller et al., 2008). Topham et al. (2010) assessed the role of other potential moderating factors (i.e., Socio-Economic Status (SES) and maternal depression) on the relationship between general parenting style (authoritative, authoritarian, permissive) and child obesity. They hypothesized that maternal depression and high SES would aggravate the impact of authoritarian parenting as well as permissive parenting on child obesity. Findings indicated that only for permissive parenting there was an interaction with maternal depression and SES. Both depressed mothers and high SES mothers had children who are more likely to be obese when they had permissive parenting styles. Finally, Berge et al. (2010a) found in a large ethnically diverse group of US teens, who reported about the parenting styles of their parents, that the combination of maternal authoritarian parenting and paternal neglectful parenting was related with a high BMI in sons, but not in daughters. 


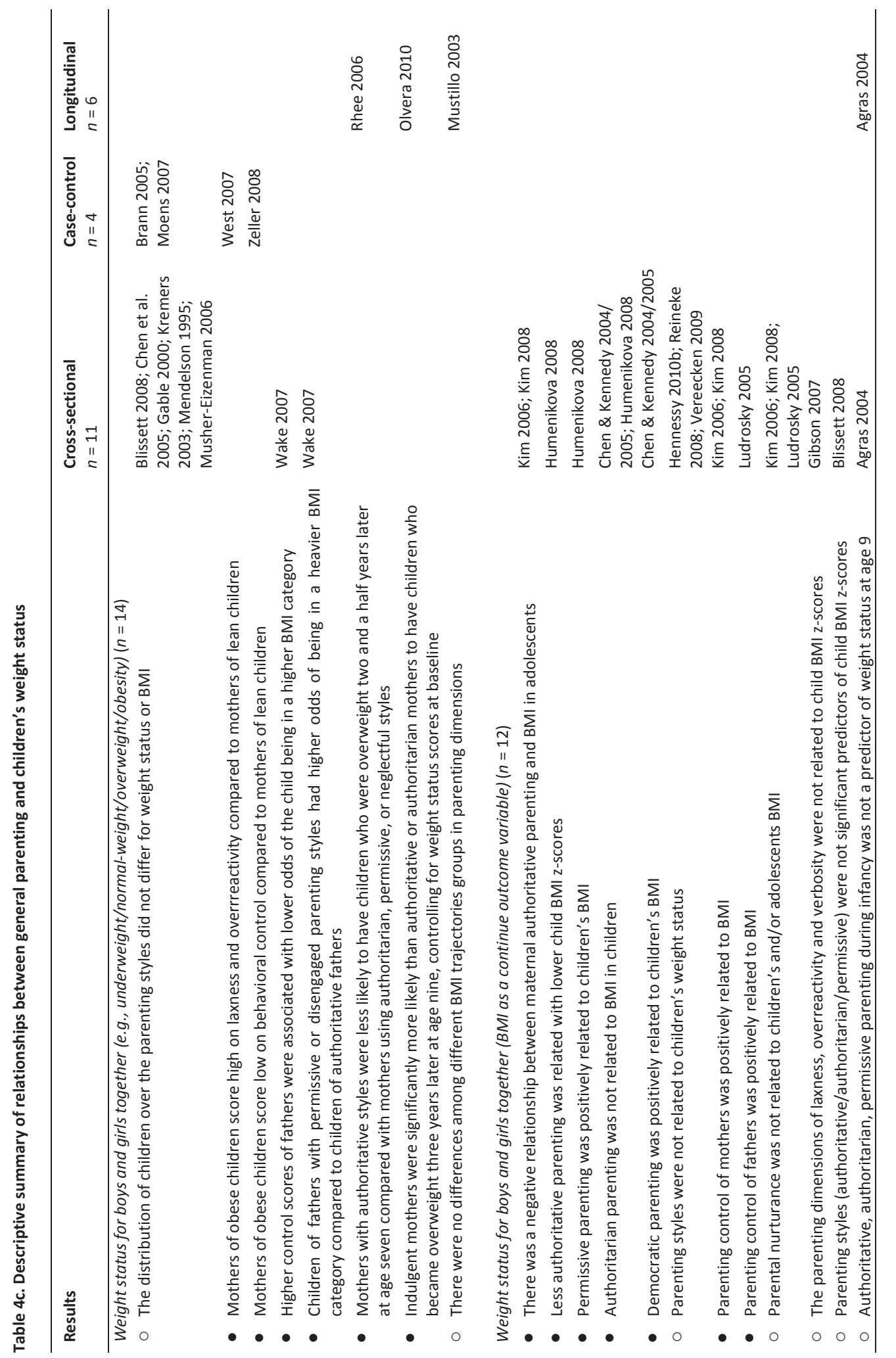




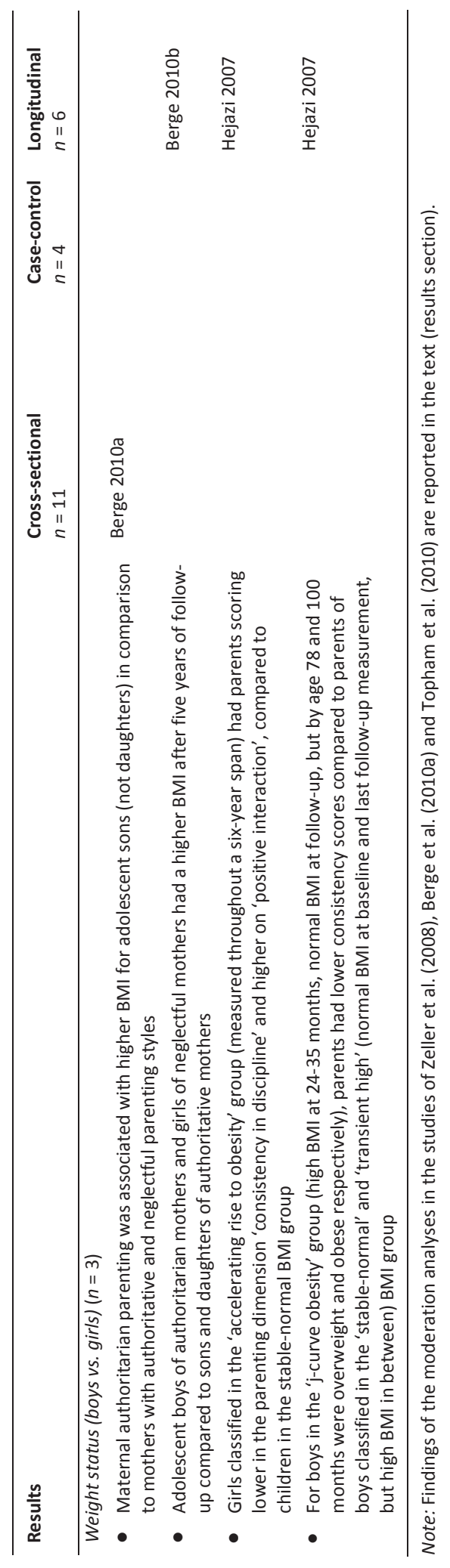




\section{Discussion}

The objective of the present review was to evaluate the existing literature examining the relationship between general parenting and children's weight-related outcomes. Some results were inconsistent. Generally, in many studies where a significant association with general parenting was found, children raised in authoritative homes were found to eat more healthily, to be more physically active, and to have lower BMI scores compared to children who were raised with a different style. An authoritative style is characterized by a family context of expressing warmth and emotional support, together with using clear, bidirectional communication (Maccoby \& Martin, 1983). These results are strengthened by prospective findings which inform us about the direction of causality. Five of the seven longitudinal studies show that general parenting at an early age has an impact on weight-related outcomes at a later date (Berge et al., 2010b; Hejazi, 2007; Lohaus et al., 2009; Olvera \& Power, 2010; Rhee et al., 2006). Furthermore, the findings of some moderation studies indicate that general parenting can have differential impact on children's weight status and related health behaviors, depending on characteristics of the child and the parents.

\section{Discrepancies in study results}

The parenting dimension 'nurturance' was typically positively related to overweight preventing behaviors of the child (e.g., high levels of physical activity) and negatively related to the child's obesity-inducing behaviors (e.g., fat and caloric intake). For the dimension 'control' inconsistent findings were reported. Probably this is caused by different conceptualizations of controlling parenting. This dimension has been referred to as psychological control versus psychological autonomy, but also as lax control versus behavioral/firm control. Psychological control (opposite of psychological autonomy) was assessed in few of the included studies (Kim, 2006; Kim et al., 2008; Lohaus et al., 2009; Zeller et al., 2008). It is defined as 'parental behaviors (such as guilt-induction, love withdrawal or contingent love, instilling anxiety, and invalidation of the child's perspective) that are intrusive and manipulative of children's thoughts, feelings, and attachments to parents' (Barber \& Harmon, 2002). This construct was related to negative behavioral outcomes (e.g., high intake of calories and fat) (Kim, 2006; Kim et al., 2008). Although this type of control by mothers was related to BMI z-scores of the children in the study by Kim (2006/2008), there was no difference between obese and non-overweight youth in reported psychological control in the study of Zeller and others (2008). Using another operationalization, Lohaus et al. (2009) created the four prototypes of parenting based on three dimensions of parental behavior; besides 'warmth' and 'behavioral control' incorporating 'psychological pressure' in the classification of parenting typologies. Authoritative parenting was characterized by high levels of behavioral control and low levels of psychological pressure and related to the most positive health outcomes (Topham et al., 2010). Very closely related to the construct of psychological control is the restrictiveness dimension assessed in the Child Rearing Practices Report (Block, 1981). Ludrosky (2005) found that paternal restrictiveness was positively related to children's BMI and caloric intake. Lax control, defined as inconsistent discipline (chaos) has been assessed in some studies (Gibson et al., 2007; Hejazi, 2007; Mustillo et al., 2003; West, 2007; Zeller et al., 2008), usually indicating 
higher scores on inconsistent discipline of parents in the obese group compared to parents of nonoverweight children (West, 2007; Zeller et al., 2008) and for parents of children with rapid excessive weight gain (Hejazi, 2007).

Besides the repeated findings for the negative influence of psychological and lax control on children's health behaviors, positive effects were reported for behavioral control e.g., (Lohaus et al., 2009; Wake et al., 2007; Zeller et al., 2008). However, controversies exist regarding the optimal level of control. For instance, Van der Horst et al. (2007) found evidence that general parenting modified the relationship between restrictive feeding practices and adolescents' sugar-sweetened beverage consumption: restrictive feeding was associated with lower intake of sugar-sweetened beverages when parents were highly involved, but moderately controlling (Van der Horst et al., 2007). Such results could indicate that both low control and very strict, overcontrolling types of parenting are counterproductive, indicating a U-shaped relationship between parental control and child weight. Despite the availability of a large number of parenting instruments, measurement tools assessing the apparent broad range of controlling dimensions are currently lacking. Therefore, one should be very cautious in comparing and interpreting the study results of the included studies.

Differences in conceptualization of parenting constructs may also explain other inconsistent findings regarding the relationship between general parenting and children's weight-related outcomes. In some studies instruments were used that can assess parenting style without crossing scores on separate parenting dimensions (Agras et al., 2004; Blissett \& Haycraft, 2008; Brann \& Skinner, 2005; Chen \& Kennedy, 2004/2005; Chen et al., 2005/2008; Gable \& Lutz, 2000; Humenikova \& Gates, 2008; Lytle et al., 2003; Mendelson et al., 1995; Musher-Eizenman \& Holub, 2006; Reineke, 2008; Schmitz et al., 2002; Topham et al., 2010). In other studies parenting styles were constructed based on the scores on separate parenting dimensions (Berge et al., 2010a/b; De Bourdeaudhuij et al., 2009; Hennessy et al., 2010b; Kim, 2006; Kim et al., 2008; Kremers et al., 2003; Lohaus et al., 2009; Olvera \& Power, 2010; Pearson et al., 2010; Rhee et al., 2006; Vereecken et al., 2009; Wake et al., 2007). Typically, parenting styles were categorized into four prototypes (authoritative, authoritarian, indulgent/permissive, and neglectful/uninvolved) based on splits of two parenting dimensions (e.g., nurturance/warmth and amount of control, involvement and strictness, sensitive to child's need and expectations for self-control, responsiveness and demandingness). In six of these studies (De Bourdeaudhuij et al., 2009; Hennessy et al., 2010b; Olvera \& Power, 2010; Pearson et al., 2010; Rhee et al., 2006; Vereecken et al., 2009) this categorization was based on median splits of both dimensions, a method which has to be applied carefully. For instance, if all parents of a study sample score very low on authoritarian control, using median splits, it is possible that parents scoring in the higher end on this dimension are classified as being authoritarian, whereas these parents would not be classified as being authoritarian in a different sample. Furthermore, when using median splits to define parenting styles, it is not possible to compare the study results with other studies, since scores on various parenting dimensions may differ across samples. 
Only in one study the categorization into prototypes of parenting was based on three dimensions (warmth, control, psychological pressure) (Lohaus et al., 2009). Of 13 studies that assessed separate parenting dimensions, ten studies only reported on relationships between parenting styles and children's weightrelated outcomes (Berge et al., 2010a/b; De Bourdeaudhuij et al., 2009; Hennessy et al., 2010b; Kremers et al., 2003; Lohaus et al., 2009; Olvera \& Power, 2010; Pearson et al., 2010; Rhee et al., 2006; Vereecken et al., 2009), rather than also assessing relationships with separate parenting dimensions. In doing so, important information could be lost. For instance, parents scoring moderately on both dimensions could be falsely categorized into a parenting style. Additionally, some studies (e.g., Gibson et al., 2007; West, 2007; Zeller et al., 2008) assessed parenting constructs as being bipolar (i.e., parents scoring high on a parenting construct are expected to score low on its opposite). Recently, however, Skinner et al. (Skinner, Johnson, \& Snyder, 2005) provided empirical support for the multidimensionality of parenting constructs rather than treating those constructs as being bipolar; parents scoring high on one parenting dimension (e.g., acceptance) do not necessarily score low on its conceptual opposite (i.e., rejection). Future researchers should take into account this multidimensionality.

Study results could differ according to the person completing the parenting instruments. The current review revealed that this could be done by both parents separately, one of the parents, or the child. Especially parental self-reporting could be a limitation of some of the studies, which may be biased because of social desirability. This may also decrease comparability with other studies which measure general parenting constructs via children or adolescents, although these constructs are also measured via questionnaires and thus subjective measures. Differences in the relationship between general parenting and children's weight-related outcomes were found for mothers and fathers (e.g., Lytle et al., 2003; Schmitz et al., 2002; Kim, 2006; Kim et al., 2008; Ludrosky, 2005; Lohaus et al., 2009). It also seems that differences among the samples (e.g., sample size, child gender, age and ethnicity) may explain some of the inconsistencies. Study findings should be interpreted with caution as the variables (i.e., general parenting and children's weight-related outcomes) are all measured in different ways. However, most outcome variables were measured with validated instruments. It was not possible to calculate power and effect sizes of each study because of the heterogeneity of measurements across the studies and lacking information (e.g., regarding distribution of independent and outcome variables) in many studies.

Furthermore, several variables (e.g., socio-economic status, maternal depression, child temperament) have been found to interact with certain parenting styles or dimensions in predicting children's weight status. Two studies examined the role of general parenting as a contextual factor that can influence the effectiveness of food-related parenting practices in predicting children's dietary intake behaviors (MusherEizenman \& Holub, 2006; Van der Horst, 2007). In line with this, we came across studies assessing parentchild interactions in relation to children's weight status in a broader context, e.g., quality of a child's environment (Beck \& Terry, 1985; Strauss \& Knight, 1999; Turner, Rose, \& Cooper, 2005; Lissau \& Sørensen, 1994; Zeller et al., 2007), parental stress (Stenhammar et al., 2010), attachment (Stenhammar et al., 2010; Trombini, Baldaro, Bertaccini, Mattei, Montebarocci, \& Rossi, 2003). Since we were specifically interested in parenting style (dimensions), these studies were not included in the present review. 


\section{Recommendations for future research}

Additional research is needed to further study the influence of mediating and moderating factors influencing the general parenting - child weight relationship, preferably employing a longitudinal design with more extended follow-up periods to establish causation. The proposed conceptual model (see Figure 1) could be used in order to guide determinant research, thereby enabling better understanding of the general parenting - child weight relationship. There is a need to conduct determinant studies using diverse ethnic samples and age groups. Moreover, larger samples of fathers should be included to allow for comparisons between mothers and fathers.

Increasingly, intervention studies address general parenting in the prevention and treatment of childhood overweight and obesity (Gerards, Sleddens, Dagnelie, De Vries, \& Kremers, 2011). We recommend intervention developers to increase their attention to the family context as it is an important factor influencing outcomes of overweight and obesity interventions for children. The primary goal of these types of interventions should be to create authoritative environments characterized by parental encouragement of instrumental competence in children by helping them in balancing other-oriented, rule-following tendencies with individualistic, autonomous active thinking (Baumrind, 1978; Newman, Harrison, Dashiff, \& Davies, 2008; Spera, 2005). 


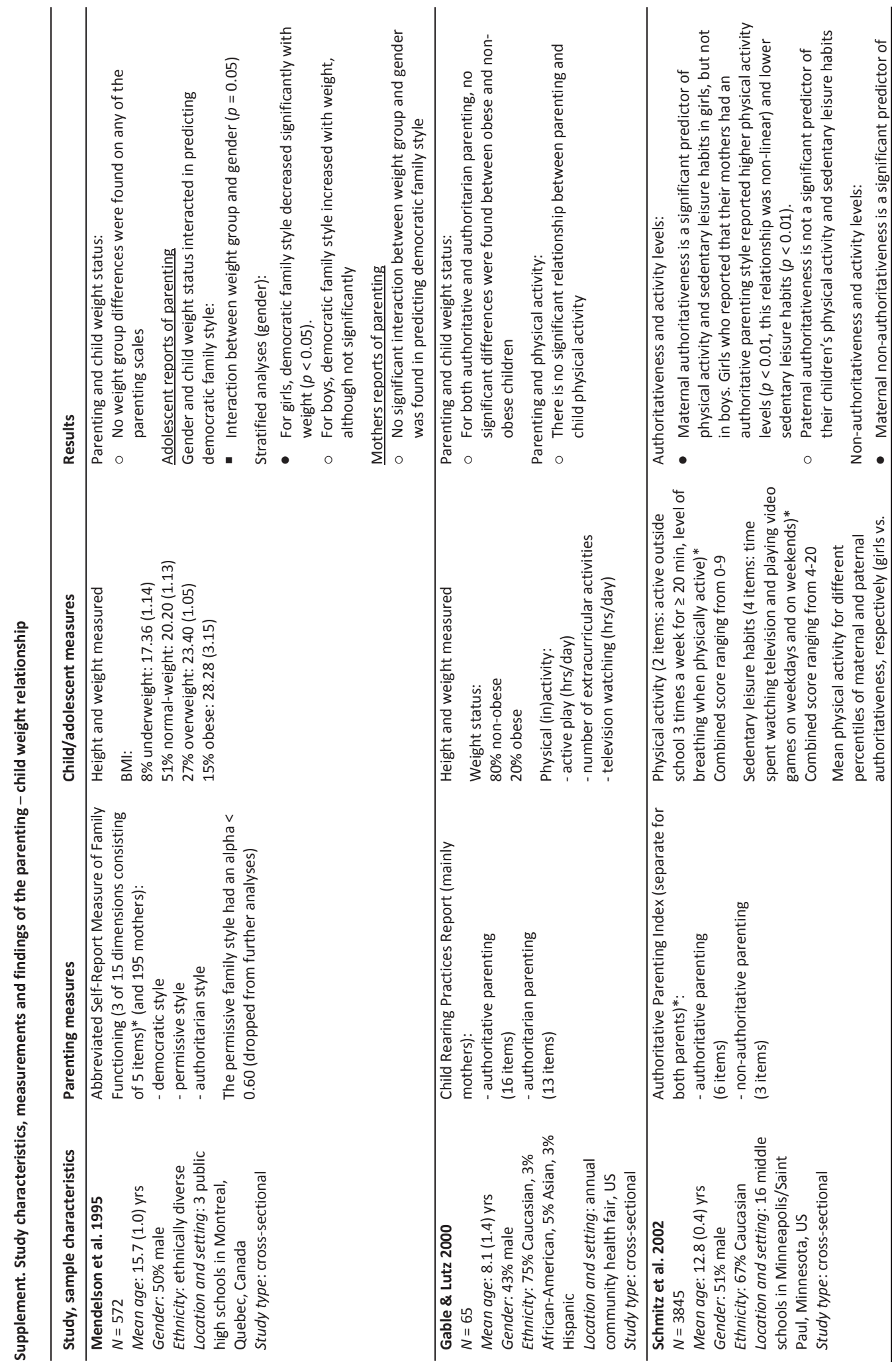




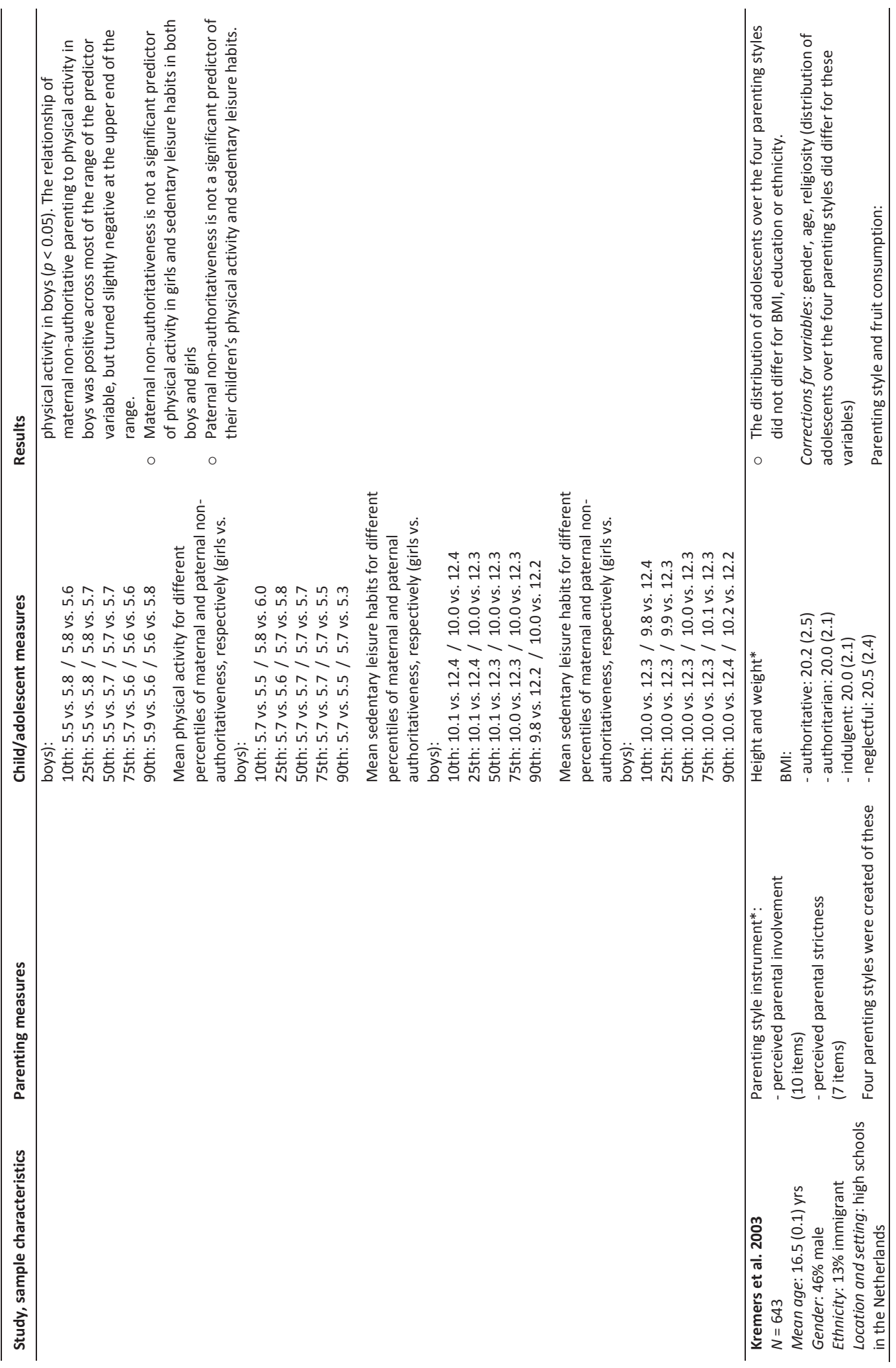




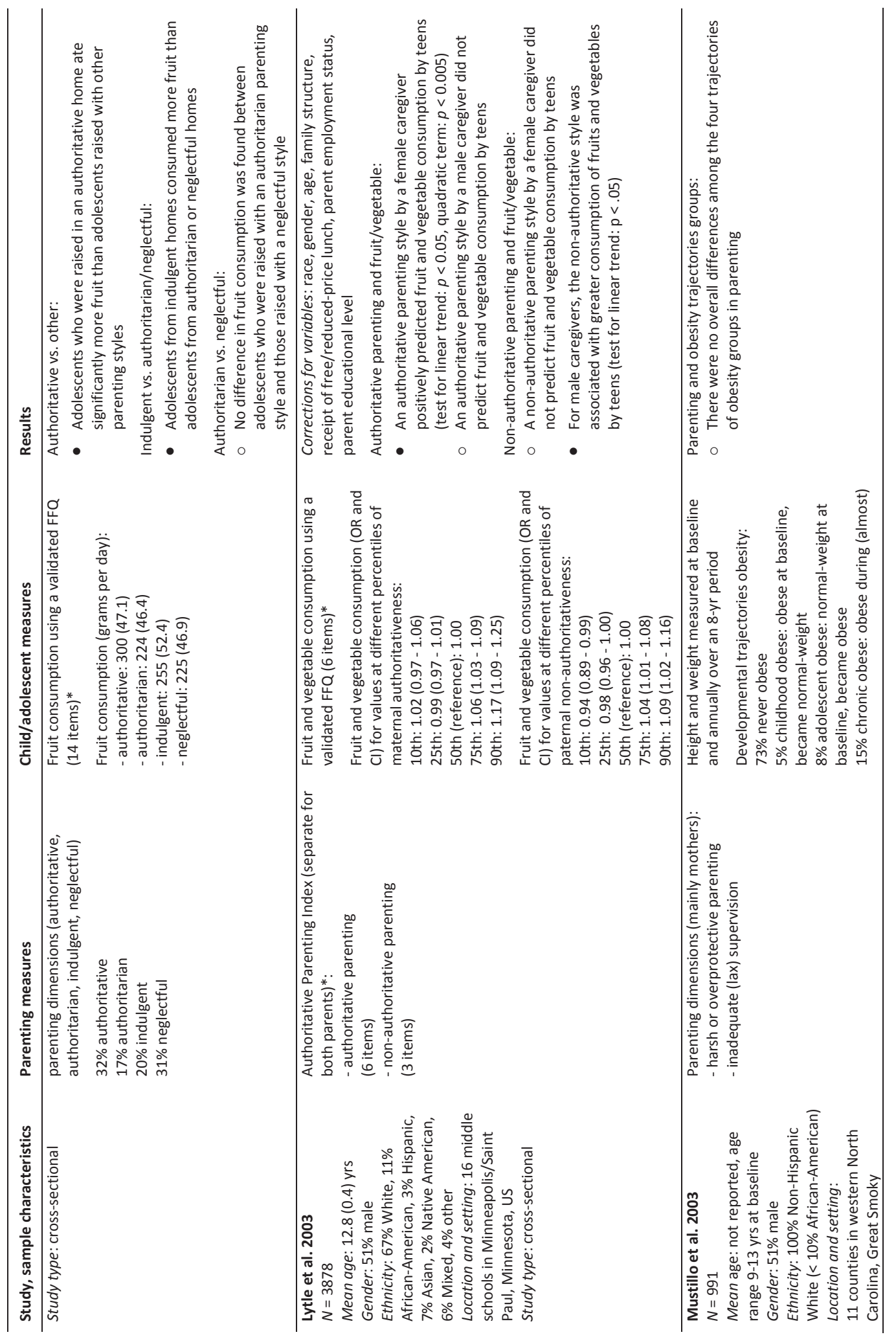




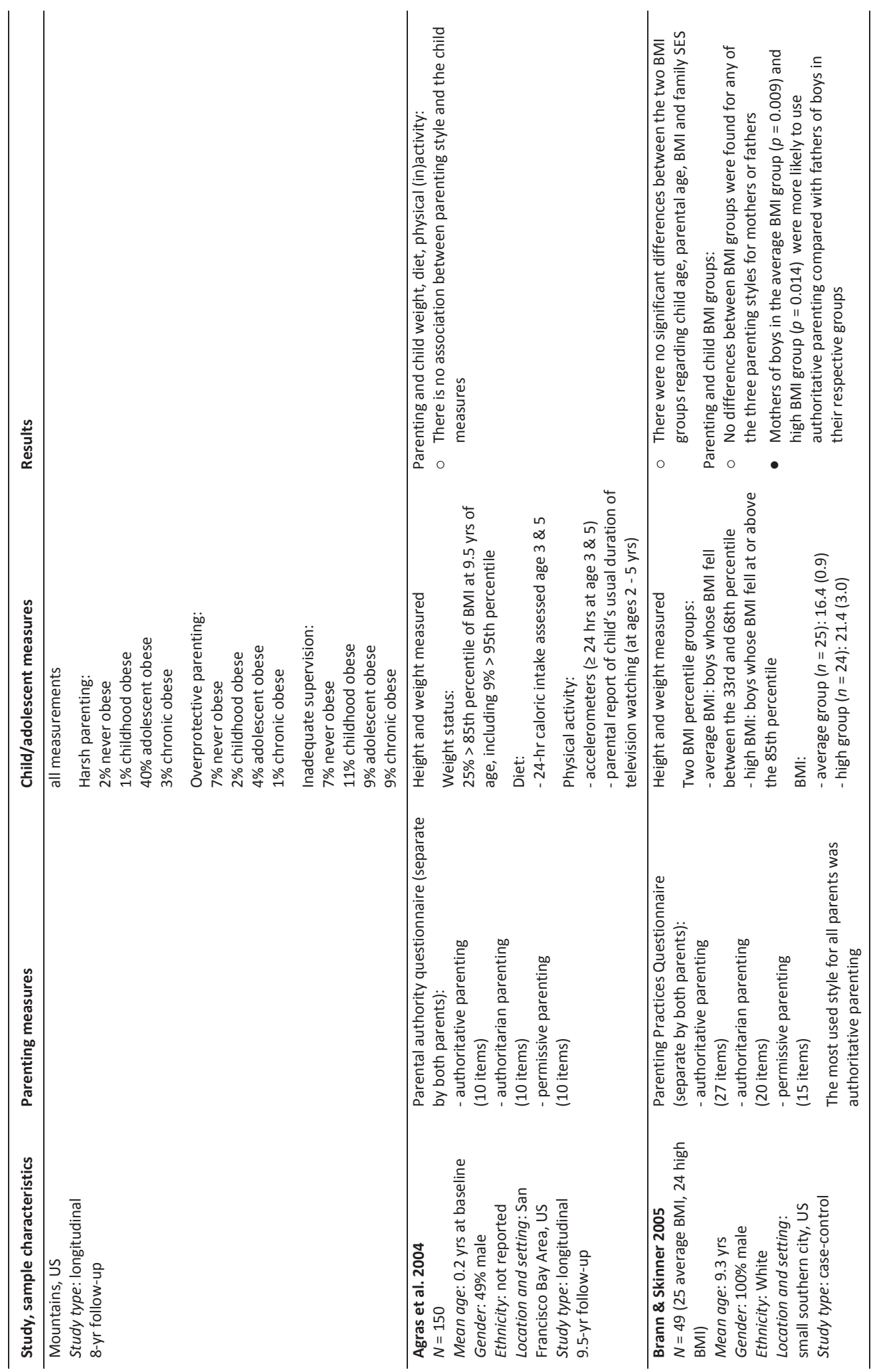




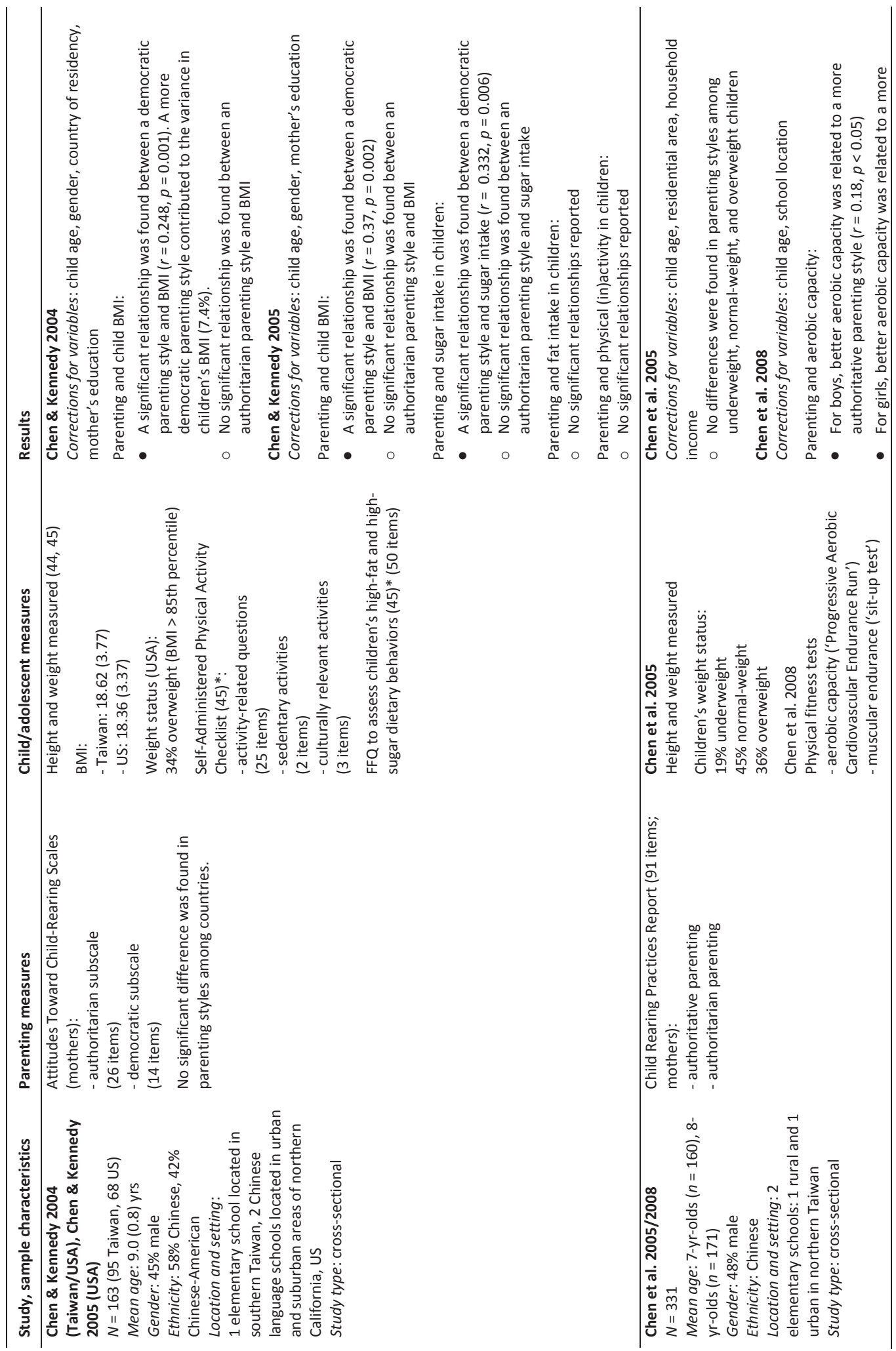




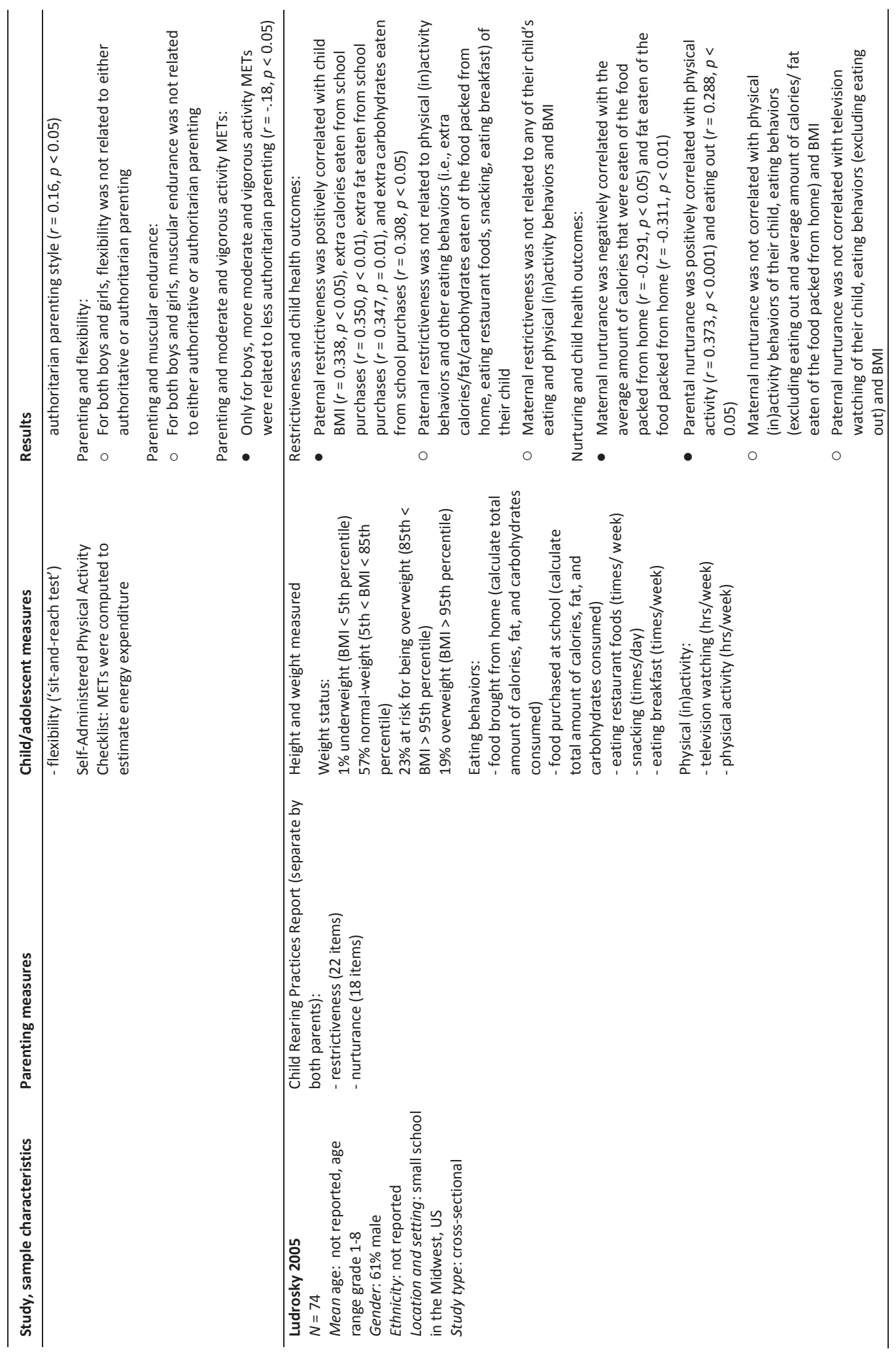




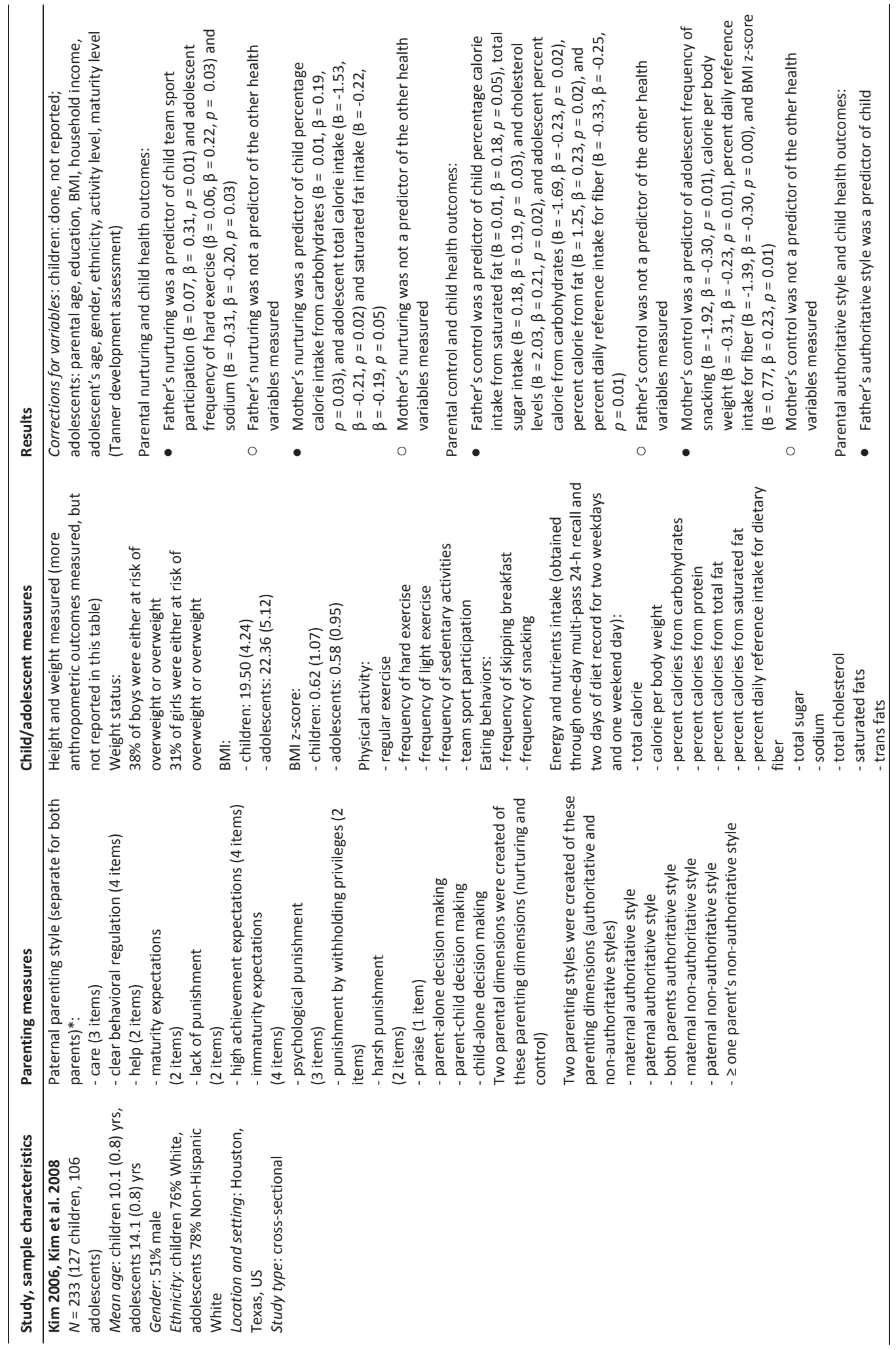




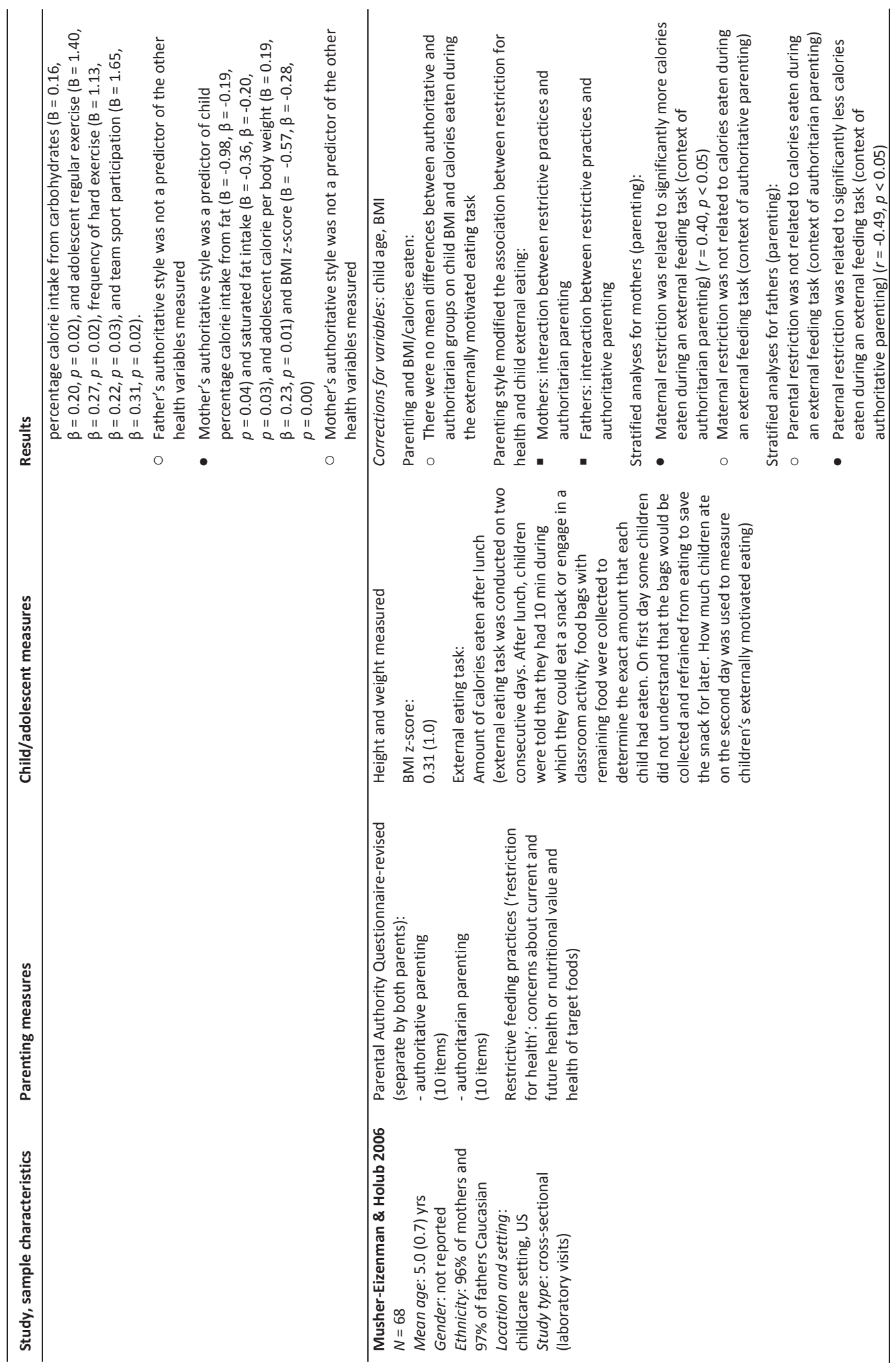




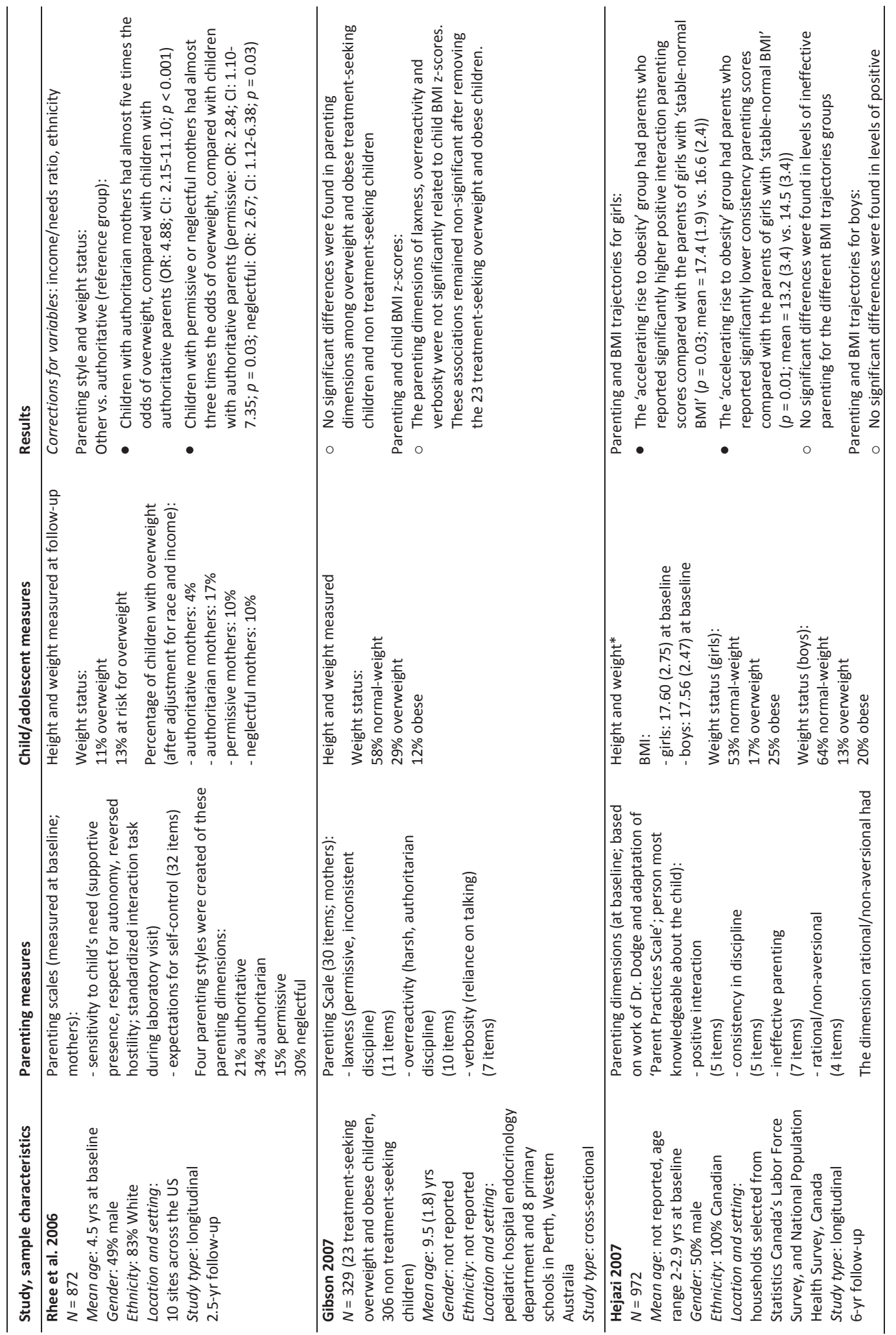




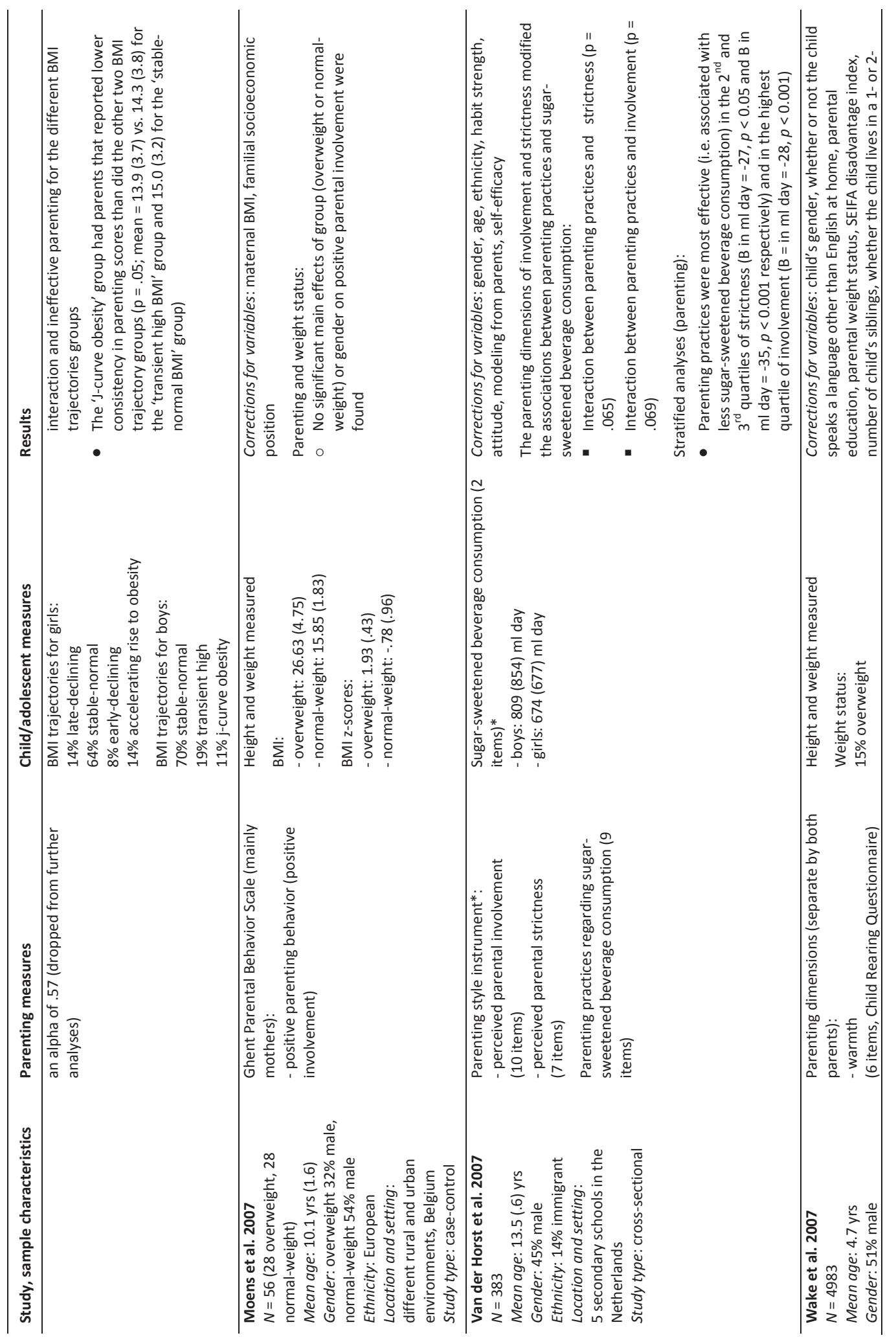




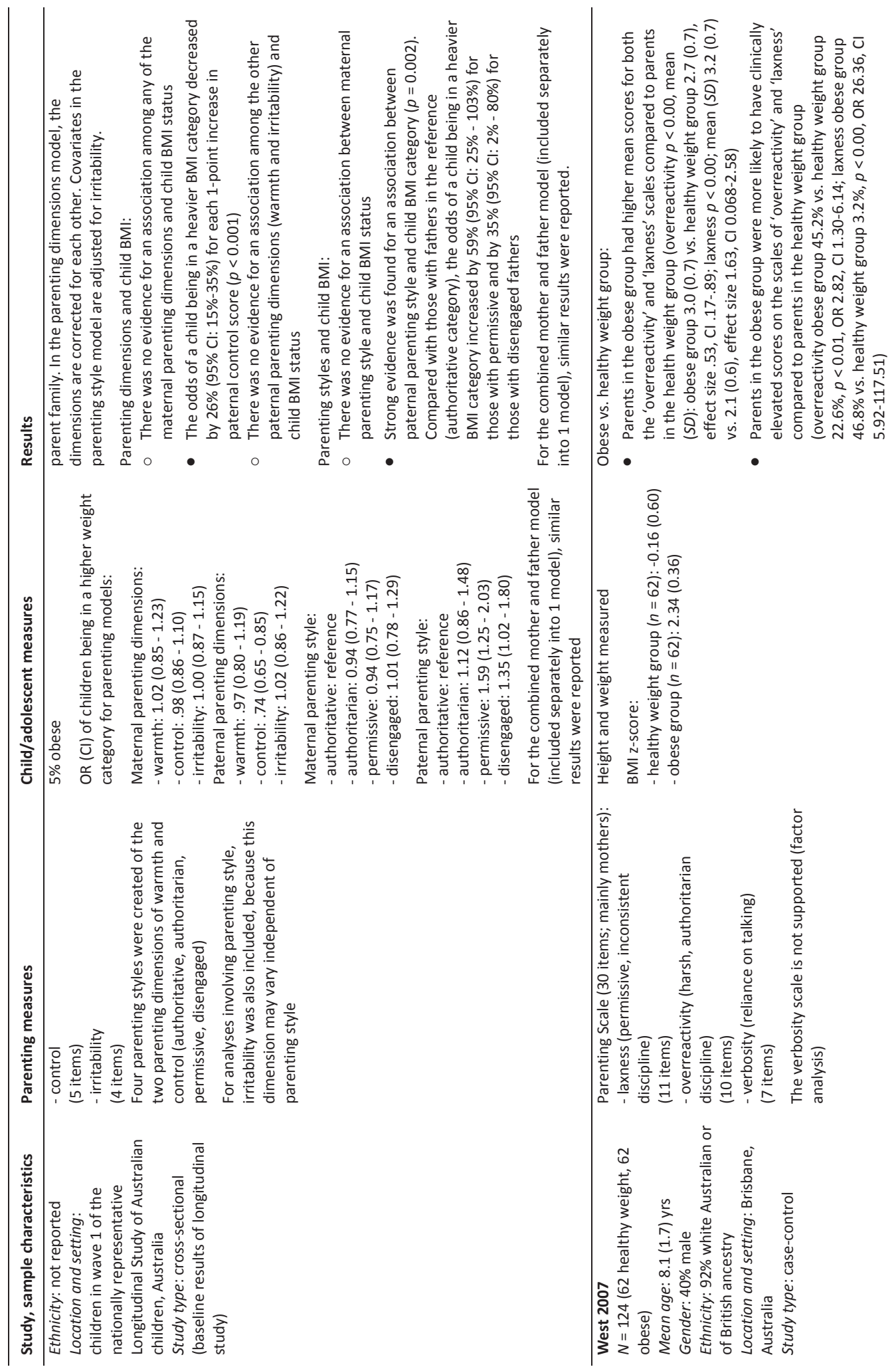




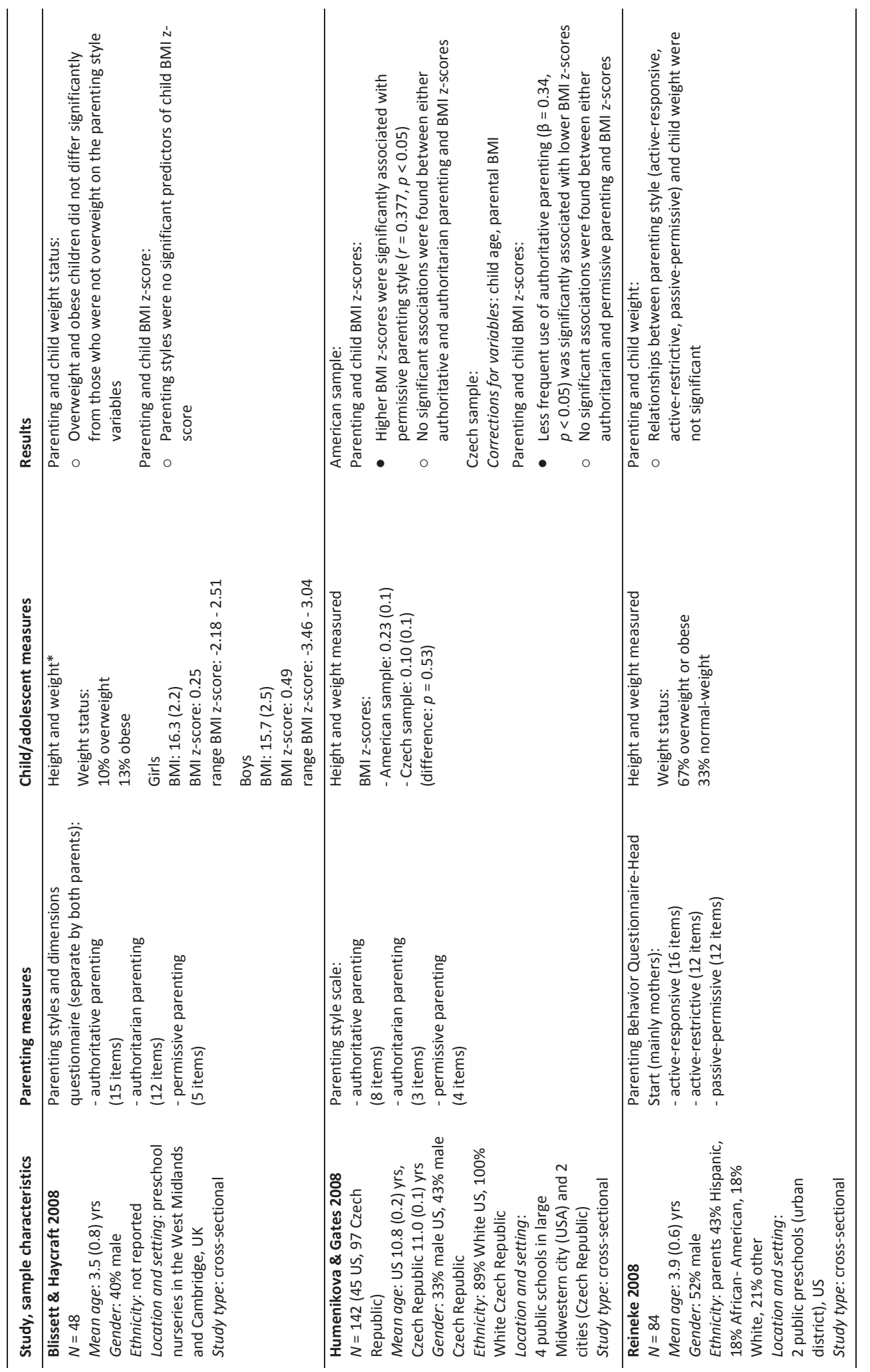




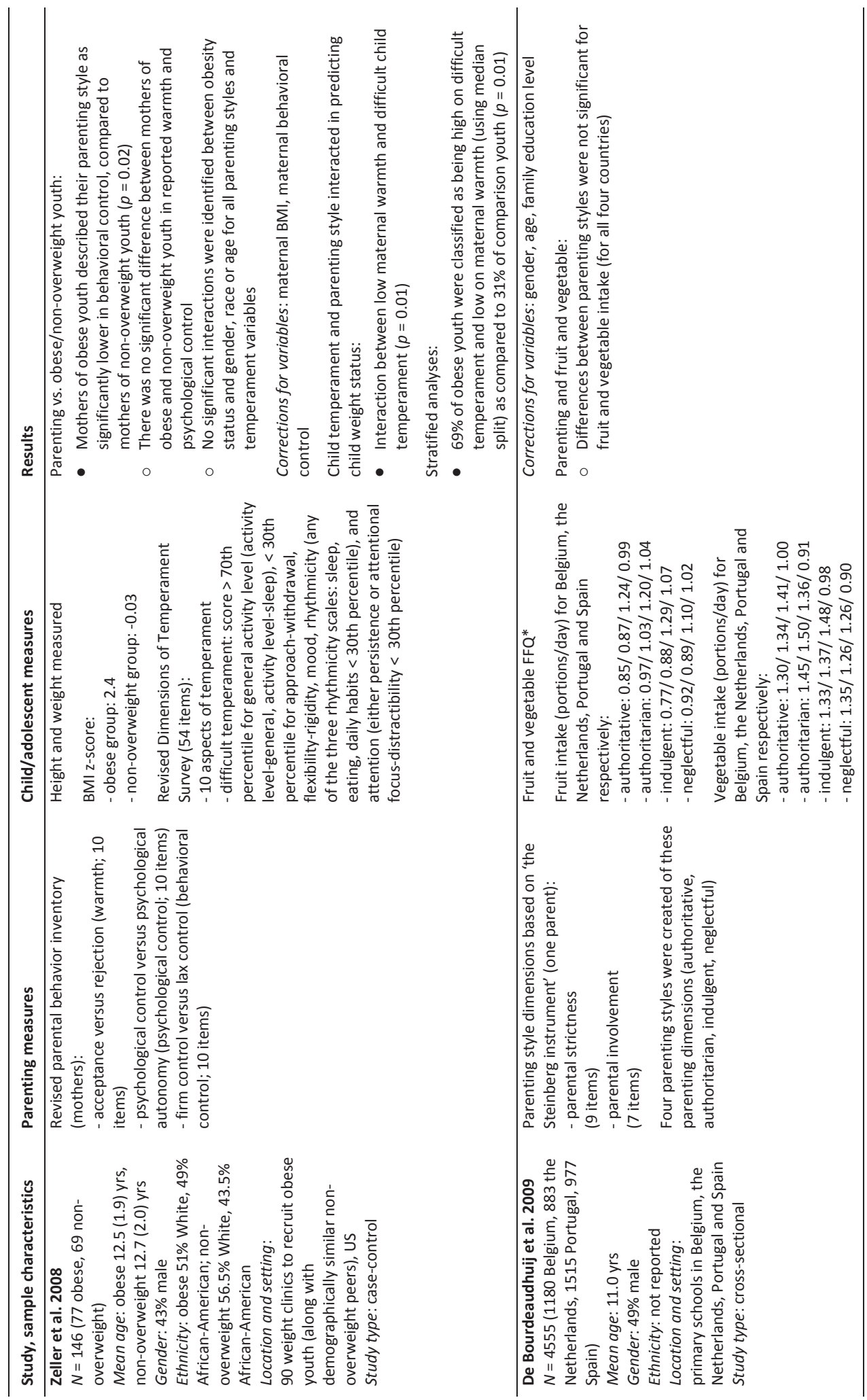




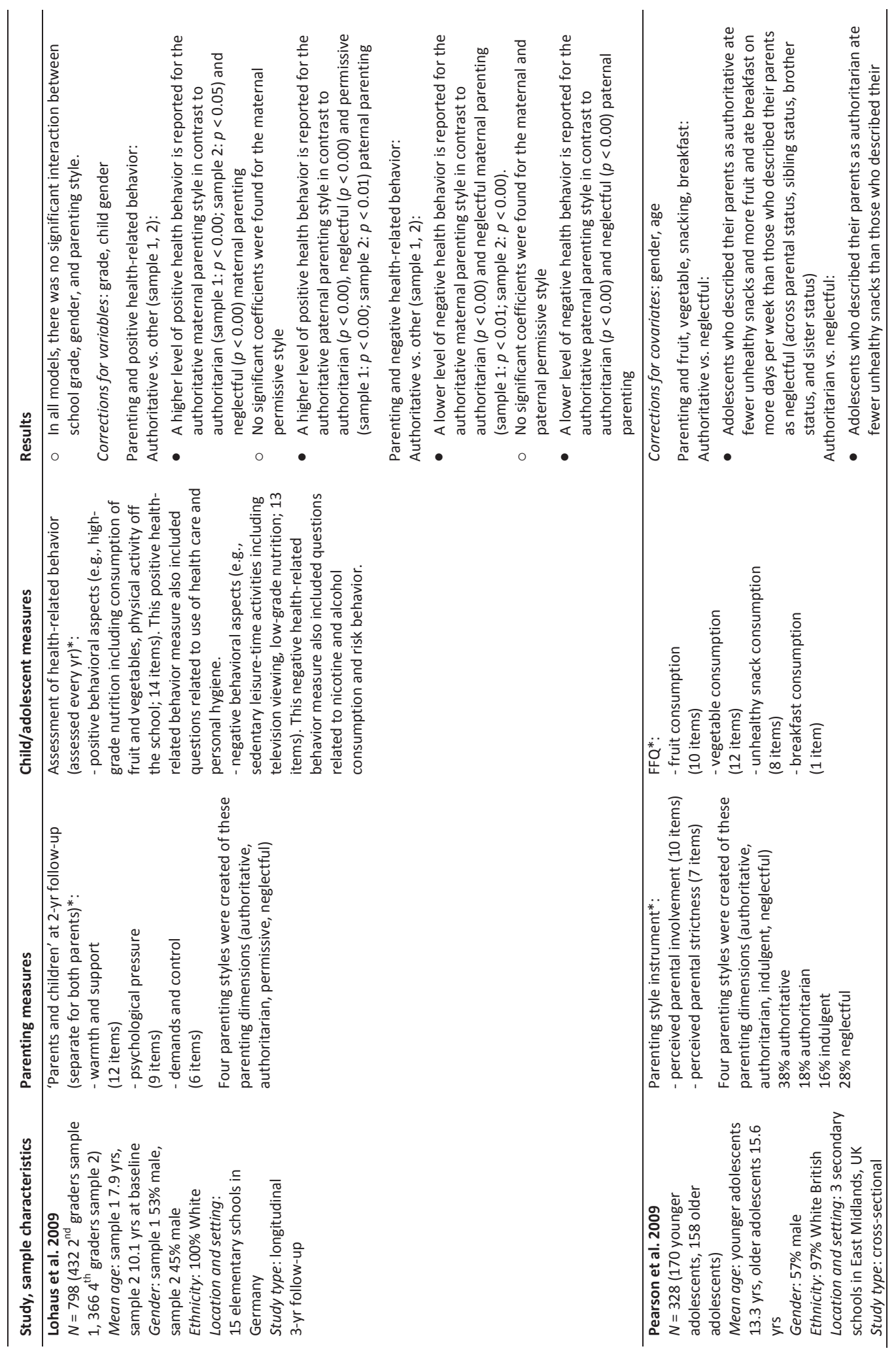




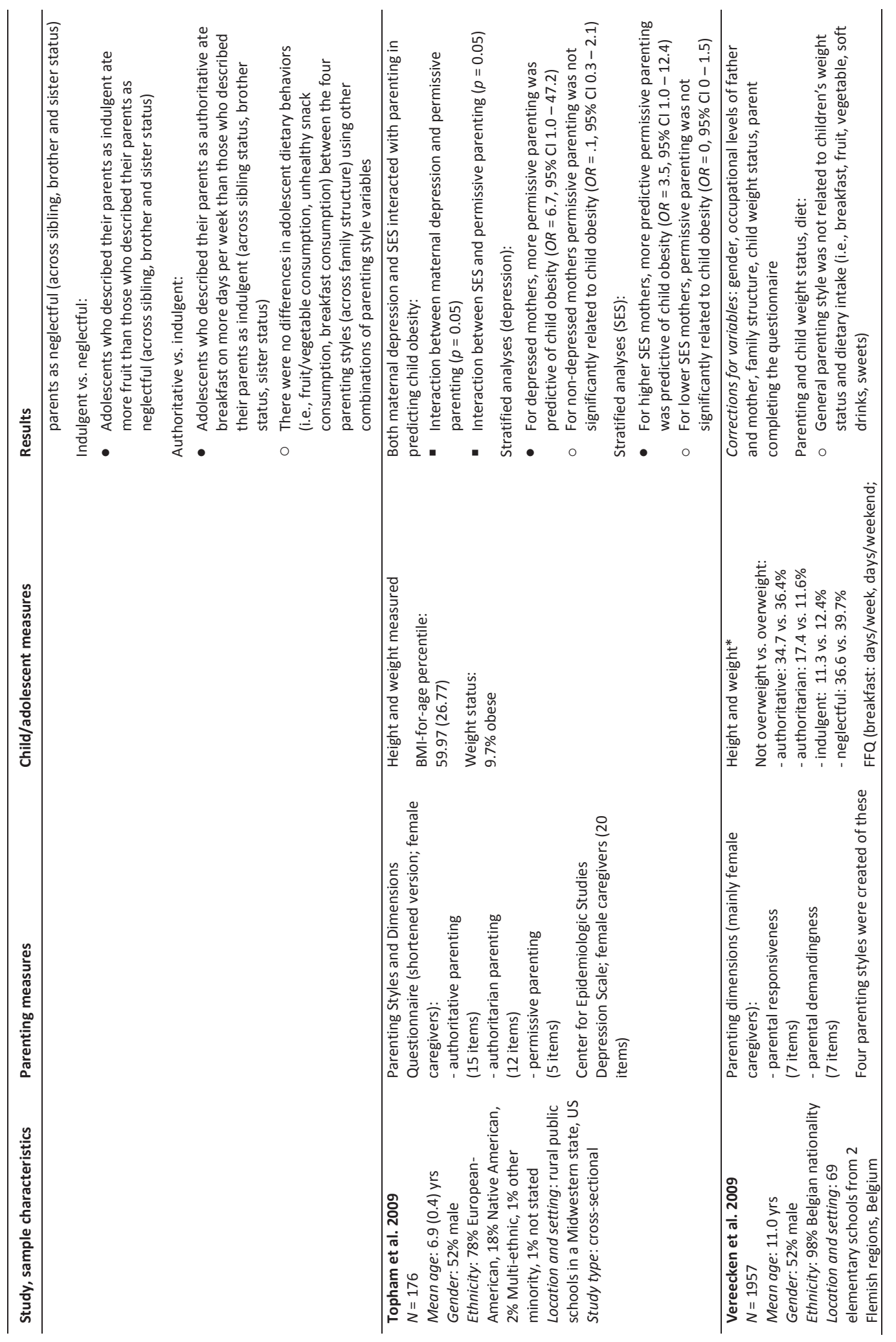




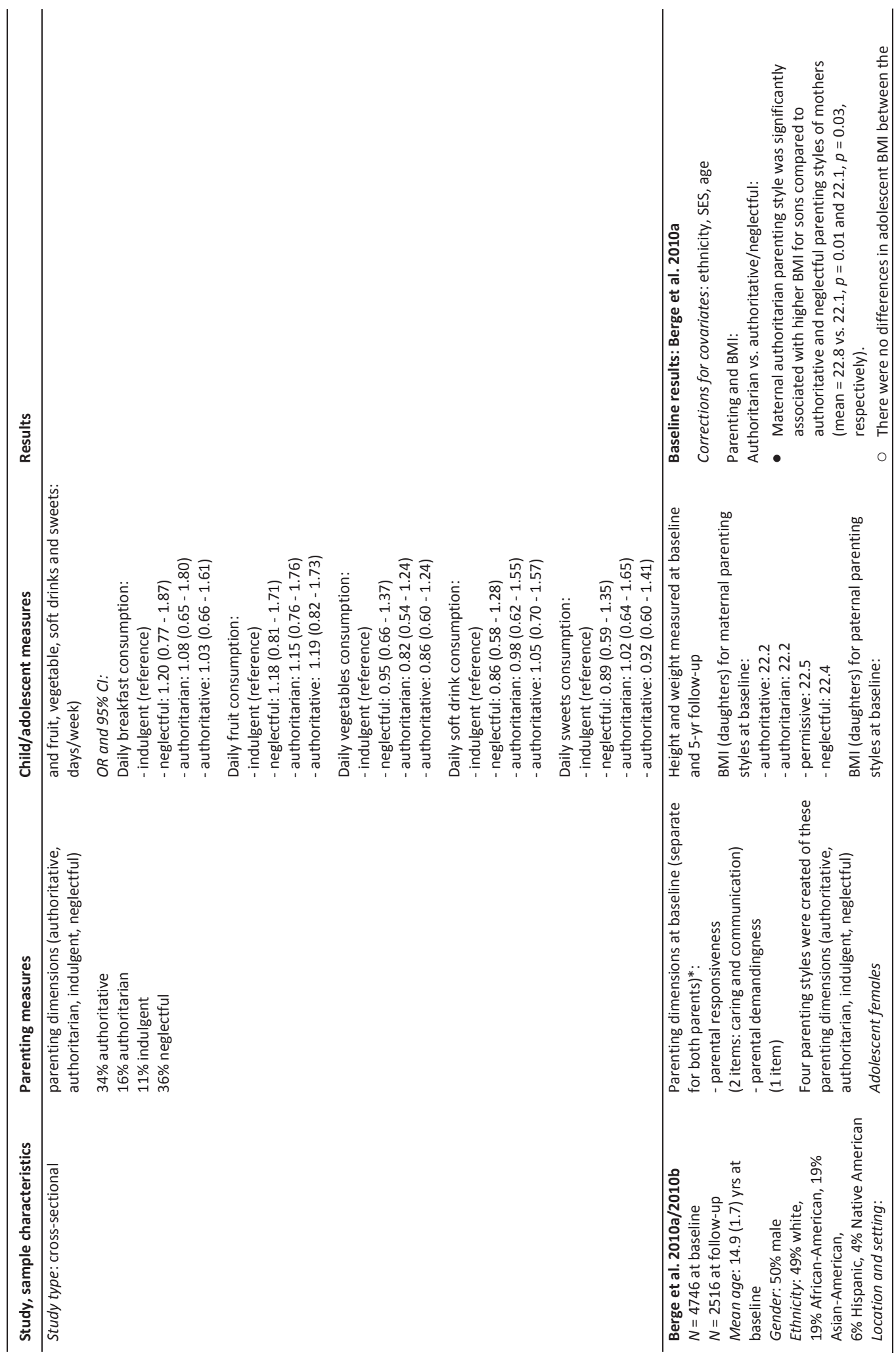




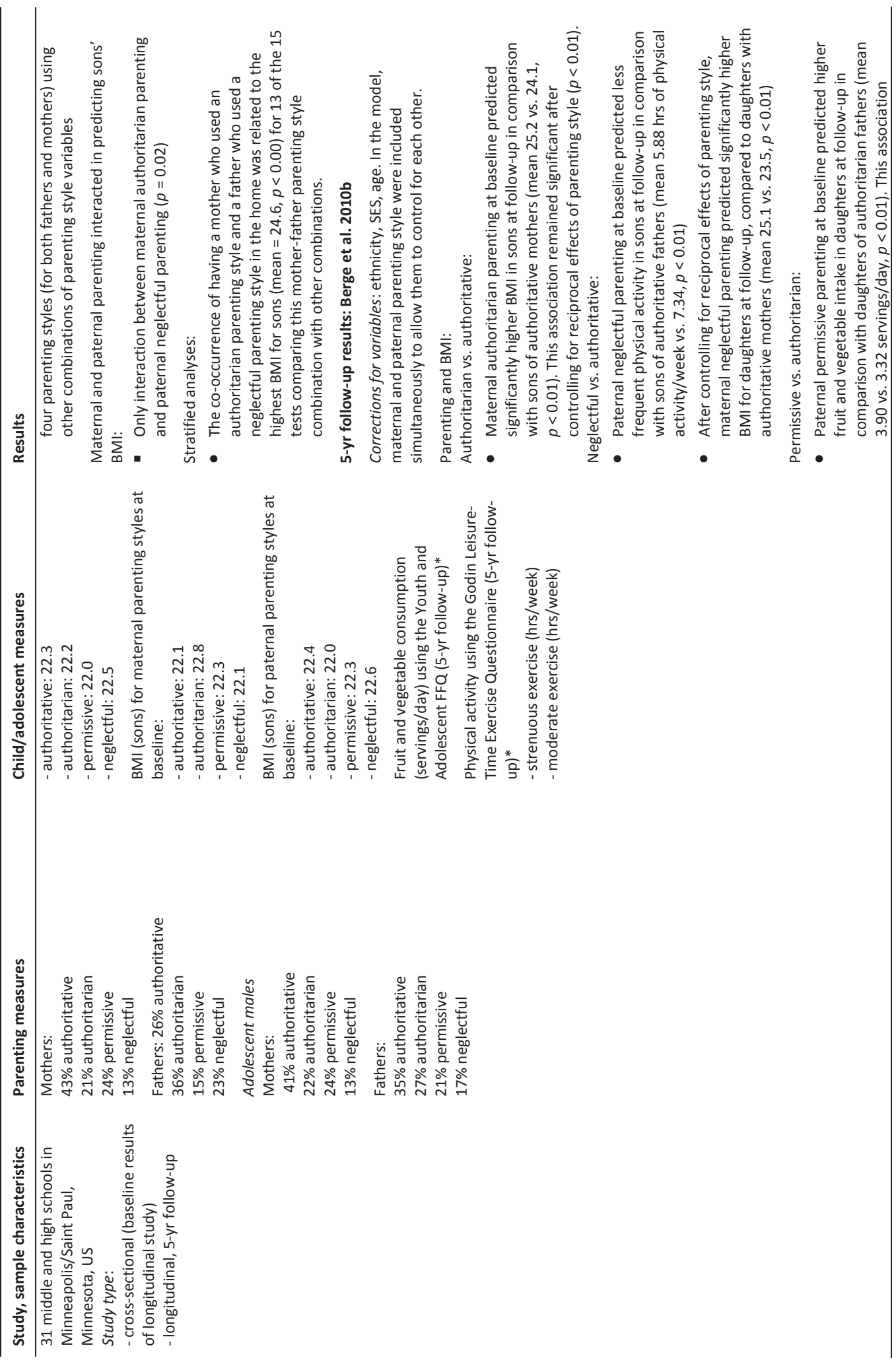




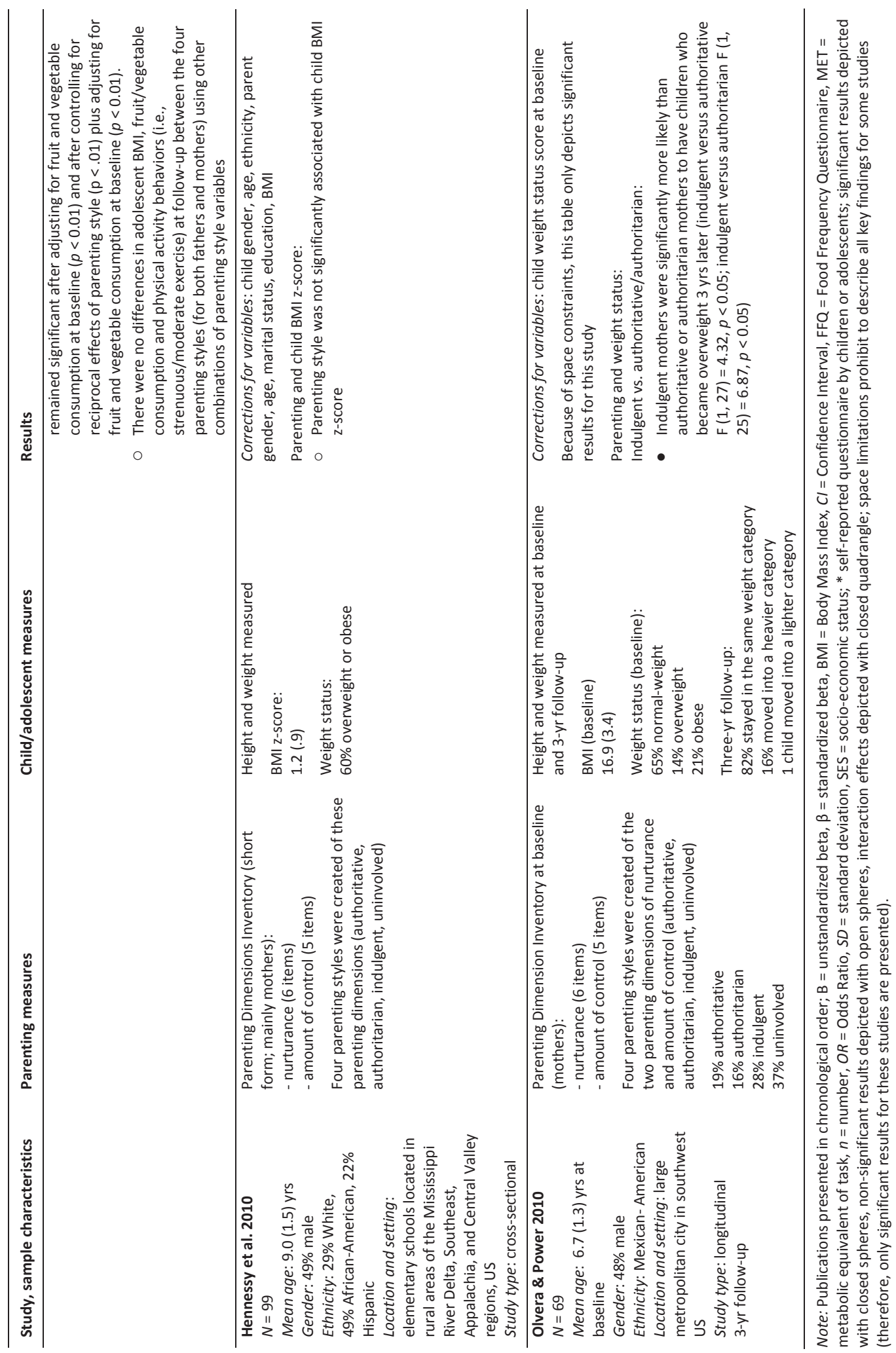




\section{CHAPTER 3}

Interventions addressing general parenting to prevent or treat childhood obesity

Sanne MPL Gerards

Ester FC Sleddens

Pieter PC Dagnelie Nanne K De Vries Stef PJ Kremers

International Journal of Pediatric Obesity, 2011 


\begin{abstract}
Observational studies increasingly emphasize the impact of general parenting on the development of childhood overweight and obesity. The aim of the current literature review was to provide an overview of interventions addressing general parenting in order to prevent or treat childhood obesity. Electronic literature databases were systematically searched for relevant studies. Seven studies were eligible for inclusion. The studies described four different general parenting programs, which were supplemented with lifestyle components (i.e., physical activity and nutrition). All studies showed significant small to moderate intervention effects on at least one weight-related outcome measure. The current review shows that despite the emerging observational evidence for the role of parenting in children's weight-related outcomes, few interventions have been developed that address general parenting in the prevention of childhood obesity. These interventions provide evidence that the promotion of authoritative parenting is an effective strategy for the prevention and management of childhood obesity.
\end{abstract}




\section{Introduction}

Overweight and obesity in children is a significant public health problem (Wang \& Lobstein, 2006; World Health Organization, 2006). The current prevalence of overweight and obesity varies considerably across parts of the world, with North America, Europe and parts of the Western Pacific having the highest prevalence of overweight among children (approximately 20-30\%) (Wang \& Lobstein, 2006). Increasingly, children become overweight at a relatively young age. Being over-weight as a child increases the risk of becoming an overweight adult, compared to normal-weight children (Freedman, Khan, Serdula, Dietz, Srinivasan, \& Berenson, 2005; Magarey, Daniels, Boulton, \& Cockington, 2003; Singh, Mulder, Twisk, Van Mechelen, \& Chinapaw, 2008). Obesity in childhood is also associated with health consequences like cardiovascular diseases and type 2 diabetes, and social consequences such as teasing and discrimination (Must \& Strauss, 1999; Strauss, 2000).

A large number of interventions to prevent or treat overweight in childhood have been developed, which have been extensively described in various systematic reviews (Bluford, Sherry, \& Scanlon, 2007; Campbell \& Hesketh, 2007; Doak, Visscher, Renders, \& Seidell, 2006; Oude Luttikhuis et al., 2009; Saunders, 2007; Summerbell, Waters, Edmunds, Kelly, Brown, \& Campbell, 2005). These reviews show that the majority of the interventions focus on changing so-called energy balance-related behaviors, that is, improving children's dietary intake and increasing their levels of physical activity (Kremers, Visscher, Seidell, Van Mechelen, \& Brug, 2005). However, there is still debate on the best way to design an intervention to achieve maximal and sustainable effects on child outcomes (Summerbell et al., 2005).

Intervention research has increasingly highlighted the importance of parents and family involvement in child obesity treatment and prevention (Kitzmann \& Beech, 2006; Nowicka \& Flodmark, 2008; Young, Northern, Lister, Drummond \& O'Brien, 2007). Parents determine their child's lifestyle to a large extent, especially in the early years of life, and several intervention studies have demonstrated that involving the family in the treatment of childhood overweight is an effective approach. For example, Epstein, McCurley, Wing, and Valoski (1990) showed that including parents as active participants in habit change and weight loss was effective in terms of weight control among children at five-year follow-up; these effects were maintained over extended periods from childhood through adolescence and adulthood (Epstein, Valoski, Wing, \& McCurley, 1990/1994). Golan (2006) showed that targeting parents as exclusive moderators resulted in greater reduction in children's percentage overweight than targeting both parents and children or targeting children alone. Similarly, prevention studies have shown that parents can be supported in achieving effective behavioral changes that seem likely to reduce the degree of overweight in their children (Campbell \& Hesketh, 2007).

A distinction between specific and general family-based intervention goals has been made in the existing literature (Kitzmann \& Beech, 2006). A specific intervention focus involves parents in changing specific child behaviors related to eating and exercise, i.e., changing specific parenting practices. A general intervention aims at changing the broader family context or family functioning (Kitzmann \& Beech, 2006). One 
important dimension of the family context concerns 'general parenting' or 'parenting styles', which is the focus of the current paper. It describes parent-child interactions across a wide range of situations, and can be regarded as the context of behavior-specific parenting (Darling \& Steinberg, 1993). Parenting styles are often rated using two dimensions: an index of parental responsiveness (warmth, acceptance or involvement) and an index of parental demandingness (control or strictness) (Maccoby \& Martin, 1983). The combination of these dimensions results in a fourfold classification of parenting: authoritative, authoritarian, indulgent (similar to permissive), and neglectful (similar to uninvolved).

While originally (and successfully) applied in the prediction of childhood problem behaviors, to date multiple observational studies have also provided evidence for the impact of general parenting on children's weight status and related health behaviors (Sleddens, Gerards, Thijs, De Vries, \& Kremers, 2011). Such observational studies have indicated the potential of general interventions addressing these general parenting skills in attempts to prevent or treat childhood obesity. Although some researchers already underlined the importance of targeting the broader family context in the prevention or treatment of childhood obesity (Kitzmann \& Beech, 2006; Kitzmann, Dalton, \& Buscemi, 2008), to date no reviews have focused exclusively on interventions that incorporated general parenting. The aim of the current review was therefore to provide an overview of interventions which address general parenting in order to prevent or treat obesity in youth (0-18 years).

\section{Method}

Studies that were eligible for the present systematic review were searched for in the computerized databases Pubmed, PsycINFO and Scopus, using combinations of the following keywords: parents, parenting, child, infant, overweight, obesity, weight gain, intervention, and prevention. We included dissertations and studies published in peer-reviewed journals until February 2010.

Studies were first assessed on their title and then on their abstract, by two reviewers (SG and ES) independently of each other. To be included in the present study, intervention studies had to meet the following inclusion criteria:

(1) The intervention study had to focus on children or adolescents (aged 0 to 18).

(2) The primary aim of the intervention had to be the prevention or treatment of overweight or obesity.

(3) The study had to describe intervention components addressing general parenting.

(4) The study had to have used anthropometric measurements at baseline and follow-up to describe the effectiveness of the intervention.

(5) The article had to be in English.

Studies which aimed to change to the children's broader environment, e.g., the emotional climate of the family were excluded from the present review. 
In total, 1057 papers were initially identified in Pubmed, PsycINFO and Scopus using the selected keywords. These were all screened on title, leading to 118 eligible hits. Of these, 55 were eliminated based on abstract evaluation. The remaining 63 papers were retrieved for reading the full-text. This resulted in four studies which were considered eligible for inclusion. Major reasons for studies not being included in the current review were: no intervention studies $(n=757)$, interventions not aimed at treatment or prevention of childhood overweight $(n=133)$, interventions not targeting children or not including parents $(n=69)$, no intervention components on general parenting $(n=69)$, no intervention effects reported $(n=14)$, and paper not in English available $(n=15)$. After the electronic literature search, we applied reference tracking, which led to three additional references, leading to seven eligible studies.

\section{Data extraction and analysis}

The data extraction process of the included studies consisted of three steps. First, we described the general characteristics of the studies: location, inclusion and exclusion criteria of the study participants, design, treatment groups, follow-up, drop-out rates, and characteristics of the study sample. Second, the intervention used in the included studies was outlined by describing the duration, the target group, the components of the intervention targeting general parenting, and the components of the intervention addressing physical (in)activity and nutrition. The final part of the qualitative data extraction process regarded the results of the interventions, including the anthropometric measures of overweight. If available, measures of physical activity (or inactivity) and nutrition and parenting measures were also recorded. These data were abstracted by the first author (SG) and checked by the second author (ES).

In addition, effect sizes (Cohen's $d$ ) were calculated for tests of differential change in weight measures across the intervention and control conditions. Cohen's $d$ is calculated by dividing weight changes by the pooled standard deviation of the baseline weight of the study population (Cohen, 1992). In case of multiple intervention groups, the effect sizes of all interventions were assessed. In studies without an appropriate control group, Cohen's d was calculated by dividing the mean weight change by the standard deviation of the baseline weight. Effect sizes (ES) were interpreted using the classification defined by Lipsey (1990): small effect (ES 0.00 to 0.32 ), moderate effect (ES 0.33 to 0.55 ), or large effect (ES >0.56).

\section{Results}

\section{General study characteristics}

Seven intervention studies, described in nine papers, were identified as meeting the inclusion criteria (Aragona, Cassady, \& Drabman, 1975; Golley, 2005; Golley, Magarey, Baur, Steinbeck, \& Daniels, 2007a; Golley, Perry, Magarey, \& Daniels, 2007b; Harvey-Berino \& Rourke, 2003; Israel, Guile, Baker, \& Silverman, 1994; Israel, Stolmaker, \& Andrian, 1985; Robertson, Friede, Blissett, Rudolf, Wallis, \& Stewart-Brown, 2008; West, 2007). General study characteristics are described in Table 1. The studies were published between 1975 and 2008. Three studies had been conducted in the United States (US; Aragona et al., 1975; 
Israel et al., 1994; Israel et al., 1985), one in the US and Canada (Harvey-Berino \& Rourke, 2003), two in Australia (Golley et al., 2007a; West, 2007) and one in the United Kingdom (Robertson et al., 2008). All authors reported inclusion and/or exclusion criteria to determine who could participate in the intervention. An age limit was reported in each study as an inclusion criterion and five studies exclusively included overweight children (Golley et al., 2007a; Israel et al., 1994; Israel et al., 1985; Robertson et al., 2008; West, 2007). Six studies were based on a Randomized Controlled Trial (RCT), with participants randomly allocated to either two or three different experimental groups. One study (Robertson et al., 2008) made use of a pretest-posttest design in which all participants received the same treatment. Participants in all studies were assessed at baseline and immediately at the end of the intervention. With the exception of the study of Harvey-Berino and Rourke (2003), study participants were also assessed after a period of no further intervention, to indicate maintenance of the intervention effects. These follow-up periods ranged from 20 weeks to three years. The drop-out rate of participants in the studies was reported per group at each measurement, ranging from 0\% (Aragona et al., 1975; Harvey-Berino \& Rourke, 2003) to 60\% (Aragona et al., 1975). The drop-out rate did not differ significantly between the experimental and control groups, except for Aragona et al. (1975) and Harvey-Berino and Rourke (2003). 


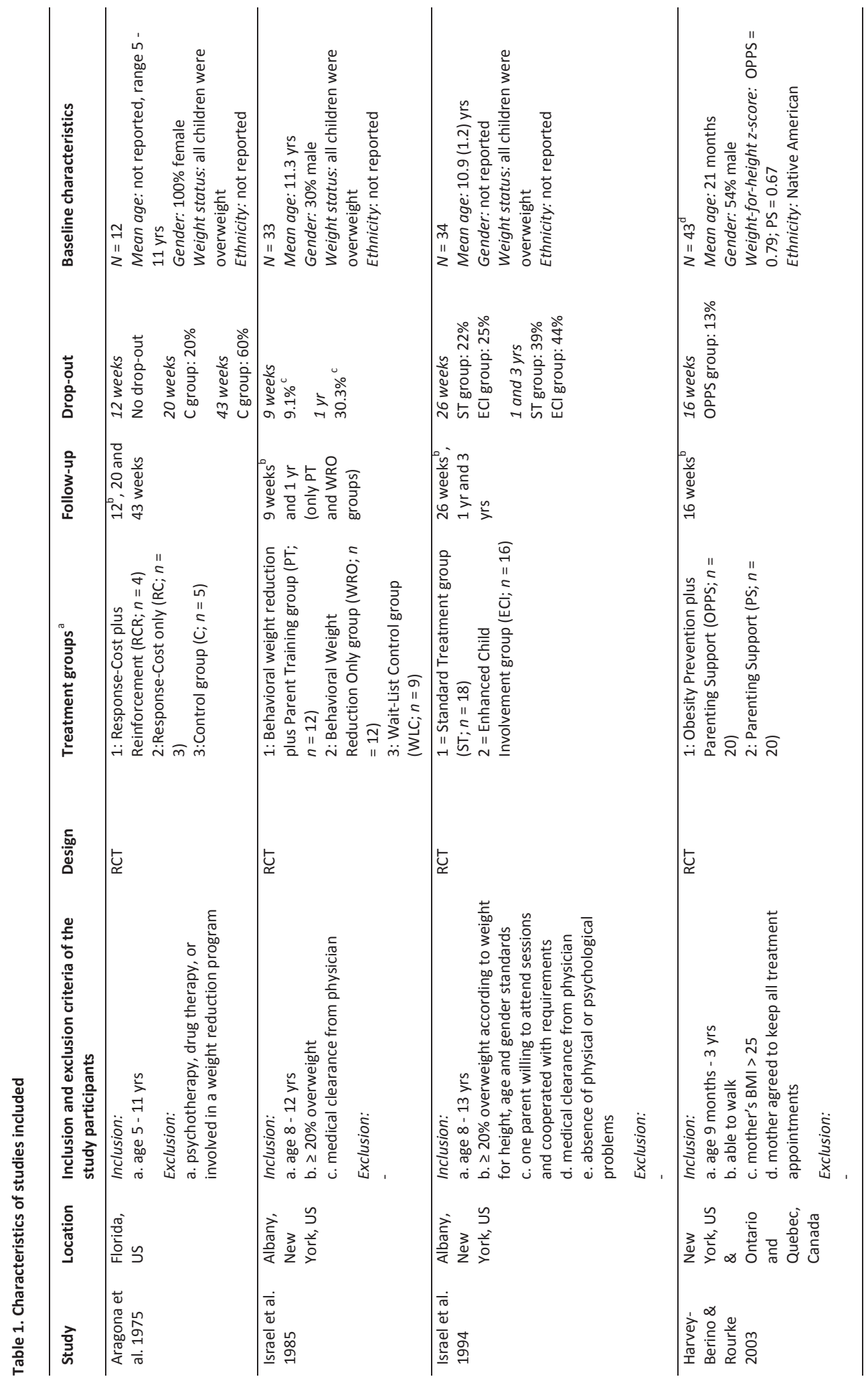




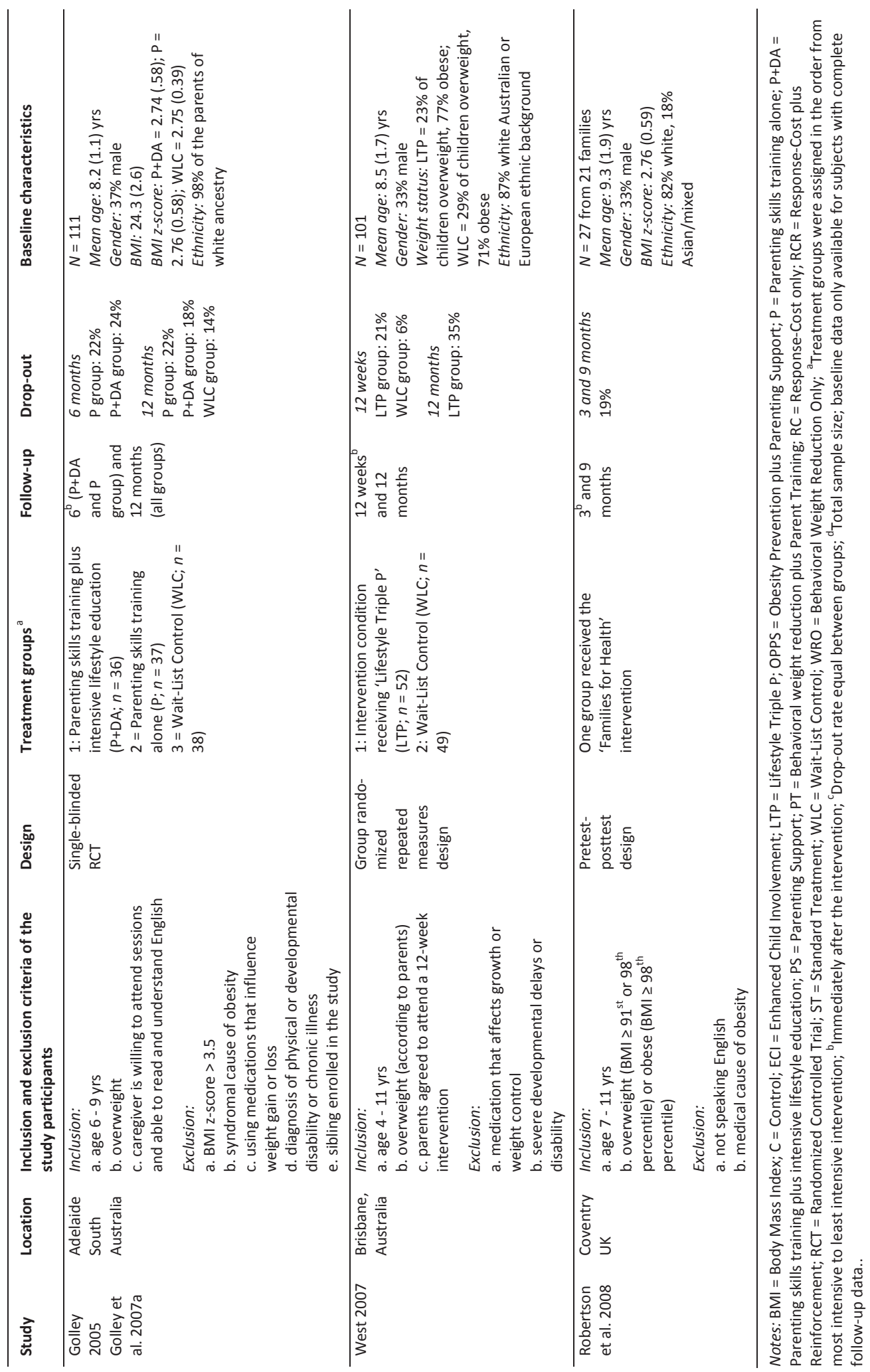


Five studies included less than 50 participants, whereas the two remaining studies included more than 100 children (mean $=52$ participants). Only the study of Golley (2005) reported a sample size calculation. The unweighted mean age of the participants was 8.3 years; one study included preschool children, five studies included children aged between 5 and 11 years and one study included children older than 11 years. Most studies primarily included girls (Aragona et al. 1975; Golley et al., 2007a; Israel et al., 1985; Robertson et al., 2008; West, 2007). In six studies, all participants were overweight or obese, while one study (Harvey-Berino \& Rourke, 2003) also included normal-weight children. The ethnicity of the participants was reported in four studies: three included mainly Caucasians (Golley et al., 2007a; Robertson et al., 2008; West, 2007), and one study included only Native Americans (Harvey-Berino \& Rourke, 2003).

\section{Intervention description}

The interventions are described in Table 2. The duration of the interventions ranged from nine weeks to six months (mean = approximately 16 weeks). Four studies had separate intervention components for both parents and children: three of them offered children a multi-component program with group sessions as well as exercise components (Israel et al., 1994; Israel et al., 1985; Robertson et al., 2008); and in one study children were provided with an intensive exercise program (Aragona et al., 1975). Two studies mainly focused on the parents but did include the children (active game sessions) (Golley et al., 2007a; West, 2007), and one study did not include the children at all (Harvey-Berino \& Rourke, 2003). The interventions were delivered by different methods, but they all incorporated group sessions for parents. In four studies, the intervention was delivered by intensively trained facilitators, who were supervised during the training (Golley, 2005; Harvey-Berino \& Rourke, 2003; Robertson et al., 2008; West, 2007). In every study, the content of the intervention consisted of components relating to general parenting and specific parenting practices related to physical (in)activity and/or nutrition. The included studies used four different standardized general parenting programs, which are described below.

The first program identified was based on the book 'Living with Children' by Patterson (Patterson, 1976; Patterson \& Gullion, 1968). Based on Social Learning concepts from Skinner (1965) and Bandura (1971), the book gives parents brief, very specific instructions on how to change behaviors which almost every parent encounters. It offers programmed instructions, in which the main ideas in the book are broken down into small items on which parents have to respond by writing down an answer.

Three different studies described an intervention that provided parents with 'Living with Children' to help them acquire child management skills. Aragona et al. (1975) used the book as guidance for the content of the group sessions with parents, while the control group did not receive the book. Israel et al. $(1985,1994)$ performed two studies in which they based the lectures in their intervention on the content of the book, while the parents were simultaneously required to read the book. In their 1985 study (Israel et al., 1985), only the intervention group attended sessions and read the book, whereas the control group did not receive any information about general parenting. In contrast, their 1994 study (Israel et al., 1994) involved both the experimental and control groups attending the parent training about general child management 
and reading the book. The difference between the groups was that parents in the standard treatment condition were made responsible for their child's motivation and compliance with the program, whereas in the enhanced child involvement condition, the children were encouraged to manage their own weight loss efforts.

The second general parenting program used in the intervention studies was the Positive Parenting Program (Triple P) (Golley et al., 2007a; West, 2007). Triple P is a standardized general parenting program (Sanders, Markie-Dadds, \& Turner, 2003), based on social learning principles (Patterson, 1982), which aims to promote the parents' competence to manage their child's behavior. Self-management is fostered through self-evaluation and problem solving. Triple $\mathrm{P}$ tries to enhance the knowledge, skills and confidence of parents in order to prevent behavioral, emotional, and developmental problems in their children. The basis of Triple $P$ is formed by five core parenting principles: (a) ensuring a safe and engaging environment, (b) creating a positive learning environment, (c) using assertive discipline, (d) having realistic expectations, and (e) taking care of oneself as a parent. These principles are translated into a range of positive parenting strategies: e.g., spend quality time with your child; provide engaging activities; set developmentally appropriate goals; set a good example; establish clear ground rules; give clear and calm instructions; back up instructions with logical consequences, quiet time, or time-out (Sanders et al., 2003).

Golley and colleagues (Golley, 2005; Golley et al., 2007a/b) were the first to evaluate the effects of a Triple P intervention on childhood obesity, in which parents took part in group sessions. Two experimental groups in their study received Triple P; one of these received additional intensive lifestyle support group sessions which focused on lifestyle knowledge and skills, and their children attended structured supervised activity sessions, while the other group only received a healthy lifestyle pamphlet on top of Triple P. The waiting list control group received only the healthy lifestyle pamphlet. West (2007) developed a new version of this Triple $\mathrm{P}$ intervention, called Lifestyle Triple $\mathrm{P}$, with a specific focus on increasing physical activity and promoting healthy eating in children. The intervention group, which was provided with Lifestyle Triple P, was compared with a waiting list control group who did not receive any intervention.

The third general parenting program applied in childhood overweight interventions is the Active Parenting curriculum (Mullis, 1999), which emphasizes the child's psychological and behavioral goals, logical and natural consequences, mutual respect, and encouragement techniques. Harvey-Berino and Rourke (2003) based their intervention on this curriculum. Two groups received the parenting program: a 'parenting support' group, which involved limited discussions on eating and exercise behaviors, and an 'obesity prevention plus parenting support' group, in which the lessons focused exclusively on improvement of parenting to facilitate exercise and healthy eating behaviors.

Finally, one study used the Family Links Nurturing Program, which was originally developed and evaluated for the treatment and prevention of child abuse and neglect (Hunt, 2003). The program consists of two parts: a parenting program offered to parents and a school-based intervention. The parenting program is based on four principles: (a) helping parents to develop appropriate expectations, (b) self-awareness and self-esteem, (c) a positive approach to discipline and (d) empathy (Barlow, \& Stewart-Brown, 2006). 
Robertson et al. (2008) used elements of the parenting part of the Family Links Nurturing Program and of family lifestyle programs. 


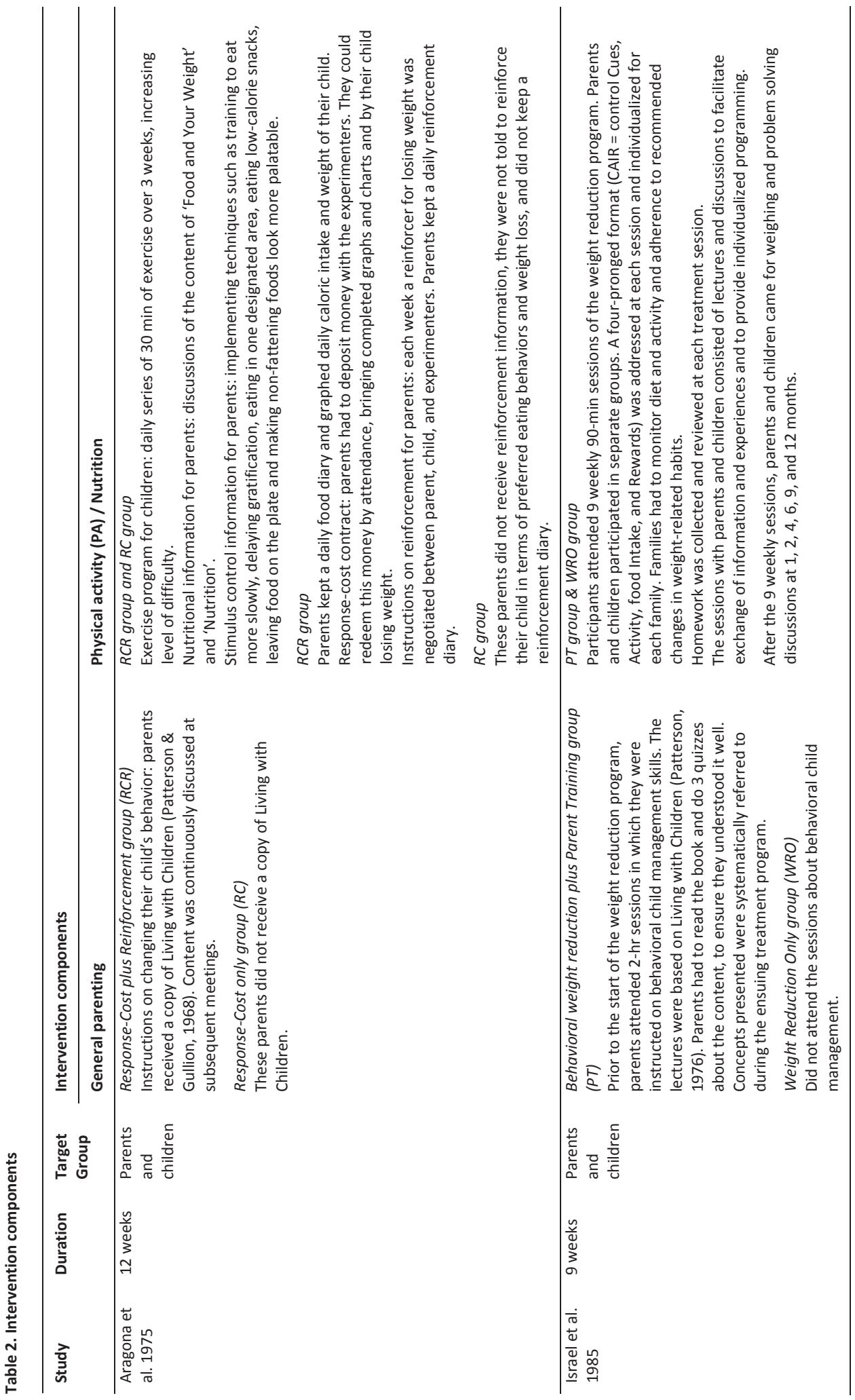




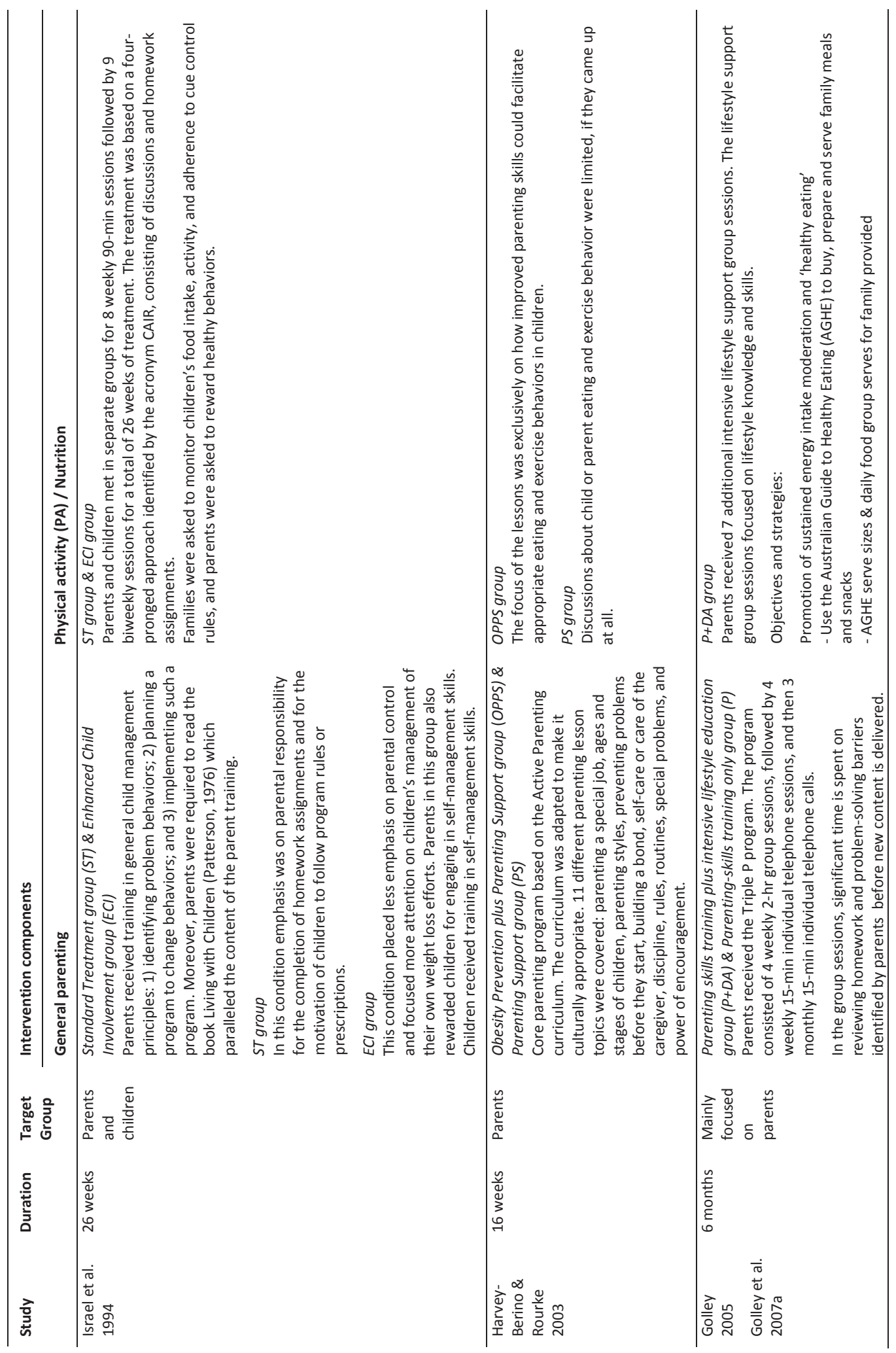




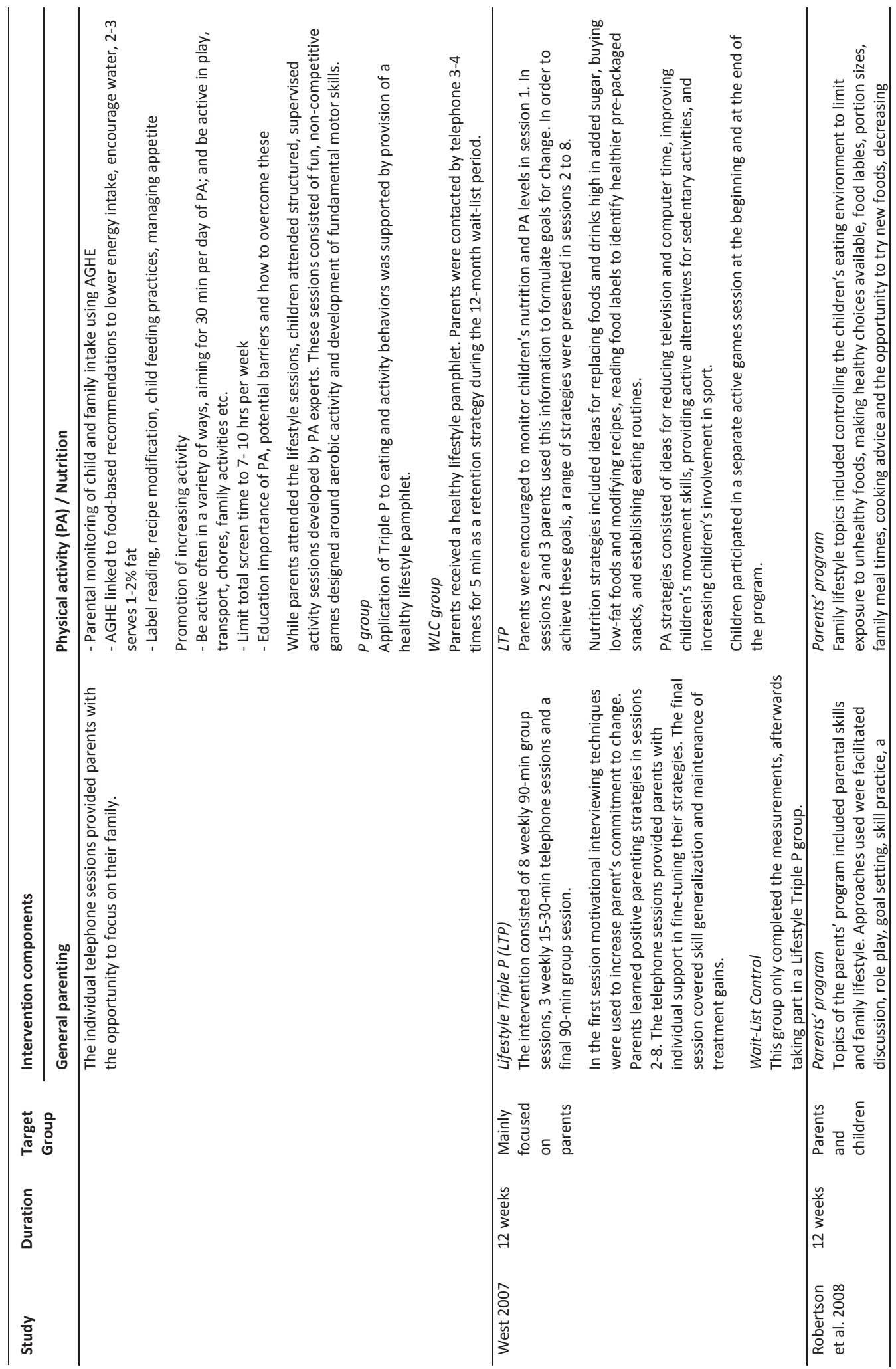




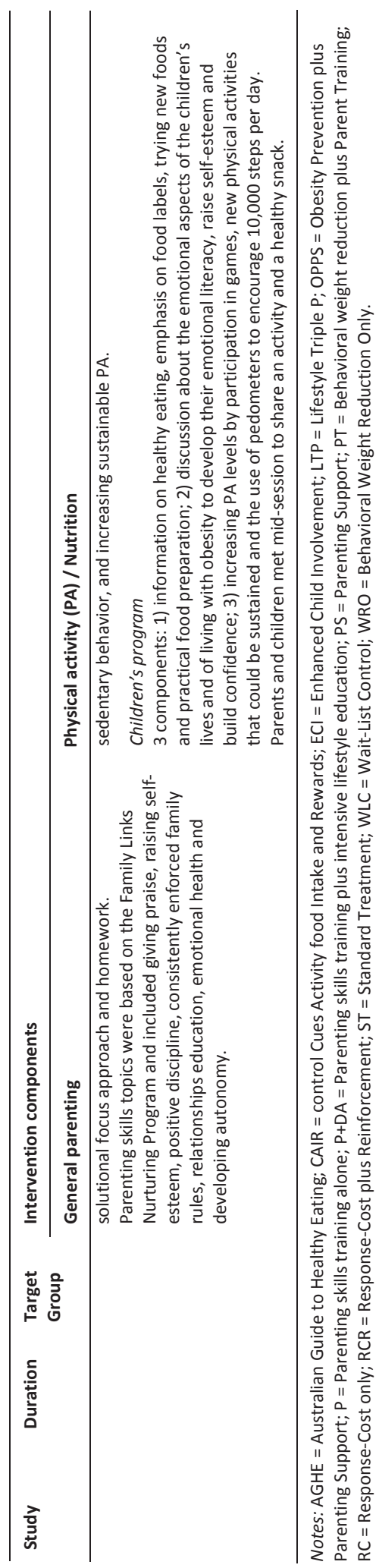




\section{Results of the interventions}

The results of the interventions are summarized in Tables $3 a$ and $3 b$. All studies found statistically significant intervention effects on one or more anthropometric outcome measures. For five studies, the effect sizes were calculated in terms of differences in weight change between groups, based on the information provided in the published papers. The magnitude of the effect of these studies was on average small to moderate, ranging from -0.20 to 0.60 . For the remaining two studies, which lacked an appropriate control group, the effect sizes were calculated in terms of change over time. The magnitude of the weightrelated outcomes in these studies was on average moderate; with effect sizes ranging from 0.28 to 1.22 .

Four studies assessed intervention effects on energy balance-related lifestyle behaviors (physical activity, sedentary behavior and nutrition) (Golley, 2005; Harvey-Berino \& Rourke, 2003; Robertson et al., 2008; West, 2007); all of these studies found significant positive effects on at least one of the behaviors measured. These positive effects were reported on energy intake, intake of extra food (high energy, fat, sugar, and/or salt food), time spent in small screen activities, time spent in active play, activity/inactivity balance, use of active transport to and from school, weekend-day sedentary activity, and number of steps counted. Eating and/or physical activity styles were measured in two studies (Israel et al., 1994; Israel et al., 1985). First, Israel et al. (1985) used the Eating Habit Checklist, a parental report of the degree to which the child engages in the type of eating behavior recommended by a behavioral weight reduction program, and reported a significant improvement due to the intervention. The second study (Israel et al., 1994) was one in which children's self-control regarding eating and activity-related behaviors was measured, and reported an increase in self-control in both experimental groups due to the intervention. Parenting practice measures were reported in four studies (Golley, 2005; Harvey-Berino \& Rourke, 2003; Israel et al., 1994; Robertson et al., 2008): parental control regarding weight-related behaviors was increased in both conditions in the study of Israel et al. (1994), a significant decrease of restrictive child feeding practices was measured in one intervention condition (obesity prevention plus parenting support group) by HarveyBerino et al. (2003), children's access to television after school and on weekend days was stable over time in all conditions in the study of Golley (Golley, 2005), and Robertson et al. (2008) reported a significant reduction of exposure to unhealthy foods in the home in their study group. Finally, the four studies that assessed general parenting (Golley, 2005; Israel et al., 1985; Robertson et al., 2008; West, 2007) all described positive effects of the intervention on this intermediary outcome. 


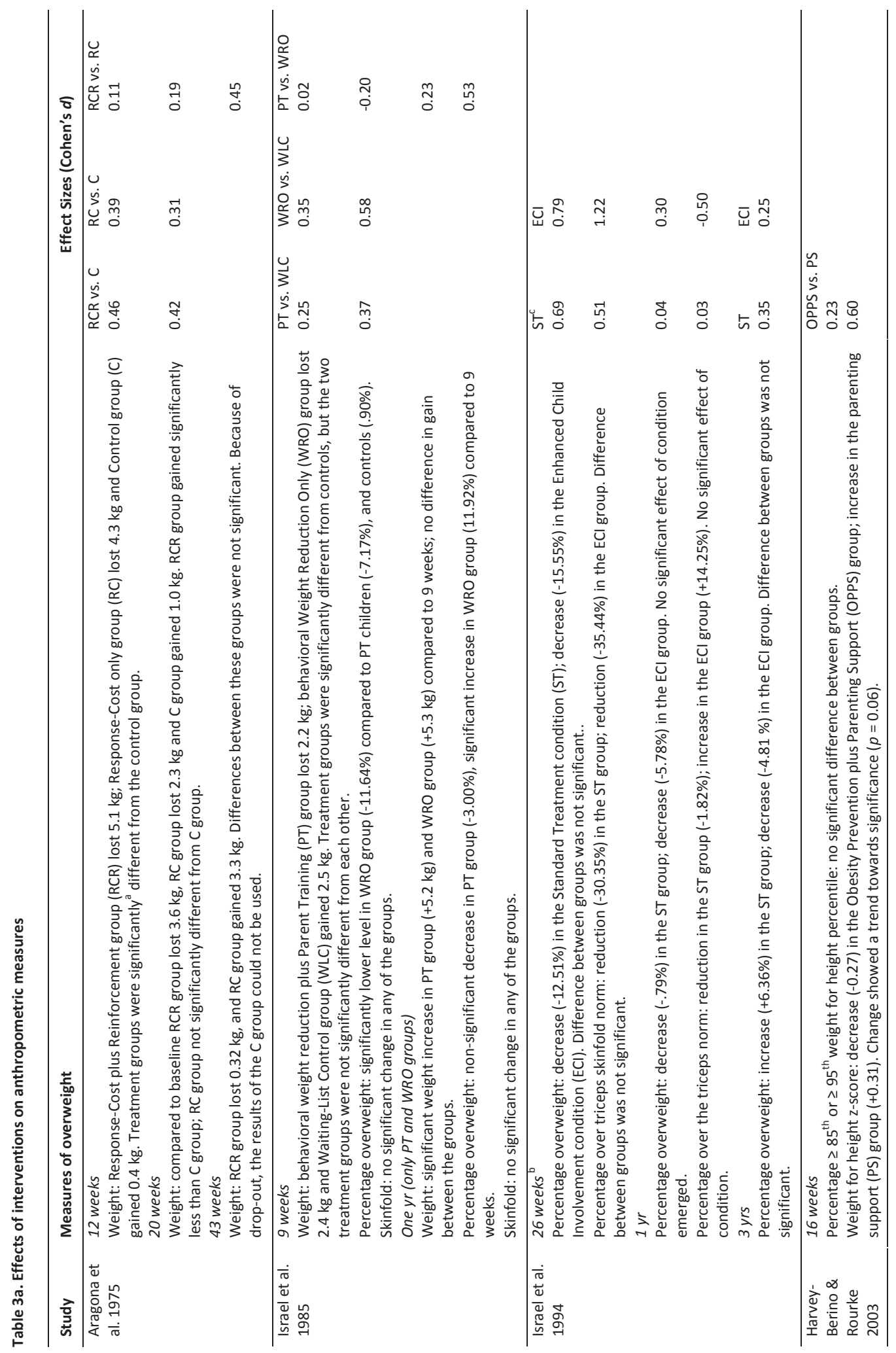




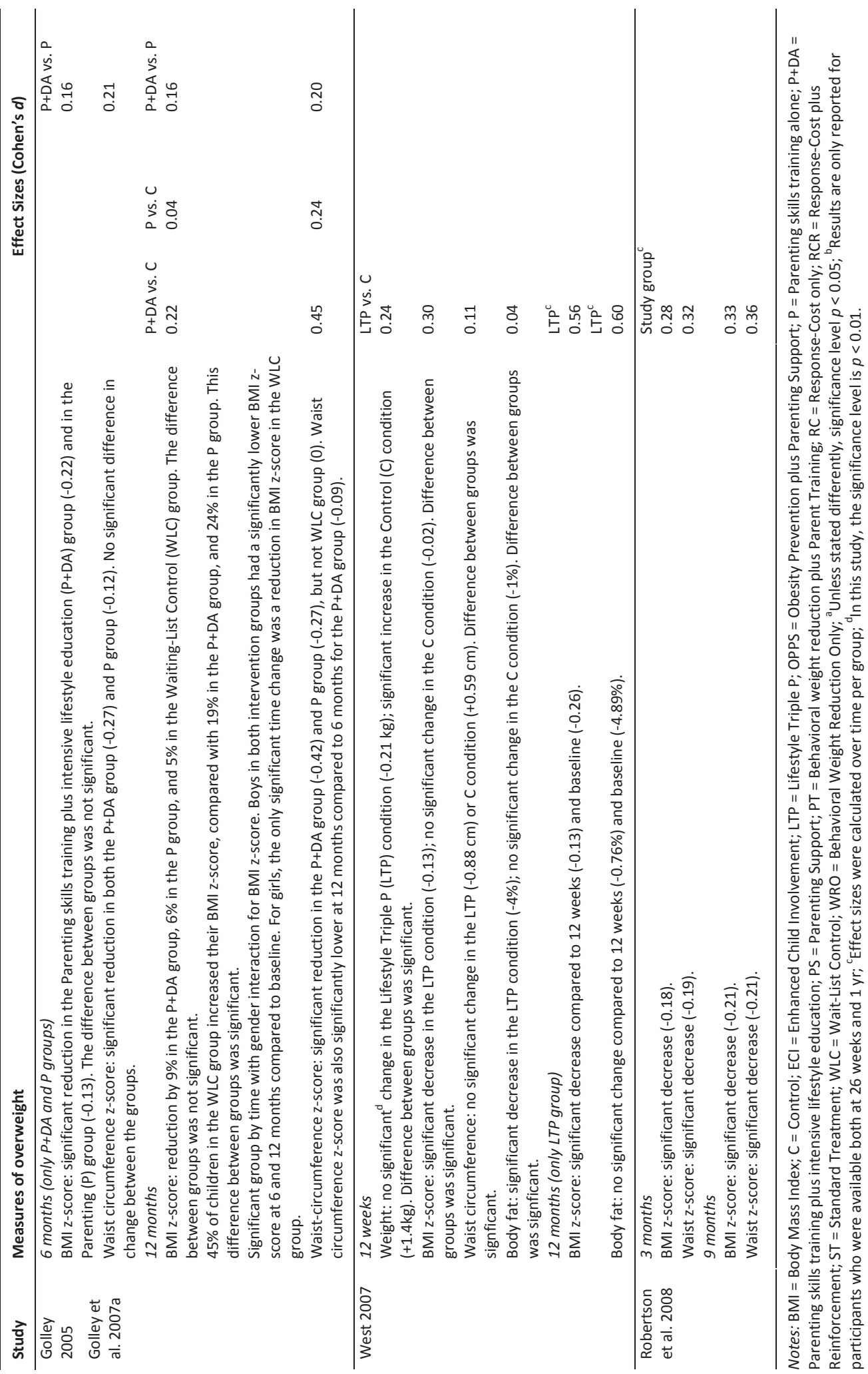




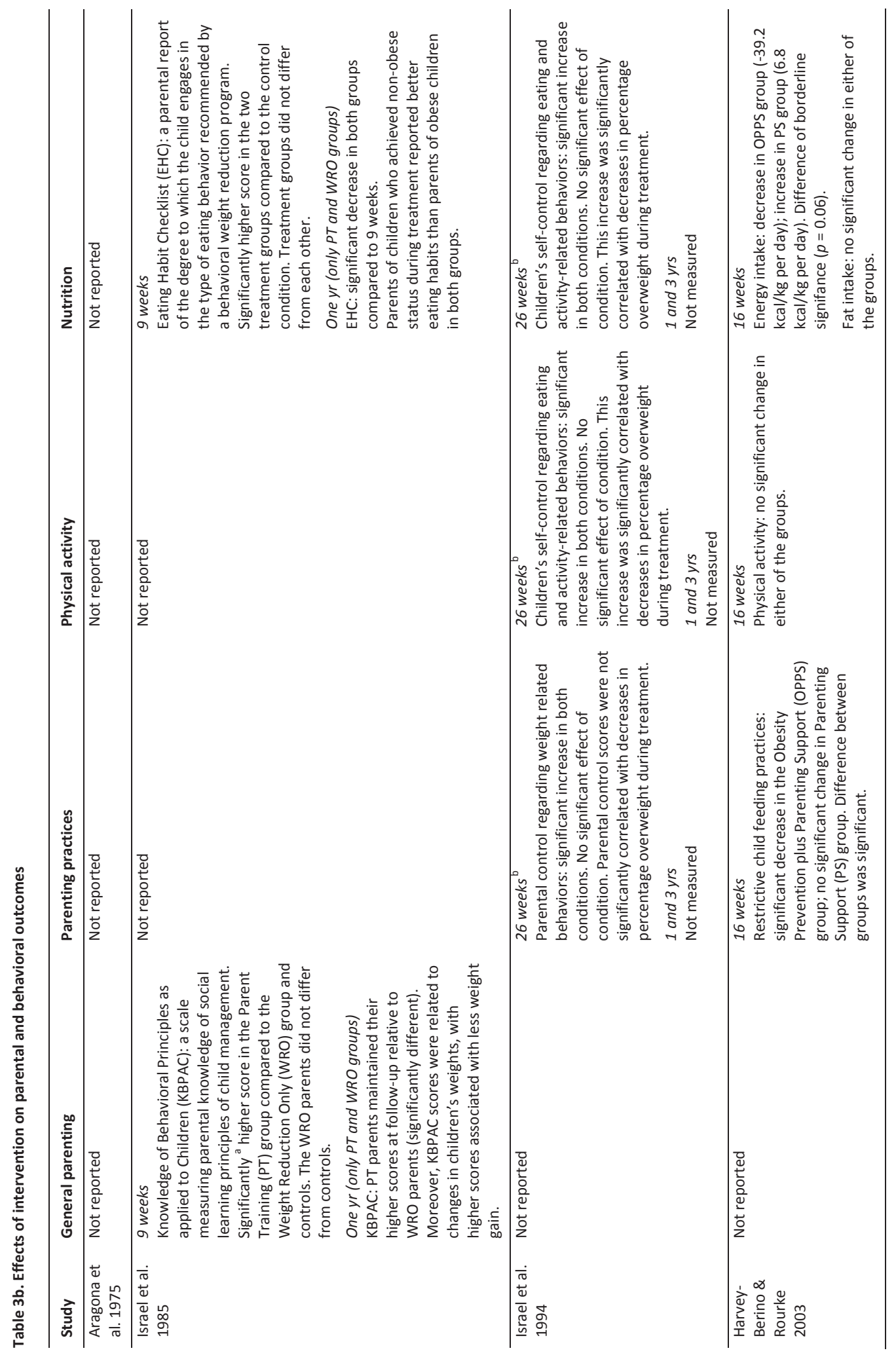




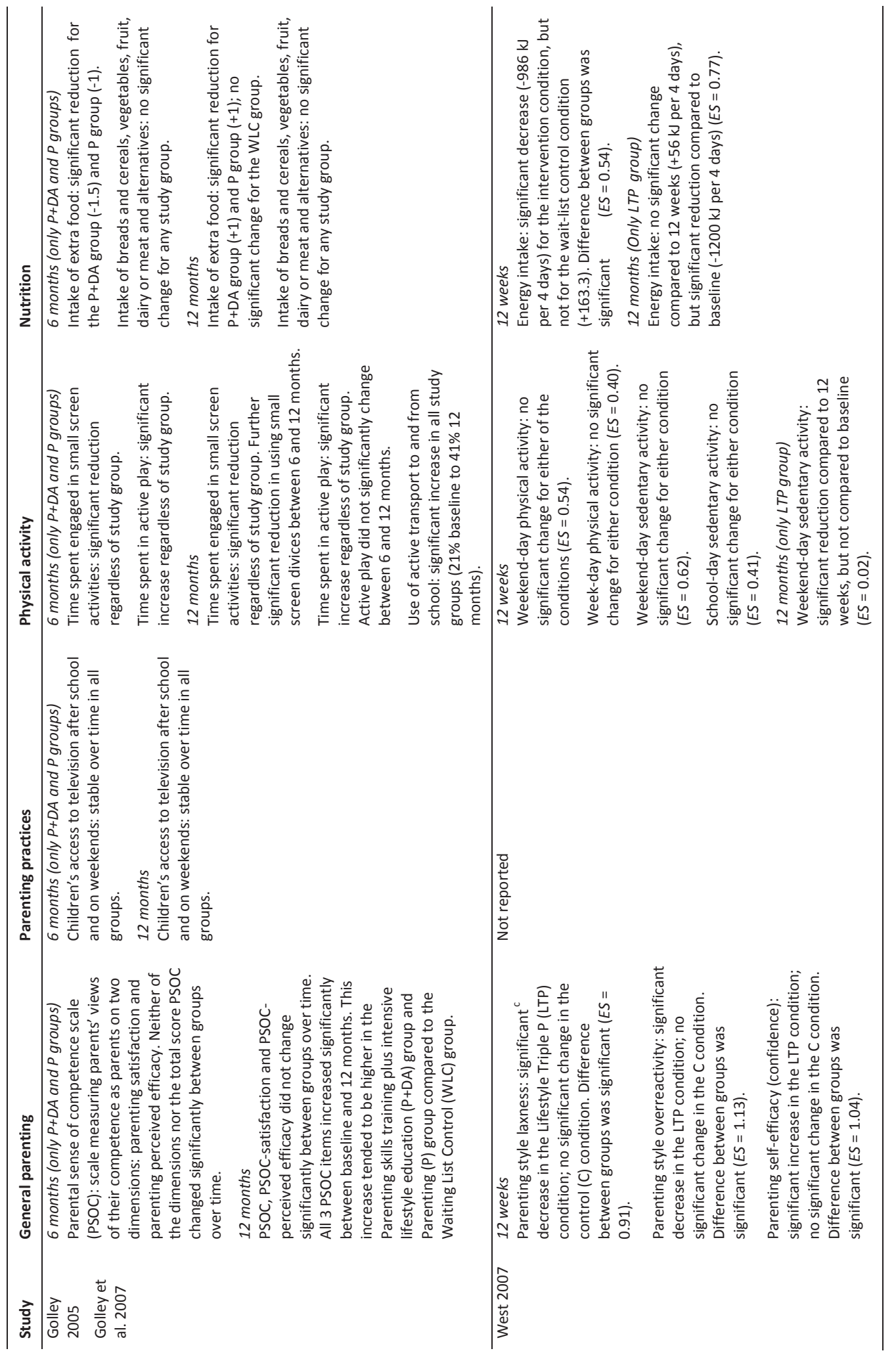




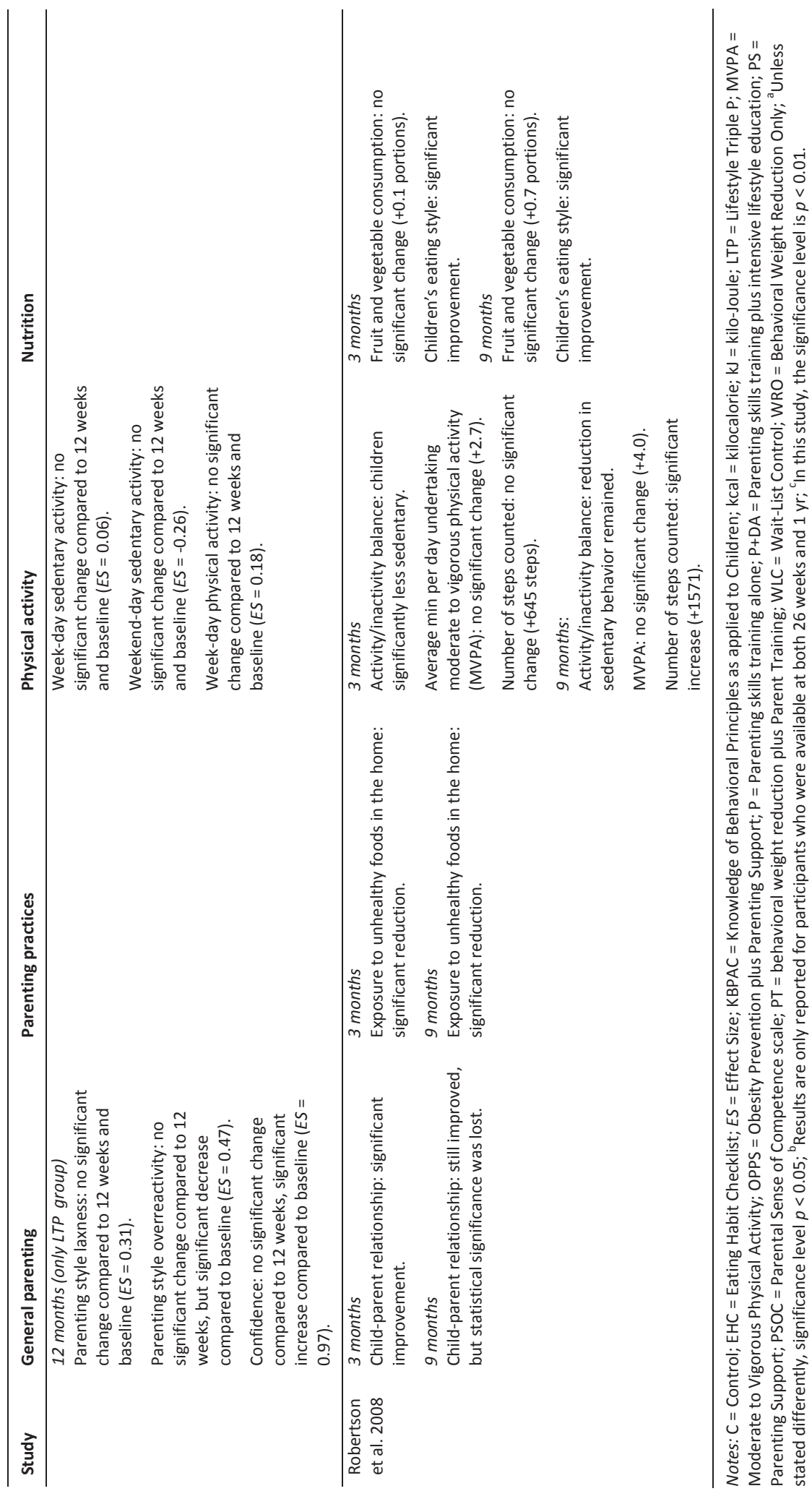




\section{Discussion}

Parenting has an important influence on the development of children's health in general (Waylen, Stallard, \& Stewart-Brown, 2008), and children's weight patterns in particular (Kitzmann et al., 2008). The current review aimed to provide an overview of interventions that address general parenting in order to prevent or treat obesity in youth. All studies showed significant small to moderate intervention effects on at least one weight-related outcome measure.

Only seven studies met the inclusion criteria for this review. However, observational studies in the field of pediatric obesity provide increasing evidence for the important role of general parenting in the development of energy balance-related behaviors and children's weight (Sleddens, Gerards et al., 2011). A reason for this apparent discrepancy may be that observational research addressing general parenting in the area of pediatric obesity has a very short history: the first study investigating parenting style as a determinant of child weight status was published in 2000 (Gable \& Lutz, 2000), and the majority of studies were published between 2007 and 2010 (Sleddens, Gerards et al., 2011). Papers on the evaluation of systematically designed interventions in this novel field would typically (and logically) follow these studies with a time-lag of several years.

As regards the publication year of the intervention studies included in our review, it is remarkable that three studies were published more than 15 years ago (1975, 1985 and 1994) (Aragona et al., 1975, Israel et al., 1994; Israel et al., 1985), whereas the other four studies were conducted recently, between 2003 and 2008 (Golley, 2005; Harvey-Berino \& Rourke, 2003; Robertson et al., 2008; West, 2007). The older studies all related to obesity treatment, whereas some of the more recent studies also focused on obesity prevention. This may reflect the early awareness of the importance of parenting among professionals working in the treatment of childhood obesity (tertiary prevention), whereas it took some years before a similar awareness arose in the area of primary and secondary prevention. Indeed, we identified several descriptive papers on ongoing preventive intervention studies (Daniels et al., 2009; Wolman, Skelly, Kolotourou, Lawson, \& Sacher, 2008; and A Vaughn, unpublished data, 2010), as well as studies registered in the International Standard RCT Number Register that report incorporating general parenting in their interventions. Furthermore, the current acknowledgment of the importance of general parenting is indicated by an increasing number of childhood prevention studies assessing parenting style as an outcome measure or as a potential moderator of the intervention effect (Golan, Kaufman, \& Shahar, 2006; Shelton, Le Gros, Norton, Stanton Cook, Morgan, \& Masterman, 2007; Stark et al., 2011; Stein, Epstein, Raynor, Kilanowski, \& Paluch, 2005). In addition, even though they did not explicitly address general parenting, we have come across studies that addressed the wider context in which parenting practices take place (for example emotional climate, e.g., Epstein, Gordy, Raynor, Beddome, Kilanowski, \& Paluch, 2001; Epstein, McKenzie, Valoski, Klein, \& Wing, 1994; Epstein, Paluch, \& Raynor, 2001; Epstein, Wing, Koeske, \& Valoski, 1985; Flodmark, Ohlsson, Rydén, \& Sveger, 1993; Golan, 2006; Golan, Fainaru, \& Weizman, 1998; Golan et al., 2006; Golan, Weizman, \& Apter, 1998; Janicke et al., 2008; Kalarchian et al., 2009; Nowicka, Hoglund, Pietrobelli, Lissau, \& Flodmark, 2008; Nowicka, Pietrobelli, \& Flodmark, 2007). 
Another reason for the observed lack of childhood obesity programs targeting general parenting may be that intervention developers are unaware or not convinced of the modifiability of general parenting. However, the intervention studies in the current review that measured parenting found large effects for this outcome measure (Golley, 2005; Israel et al., 1985; Robertson et al., 2008; West, 2007), indicating that general parenting is indeed modifiable. Research in other fields also provides evidence for the modifiability of general parenting (Spoth, Redmond, \& Shin, 1998).

We found effect sizes indicating a moderate effect on one or more outcome measures in all studies; which are considered clinically meaningful. However in a number of these studies, the changes were not statistically significant. This may also reflect issues of power and measurement precision. The results highlight that further work is needed in this area before firm conclusions can be drawn. The interventions seemed to report a relatively larger effect on general parenting. Note that general parenting is a broad concept, which determines the context of behavior-specific parenting. A positive change in this variable can affect the impact on a broad range of specific parenting practices, regarding multiple child outcomes (Kitzmann \& Beech, 2006). Changes in general parenting may therefore indicate a potentially large public health effect (Spoth et al., 1998). However, it is expected that general parenting interventions are especially effective in younger children (Stice, Shaw, \& Marti, 2006). Parental influence will decrease with advancing age of their children, which makes it more difficult to intervene with these types of interventions on older adolescents. Furthermore, it is expected that parenting behaviors of parents of older children are more difficult to change because they are more likely to perform routine behaviors.

Although the content of the intervention studies included in our review varied, they did show various similarities. They all aimed to promote a parenting style that encourages instrumental competence in children by helping them balance other-oriented, rule-following tendencies with individualistic, autonomous active thinking (Baumrind, 1978). This parenting style is typically referred to as authoritative parenting (Baumrind, 1978). Second, all interventions reviewed used group sessions for parents, meaning that they are all high-intensity interventions in which parents were seen multiple times and parental interaction played a key role. Also, all intervention studies combined components of parenting styles with lifestyle education. Two studies compared an intervention primarily focusing on general parenting with an intervention aimed at general parenting as well as physical activity and nutrition components (Golley, 2005; Harvey-Berino \& Rourke, 2003). These studies showed that interventions combining general parenting components with lifestyle components may lead to better results than interventions focusing exclusively on general parenting. Note that several studies (Harvey-Berino \& Rourke, 2003; Israel et al., 1994; Robertson et al., 2008) did not include a control condition that excluded the general parenting program, which makes the reported results difficult to interpret in terms of the 'true' impact of adding general parenting to the intervention content.

Some limitations of the studies included in the current review should be acknowledged. Most studies used self-reported outcome measures which may evoke social desirability bias. Another limitation of the included studies was that some tools to assess energy balance-related behaviors were not validated. 
Furthermore, we expected that the studies which focused on general parenting also would measure general parenting as an outcome measure. This appeared not to be the case in three studies, however. We consider this apparent lack of focus on mediating mechanisms of intervention effects as undesirable. Also, the methodological quality of the included studies varied. For example, some studies had relatively small sample sizes and a relatively short follow-up period. Some studies did not apply an RCT design, while some RCTs did not include a non-intervention control group, indicating that the study quality was heterogeneous which makes it relatively difficult to compare the studies to each other. Future studies should adopt a control condition excluding the general parenting component, and include long-term follow-up. Evaluation models should include mediating concepts such as general parenting.

The current review indicates that the promotion of authoritative parenting is a valuable addition to childhood obesity prevention and treatment. Given the lack of current intervention studies addressing general parenting, further development and testing of theory- and practice-based interventions is strongly recommended. 


\title{
CHAPTER 4
}

Physical activity parenting:

A systematic review of existing questionnaires and their associations with child activity levels

\author{
Ester FC Sleddens \\ Stef PJ Kremers \\ Sheryl O Hughes \\ Matthew B Cross \\ Carel Thijs \\ Nanne K De Vries \\ Teresia M O’Connor
} Obesity Reviews, 2012 


\begin{abstract}
Insufficient physical activity (PA) is considered a critical contributor to childhood overweight. Parents are key in influencing their child's PA through various mechanisms of PA parenting, including support, restriction of PA and facilitation of enrolment in PA classes or activities. However, study findings are difficult to compare because instruments vary in terms of constructs, psychometric assessment and type of PA assessed. The goal of the current review was to identify existing PA parenting questionnaires and report on the validation of these measures through findings of their psychometric performance and correlation to youth's PA. The search of eligible studies was restricted to instruments with multiple items. Eleven unique PA parenting questionnaires were identified, and 46 studies that used these instruments were included. Extracted data include sample characteristics, as well as type and assessment methods of parental influence and PA. Findings highlight the tremendous variation in the conceptualization and measurement of PA parenting, common use of non-validated instruments and lack of comprehensive measures. The development of theory-based PA parenting measures (preferably multidimensional) should be prioritized to guide the study of the parental role in promoting child's PA as well as the design of family-based PA interventions.
\end{abstract}




\section{Introduction}

Insufficient physical activity (PA) is widely considered an important contributor to low cardiorespiratory fitness (Parikh \& Stratton, 2011) and the overweight epidemic (Parikh \& Stratton, 2011; Riddoch et al., 2009), partly also through its relation with unhealthy dietary behaviors (Pearson \& Biddle, 2011). The development of overweight and related disorders starts at an early age, resulting from a complex interaction between genes and the environment. Formed during childhood, PA behaviors are likely to become habitual and therefore difficult to modify at a later age. Sedentary (Biddle, Pearson, Ross, \& Braithwaite, 2010) and PA behaviors (Hallal, Victora, Azevedo, \& Wells, 2006; Telama, 2009) have a tendency to track into later life. Currently, a considerable number of children fail to achieve the daily PA recommendations (Foltz et al., 2011; Li, Treuth, \& Wang, 2010) and it may be necessary to increase some children's PA and prevent the decline of other children's PA to promote healthy weight status and overall health. Identifying PA correlates is of high priority, and providing an empirical basis will benefit the development of effective PA intervention programs for children and adolescents. Sallis, Prochaska, and Taylor (2000) conducted the first comprehensive review summarizing studies assessing different correlates of youth PA behavior (i.e., demographic/biological, psychological, behavioral, social/cultural, and environmental). Several research groups have updated this review during the last decade (Biddle, Whitehead, O'Donovan, \& Nevill, 2005; Hinkley, Crawford, Salmon, Okely, \& Hesketh, 2008; Van der Horst, Chin A. Paw, Twisk, \& Van Mechelen, 2007). For a brief overview of these study findings, we refer to a recently published review providing an overview of systematic reviews to identify factors associated with children's and adolescents' PA (Biddle, Atkin, Cavill, \& Foster, 2011).

Caregivers, including parents, are in part responsible for children's PA behaviors. Parents influence their child's PA levels through various mechanisms including encouragement, social support, involvement, restriction of PA, facilitation such as provision of transportation and sport enrollment, and role modeling. Several literature reviews have assessed parental influence on activity levels in youth (e.g., Beets, Cardinal, \& Brandon, 2010; Biddle et al., 2005; Edwardson \& Gorely, 2010; Ferreira, Van der Horst, Wendel-Vos, Kremers, Van Lenthe, \& Brug, 2006; Gustafon \& Rhodes, 2006; Hinkley et al., 2008; Mitchell et al., 2011; Pugliese \& Tinsley, 2007; Sallis et al., 2000; Trost \& Loprinzi, 2011; Van der Horst et al., 2007). These studies provide evidence for the key role parents play in promoting or deterring activity levels in their offspring. Generally, parental support has been found to be positively related to PA, and active parents (especially active fathers) were more likely to have active children (Biddle et al., 2011).

Parental PA influences have been assessed with a variety of questionnaires consisting of one or more items. For instance, McGuire, Hannan, Neumark-Sztainer, Cossrow, and Story (2002) assessed the motivational aspect of parental support (i.e., encouragement) with a one-item measure. Similarly, Beets, Vogel, Forlaw, Pitetti, and Cardinal (2006) used single items to measure support but assessed multiple dimensions of this construct (i.e., instrumental, motivational, and conditional), whereas Davison, Cutting, and Birch (2003) used multiple items for assessing instrumental support. Additionally, some studies examined supportive parental behaviors as a composite score (e.g., Heitzler, Lytle, Erickson, Barr-Anderson, Sirard, \& Story, 2010; 
Trost, Sallis, Pate, Freedson, Taylor, \& Dowda, 2003), thereby losing the ability to discover unique associations between subtypes of support and activity. Instruments have also measured parental support using a generic reference to PA by including specific sports and PA together, although these domains are conceptually distinct and require different types of support to facilitate activity (Heitzler, Martin, Duke, \& Huhman, 2006). To summarize, the available instruments measuring parental influences on child PA vary in scope (i.e., constructs and type of PA assessed) and length, and may or may not use summated scale responses in their analyses. Having multiple PA parenting instruments limits the ability to compare findings across studies. Clarity on existing questionnaires and the degree to which these instruments can validly and reliably provide a detailed and comprehensive assessment of parental PA influence is needed. Therefore, the goal of the current review was to give an overview of the PA parenting questionnaires available and report on the validation of these measures through findings of the instruments' psychometric performance (validity and reliability) and correlation to youth PA (taking into account different PA contexts and assessment methods).

\section{Method}

\section{Search strategy and eligibility criteria}

MC conducted the literature search and obtained 3310 citations using PubMed $(n=2846), \operatorname{CINAHL~}(n=10)$, Scopus ( $n=296)$, PsycINFO (EBSCO) ( $n=148)$, and PsycINFO (Ovid) $(n=10)$ databases using the following search terms: exercise parenting, parental PA socialization, PA parenting (dimensions), parent (child relations) PA, and activity-related parenting. In the context of the current review, physical activity was defined as any bodily movement that requires energy expenditure, categorized as both organized (e.g., sports) and non-organized (e.g., free play) physical activities. Physical activity parenting was defined as parental behaviors intended to influence their child's PA, either to increase or decrease. The inclusion criteria were as follows: (a) studies assessing parenting behaviors regarding children's PA (i.e., PA parenting questionnaires; either parent-report, spouse reporting on the other parent, or child-report) among families with children between the ages of 2 and 18 years old, (b) studies published between January 1, 1990 and May 31, 2012 in the English language, and (c) published peer-reviewed empirical articles. Exclusion criteria were: (a) one-item PA parenting questionnaires and multiple-item PA parenting questionnaires using separate single items for their analyses, (b) instruments that focused only on restricting sedentary behaviors because sedentary behaviors and PA are conceptually different constructs, and only limited support has been found for the displacement hypothesis in which sedentary behaviors are thought to displace PA (Biddle, Gorely, Marshall, Murdey, \& Cameron, 2003; Marshall, Biddle, Gorely, Cameron, \& Murdey, 2004; Wong \& Leatherdale, 2009), (c) instruments assessing home PA environment such as equipment (e.g., jumping rope, basketball) or related factors (e.g., numbers of cars, yard size, dog ownership), (d) instruments assessing parental attitudes/beliefs regarding child PA including benefits and barriers to PA, (e) instruments assessing parents' own PA behavior without information of the child being present, (f) studies solely assessing special populations such as chronically ill, institutionalized patients, children with autism, and cancer survivors; and (g) theses, dissertations, book chapters, non peer-reviewed 
articles, conference proceedings, review articles, case studies, observational studies such as videotaped parent-child interactions and experimental studies, and qualitative studies. Instruments (i.e., multiple scales) assessing PA parenting that included items or scales on parenting regarding sedentary behavior were included in order to describe the overall instrument. To best describe the development and validation of the PA parenting questionnaires, all related articles were identified including development steps, validation and cross-validation studies, articles reporting revisions of the original questionnaire, and articles that have used the questionnaires as part of PA studies.

Duplicate citations $(n=321)$ were removed, resulting in 2989 unique citations. ES screened all titles $(N=$ 2989) of the citations for relevance. This procedure was repeated by SK, TO and SH, who independently screened one-third of the titles, such that all titles were screened by at least two authors. Any disagreement was resolved by discussion between these reviewers. Following title screening, 1956 citations were removed. Subsequently, full-texts of the remaining 1033 citations were retrieved for further screening. ES screened all 1033 abstracts, and about half were also independently screened by the coauthors; SK $(n=76)$, TO $(n=238)$, and SH $(n=187)$. Another 862 citations were removed, resulting in 171 articles for full-text inspection. In case of doubt, ES and SK discussed potential inclusion. Studies that did not meet the inclusion criteria $(n=131)$ were removed. Figure 1 displays the reasons for exclusion. Additionally, the reference lists of all studies selected for inclusion $(n=40)$ (Adkins, Sherwood, Story, \& Davis, 2004; Anderson, Mâsse, Zhang, Coleman, \& Chang, 2009/2011; Beets, Pitetti, \& Forlaw, 2007; Cleland, Timperio, Salmon, Hume, Baur, \& Crawford, 2010; Corder et al., 2011; Corder, Van Sluijs, McMinn, Ekelund, Cassidy, \& Griffin, 2010; Crawford et al., 2010; Davison, 2004; Davison et al., 2003; Davison \& Deane, 2010; Davison, Downs, \& Birch, 2006; Davison \& Jago, 2009; Davison, Li, Baskin, Cox, \& Affuso, 2011; Duncan SC, Duncan TE, \& Strycker, 2005; Edwardson \& Gorely, 2010; Fisher, Saxton, Hill, Webber, Purslow, \& Wardle, 2010; Gattshall, Shoup, Marshall, Crane, \& Estabrooks, 2008; Gubbels et al., 2011; Heitzler et al., 2010; Hennessy, Hughes, Goldberg, Hyatt, \& Economos, 2010a; Huang, Becerra, Golnari, Fernandez, Opalach, \& Andres del Valle, 2009; levers-Landis, Burant, Drotar, Morgan, Trapl, \& Kwoh, 2003; King et al., 2011; Jago, Davison, Brockman, Page, Thompson, \& Fox, 2011; Jago, Fox, Page, Brockman, \& Thompson, 2009; Kahan, 2005; Kirby, Levin, \& Inchley, 2011; Loprinzi \& Trost, 2010; McMinn, Van Sluijs, Wedderkopp, Froberg, \& Griffin, 2008; Neumark-Sztainer et al., 2010; Nickelson, Alfonso, McDermott, Bumpus, Bryant, \& Baldwin, 2011; O’Connor, Hilmers, Watson, Baranowski, \& Giardino, 2011; Paez, Maloney, Kelsey, Wiesen, \& Rosenberg, 2009; Prochaska, Rodgers, \& Sallis, 2002; Raudsepp, 2006; Timperio et al., 2008; Trost et al., 2003; Williams \& Mummery, 2011; Zecevic, Tremblay, Lovsin, \& Michel, 2010) were scanned for further relevant references. This reference tracking technique resulted in 6 additional articles appropriate for inclusion (Beets, Vogel, Forlaw, Pitetti, \& Cardinal, 2006; Cleland, Timperio, Salmon, Hume, \& Telford, 2011; Davison \& Schmalz, 2006; Ommundsen, Klasson-Heggebø, \& Anderssen, 2006; Ommundsen, Page, Ku, \& Cooper, 2008; Zabinski, Saelens, Stein, Hayden-Wade, \& Wilfley, 2003). The majority of the studies identified by reference tracking used a particular PA parenting instrument (Sallis et al., 1989; Sallis, Alcaraz, McKenzie, Hovell, Kolody, \& Nader, 1992; Sallis, Wendell, Dowda, Freedson, \& Pate, 2002; Taylor, Sallis, Dowda, Freedson, Eason, \& Pate, 2002). In total, 46 studies were considered eligible for the current review. Figure 1 summarizes the manuscript selection process. 


\section{Data extraction}

The first author (ES) extracted data from the selected studies including sample characteristics (sample size, child age, gender, ethnicity, and study location), PA parenting constructs assessed, type and assessment method of parental influence, psychometric properties of the PA parenting scales and/or subscales, association of PA parenting with child PA behaviors, and how child PA was assessed.

To give a complete overview of the questionnaire's validation, we reported on the qualitative development of the instruments (e.g., focus group interviews, expert meetings) and range of available indicators for appropriate validation (e.g., factor analysis, internal consistency, test-retest reliability, inter-correlations between an instrument's subscales). We accounted for potential methodological aspects affecting the PA parenting - child PA relationship (e.g., reporting PA parenting associations with boys' and girls' PA separately, if available). 


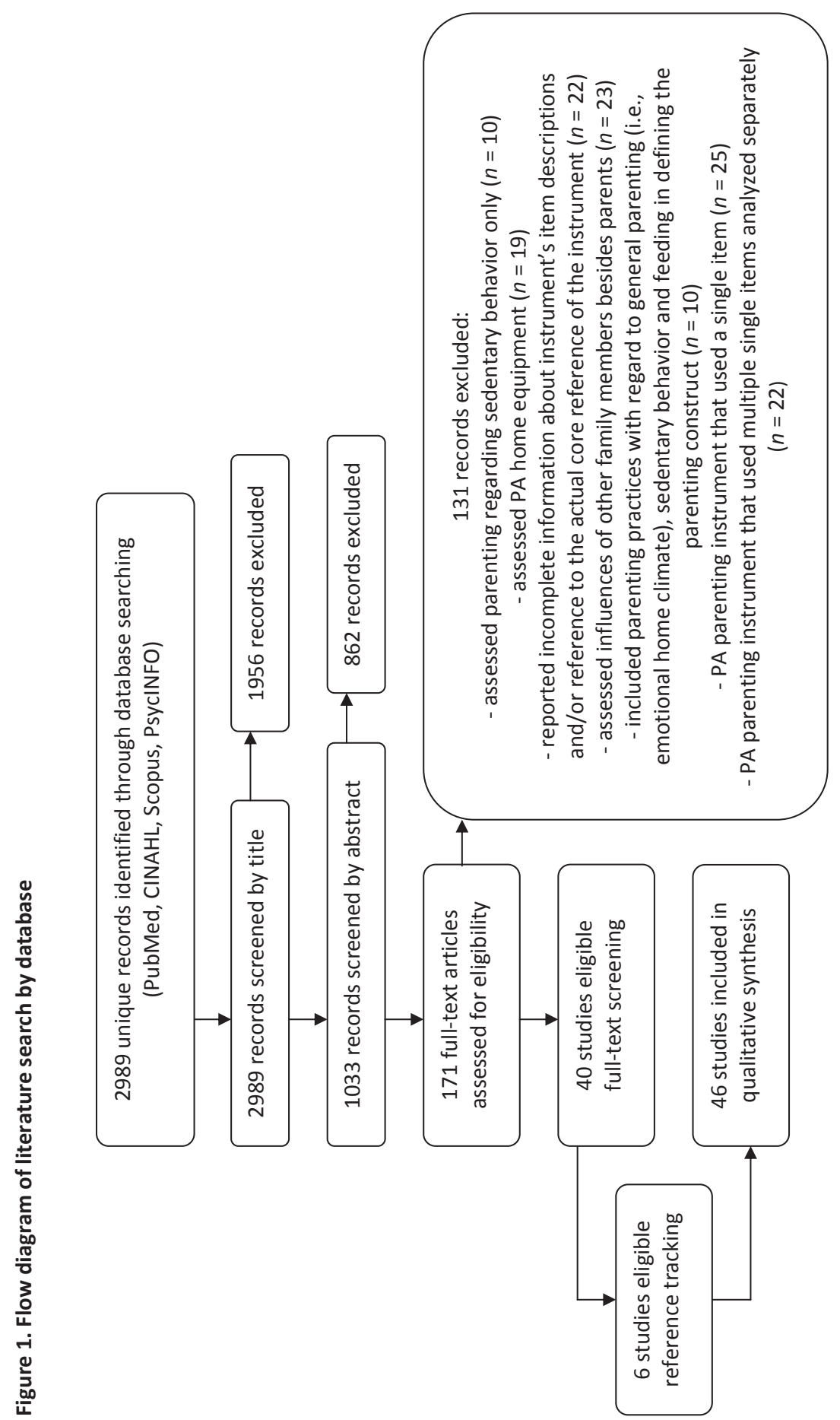




\section{Results}

Forty-six studies were eligible for inclusion (Adkins et al., 2004; Anderson et al., 2009/2011; Beets et al., 2007; Beets et al., 2006; Cleland et al., 2010/2011; Corder et al., 2010/2011; Crawford et al., 2010; Davison, 2004; Davison et al., 2003/2006/2011; Davison \& Deane, 2010; Davison \& Jago, 2009; Davison \& Schmalz, 2006; Duncan et al., 2005; Edwardson \& Gorely, 2010; Fisher et al., 2010; Gattshall et al., 2008; Gubbels et al., 2011; Heitzler et al., 2010; Hennessy et al., 2010a; Huang et al., 2009; levers-Landis et al., 2003; Jago et al., 2009/2011; Kahan, 2005; King et al., 2011; Kirby et al., 2011; Loprinzi \& Trost, 2010; McMinn et al., 2008; Neumark-Sztainer et al., 2010; Nickelson et al., 2011; O'Connor et al., 2011; Ommundsen et al., 2006/2008; Paez et al., 2009; Prochaska et al., 2002; Raudsepp, 2006; Timperio et al., 2008; Trost et al., 2003; Williams \& Mummery, 2011; Zabinski et al., 2003; Zecevic et al., 2010), representing studies using eleven unique questionnaires that measured some aspect of PA parenting. Table 1 provides a complete overview of the different constructs, definitions, and items of the included instruments clustered by type of PA parenting questionnaire (starting with the earliest instrument).

\section{Study characteristics}

Table 2 shows the study characteristics (i.e., study year, sample size, age, gender, ethnicity, and country). The majority of the included studies were conducted in the United States (US; $n=26$ ), followed by the United Kingdom (UK; $n=7)$, Australia $(n=6)$, Estonia, Norway and Denmark $(n=2)$, and Canada, Scotland, the Netherlands, and Portugal $(n=1)$. One study (Ommundsen et al., 2008) included study populations from four countries: Estonia, Norway, Denmark and Portugal. Samples from the US consisted of different ethnic minorities, including participants with Hispanic, African-American, American Indian and Asian backgrounds, although the majority was predominantly white. Sample sizes of the included studies ranged from 52 to almost 4000, representing the absolute number of caregivers and/or children who participated in the study. More girls than boys were included in the studies largely because some studies selected only girls for inclusion (Adkins et al., 2004; Beets et al., 2007; Davison et al., 2003; Davison \& Deane, 2010; Davison et al., 2006; Davison \& Jago, 2009; levers-Landis et al., 2003; Neumark-Sztainer et al., 2010). Mean child age ranged from 3.7 to 15.8 years. 


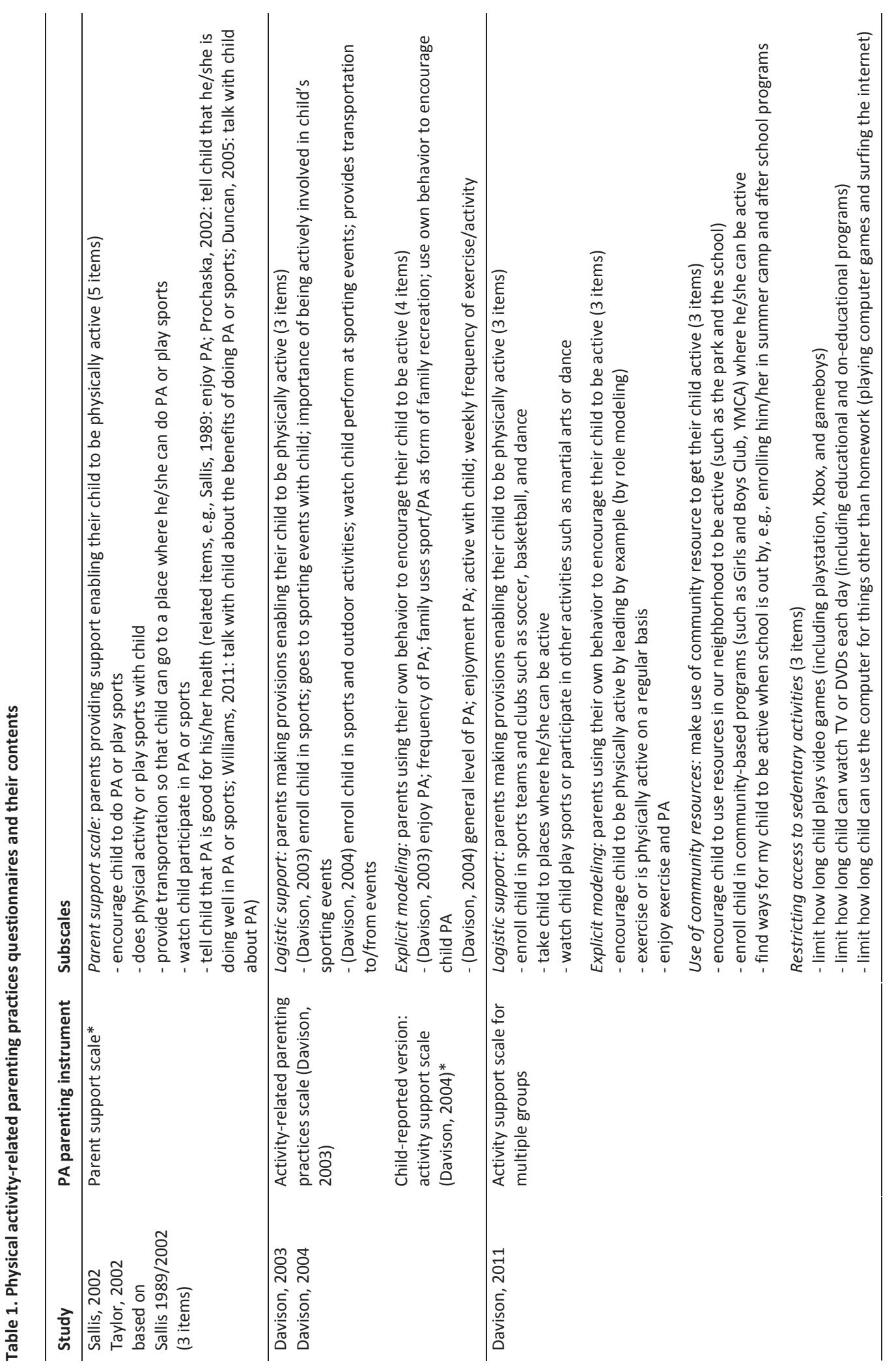




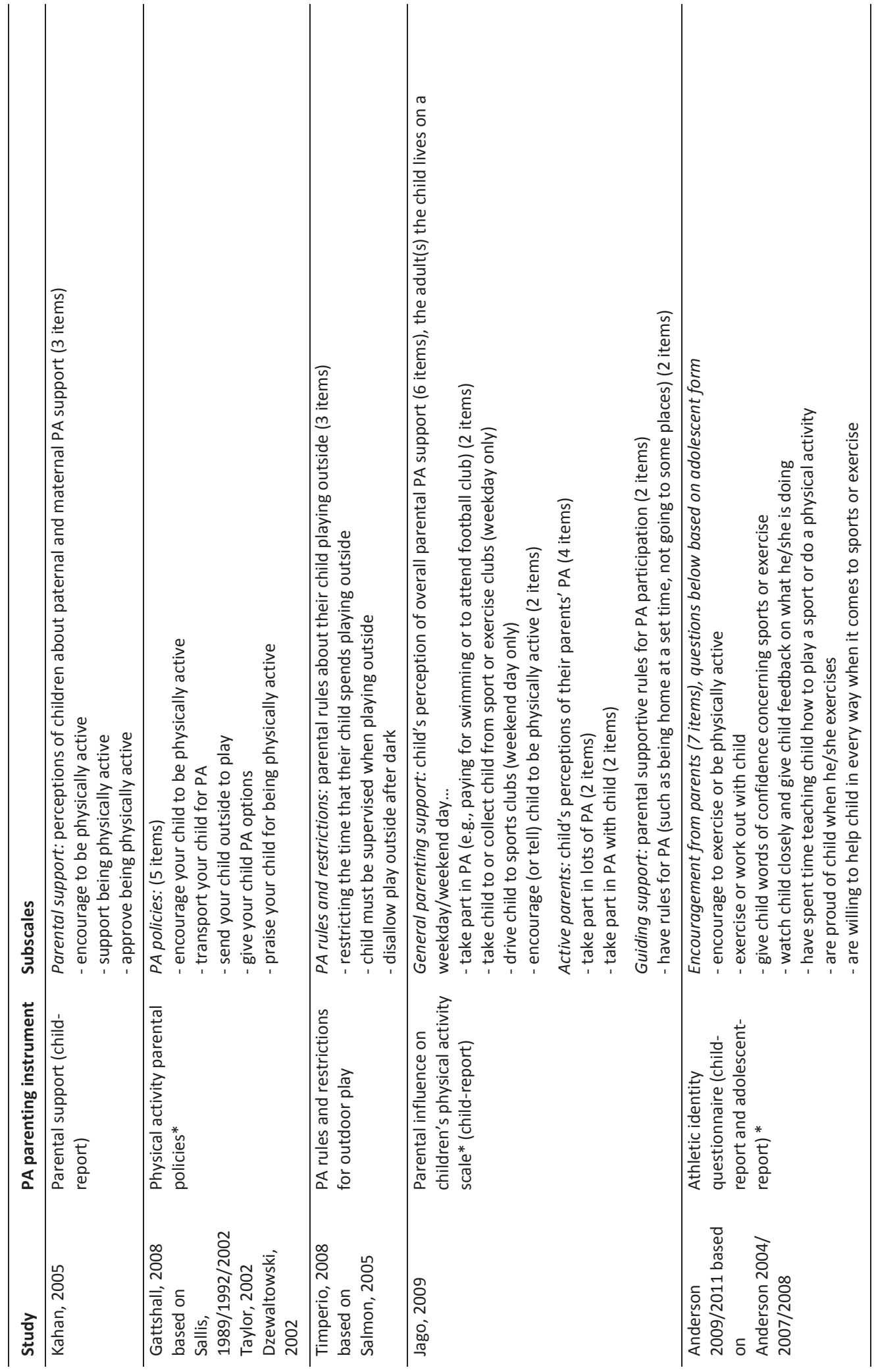




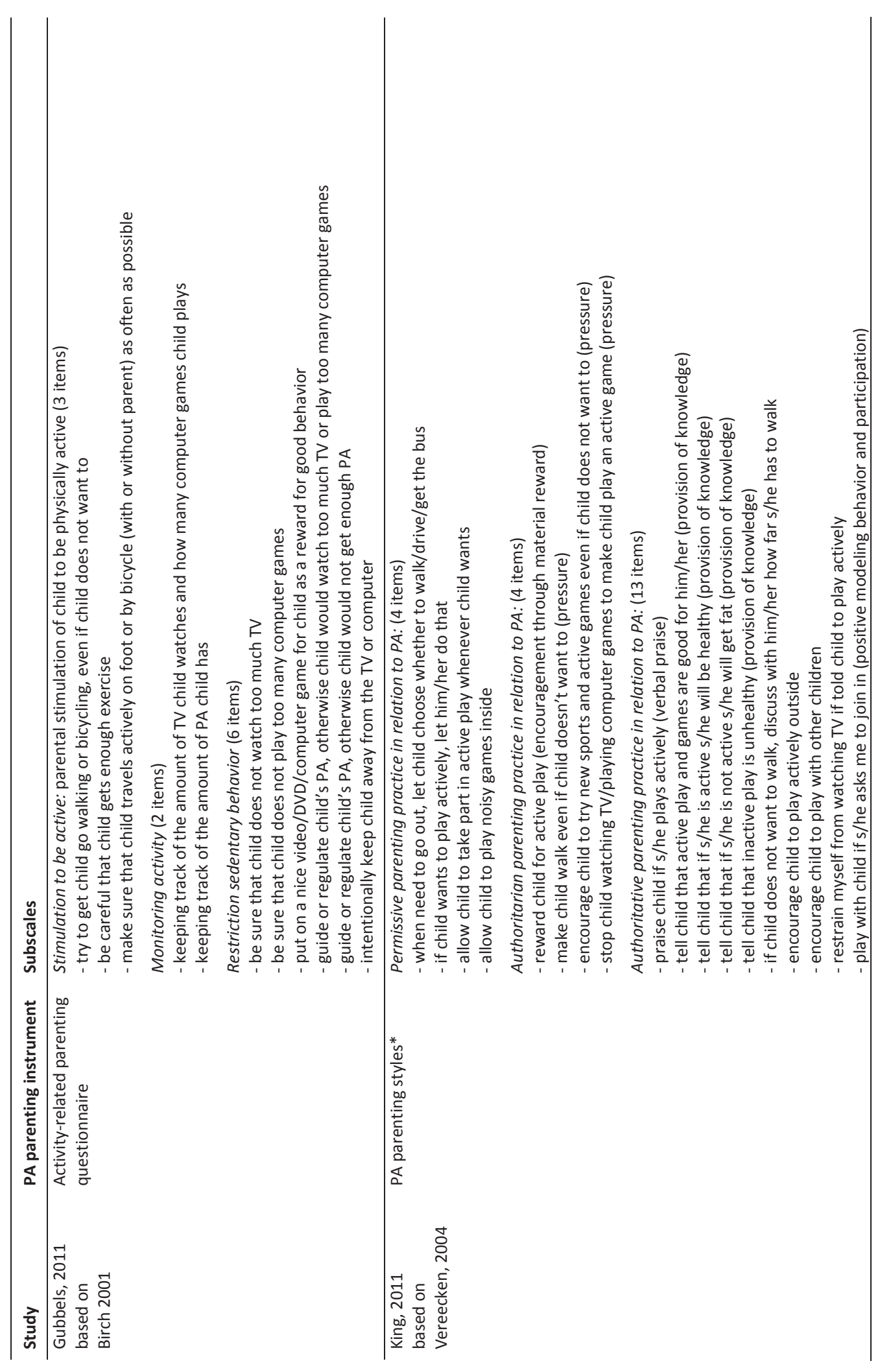




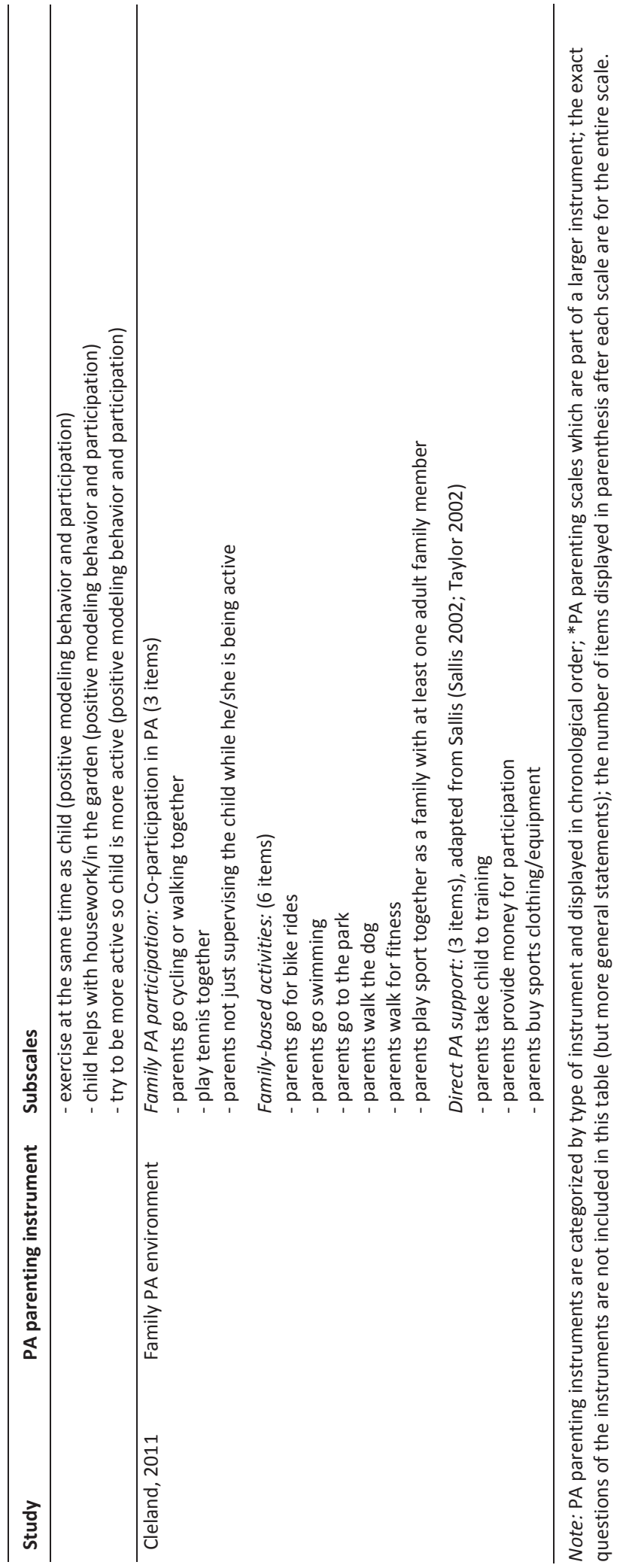




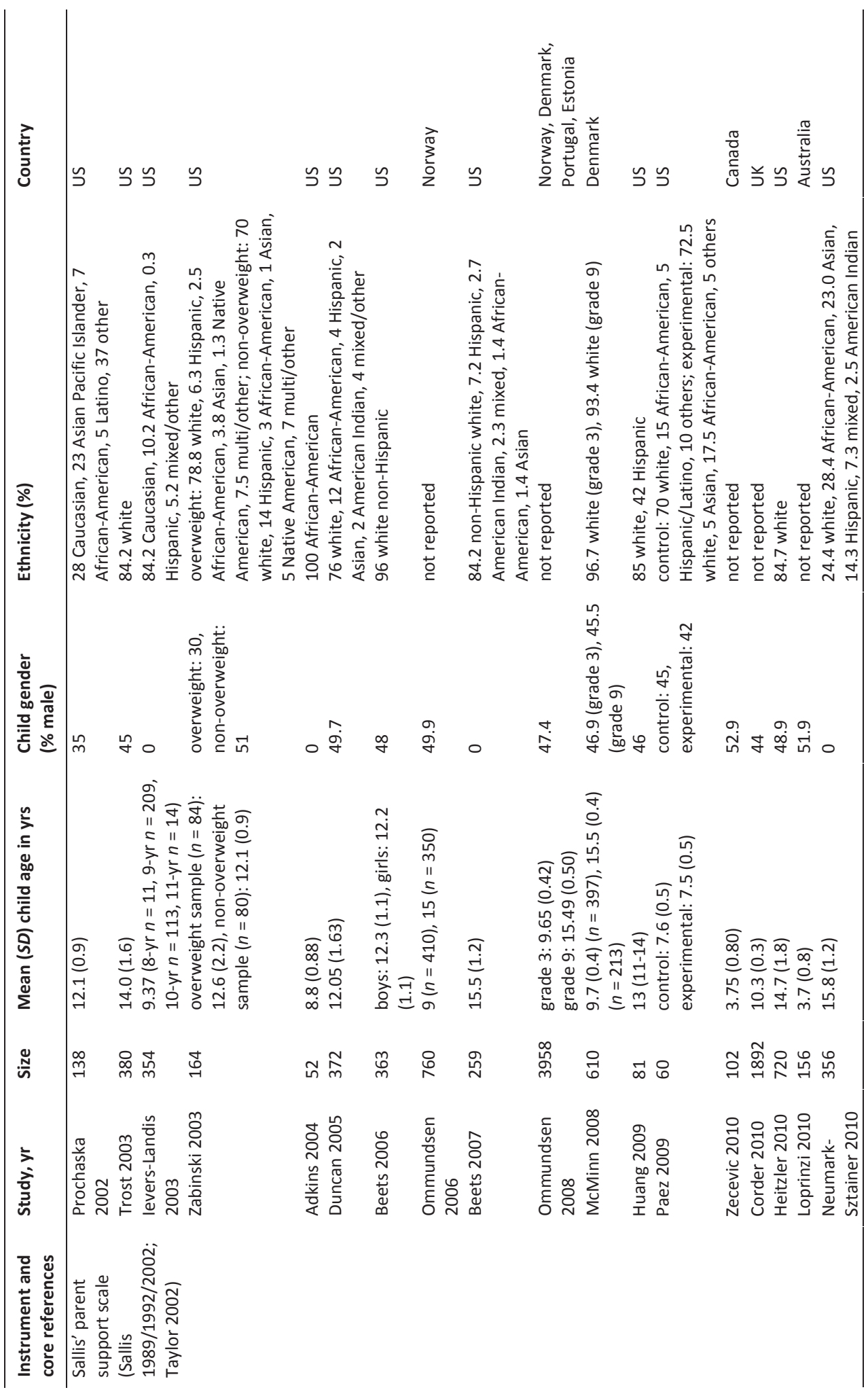




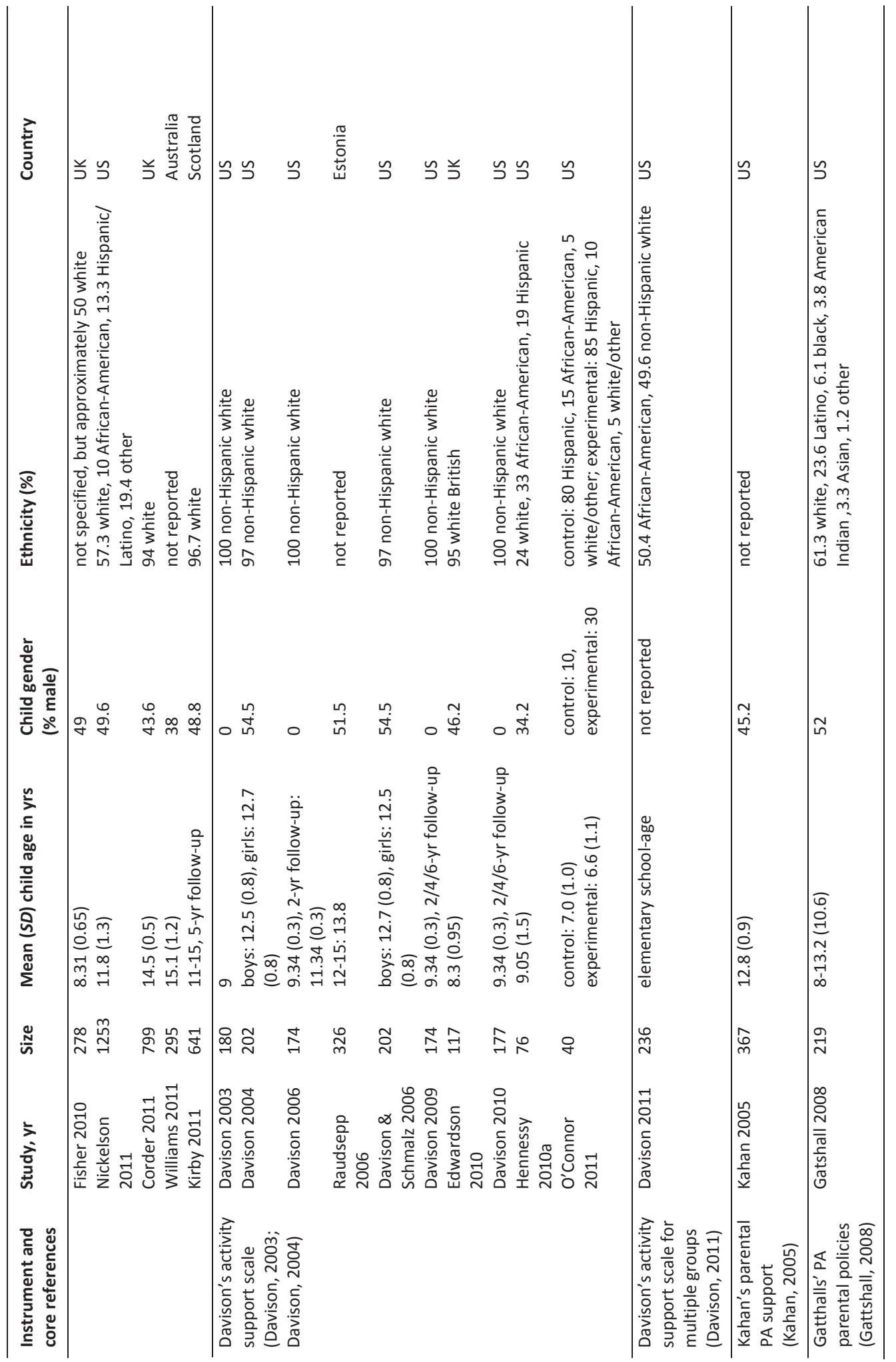




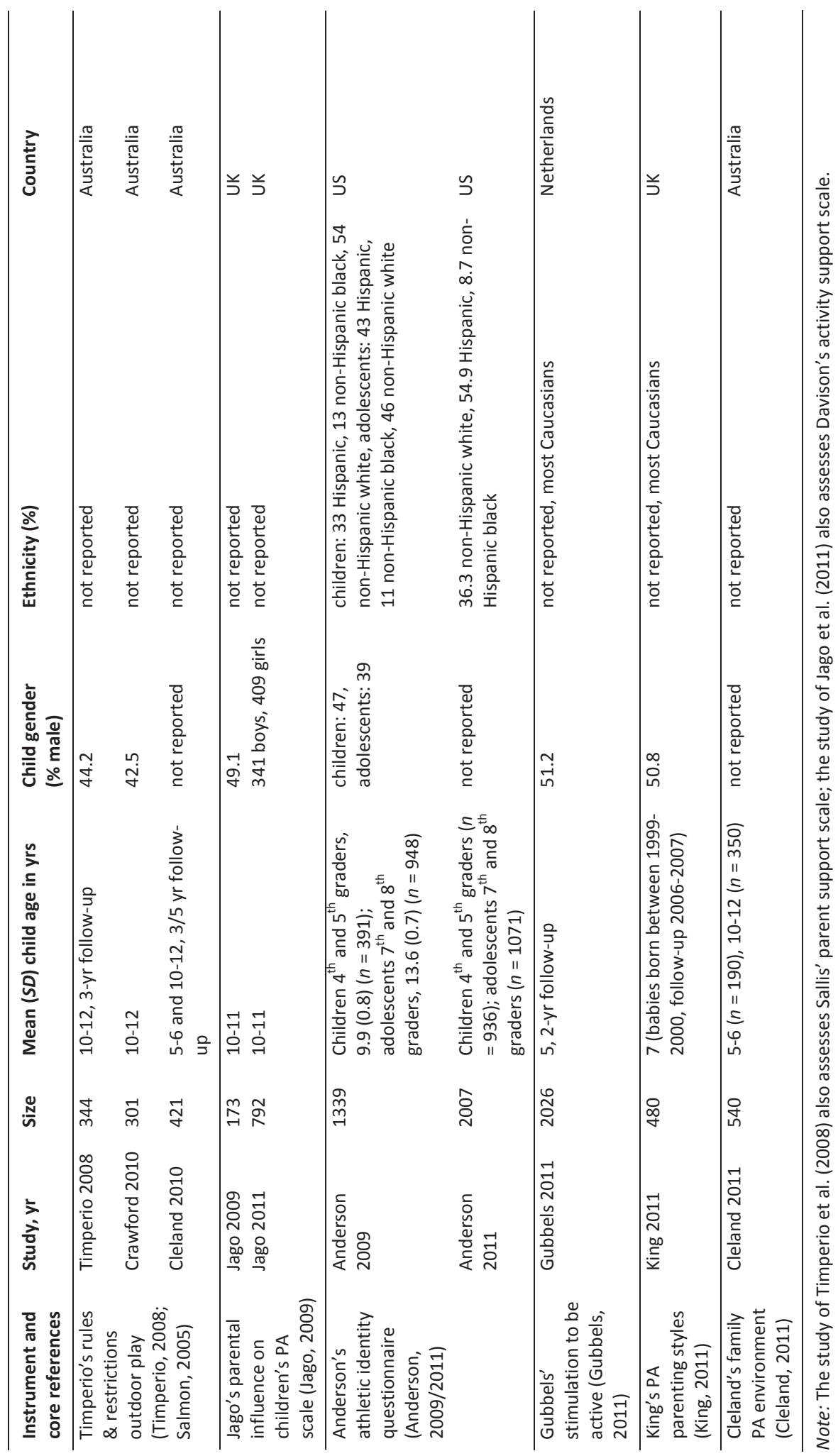




\section{Findings per PA parenting instrument}

Below, we give an overview of development and validation steps of the instruments. Additionally, we report on associations of the main constructs of the identified PA parenting questionnaires and child PA levels (see also Table 3 for a brief overview of these findings and the Supplement on page 112-132 for an in-depth description of the measured variables/outcomes and complete overview of the study findings).

\section{Sallis' parent support scale}

Development and validation. The Parent Support Scale is a 5-item survey questionnaire on parental support for child activity (e.g., 'encourage child to do PA or play sport' and 'watch child participate in PA or sports') reported by Sallis et al. (2002) and Taylor et al. (2002), which has frequently been used in later studies. The original questionnaire consisted of 19 items measuring family supporting influences on child PA levels, including 5 items each about mother's and father's support (assessed separately), and the 9 remaining items assessing support provided by siblings and other family members (Sallis et al., 2002; Taylor et al., 2002). The instrument has been administered as a parent-report survey (Sallis et al., 2002) or a child-report survey (Taylor et al., 2002). Through reference tracking, we retrieved the original articles from 1989 (Sallis et al., 1989) and 1992 (Sallis et al., 1992) that described the precursors of the 'family support scale,' consisting of three items. Detailed information about the exact development of the scale was lacking. The earliest article (Sallis et al., 1989) described that the selection of items originated from operant and social learning theories (Bandura, 1977; Skinner, 1953), and previous research that identified associations between parental influences, including PA encouragement, support, and modeling, and child PA (Dishman, Sallis, \& Orenstein, 1985; Gottlieb \& Baker, 1986). Pretests were conducted to test comprehensiveness of the items (Sallis et al., 2002; Taylor et al., 2002). Internal reliability coefficients for these scales were 0.78 and 0.81 , and Intra-Class Correlation (ICC) coefficients were 0.81 and 0.88 for the studies of Sallis et al. (2002) and Taylor et al. (2002), respectively. In both studies principal components analyses were conducted to check for unidimensionality. We focus on the 'parental support scale,' as described in Table 1. We identified 24 additional studies using this scale (Adkins et al., 2004; Beets et al., 2006/2007; Corder et al., 2010/2011; Davison \& Schmalz, 2006; Duncan et al., 2005; Fisher et al., 2010; Heitzler et al., 2010; Huang et al., 2009; levers-Landis et al., 2003; Kirby et al., 2011; Loprinzi \& Trost, 2010; McMinn et al., 2008; Neumark-Sztainer et al., 2010; Nickelson et al., 2011; Ommundsen et al., 2006/2008; Paez et al., 2009; Prochaska et al., 2002; Timperio et al., 2008; Trost et al., 2003; Williams \& Mummery, 2011; Zabinski et al., 2003; Zecevic et al., 2010). Half of these studies reported scale reliability estimates (Beets et al., 2007; Corder et al., 2011; Heitzler et al., 2010; levers-Landis et al., 2003; Loprinzi \& Trost, 2010; McMinn et al., 2008; Neumark-Sztainer et al., 2010; Nickelson et al., 2011; Ommundsen et al., 2006; Prochaska et al., 2002; Zabinski et al., 2003; Zecevic et al., 2010), ranging from 0.65 to 0.85 .

Association with child PA. The 19 studies that assessed associations of the scale with child's PA (Adkins et al., 2004; Beets et al., 2006/2007; Corder et al., 2010/2011; Duncan et al., 2005; Fisher et al., 2010; Heitzler et al., 2010; levers-Landis et al., 2003; Kirby et al., 2011; Loprinzi \& Trost, 2010; McMinn et al., 2008; Ommundsen et al., 2006/2008; Paez et al., 2009; Prochaska et al., 2002; Trost et al., 2003; Williams \& 
Mummery, 2011; Zecevic et al., 2010), all except one (Kirby et al., 2011) cross-sectional, used a variety of child PA assessments, including parent-reports (Loprinzi \& Trost, 2010; Zecevic et al., 2010), child-reports (Adkins et al., 2004; Beets et al., 2006/2007; Corder et al., 2010/2011; Duncan et al., 2005; levers-Landis et al., 2003; Kirby et al., 2011; Ommundsen et al., 2006; Prochaska et al., 2002; Trost et al., 2003; Williams \& Mummery, 2011), pedometers (Duncan et al., 2005), accelerometers (Adkins et al., 2004; Corder et al., 2010/2011; Fisher et al., 2010; Heitzler et al., 2010; Loprinzi \& Trost, 2010; McMinn et al., 2008; Ommundsen et al., 2008; Paez et al., 2009; Prochaska et al., 2002), and physical fitness (Beets et al., 2007). Positive relationships were found among the whole sample or some sub-groups in 15 studies (Adkins et al., 2004; Corder et al., 2010/2011; Fisher et al., 2010; Heitzler et al., 2010; levers-Landis et al., 2003; Kirby et al., 2011; Loprinzi \& Trost, 2010; McMinn et al., 2008; Ommundsen et al., 2006/2008; Prochaska et al., 2002; Trost et al., 2003; Williams \& Mummery, 2011; Zecevic et al., 2010), another 15 reported no associations with child PA in the whole sample or some sub-groups (Beets et al., 2006/2007; Corder et al., 2010; Duncan et al., 2005; Fisher et al., 2010; Heitzler et al., 2010; levers-Landis et al., 2003; Kirby et al., 2011; Loprinzi \& Trost, 2010; McMinn et al., 2008; Ommundsen et al., 2006/2008; Paez et al., 2009; Prochaska et al., 2002; Zecevic et al., 2010), whereas one study reported a negative association with active commuting to and from school (i.e., higher score indicating more active commuting) only (Ommundsen et al., 2006). These findings seem to differ depending on child gender (Fisher et al., 2010; Kirby et al., 2011), maternal or paternal PA parenting practice (Kirby et al., 2011), method of PA measurement (parent- or child-reported and/or objectively assessed child PA) and parenting support being assessed as part of a larger multivariate model (Beets et al., 2006/2007; Corder et al., 2010; Duncan et al., 2005; Fisher et al., 2010; Heitzler et al., 2010; levers-Landis et al., 2003; Loprinzi \& Trost, 2010; Ommundsen et al., 2006; Prochaska et al., 2002; Trost et al., 2003; Zecevic et al., 2010) (see Table 3). Two studies did not use composite support scores in their analyses (Paez et al., 2009; Williams \& Mummery, 2011). Paez et al. (2009) dichotomized subjects into low and high parental support groups and found that mean child MVPA levels were not significantly different between the two groups. Williams and Mummery (2011) classified subjects into three parent support groups; mothers classified into the moderate and highest supportive group were found to be more likely to have sufficiently active children compared to low supportive mothers. Ommundsen et al. (2008) dichotomized child PA. Higher mean levels for the high PA group were retrieved for parental support (composite scores of 3 items) but not for parental encouragement (composite score of 2 items). Two studies (Corder et al., 2010/2011) used accelerometry and perceptions about child PA to define PA awareness groups, from subjects who accurately reported, overestimated, or underestimated activity levels. In general, those who accurately reported their activity levels had higher parent support scores.

\section{Davison's activity support scale and activity support scale for multiple groups}

Development and validation. Davison et al. (2003) developed the 7-item 'activity-related parenting practices scale' in 2003. Mothers and fathers of 180 7-year-old non-Hispanic white girls taking part in a longitudinal observational study of health and development participated in the piloting phase of this study. Low internal consistency led the researchers to add extra items. The same parents completed the revised 
version when girls were 9 years old (Davison et al., 2003). Factor analysis identified two conceptually distinct factors: logistic support (3 items: making provisions enabling children to be physically active) and explicit modeling (4 items: using own behavior to encourage children to be active), assessed separately for mothers and fathers, for which acceptable model fit was reported. Cronbach's alphas for the scales ranged from 0.61 to 0.75 . Mean scores of the two scales were positively correlated, and both mean scores of fathers and mothers for logistic support and explicit modeling were significantly correlated. In 2004, Davison et al. developed a child-report version of the instrument; the 'activity support scale' (ACTS). The instrument comprised 27 items, of which maternal and paternal PA support was assessed by 14 items: 3 items on logistic support (reported separately for each parent), and 4 items on explicit modeling (reported separately for each parent). The remaining 13 items of this child-reported version assessed constructs beyond the scope of our review (general familial support, peer support, and sibling support). Similar to the original reporting of the 'activity-related parenting practices scale' (Davison et al., 2003), acceptable fit was reported for both scales following confirmatory factor analysis. Cronbach's alphas ranged from 0.74 to 0.76 and all sources of support (the two scales for mothers and fathers) were positively correlated. Recently, the applicability of the ACTS was extended to African-American parents of elementary school-aged children, the 'activity support scale for multiple groups' (ACTS-MG) (Davison et al., 2011). Its modification started by conducting five focus groups with 27 parents to assess for cultural relevance, clarity, and completeness of the items covering the topic of PA parenting. This method resulted in the addition and revision of items, and the development of two new scales named 'use of community resources' and 'restricting access to sedentary activities.' Thereafter, a group of African-American and non-Hispanic white parents completed the questionnaire. Model fit for the groups were acceptable to good, and results supported the factorial invariance across ethnicity. The four scales were internally consistent (Cronbach's alphas ranging from 0.69 to 0.88$)$. The correlations of mean scores of all scales were significant and positive. We identified nine additional studies using one of Davison's PA parenting measures (Davison et al. 2006; Davison \& Deane, 2010; Davison \& Jago, 2009; Davison \& Schmalz, 2006; Edwardson \& Gorely, 2010; Hennessy et al., 2010a; Jago et al., 2011; O’Connor et al., 2011; Raudsepp, 2006). All but one (O'Connor et al., 2011) reported internal reliability estimates of their samples, ranging from 0.55 for explicit modeling (Nennessy et al., 2010) to 0.86 for one of the subscales (not reported which one) (Edwardson \& Gorely, 2010).

Association with child PA. All studies provided support for the positive relationship between some aspects of parental support (i.e., logistic support, explicit modeling and/or total parental support scale) and child PA (Davison, 2004; Davison et al., 2003/2006; Davison \& Jago, 2009; Davison \& Schmalz, 2006; Edwardson \& Gorely, 2010; Hennessy et al., 2010a; Jago et al., 2011; Raudsepp, 2006), including a longitudinal cohort study of girls (Davison et al., 2006; Davison \& Jago, 2009). Those studies used either the 'activity-related parenting practices scale' (Davison et al., 2003/2006; Davison \& Jago, 2009; Edwardson \& Gorely, 2010; Hennessy et al., 2010a; Raudsepp, 2006) or the child-report version of the instrument (Davison, 2004; Davison \& Schmalz, 2006; Jago et al., 2011). Findings differ depending on child gender and type of caregiver (i.e., mother or father) applying the PA parenting practice. Raudsepp (2006) found that maternal and paternal logistic support and explicit modeling were related to child-reported PA in both boys and girls among 326 parents of 12 to 15 year olds. Edwardson and Gorely (2010) did not find any significant 
relationships among 117 parents of 7 to 10 year olds, with the exception of the positive relationship between paternal explicit modeling and MVPA/VPA in boys. Hennessy et al. (2010) only found that logistic support was positively related to child PA. Jago et al. (2011) also found explicit modeling was unrelated to child PA, while maternal logistic support was related to PA in girls only (2011). This finding concurs with Davison's et al.'s earlier study (23) among a sample of parent-daughter dyads. Paternal logistic support was not related to PA in girls (Davison et al., 2003; Jago et al., 2011) but was related to PA in boys (Jago et al., 2011). In contrast to logistic support, paternal explicit modeling was related to girls PA, whereas no association was found between maternal explicit modeling and girls PA (Davison et al., 2003). Studies using different categorizations of PA levels reported mixed results (Davison, 2004; Davison \& Jago, 2009; Edwardson \& Gorely, 2010), with either no differences between activity groups in mean levels of parental support (Edwardson \& Gorely, 2010), or some significant results depending on child gender and type of caregiver (Davison, 2004) or developmental period (age 9 to 15) (Davison, 2009). Three studies classified subjects into different levels of parental support groups (including both logistic support and explicit modeling) (Davison, 2004; Davison et al., 2003; Hennessy et al., 2010a). All found that children in families applying high levels of support were more physically active than those in families applying low levels of support. In Hennessy et al. (2010) this was only the case for MPA and MVPA. Similarly, the composite score of parental support was correlated with higher activity levels among children (Davison, 2004; Davison et al., 2006; Davison \& Schmalz, 2006), but Edwardson and Gorely (2010) found no association in either boys or girls. To date, no studies have used the ACTS-MG for assessing the relationship between PA parenting and child activity levels.

\section{Kahan's parental PA support}

Development and validation. Kahan's 3-item child-reported scale (2005) distinguished between maternal and paternal support of, encouragement of, and approval of their children's PA behavior, based on previous work (Taylor, Baranowski, \& Sallis, 1994). Factor analysis confirmed the parental support scale, distinguishing between maternal and paternal support. Cronbach's alphas for the maternal support and paternal support scales, both consisting of 3 items reported separately for each parent, were 0.79 and 0.82 , respectively, in a sample of 367 adolescents.

Association with child PA. Associations with child PA have not been reported.

\section{Gatthalls' PA parental policies}

Development and validation. The 5-item 'PA parental policies' scale is part of a larger survey consisting of 126 items divided into 10 scales with different constructs (Gattshall et al., 2008). Before designing the instrument, a theoretical framework was developed using Golan's model of environmental influence (Golan \& Weizman, 2001) by extending Golan's model to include the physical and social home environment related to food and PA as concepts affecting child body mass index (BMI). The PA parental policies scale items were based on adaptations from Sallis' 'parental support scale' (Sallis et al., 2002; Taylor et al., 2002) 
and a measure developed by Dzewaltwoski and Ryan (2002). Cronbach's alpha for the scale was 0.79, and test-retest reliability was 0.80 . Inter-rater reliability, assessed by having both parents complete the questionnaire, was modest $(r=0.24)$.

Association with child PA. Only one study reported on the association of this scale and child PA (Gattshall et al., 2008). Significant positive associations were reported with child PA as measured by accelerometry (total minutes of MVPA per day; $r=0.21, p<0.01)$ and parent report of child PA $(r=0.16, p<0.05)$.

\section{Timperio's rules and restrictions for outdoor play}

Development and validation. In 2008, Timperio et al. (2008) reported a 3-item 'parental rules and restrictions' scale for outdoor play (see Table 1). This instrument was based on Salmon et al.'s (Salmon, Timperio, Telford, Carver \& Crawford, 2005) 'rules and restrictions' scale used to assess parental behavior related to their child screen-based behaviors. No reports about the qualitative development of the instrument are available. The questionnaire was used in two later studies (Cleland et al., 2010; Crawford et al., 2010), the latter one (Cleland et al., 2010) using only the sum of two items.

Association with child PA. Two studies (Cleland et al., 2010; Crawford et al., 2010) have assessed relationships with child activity levels. The first $(N=301)$ (Crawford et al., 2010) indicated positive relationships with MVPA in girls only as assessed by accelerometry, whereas the latter study $(N=421)$ (Cleland et al., 2010) found no significant relationships with child PA as assessed by parent-report of child PA in a 5-year longitudinal study.

\section{Jaqo's parental influence on children's physical activity scale}

Development and validation. In 2009, Jago et al. (2009) reported the development and validation of the 'parental influence on children's PA scale.' Seventeen focus groups aimed at discovering strategies parents use to help their children be more physically active were conducted with 113 primary school aged children (10 to 11 years of age) (Brockman, Jago, Fox, Thompson, Cartwright, \& Page, 2009). Findings indicated that parents influence child PA behavior through encouragement of PA, with low SES families relying more on verbal encouragement and middle and high SES families relying more on logistical support, financial support, co-participation and modeling. The first author (Jago et al., 2009) generated items that captured all issues addressed in the focus group interviews, and expert meetings with the co-authors resulted in a version used in a pilot test. Because children who participated in the focus groups reported that the caregivers with whom they live differ for weekdays and weekend days, the measure accommodates for this difference (Crawford et al., 2010; Jago et al., 2011). The 'parental influence on PA scale' consists of 4 subscales: general parenting support, active parents, past parental activity, and guiding support (see Table 1). The construct of past parental activity was excluded from our review, as it does not reflect our definition of PA parenting practice. Reported Cronbach's alphas were 0.83 for general parenting support, 0.84 for active parents, and 0.82 for guiding support (Jago et al., 2009). ICCs for all items ranged from 0.60 to 0.80 . 
Inter-correlations between the scales only showed significant results for the relationship between general parenting support and active parents $(r=0.26, p<0.01)$.

Association with child PA. Two studies have evaluated the association with objectively measured PA via accelerometers in UK schoolchildren aged 10 to 11 years. The active parents scale (characterized by participation in high amount of PA, and taking part in PA with the child) was positively related to light intensity PA in 173 children ( $r=0.18, p=0.04$ ) in one study (Jago et al., 2009). For the other scales, no significant relationships with child PA were found, nor for boys and girls when analyzed separately. In the second study ( $N=792$ ) (Jago et al., 2011), a higher level of guiding support was associated with higher daily MVPA in girls only.

\section{Anderson's athletic identity questionnaire}

Development and validation. Anderson et al. developed the 40-item Athletic Identity Questionnaire (AIQ) child-report in 2007 (Anderson, Mâsse, \& Hergenroeder, 2007) and adolescent-report in 2008 (Anderson \& Coleman, 2008), consisting of four dimensions: appearance, competence, importance of activity, and encouragement from family, friends and teachers/other adults. However, both studies measured family encouragement instead of parent encouragement, and were therefore excluded from this review. In 2009 and 2011, other studies conducted by Anderson et al. were published (Anderson et al., 2009/2011) that assessed encouragement from parents, not other family members, and were therefore included in the current review. The parent PA encouragement scale from the AIQ, applicable for the current review, included 7 items (Anderson et al., 2009/2011).

The first formative study for the AIQ was published in 2004, when Anderson reported on the development and validation of the questionnaire (21 items total, 4 items for the family encouragement scale) in adults (Anderson, 2004). The questionnaire was based on Anderson's model of athletic identity (Anderson, 2004) including the four dimensions. Validation studies, using confirmatory methods, have supported this structure in adults and its relation with PA (Anderson, 2004). After testing the hypothesized factorial dimensions and rewording of items for adolescents (Anderson et al., 2007) and children (Anderson \& Coleman, 2008), a 40-item questionnaire was developed. Among two multi-ethnic samples of adolescents (Anderson et al., 2007) and children (Anderson \& Coleman, 2008), the factorial and construct validity of the parent encouragement scale was supported.

Association with child PA. Associations with child and adolescent PA were reported in 2009 (Anderson et al., 2009). Significant positive effects were found for encouragement from parents and children's PA, but not adolescent's PA. No significant associations with child or adolescent team sport participation were found. 


\section{Gubbels' activity-related parenting questionnaire}

Development and validation. Gubbels et al. (2011) adapted the Child Feeding Questionnaire (CFQ) (Birch, Fisher, Grimm-Thomas, Markey, Sawyer, \& Johnson, 2001) for applicability in the activity context, the 'activity-related parenting questionnaire,' and assessed it among a sample of parents of 5-year-old children ( $N=2026)$. It consists of three scales: 'stimulation to be physically active' ( 3 items), 'monitoring activity' (2 items), and 'restriction of sedentary behavior' ( 6 items). Internal consistency estimates were $0.57,0.65$, and 0.59 for the three scales, respectively. The 'stimulation to be active' scale was based on the 'pressure to eat' scale in the CFQ (Birch et al., 2001) but then framed to include activity behavior.

Association with child PA. To date, no other studies have used the 'activity-related parenting questionnaire.' Gubbels et al. (2011) indicated that children who were stimulated to be physically active had higher levels on the parent-reported activity style construct (characterized by high levels of activity and low frequency of sitting down quietly) ( $B=0.06, p<0.05$, as main effect in a multivariate model). Stimulation to be active was also positively associated with parent-reported child PA $(B=0.12, p<0.001)$. Children whose access to sedentary behaviors was restricted had lower levels on the activity style construct ( $B=-0.13, p<0.001$, as main effect in a multivariate model) (65). This scale was also negatively associated with parent-reported child PA $(B=-0.19, p<0.001)$. No association was reported with child activity style or PA for parental monitoring of PA (Gubbels et al., 2011) (see Table S1 for complete results).

\section{King's PA parenting styles}

Development and validation. King et al. (2011) developed the PA parenting styles questionnaire, measuring authoritative (13 items), authoritarian ( 4 items) and permissive (4 items) forms of PA parenting (see Table 1). The instrument was adapted from Vereecken et al. (Vereecken, Keukelier, \& Maes, 2004), who focused on parenting in regard to feeding. Vereecken's parenting feeding items originated from scientific literature and discussion with a small group of parents of preschool-aged children. King et al. (2011) presented no information about the validation of the instrument or for its applicability to an older age group.

Association with child PA. Only the original study has reported on the scale's association with children's PA (2011) among 7-year-olds $(N=480)$. No significant associations were found with child PA as measured by accelerometry.

\section{Cleland's family PA environment}

Development and validation. The 'family PA environment' instrument developed by Cleland et al. (2011) consists of five scales. Three scales were eligible for the current review: family PA participation, familybased activities, and direct PA support. The role modeling scale (i.e., parents' self-reported PA and parent reported PA of their spouse) was beyond the scope of our review not reflecting PA parenting. Additionally, the one-item scale of PA reinforcement was excluded. The measure was partially based on key constructs from the Family Influence Model (Kimiecik \& Horn, 1998; Kimiecik, Horn, \& Shurin, 1996), which considers 
family behavior one of the fundamental influences on children's behaviors (e.g., operationalized as participation in PA with family members). The direct support scale is adapted from Sallis et al.'s (Sallis et al., 1989/1992/2002; Taylor et al., 2002) PA support scale. The only validation reported for this scale is the internal consistency coefficient of 0.63 for the family-based activities scale (Cleland et al., 2011).

Association with child PA. Cleland et al.'s (2011) original study reported on associations between the family PA environment (parents in particular) and child PA levels as assessed by parents in a sample of 5 to 12 year olds $(N=540)$. Most of the PA parenting scales were positively related to child activity, but results depended on child gender and age (see Cleland et al., 2011 and the Supplement (page 112-132) for complete results). 


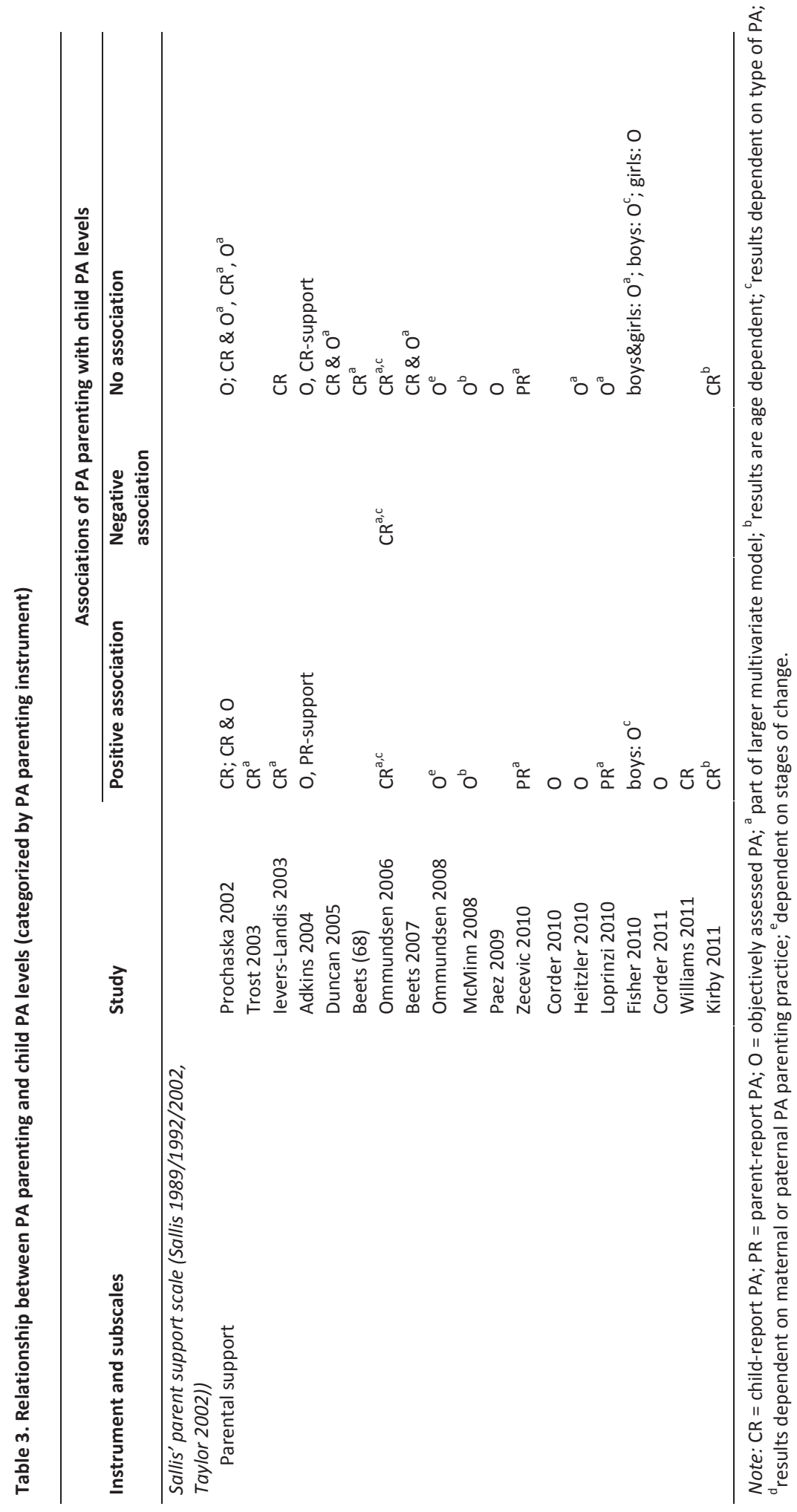




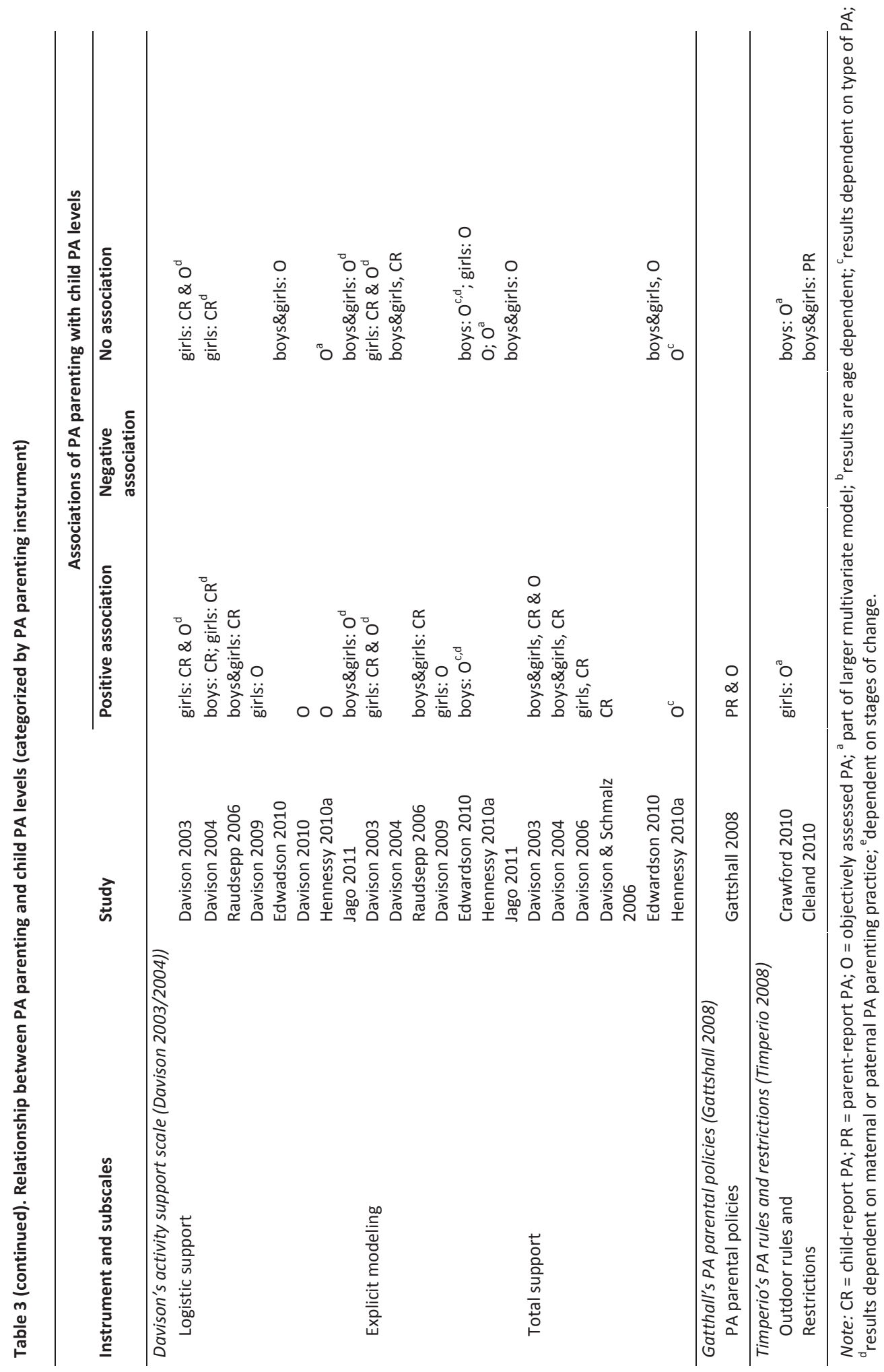




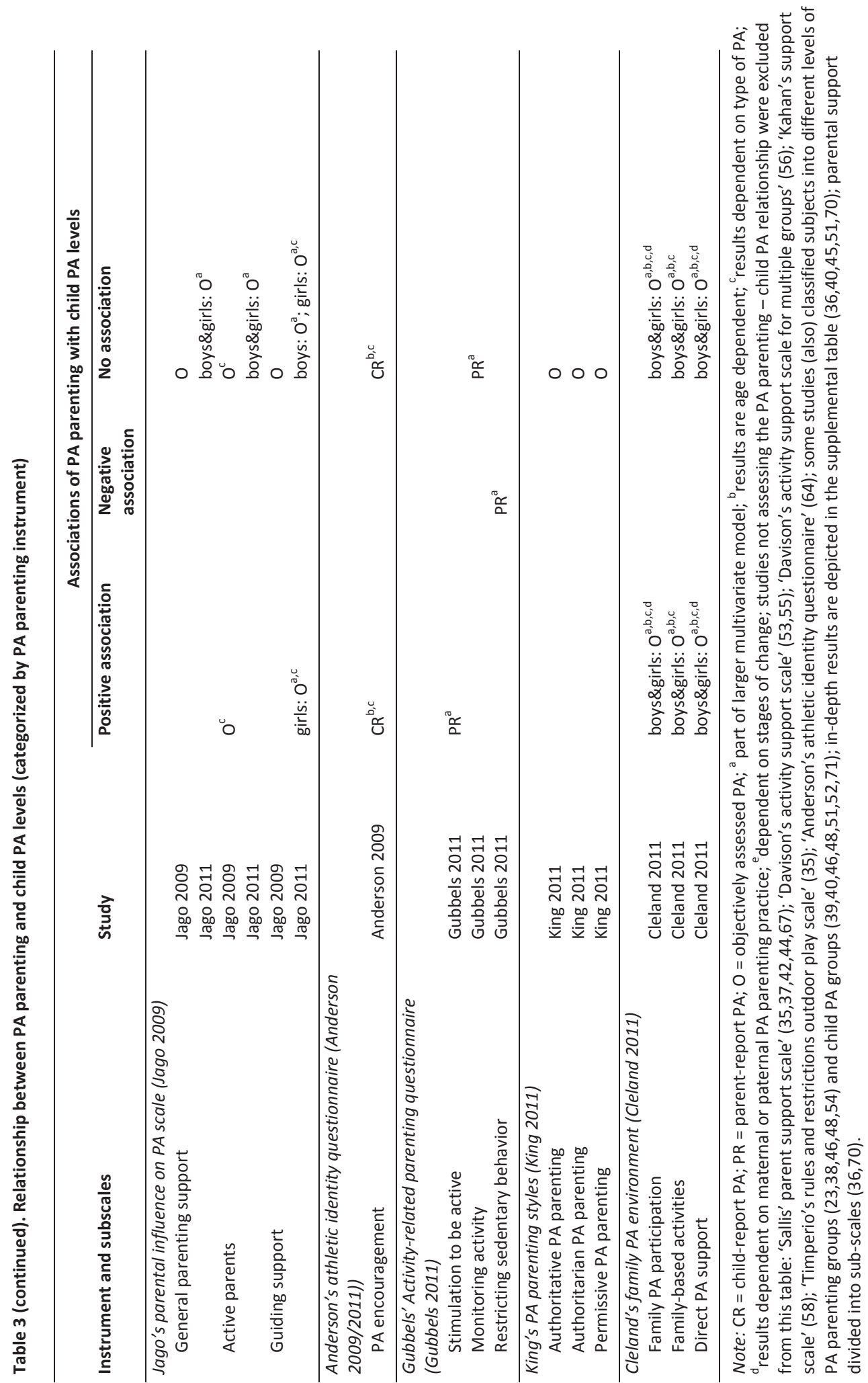




\section{Discussion}

The objectives of the present review were to identify existing questionnaires measuring PA parenting, to report the measures' development and psychometric properties, and to identify relationships between the constructs assessed by these questionnaires and child PA behaviors and/or intensity levels. In contrast to the literature on food parenting measurement (e.g., Faith, Scanlon, Birch, Francis, \& Sherry, 2004; Hurley, Cross, \& Hughes, 2011; Ventura \& Birch, 2008; Wardle \& Carnell, 2006), literature on parenting assessment in relation to child PA is still in its infancy. We identified eleven PA parenting instruments eligible for the current review. Findings highlight the tremendous variation in the conceptualization and measurement of PA parenting and widespread use of non-validated instruments. This makes it difficult to recommend one particular PA parenting questionnaire for use in research studies. Moreover, most of the included studies combine subtypes of PA parenting into one composite score, thereby losing the ability to examine specific effects of type of parenting. Concerning the relationship between PA parenting and child activity behavior, we can conclude that supporting PA behaviors (including modeling) were positively related with child PA levels (see Table 3 ). There are some indications that results differ depending on child age, gender, and ethnicity; method of PA assessment (objective, parent-report or child-report), maternal or paternal PA parenting practice, type of PA parenting construct, and generic reference to PA or making distinctions between context of PA (e.g., sports, free play).

The earliest and most frequently used instrument is Sallis et al.'s (Sallis et al., 1989/1992/2002; Taylor et al., 2002) 'parent support scale.' Some of the other reviewed PA parenting questionnaires are partly based on this measure (i.e., Adkins' (Adkins et al., 2004) 'parent support of child PA level' and 'child perception of parental support' scale categorized as belonging to Sallis' parent support scale despite slight deviations, Kahan's (Kahan, 2005) 'parental support' scale, Gattshall's (Gattshall et al., 2008) 'PA parental policies' scale, and Cleland's (Cleland et al., 2011) 'direct PA support' scale). All these instruments have one feature in common -- that one global PA parenting construct, social support, is measured with multiple items. One instrument measured only a single PA parenting scale: Timperio's 'PA rules and restrictions' scale (Timperio et al., 2008). Only one of the four scales in Anderson's (Anderson et al., 2009/2011) AIQ was applicable for the review, 'encouragement from parents'. The remaining identified PA parenting instruments assessed multiple dimensions of PA parenting (i.e., explicit modeling and logistic support by Davison (Davison, 2004; Davison et al., 2003); explicit modeling, logistic support, use of community resources, and restricting access to sedentary activities by Davison (Davison et al., 2011); general parenting support, active parents, and guiding support by Jago (Jago et al., 2009); stimulation to be active, monitoring activity, and restriction of sedentary behavior by Gubbels (Gubbels et al., 2011); authoritative, authoritarian and permissive PA parenting by King (King et al., 2011); and family PA participation, family-based activities and direct PA support by Cleland (Cleland et al., 2011); see Table 1).

We identified several studies that did not meet the inclusion criteria. For instance, some used observations to assess PA parenting in the child's home environment (Klesges, Eck, Hanson, Haddock, \& Klesges, 1990; Sallis et al., 1993). Others did use questionnaires but did not meet inclusion criteria for this review, such as 
one that identified a questionnaire using multiple PA parenting scales that consisted of single items of PA parenting (besides also assessing 'sedentary' parenting) (Arredondo, Elder, Ayala, Campbell, Baquero, \& Duerksen, 2006; Larios, Ayala, Arredondo, Baquero, \& Elder, 2009). Another frequently used questionnaire (e.g., Brustad, 1993; Schaben, Welk, Joens-Matre, \& Hensley, 2006; Welk, Wood, \& Morss, 2003) was ineligible due to incomplete information about the contents of the instrument and lack of the reference reporting the development and psychometrics of the instrument. Finally, PA influence questionnaires focusing on the influence of other family members (e.g., including siblings), such as the 'social support for exercise survey' (Sallis, Grossman, Pinski, Patterson, \& Nader, 1987), were excluded.

\section{Methodological issues}

\section{$\underline{\text { Development and validation }}$}

The majority of the identified PA parenting questionnaires lacked a theoretical framework to conceptualize the PA parenting constructs that were being assessed, with only a few reporting about theories supporting scale development (Sallis' 'parent support scale' (Sallis et al., 1989); Gatthalls' 'PA parental policies' scale (Gattshall et al., 2008); Cleland's 'family PA environment' scale (Cleland et al., 2011); Anderson's AlQ (Anderson, 2004)) or qualitative methods to unravel parental strategies aimed at increasing their child's activity behaviors (Jago's 'parental influence on PA' scale (Jago et al., 2009) and the 'ACTS-MG' (Davison et al., 2011)). Psychometric validation was also lacking in most studies. The most extensive validation was reported in the studies of Davison et al. (Davison, 2004; Davison et al., 2003/2011) and Jago et al. (2009), both of which discussed extensive factor analytic procedures beyond more classical test theory approaches (e.g., internal consistency estimates, inter-correlations between scales).

\section{Conceptualization and measurement of PA parenting}

One of the major limitations of PA parenting instruments was the tremendous variation in the conceptualization and inconsistent measurement of PA parenting constructs. Several of the PA parenting instruments distinguishes between different and conceptually unique types of social support (Sallis' 'parent support scale' (Sallis et al., 1989/1992/2002, Taylor et al., 2002), Gatthalls' 'PA parental policies' scale (Gattshall et al., 2008), and Jago's 'general parenting support' scale (Jago et al., 2009)). Beets et al. (Beets et al., 2010) identified two mechanisms of social support: 1) tangible support consisting of two types: 1a) instrumental (including payment of fees and transportation) and 1b) conditional (doing activity with child and supervision) and 2) intangible support consisting of two types: 2a) motivational (encouragement and praise) and $2 \mathrm{~b}$ ) informational (discussing benefits of PA). The instruments referred to above (Gattshall et al., 2008; Jago et al., 2009; Sallis et al., 1989/1992/2002, Taylor et al., 2002) cover both tangible and intangible aspects of social support. However, most of the studies combine these into one composite measure (sometimes also combining mother and father scores), thereby losing the ability to examine specific effects of type of support provided. Thus, we recommend using composite scores only for conceptually similar types of PA parenting constructs, separated for mothers and fathers. 


\section{$\underline{\text { Longitudinal research designs }}$}

Much of the research on PA parenting has been cross-sectional, limiting the possibility to draw causal inferences. For instance, parents may adapt a more supporting PA parenting practice when they find that their child is not active enough. Only three studies identified in the present review used longitudinal designs to assess the PA parenting - child PA relationship over time (Cleland et al., 2010; Davison et al., 2006; Davison \& Jago, 2009); the first two studies using Davison's 'activity-related parenting practices scale' (Davison et al., 2006; Davison \& Jago, 2009) found positive association on child PA for the scale of parental support, and the latter study (Cleland et al., 2010) found no association on child PA for Timperio's scale of PA rules and restrictions.

\section{Independent measurement of PA parenting and children's PA behavior}

Most studies relied on reporting of PA parenting and PA behavior both by the same respondent (usually parents, see Table 3). The use of child-report of PA or objective measurements by pedometers or accelerometers makes the measurements mutually independent, with the advantage of reducing influences from parental expectations or rationalizations.

\section{Directions for future research}

Despite the emerging literature on the role of parents in shaping their children's PA behaviors, the methodological challenges related to the theory-based conceptualization and measurement of PA parenting and use of non-validated measures has hampered progress in this area. The exact mechanisms of parental PA influence on child PA behaviors still need to be unraveled in order to develop effective interventions to encourage PA among children and families.

In conclusion, although several different PA parenting instruments are used worldwide, comprehensive and accepted measures appear to be lacking. Future research should validate existing instruments and may necessitate the development of PA parenting measures that are more comprehensive (multidimensional) and theory-based using thorough validation methods. Longitudinal designs with independent measurements of PA parenting and child PA behavior may be needed for causal interpretation. Such studies should account for possible moderating factors affecting the PA parenting - child PA relationship. The influence on child PA levels in a broader social context, e.g., including other family members such as siblings, or including members of the school environment such as peers and teachers, was beyond the scope of the present review but will potentially add to the ability to explain child PA. Parental attitudes and beliefs, as predictors of parenting behavior, were also beyond the scope of this review. 


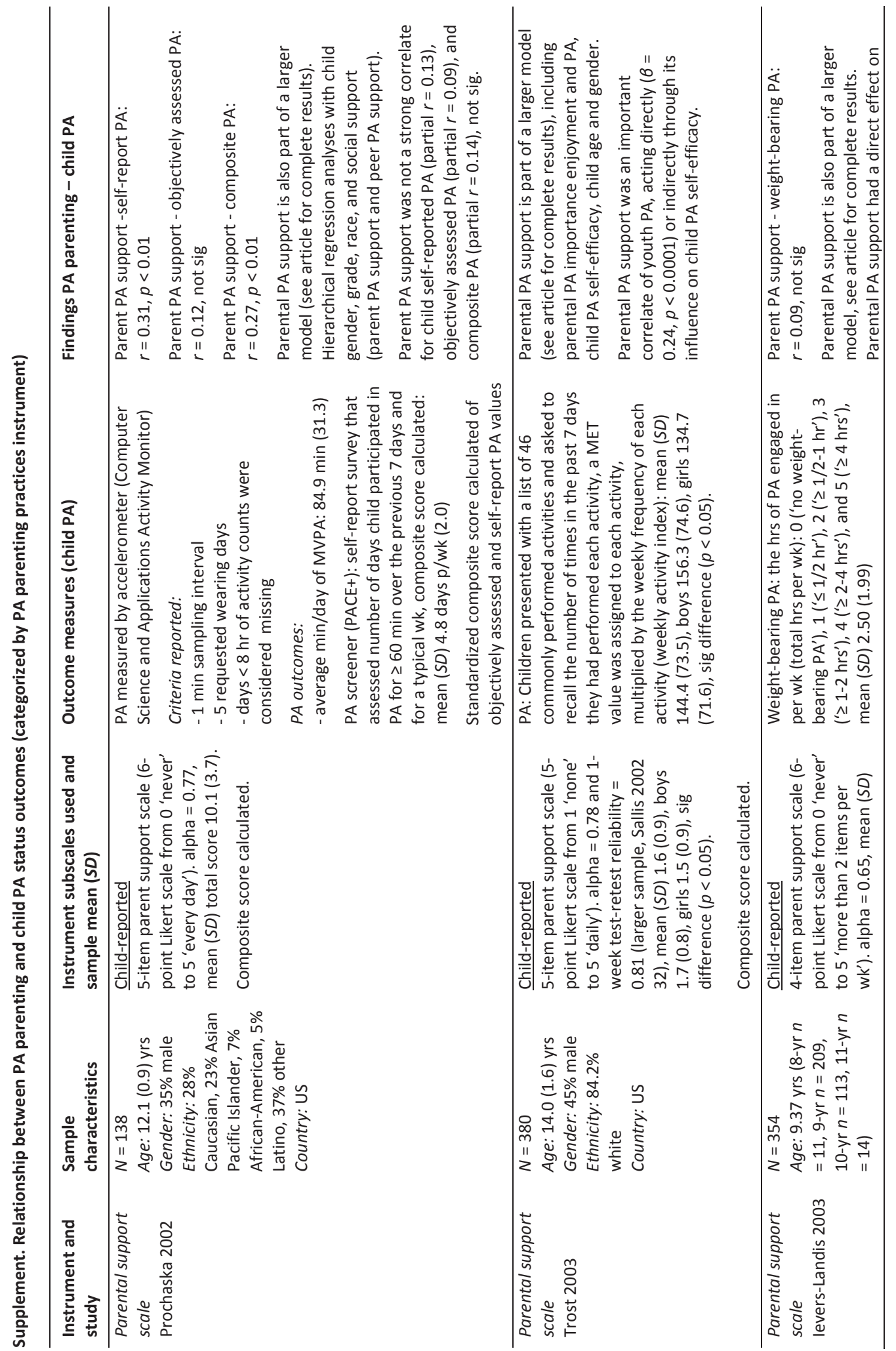




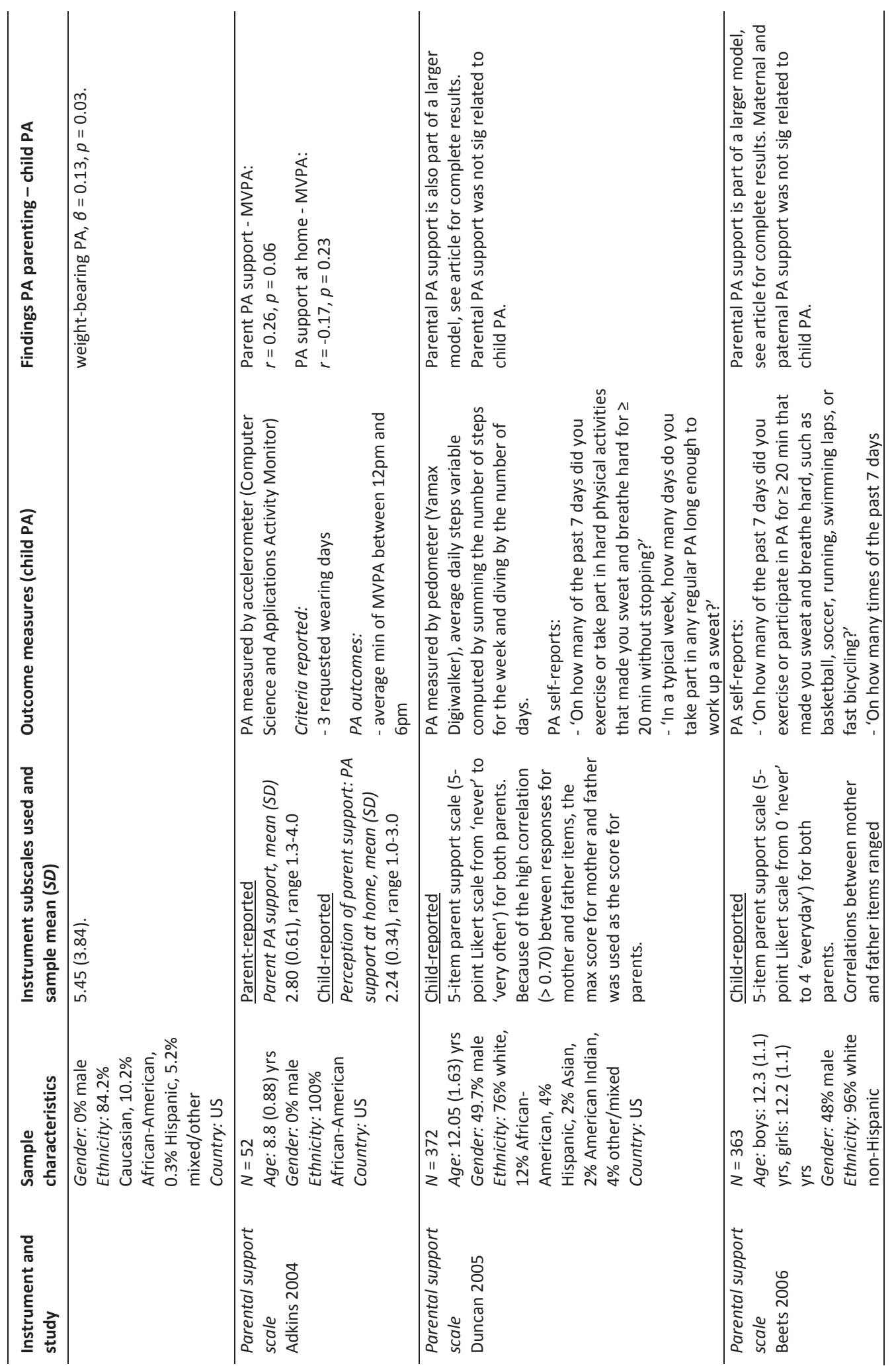




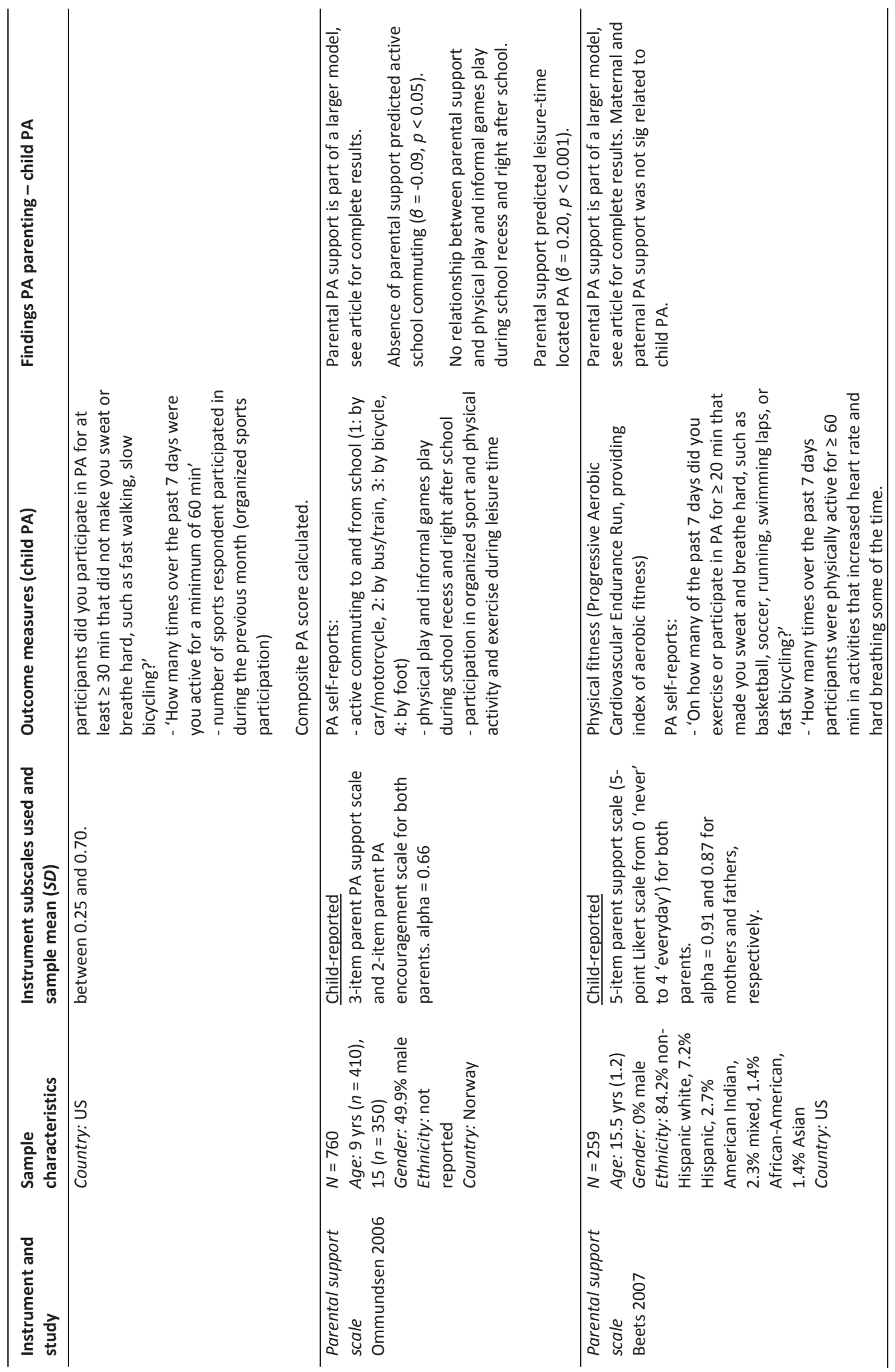




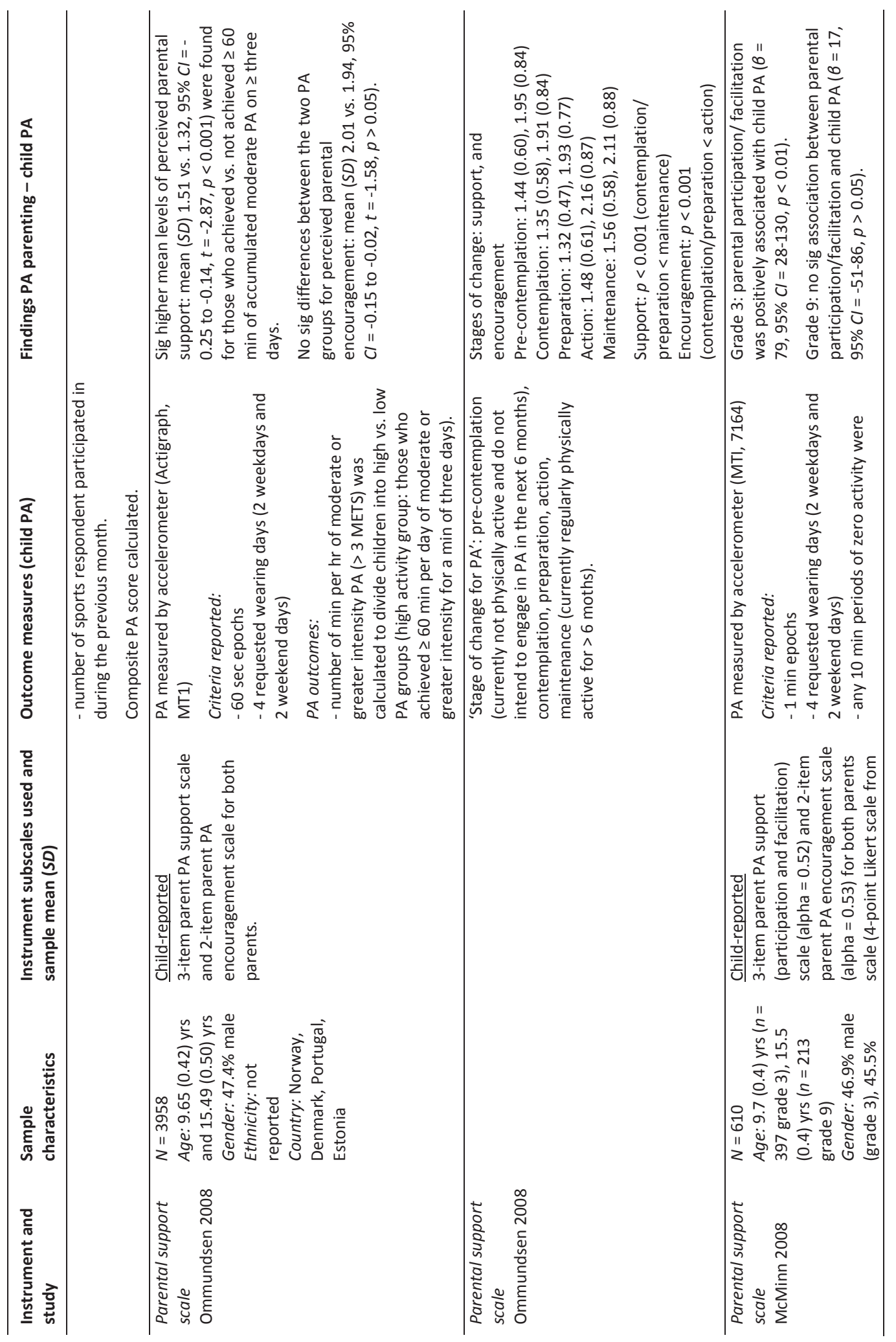




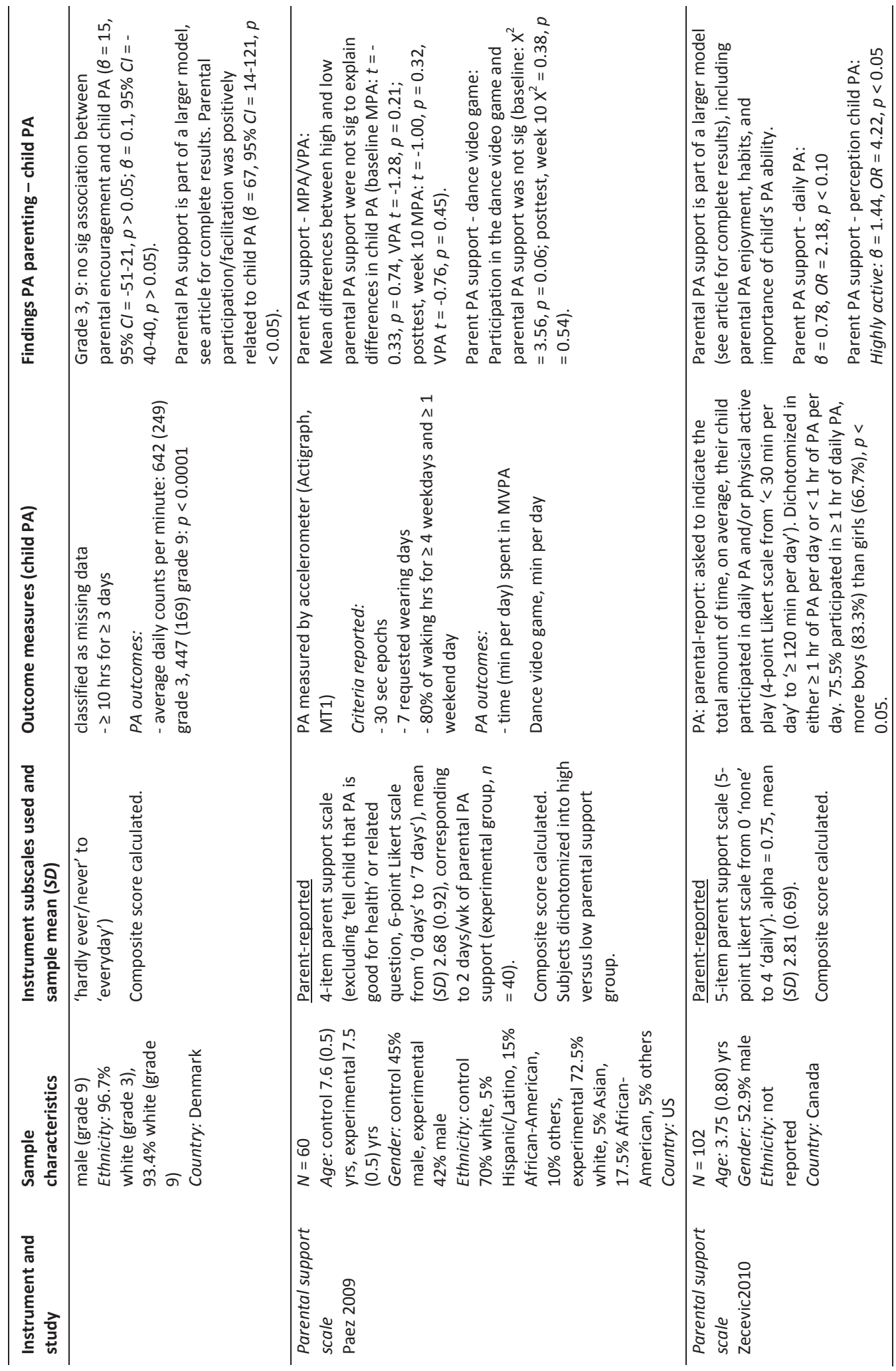




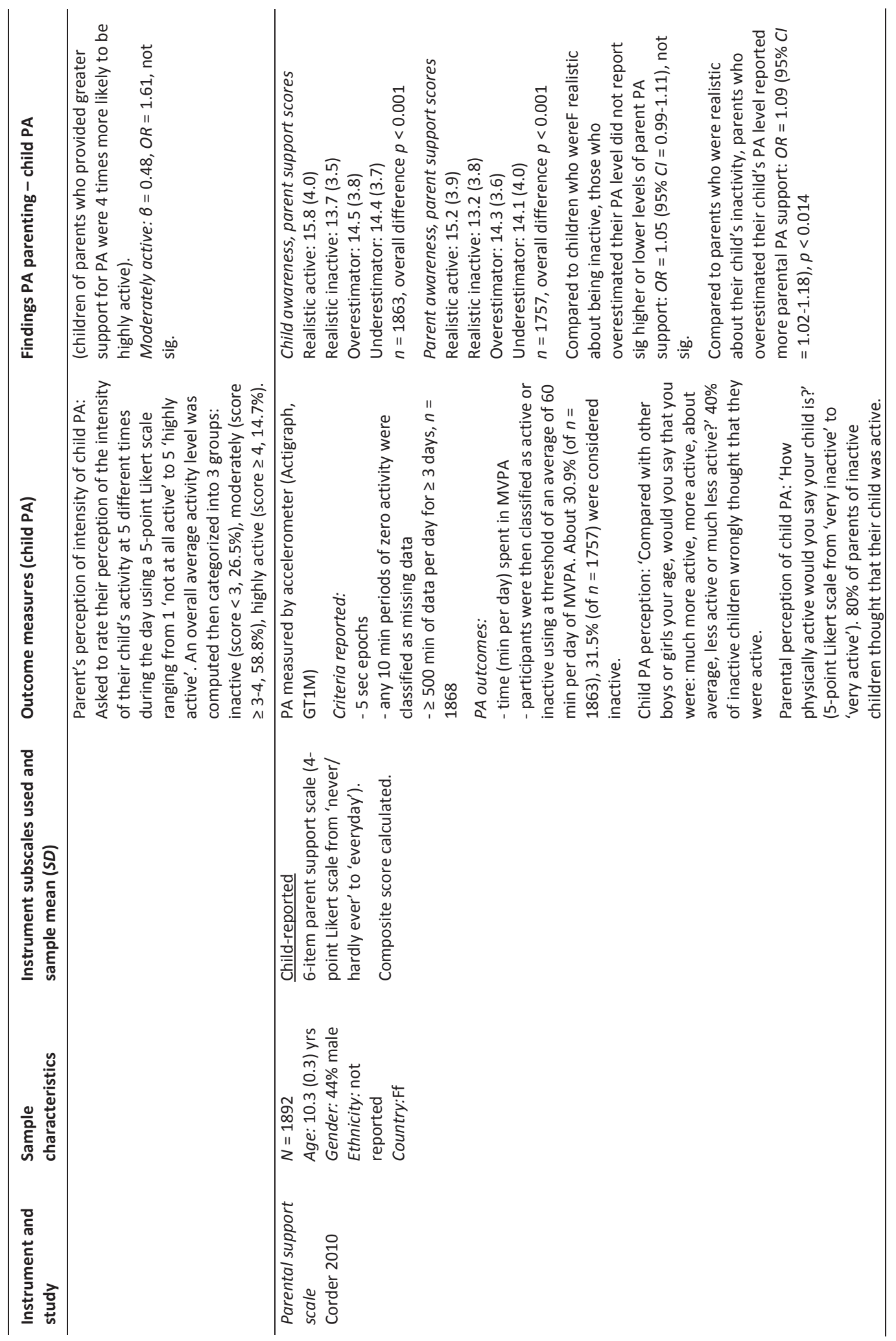




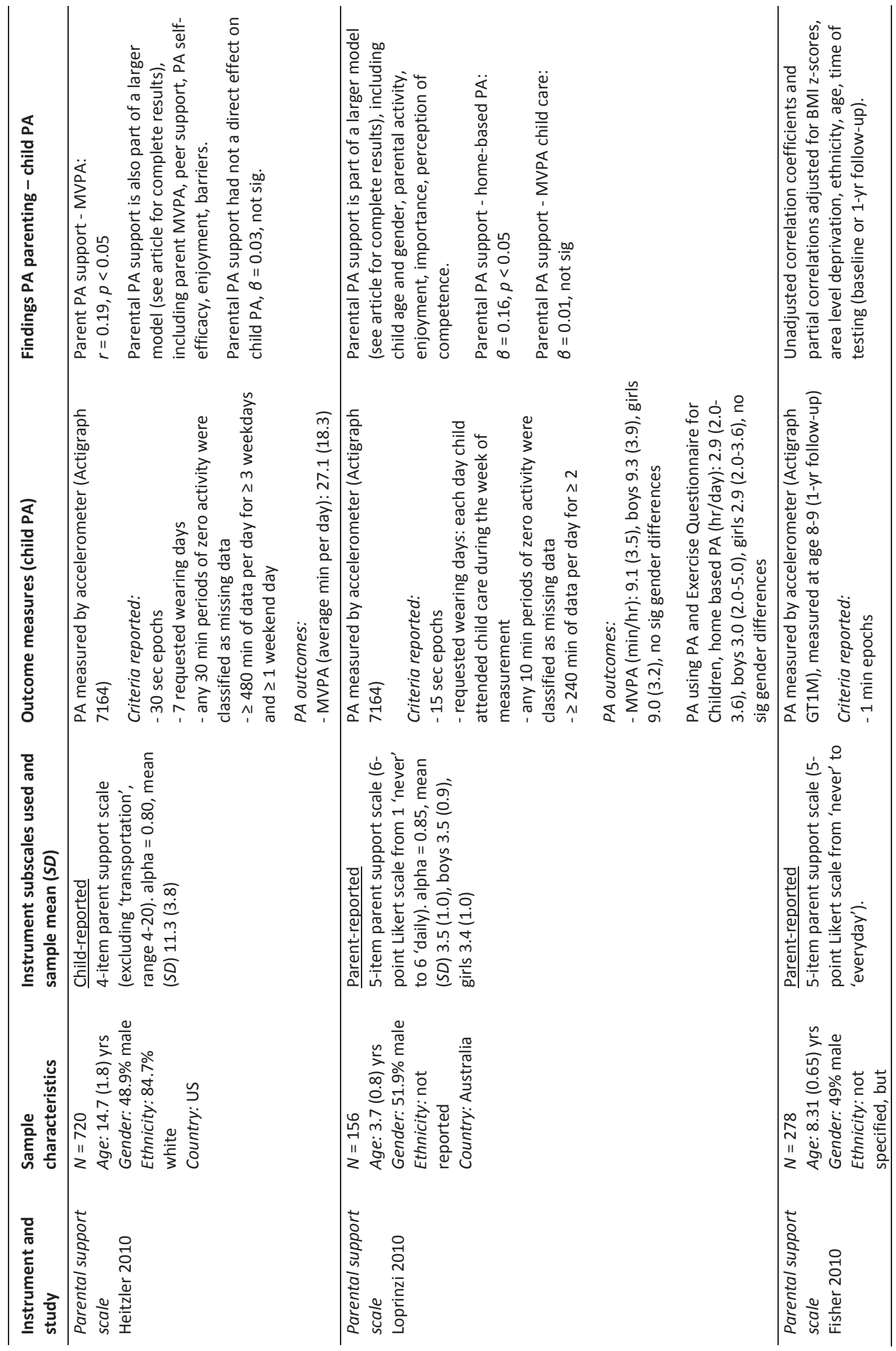




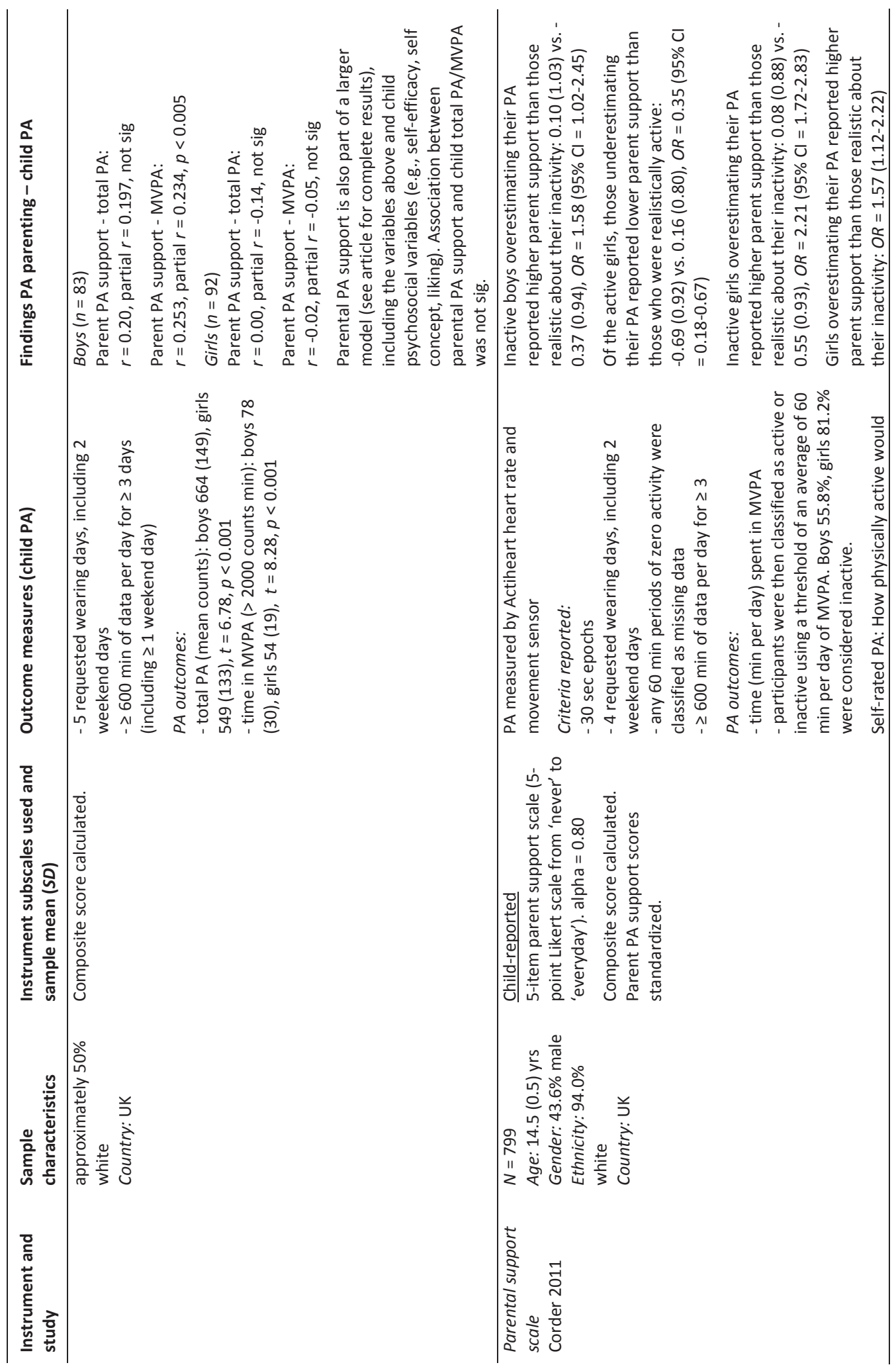




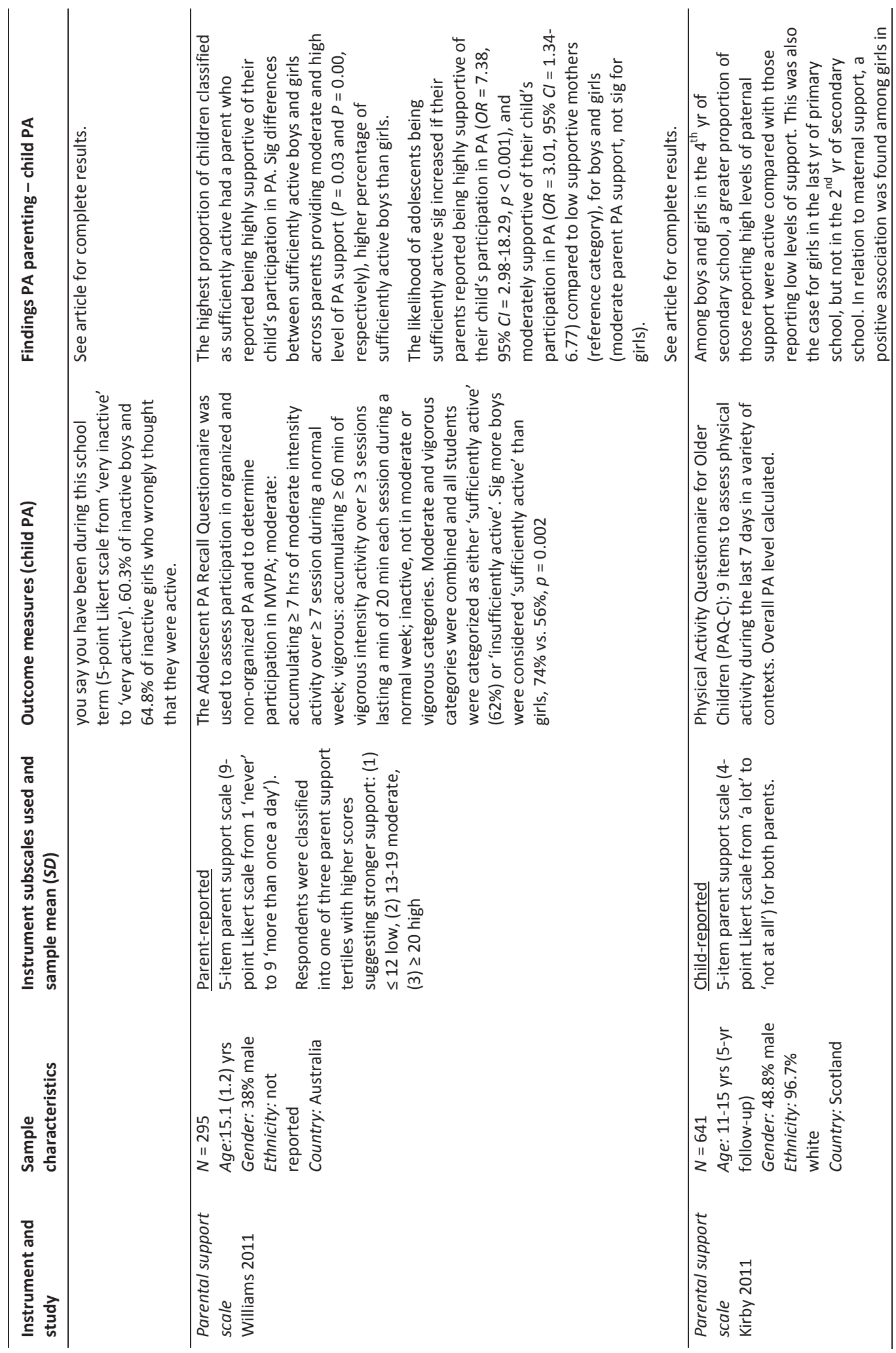




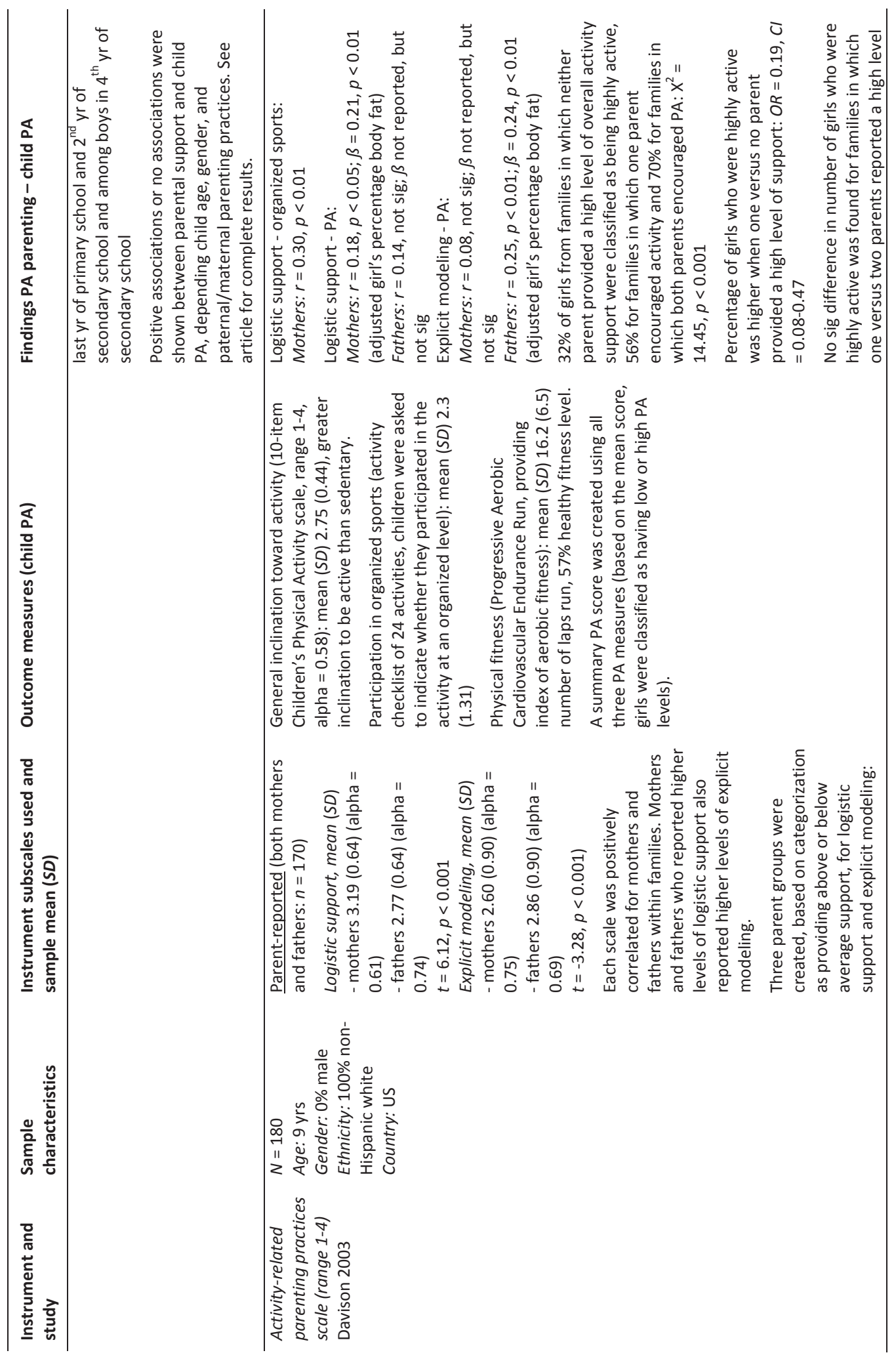




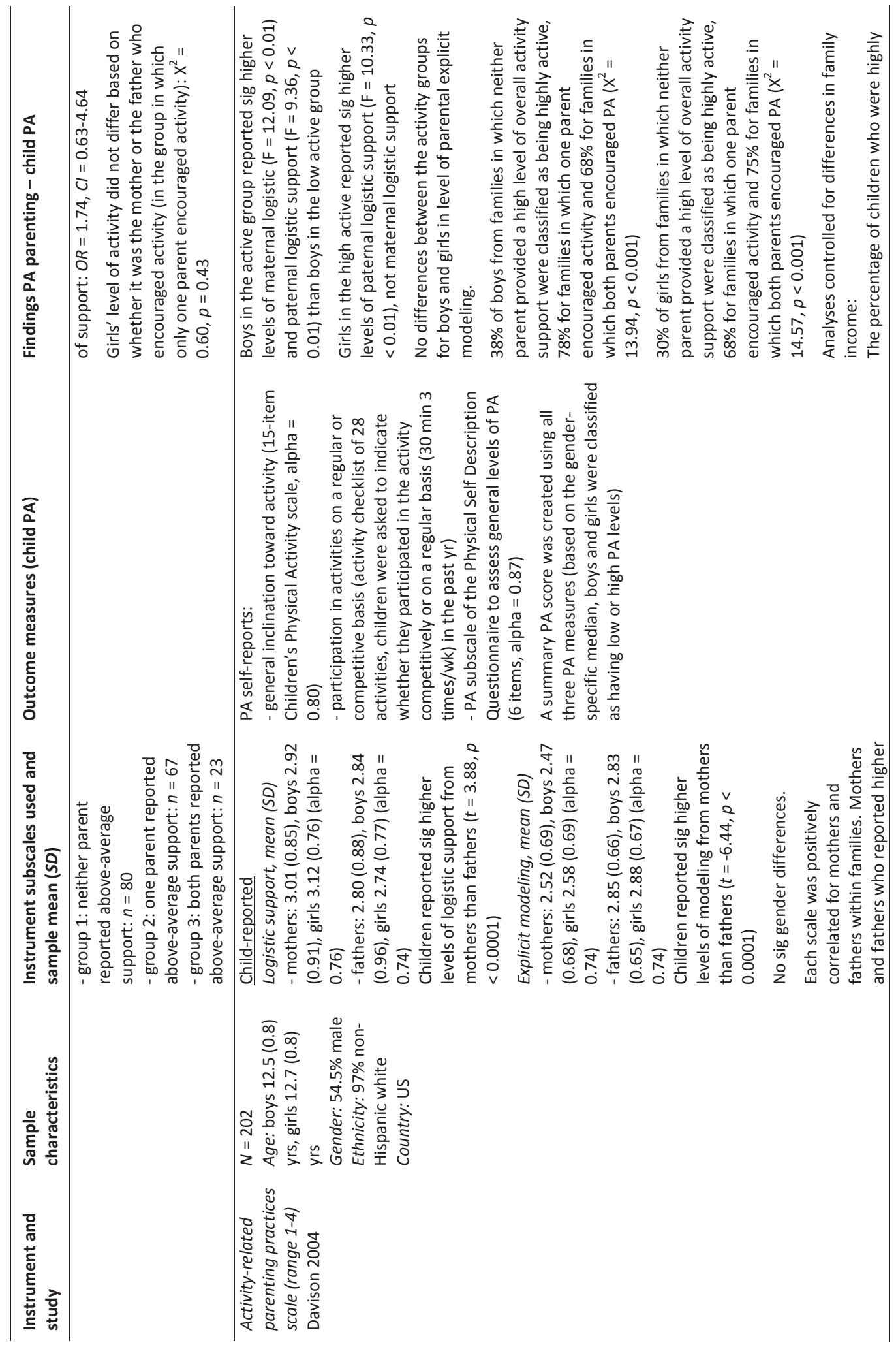




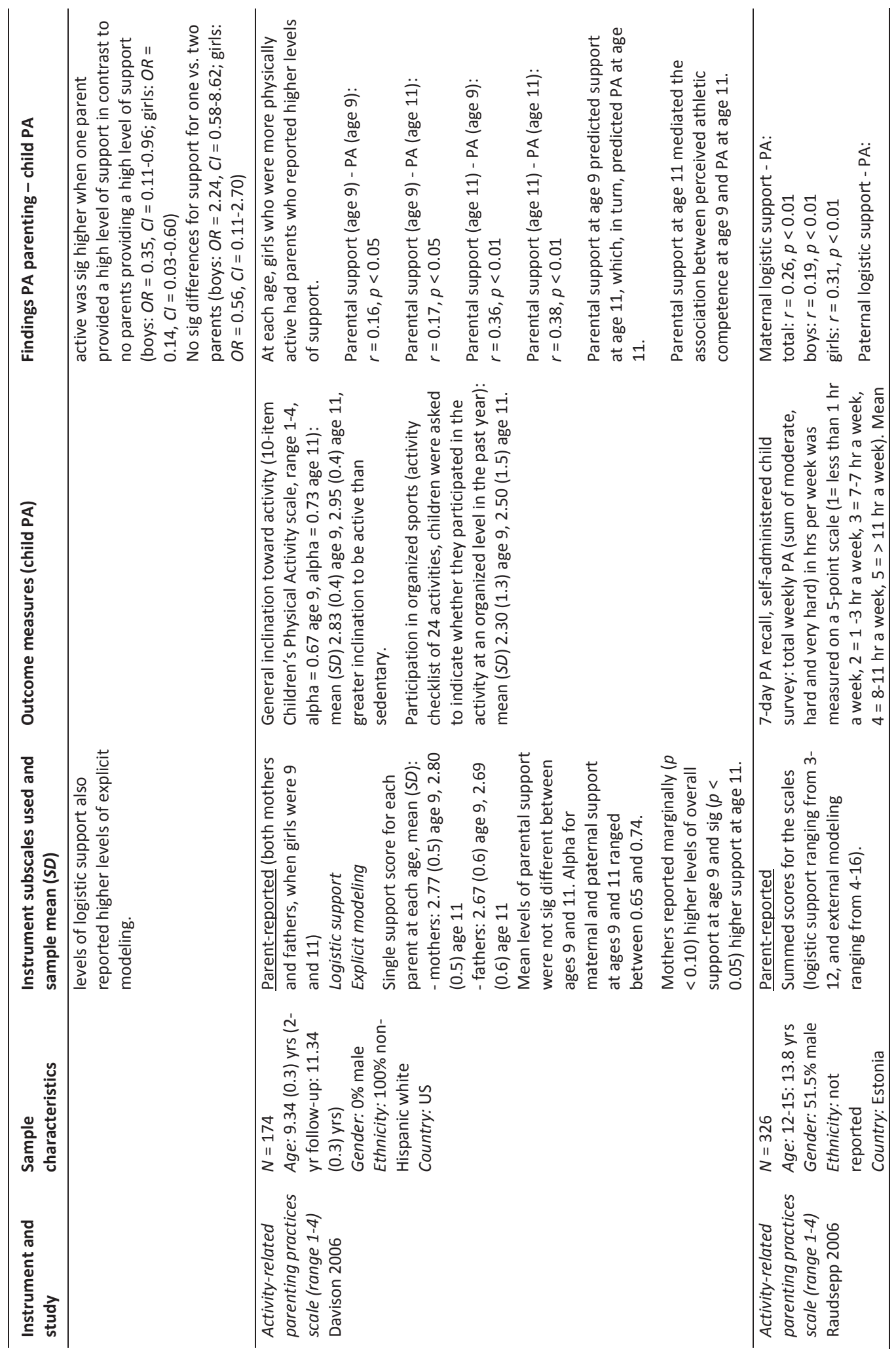




\begin{tabular}{|c|c|c|}
\hline & 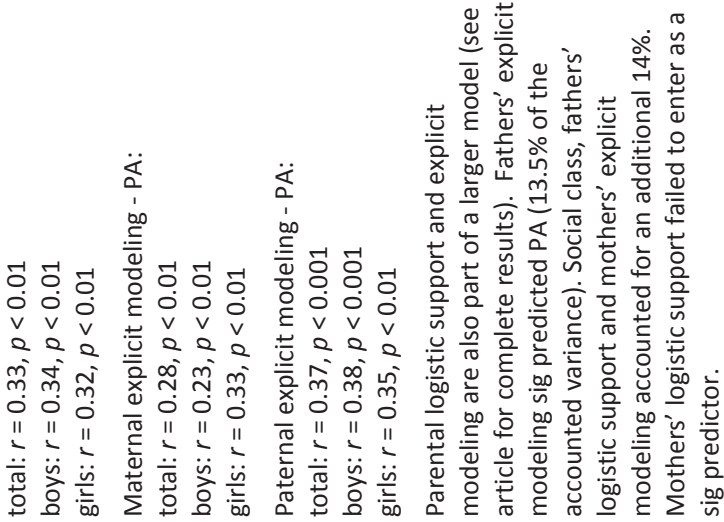 & 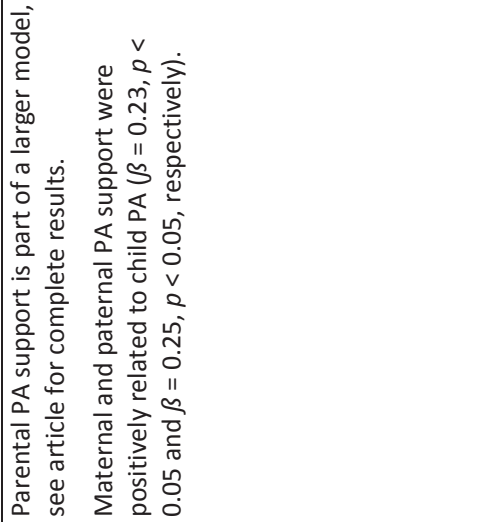 \\
\hline & 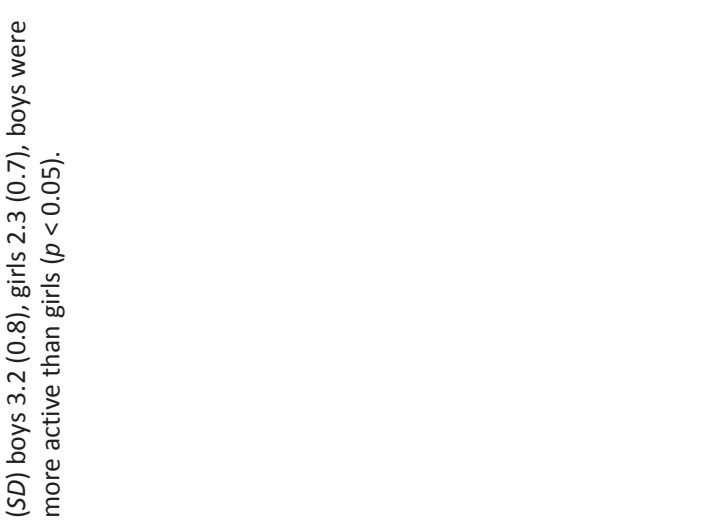 & 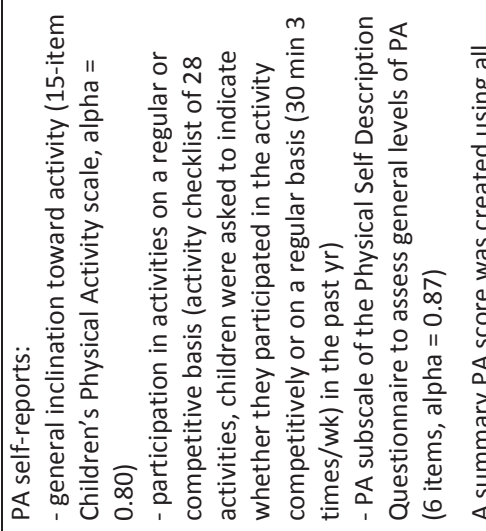 \\
\hline 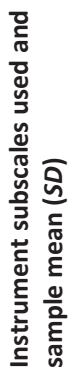 & 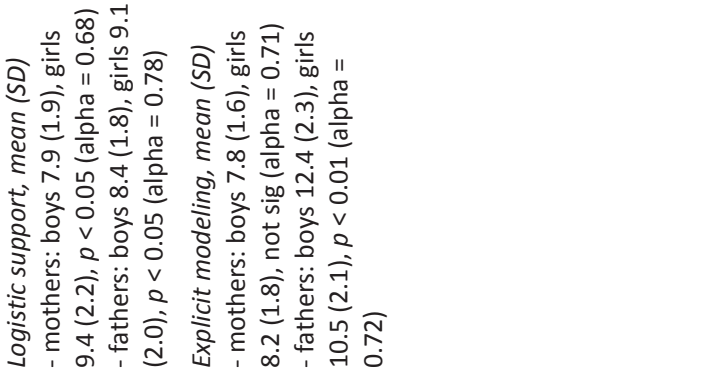 & 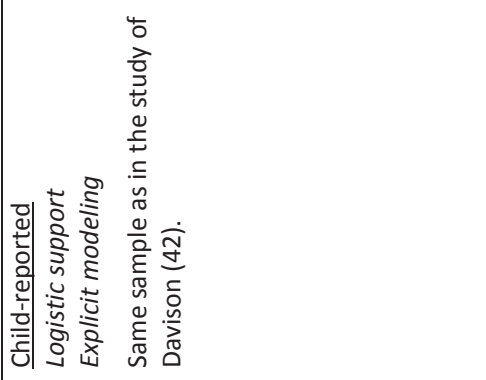 \\
\hline 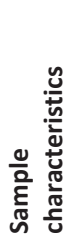 & & 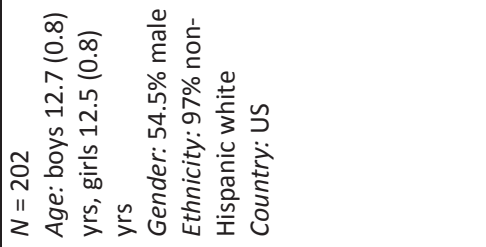 \\
\hline 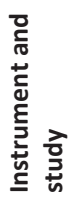 & & 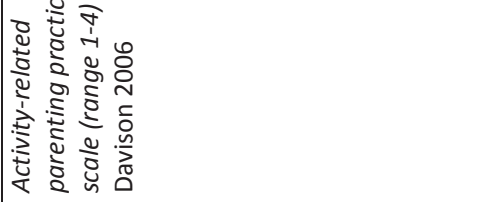 \\
\hline
\end{tabular}




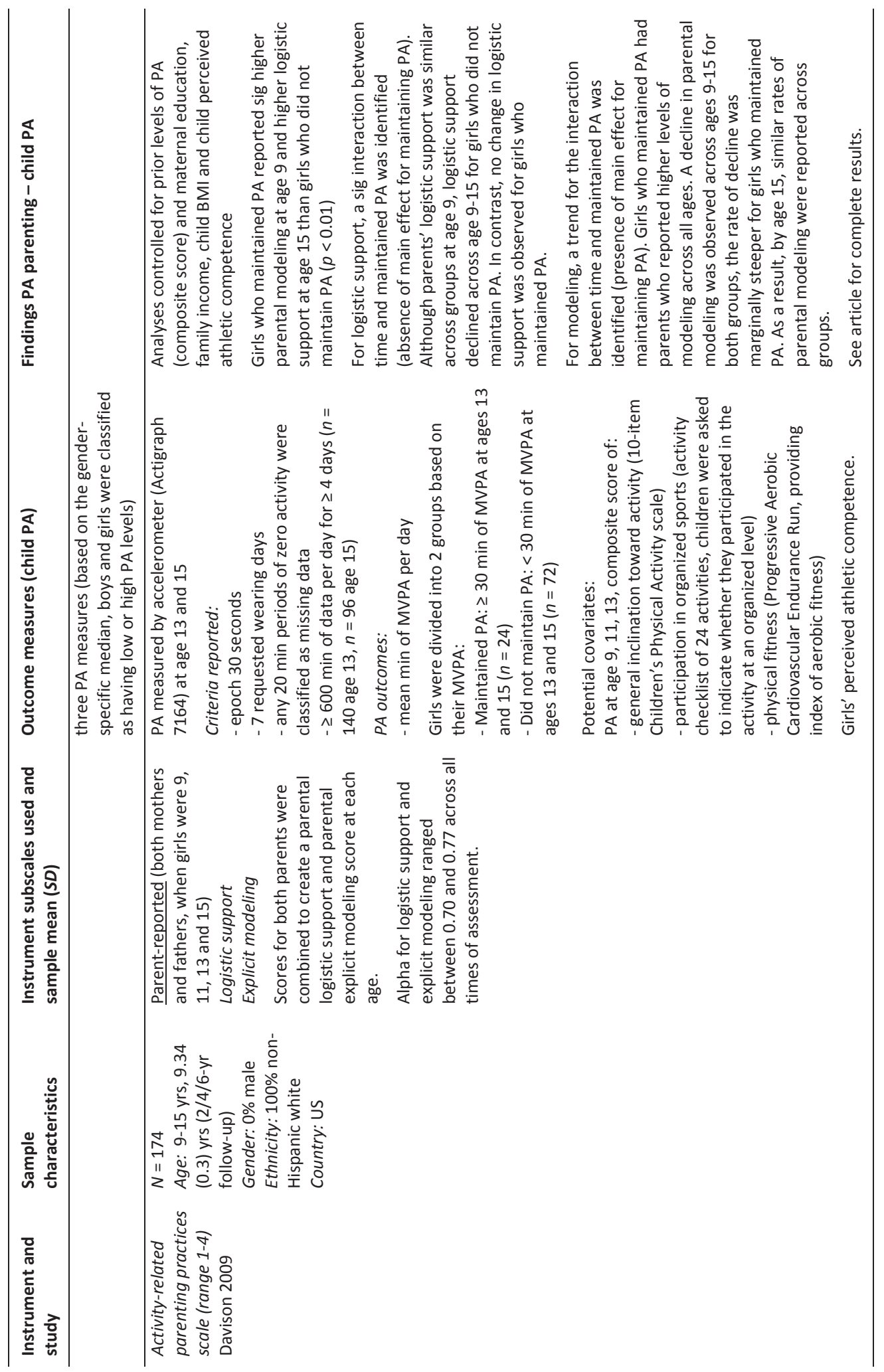




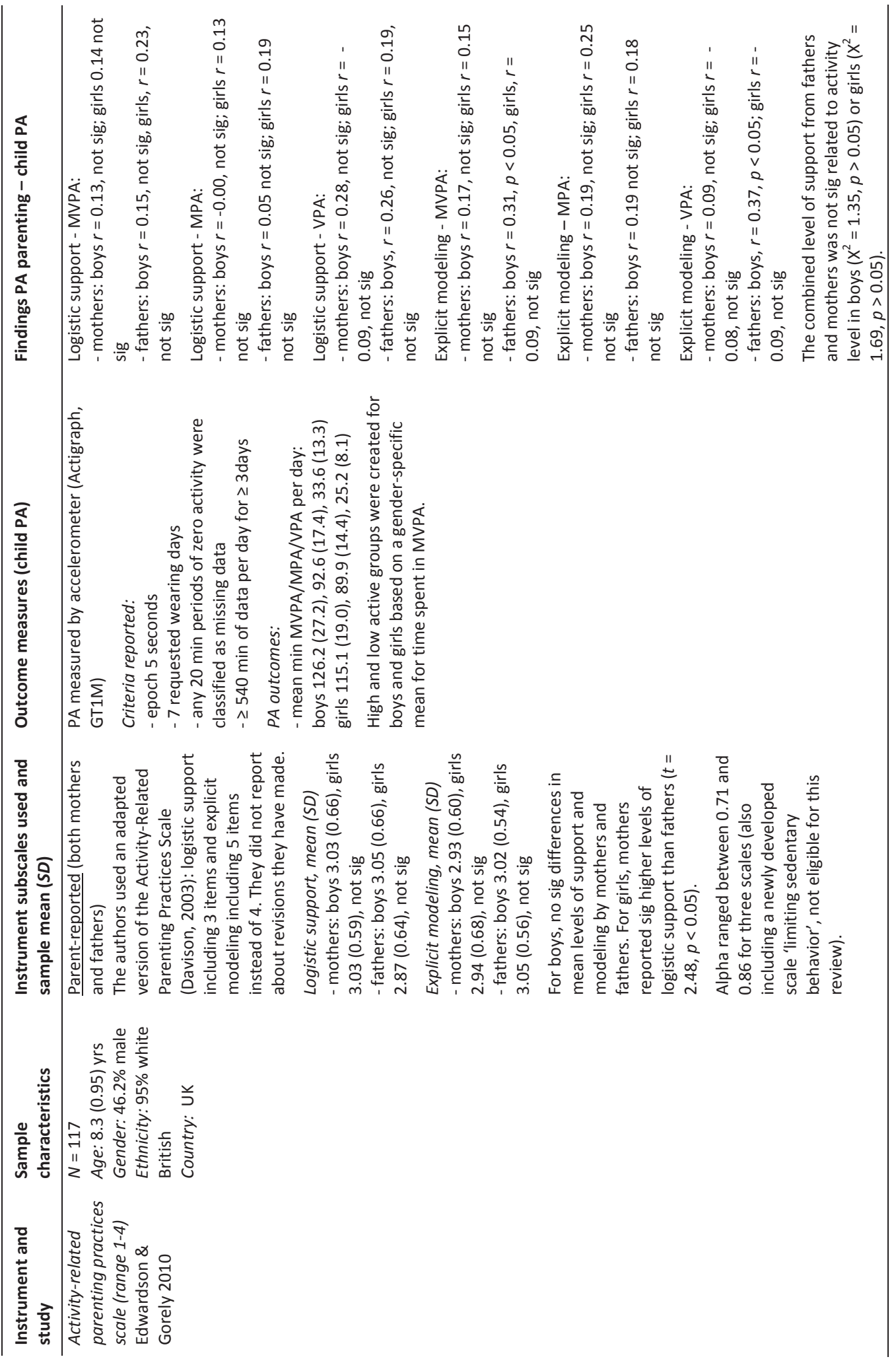




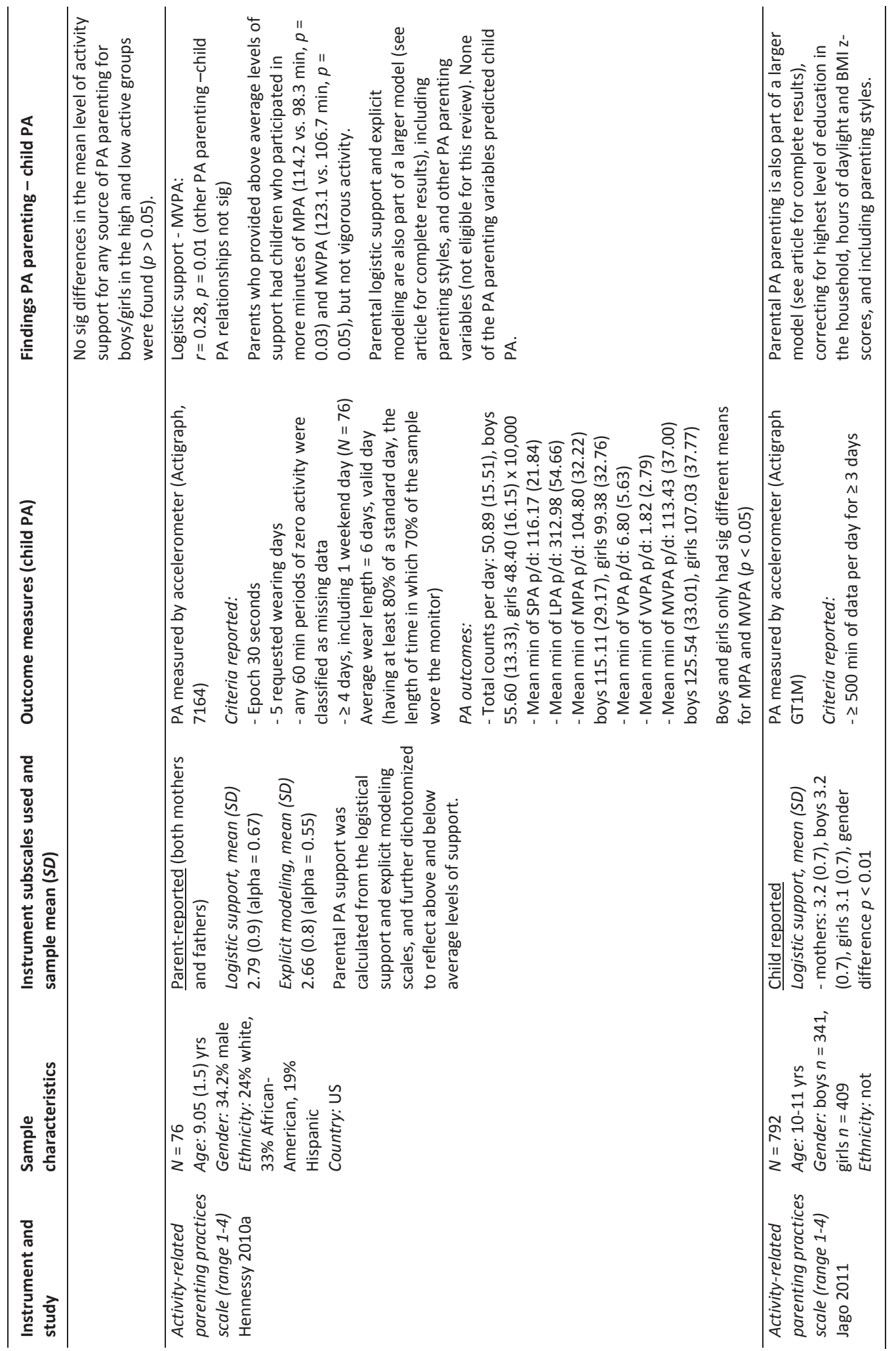




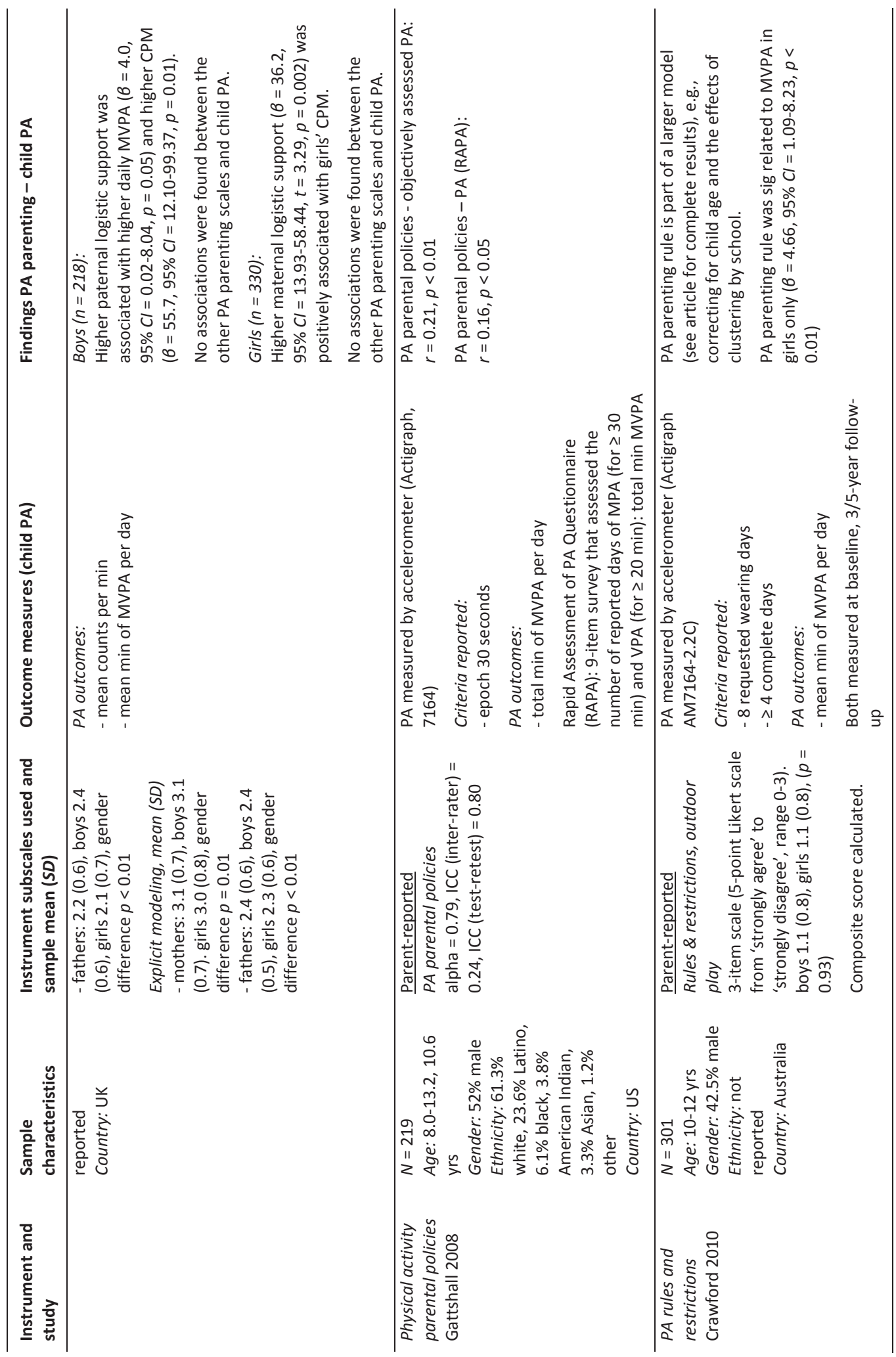




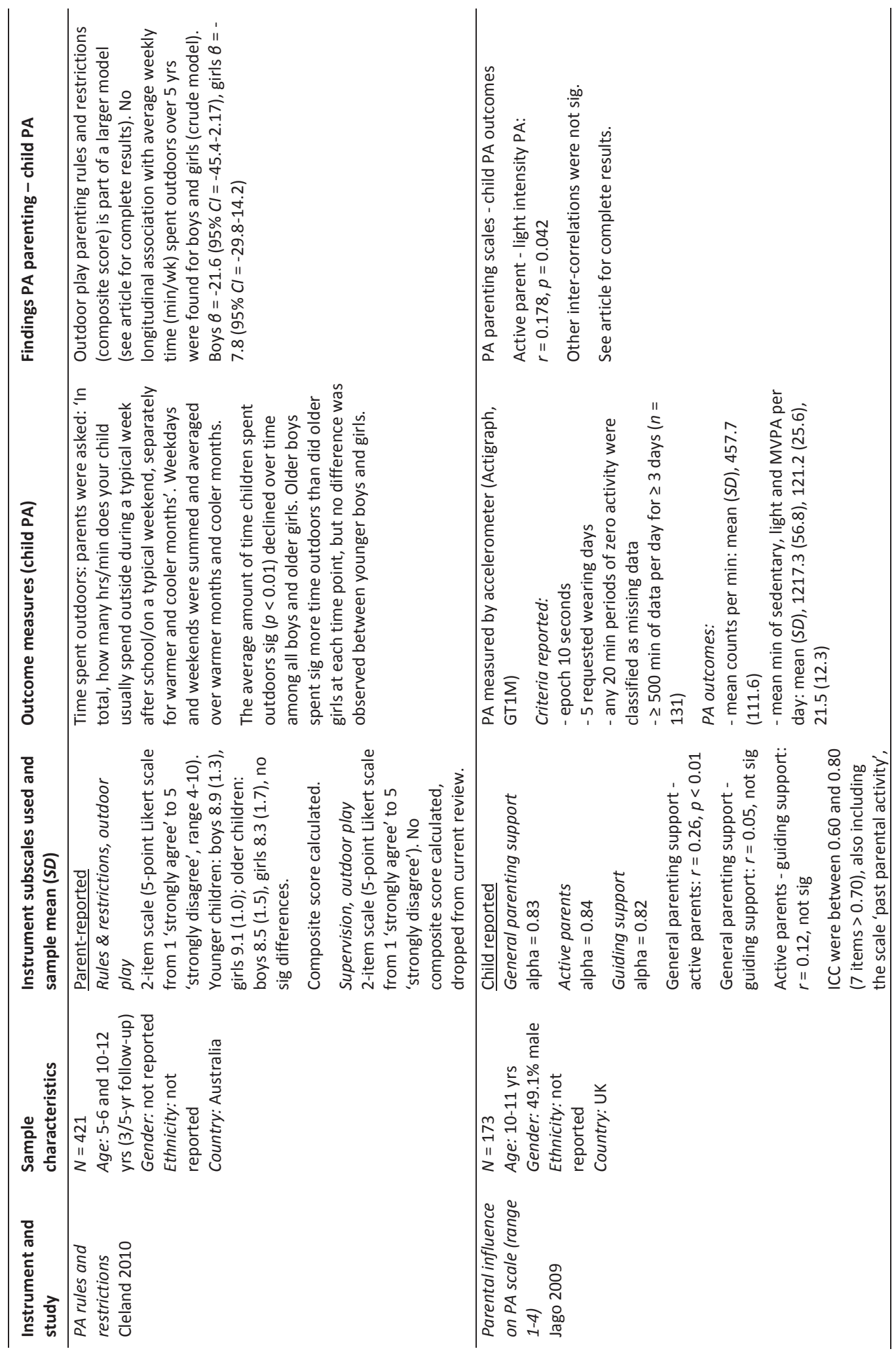




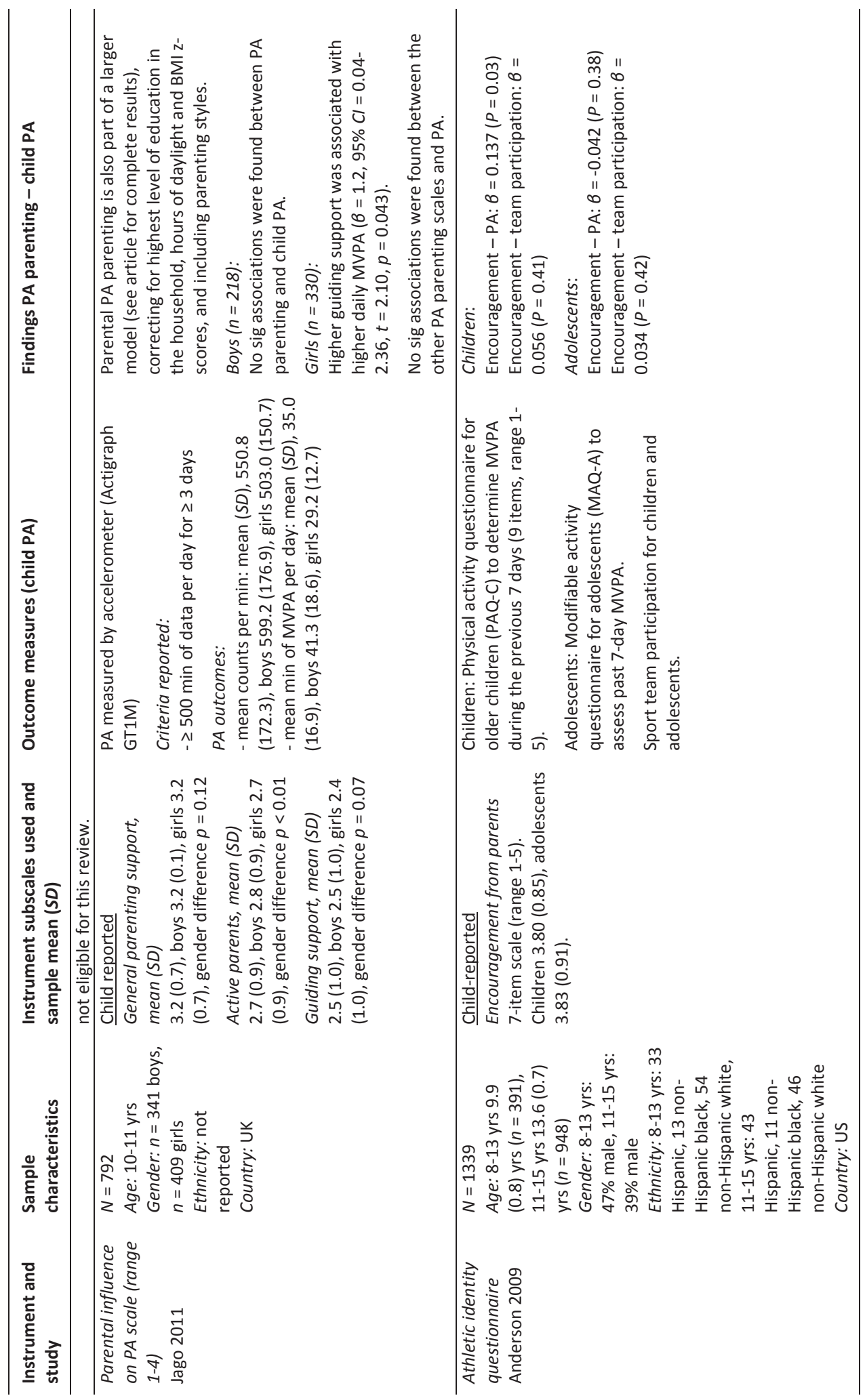




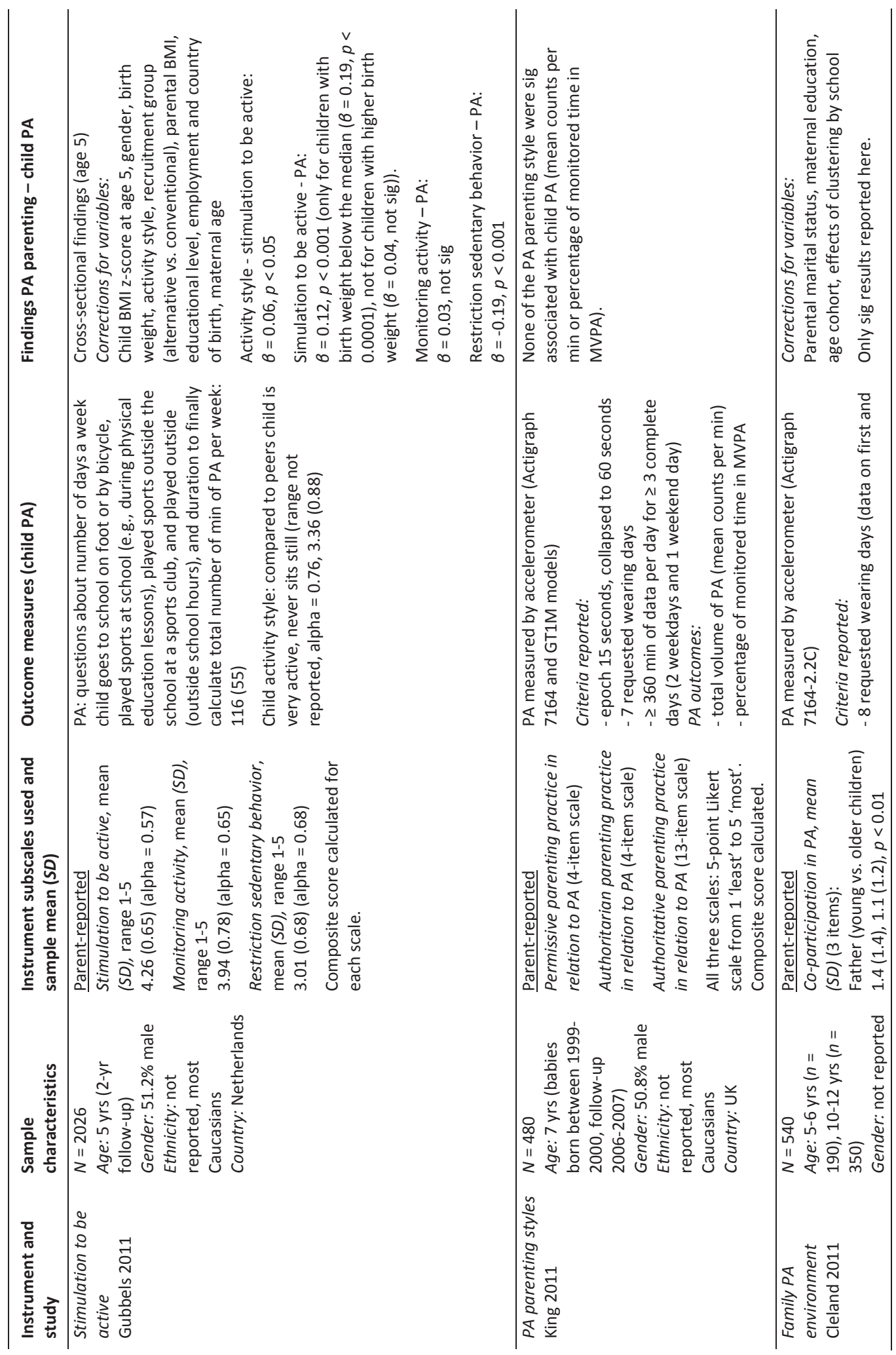




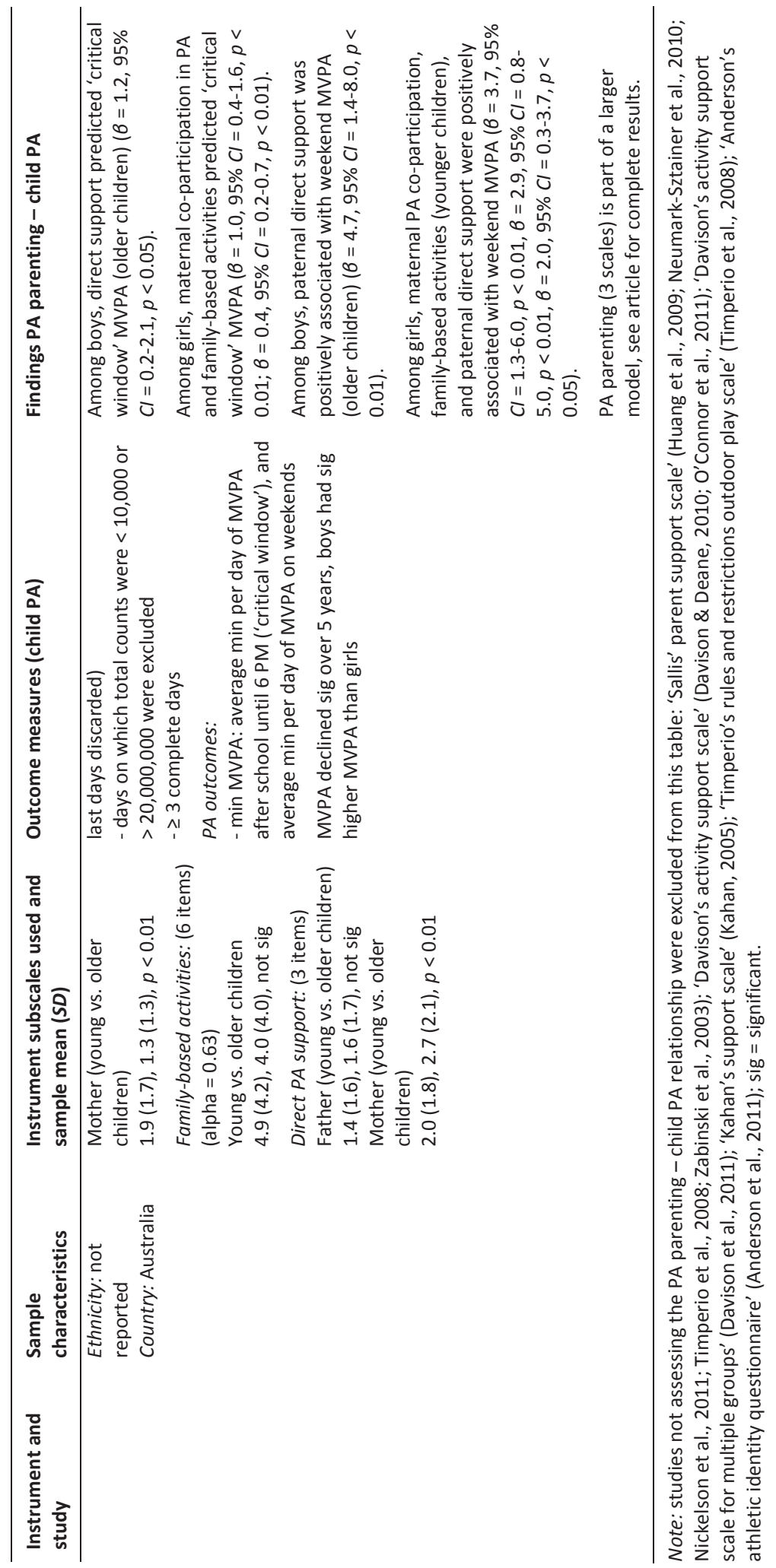




\section{CHAPTER 5}

\section{Validating the Children's Behavior Questionnaire in Dutch children:}

Psychometric properties and a cross-cultural comparison of factor structures

Ester FC Sleddens

Stef PJ Kremers Math JJM Candel Nanne K De Vries Carel Thijs

Psychological Assessment, 2011 


\begin{abstract}
In this article, we examined the factorial validity of the Dutch translation of the Children's Behavior Questionnaire ( $\mathrm{CBQ}$ ) and the Very Short Form scores. In addition, we conducted cross-cultural comparisons of temperament structure. In total, 353 parents of 6- to 8-year-olds completed the instrument. The original higher order factor structure of the different $\mathrm{CBQ}$ forms was generally replicated and represented the three broad dimensions of temperament: Surgency/Extraversion, Negative Affectivity, and Effortful Control. For the Standard Form, results demonstrated a relatively high degree of factor similarity of the Dutch sample with other cultures (e.g., China and Japan). The findings provide evidence for applicability of the CBQ in Western Europe, as a promising instrument to comprehensively assess reactive and self-regulative temperamental dimensions in young children.
\end{abstract}




\section{Introduction}

Temperament has been defined as 'constitutionally based, individual differences in reactivity and selfregulation' (Rothbart, Ahadi, Hershey, \& Fisher, 2001, page 1395). The term constitution refers to the biological basis of temperament influenced by interactions with the environment (e.g., parenting, experience and maturation). Temperamental characteristics are distinctive across individuals, since they differ in reactivity (i.e., individual's reaction to changes in the environment as reflected in the arousability of motor, affective, and sensory response systems) and self-regulation (i.e., processes that serve to modulate reactivity, such as attentional strategies and behavioral avoidance). Various adverse developmental outcomes, including mental as well as physical health problems are likely to be partially influenced by individual differences in reactivity and self-regulation (e.g., Goodyer, Ashby, Altham, Vize, \& Cooper, 1993; Lindhout, Markus, Hoogendijk, \& Boer, 2009; Martin, Wertheim, Prior, Smart, Sanson, \& Oberklaid, 2000; Mâsse \& Tremblay, 1997; Zeller, Boles, \& Reiter-Purtill, 2008). For example, Martin et al. (2000) found that the temperament characteristics of negative emotionality and low persistence in childhood were associated with risk of developing eating and body concerns in early adolescence, particularly in girls. Lindhout et al. (2009) recently found that levels of emotionality and shyness were higher in anxiety-disordered children compared with controls who were not diagnosed with an anxiety disorder.

A caregiver report measure, the Rothbart's Children's Behavior Questionnaire (CBQ; Ahadi, Rothbart, \& Ye, 1993; Rothbart et al., 2001) was developed in the United States (US) to assess a broad range of temperamental behavior patterns (positive as well as negative emotional reactivity and self-regulatory mechanisms) in 3- to 8-year-old children. The $\mathrm{CBQ}$ is generally regarded as one of the most comprehensive instruments, incorporating all components thought to underlie temperament (arousal, affect, and selfregulation).

Previous studies using the $\mathrm{CBQ}$ have attempted to capture the structure of temperament for children (Ahadi et al., 1993; Rothbart et al., 2001) in different countries (the US, China, Japan). Validating the crosscultural similarities of the temperament construct has been subject to research with the aim of constructing a uniform measure. Following factor-analytic procedures on CBQ responses, higher order temperamental traits were identified, represented by three factors: Surgency/Extraversion (SR), Negative Affectivity (NA), and Effortful control (EC). This three-factor structure was replicated by means of factor analysis on the Very Short Form (36 items) of the CBQ (Putnam \& Rothbart, 2006). The overarching factor, $\mathrm{SR}$, involves the tendency to perform impulsive and active behavior and involves positive affectivity that can be characterized, for instance, by having pleasure in situations involving high-intensity stimuli (Rothbart et al., 2001). The NA factor refers to the predisposition to experience negative affective states (automatic or involuntary), including feelings of anger, discomfort, fear, sadness and difficulty to be soothed (Rothbart et al., 2001). These two factors determine children's reactive behaviors in response to environmental changes. The third factor, EC, can be regarded as the self-regulation aspect of temperament and involves processes such as voluntary regulation of attention and behavior. This factor has been defined as 'the 
ability to inhibit a dominant response to perform a subdominant response' (Rothbart \& Bates, 1998, page 137). For adults, largely similar structural models of personality have been found. For instance, Rothbart, Ahadi, and Evans (2000) have found evidence that the CBQ dimensions of SR, NA, and EC appear to be highly related to the major Big Five dimensions of adult personality labeled Extraversion, Neuroticism, and Conscientiousness, respectively (Saucier, 1994). As Caspi, Roberts, and Shiner (2005) proposed, temperamental characteristics may track into adulthood. Indeed, longitudinal studies have shown that temperamental traits of children were relatively stable across adolescence and adulthood (e.g., Asendorpf, Denissen, \& Van Aken, 2008; Caspi, Harrington, Milne, Amell, Theodore, \& Moffitt, 2003). Unfortunately, few studies exist that assess continuity and changes in personality from childhood to adulthood (Caspi et al., 2005; McAdams \& Bradley, 2010).

To our knowledge, the 36-item form of the CBQ has not been validated in cultures other than the US. However, using the Standard Form, the structure of child temperament has been investigated crossculturally for large samples of Chinese and Japanese children using a factor-analytic approach (Ahadi et al., 1993; Rothbart et al., 2001). Results demonstrated the factorial invariance of temperamental structure across these Asian countries, although subtle deviations in factor structure to the original US study were reported. Cross-cultural research to measure the factorial invariance of child temperament has not been widespread but is valuable, since cultures differ in socialization patterns (Kohnstamm, 1989). While potential cross-cultural variations in temperament characteristics are to be expected as a result of biological differences among populations, parents perceive and guide their actions toward children in accordance with their culture's constructions of childhood (Cervera \& Méndez, 2006). Cross-cultural comparisons of child temperament measures may shed more light on the extent to which self-regulatory and reactive components of temperament interact under the influence of culture (Ahadi et al., 1993).

Gartstein, Knyazev, and Slobodskaya (2005) found that temperamental structure of infants using the revised version of the Infant Behavior Questionnaire (Gartstein \& Rothbart, 2003) was generally consistent across cultures (US and Russian samples). In another study, Windle, Iwawaki, and Lerner (1988) found invariance in factor structure of the Revised Dimensions of Temperament Survey (Windle \& Lerner, 1986) across a sample of American and Japanese preschoolers. Furthermore, some attempts have been made to assess the factorial structure of the CBQ in European countries, for instance, in Belgium by Eggers, De Nil, and Van den Bergh (2009, $N=259)$, and Van den Bergh and Ackx (2003, $N=71)$, in the Netherlands by Majdandžić and Van den Boom (2007, N =94), and in Norway by Nygaard, Smith, and Torgersen (2002, N= 243). The studies of Nygaard et al. (2002) and Eggers et al. (2009) were conducted to determine whether the underlying temperamental structure was identical for three groups of children. In the Norwegian study, structure of temperament was assessed for children with the Down syndrome $(n=55)$, prematurely born children ( $n=97)$ and normally developing children ( $n=91$; Nygaard et al., 2002), whereas in the Belgian study this was assessed for children who stutter $(n=69)$, voice disordered children $(n=41)$ and normal speaking children ( $n=146$; Eggers et al., 2009). Since these studies were conducted using a small number of participants, further validation of the $\mathrm{CBQ}$ in these countries is needed, using larger sample sizes comparable to that of the original study $(N=341)$. The present study overcomes this limitation using a large 
sample of Dutch children. Furthermore, these findings emphasize the need for further research to assess cross-cultural applicability of temperament measurements to provide additional evidence of validity and reliability.

The overall aim of the present study was three-fold. The first aim was to evaluate factorial validity and psychometric properties of the scores on the Dutch version of the CBQ and the Very Short Form (Putnam \& Rothbart, 2006) in a large sample of Dutch children ages 6 to 8 years. Second, cross-cultural differences in temperament structures were examined by comparing scale loadings on each of the three higher order temperament factors between our study and the US and Asian (i.e., Chinese and Japanese) studies, respectively. We hypothesized that the factorial structure would closely resemble findings of the original US study. In line with findings of previous studies (Ahadi et al., 1993; Rothbart et al., 2001), deviations in temperament structure in terms of the size of factor loadings on the three higher order dimensions were hypothesized to be larger when comparing our results with those of Asian countries (i.e., China and Japan). Third, we aimed to examine the congruence between responses on the three higher order dimensions of the Standard and the Very Short Form $\mathrm{CBQ}$; the scores on the latter version were extracted from the same data. We applied Levy's (1967) correction to achieve true score correlations between the two versions. In accordance with prior study results of Putnam and Rothbart (2006), we expected these correlations to be relatively high.

\section{Method}

\section{Overview of procedures and participants}

In total, 353 questionnaires were collected; 163 parents of children in eight primary schools of Maastricht and surroundings (south of the Netherlands), and 190 parents of children in seven primary schools of Veenendaal and surroundings (center region of the Netherlands) completed the Standard Form of the instrument. The response rate per primary school ranged from $24.2 \%$ to $47.4 \%$ (mean $=29.7 \%$ ) in Maastricht and ranged from $28.2 \%$ to $61.2 \%$ (mean $=45.9 \%$ ) in Veenendaal and surroundings. Only parents of 6- to 8-year-olds who reported holding Dutch nationality were included in the study. Fourteen questionnaires were excluded for this reason. Parents could decide which of them would complete the questionnaire; most often, it was the mother $(n=290)$. Twenty-eight fathers filled out the questionnaire, and 32 families reported that both parents completed the questionnaire together. Three families did not indicate which family member completed the questionnaire. In general, $33.5 \%$ and $41.0 \%$ of the mothers and fathers, respectively, had received a college or university degree. The mean age of the participating mothers and fathers was $38.0(S D=4.1)$ years and $40.4(S D=5.0)$ years, respectively.

Our sample consisted of three age groups: 6-year-olds $(n=174)$, 7-year-olds $(n=160)$, and 8-year-olds $(n=$ 15 ; in four cases the age was unknown). The mean age of the participating children in our sample was 84.2 $(S D=7.1)$ months. Gender was almost equally represented with 187 girls (53\%) and 166 boys (47\%). 


\section{Data sources of cross-cultural CBQ samples}

To evaluate cross-cultural differences in the structure of temperament and psychometric properties of the scores on the $\mathrm{CBQ}$, we used data of three cultural samples: the US, China, and Japan. For comparison with the US sample, data on North American 6- and 7-year-old children were extracted from a study conducted by Rothbart et al. (2001). The US sample consisted of 341 children, and the CBQ questionnaire data of this sample were generated by parents who participated in studies conducted by Goldsmith and Rothbart at the University of Oregon (Goldsmith, Buss, \& Lemery, 1997; $n=158$ ) and by Fagot and Fisher at the Oregon Social Learning Center ( $n=183$; Fagot \& Leve, 1998; Fisher, 1993). For comparison with the Chinese sample, data on 468 Chinese children ages 6 and 7 were extracted from a study conducted by Ahadi et al. (1993). The Japanese sample consisted of 372 children ages 3 to 6 (Kusanagi, 1993; Rothbart et al., 2001).

\section{Description of the CBQ format}

Individual $\mathrm{CBQ}$ items were theoretically derived from research into temperamental characteristics of children until the age of 7 years, adapting existing measures, and from parental interviews and pilot questionnaires (Rothbart et al., 2001). The standard version of the CBQ consists of 195 statements assessing a broad range of temperamental characteristics represented by 15 scales, each containing nine to 13 items. These 15 scales can be grouped into three higher order temperament dimensions of SR (Activity Level, High Intensity Pleasure, Impulsivity, Positive Anticipation, and reversed dimension of Shyness), NA (Anger, Discomfort, Fear, Sadness, and reversed dimension of Soothability), and EC (Attentional Focusing, Inhibitory Control, Low Intensity Pleasure, Perceptual Sensitivity, and Smiling/Laughter). The scale content and sample items of the individual scales included in each of the higher order dimensions are displayed in the Appendix on page 148. Parents are asked to indicate on a seven-point Likert scale ranging from 1 (Extremely untrue of your child) to 7 (Extremely true of your child) how well each statement describes their child's reaction to a given situation within the past 6 months. Moreover, a 'not applicable' answer option is provided to be used when parents could not answer because they had not seen their child in the particular situation described. The CBQ (Ahadi et al., 1993; Rothbart et al., 2001) has been translated into Dutch by a team of experts on child rearing (Majdandžić \& Van den Boom, 2007).

Following Rothbart's advice, three CBQ items of this Standard Form were no longer included as well as five items of the Attentional Shifting scale (Rothbart et al., 2001). To allow comparison with the original study, we therefore decided to perform the present analyses on 187 items of the instrument. In this report, we refer to the 187-item form as the Standard Form. The Very Short Form of the CBQ, consisting of 36 items, was constructed following the factor pattern of the Standard Form and consists of a subset of questions of the Standard Form (Putnam \& Rothbart, 2006).

\section{Psychometric properties of the CBQ}

For the scores on the Standard Form (Rothbart et al., 2001), good internal consistency was reported for North American samples; Cronbach's alpha ranging from 0.67 to 0.92 for scores on the 15 scales in the 
Oregon Social Learning Center sample of 6- and 7-year-olds ( $n=183$ ), with a mean of 0.75 (Rothbart et al., 2001). Similar internal consistency estimates were obtained for the scores on CBQ scales in other US samples (Ahadi et al., 1993; Kochanska, De Vet, Goldman, Murray, \& Putnam, 1994). Moreover, adequate average corrected item-total correlations were reported indicating homogeneity of the items contained in each of the scales (Rothbart et al., 2001). Cross-cultural comparison of scale reliabilities with Asian countries revealed generally similar alphas (Chinese sample 0.43 to 0.85 , Ahadi et al., 1993; Japanese sample 0.54 to 0.93 , Kusanagi, 1993), although some scales were considered unreliable (Cronbach's alpha < 0.60). This may possibly be due to translation problems or cultural differences in temperament. Finally, the scores on the very short version had adequate internal consistency estimates of $0.75,0.72$, and 0.74 for the dimensions SR, NA, and EC, respectively (Putnam \& Rothbart, 2006). For these calculations a different sample consisting of 590 US children with a mean age of 54.4 months was used.

\section{Factorial structure of the $C B Q$}

Regarding the factor structure of the $\mathrm{CBQ}$ scales, the higher order three-factor structure has repeatedly been confirmed in different US samples (Ahadi et al., 1993; Kochanska et al., 1994; Rothbart et al., 2001). Moreover, the factor solution was replicated in other cultures, such as China (Ahadi et al., 1993), Japan (Kusanagi, 1993), Norway (Nygaard et al., 2002), Belgium (Eggers et al., 2009; Van den Bergh \& Ackx, 2003), and the Netherlands (Majdandžić \& Van den Boom, 2007), although the samples of the three European countries were rather small.

\section{Rater/measure convergence of the $C B Q$}

Parental agreement for the CBQ scales was earlier reported to be satisfactory (Majdandžić \& Van den Boom, 2007; Majdandžić, Van den Boom, \& Heesbeen, 2008; Rothbart et al., 2001). Majdandžić and Van den Boom (2007) reported that agreement was highest at two different time points for externalizing traits including High Intensity Pleasure and Impulsivity and for easily observable internalizing traits including Shyness and Attentional Focusing among 4-year-olds (with correlations ranging from 0.49 to 0.76 ), and lowest for Smiling/Laughter and Low Intensity Pleasure (with correlations ranging from 0.19 to 0.34 ). Moreover, largely similar correlations between parents were found in the other Dutch study of Majdandžić et al. (2008), and for different samples in the study of Rothbart et al. (2001).

Correlations between laboratory observations of children's temperamental behavior and caregiver responses to the CBQ were generally moderate (e.g., Kochanska, Murray, Jacques, Koenig, \& Vandegeest, 1996; Majdandžić \& Van den Boom, 2007; Majdandžić et al., 2008). Kochanska et al. (1996) found moderate convergence between observational scores of Inhibitory Control and the CBQ scores on Inhibitory Control $(r$ $=0.30$ at age 3 and $r=0.42$ at age 4$)$ and reversed on Impulsivity ( $r=-0.26$ at age 3 and 4$)$. In the Dutch studies (Majdandžić \& Van den Boom, 2007; Majdandžić et al., 2008) other CBQ dimensions (i.e., Surgency, Fear, Shyness, Attentional Focusing, Anger, Sadness, and Activity Level) were matched to corresponding 
laboratory scores at appropriate levels of aggregation. In these studies, measurement convergence was low to moderate, varying across dimensions and over time.

\section{Statistical analysis}

\section{Study aim 1: Dutch assessment CBQ factor analyses and internal consistency}

In the current sample, a Principal Factor Analysis with oblique rotation was performed on the mean scores of the $15 \mathrm{CBQ}$ scales to see whether the original higher order factor solution would be replicated in our sample. The mean scale scores were calculated by summing the relevant scale items divided by the number of items receiving a numerical response (i.e., we did not include items marked 'does not apply' or items receiving no response in determining the number of items). The factor structure of the Very Short Form of the $\mathrm{CBQ}$ was also assessed by performing a principal factor analysis. Items receiving no numerical response were imputed with the mean score of the particular scale the item belongs to. In addition, to study the robustness of the results for the short form, a principal factor analysis was performed only on complete cases ( $n=284)$, thus excluding 70 respondents with missing values. Horn's parallel analysis was performed using a computer program (Monte Carlo principal components analysis for parallel analysis) developed by Watkins $(2000,2006)$ to determine accurately the number of factors to retain in the factor analysis. We forced the program to generate 1,000 samples of random number matrices and corresponding eigenvalues, each of which were based on 353 cases and 15 and 36 variables for the Standard and Very Short Form, respectively. The eigenvalues resulting from Horn's parallel procedure were compared with the eigenvalues found following principal factor analysis on our data.

Internal consistency was evaluated by calculating both Cronbach's alpha and average corrected item-total correlations. Following the guidelines proposed by Nunnally (1978) to define the minimum levels of itemscale correlations that are acceptable, we used cut-off points of 0.15 and 0.30 . Correlations above 0.30 are considered good and correlations below 0.15 are considered unreliable since they would indicate lack of homogeneity of the items within a scale. The reliability estimates were compared cross-culturally.

\section{Study aim 2: Cross-cultural differences in temperament structure}

To provide a quantitative indicator of how well the factor solution of the standard CBQ version found by Rothbart et al. (2001) in the US sample was replicated in the current Dutch sample, factor congruence coefficients were computed for the pairwise comparisons of scale loadings on each factor between the two studies (Watkins, 2005). In addition, comparisons of the factor structure of our sample with the Chinese and Japanese samples were performed. Coefficients of congruence were computed using the computer program Coefficient of Congruence, developed by Watkins (2005). These coefficients can be interpreted as correlation coefficients, ranging from a minimum of 0 (indicating no similarity) to 1.0 (indicating perfect correspondence between pairs of factors). The literature recommends different criteria to express the extent of congruence. In line with Fisher, Schaefer, Watkins, Worrell and Hall (2006), we considered 
absolute coefficients of congruence values equal to or above 0.90 to be indicative of a high degree of factor similarity.

\section{Study aim 3: Comparison of the standard and very short version}

To examine congruence between responses on the three higher order dimensions of the Standard and Very Short Form $\mathrm{CBQ}$, overall mean differences and relative absolute differences were calculated. Moreover, we applied Levy's correction (1967) to determine the correlation between the scores on the Standard and the Very Short Form of the $\mathrm{CBQ}$, using a computer program developed by Barrett (2005).

\section{Results}

\section{Factor analysis of the Standard Form}

A principal factor analysis on the scores of the $15 \mathrm{CBQ}$ scales confirmed the postulated three-factor structure. The factor pattern matrix for the Dutch sample of 6- to 8-year-olds is presented in Table 1. Similar to Rothbart et al. (2001), three factors with eigenvalues greater than 1 were identified, representing the three higher order factors that could be labeled SR, NA, and EC. These factors explained $50.5 \%$ of the variance. In addition, results of the Horn's parallel analysis (eigenvalues were lower than the eigenvalues of the three factors we found following principal factor analysis) led to the retention of the three-factor structure. However, in contrast to Rothbart et al. (2001), we found that more of the variance in the current analysis was explained by the factor SR $(21.9 \%)$ than the factor NA (19.0\%). The first factor in our sample, SR, was defined by positive loadings for the scales of Impulsivity, Activity Level, High Intensity Pleasure, Approach/Anticipation and negative loadings for the scale Shyness. In accordance with the US sample and the previous study conducted in the Netherlands, Smiling/Laughter also loaded on this factor (Majdandžić \& Van den Boom, 2007; Rothbart et al., 2001). However, this factor loaded most highly on the EC factor in our sample (factor loading 0.62 ), comparable to the US sample (factor loading 0.72 ). The second factor was defined primarily by loadings for the scales Sadness, Anger/Frustration, Discomfort, Fear, and negative loadings for the scale Soothability. This factor could be interpreted as NA. The third factor could be interpreted as EC and was defined by loadings for the scales of Low Intensity Pleasure, Smiling/Laughter, Perceptual sensitivity, Inhibitory Control, and Attentional Focusing. The structural correlations between the three factors were relatively small. The correlation of the SR factor with the NA factor and EC factor was near 0 ( $r=-0.02$ and $r=-0.05$, respectively). The NA factor correlated positively with the EC factor $(r=0.11)$. 
Table 1. Factor pattern of Children's Behavior Questionnaire scales of the standard version

\begin{tabular}{|c|c|c|c|}
\hline \multirow[b]{2}{*}{ Scale } & \multicolumn{3}{|c|}{ Factor } \\
\hline & 1. Surgency/Extraversion & 2. Negative Affectivity & 3. Effortful Control \\
\hline Approach/anticipation & 0.59 & -0.25 & 0.27 \\
\hline High intensity pleasure & 0.63 & 0.30 & 0.04 \\
\hline Smiling and laughter & 0.32 & 0.25 & 0.62 \\
\hline Activity level & 0.81 & -0.04 & -0.09 \\
\hline Impulsivity & 0.88 & 0.16 & 0.05 \\
\hline Shyness & -0.33 & -0.47 & -0.18 \\
\hline Discomfort & -0.09 & -0.64 & 0.13 \\
\hline Fear & -0.03 & -0.61 & 0.08 \\
\hline Anger/frustration & 0.37 & -0.65 & -0.06 \\
\hline Sadness & 0.04 & -0.76 & 0.11 \\
\hline Falling reactivity \& soothability & -0.08 & 0.69 & 0.22 \\
\hline Inhibitory control & -0.59 & 0.26 & 0.40 \\
\hline Attentional focusing & -0.45 & 0.13 & 0.31 \\
\hline Low intensity pleasure & -0.18 & -0.01 & 0.67 \\
\hline Perceptual sensitivity & 0.00 & -0.22 & 0.52 \\
\hline
\end{tabular}

Note: $N=353$; Loadings greater than or equal to 0.25 are presented in bold.

\section{Factor analysis of the Very Short Form}

Principal factor analysis of the very short version yielded 10 factors with eigenvalues greater than 1 . After forcing a three-factor solution, a more interpretable and plausible factor structure was found, representing the three higher order factors previously mentioned (see Table 2). The three-factor solution of the very short version of the $\mathrm{CBQ}$ accounted for $24.0 \%$ of the total explained variance. Most of the variance was explained by the NA factor (9.8\%), in line with the study of Putnam and Rothbart (2006). Whereas these authors found that the second and third factors were SR and EC respectively, we found a reversed pattern (EC explaining $8.6 \%$ of the variance and SR explaining $5.6 \%$ of the variance). When we performed factor analyses on the complete cases $(n=284)$, largely similar results were obtained (data not reported). We compared these results with the factor analytic results in the US sample (Putnam \& Rothbart, 2006), and the pattern of factor loadings turned out to be largely similar. However, some scales loaded on other factors than expected (see Table 2). One scale that deserves special attention is Shyness. The three items representing Shyness loaded most highly onto the NA factor (with factor loadings of $-0.54,-0.44$, and -0.41 ), instead of the SR factor. Comparable findings were found with an item of the scale Impulsivity, which also loaded most highly onto the NA factor (-0.59). Another item of the Impulsivity scale loaded onto the EC factor (0.44), but on theoretical grounds belongs to the factor SR (0.35). Additionally, there were some items that loaded primarily onto the factor on which they were supposed to load, but also had loadings (greater than 0.25 ) on another factor (see Table 2). Finally, two items loaded primarily onto the NA factor, on which they were supposed to load, but had loadings below 0.25 . 
Table 2. Factor loadings on higher order dimensions for the Children's Behavior Questionnaire very short version

\begin{tabular}{|c|c|c|c|c|}
\hline Item no. & Scale & Negative affect & Effortful control & Surgency/Extraversion \\
\hline 62 & Anger/frustration & 0.44 & & \\
\hline 78 & Anger/frustration & 0.48 & & \\
\hline 101 & Discomfort & 0.28 & & \\
\hline 44 & Sadness & 0.38 & & \\
\hline 55 & Sadness & 0.42 & & \\
\hline 61 & Discomfort & 0.43 & & \\
\hline 68 & Falling reactivity \& soothability & 0.51 & & \\
\hline 150 & Falling reactivity \& soothability & 0.48 & & \\
\hline 40 & Fear & 0.42 & & \\
\hline 190 & Discomfort & 0.20 & & \\
\hline 70 & Fear & 0.23 & & \\
\hline 64 & Sadness & 0.40 & & \\
\hline 59 & Impulsivity & -0.29 & & 0.49 \\
\hline 71 & Impulsivity & -0.59 & & 0.26 \\
\hline 90 & Impulsivity & & 0.44 & 0.35 \\
\hline 143 & Shyness (reversed) & -0.41 & & 0.28 \\
\hline 23 & Shyness (reversed) & -0.54 & & 0.29 \\
\hline 74 & Shyness (reversed) & -0.44 & & 0.17 \\
\hline 1 & Activity level & 0.27 & & 0.47 \\
\hline 102 & Activity level & & & 0.48 \\
\hline 8 & High intensity pleasure & & & 0.54 \\
\hline 172 & Activity level & & & 0.52 \\
\hline 159 & High intensity pleasure & & & 0.57 \\
\hline 139 & High intensity pleasure & & & 0.49 \\
\hline 136 & Inhibitory control & -0.34 & -0.40 & \\
\hline 147 & Inhibitory control & & -0.41 & -0.33 \\
\hline 63 & Inhibitory control & & -0.41 & \\
\hline 125 & Attentional focusing & & -0.44 & \\
\hline 151 & Low intensity pleasure & & -0.58 & \\
\hline 31 & Perceptual sensitivity & & -0.44 & \\
\hline 98 & Perceptual sensitivity & & -0.49 & \\
\hline 144 & Attentional focusing & & -0.34 & \\
\hline 65 & Perceptual sensitivity & & -0.51 & \\
\hline 146 & Low intensity pleasure & & -0.43 & \\
\hline 186 & Attentional focusing & & -0.37 & \\
\hline 164 & Low intensity pleasure & & -0.34 & \\
\hline
\end{tabular}

Note: $N=353$; Loadings greater than or equal to 0.25 in bold (for items loading on another factor than intended).

\section{Internal consistency}

In calculating the mean scores and Cronbach's alphas for the three higher order factors of both CBQ forms, the scales Positive Anticipation and Smiling/Laughter were not included. This is based on previous study findings showing that these scales often loaded highly on more than one factor (Rothbart et al., 2001). Since the CBQ was not designed with this structure in mind (Putnam \& Rothbart, 2006), these scales were not included in alpha calculations for the higher order dimensions. Cronbach's alphas of the scores on the Standard and Very Short Form are provided in Table 3. Similar results emerged as in the US sample (Rothbart et al., 2001). The average item-total correlations of the 15 scales (after correction for overlap) are also presented in Table 3, suggesting adequate consistency of the items. In this table the mean, SD, and ranges for the scores on the individual $\mathrm{CBQ}$ scales and higher order dimensions of the standard version are 
displayed. For the very short version, we presented mean scores, standard deviations, and ranges for the scores on the three higher order dimensions.

Table 3. Descriptives, internal consistency and average corrected item-total correlations for the Children's Behavior Questionnaire scales and the higher order dimensions

\begin{tabular}{|c|c|c|c|c|c|c|}
\hline & No. Items & Mean (SD) & Min. & Max. & $\begin{array}{l}\text { Cronbach's } \\
\text { alpha }\end{array}$ & $\begin{array}{l}\text { Average corrected } \\
\text { item-total } \\
\text { correlations }\end{array}$ \\
\hline \multicolumn{7}{|l|}{ Standard version (15 Scales) } \\
\hline Approach/anticipation & 13 & $4.64(0.77)$ & 2.46 & 6.62 & 0.75 & 0.37 \\
\hline High intensity pleasure & 13 & $4.67(0.97)$ & 2.08 & 6.83 & 0.83 & 0.47 \\
\hline Smiling and laughter & 13 & $5.65(0.69)$ & 2.62 & 7.00 & 0.81 & 0.45 \\
\hline Activity level & 13 & $4.30(0.88)$ & 1.92 & 6.54 & 0.81 & 0.44 \\
\hline Impulsivity & 13 & $4.25(0.81)$ & 2.15 & 6.85 & 0.77 & 0.40 \\
\hline Shyness & 13 & $3.08(1.12)$ & 1.00 & 6.00 & 0.91 & 0.63 \\
\hline Discomfort & 12 & $3.32(0.90)$ & 1.45 & 6.50 & 0.76 & 0.40 \\
\hline Fear & 12 & $3.19(0.97)$ & 1.00 & 6.83 & 0.76 & 0.40 \\
\hline Anger/frustration & 13 & $3.76(0.84)$ & 1.54 & 6.08 & 0.78 & 0.41 \\
\hline Sadness & 12 & $3.57(0.80)$ & 1.33 & 5.58 & 0.73 & 0.36 \\
\hline Falling reactivity \& soothability & 13 & $5.12(0.84)$ & 2.54 & 6.75 & 0.79 & 0.43 \\
\hline Inhibitory control & 13 & $4.91(0.83)$ & 2.54 & 6.69 & 0.82 & 0.46 \\
\hline Attentional focusing & 9 & $4.75(0.93)$ & 1.11 & 6.67 & 0.77 & 0.45 \\
\hline Low intensity pleasure & 13 & $5.34(0.68)$ & 3.38 & 7.00 & 0.70 & 0.33 \\
\hline Perceptual sensitivity & 12 & $5.17(0.73)$ & 1.78 & 6.92 & 0.65 & 0.31 \\
\hline \multicolumn{7}{|l|}{ Standard version (3 Factors) } \\
\hline Negative Affectivity & 62 & $3.35(0.65)$ & 1.85 & 5.66 & 0.90 & 0.35 \\
\hline Surgency/Extraversion & 52 & $4.53(0.72)$ & 2.48 & 6.40 & 0.90 & 0.37 \\
\hline Effortful Control & 47 & $5.06(0.55)$ & 3.52 & 6.49 & 0.85 & 0.31 \\
\hline \multicolumn{7}{|l|}{ Very short version (3 Factors) } \\
\hline Negative Affectivity & 12 & $3.42(0.83)$ & 1.42 & 5.92 & 0.72 & 0.36 \\
\hline Surgency/Extraversion & 12 & $4.51(0.84)$ & 2.08 & 6.67 & 0.76 & 0.39 \\
\hline Effortful Control & 12 & $5.26(0.74)$ & 3.17 & 7.00 & 0.72 & 0.37 \\
\hline
\end{tabular}

Note: $N=353$; CBQ standard version (187 items, 15 scales, 3 higher order factors); CBQ very short version (36 items, 3 higher order factors); based on theoretical grounds as delineated in the US studies of Putnam and Rothbart (2006), and Rothbart et al. (2001), the Positive Anticipation and Smiling/Laughter scales were not included to calculate mean scores for the higher order factors of SR and EC, respectively.

\section{Cross-cultural differences in temperament structure}

The similarity of the factor loadings obtained by Rothbart et al. (2001) for the Standard Form and the factor loadings of the current sample was assessed by computing the coefficient of congruence. Since we replicated the three-factor solution found by Rothbart et al. (2001), exploration of factor similarities across samples was possible. The congruence coefficients, 0.96 (SR), 0.95 (NA), and 0.98 (EC), indicate a high degree of similarity with the US sample. Coefficients of congruence between the Asian studies and loadings in our study were as follows: 0.87 (SR), 0.95 (NA), 0.83 (EC) for the Chinese sample, and 0.91 (SR), 0.88 (NA), and 0.96 (EC) for the Japanese sample. The results indicate relatively high congruity for most of the higher order factors, with the highest congruity for the US sample. 


\section{Comparison of the standard and very short version}

The overall mean differences between three higher order dimensions (SR, NA, and EC) of the Standard and Very Short CBQ forms are $-0.02,0.07$, and 0.20 respectively, on a seven-point Likert scale (see Table 4). This table also presents the percentages of respondents falling into five different categories of relative absolute differences. The percentage of respondents with relative absolute differences greater than 0.15 is as follows: $4.2 \%$ for SR, $21.2 \%$ for NA, $8.5 \%$ for EC.

Table 4. Comparison of the standard and short Children's Behavior Questionnaire form on the higher order dimensions

\begin{tabular}{|c|c|c|c|c|c|c|c|c|c|}
\hline & \multicolumn{2}{|c|}{ Mean } & \multirow{2}{*}{$\begin{array}{c}\text { Overall mean } \\
\text { difference }\end{array}$} & \multicolumn{6}{|c|}{ Percentage of respondents with a relative absolute difference (D) } \\
\hline & Standard & Short & & $<0.05$ & $\begin{array}{c}0.05<\mathrm{D}< \\
0.10\end{array}$ & $\begin{array}{c}0.10<D< \\
0.15\end{array}$ & $\begin{array}{c}0.15<D< \\
0.20\end{array}$ & $\begin{array}{c}0.20<D< \\
0.25\end{array}$ & $>0.25$ \\
\hline SR & 4.53 & 4.51 & -.02 & $53.4 \%$ & $29.1 \%$ & $13.3 \%$ & $3.1 \%$ & $0.8 \%$ & $0.3 \%$ \\
\hline NA & 3.35 & 3.42 & .07 & $28.5 \%$ & $29.7 \%$ & $20.6 \%$ & $11.6 \%$ & $6.2 \%$ & $3.4 \%$ \\
\hline EC & 5.06 & 5.26 & .20 & $41.2 \%$ & $32.2 \%$ & $18.1 \%$ & $5.6 \%$ & $2.3 \%$ & $0.6 \%$ \\
\hline
\end{tabular}

Note: $\mathrm{SR}=$ Surgency/Extraversion; NA = Negative Affectivity; $\mathrm{EC}=$ Effortful Control.

We applied Levy's correction (1967) to determine the relationship between the three higher order dimensions of the standard and the very short $\mathrm{CBQ}$ scores. This correction factor partials out the common error from the scores of the two forms to achieve true score correlations between the longer version and the shorter version that includes the same items. The resulting corrected correlations were 0.82 for NA, 0.86 for $\mathrm{SR}$, and 0.75 for $\mathrm{EC}$.

\section{Discussion}

The present study aimed to assess factorial validity and psychometric properties of the CBQ scale scores in a Dutch sample and to examine cross-cultural differences in temperament structure with large samples from the US, China and Japan. The factors found in the Dutch version of the Standard CBQ Form are similar to the three factors found previously and were accordingly labeled as SR, NA, and EC. Moreover, the present study showed good psychometric properties of the Dutch translation of the CBQ scores in terms of reliability estimates and consistency of item content within the scales, which were very close to the US studies (Ahadi et al., 1993; Rothbart et al., 2001). For the Dutch sample of 6- to 8-year-olds, the threefactor solution had excellent congruence with the US sample. Findings of the Dutch study of Majdandžić and Van den Boom (2007) indicated a lower congruency coefficient for one factor (coefficients ranged from 0.88 to 0.98 , with a mean of 0.95 ). However, this lower degree of congruity is most probably due to the small sample size of that study $(\mathrm{N}=94)$ or related to the inclusion of a slightly younger age group. Nevertheless, the cross-national robustness of the scale is supported because both Dutch studies yielded 
the three-factor structure as proposed by Rothbart et al. (2001). For the two Asian samples, the congruence coefficients were a little lower, slightly above or below the value of 0.90 .

Although the structural model was highly consistent with the structure identified in different cultural samples, there were some differences in terms of factor loadings. For a visual comparison of the factorial structure across the US, China, and Japan we refer to the work of Ahadi et al. (1993) and Rothbart et al. (2001). First, whereas Smiling/Laughter loaded only on the SR factor in the Chinese sample, it loaded primarily on the factor EC and moderately on the factor SR in the Dutch, US and Japanese sample. Rothbart et al. (2001) hypothesized that 'in cultures that encourage expression of positive effect, Smiling/Laughter may be related to EC as well as to positive affect' (page 1404). Chinese socialization patterns may differ in this respect, in that children are subject to a highly disciplined socialization process to meet adult expectations (Ho, 1986). The factor loading of the scale Approach/Anticipation in our sample is high for the factor SR, in line with findings of the Chinese study. In contrast to these results, the loading on this scale for the US sample is not as strong and is more evenly distributed across all three factors. For the Japanese sample, the loading of the Approach/Anticipation scale is high for the factor NA, whereas the role of this scale in defining the SR factor is moderate. More research is needed to understand these results. With respect to Shyness in our Dutch sample, this scale loaded higher on the factor NA $(-0.47)$ than on the SR factor (-0.33), where the factor originally belongs, in contrast to the US, Chinese and Japanese samples. This finding is replicated in our factor analysis of the 36 -item form. From these findings, we might conclude that in our Dutch sample the scale of Shyness better fits the higher order dimension of NA instead of SR. More research is needed on numerous Dutch samples to replicate these findings. The last difference was the higher factor loadings of the scales Inhibitory Control and Attentional Focusing in our sample for the factor SR (-0.59 and -0.45$)$ compared to the factor EC (0.40 and 0.31 , respectively). For the other three countries these two scales had the highest factor loading on the factor EC.

The variation in factor loadings between the different samples is possibly due to cultural differences and translation problems for the Japanese version (because of lower scale reliabilities), thereby explaining the outcomes with regard to the congruence coefficients found between the different cultural samples. Moreover, differences in structural relations between the three higher order dimensions across countries may account for small variations in congruence coefficients. Although three congruence coefficients were slightly below 0.90 (Ahadi et al., 1993; Rothbart et al., 2001), these values are still relatively high. Therefore, we can conclude that the factor structure was largely invariant across the cultures. This result is in line with Rothbart et al.'s (2001) hypothesis that the basic structure of temperament characteristics among children is relatively invariant across cultures. Temperament, in that sense, reflects the essential sameness of inborn capacities of humans and the common biology-based mechanisms from which individual differences in temperament arise (Ahadi et al., 1993). Future research is necessary to better understand the differences in factor loadings between countries.

Furthermore, the factorial validity and psychometric characteristics of the very short CBQ form scale scores were assessed. Although the factor structure was not perfectly replicated, as indicated by the 10-factor 
structure, the forced three factors had adequate internal consistency coefficients and a relatively high degree of correspondence with the Standard Form higher order factors. In the future, the Very Short Form needs to be administered in isolation from the longer version to confirm our (and other) findings regarding factor structure and psychometric properties of this $\mathrm{CBQ}$ version. Since questionnaire length is reduced by more than $80 \%$ when administering the Very Short Form, the time needed to complete the questionnaire is greatly reduced from approximately $1 \mathrm{hr}$ to no more than $15 \mathrm{~min}$. In choosing between the two forms, it is important to consider that the Standard Form CBQ gives more precise estimates of the temperamental behavior patterns than does the Very Short Form. However, in case of time constraints the Very Short Form $\mathrm{CBQ}$ would be a valid and appropriate alternative.

A major strength of our study was the large sample size $(N=353)$, comparable to the validation study of the original sample. However, generalization of our findings regarding temperamental factor structure to age groups below the age of 6 in the Netherlands is not possible. In the future, the CBQ should be administered to these younger age samples. Additionally, caution is warranted in generalizing our results to the larger population due to the low response rate. Nevertheless, we have no reason to suspect selective nonresponse of parents. The sample was representative for the Dutch population in terms of gender, marital status, parental employment status, and mother's educational level. A slightly higher percentage of fathers achieved a college or university degree in our sample (41.1\%) compared to the Dutch population of males between the ages 35 and 45 (38.5\%; Centraal Bureau voor de Statistiek, 2010). On the basis of the demographic characteristics of our sample, we do not expect the response rate to have biased our findings. To conclude, the $\mathrm{CBQ}$ is a promising instrument for reliable and valid measurement of temperament in Western European children. Since the CBQ is easily administered, it can be readily employed as a research tool to assess a broad range of temperament characteristics in young children. 


\section{Appendix}

\section{Children's Behavior Questionnaire scale definitions and sample items by higher order temperamental trait}

\section{Surgency/Extraversion}

Activity level: Gross motor activity, including rate and extent of locomotion. 'Seems always in a big hurry to get from one place to another'

High intensity pleasure: Pleasure or enjoyment related to situation involving high stimulus intensity, rate, complexity, novelty, and incongruity. 'Likes going down high slides or other adventurous activities'

Impulsivity: Speed of response initiation. 'Usually rushes into an activity without thinking about it'

Positive anticipation: Amount of excitement and anticipation for expected pleasurable activities. 'Gets so worked up before an exciting event that $s($ he) has trouble sitting still'

Shyness (vs. social approach) reversed: Slow or inhibited (versus rapid) speed of approach and discomfort (versus comfort) in social situations. 'Often prefers to watch rather than join other children playing'

\section{Negative Affectivity}

Anger/Frustration: Negative affectivity related to interruption of ongoing tasks or goal blocking. 'Has temper tantrums when $s($ he) doesn't get what $s($ he) wants'

Discomfort: Negative affectivity related to sensory qualities of stimulation, including intensity; rate; or complexities of light, movement, sound, and texture. 'Is not very bothered by pain'

Fear: Negative affectivity, including unease, worry, or nervousness, which is related to anticipated pain or distress and/or potentially threatening situations. 'Is not afraid of large dogs and/or other animals'

Sadness: Negative affectivity and lowered mood and energy related to exposure to suffering, disappointment, and object loss. 'Cries sadly when a favorite toy gets lost or broken'

Soothability (and Falling Reactivity) reversed: Rate of recovery from peak distress, excitement, or general arousal. 'Has a hard time settling down for a nap'

\section{Effortful Control}

Attentional focusing: Capacity to maintain attentional focus on task-related channels. 'When picking up toys or other jobs, usually keeps at the task until it's done'

Inhibitory control: Capacity to plan and to suppress inappropriate approach responses under instructions or in novel or uncertain situations. 'Can lower his/her voice when asked to do so'

Low intensity pleasure: Pleasure or enjoyment related to situations involving low stimulus intensity, rate, complexity, novelty, and incongruity. 'Rarely enjoys just being talked to'

Perceptual sensitivity: Detection of slight, low-intensity stimuli from the external environment. 'Notices the smoothness or roughness of objects $s($ he) touches'

Smiling/Laughter: Positive affect in response to changes in stimulus intensity, rate, complexity, and incongruity.

'Laughs a lot at jokes and silly happenings'

Source. Adapted from 'Children's temperament in the United States and China: Similarities and differences', by S. A. Ahadi, M. K. Rothbart, \& R. Ye, 1993, European Journal of Personality, 7, pp. 359-377. Copyright 1993 by John Wiley \& Sons. Adapted with permission. 


\section{CHAPTER 6}

The Children's Behavior Questionnaire very short form:

Psychometric properties and validation of a one-item temperament scale

Ester FC Sleddens

Sheryl O Hughes Teresia M O'Connor Alicia Beltran Janice C Baranowski Theresa A Nicklas

Tom Baranowski

Psychological Reports, 2012 


\begin{abstract}
Little research has been conducted on the psychometrics on the very short scale (36 items) of the Children's Behavior Questionnaire (CBQ), and no one-item temperament scale has been tested for use in applied work. In this study, 237 United States caregivers completed a survey to define their child's behavioral patterns (i.e., Surgency, Negative Affectivity, Effortful Control) using both scales. Psychometrics of the 36-item CBQ was examined using classical test theory, principal factor analysis, and item response modeling. Classical test theory analysis demonstrated adequate internal consistency and factor analysis confirmed a three-factor structure. Potential improvements to the measure were identified using item response modeling. A one-item (three response category) temperament scale was validated against the three temperament factors of the 36 -item scale. The temperament response categories correlated with the temperament factors of the 36 -item scale, as expected. The one-item temperament scale may be applicable for clinical use.
\end{abstract}




\section{Introduction}

Children make important contributions to their interpersonal relationships. Child temperament is one child characteristic that has been shown to strongly affect the development of appropriate social interactions in children (Putnam, Sanson, \& Rothbart, 2002). Temperament has been defined as a function of biological make-up, reactivity to stimuli (arousability of motor, affective, and sensory response systems), and selfregulation (including attentional strategies and behavioral avoidance) (Rothbart \& Bates, 1998; Rothbart, Ahadi, Hershey, \& Fisher, 2001). The Children's Behavior Questionnaire (CBQ; Rothbart et al., 2001) is one of the most well-known and comprehensive instruments assessing individual differences in facets of temperament in children ages three to eight years old.

Progressively shorter versions of the $\mathrm{CBQ}$ have been validated for use in research, including the standard (195 items), short (94 items) and very short scales (36 items) (Putnam \& Rothbart, 2006; Rothbart et al., 2001). All scales capture three broad temperamental traits: Surgency or Extraversion (in this paper referred to as Surgency) (SR), Negative Affectivity (NA), and Effortful Control (EC), which are conceptually similar to three of the major 'Big Five' dimensions of adult personality, labeled Extraversion, Neuroticism, and Conscientiousness, respectively (Rothbart, Ahadi, \& Evans, 2000). Whereas the first two traits are characterized by behaviors reactive to environmental changes, the third is the self-regulation aspect of temperament, involving processes that moderate reactivity. These higher order temperament factors have been repeatedly confirmed using factor analytic procedures in the United States (US; Putnam \& Rothbart, 2006; Rothbart et al., 2001) and European (Eggers, De Nil, \& Van den Bergh, 2009/2010; Majdandžić \& Van den Boom, 2007; Nygaard, Smith, \& Torgersen, 2002; Sleddens, Kremers, Candel, De Vries, \& Thijs, 2011; Van den Bergh \& Ackx, 2003) and Asian countries (Ahadi, Rothbart, \& Ye, 1993; Rothbart et al., 2001).

Although the standard scale of the Children's Behavior Questionnaire gives better estimates of temperamental behavior patterns, administering the longer scale is not always feasible due to time constraints or participant burden. The three higher order temperament factors, however, have been invariant across the two shorter versions (Putnam \& Rothbart, 2006; Sleddens, Kremers et al., 2011), and other psychometric results (e.g., internal reliability coefficients) were promising for the 94-item scale (Healey, Brodzinsky, Bernstein, Rabinovitz, \& Halperin, 2010; Putnam \& Rothbart, 2006; Tumanova, Zebrowski, Throneburg, \& Kulak Kayikci, 2011) and the 36-item scale (Hughes, Shewchuk, Baskin, Nicklas, \& Qu, 2008; Miller et al., 2009; O'Connor et al., 2010; Putnam \& Rothbart, 2006; Sleddens, Kremers et al., 2011; Ward, Gay, Alkon, Anders, \& Lee, 2008).

The very short scale of the $\mathrm{CBQ}$, consisting of items extracted from the standard scale, has been more frequently administered during the last few years (Hughes et al., 2008; Miller et al., 2009; O'Connor et al., 2010; Ward et al., 2008). However, the factorial validity of this 36 -item scale has only been established when administered as part of the longer scales of the CBQ (Putnam \& Rothbart, 2006; Sleddens, Kremers et al., 2011). In addition, advanced psychometric procedures, e.g., item response modeling, have not been reported for the $\mathrm{CBQ}$. In the current study, the 36-item scale was administered to evaluate its psychometric 
properties using classical test theory (internal consistency and corrected item-total correlation) and factor analysis (Putnam \& Rothbart, 2006) in an ethnically diverse sample. Item response modeling was also applied to extend the classical test theory approach, a method which examines whether the item content covers the range of respondents' answers about the construct of interest (Heesch, Mâsse, \& Dunn, 2006; Wilson, Allen, \& Corser, 2006a/b).

It has been shown that an incompatibility or 'poor fit' between the temperamental characteristics of the child and expectations and attitudes of caregivers may lead to poor outcomes for children (Putnam et al., 2002). Interventions targeting parenting may benefit from being tailored to the child's temperament (e.g., Bradley \& Corwyn, 2008; Gubbels et al., 2009; Kochanska, Aksan, \& Joy, 2007; Van Zeijl et al., 2007; Wu, Dixon, Dalton, Tudiver, \& Liu, 2010; Zeller, Boles, \& Reiter-Purtill, 2008), but a simple way to assess a child's temperament would be needed. For example, researchers using game technology to develop an interactive electronic game to teach parents strategies to increase vegetable consumption in preschoolers used a short assessment of child temperament to tailor parenting strategies to the temperament of their child (Baranowski et al., in press; Beltran et al., in press). Such a game that simulates a child with whom parents can interact during feeding scenarios may benefit from having the child character reflect the temperament of the player's child, making the interaction more realistic. Within the context of a video game, the assessment of the player's child's temperament needs to be done rapidly, or else the player's attention and interest will be lost, and parents need to identify the single temperament characteristic they feel is most dominant in their child to make tailoring of the game feasible. A single item assessment of the player's child's temperament would work well in such a situation, but the single item must be validated. This approach increases the likelihood of parents participating in the game and benefiting from the strategies proposed. Hence, besides assessing the psychometrics of the 36 -item temperament scales, this study aimed to assess the validity of a one-item scale, wherein parents chose from one of three vignettes to describe their child's temperament. It was hypothesized that parents selecting the one best vignette to describe the dominant temperament characteristic of their child would provide the highest mean scores on the corresponding temperament factor when rating their child on the 36 -item scale.

\section{Method}

\section{Respondents and Procedures}

US English-speaking caregivers of children ages 3 to 5 years were recruited for this cross-sectional online survey about child temperament, in which both the 36-item and the one-item temperament scale were administered. Potential participants were approached from July 2010 until February 2011 by (a) posting flyers in the vicinity of the Texas Medical Center, community centers, public libraries, universities, sports centers and museums throughout Houston, Texas; (b) posting the study on the website of Baylor College of Medicine and the Children's Nutrition Research Center (CNRC); and (c) listing the study in the CNRC's nationally distributed newsletter and recruiting from the participant database. Completion time for the survey was approximately 10 minutes. From all completed entries, two drawings were conducted for two 
$\$ 100$ gift cards. Only participants who agreed to take part in the raffles $(n=224)$ had a chance to win one of the gift cards.

A total of 301 participants entered the website. Of those, 13 were disqualified because they did not agree to participate $(n=2)$, did not have a 3- to 5-year-old child $(n=10)$, or did not live with the child during most of the week $(n=1)$. Another 51 entries were deleted from the database: 11 were duplicates (assessed by checking email and IP addresses) and 40 participants did not complete the 36-item CBQ scale. The final data set contained 237 participants, mostly female caregivers (93.2\%). Children's mean age was $3.9(S D=0.8)$ years, and gender of the child was almost equally divided (boys: $53.6 \%$, girls: $46.4 \%$ ). The study was reviewed and approved by the Institutional Review Board of the Baylor College of Medicine; all caregivers completed online informed consent prior to data collection.

\section{Measures}

\section{$\underline{\text { Background characteristics }}$}

Parents or guardians were asked to report some family demographics in the beginning of the online survey including child's age, gender, and their relationship to the child. The participant's gender, race, living situation, highest educational level attained, employment, and annual household income were assessed at the end of the survey.

\section{Temperament scales}

The 36-item scale of the CBQ (Putnam \& Rothbart, 2006) was used to assess the three broad factors of temperament: SR, NA, and EC, consisting of 12 statements for each trait. Parents were asked to report using a seven-point scale, with anchors 1 (Extremely untrue of your child) and 7 (Extremely true of your child), on how well each statement described their child's reaction to a given situation within the past 6 months; a 'not applicable' option was provided if parents could not answer because they had not seen their child in the situation described. The $\mathrm{CBQ}$ higher order factors have been replicated across multiple age samples among different countries and showed adequate internal consistency reliability ranging from 0.61 to 0.94 (e.g., Ahadi, Rothbart, \& Ye, 1993; Kochanska, De Vet, Goldman, Murray, \& Putnam, 1994; Majdandžić \& Van den Boom, 2007; Rothbart et al., 2001; Sleddens, Kremers et al., 2011). The very short scale (Hughes et al., 2008; O'Connor et al., 2010; Putnam \& Rothbart, 2006; Sleddens, Kremers et al., 2011; Ward et al., 2008) also showed adequate internal consistency (Cronbach's alphas ranging from 0.63 to 0.76 for SR, from 0.65 to 0.79 for NA, and from 0.67 to 0.74 for EC). Convergent validity included parental agreement (Rothbart et al., 2001; Majdandžić \& Van den Boom, 2007; Majdandžić, Van den Boom, \& Heesbeen, 2008) and prediction of social (Rothbart, Ahadi, \& Hershey, 1994; Rotbhart et al., 2001) and laboratory behavior patterns (Kochanska, Murray, Jacques, Koenig, \& Vandegeest, 1996; Majdandžić \& Van den Boom, 2007; Majdandžić et al., 2008). 
The one-item temperament scale included three response options reflecting the three higher order temperament factors (SR, NA, and EC) from the CBQ. For a description of the one-item scale and operational definitions, see Table 1. This one-item scale was developed by two of the authors (SH and TO) and agreed upon by the whole group. The two co-authors (SH and TO), one an expert in child development (SH) and the other a pediatrician (TO), wrote single item statements that reflected the general theory and the items (Putnam \& Rothbart, 2006; Rotbhart et al., 2001) for each of the three major child temperament factors. Each vignette attempted to capture the original subscales defined by the higher order factor (Rothbart et al., 2001): SR by Impulsivity, lack of Shyness, Activity Level, and High Intensity Pleasure; NA by Anger, Discomfort, Sadness, difficulty to Sooth, and Fear; and EC by Inhibitory Control, Attentional Focusing, Low Intensity Pleasure, and Perceptual Sensitivity. Each statement was reviewed and revised by the co-authors until consensus was reached about optimal wording. A single item was selected to identify the dominant temperament characteristic of the child in order to simplify the task of tailoring an intervention to the child's temperament.

\section{Table 1. One-item temperament scale}

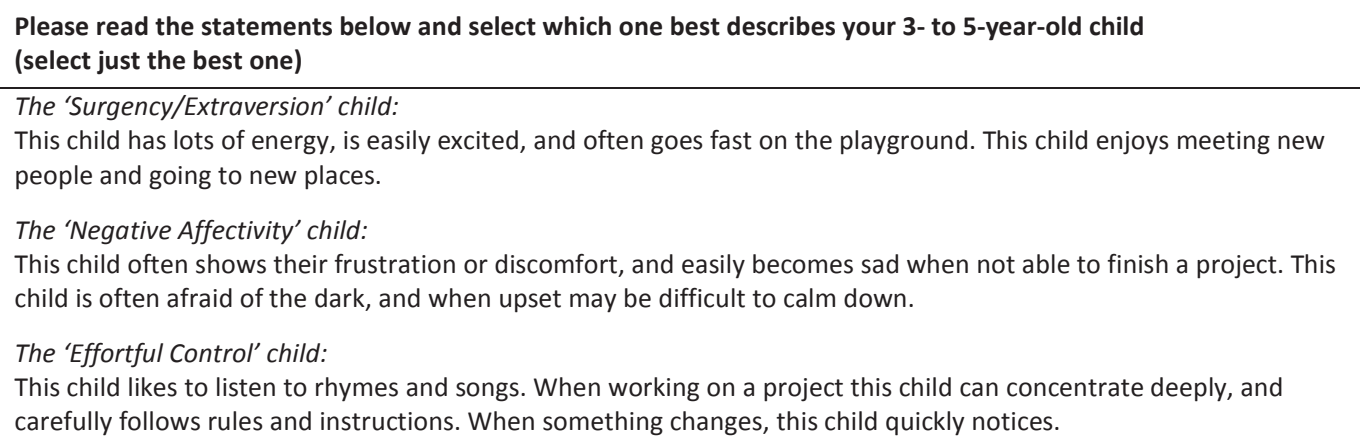

Note: Operational definition of the three higher order temperament factors: SR (tendency to perform impulsive and active behavior), NA (predisposition to experience negative affective states), EC (the ability of a child to control attentional processes and behavior).

\section{Statistical Analyses}

\section{Classical test theory item analysis, 36-item temperament scale}

Relevant scale items of the 36-item scale were combined to create mean factor scores for SR, NA, and EC. Items marked 'does not apply' were not included in the scores. Missing data were not present because the participants were forced to answer all of the questions or the computer would not progress. Internal consistency reliability was evaluated by calculating both Cronbach's alpha and average corrected item-total correlations. The minimum acceptable cut-off point for Cronbach's alphas was 0.70 and of item-scale correlations were between 0.15 and 0.30 (Nunnally \& Bernstein, 1994). Correlations of 0.30 or above were 
considered good, and below 0.15 were considered unreliable since they would indicate lack of homogeneity of the items within a scale.

\section{Principal Factor Analysis, 36-item temperament scale}

Principal Factor Analysis (exploratory in nature) with oblique rotation yielded factor loadings for each item as well as the percent variance explained by each factor. In accordance with the original validation studies (Putnam \& Rothbart, 2006; Rothbart et al., 2001) a factor loading of 0.25 or higher was an indication of a reasonably high factor loading. Items with no numerical response were imputed with the mean score of the factor to which the item belonged. On average, respondents chose the 'not applicable' option less than $1 \%$ of the time. The frequency of 'not applicable' responses was identified for each item. Item 6 ('Prepares for trips and outings by planning things he/she will need') had the largest number of 'not applicable' answers ( $n=12,5.1 \%$ ). To verify the robustness of the results, a principal factor analysis was performed on only complete cases $(n=183)$, excluding 54 respondents with missing values. Parallel analysis was performed using the SPSS syntax (O'Connor, 2000) to determine accurately the number of factors to retain in the factor analysis. The program was forced to generate 1,000 samples of random number matrices and corresponding eigenvalues, each of which was based on 183 cases and 36 variables. The eigenvalues resulting from this procedure were compared with the eigenvalues found following principal factor analysis on the data. Factors were retained when eigenvalues from the actual data (following principal factor analysis) were greater than the eigenvalues from the random data.

\section{Item response modeling analyses, 36-item temperament scale}

Rasch modeling (partial credit model) was performed on all cases using the 'ConQuest' software program (Wu, Adams, \& Haldane, 2007) which provided item parameter difficulty estimates, item fit statistics, Wright maps, and reliability indices. Item difficulty refers to the item's location on the underlying temperament trait, and reflects how difficult it was for a person to respond affirmatively to an item (higher values $=$ more difficult). Item fit was determined by computing weighted mean square fit statistics for each item, which indicated whether residuals varied as much as expected given the observed distribution. Items with a weighted fit statistic $<0.75$ or $>1.33$, and for which the weighted $t$ statistic was $<-2.00$ or $>2.00$, were considered to fit poorly (Adams \& Khoo, 1996). The Wright map visually linked the distribution of respondents (indicated by Xs on the left side of the Wright map) on the latent temperament factors to the distribution of individual item difficulties (represented on the right side by item number), using the same metric, i.e., a logit scale centered at a mean of zero. The items and respondents should be located between -3 and +3 logits. Also included in the Wright map is the location of the items by threshold (the seven-point Likert scale is separated by six threshold points, where Threshold 1 refers to the threshold between response option 1 (Extremely untrue of your child) and response option 2 (Quite untrue of your child). Item separation reliability, which indicated 'how well the sample of subjects had spread the items along the measure of the test' (Fisher, 1992, page 238), was calculated for the three factors. Finally, visual examination of the item response functions evaluated the functioning of the seven-point response format. 


\section{Analysis of variance and pearson chi-square analyses of the one-item temperament scale}

The average scores of the three temperament factors (SR, NA, EC) were compared for each of the three temperament groups as defined by the one-item temperament scale. This was conducted by means of a three-level group ANOVA (SR, NA, EC), followed by Bonferroni and Tukey's HSD post hoc analyses, adjusting $p$ values for multiple testing to detect differences in mean scores on each of the three temperament factors between the three groups. Pearson chi-square analyses with categorical variables were used with a $3 \times 3$ contingency table comparing temperament characteristics of the children, defined by parents using the one-item scale, and the 36-item scale. Mean scores were calculated for the three temperament factors of $\mathrm{SR}, \mathrm{NA}$, and EC, and a child was characterized by a temperament characteristic based on the highest mean score across the three factors.

\section{Results}

The study sample was ethnically diverse (Table 2). The majority was White (39.2\%), but Hispanics (25.3\%) and African-Americans (23.6\%) were also represented. A minority of the participants were combined into 'other', consisting of American Indians, Native Hawaiians, Pacific Islanders, and Asians (10.5\%). In total, 209 participants (88.2\%) were from Texas (US). The other 28 participants reported living in other states within the US $(n=24)$, Canada $(n=1)$, or these data were missing $(n=3)$. Most participants indicated they were married or lived with a significant other (75.5\%). A majority reported higher levels of education $(59.1 \%$ indicated having a college degree or higher) and high annual household income $(46.0 \%$ indicated a household income above $\$ 60,000$ a year). 
Table 2. Participant characteristics $(N=237)$

\begin{tabular}{|c|c|c|}
\hline Description & $n$ & $\%$ \\
\hline \multicolumn{3}{|l|}{ Gender } \\
\hline Male & 16 & 6.8 \\
\hline Female & 221 & 93.2 \\
\hline \multicolumn{3}{|l|}{ Relationship to child } \\
\hline Parent & 227 & 95.8 \\
\hline Grandmother & 5 & 2.1 \\
\hline Aunt & 4 & 1.7 \\
\hline Female guardian & 1 & 0.4 \\
\hline \multicolumn{3}{|l|}{ Race } \\
\hline Black or African-American & 56 & 23.6 \\
\hline White or Euro-American & 93 & 39.2 \\
\hline Hispanic & 60 & 25.3 \\
\hline Other & 25 & 10.5 \\
\hline No response & 3 & 1.3 \\
\hline \multicolumn{3}{|l|}{ Living situation } \\
\hline Married or living with a significant other & 179 & 75.5 \\
\hline Single, never married & 21 & 8.9 \\
\hline Divorced, separated or widowed & 35 & 14.8 \\
\hline No response & 2 & 0.8 \\
\hline \multicolumn{3}{|l|}{ Education } \\
\hline Attended some high school & 12 & 0.8 \\
\hline High school graduate or GED & 17 & 7.2 \\
\hline Technical school & 12 & 5.1 \\
\hline Some college & 64 & 27.0 \\
\hline College graduate & 72 & 30.4 \\
\hline Post graduate study & 68 & 28.7 \\
\hline No response & 2 & 0.8 \\
\hline \multicolumn{3}{|l|}{ Employed } \\
\hline Yes & 160 & 67.5 \\
\hline No & 75 & 31.6 \\
\hline No response & 2 & 0.8 \\
\hline \multicolumn{3}{|l|}{ Annual household income } \\
\hline Less than $\$ 10.000$ & 10 & 4.2 \\
\hline$\$ 10.000-\$ 19.999$ & 15 & 6.3 \\
\hline$\$ 20.000-\$ 39.999$ & 45 & 19.0 \\
\hline$\$ 40.000-\$ 59.999$ & 56 & 23.6 \\
\hline$\$ 60.000$ or more & 109 & 46.0 \\
\hline No response & 2 & 0.8 \\
\hline
\end{tabular}

Psychometric properties of the 36-item temperament scale

\section{Classical test theory item analysis}

Internal consistency (Cronbach's alpha) ranged from 0.69 to 0.78 (Table 3). The average item-total correlations among the three factors suggested good consistency of the items $(S R=0.41, N A=0.37, E C=$ 0.33). For the SR factor the corrected item-total correlations of all items were above the value of 0.30 . For the two other higher order factors, some items fell below the value of 0.30 , but still above the critical cut- 
off point of 0.15 (NA = two items; $E C=$ five items). One EC item had an item-total correlation (after correlation for overlap) of 0.05 , far below the critical value of 0.15 . Cronbach's alpha increased from 0.69 to 0.71 when this item was removed.

\section{Principal factor analysis}

Principal factor analysis revealed 12 factors having eigenvalues greater than 1 . Forcing a three-factor structure, as proposed by Rothbart et al. (2001) and Putnam and Rothbart (2006), revealed the three higher order factors SR, NA, and EC (Table 3). Results of the parallel procedure (eigenvalues from random data were lower than the eigenvalues of the three factors found following principal factor analysis) supported the retention of the three-factor structure. The three-factor solution accounted for $25.1 \%$ of the total variance. Most of the variance was explained by the SR factor (9.4\%) with EC explaining $8.4 \%$ and NA explaining 7.3\%, respectively. Item 30 on EC ('My child approaches places he/she has been told are dangerous slowly and cautiously') had a factor loading below 0.25 (factor loading of 0.09). Factor analyses on the complete cases $(n=183$ ) obtained similar results (data not reported in table), and the factor loading of Item 30 only slightly improved from 0.09 to 0.11 . 
สุ

ซ

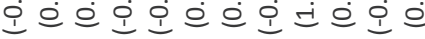

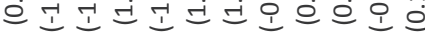

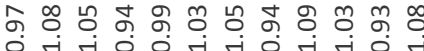
ष्ष

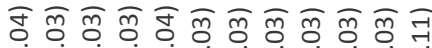

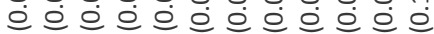

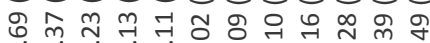

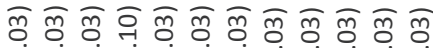

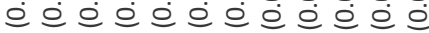
กี $\begin{aligned} & m \\ & 0\end{aligned}$ i

o

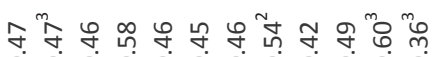

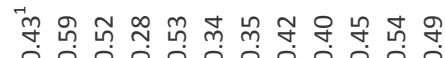
000000000000 0000000000000

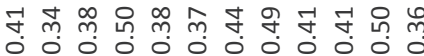

至员

ธิ

দ্口

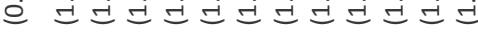
กิ $\infty$ 뉴ำ

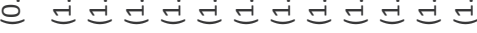
ம்

尚

:

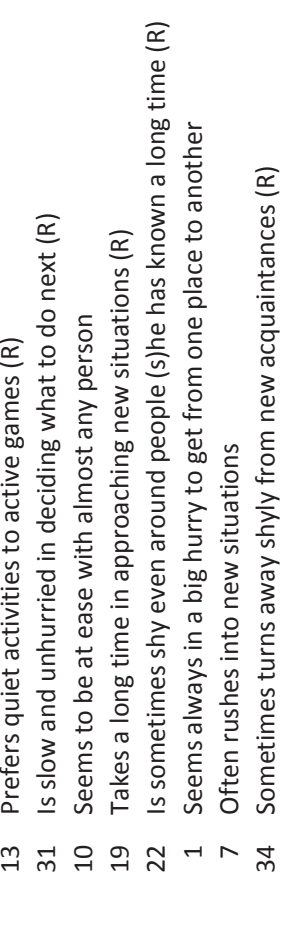

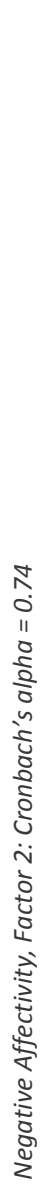

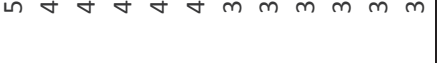

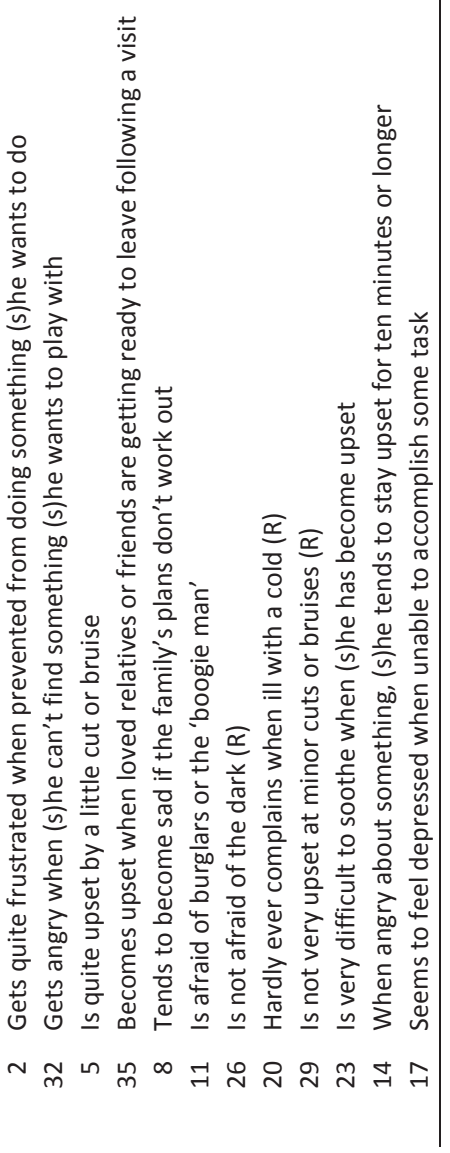




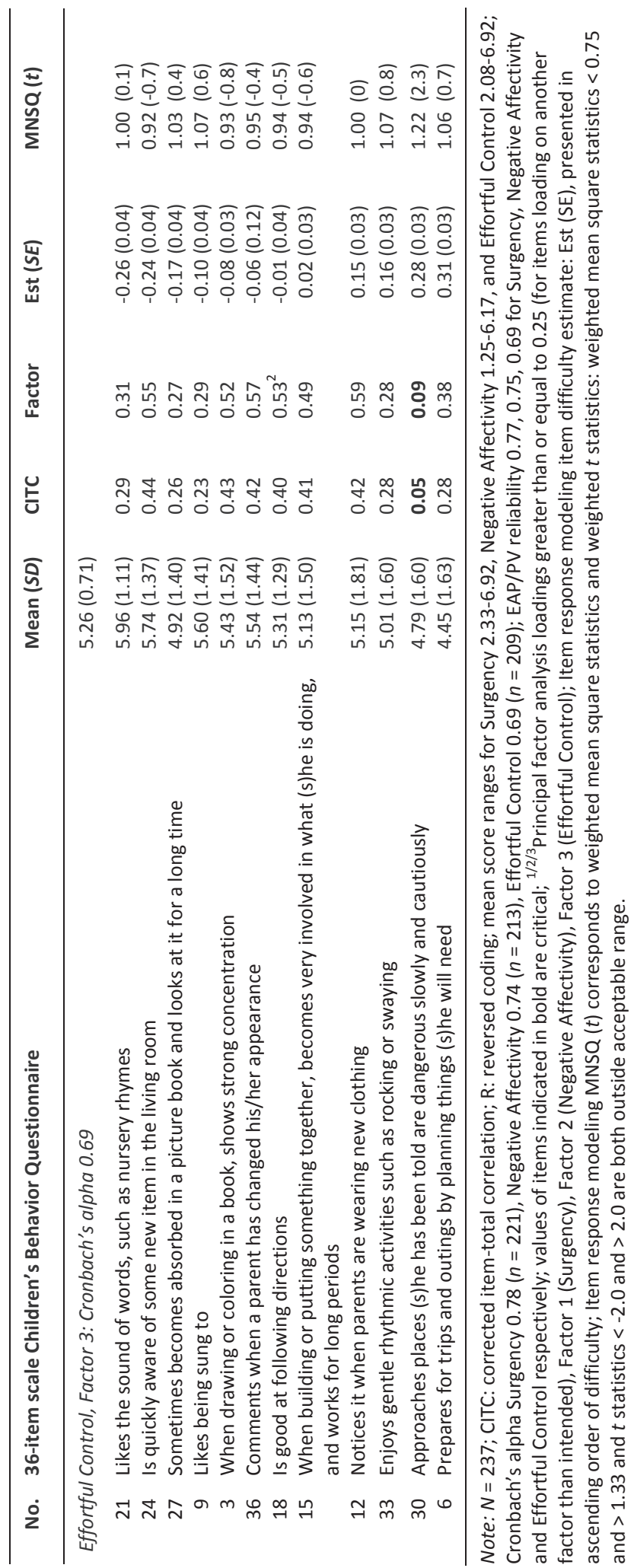




\section{Item response modeling analyses}

All items of the three higher order factors (SR, NA, and EC) had both weighted mean square statistic and $t$ statistic within the acceptable ranges (Table 3). Figure 1 presents the item-respondent Wright map for the three higher order temperament factors (SR: Figure 1a, NA: Figure 1b, EC: Figure 1c). The distribution of items indicated no item difficulty estimates covered the scores that were extremely easy or extremely difficult (the logits ranged from -0.69 to 0.49 ). The best distributional results were for SR (Figure 1a). However, the upper end of the continuum remained uncovered by items with higher difficulty. The distribution of NA items (Figure 1b) was clustered at the middle: the 12 items did not adequately distinguish among parents with children rated high and low on NA, and several items had overlapping difficulty estimates. EC had an especially poor distribution of items (Figure 1c). The items covered a restricted portion of the range of respondents' answers suggesting inadequate content validity for children at the more-difficult-to-agree end of the scale. Several items occupied the same location on the EC latent variable, indicating they had the same difficulty and could be removed from the scale without affecting content validity.

For all three latent temperament variables, every item had the first threshold targeting none of the respondents, suggesting the 'extremely untrue of your child' response option was not chosen by the respondents. This indicated that the scale had skewed content representation of the construct and Threshold 1 could be removed. Moreover, the second threshold targeted an EC of -0.2 and less, but only approximately four respondents had this score, suggesting also that the second threshold could be eliminated. For all 12 items of NA, the sixth threshold targeted none of the respondents, indicating that the response option 'extremely true of your child' was not chosen by the respondents. The item separation reliability estimates were $0.77,0.75$ and 0.69 for SR, NA and EC, respectively. Finally, the functioning of the seven-point response scale was assessed by visual examinations of the item response functions. The pattern of response options suggested that several items functioned more like a five-point rather than a seven-point response format. 
Figure 1a. Item-respondent map for Surgency

Logits Resp Items *Items by threshold

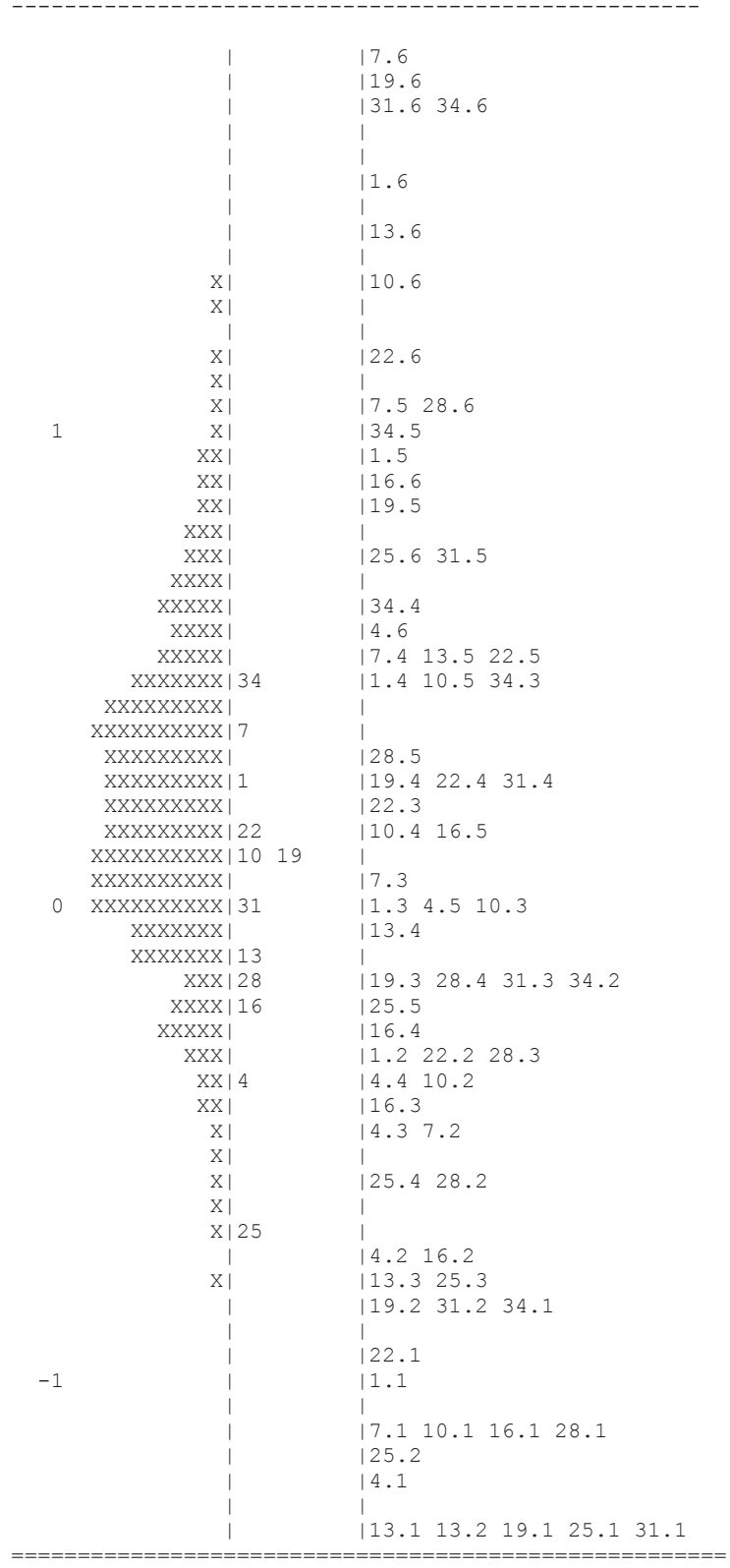

Note: $N=237$; Each ' $X$ ' represents 1.4 cases; *items by thresholds show the item first followed by the threshold (e.g., 7.4 refers to item 7 and Threshold 4). 
Figure 1b. Item-respondent map for Negative Affectivity

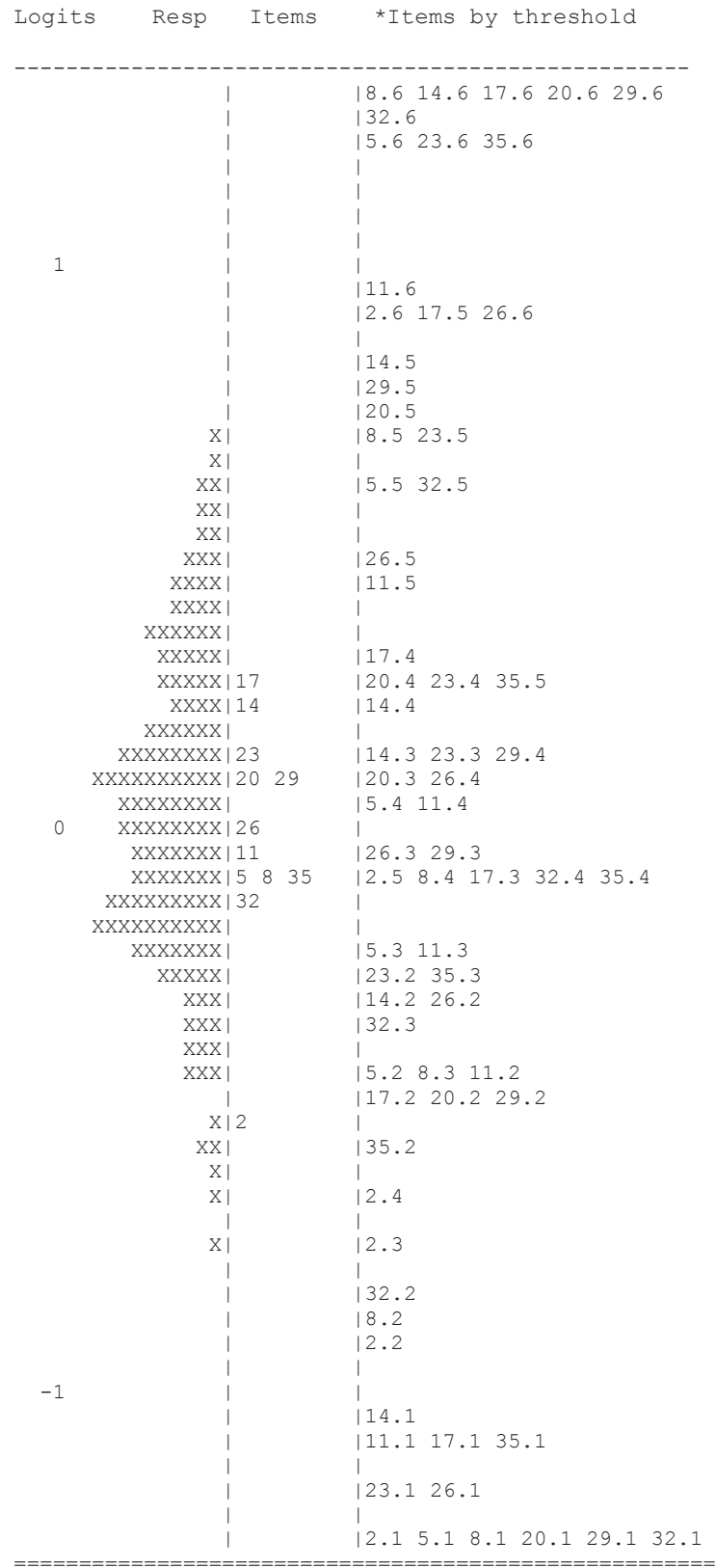

Note: $N=237$; Each ' $X$ ' represents 1.6 cases; ${ }^{*}$ items by thresholds show the item first followed by the threshold (e.g., 8.4 refers to item 8 and Threshold 4). 
Figure 1c. Item-respondent map for Effortful Control

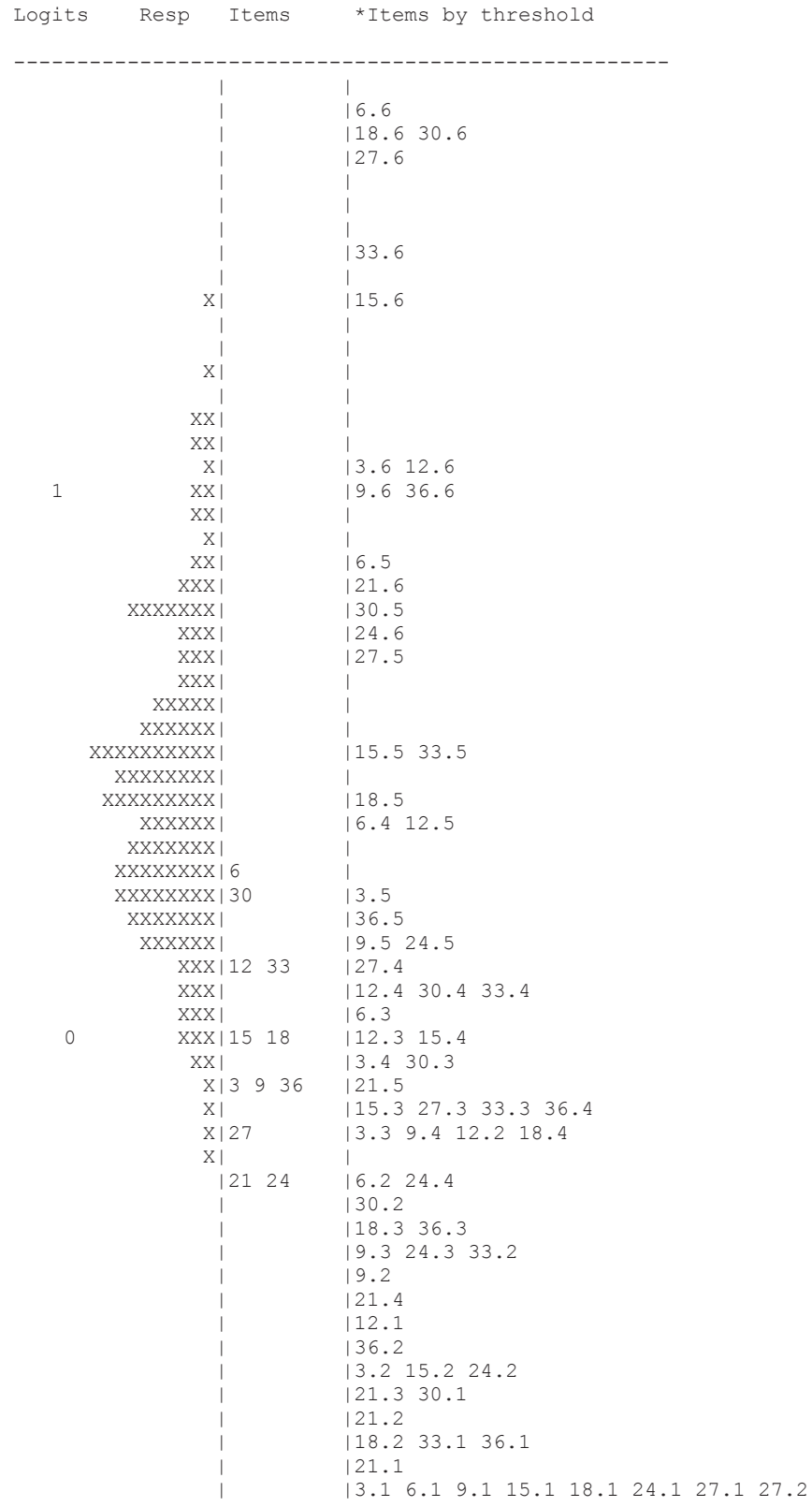

Note: $N=237$; Each ' $\mathrm{X}$ ' represents 1.8 cases; ${ }^{*}$ items by thresholds show the item first followed by the threshold (e.g., 9.4 refers to item 9 and Threshold 4). 


\section{Validation of a one-item temperament scale using the 36-item temperament scale}

ANOVAs across the three temperament groups defined by the one-item temperament scale $(S R, n=108$; $\mathrm{NA}, n=30$; EC, $n=99$ ) indicated significant differences among the means on the three temperament factors (SR: $F_{2,236}=31.35, p<0.001, \eta^{2}=0.46 ; N A: F_{2,236}=8.01, p<0.001, \eta^{2}=0.25 ; E C: F_{2,236}=8.26, p<0.001, \eta^{2}=$ $0.26)$.

Bonferroni post hoc tests revealed that parents who selected the 'SR' response options had higher scores on the SR factor (using the 12-item scale; mean $=5.02, S D=0.72$ ) compared to the scores on this factor for parents selecting the other options (NA: mean $=4.30, S D=0.96$; $\mathrm{EC}$ : mean $=4.22, S D=0.75)(p s<0.001)$. No significant differences in mean scores on SR were present between parents classifying their child as having either a 'NA' or an 'EC' temperament. Similar findings were obtained for the other two temperament factors (NA and EC; Table 4). When using Tukey alpha for adjusting post hoc multiple comparisons to identify significant mean differences, similar results were found (data not reported).

Table 4. Average scores on the three factors of the 36-Item temperament scale for parents classifying their children on the one-item scale and $p$-values for pairwise between-group comparisons

\begin{tabular}{|c|c|c|c|c|c|c|}
\hline \multirow[b]{2}{*}{$\begin{array}{l}\text { 36-item scale } \\
\text { (3 factors) }\end{array}$} & \multicolumn{3}{|c|}{ One-item temperament scale } & \multirow[b]{2}{*}{$(1-2)$} & \multirow[b]{2}{*}{$(1-3)$} & \multirow[b]{2}{*}{$(2-3)$} \\
\hline & $\begin{array}{c}\text { Surgency } \\
\begin{array}{c}(n=108) \\
\text { Mean (SD) (1) }\end{array}\end{array}$ & $\begin{array}{c}\text { Negative } \\
\text { Affectivity } \\
(n=30) \\
\text { Mean (SD) (2) }\end{array}$ & $\begin{array}{c}\text { Effortful } \\
\text { Control } \\
(n=99) \\
\text { Mean (SD) (3) }\end{array}$ & & & \\
\hline Surgency & $5.02(0.72)$ & $4.30(0.96)$ & $4.22(0.75)$ & $<0.001^{*}$ & $<0.001^{*}$ & 1.0 \\
\hline Negative Affectivity & $4.08(0.83)$ & $4.68(0.78)$ & $4.00(0.84)$ & $0.002^{*}$ & 1.0 & $<0.001^{*}$ \\
\hline Effortful Control & $5.18(0.68)$ & $4.92(0.72)$ & $5.45(0.70)$ & 0.209 & $0.014^{*}$ & $0.001^{*}$ \\
\hline
\end{tabular}

Note: $N=237$; using Bonferroni correction for adjusting post hoc multiple comparisons; statistical significance was assigned at the $p<0.05$ level; ${ }^{*} p$ value remained significant using Tukey alpha, 36 -item temperament scale answer options ranging from 1 to 7 .

Three participants had similar high mean scores on two of the three factors of the 36-item temperament scale and these were deleted because they could not be properly categorized into a single temperament trait (Table 5). The Pearson chi-square statistic was $70.79(p<0.001)$, suggesting an association between the temperament constructs as measured by the two methods. A sensitivity score was calculated to identify the percentage of caregivers identifying their child on a particular temperament trait with both temperament scales (36-item scale is the 'gold standard'). Thus, it is the probability a parent correctly identifies their child on a particular temperament trait. This score ranged from $54.17 \%$ for NA to $76.79 \%$ for SR. However, a substantial number of participants $(n=56)$ characterized their child as 'SR' on the one-item temperament scale, but the highest mean score was for the EC factor (Table 5). 
Table 5. Parental characterization of child temperament based on the one-item and 36-item temperament scale

\begin{tabular}{lccccc}
\hline & \multicolumn{3}{c}{ one-item temperament scale } & & \\
\cline { 2 - 5 } 36-item scale & Surgency & $\begin{array}{c}\text { Negative } \\
\text { Affectivity }\end{array}$ & $\begin{array}{c}\text { Effortful } \\
\text { Control }\end{array}$ & Total & $\begin{array}{c}\text { Sensitivity } \\
\text { (percentage) }\end{array}$ \\
\hline Surgency & 43 & 4 & 9 & 56 & $76.79 \%$ \\
Negative Affectivity & 6 & 13 & 5 & 24 & $54.17 \%$ \\
Effortful Control & 56 & 13 & 85 & 154 & $55.19 \%$ \\
Total & 105 & 30 & 99 & 234 & \\
\hline
\end{tabular}

Note: $n=234$; a respondent with similar mean scores (and the highest mean scores) on two of the three CBQ higher order factors was excluded from the analyses $(n=3) ; \mathrm{X}^{2}=70.79, d f=4, p<0.001$; statistical significance was assigned at the $p<0.05$ level; sensitivity was calculated to identify the percentage of caregivers identifying their child on a particular temperament trait with both temperament scales (36-item scale is the 'golden standard').

Secondary analyses showed that for this group of children whose parents chose 'SR' on the one-item scale, the means of the three temperament factors were: SR $4.71(S D=0.56), N A 4.04(S D=0.77)$, EC $5.61(S D=$ 0.44). Pearson's correlation analysis showed that SR and EC were related $(r=0.35, p<0.01)$, whereas the correlations between EC-NA, and SR-NA were not statistically significant. Thirteen participants identified their child's temperament as 'NA' on both scales. Thirteen other participants also described their child's temperament as being ' $N A$ ' using the one-item scale, but had a higher mean score on EC using the 36-item $\mathrm{CBQ}$ scale (Table 5). Secondary analyses showed that the means for this group of children for the three temperament factors were as follows: SR $3.76(S D=0.68)$, NA $4.29(S D=0.81)$, EC $5.45(S D=0.62)$ on the one-item scale. Findings of the correlation analysis showed that none of the temperament factors were significantly correlated.

\section{Discussion}

The psychometric properties of the very short scale of the $\mathrm{CBQ}$, using classical test theory, principal factor analysis and item response modeling, were found to be acceptable to outstanding. These methods complement each other and are needed for a thorough psychometric evaluation of the temperament scale. The factor analytic results closely resembled those of the original US (Putnam \& Rothbart, 2006) and Dutch studies (Sleddens, Kremers et al., 2011), after forcing the principal factor analysis to retrieve three factors. Most of the variance in the present study was explained by SR, in contrast to the study of Putnam and Rothbart (2006) and the Dutch study of Sleddens, Kremers et al. (2011), where NA was the factor accounting for the highest variance. Factor loadings in the present study were slightly higher than in the Dutch study, which may have been due to the CBQ in this study being administered in isolation from the longer scales of the $\mathrm{CBQ}$, or to differences in culture. Better psychometric properties might be expected when solely administering the 36 items, because respondents may be more inclined to be conscientious when completing a shorter scale (Putnam \& Rothbart, 2006). 
The classical test theory results indicated that internal reliability estimates were acceptable and most items discriminated well. However, Item 30 ('My child approaches places he/she has been told are dangerous slowly and cautiously') had a corrected item-total correlation of 0.05 which is far below the critical cut-off point of 0.15 (Nunnally \& Bernstein, 1994). In addition, this item had a low factor loading following principal factor analysis (0.09), but the item did not have a significant loading on one of the two other higher order factors. These results are not consistent with other studies (Putnam \& Rothbart, 2006; Sleddens, Kremers et al., 2011), and must be replicated before definitive conclusions can be drawn. When comparing the internal reliability estimates of the current study to other studies in which the 36-item scale was administered in isolation from the longer versions (Hughes et al., 2008; O'Connor et al., 2010; Ward et al., 2008), slight deviations were found. In this study, Cronbach's alpha for SR was higher compared to the others. Cronbach's alpha for EC was higher in the studies of Hughes et al. (2008) and O'Connor et al. (2010); both studies reported samples of predominantly African-American and Hispanic Head Start preschoolers. Ward et al. (2008) found a slightly higher internal reliability coefficient for NA in a small group of preschoolers.

The item response modeling analyses provided a complementary in-depth assessment of the psychometric properties of the very short scale of the $\mathrm{CBQ}$. Although fit indices were within acceptable ranges and reliability was adequate, the findings of the Wright map were less optimistic. The distribution of participants along the continuum appeared to be normal; however, the items were not well distributed along the latent variables, and no item difficulty estimates covered the scores that were extremely easy or difficult. Especially for the EC latent variable, only a restricted portion of the distribution was covered by items with lower difficulty, suggesting inadequate content validity. Moreover, the seven-point response format did not work well for several items. Future research needs to use the item response modeling approach on the CBQ using different samples including countries outside the US, and the longer scales of the $C B Q$ should be evaluated to identify items that enhance the distributional properties of the scale, and remove redundant items. This is the first study reporting the factorial validity of the 36-item CBQ scale when administered in isolation from the longer versions. Moreover, this study is the first using item response modeling approaches for analyzing the $\mathrm{CBQ}$, beyond more classical test theory approaches for psychometric evaluation.

The newly developed one-item temperament scale is a reasonably valid alternative measure to use in studies where brief and quick assessment of child temperament is desired, such as tailoring interventions. The vignettes contained the information to correctly identify child temperament (sensitivity percentages ranging from 54 to $77 \%$ ), despite some violations of the hypothesis, i.e., a large group of parents characterizing their child's temperament as characterized by SR or NA on the one-item scale, having higher mean scores on one of the other temperament factors using the 36 -item scale of the CBQ. Such a brief, single item scale is not intended to replace the multi-dimensional scales for assessing child temperament for observational studies, but rather will allow scientists to translate the findings from decades of research on child temperament to develop novel interventions tailored on the child's behavioral characteristics that parents perceive to most exemplify their child's temperament. Brief vignettes, as used in this study, were 
more effective when including fewer details or texts for participants to attend to (Sha \& Pan, 2009). Therefore, when there are time constraints and when a large battery of questionnaires needs to be administered, the one-item scale would be an appropriate alternative to assist in tailoring interventions.

The strengths of this study include the in-depth assessment of psychometric properties of the CBQ very short scale administered in isolation from the longer scales, and using item response modeling approaches in addition to more traditional approaches. Moreover, the sample was ethnically diverse. The weaknesses include a potential sample bias since a large group of participants reported a household income of $\$ 60,000$ or more a year (46\%). Putnam and Rothbart (2006), however, reported that internal consistency for lowincome participants was only lower for the 94-item short scale, not the very short Scale. The majority of participants lived in Texas, which lowered generalizability to other populations. Another limitation is the loss of some important information when the very short scale, and more specifically the one-item temperament scale, was administered instead one of the more precise longer scales of the CBQ. However, given the findings of this study, these short scales are appropriate to use in case of time constraints and to reduce participant burden.

\section{Implications for Research and Practice}

The current study showed adequate psychometric properties of the very short scale $\mathrm{CBQ}$ among a sample of US parents of 3- to 5-year-old children. Item response modeling indicated lack of item coverage among respondents on the extremes of the latent temperament variables. Future cross-cultural research is necessary, applying item response modeling to other samples that completed the 36 -item scale of the $\mathrm{CBQ}$. Item response modeling approaches should also be applied to longer scales of the CBQ to generate additional items at different levels of difficulties of the temperament variables to improve assessment of the full range of these constructs with non-redundant items. Efforts to use these more advanced statistical techniques are upcoming in health behavior research, and needed for proper development and validation of questionnaires. The newly developed one-item temperament scale has promising results (i.e., moderate predictive value) and is potentially useful in intervention studies tailoring to child temperament to decrease participant burden. 


\section{CHAPTER 7}

Measuring child temperament:

Validation of a 3-item temperament scale and 13-item impulsivity scale

Ester FC Sleddens

Stef PJ Kremers

Nanne K De Vries

Carel Thijs

European Journal of Developmental Psychology, 2012 


\begin{abstract}
A 3-item temperament measure and a 13-item impulsivity scale were tested against the 36-item Children's Behavior Questionnaire ( $\mathrm{CBQ}$ ). Overall, 229 caregivers completed the $\mathrm{CBQ}$ and the 3 -item temperament measure based on the traits of Surgency, Negative Affectivity and Effortful Control as measured by the $\mathrm{CBQ}$. Their children completed the impulsivity scale. Psychometrics of the CBQ and the impulsivity scale were examined, which were considered good in terms of internal consistency and factorial structure. The three temperament items correlated with the average scores on the corresponding CBQ factors. Furthermore, Surgency was highly related to Impulsivity. Findings provide evidence for the applicability of the Impulsivity and temperament measures for research.
\end{abstract}




\section{Introduction}

Temperament has been shown to be a key factor impacting on the social development of children (Rothbart, Ahadi, \& Evans, 2000), being defined as 'biologically based individual differences in reactivity and self-regulation' (Rothbart, Ahadi, Hershey, \& Fisher, 2001). 'Reactivity' refers to the arousability of motor, affective, and sensory response systems as a result of changes in the environment. 'Self-regulation' includes those processes that serve to modulate one's reactivity. The Children's Behavior Questionnaire (CBQ) is a caregiver report instrument which captures these reactive and effortful processes to measure the broad temperamental traits of Surgency/Extraversion (SR; tendency to perform impulsive and active behavior), Negative Affectivity (NA; predisposition to experience negative affective states) and Effortful Control (EC; ability to control attentional processes and behavior) (Rothbart et al., 2001). The 36-item CBQ (Putnam \& Rothbart, 2006) has been developed in case of time constraints. Recent studies support the use of this short version (Sleddens, Hughes et al., 2012a; Sleddens, Kremers, Candel, De Vries, \& Thijs, 2011), confirming the three-dimensional temperament structure. Recently, Sleddens, Hughes et al. (2012a) developed the one-item temperament measure, which consists of three vignettes reflecting the temperament factors from the $\mathrm{CBQ}$. Such a brief measurement would work well in tailored interventions to child temperament, for which rapid assessment is needed (Baranowski et al., 2012). The one-item measure correlated with the three dimensions of the CBQ (Sleddens, Hughes et al., 2012a), validating this simplified approach to identifying dominant temperament for use in applied research. However, asking parents to score their child on only one of the traits may provide insufficient information regarding the threedimensionality of temperament.

One of the temperament characteristics frequently present in personality measures is impulsivity. This construct refers to high speed or response initiation (Rothbart et al., 2001) and is partially assessed by the trait of SR included in the $\mathrm{CBQ}$ and other temperament instruments such as the Temperament in Middle Childhood Questionnaire (TMCQ; Simonds \& Rothbart, 2004).

We adapted the one-item temperament measure (Sleddens, Hughes et al., 2012a), to develop a 3-item (three response category) temperament measure based on the three traits as measured by the CBQ. For every vignette, parents are asked to select how much it applied to their child. This instrument, complying with the $\mathrm{CBQ}$ dimensional approach, might be valuable in observational studies with significant time constraints. The current study also assessed children's self-reported impulsivity as measured by the TMCQ (13 items). We aimed to provide evidence for applicability of the 3-item temperament measure and the impulsivity scale. In this process, we first assessed the psychometrics of the CBQ and impulsivity scale. Second, the 3-item temperament measure and impulsivity scale were tested against the CBQ. 


\section{Method}

\section{Overview of participants and measurements}

The current study is part of the KOALA Birth Cohort Study (Kummeling et al., 2005). From a subsample of 229 children we collected data regarding children's behavior patterns at two time points. Around the child's age of 6 to 7 years parents completed the CBQ (mean age $6.8(S D=0.6)$ years). Two years later the same sample of parents completed a 3-item temperament measure, and their participating child completed an impulsivity scale (mean age $8.7(S D=0.6)$ years). The sample consisted of 112 boys and 117 girls. Ethical approval was obtained and all parents signed informed consent.

\section{$\underline{\text { Temperament scales }}$}

The $\mathrm{CBQ}$ assesses the three temperament factors. For each statement parents indicated how well it described their child's reaction to a given situation within the past six months, on a scale ranging from 1 (Extremely untrue of your child) to 7 (Extremely true of your child). The 3-item temperament measure (adapted from Sleddens, Hughes et al., 2012a) comprised three vignettes reflecting the temperament factors from the CBQ (see Table 1).

\section{Table 1. Three-item temperament measure}

\section{Please read the general statements below and select for every statement how much it describes your child (response options: 'Extremely untrue of your child'; 'Slightly true of your child'; 'Partially true/partially untrue of your child'; 'Quite true of your child'; or 'Extremely true of your child').}

The 'Surgency/Extraversion' child:

This child has lots of energy, is easily excited, and often goes fast on the playground. This child enjoys meeting new people and going to new places.

The 'Negative Affectivity' child:

This child often shows their frustration or discomfort, and easily becomes sad when not able to finish a project. This child is often afraid of the dark, and when upset may be difficult to calm down.

The 'Effortful Control' child:

This child likes to listen to rhymes and songs. When working on a project this child can concentrate deeply, and carefully follows rules and instructions. When something changes, this child quickly notices.

\section{$\underline{\text { Impulsivity scale }}$}

For the measurement of impulsivity we used the impulsivity scale from the child-reported version of the TMCQ (Simonds \& Rothbart, 2004), adapted from the CBQ. Translation into Dutch was conducted. A back translation was very similar to the original $\mathrm{CBQ}$ scale. Any disagreement about proper wording was resolved by discussion. Children were asked to respond to statements describing impulsive behaviors and had to choose the answer that applied to them most, using a five-point Likert scale ranging from 1 (Never) to 5 (Always), see Table 2 . 


\section{Statistical analyses}

Average scores were composed for the three factors of the $\mathrm{CBQ}$, as well as the impulsivity scale. Internal consistency was evaluated by computing both Cronbach's alphas and corrected item-total correlations. The minimum acceptable levels of cut-off points of Cronbach's alphas were 0.70 . Item-scale correlations of $\geq$ 0.30 were considered good, and $<0.15$ were considered unreliable (Nunnally \& Bernstein, 1994).

A principal components analysis was employed for the impulsivity scale $(n=226)$. For the $C B Q$, a principal factor analysis with oblique rotation was performed in accordance with the original validation studies (Putnam \& Rothbart, 2006; Rothbart et al., 2001) ( $n=229)$, to see whether the three-factor structure replicated in our sample.

Item-response modeling (IRM; partial credit model) was conducted, extending the classical test theory approach (Wilson, Allen, \& Li, 2006b). The findings retrieved were item difficulty estimates, item infit statistics (items with weighted fit statistics $>0.75$ and/or $<1.33$ or weighted $t$ statistics $>-2.00$ or $<2.00$ were considered fitting well; Adams \& Khoo, 1996), item-respondent Wright maps, and item separation reliability.

Pearson's correlations assessed relationships between the average scores on the three as measured by the 3- and 36-item temperament measures and the average score on the impulsivity scale. Interpretations were based on Cohen's descriptive guidelines (1998): correlations between 0.1 and 0.3 were considered as small, between 0.3 and 0.5 as medium, and between 0.5 and 1.0 as large.

\section{Results}

\section{Psychometric properties of the impulsivity scale}

Table 2 describes the psychometric properties for the impulsivity scale. Cronbach's alpha (0.78) was acceptable, as well as the corrected item-total correlations. The average item-total correlation (0.41) suggested good consistency of the items.

Principal components analysis was forced to retrieve one factor, which accounted for $29 \%$ of the total variance. Factor loadings were generally high (10 items had loadings $\geq 0.50$ ).

None of the items of the impulsivity scale had unacceptable values for both the weighted mean square statistic and $t$ statistic. After examination of the item-respondent Wright map (graph not shown) the distribution of items showed no coverage of item difficulty estimates scores that were extremely easy or extremely difficult (the logits ranged from -0.86 to 1.04 ). The items covered a restricted portion (middle and upper end) of the range of respondents' answers suggesting inadequate content validity for children at the end of the scale denoting lower levels of difficulty. Several items occupied the same location on the 
latent variable, indicating they had the same level of difficulty and could be removed from the scale without affecting content validity. Item separation reliability estimate was 0.77 . 


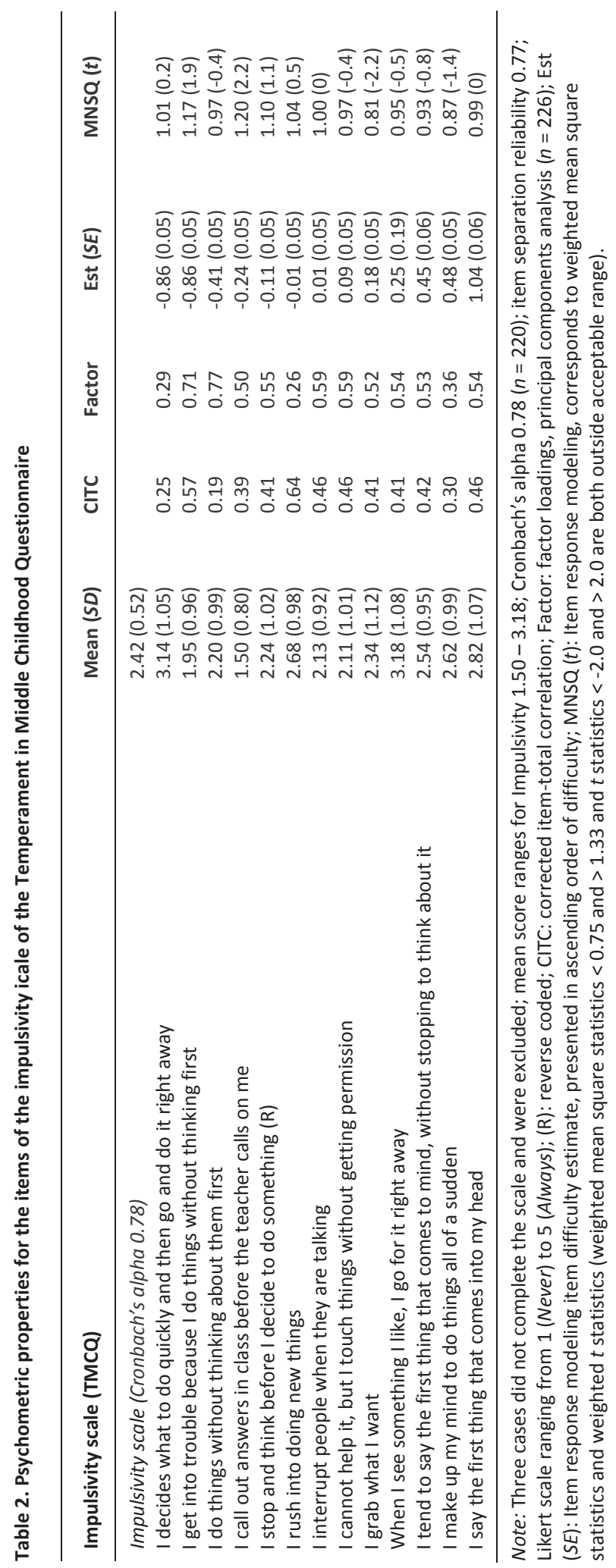




\section{Psychometric properties of the CBQ}

Table 3 describes the psychometric properties for the three scales of the CBQ. Cronbach's alphas ranged from 0.74 to 0.78 . The average item-total correlations among the three factors suggest good consistency of the items $(\mathrm{SR}=0.38, \mathrm{NA}=0.35, \mathrm{EC}=0.36)$.

Principal factor analysis revealed eleven factors having eigenvalues greater than 1 . Forcing a three-factor structure, as proposed by Rothbart et al. (2001) and Putnam and Rothbart (2006), revealed the factors SR, NA, and EC. This factor solution accounted for $27.6 \%$ of the total variance. Most of the variance was explained by the SR factor (10.7\%), followed by NA (9.8\%) and EC (7.1\%).

None of the items of the three factors had unacceptable values for both the weighted mean square statistic and $t$ statistic. After examination of the item-respondent Wright maps (graphs not shown) the distribution of items showed no coverage of item difficulty estimates scores that were extremely easy or extremely difficult (the logits ranged from -0.58 to 0.94 ). In contrast to the study conducted by Sleddens, Hughes et al. (2012a) on the CBQ, the items were more evenly spaced across the continuum, suggesting that the content better matched the distribution of the respondents. Some items had overlapping difficulty estimates (see Table 3). The item separation reliability estimates were $0.78,0.76,0.75$ for SR, NA and EC, respectively. 


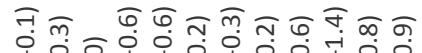

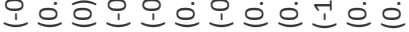

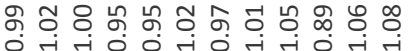



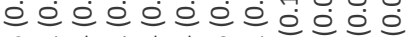

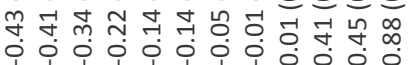

䓛

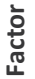

సุำ ํำ

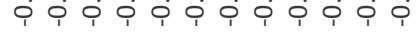

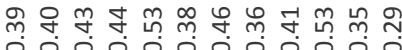

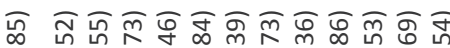

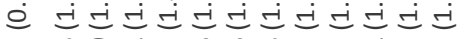
ㅇํำ

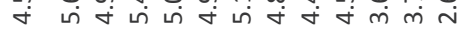

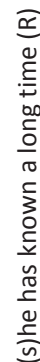

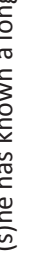

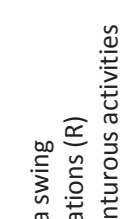

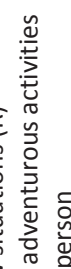

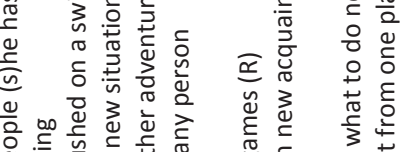

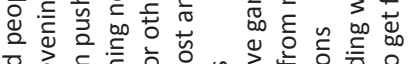

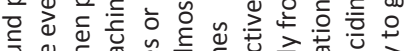

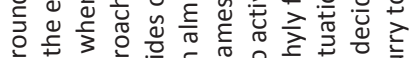

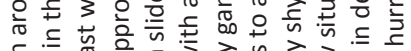

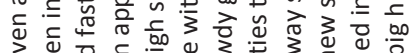

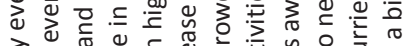

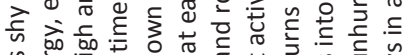

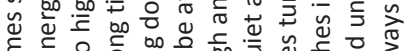
纯 है

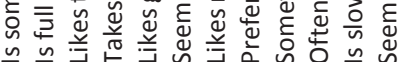

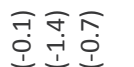

ลุ

शे कू

옹 ำ

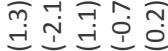

\section{庹守}

응

ํํำ นnํำ

ఫ守

응ํㅇ

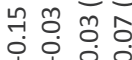

2 d d d

¿ $\doteq \dot{0} \doteq 0$

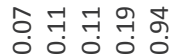

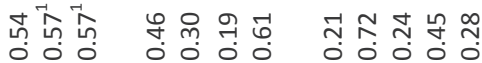

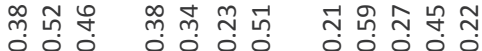

\begin{tabular}{|c|c|}
\hline 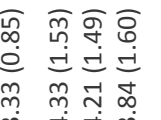 & 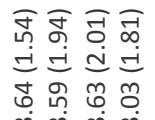 \\
\hline
\end{tabular}

$\sqrt{2}$

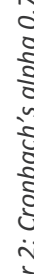

过 


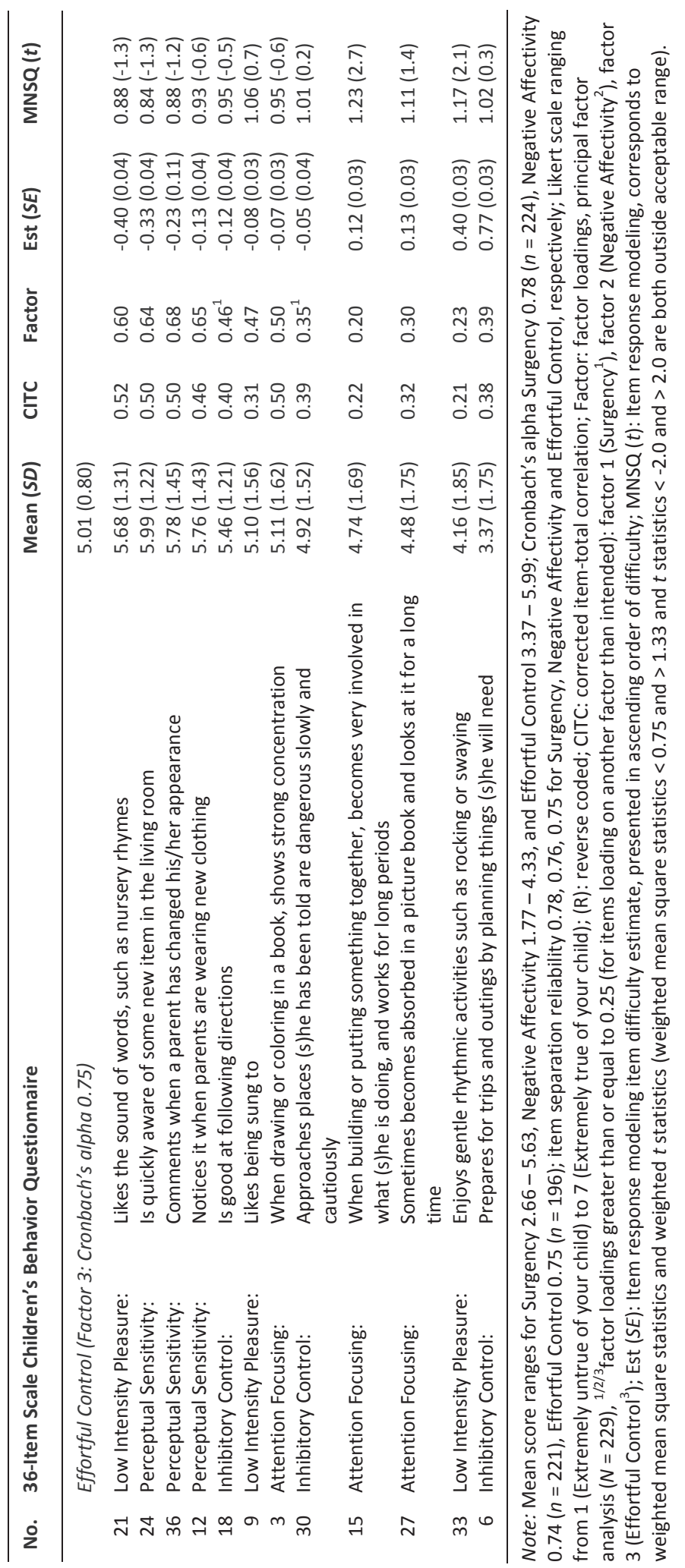




\section{Correlations between the temperament measures and impulsivity scale}

The correlation between the SR factors of the one-item and 12-item Surgency scale of the CBQ was positive, with a large effect size of $0.52(p<0.001)$. Similar correlations were found for the NA and EC factors, respectively. Impulsivity was related to the SR factor of both the 3- and 36-item temperament measures, with correlations of 0.30 and 0.28 , respectively. Impulsivity was positively correlated with NA and negatively with EC of both measures, but with small effect sizes (Table 4).

Table 4. Pearson's correlations between the average scores on the three factors of the 3- and 36-item Children's Behavior Questionnaire and the impulsivity scale

\begin{tabular}{|c|c|c|c|c|c|c|c|}
\hline & & \multicolumn{3}{|c|}{ 3-item measure } & \multicolumn{3}{|c|}{ 36-item measure } \\
\hline & & SR & NA & EC & SR & NA & EC \\
\hline \multirow{3}{*}{$\begin{array}{l}\text { 3-item } \\
\text { measure }\end{array}$} & SR & - & & & & & \\
\hline & NA & -0.07 & - & & & & \\
\hline & EC & $-0.14^{*}$ & -0.04 & - & & & \\
\hline \multirow{3}{*}{$\begin{array}{l}36 \text {-item } \\
\text { measure }\end{array}$} & SR & $0.52 * * *$ & -0.12 & $-0.17 * *$ & - & & \\
\hline & NA & -0.11 & $0.54^{* * *}$ & 0.13 & $-0.14^{*}$ & - & \\
\hline & EC & -0.06 & -0.08 & $0.45^{* * *}$ & $-0.21 * *$ & 0.03 & - \\
\hline Impulsivity & & $0.30 * * *$ & $0.16^{*}$ & $-0.22 * *$ & $0.28 * * *$ & $0.14^{*}$ & $-0.23 * *$ \\
\hline
\end{tabular}

Note: $\mathrm{SR}=$ Surgency/Extraversion, NA $=$ Negative Affectivity, $\mathrm{EC}=$ Effortful Control; ${ }^{*} p \leq 0.05,{ }^{* *} p \leq 0.01, * * * p \leq 0.001$ (two-sided).

\section{Discussion}

The psychometric properties of the $\mathrm{CBQ}$ and impulsivity scale were considered good. The CBQ factor analytic results confirmed the temperament structure as suggested by Rothbart et al. (2001), and closely resembled the findings of previous studies (Putnam \& Rothbart, 2006; Sleddens, Kremers et al., 2011; Sleddens, Hughes et al., 2012a). Results of the IRM analyses on the CBQ and impulsivity scale found fit indices within acceptable ranges. The items were generally well distributed, but none covered extreme scores. We suggest IRM be applied to longer CBQ forms and multiple samples to identify easy and difficult items, and remove redundant items.

Findings of the correlation analyses provide evidence for the applicability of both the 3-item temperament measure as well as the impulsivity scale. The 3-item temperament response categories correlated with the average scores on the CBQ factors. Furthermore, the factor of SR measured with the two temperament measures was highly related to impulsivity. As both SR and impulsivity have been found to show stability when measured at different points across time (Putnam, Rothbart \& Gartstein, 2008), since SR-related characteristics mature early (Putnam, 2011), the impulsivity scale has proven to support score inferences. This stability over time is also confirmed by the high correlation between both temperament measures despite a time lag of almost two years. This time lag further guaranteed that parents were blinded for their previous assessments. 


\section{CHAPTER 8}

The Children's Eating Behavior Questionnaire:

Factorial validity and association with Body Mass Index in Dutch children aged 6 to 7

Ester FC Sleddens Stef PJ Kremers

Carel Thijs

International Journal of Behavioral Nutrition and Physical Activity, 2008 


\begin{abstract}
The Children's Eating Behavior Questionnaire (CEBQ) is a parent-report measure designed to assess variation in eating style among children. In the present study we translated the CEBQ and examined its factor structure in a sample of parents of 6- and 7-year-old children in the Netherlands. Additionally, associations between the mean scale scores of the instrument and children's Body Mass Index (BMI) were assessed. In total, 135 parents of primary school children aged 6 and 7 completed the questionnaire (response rate $41.9 \%$ ). Children's BMI was converted into standardised z-scores, adjusted for child gender and age to examine the association between mean scale scores and child weight status. Results generally confirmed the theoretical factor structure, with acceptable internal reliability and between-subscale correlations. Linear regression analyses revealed that BMI z-scores were positively associated with the 'food approach' subscales of the CEBQ (food responsiveness, enjoyment of food, emotional overeating) ( $\beta$ 's 0.15 to 0.22 ) and negatively with 'food avoidant' subscales (satiety responsiveness, slowness in eating, emotional undereating, and food fussiness) ( $\beta$ 's -0.09 to -0.25$)$. Significant relations with child BMI z-scores were found for food responsiveness $(p=0.02)$, enjoyment of food $(p=0.03)$, satiety responsiveness $(p=$ $0.01)$ and slowness in eating $(p=0.01)$. The results support the use of the CEBQ as a psychometrically sound tool for assessing eating behaviors in Dutch children and the study demonstrates its applicability in overweight-related studies.
\end{abstract}




\section{Background}

Especially during the last few decades the prevalence rates of childhood overweight and obesity have reached epidemic proportions worldwide (Wang \& Lobstein, 2006), and also in the Netherlands (Schokker, Visscher, Nooyens, Van Baak, \& Seidell, 2007). Obese children face difficulties in their social life and run a substantially increased risk of becoming our future generation of obese, chronically diseased adolescents and adults (Must \& Strauss, 1999; Whitaker, Wright, Pepe, Seidel, \& Dietz, 1997). Despite widely held beliefs regarding the importance of factors promoting excessive weight gain in children, it still remains a challenge to discover the underlying child behaviors that might contribute to differences in weight status across children (Carnell \& Wardle, 2007; Viana, Sinde, \& Saxton, 2008; Wardle, Guthrie, Sanderson, \& Rapoport, 2001b). Unraveling these factors will inform the development of evidence-based intervention programs to prevent overweight and obesity in children.

In the past, a number of psychometric instruments have been developed to assess eating behavior in children, including the Children's Eating Behavior Questionnaire (CEBQ) (Wardle et al., 2001b), the Dutch Eating Behavior Questionnaire (DEBQ) (Van Strien, Frijters, Bergers, Defares, 1986; Van Strien \& Oosterveld, 2008), the Children's Eating Behavior Inventory (CEBI) (Archer, Rosenbaum, \& Streiner, 1991) and the BATMAN (Bob and Tom's Method of Assessing Nutrition) (Babbitt, Edlen-Nezien, Manikam, Summers, \& Murphy, 1995). The CEBQ is generally regarded as one of the most comprehensive instruments in assessing children's eating behavior. The instrument was developed and validated in the United Kingdom (UK), and recently the instrument has been validated in a Portuguese sample (Viana et al., 2008). To our knowledge, no other validation studies have been performed on the CEBQ, but the instrument has been used for different research purposes, e.g., to examine associations with child body mass index (BMI) (Carnell \& Wardle, 2008; Powers, Chamberlin, Van Schaick, Sherman, \& Whitaker, 2006; Viana et al., 2008); to compare appetite preferences in children of lean and obese parents (Powers et al., 2006; Wardle, Guthrie, Sanderson, \& Plomin, 2001a); to discover continuity and stability in children's eating behaviors across time (Ashcroft, Semmler, Carnell, Van Jaarsveld, \& Wardle, 2007); and to examine eating behaviors of children with idiopathic short stature (Wudy et al., 2005).

The CEBQ consists of the following eight scales. The scales food responsiveness (FR) and enjoyment of food (EF) reflect eating in response to environmental food cues. In response to these cues appetitive responses and eating rate have been found to strongly increase in overweight or obese children (Carnell \& Wardle, 2007/2008; Wardle et al., 2001b). The scale desire to drink (DD) reflects the desire of children to have drinks to carry around with them, usually sugar-sweetened drinks (Wardle et al., 2001b). Several studies found that BMI was positively associated with frequent consumption of sugar-sweetened drinks (Ludwig, Peterson, \& Gortmaker, 2001; Utter, Scragg, Schaaf, Fitzgerald, \& Wilson, 2007) and a decline in soft drink consumption would result in a reduction of overweight and obese children (James, Thomas, Cavan, \& Kerr, 2004). Satiety responsiveness (SR) represents the ability of a child to reduce food intake after eating to regulate its energy intake. Infants tend to be highly responsive to internal hunger and satiety cues, whereas this level of responsiveness decreases with advancing age (Carnell \& Wardle, 2007/2008; Cecil et al., 2005). 
Thus, during childhood, children will gradually lose the ability to effectively self-regulate energy intake, thereby promoting episodes of over-consumption and subsequently excessive weight gain. High scores on the scale slowness in eating (SE) is characterized by a reduction in eating rate as a consequence of lack of enjoyment and interest in food. Compared to their leaner counterparts, obese children have an increased consumption and have less reduction of their eating rate during the end of a meal (Barkeling, Ekman \& Rössner, 1992). Food fussiness (FF) is usually defined as rejection of a substantial amount of familiar foods as well as 'new' foods, thereby leading to the consumption of an inadequate variety of foods (Dovey, Staples, Gibson, \& Halford, 2008). This type of eating style is characterized by a lack of interest in food (Carruth, Skinner, Houck, Moran, Coletta, \& Ott, 1998), and slowness in eating (Reau, Senturia, Lebailly, \& Christoffel, 1996). Conflicting findings regarding the relationship between fussy eating and BMI in children have been found (Carruth \& Skinner, 2000; Carruth et al., 1998; Dubois, Farmer, Girard, Peterson, \& Tatone-Tokuda, 2007; Rydell, Dahl, \& Sundelin, 1995). The scales emotional overeating (EOE) and emotional undereating (EUE) can be characterized by either an increase or a decrease in eating in response to a range of negative emotions, such as anger and anxiety. Emotional overeating has been found to be positively related to child BMI, whereas emotional undereating was negatively related to child BMI (Braet \& Van Strien, 1997; Viana et al., 2008).

The original CEBQ scale has been shown to have good internal consistency (Cronbach's alphas ranging from 0.72 to 0.91 ) (Wardle et al., 2001b), adequate two-week test-retest reliability (correlation coefficients ranging from 0.52 to 0.87 ) (Wardle et al., 2001b) and construct validity (Carnell \& Wardle, 2007). Principal components analyses showed that each scale had a single factor, which explained $50-84 \%$ of the variance, and an overall factor analysis resulted in a verification of the hypothesized (theoretical) scales (Wardle et al., 2001b).

The present study aimed to examine the factorial nature of the CEBQ in a Dutch sample of 6- and 7-year-old children. Specific objectives were to translate the CEBQ into the Dutch language, to assess its psychometric properties and to compare them with the original CEBQ, and to demonstrate its application in overweightrelated studies by examining its association with the child's BMI. We hypothesized that overweight and obese children would have higher scores on 'food approach' subscales (i.e., FR, EF, EOE) and lower scores on 'food avoidant' subscales (i.e., SR, SE, EUE, FF) of the CEBQ.

\section{Method}

\section{Overview of procedures and participants}

In total, 334 questionnaires were distributed among parents with the Dutch nationality by teachers of third graders (6- to 7-year-olds) of seven primary schools in Maastricht and surroundings, the Netherlands. Overall, 140 completed questionnaires were returned (41.9\%). The response rate per primary school ranged from $15.0 \%$ to $60.7 \%$. Five children were excluded, because the parents did not have the Dutch nationality. The mean age of the participating children was $6.5(S D=0.5)$ years, consisting of two 
approximately equal-sized age groups: 6 -year-old children $(n=71)$, and 7-year-old children $(n=62)$, two cases with no age indicated. Gender was equally divided across our sample, girls $(n=67)$ and boys $(n=68)$. With respect to parental education, seven levels were distinguished. A total of 24 parents $(9.2 \%)$ completed lower general secondary education as highest educational level ( $n_{\text {father }}=12 ; 9.4 \%, n_{\text {mother }}=12 ; 9.0 \%$ ). Other educational levels that were distinguished (in ascending order) were intermediate general secondary education $\left(n_{\text {father }}=7 ; 5.5 \%, n_{\text {mother }}=6 ; 4.5 \%\right)$, intermediate vocational education $\left(n_{\text {father }}=36 ; 28.1 \%, n_{\text {mother }}=\right.$ $45 ; 33.6 \%)$, intermediate/high general secondary education $\left(n_{\text {father }}=9 ; 7.0 \%, n_{\text {mother }}=10 ; 7.5 \%\right)$, higher general secondary education $\left(n_{\text {father }}=1 ; 0.8 \%, n_{\text {mother }}=1 ; 0.7 \%\right)$, higher vocational education, college $\left(n_{\text {father }}\right.$ $\left.=37 ; 28.9 \%, n_{\text {mother }}=43 ; 32.1 \%\right)$, and higher vocational education, university $\left(n_{\text {father }}=26 ; 20.3 \%, n_{\text {mother }}=17\right.$; $12.7 \%)$.

\section{Measures}

The CEBQ was translated into Dutch by a team of four experts on eating behavior at Maastricht University (the Netherlands) who are Dutch native speakers and fluent speakers of the English language (the two authors of this manuscript ES and SK, and two colleagues of the Department of Health Education and Promotion). Translations were cross-checked by this team and in case of inconsistencies between the translations, team meetings were held to discuss the particular item; for some issues, we contacted the developer of the instrument (Prof. Wardle) (Wardle et al., 2001b). All translators approved the final translation.

The CEBQ consists of 35 items comprising eight subscales, each containing 3 to 6 items. Parents are asked to rate their child's eating behavior on a five-point Likert scale (Never, Rarely, Sometimes, Often, Always; 15). Sample scale items include for example 'Given the choice, my child would eat most of the time', and 'My child leaves food on his/her plate at the end of a meal'. In Table 1, all items of the CEBQ are displayed.

\section{Body Mass Index}

Parents were asked to report their children's height and weight to calculate BMI. Specific age and gender BMI cut-off points were used to define underweight (Cole, Flegal, Nicholls, \& Jackson, 2007) and overweight/ obesity (Cole, Bellizzi, Flegal, \& Dietz, 2000). Additionally, a child's BMI was converted to a standardized z-score, adjusting for age and gender, based on reference data of the Fourth Dutch National Growth Study (1997) (Netherlands Organization for Applied Scientific Research, 1997). Parental reported weight and height of their children was available for 115 (85.2\%) respondents.

\section{Statistical procedures}

A principal components analysis with Varimax rotation was performed on all items of the CEBQ to determine if the original eight-factor structure (CEBQ) (Wardle et al., 2001b) would be replicated in our sample. 
Both internal reliability coefficients (Cronbach's alphas) and (average) corrected item-total correlations were calculated. Guidelines exist to interpret (average) corrected item-total correlations, which correct for the contribution of the items to the scale. For the present study, we used the guidelines by Nunnally, who considered that correlations above 0.30 are 'good' and correlations below 0.15 may be unreliable (i.e. because they are wrongly interpreted by the study participants and/or do not measure the same construct as the subscale) (Nunnally, 1978). The reliability estimates were compared with those found by previous validation studies (Viana et al., 2008; Wardle et al., 2001b).

Pearson's correlations were computed to evaluate relationships between mean item scale scores on each of the eight factors of the CEBQ originally found by Wardle et al. (2001b). Interpretations were based on Cohen's descriptive guidelines (Cohen, 1998), correlations between 0.5 and 1.0 being considered as large, correlations between 0.3 and 0.5 as medium, and correlations between 0.1 and 0.3 as small.

Gender and age differences between scores were calculated using independent samples t-tests. A series of multiple linear regression analyses was conducted to examine associations between scores on the subscales of the CEBQ with children's BMI z-scores as the dependent variable. Every subscale of the questionnaire was entered into the analysis separately with the following co-variables to correct for potential confounding: child's gender and age; parental education, ranging from 1 (lowest level of education) to 7 (highest level of education); and parental employment status, dichotomized into 1 (employed) and 2 (non-employed). Missing anthropometric data was present for 20 children, and therefore BMI z-scores of these children could not be calculated. Those missing BMI z-scores were replaced using the mean imputation method. The sample size of the current study $(N=135)$ enables the detection of an additional explained variance of $6 \%\left(\Delta R^{2}=0.06\right)$ in the prediction of one unit change in BMI z-score, with a power of 0.80 (alpha 0.05 ). In addition, one-way analysis of variance for comparison by weight status was used to examine differences in scale scores by child BMI groups and to assess the possibility of a non-linear relationship between $\mathrm{BMI}$ and eating style constructs. BMI was categorized into three weight categories, underweight ( $n=20 ; 17.4 \%)$, normal weight ( $n=83 ; 72.2 \%)$, and over-weight/obesity $(n=12 ; 10.4 \% ; 10$ overweight and 2 obese children grouped together to increase the statistical power).

\section{Results}

\section{Factor analysis}

The factor analysis revealed a seven-factor solution, presented in Table 1. The seven factors accounted for $62.8 \%$ of the total variance. The items from two scales (EOE and FR) loaded onto the same factor, which we propose to name 'overeating' (Table 2). Most of the scale items loaded as expected and their factor loadings were comparable to those obtained in the original study by Wardle et al. (2001b) and the study by Viana et al. (2008). However, four items deserve special attention. First of all, the item 'my child is always asking for food' did not load onto the expected factor FR, but on EF. Second, the item 'my child eats more when annoyed' loaded most highly onto the EUE factor (0.55), but has been retained on the EOE scale on 
theoretical grounds (factor loading 0.47 ). The item 'my child eats more and more slowly during the course of a meal' loaded most highly onto the SR factor (0.63), but has been retained on the SE factor (0.39). Separate principal components analyses on the seven final scales showed that six of them constituted a single factor with an eigenvalue greater than 1, accounting for $51-70 \%$ of the variance across the scales. One exception was the overeating scale, which had two factors with an eigenvalue greater than 1 (revealing the original FR and EOE scales), accounting for $42 \%$ of the variance across the seven scales. In spite of our seven-factor solution, we performed further statistical analyses on the eight subscales as defined by Wardle et al. (2001b), in order to allow comparison with the original subscales and in line with the previous Portuguese study (2008). 
Table 1. Factor loadings on Varimax rotated solution of Principal Components Analysis (CEBQ, $N=135)$

\begin{tabular}{|c|c|c|c|}
\hline Scale name and items & Loading & Scale name and items & Loading \\
\hline \multicolumn{2}{|l|}{ Food fussiness (Factor $1 ; 13.2 \%$ variance) } & \multicolumn{2}{|l|}{ Satiety responsiveness (Factor 4; 8.8\% } \\
\hline My child refuses new foods at first & 0.83 & variance) & \\
\hline My child enjoys tasting new foods & 0.87 & My child has a big appetite & 0.32 \\
\hline \multirow{2}{*}{$\begin{array}{l}\text { My child enjoys a wide variety of foods } \\
\text { My child is difficult to please with meals }\end{array}$} & 0.77 & \multirow{2}{*}{$\begin{array}{l}\text { My child leaves food on his/her plate at the } \\
\text { end of a meal }\end{array}$} & \multirow{2}{*}{0.69} \\
\hline & 0.56 & & \\
\hline $\begin{array}{l}\text { My child is interested in tasting food s/he } \\
\text { hasn't tasted before }\end{array}$ & 0.88 & $\begin{array}{l}\text { My child gets full before his/her meal is } \\
\text { finished }\end{array}$ & 0.70 \\
\hline \multirow{2}{*}{$\begin{array}{l}\text { My child decides that s/he doesn't like } \\
\text { food, even without tasting it }\end{array}$} & \multirow[t]{2}{*}{0.75} & My child gets full up easily & 0.65 \\
\hline & & $\begin{array}{l}\text { My child cannot eat a meal if } s / \text { he has had a } \\
\text { snack just before }\end{array}$ & 0.55 \\
\hline \multicolumn{4}{|l|}{ Enjoyment of food (Factor 2; $10.5 \%$} \\
\hline variance) & & \multicolumn{2}{|l|}{ Emotional undereating (Factor 5; 8.7\% } \\
\hline My child loves food & 0.69 & variance) & \\
\hline My child is interested in food & 0.66 & My child eats less when s/he is angry & 0.78 \\
\hline My child is always asking for food ${ }^{(b)}$ & 0.53 & My child eats less when $\mathrm{s} /$ he is tired & 0.77 \\
\hline My child enjoys eating & 0.62 & My child eats more when s/he is happy & 0.71 \\
\hline My child looks forward to mealtimes & 0.56 & My child eats less when $s /$ he is upset & 0.72 \\
\hline \multicolumn{2}{|l|}{ Food responsiveness/Emotional } & \multicolumn{2}{|l|}{ Desire to drink (Factor 6; 6.3\% variance) } \\
\hline overeating $^{(a)}$ (Factor 3; 9.3\% variance) & 0.43 & My child is always asking for a drink & 0.74 \\
\hline \multicolumn{2}{|l|}{ My child eats more when worried } & \multirow{4}{*}{$\begin{array}{l}\text { If given the chance, my child would drink } \\
\text { continuously throughout the day } \\
\text { If given the chance, my child would always } \\
\text { be having a drink }\end{array}$} & \multirow[t]{2}{*}{0.83} \\
\hline My child eats more when annoyed ${ }^{(c)}$ & 0.47 & & \\
\hline \multirow{2}{*}{$\begin{array}{l}\text { If allowed to, my child would eat too much } \\
\text { My child eats more when anxious }\end{array}$} & 0.73 & & \multirow[t]{2}{*}{0.81} \\
\hline & 0.61 & & \\
\hline \multirow{2}{*}{$\begin{array}{l}\text { Given the choice, my child would eat } \\
\text { most of the time }\end{array}$} & 0.65 & \multirow[b]{2}{*}{ Slowness in eating (Factor $7 ; 6.0 \%$ variance) } & \\
\hline & & & \\
\hline \multirow{2}{*}{$\begin{array}{l}\text { My child eats more when s/he has } \\
\text { nothing else to do }\end{array}$} & \multirow[t]{2}{*}{0.67} & My child finishes his/her meal very quickly & 0.66 \\
\hline & & My child eats slowly & 0.71 \\
\hline $\begin{array}{l}\text { Even if my child is full up, s/he finds room } \\
\text { to eat his/her favorite food }\end{array}$ & 0.38 & $\begin{array}{l}\text { My child takes more than } 30 \text { minutes to } \\
\text { finish a meal }\end{array}$ & 0.51 \\
\hline $\begin{array}{l}\text { If given the chance, my child would } \\
\text { always have food in his/her mouth }\end{array}$ & 0.72 & $\begin{array}{l}\text { My child eats more and more slowly during } \\
\text { the course of a meal }{ }^{(\mathrm{d})}\end{array}$ & 0.39 \\
\hline
\end{tabular}

Note: ${ }^{(a)}$ FR and EOE loaded onto the same factor in the final solution, so one scale was developed which we propose to name 'overeating' (OE); ${ }^{(b)}$ The item 'My child is always asking for food' loaded most highly onto the EF factor (0.53) than on the FR factor (0.05), where the factor originally belongs. Therefore, this item was incorporated in the factor $\mathrm{EF}$; ${ }^{(\mathrm{c})}$ The item 'My child eats more when annoyed' loaded most highly onto the EUE factor (0.55), but on theoretical grounds has provisionally been retained on the EOE scale, which is part of the newly developed factor OE; ${ }^{(d)} \mathrm{The} \mathrm{item}^{(\mathrm{d}}$ 'My child eats more and more slowly during the course of a meal' loaded most highly onto the SR factor (0.63), but has provisionally been retained on the SE factor, to provide better comparability with the original factor structure of the CEBQ; ${ }^{(\mathrm{e})}$ The item 'My child is difficult to please with meals' loaded also onto the factor SR (0.44). 


\section{Reliability}

Reliability coefficients (Cronbach's alphas) for the different scales of the instrument are presented in Table 2. The coefficients ranged from 0.75 to 0.91 for the CEBQ subscales, which are all within acceptable ranges. The average item-total correlations, correcting for the contribution of the items to the scale, suggested adequate consistency of item content within the CEBQ subscales $(0.51-0.75)$ (Table 2). Moreover, all corrected item-total correlations are considered 'good' (ranging from 0.39 to 0.84 ) (Nunnally, 1978).

Table 2. Factor structure and internal consistency of the CEBQ $(N=135)$

\begin{tabular}{lcccc}
\hline & $\begin{array}{c}\text { Number of factors } \\
\text { with eigenvalue >1 }\end{array}$ & $\begin{array}{c}\text { Percentage of } \\
\text { variance factor 1 }\end{array}$ & $\begin{array}{c}\text { Cronbach's } \\
\text { alpha }\end{array}$ & $\begin{array}{c}\text { Average corrected item-total } \\
\text { correlation (range) }\end{array}$ \\
\hline Food fussiness & 1 & 70 & 0.91 & $0.75(0.64-0.84)$ \\
Enjoyment of food & 1 & 57 & 0.80 & $0.60(0.39-0.67)$ \\
Overeating & 2 & 42 & 0.78 & $0.51(0.39-0.64)$ \\
${ }^{*}$ Food responsiveness & 1 & 52 & 0.72 & $0.54(0.38-0.65)$ \\
*Emotional overeating $_{\text {SEtiety responsiveness }}$ & 1 & 52 & 0.67 & $0.50(0.39-0.61)$ \\
Emotional undereating & 1 & 51 & 0.76 & $0.54(0.45-0.66)$ \\
Desire to drink & 1 & 63 & 0.81 & $0.62(0.54-0.72)$ \\
Slowness in eating & 1 & 67 & 0.75 & $0.59(0.44-0.69)$ \\
\hline
\end{tabular}

Note: *The items from two scales (FR and EOE) loaded onto the same factor, which we propose to name 'overeating'; when performing separate principal components analyses on the factor overeating, the two original factors, FR and EOE, were identified both with an eigenvalue $>1$ (stated in italics). In this Table, item 12 'My child is always asking for food', originally belonging to the FR scale, was removed from this scale and incorporated in the factor EF.

\section{Age and gender differences}

Independent samples t-tests were conducted to examine age and gender variations in children's eating behavior (Table 3). There were no statistically significant differences in parental responses regarding 6-year old children compared to parents of 7-year-olds. Significant gender differences were found. Boys scored higher on fussy eating (FF) than girls (mean $3.1(S D=0.9)$ versus $2.6(S D=0.9), p=0.000)$. Higher mean EOE values were found among boys $(1.6(S D=0.5))$ than among girls $(1.3(S D=0.4))(p=0.003)$ and mean values for EF were higher for girls than for boys (girls $3.5(S D=0.6$ ) versus boys $3.3(S D=0.7), p=0.024)$. 
Table 3. Mean $(S D)$ of CEBQ subscale scores by gender $(N=135)$ and age group $\left(n=133^{*}\right)$

\begin{tabular}{|c|c|c|c|c|}
\hline \multirow[b]{2}{*}{ CEBQ scales } & \multicolumn{2}{|c|}{ Gender } & \multicolumn{2}{|c|}{ Age group } \\
\hline & $\begin{array}{c}\text { Girls } \\
(n=67)\end{array}$ & $\begin{array}{c}\text { Boys } \\
(n=68)\end{array}$ & $\begin{array}{c}\text { 6-years-old } \\
\quad(n=71)\end{array}$ & $\begin{array}{c}\text { 7-years-old } \\
(n=62)\end{array}$ \\
\hline Food responsiveness & $1.8(0.5)$ & $2.0(0.6)$ & $1.8(0.5)$ & $2.0(0.6)$ \\
\hline Enjoyment of food & $3.5(0.6)$ & $3.3(0.7)$ & $3.4(0.7)$ & $3.4(0.7)$ \\
\hline Emotional overeating & $1.3(0.4)$ & $1.6(0.5)$ & $1.4(0.5)$ & $1.5(0.5)$ \\
\hline Desire to drink & $2.3(0.8)$ & $2.5(0.7)$ & $2.3(0.7)$ & $2.5(0.8)$ \\
\hline Satiety responsiveness & $2.8(0.6)$ & $2.8(0.7)$ & $2.8(0.7)$ & $2.8(0.6)$ \\
\hline Slowness in eating & $2.6(0.6)$ & $2.8(0.8)$ & $2.8(0.7)$ & $2.6(0.7)$ \\
\hline Emotional undereating & $2.2(0.8)$ & $2.3(0.8)$ & $2.2(0.8)$ & $2.3(0.8)$ \\
\hline Food fussiness & $2.6(0.9)$ & $3.1(0.9)$ & $2.9(0.9)$ & $2.8(0.9)$ \\
\hline
\end{tabular}

Note: *Information on age was missing in 2 children.

\section{Correlations between scales}

The correlations between subscales of the CEBQ (Table 4) indicate that the 'food approach' subscales (FR, $\mathrm{EF}$, and $\mathrm{EOE}$ ) and the 'food avoidant' subscales (SR, SE, EUE, and FF) tend to be positively inter-correlated. For the 'food approach' subscales, especially the FR-EF and FR-EOE correlations were found to have a large effect size. Moreover, a large correlation was found between the 'food avoidant' subscales SR and SE, whereas medium correlations were found for SR-FF and SE-FF. The 'food approach' subscales and the 'food avoidant' subscales were found to be negatively correlated. Large negative correlations were found for EF$\mathrm{SR}, \mathrm{EF}-\mathrm{SE}$, and EF-FF, whereas medium correlations exist for FR-SR and FR-SE. The only exception among these negative correlations was the medium-sized positive correlation between the 'food approach' EOE factor and the 'food avoidant' EUE factor. The correlations coefficients were compatible with the findings of Wardle et al. (2001b) and Viana et al. (2008).

Table 4. Pearson's correlations between the CEBQ subscales $(N=135)$

\begin{tabular}{|c|c|c|c|c|c|c|c|c|}
\hline CEBQ scales & $\begin{array}{l}1 \\
F R\end{array}$ & $\begin{array}{l}2 \\
E F\end{array}$ & $\begin{array}{l}3 \\
\text { EOE }\end{array}$ & $\begin{array}{l}4 \\
\mathrm{DD}\end{array}$ & $\begin{array}{l}5 \\
S R\end{array}$ & $\begin{array}{l}6 \\
\text { SE }\end{array}$ & $\begin{array}{l}7 \\
\text { EUE }\end{array}$ & $\begin{array}{l}8 \\
F F\end{array}$ \\
\hline 1 Food responsiveness (FR) & - & & & & & & & \\
\hline 2 Enjoyment of food (EF) & $0.50 * * *$ & - & & & & & & \\
\hline 3 Emotional overeating (EOE) & $0.54 * * *$ & 0.17 & - & & & & & \\
\hline 4 Desire to drink (DD) & $0.18^{*}$ & 0.00 & 0.16 & - & & & & \\
\hline 5 Satiety responsiveness (SR) & $-0.36 * * *$ & $-0.59 * * *$ & -0.13 & 0.09 & - & & & \\
\hline 6 Slowness in eating (SE) & $-0.38 * * *$ & $-0.53 * * *$ & -0.16 & 0.07 & $0.61 * * *$ & - & & \\
\hline 7 Emotional undereating (EUE) & 0.13 & -0.02 & $0.41 * * *$ & 0.05 & $0.22 *$ & $0.21 *$ & - & \\
\hline 8 Food fussiness (FF) & $-0.18^{*}$ & $-0.62 * * *$ & 0.00 & 0.15 & $0.48 * * *$ & $0.44 * * *$ & 0.14 & - \\
\hline
\end{tabular}

Note: ${ }^{*} p<0.05 ;{ }^{* *} p<0.01 ;{ }^{* *} p<0.001$ (two-sided); highlighted area upper-left corner: inter-correlations between 'food approach' subscales; highlighted area bottom-right corner: inter-correlations 'food avoidant' subscales. 


\section{Weight differences}

A series of independent regression analyses was used to model each subscale of the CEBQ separately with child BMI z-scores entered as a continuous dependent variable, while correcting for potential confounding variables (child's gender and age, parental educational level, and parental employment status). In general, child BMI z-scores showed a linear increase with the 'food approach' subscales of the CEBQ ( $\beta 0.15$ to 0.22 ), and a decrease with 'food avoidant' subscales ( $\beta-0.09$ to -0.25 ) (Table 5). Significant relationships were found for FR, EF $(p<0.05)$, and SR, SE $(p<0.01)$.

Table 5. Hierarchical linear regression analyses for BMI z-scores on CEBQ subscales $(N=135)$

\begin{tabular}{|c|c|c|c|c|c|}
\hline \multirow{2}{*}{ 'Food approach' scales } & \multirow[t]{2}{*}{ Mean (SD) } & \multirow[t]{2}{*}{$\begin{array}{c}\text { Standardized } \beta \\
\text { coefficient }\end{array}$} & \multicolumn{2}{|c|}{$\begin{array}{c}95 \% \mathrm{Cl} \text { for standardized } \beta \\
\text { (lower bound - upper bound) }\end{array}$} & \multirow[t]{2}{*}{$p$-value } \\
\hline & & & & & \\
\hline Food responsiveness & $1.88(0.56)$ & 0.217 & 0.042 & 0.392 & 0.016 \\
\hline Enjoyment of food & $3.40(0.69)$ & 0.207 & 0.025 to & 0.389 & 0.027 \\
\hline Emotional overeating & $1.47(0.48)$ & 0.145 & -0.036 to & 0.326 & 0.118 \\
\hline \multicolumn{6}{|l|}{ 'Food avoidant' scales } \\
\hline Satiety responsiveness & $2.77(0.65)$ & -0.240 & -0.409 to & -0.071 & 0.006 \\
\hline Slowness in eating & $2.73(0.75)$ & -0.248 & -0.421 to & -0.075 & 0.006 \\
\hline Emotional undereating & $2.27(0.79)$ & -0.088 & -0.269 to & 0.093 & 0.344 \\
\hline Food fussiness & $2.84(0.90)$ & -0.103 & -0.284 to & 0.078 & 0.270 \\
\hline
\end{tabular}

Note: Child gender and age, maternal and paternal education level, and maternal and paternal employment status were forced into the models before adding each of the CEBQ scales separately. Standardized $\beta$ coefficients ( $p$-values) were $0.033(p=0.715), 0.030(p=0.734),-0.021(p=0.852),-0.051(p=0.658), 0.122(p=0.190)$ and $0.029(p=0.752)$ for the control variables respectively.

The results regarding differences in scale scores across child BMI groups (one-way analysis of variance) are graphically displayed in Figures 1 and 2, illustrating mean 'food approach' and mean 'food avoidant' scores by weight status category. Significant differences between weight categories were found for the factors SR $(\mathrm{F}=3.69, p<0.05)$ and $\mathrm{SE}(\mathrm{F}=3.86, p<0.05)$. Normal-weight subjects scored significantly higher on the SR scale than the over-weight/obese subjects (mean score normal-weight subjects $2.8(S D=0.7)$ versus overweight/obese subjects $2.3(S D=0.7), p=0.02)$. For the SE scale significant differences were found between underweight and overweight/obese children, with underweight subjects scoring higher (mean 3.0 $(S D=0.5)$ versus $2.2(S D=0.5), p=0.02)$. 
Figure 1. Mean 'food approach' scores by Body Mass Index category

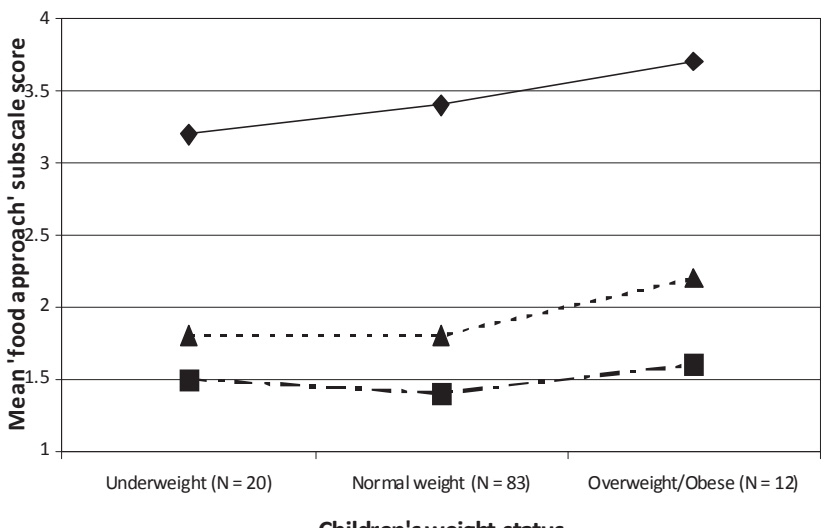

Note: Children's Eating Behavior Questionnaire subscales:

- . . . . . . . f food responsiveness;

- - - - - - - emotional overeating;

- enjoyment of food

Figure 2. Mean 'food avoidant' scores by Body Mass Index category

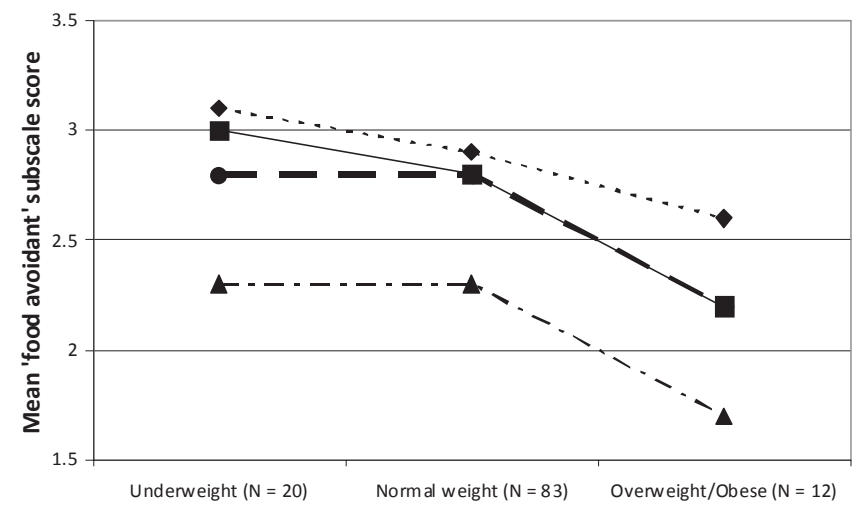

Children's weight status

Note: Children's Eating Behavior Questionnaire subscales:

- - - - - satiety responsiveness;

$\begin{array}{ll} & \begin{array}{l}\text { slowness in eating; } \\ \text { emotional undereating; }\end{array} \\ \ldots \ldots \ldots \ldots-\ldots & \text { food fussiness }\end{array}$ 


\section{Discussion}

The present study showed good psychometric properties of the Dutch translation of the CEBQ in terms of factor structure, internal reliability and correlations between subscales corresponding very closely to the original study (Wardle et al., 2001b) and a recent Portuguese validation study of the CEBQ (Viana et al., 2008). In our sample of 6- and 7-year-old Dutch children a seven-factor structure was the best interpretable solution, which explained $62.8 \%$ of the variance. In parallel with earlier studies (Viana et al., 2008; Wardle et al., 2001b), the original eight-factor structure could not perfectly be replicated. In comparison to the original factor structure (Wardle et al., 2001b), the scales of FR and EOE were clustered together in the present Dutch sample to ascertain the psychometric properties of this study. The FR and EOE scales were highly correlated, and combining them into one scale ('overeating') increased the internal consistency coefficient. However, caution is needed when combining those two scales, since they may differentiate in older age groups and it should be noted that the original FR and EOE scales were revealed in a separate Principal Components Analysis on the combined scale.

Cross-sectional associations between the mean scale scores and BMI showed that overweight children displayed weaker satiety responses and stronger appetite responses to food compared to their leaner counterparts. This result is in line with the Portuguese study (Viana et al., 2008). In addition, overweight children appeared to apply poorer eating regulatory mechanisms and to have an increased eating rate compared to normal-weight children. The positive association of the scales FR and EF with child's BMI zscore is consistent with research demonstrating that children with a higher $\mathrm{BMI}$ are highly responsive to environmental food cues (e.g., Braet \& Van Strien, 1997; Carnell \& Wardle, 2007/2008; Viana et al., 2008; Wardle et al., 2001b). SR and SE were inversely associated with child BMI z-score similar to the recently published study of Carnell and Wardle (2008) and Viana et al. (2008). In the current study, EUE and FF were found to have the weakest associations with the BMI z-score. This result parallels those reported by Viana et al. (2008), suggesting that these eating behaviors are less strongly related to child weight. Moreover, this low non-significant association of fussiness with the child's BMI resembled findings of other studies (Carruth \& Skinner, 2000; Carruth et al., 1998; Rydell et al., 1995). More studies are needed applying the CEBQ cross-culturally to confirm these findings.

A recently published study in the Netherlands (Van Strien \& Oosterveld, 2008) suggested that emotional undereating was a more salient dimension for young children than emotional overeating. Young children react to emotional distress (loss of appetite when feeling e.g., upset or anxious) with a biologically natural response, which includes a reduction of gut activity thereby reducing children's food intake (Van Strien \& Ouwens, 2007). Indeed, consistent with findings from previous research (Van Strien \& Oosterveld, 2008; Wardle et al., 2001b), we found a low mean scale score on the EOE scale, confirming that eating in response to emotional stressors is quite abnormal in young children. In addition, our results support the psychosomatic theory (Bruch, 1973; H.I. Kaplan \& H.S. Kaplan, 1957), which posits that people overeat as a way of coping with emotional stressors based on experiences learned early in life. Our study indicates that 
this learned response to distress is not yet well-established in children as young as 7 years of age (see also Ashcroft et al., 2007).

In contrast to the studies of Wardle et al. (2001b) and Ashcroft et al. (2007), no age effects were found for the CEBQ subscales. This may well be due to the narrow age range in our study ( 29 months), whereas the age range in the study of Wardle et al. (2001b) and Ashcroft et al. (2007) was at least 4 and 6 years respectively. Similar to the findings reported by Wardle et al. (2001b), we found gender differences for FF, with boys scoring higher on fussy eating than girls. However, we also found significant differences for EOE (boys emotionally overeat more often than girls) and EF (girls enjoying food more often than boys). Since many differences in eating behaviors are detected during the teenage years among boys and girls, it would be advisable to track the development of gender differences in eating styles from early childhood onwards. Additionally, more research is needed to assess the exact role of gender in child eating behaviors, possibly in interaction with parental feeding styles (Kremers, De Bruijn, Visscher, Van Mechelen, De Vries, \& Brug, 2006).

Recently, evidence has been found regarding heritability of certain appetitive traits known to be related to the development of obesity. Carnell, Haworth, Plomin, and Wardle (2008) found evidence for a strong genetic influence of satiety and food cue responsiveness in children. In addition, Wardle, Carnell, Haworth, Farooqi, O'Rahilly, and Plomin (2008) have shown that genetic variants could contribute to lower sensitivity to satiety cues. These genetic influences on children's appetite responses indicate the importance of identifying high-risk children in early childhood, since they are more likely to overeat when encountering obesogenic environments.

The present study has several limitations that should be acknowledged. First, factor-analytic procedures have to be repeated on a larger sample of Dutch 6- and 7-year olds to replicate our findings. In addition, considering the small sample size, confirmation regarding the associations between various eating styles and BMI in Dutch children age 6 and 7 is needed. Second, the response rate was relatively low (mean 41.9\%) and families with lower levels of education were relatively underrepresented in the current study. Another limitation was that the children's weight and height were parentally reported and not directly measured. Compared with measured weight and height, parents of 4-year-old children have been shown to slightly underestimate their children's weight and overestimate height, especially if their child was overweight or obese, whereas parents of underweight children tended to overestimate weight (Scholtens et al., 2007). Hence, our study reported slightly lower percentages of overweight/obesity (10.4\%) compared to the Dutch reference population of children aged 6 and 7 (2002-2004: ranging from 12.5\% to 18.7\%) (Van den Hurk, Van Dommelen, De Wilde, Verkerk, Van Buuren, \& HiraSing, 2006). It is likely that the present study yielded underestimates of associations between the instruments' scale scores and BMI, because of the parental reported nature of this study. In addition, there is a potential bias if parents who did not complete the questions regarding their children's weight and height had responded differently to distinct subscales than parents who completed those questions. However, except for DD, with slightly higher DD scores in those with missing height and weight data than in those with data present, no differences on any 
of the subscales were present. Finally, due to the cross-sectional nature of the study, inferences regarding causality cannot be made. Longitudinal and experimental study designs are needed to strengthen inferences, and assess the exact role of children's eating behaviors in the etiology of obesity.

\section{Conclusion}

This study is the first to evaluate the factor structure of the CEBQ in a Dutch population among parents of children aged 6 or 7 . In summary, the findings of the present study suggest that the instrument is valuable for identifying specific eating styles, which can be seen as important and modifiable determinants implicated in the development and maintenance of overweight and obesity. The identification of such variables is a prerequisite to gain insight into the behavioral pathways to obesity, and subsequently for the development of evidence-based intervention programs to prevent obesity in young children. Further longitudinal studies are needed to assess the role of eating behaviors in the development of obesity during childhood and into adulthood. 


\section{CHAPTER 9}

Relationship between parental feeding styles and eating behaviors of Dutch children aged 6 to 7

Ester FC Sleddens Stef PJ Kremers Nanne K De Vries Carel Thijs

Appetite, 2010 


\begin{abstract}
The present study assessed the relationship between parental feeding styles and dietary intake behaviors of Dutch children aged 6 to 7. Associations between feeding styles and dietary behaviors of the parents were also examined. We translated the validated 'Parental Feeding Style Questionnaire' and evaluated its factor structure. A cross-sectional survey was completed by one of the parents of 135 children. Results indicated considerable similarity of factor structure, internal reliability and between-subscale correlations with the original instrument. The parental feeding dimensions of 'instrumental feeding' (i.e., using food as a reward) and 'emotional feeding' (i.e., feeding in response to children's emotional distress) were positively related to children's snacking behavior. The feeding style 'encouragement to eat' was negatively associated with children's snacking behavior. Various feeding styles were found to be related to parental dietary behaviors. Findings indicate the importance of acknowledging parental feeding styles in future research efforts as well as in the development of family-based interventions promoting healthy eating habits among children.
\end{abstract}




\section{Introduction}

The key role that parents play in the development of obesity-inducing eating habits in their offspring is a topic of increasing interest worldwide (Golan \& Crow, 2004). Several dietary behaviors have been shown to contribute to excessive weight gain in children. Obesity-inducing behaviors include the consumption of sugar-rich and energy-dense snacks (e.g., Jebb, 2005), and sugar-sweetened drinks (e.g., James \& Kerr, 2005; Ludwig, Peterson, \& Gortmaker, 2001; Vartanian, Schwartz, \& Brownell, 2007). On the contrary, fruit consumption (Lock, Pomerleau, Causer, Altmann, \& McKee, 2005; Tohill, Seymour, Serdula, Kettel-Khan, \& Rolls, 2004) and daily breakfast consumption (Dubois, Girard, Potvin Kent, Farmer, \& Tatone-Tokuda, 2008; Ortega et al., 1998; Rampersaud, Pereira, Girard, Adams, \& Metzl, 2005; Ruxton, \& Kirk, 1997) have been found to be associated with having a healthy body weight. Given the persistence of obesity and related comorbidities in later life, leading international institutions such as the World Health Organization (2000) and the International Obesity Task Force (Lobstein, Baur, \& Uauy, 2004) have set the prevention of weight gain at early age as a priority. These institutions call for research into the influence of specific parenting practices on children's health behaviors.

Wardle, Sanderson, Guthrie, Rapoport and Plomin (2002) designed an instrument, the Parental Feeding Style Questionnaire (PFSQ), to assess four aspects of feeding style (i.e., instrumental feeding, control, encouragement to eat, and emotional feeding). The PFSQ is one of the few psychometrically sound tools available to assess parental feeding styles (Wardle et al., 2002). The instrument was developed and validated in the UK, and has proved to possess adequate to good internal consistency (Cronbach's alpha ranging from 0.67 to 0.83 ) and excellent two-week test-retest reliability ( $r=0.76-0.83$ ) (Wardle et al., 2002).

To date, each of the four aspects of parental feeding styles applying various instruments have been examined in relation to variations in children's eating behaviors and weight status. For instance, an experimental study demonstrated that parents' use of the instrumental feeding style (i.e., using food as a reward) has an impact on children's food preferences; using a particular food as a means to get the reward (in this case another snack product) leads to a devaluation of the means food relative to the reward snack, implying that a child's preference for healthy snacks could decrease (Newman \& Taylor, 1992). Moreover, other studies also reported that requiring children to eat a food in order to get a reward has been shown to reduce a child's liking for that food (e.g., Birch LL, Birch D, Marlin, \& Kramer, 1982; Birch, Marlin, \& Rotter, 1984). Using foods as rewards for regulating a child's behavior have been shown to increase children's preferences for these products (Birch, Zimmerman, \& Hind, 1980). However, a few studies found contradictory results regarding the effects of instrumental feeding on child's preferences and behavior (Horne, Tapper, Lowe, Hardman, Jackson, \& Woolner, 2004; Lowe, Horne, Tapper, Bowdery, \& Egerton, 2004; Moore, Tappen, \& Murphy, 2007). Regarding the influence of the instrumental feeding scale of the PFSQ on children's weight status, Carnell and Wardle (2007) failed to find an association with children's adiposity at the ages of three to six. Musher-Eizenman, De Lauzon-Guillain, Holub, Leporc, and Charles 
(2009) reported that using food as a reward for child behavior was positively related to child Body Mass Index (BMI) in the United States, but was inversely related to child BMI in France.

Mixed results regarding the impact of feeding styles on children's dietary behaviors and ultimately weight status were also reported for parental use of controlling feeding styles. Following parental restriction, a child's preferences for the forbidden foods have been shown to increase (Fisher \& Birch, 1999a/b; Jansen E, Mulkens, \& Jansen A, 2007; Liem, Mars, \& De Graaf, 2004), even in the absence of hunger. This may clarify the finding that parental restriction of highly palatable snack foods has been found to be related to higher levels of eating (e.g., Birch, Fisher, \& Davison, 2003; Fisher \& Birch, 1999a/b; Musher-Eizenman \& Holub, 2006), and excessive weight gain among children (e.g., Faith, Berkowitz, Stallings, Kerns, Storey, \& Stunkard, 2004; Faith, Scanlon, Birch, Francis, \& Sherry, 2004). In contrast, other studies found that controlling feeding styles were not linked to children's intake of energy-dense foods (e.g., Montgomery, Jackson, Kelly, \& Reilly, 2006), children's BMI (e.g., Brann \& Skinner, 2005; Carnell \& Wardle, 2007; Haycraft \& Blissett, 2008; Montgomery et al., 2006) or body fatness (Spruijt-Metz, Lindquist, Birch, Fisher, \& Goran, 2002). Others reported that parental control was inversely related to children's intake of snack foods and soft drinks (Gubbels, Kremers, Stafleu, Dagnelie, Goldbohm, \& De Vries, 2009) or children's adiposity (e.g., Robinson, Kiernan, Matheson, \& Haydel, 2001).

Studies examining parental prompting to eat in relation to children's eating behavior and weight status are relatively sparse and have reported contradictory findings. Small positive correlations between the PFSQ scale of encouragement and children's BMI of first-borns have been found, suggesting that parents of thinner children reported less prompting (Wardle et al., 2002). Drucker, Hammer, Agras, \& Bryson (1999) reported that more maternal prompting to eat has been related to increased intake of calories among young children. In contrast, Vereecken, Legiest, De Bourdeaudhuij, and Maes (2009) showed that parental encouragement through negotiation had a positive impact on dietary habits among sixth graders, and increased the likelihood of vegetable consumption. In addition, maternal encouragement to promote the intake of a variety of foods and healthy foods was found to be related to lower child BMI (MusherEizenman et al., 2009). However, others found no association between prompts to eat and children's weight status (e.g., Carnell \& Wardle, 2007; Koivisto, Fellenius, \& Sjödén, 1994).

Regarding the fourth, and final, dimension of parental feeding style, emotional feeding, only few studies have been conducted to examine its impact on child dietary behavior and weight status. Two studies found no relationship between the PFSQ dimension of emotional feeding and children's BMI (Carnell \& Wardle, 2007; Musher-Eizenman et al., 2009). At best, the relation between parental feeding styles and eating behavior and overweight of children is inconsistent.

Studies examining parental feeding styles in relation to eating behaviors of parents are relatively sparse (Birch \& Fisher, 2000; De Lauzon-Guillain, Musher-Eizenman, Leporc, Holub, \& Charles, 2009; Fisher \& Birch, 1999a/b; Francis, Hofer, \& Birch, 2001; Tiggemann \& Lowes, 2002; Ventura \& Birch, 2008). Results of those studies showed that restrained eating among parents is linked to parental use of restriction as a 
feeding style (Birch \& Fisher, 2000; De Lauzon-Guillain et al., 2009; Fisher \& Birch, 1999a/b; Francis et al., 2001; Tiggemann \& Lowes, 2002). Other studies have found that using food for non-nutritive purposes, including emotional feeding (Wardle et al., 2002) and using food as a reward (De Lauzon-Guillain et al., 2009), were positively associated with parental emotional eating. Additionally, external eating among mothers was characterized by higher levels on the instrumental feeding scale in the study of Wardle et al. (2002). Studies regarding the relationship between feeding styles and specific dietary behaviors of parents (e.g., snacking, soft drink consumption, fruit consumption and breakfast consumption) are currently lacking.

Whereas many studies measuring parental feeding styles have mainly focused on examining the association with children's BMI (e.g., Carnell \& Wardle, 2007; Haycraft \& Blissett, 2008; Montgomery et al., 2006; Wardle et al., 2002), the current study aimed to assess a comprehensive set of dietary behaviors as more proximal predictors of children's adiposity. The aim of the present study was three-fold. First, we translated the PFSQ and evaluated its factorial validity and psychometric characteristics in a Dutch sample of 6- and 7year-olds. Second, we assessed associations of parental feeding styles with eating behaviors of children aged 6 to 7. Third, we examined associations of parental feeding styles with actual parental dietary behaviors.

\section{Method}

\section{Procedures and participants}

Seven primary schools in the town of Maastricht and its surrounding area (the Netherlands) agreed to take part in this study. In total, 334 questionnaire packages were distributed among parents of 6- and 7-year-old children at these schools. This package included the PFSQ and items assessing children's and parental snacking behavior, soft drink consumption, fruit consumption and breakfast consumption. We received 140 completed questionnaires (41.9\%). The response rate per school ranged from $15.0 \%$ to $60.7 \%$. Five children were excluded, because the parents did not have Dutch nationality. Parents could decide which of the parents completed the questionnaire. Most often mothers completed the questionnaire $(n=122)$. Eleven fathers filled out the questionnaire and two families reported that both parents completed the questionnaire together. The participating children consisted of two approximately equal-sized age groups: 6 -year-old children $(n=71)$ and 7-year-old children $(n=62)$. Age was not reported in two cases. Gender was evenly divided (67 girls and 68 boys). In general, parents who completed a high level of education were overrepresented $(44.8 \%$ and $49.2 \%$ of, respectively, mothers and fathers received a college or university degree).

\section{Parental Feeding Style Questionnaire}

The PFSQ was translated into Dutch by a team of four experts on eating behavior at Maastricht University (the Netherlands) who are Dutch native speakers and fluent speakers of the English language (the authors 
ES and SK, and two colleagues of the Department of Health Promotion). Translations were cross-checked by this team and in case of inconsistencies between the translations, team meetings were held to discuss the particular item; for some issues, we contacted the developer of the instrument (Prof. Wardle). All translators approved the final translation.

The PFSQ consists of 27 items representing four scales, each including $4-10$ items (Wardle et al., 2002). The four scales, measuring parental feeding styles, are 'instrumental feeding' (I), comprising four items with statements such as 'In order to get my child to behave him/herself I promise him/her something to eat'; 'control over eating' (C), comprising 10 items, such as 'I decide how many snacks my child should have'; 'emotional feeding' (EM), comprising five items, such as 'I give my child something to eat to make him/her feel better when s/he is feeling upset' and 'encouragement to eat' (EN), comprising eight items, such as 'I encourage my child to enjoy his/her food'. A complete list of all scale items is presented in Table 1 . The response format consists of a Likert scale ranging from 1 (Never) to 5 (Always).

\section{Dietary intake of children}

Dietary behaviors were assessed using several items from a validated food frequency questionnaire designed to accurately assess energy intake of Dutch children aged 2 to 12 (Brants, Stafleu, Ter Doest, Hulshof, \& Thijs, 2006). The validation study showed a correlation coefficient between the original questionnaire and the doubly labeled water method of 0.62 .

Children's snacking frequency of several sugar-sweetened and energy-dense food products (between meals) was assessed with 11 items derived from this food frequency questionnaire (Cronbach's alpha 0.61). The child's parent was asked to indicate how many days a week (normal week) their children consumed the following snacks in between meals: 1) potato crisps; 2) cake or large biscuits; 3) peanuts; 4) sausage-rolls; 5) pie or pastry; 6) ice-cream; 7) candy bars; 8) chocolates; 9) frankfurters; 10) salted biscuits; and 11) candy (e.g., lollipops, and liquorice). Answering categories were: never, less than 1 day a week, 1 day a week, 2 to 3 days a week, 4 to 5 days a week, and 6 to 7 days a week. A single score was computed for the number of snacking occasions (between meals) per week, by adding reported frequency (in days a week) of the 11 snacks. Six respondents did not respond to one of the 11 snacking items. Those missing data were replaced with the mean value of all items measuring snack consumption for that particular respondent.

Children's soft drink consumption was assessed with three items by asking one parent to indicate how many glasses $(250 \mathrm{ml})$, cans $(330 \mathrm{ml})$ and/or bottles $(500 \mathrm{ml})$ of both sugar-sweetened and diet soft drinks their child consumed on a regular day. Artificially sweetened drinks were included in the overall soft drink measure. Although artificial sweeteners do not contain any nutritional value, they may disrupt a body's natural ability to regulate food intake and will eventually lead to increased weight (Swithers, \& Davidson, 2008). Frequency and amount of soft drinks consumed were multiplied to obtain an average score for consumed soft drinks in milliliters per day. One respondent had a missing value. The missing value was 
replaced by imputing the mean value of all respondents on the particular item. A similar procedure was executed for the other dietary behaviors (i.e., fruit consumption and breakfast consumption).

Fruit consumption was assessed by asking one parent to indicate how many days a week (normal week) their children consumed fruit. Answering categories were: never, less than 1 day a week, 1 day a week, 2 to 3 days a week, 4 to 5 days a week, and 6 to 7 days a week. Additionally, the parent was asked to indicate the number of servings their children consumed on such a day, corresponding with earlier validation studies (Bogers, Van Assema, Kester, Westerterp, \& Dagnelie, 2004). We specified that 1 apple or 1 pear counted as one serving, 2 mandarins counted as one serving, and 1 bunch of grapes counted as one serving as well. Multiplying frequency with the reported usual amount calculated an average score for the number of fruit servings consumed per week. Seven respondents did not respond on the item regarding number of fruit servings consumed per day.

Breakfast consumption was assessed with 1 item by asking the parent how many days a week (normal week) their child consumed breakfast. One respondent did not respond to this item.

\section{Parental dietary behaviors}

Dietary intake of the parent was assessed by asking one of the parents to indicate whether they consumed snacks, fruit, soft drinks and breakfast regularly. Answering categories ranged from totally disagree (1) to totally agree (5). Two families had missing values on the four dietary behaviors. These missing values were not imputed.

\section{Statistical procedures}

A Principal Components Analysis with Varimax rotation was applied to the 27 items of the PFSQ to determine the underlying factor structure of the questionnaire. The reliability of the questionnaire was assessed by calculating both internal consistency coefficients (Cronbach's alphas) and (average) corrected item-total correlations (indicating the degree to which an individual item relates to the total scale score). Nunnally's guidelines were used to interpret corrected item-total correlations, with correlations above 0.30 regarded as 'good' and those below 0.15 as unreliable (Nunnally, 1978).

Pearson's correlation coefficients $(r \mathrm{P})$ were computed for a general assessment of the correlations between parental feeding styles and eating behaviors of both children and their parents. The strength of the relationship between the variables studied was assessed using effect sizes as suggested by Cohen (1983). Cohen defined three levels of effect sizes - small $(0.1-0.3)$, medium $(0.3-0.5)$, and large $(>0.5)-$ corresponding to absolute correlations of $0.1,0.3$ and 0.5 , respectively. Moreover, Partial Rank correlation coefficients ( $r \mathrm{PR}$ ) were computed, adjusted for potential co-variables (i.e., child's gender, age, parental educational level (ranging from 1: lowest level of education, to 7: highest level of education), and parental employment status, dichotomized as 1: employed or 2: non-employed). 


\section{Results}

\section{Factor analysis and psychometric evaluation of the PFSQ}

The four-scale structure identified by Wardle et al. (2002) was generally confirmed (Table 1), with the fourfactor solution explaining $44.5 \%$ of the variance in PFSQ responses. However, two of the original dimensions, instrumental feeding and emotional feeding, loaded onto one and the same factor, which resulted in the construction of a new scale which we named 'instrumental and emotional feeding'. In addition, items on the encouragement scale had high loadings on two factors, one containing four items representing 'encouragement of food variety' and four items representing 'encouragement of interest in food'. 


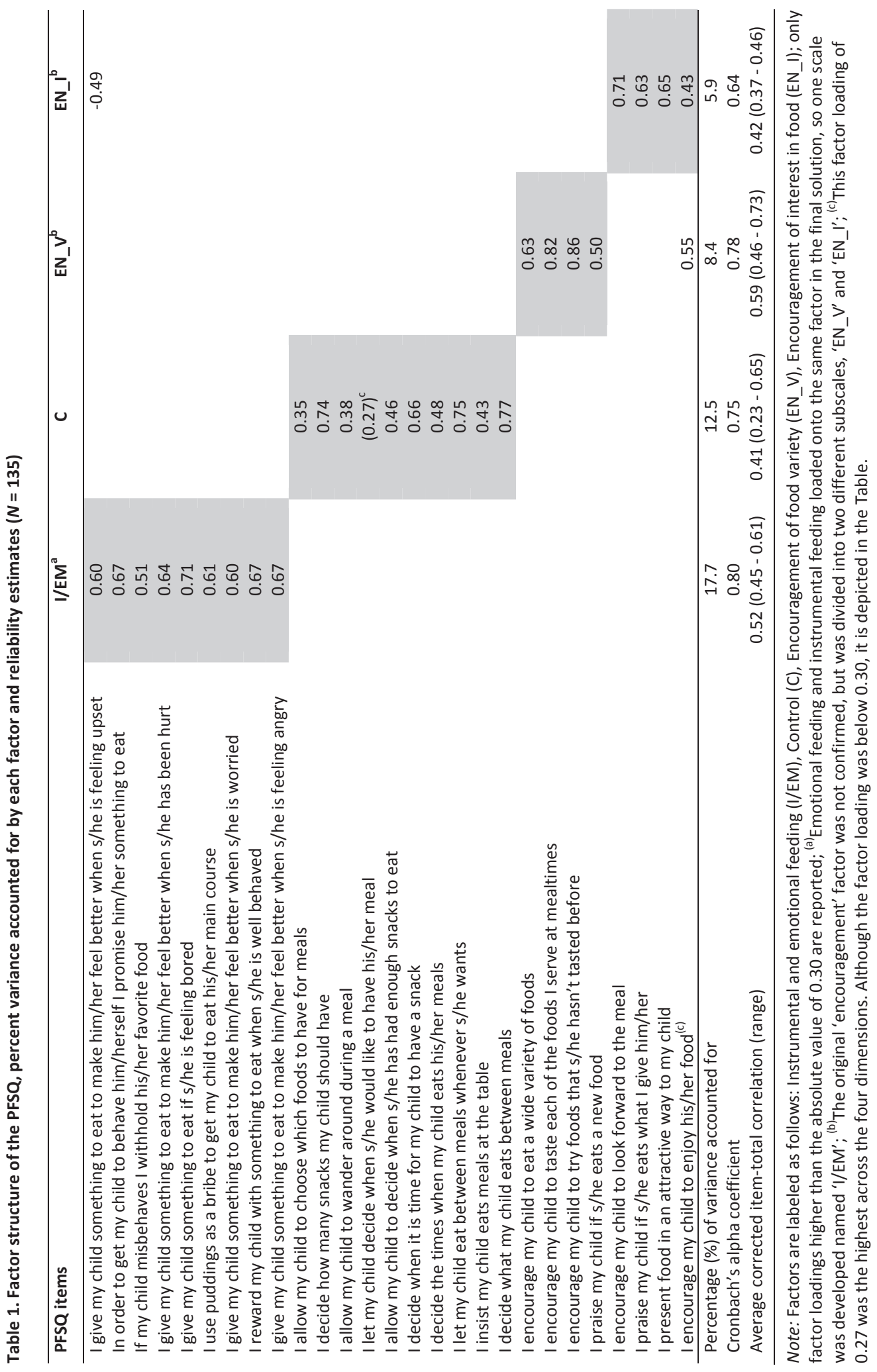


Most items loaded onto a single factor. However, a few items had loadings higher than the absolute value of 0.30 on two factors. These items with cross-loadings were 'I encourage my child to enjoy his/her food' and 'I give my child something to eat to make him/her feel better when s/he is feeling upset' (see Table 1). To enable calculation of the Cronbach's alphas and item-total correlations, these items were allocated to the factor where the theoretical fit was best. The item 'I let my child decide when s/he would like to have his/her meal', loaded most highly onto the control factor (0.27), where the item originally belongs. Despite the low loading of this item (below the value of 0.30 ), the item was retained in the final factor solution to provide better comparability with the original factor structure of the PFSQ.

Cronbach's alpha coefficients for the four scales of the PFSQ ranged from 0.64 to 0.80 (Table 1). This table also presents average corrected item-total correlations, which suggest adequate consistency of item content within the PFSQ subscales (0.41 to 0.59). Despite corrected item-total correlation values below 0.30 on three items of the control scale of $0.23,0.25$ and 0.29 , the additional corrected item-total correlations can be considered 'good' (Nunnally, 1978).

Given the reasonable approximation of the factor structure in the current sample with the original PFSQ factor structure, and to enable comparison with previous research utilizing the PFSQ, the psychometric properties of the original scales were examined. The internal consistency coefficients for the original four scales are displayed in Table 2 and can be considered adequate, ranging from 0.67 to 0.75 . The corrected item-total correlations are also within acceptable ranges for the original factor solution. Further statistical analyses were performed on the four subscales as defined by Wardle et al. (2002), in order to allow comparison with the original subscales.

Table 2. Internal consistency of the PFSQ scales $(N=135)$, based on the original four-factor solution (Wardle et al., 2002)

\begin{tabular}{lcc}
\hline PFSQ scales & Cronbach's alpha & Average corrected item-total correlation (range) \\
\hline Instrumental feeding & 0.67 & $0.46(0.40-0.50)$ \\
Emotional feeding & 0.74 & $0.53(0.46-0.58)$ \\
Control & 0.75 & $0.41(0.23-0.65)$ \\
Encouragement & 0.75 & $0.46(0.37-0.55)$ \\
\hline
\end{tabular}

\section{Correlations between key study variables}

The mean number of snacking occasions per week was $0.8(S D=0.4)$ for children. Consuming sausage-rolls accounted for the lowest number of snacking occasions per week (mean $=0.2, S D=0.3$ ) and eating candy for the largest (mean $=3.2, S D=2.3$ ). Children's mean intake of beverage was $344 \mathrm{ml}(S D=364)$ per day. The mean number of servings of fruit per week was $5.7(S D=2.8)$. A total of $89 \%$ of the parents reported that their child consumed breakfast seven days a week (mean $=6.7, S D=0.9$ ). 
Table 3 presents Pearson's correlation coefficients and Partial Rank correlation coefficients (adjusted for child's gender and age, parental education level and parental employment status) between the study variables. A large significant correlation was found between the instrumental feeding and emotional feeding subscales of the PFSQ $(r P=0.57, r P R=0.54, p<0.01)$. Small negative correlations were found between emotional feeding and the control scales of the PFSQ $(p<0.05)$, whereas a small $(r \mathrm{P})$ to medium $(r \mathrm{PR})$ positive correlation was found for control-encouragement $(p<0.01)$. Both the instrumental feeding scale and the emotional feeding scale of the PFSQ were positively correlated with children's snack consumption (small effect size). The Partial correlation between instrumental feeding and child snacking was not statistically significant. A small negative, but significant Pearson correlation was present between the encouragement scale $(p<0.05)$ and snacking behavior of children. When controlled for potential confounding variables this association was non-significant. Associations were not only present between parental feeding and children's eating behaviors, but also between parental feeding styles and their eating behaviors. To indicate, instrumental feeding was positively related to parental snacking and after adjusting for the co-variables, negatively to parental fruit consumption (small effect size, $p<0.05$ ). A positive Partial Rank correlation was also found between the control scale of the PFSQ and parental fruit consumption $(p<$ 0.05). The encouragement style was positively associated with parental fruit and breakfast consumption (small effect size, $p<0.01$ and $p<0.05$, respectively). However, the Partial Rank correlation coefficient between encouragement and parental fruit consumption was not statistically significant. 


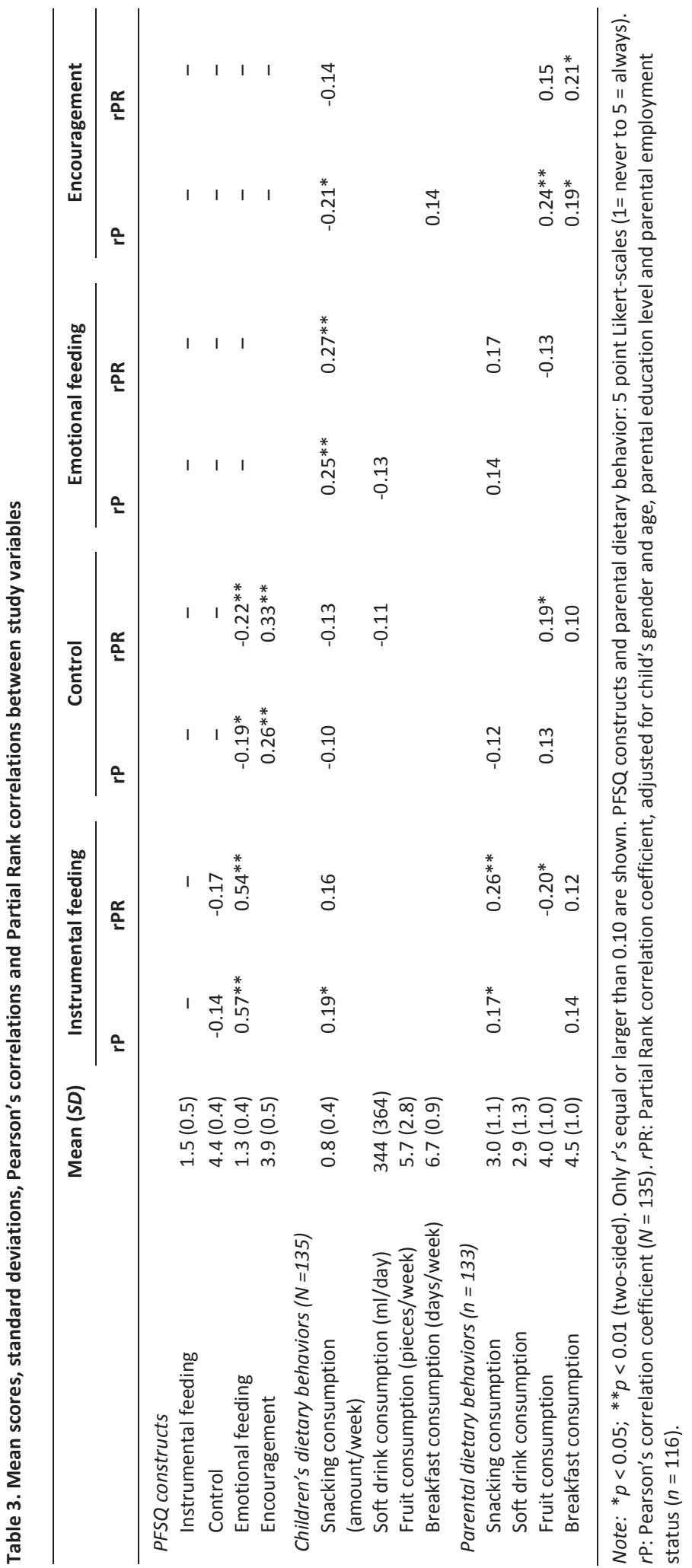




\section{Discussion}

The psychometric properties of the Dutch version of the PFSQ were comparable to those of the original instrument, with acceptable internal reliability and between-subscale correlations. Although the present study provided support for the proposed original four-factor solution, two scales of the original instrument (instrumental feeding and emotional feeding) clustered together in our study. The scales were highly correlated, and combining them into one scale ('instrumental and emotional feeding') increased the internal consistency. In contrast, the 'encouragement' scale was divided into two subscales to safeguard the psychometric properties of the questionnaire. However, since reliability estimates of the original PFSQ scales were adequate and in order to allow comparison with the original subscales, we recommend the four-factor solution as defined by Wardle et al. (2002) for general use.

Both instrumental feeding and emotional feeding styles were positively related to children's snack consumption. Parental use of snacks as rewards may increase a child's preference for the 'rewarding' food (Birch et al., 1980; Newman \& Taylor, 1992). It is possible that those children will become increasingly responsive to external eating cues. Increased liking and greater food cue responsiveness regarding the consumption of unhealthy snack products is expected to promote overeating of these products in children. Moreover, it seems that children, who are encouraged to be interested in foods and to eat a variety of foods, consume less sugar-sweetened and energy-dense food products. It is advisable for parents not to use foods to regulate children's behavior or emotions, and to encourage their children's interest and curiosity to taste and eat a variety of foods.

Previous studies have yielded conflicting results regarding the influence of the parental feeding style 'control' on children's health behavior and weight status (e.g., Birch et al., 2003; Faith, Berkowitz et al., 2004; Faith, Scanlon et al., 2004; Fisher \& Birch, 1999a/b; Haycraft \& Blissett, 2008; Liem et al., 2004; Montgomery et al., 2006; Powers, Chamberlin, Van Schaick, Sherman, \& Whitaker, 2006). Findings of the present study indicated that the PFSQ construct of 'control' was negatively associated with children's snacking behavior, but not statistically significant. Contradictory results regarding the influence of controlling practices on children's health behaviors and weight status and vice versa could be attributable to different conceptualizations of controlling feeding practices. Ogden, Reynolds, and Smith (2006) expanded the concept of parental control and developed an instrument that measures two constructs, overt and covert control. Overt control is defined as forms of parental control over children's eating that can be perceived by the child. This type of parental control is assessed by the PFSQ. Covert control is defined as forms of parental control over children's eating that are not perceived by the child, for example by avoiding having unhealthy food in the house. This construct is not assessed by the PFSQ. Ogden et al. (2006) found that overt control was not related to children's unhealthy snacking behavior, whereas covert control was negatively related to unhealthy snacking among children.

Previous research of Wardle et al. (2002) showed that instrumental feeding was positively associated with externally cued eating in mothers. External eating has been demonstrated to be associated with high 
consumption of fast food and sweets in both men and women (Burton, Smit, \& Lightowler, 2007; Elfhag, Tholin, \& Rasmussen, 2008) and low maternal consumption of fruits (Elfhag et al., 2008). In line with these earlier findings, our study showed a positive relationship between snacking habits among parents and the instrumentality of their feeding style, as well as a negative association between parental fruit intake and instrumental feeding after controlling for co-variables. An explanation for this relation could be that parents believe that their child has the same needs as themselves (e.g., parents who regularly snack also reinforce their child with food) (Wardle et al., 2002). As parents are the gatekeepers of the home food supply, unhealthy food products can be brought into the home leading to increased consumption of those foods among children (Campbell et al., 2007). Regarding the association between parental controlling feeding practices and their dietary behaviors, only the association with fruit consumption was significant after controlling for co-variables. In general there is a tendency in the current study that controlling practices are positively associated with healthy parental dietary behaviors and negatively associated with snacking among parents. Parental prompting of a child to eat was positively related to parental breakfast and fruit consumption. Parents who regularly consumed breakfast and fruits may be more inclined to prompt their children to be interested and curious to eat a wide variety of foods.

Some comments should be made on the limitations of the study. Firstly, factor-analytic procedures have to be repeated on a larger sample to replicate our findings (i.e., the subtle differences between the original factor solution found by Wardle et al. (2002) and our factor solution should be further explored, by making nuances between the 'encouragement of food variety' and 'encouragement of interest in food' scales). Secondly, the response rate was relatively low (mean 41.9\%) and families with lower levels of education were relatively underrepresented in the current study. A third limitation is that children's dietary intake behaviors were parent-reported. It is possible that parental reports underestimate actual children's snacking and soft drink consumption, as the participating children are exposed to school food environments that parents may not be fully aware of. Parents could also respond to items regarding their children's eating behaviors and their own eating patterns by giving socially desirable answers. Due to these systematic biases, correlations between parental feeding and dietary intake behaviors of both children and parents in the current sample may have been overestimated. Fourthly, the finding that no significant correlations were found for the association between parental feeding style and children's breakfast consumption could be due to the low variability in the breakfast consumption score for children. Finally, the present study was limited by its cross-sectional nature, which does not allow us to draw conclusive inferences about causality.

\section{Conclusion}

Our findings provide initial support for the use of the PFSQ among parents of 6- and 7-year-old children in the Netherlands. Identification of specific parental dietary behaviors and feeding styles, as well as more global parenting styles is useful, since children's abilities and opportunities to make healthful food choices are highly dependent on the social context in which these are made (Kremers, Brug, De Vries, \& Engels, 
2003). The current study indicated that both instrumental feeding and emotional feeding may have a detrimental impact on children's snacking behavior, in such a way that these feeding styles will increase child snacking frequency. In contrast, encouragement of the child's interest and curiosity to taste and eat a variety of foods is indicated to be beneficial in reducing a child's consumption of sugar-sweetened and energy-dense food products. Further longitudinal and experimental research is warranted to unravel the exact mechanism underlying the parental feeding - eating relationship. 


\title{
CHAPTER 10
}

Issues in the measurement of parenting style

\author{
Thomas G Power \\ Ester FC Sleddens \\ Jerica Berge \\ Lauren Connell \\ Bert Govig \\ Erin Hennessy \\ Leanne Ligget \\ Kimberley Mallan \\ Diane Santa Maria \\ Angela Odoms-Young \\ Sara M St. George
}

Submitted 


\begin{abstract}
The parenting style measurement working group at the 'International Society of Behavioral Nutrition and Physical Activity' (ISBNPA) pre-conference, 'Parenting measurement: Current status and consensus reports' (Houston, Texas, United States, May 20-22, 2012), chaired by Thomas Power and Ester Sleddens, discussed a range of issues regarding the assessment of parenting. These included: 1) general versus domain specific parenting styles and practices; 2 ) novel approaches to parenting measurement; 3 ) the role of ethnicity and culture; 4) assessing bidirectional influences; 5) broadening assessments beyond the immediate family; and 6) designing effective interventions. Numerous directions for future research were offered.
\end{abstract}


As part of the ISBNPA pre-conference on parenting measurement (Baranowski et al., under review), May 20-22, 2012, participants in the parenting style measurement working group discussed wide-ranging topics related to the assessment of general parent-child interactions. The major issues are considered below.

\section{General and domain specific parenting styles and practices}

\section{Definitions}

Parenting styles can be general or domain specific and are related to but distinct from parenting practices. These terms can be conceptualized as a nested hierarchy, with general parenting styles being the broader and more inclusive concept reflecting an approach to child-rearing across situations and domains (Baumrind, 1967). General parenting styles are a function of the parent's attitudes, beliefs, and behaviors, and, as Darling and Steinberg (1993) argue, reflect the emotional climate in which specific parenting practices are implemented. Well-known examples of parenting styles include the authoritarian, authoritative, and permissive styles first identified by Baumrind in the mid 1960's (Baumrind, 1967).

Parenting practices are the discrete, observable acts of parenting (praise, feedback, reward, punishment, reasoning, limit setting, etc.). Because they are more measurable, there has been a tendency to measure practices and use them to assign parents to parenting styles. For example, if a mother frequently reasons with her child and consistently enforces maturity demands, she will be labeled an authoritative parent. However, the relationship between parenting styles and practices is complex - a father may turn a blind eye to a disobedient child, because of a belief that positive rewards are more effective than punishments, or because he does not care. Although the behavior of these two fathers appears similar, we would label the first as indulgent and the second as uninvolved. This example illustrates how the values and attitudes of a parent create an important backdrop that gives context to and colors the interpretation of their parenting behavior. While some have argued that parenting attitudes should be studied separately from behaviors (Schaffer, 1977), our working group concluded that parenting practices should be defined in the context of other parenting practices, and in the context of the underlying beliefs, values, and attitudes of the parents. Such measurement, ideally, would require the combination of self-report and observational methods.

\section{Comparing specific and general practices and styles}

Research in social psychology demonstrates that the relationship between attitudes and specific behaviors increases as one increases the specificity of the attitude being studied (Ajzen \& Fishbein, 1977). Consistent with this theory, we would expect that parental feeding practices would correlate more highly with child eating behavior than general parenting practices and styles. Although numerous studies show that general parenting style predicts child overweight and eating behaviors (Sleddens, Gerards, Thijs, De Vries, \& Kremers, 2011), when measures of general parenting style and specific feeding practices are entered in the same regression, only feeding practices are significant predictors of child weight status and food consumption - not general parenting style (Blissett \& Haycraft, 2008; De Bourdeaudhuij, Te Velde, Maes, 
Pérez-Rodrigo, De Almeida, \& Brug, 2009; Vereecken, Legiest, De Bourdeaudhuij, \& Maes, 2009; Vereecken, Rovner, \& Maes, 2010). This suggests that the effects of general parenting style may be mediated through specific parental feeding practices. This means, for example, that authoritative parents would be less likely to have obese children because of the nature of their feeding practices.

In contrast to the above findings, Brotman et al. (2012) demonstrated that a family intervention to promote effective parenting in early childhood had a significant impact on preventing obesity in adolescence, despite the fact that the intervention did not address parental feeding practices. As the authors argue, 'obesity interventions that are narrowly focused on eating and activity without changing fundamental aspects of the early family environment are likely to be insufficient, especially for children at high risk' (page e626). It is possible that the effects of this intervention were mediated through a change in parental feeding practices, or that general parenting does have an effect over and above specific feeding practices. These findings may not be at odds. A recent analysis of seven studies suggests that interventions that focus on both parenting styles and specific practices are the most effective (Gerards, Sleddens, Dagnelie, De Vries, \& Kremers, 2011).

The results of these studies raise a fundamental question: When intervening with parents should we try to change parenting practices or parenting styles? And if the answer is both, then in what proportion? Current research does not fully answer this question. One might argue that targeting parenting styles might be more effective, because changing parental beliefs, values, and attitudes may have a broader impact on child developmental outcomes than changing parenting practices alone. Conversely, there is likely a gradient of learnability ranging from specific parenting practices to general parenting practices to parenting styles, making it easier to change specific practices. This may be an either-or question. Maybe the most effective approach would be to tailor parenting interventions to specific parenting styles. Future research should explore the relationships between specific and general practices, and parenting attitudes, values, beliefs and styles on child behavior and health.

\section{Measurement issues in parenting}

\section{New measurement options}

Traditional methods for assessing parenting include observation, parent reports, and child reports, but emerging electronic and web-based technologies open the door to a wide range of new assessment tools. Smart phones and tablet computers, for example, allow for real time collection of audio and video data, without the cost or intrusion of having an investigator in the room. The best tools for measuring parenting depend on the research question being asked and the resources available. When possible, the use of multiple measuring tools is preferable as they will likely collect complementary information. For example, reports from parents and children would likely yield useful but different data. 


\section{Short forms}

Researchers often do not include parenting assessments in their studies because instruments with good reliability and validity take too long to complete. Advanced psychometric methods including confirmatory factor analyses and item response modeling should be applied to existing parenting measures (i.e., item banks) to develop short forms and rapid assessment parenting tools. These new tools could be used for multiple purposes such as adding a short set of questions to large national surveys where parenting is not the main focus, developing screening instruments for identifying high-risk participants for targeted interventions, assessing parenting in clinical settings such as pediatric and primary care medical practices, and evaluating parenting-related interventions.

\section{Assigning parents to styles}

Parents are often assigned to styles using a median split procedure. This approach might be useful when samples are small or when previous research provides clear guidelines for assigning parents to categories. Unfortunately, the data are not always clear, and many individuals may be close to the median making assignment to one cluster or another seem arbitrary. Cluster analytic approaches using multiple dimensions may be more effective for capturing the complexity of parenting and may facilitate the exploration and discovery of parenting styles that are not driven by current theoretical positions. Also, because such approaches identify naturally occurring groups of subjects, the number of individuals at the 'cluster borders' is often smaller.

\section{Ethnicity and culture}

Parenting styles and practices are imbedded in the larger culture - theories and measurement practices need to take this critical issue into account. Baumrind (1972), for example, in her classic parenting research, identified parenting styles unique to African-American families. This led to considerable subsequent research on parenting in African-American families (Tamis-LeMonda, Briggs, McClowry, \& Snow, 2008). One finding to emerge from these studies is that authoritarian parenting, a style associated with negative child outcomes in middle class, European-American families, is not associated with negative outcomes in lowincome, African Americans (Landsford, Deater-Deckard, Dodge, Bates, \& Pettit, 2004; LeCuyer, Swanson, Cople, \& Kitzman, 2011).

\section{Measurement}

We need to develop and validate parenting measurement tools that can be used across cultural groups without excluding important cultural constructs, and examine cultural similarities and differences in the correlates of these measures. Chao (1994), for example, developed questionnaire items that assessed the Chinese concept of chiao shun - a concept related to training that is central to the practices of Chinese and 
many Chinese-American parents. The transferability and predictive validity of this construct across different cultural groups remains unclear. Similarly, Power, Kobayashi-Winata, and Kelley (1992) found that the Parenting Dimensions Inventory (PDI) (Slater \& Power, 1987), a questionnaire developed with EuropeanAmerican parents, had good psychometric properties when administered to parents in Japan, but cluster analyses identified parenting styles in the United States (US) and Japan that were markedly different. This begs a series of questions: What do these new clusters really represent? Do they have correlates in other cultures? Are we missing other constructs or behavior clusters because our tools are incomplete?

\section{Universality}

With enough research, we may be able to identify underlying universal characteristics of parenting that operate across cultures, but may be expressed in different ways. The three components of selfdetermination theory (competence, autonomy, and relatedness) (Ryan \& Deci, 2000) were given as an example of such universal characteristics in another domain. For example, although cultures clearly differ in the degree to which they place an emphasis on autonomy, Milyavskaya et al. (2009) found that the satisfaction of autonomy, competence, and relatedness needs were associated positively with adolescent adjustment in a wide range of cultural settings including Canada, China, France, and the US. If such universal characteristics could be identified in the parenting domain (with the wording for the items inclusive enough to be equally valid across cultures), parenting measures could be developed that could be used across varying cultural contexts.

\section{Parenting in perspective}

\section{Bidirectional effects}

Parenting is traditionally defined as the act of raising children. There is an inherent parent centric bias to this definition that casts parents as the principal players. Another perspective (that of the child) may define the growing up process differently and may view parent-child interactions more as the act of 'taming the parents.' In fact, the influence of children on parents is well documented (Bell \& Chapman, 1986). These 'child effects' have been demonstrated through experimental studies that vary child behavior, and in longitudinal studies where changes in child behavior predict changes in parent behavior over time (Bell \& Chapman, 1986). Children influence their parents at the same time that parents influence their children. However the complex moment-to-moment processes that operate in these behavioral transactions (Sameroff, 1975) have yet to be adequately described. This is unfortunate, since theorists have been writing about such bidirectional processes for at least 60 years (Sears, 1951). Perhaps new technology which now allows for ecological momentary assessment may be used moving forward to further explore these types of interactions. 


\section{Household Perspectives}

It is likely that child outcomes are influenced by the combined parenting practices and styles of the household, but the interactions between these styles and practices may be complex. For example, if one parent is authoritarian and the other parent is authoritative, does the authoritative parenting style 'buffer' the other parent's authoritarian style? One study (Berge, Wall, Neumark-Sztainer, \& Bauer, 2010) found that the co-occurrence of an authoritarian mother and a neglectful father was associated with higher Body Mass Index (BMI) in adolescent sons, but there was no protective effect of authoritative parenting style. Furthermore, the study found that incongruent parenting practices were associated with higher BMIs in adolescents. Specifically, when mothers modeled and encouraged healthful eating and physical activity, but fathers did not, adolescents had higher BMIs. Thus, bi-directional influences between parents and other caregivers in relation to parenting style and parenting practices are important to identify in order to capture a more comprehensive picture of the home environment when trying to assess risk and protective factors for childhood and adolescent obesity in the home environment.

Suggestions for future study included: 1 ) looking at the quality of the parent-child relationship as a moderator of the effect of parenting practices (e.g., Kochanska, Aksan, Knaack, \& Rhines, 2004); 2) examining dyadic, triadic, and broader contexts (e.g., mother, father, and child; parents, caregivers, siblings, and child) (e.g., Lindahl, Clements, \& Markman, 1997); 3) applying concepts from the social psychological literature on close relationships to parent-child relationships (e.g., Lollis \& Kuczynski, 1997); and 4) examining interactive processes from a systems science perspective (e.g., emergent processes, state changes, feedback loops) (e.g., Hollenstein \& Lewis, 2006).

\section{Community perspective}

It seems almost too obvious to state that a child's development and behavior is influenced by all of the influences in his environment (Bronfenbrenner, 1977). This includes the physical environment, siblings, primary caregivers, as well as relatives, friends, neighbors, childcare providers, teachers -in fact the community at large. The cliché, 'It takes a village to raise a child' comes into sharp focus when one observes positive outcomes in settings where traditional parenting is almost totally lacking. Little is known about parenting influences outside of the household, but this may be an interesting vein of research in a world where family and home structures are rapidly evolving. Parenting researchers should broaden their focus and include in the research designs assessments of the 'parenting' behavior of the other individuals who may play a significant role in the child's life. 


\section{Designing interventions}

\section{Challenges}

Parenting is a central element in everyone's lives, and any 'challenge' relating to parenting, could be negatively perceived. As with all interventions, unforeseen consequences (positive and negative) are inevitable. Awareness and surveillance of such effects should be the norm.

\section{Intervention Level}

Given the limited resources typically available for prevention, what populations should be targeted in parenting interventions? Gordon (1987) differentiates between universal, selective, and indicated approaches. Universal programs are designed to reach the entire population, selective programs target atrisk groups, and indicated interventions are for individuals who are beginning to show early signs of the problem behavior. Effective prevention involves intervening at all levels, such as in the well-known, evidenced-based, Triple P-Positive Parenting Program (Sanders, Turner \& Markie-Dadds, 2002). The intervention contains five levels, ranging from the use of universal media to disseminate positive parenting information to an intensive individually-tailored program which includes home visits.

\section{Strength based approaches}

Group settings are particularly attractive for parenting interventions. Parenting is fundamentally a social act that is heavily influenced by cultural and community role modeling and norms. A group setting potentially circumvents some of the issues relating to judgment by providing a built in support group. The best approaches are also strength-based - parents reflect upon their parenting goals and strengths, and build on these strengths to achieve their goals. As with behavioral change in other domains, prescriptive as opposed to restrictive goals are more likely to be effective.

\section{Timing}

Transition periods are by definition linked to change, uncertainty, and perceived or real risk, and may also be accompanied by a receptiveness to interventions. Parenting milestones that may be privileged opportunities for intervention include the birth of a child, childcare or school transitions, adiposity rebound, and puberty.

\section{Summary}

The study of parenting as a determinant of childhood behavior and health is an exciting and important field. This paper outlines general existing concepts, nomenclature, core constructs, measurement issues 
and challenges, emerging technology, new and evolving perspectives on parenting, and design issues for interventions. Collaboration within the burgeoning parenting research community is a top priority. 


\section{CHAPTER 11}

Development of the Comprehensive General Parenting Questionnaire for parents of 5 to 13 year olds

Ester FC Sleddens Teresia M O'Connor Kathleen B Watson Sheryl O Hughes Thomas G Power Carel Thijs Nanne K De Vries Stef PJ Kremers

In revision 


\begin{abstract}
Despite the large number of parenting questionnaires, considerable disagreement exists about how to best assess parenting. Most of the instruments only assess limited aspects of parenting. To overcome this shortcoming, the 'Comprehensive General Parenting Questionnaire' (CGPQ) was systematically developed. First, an item bank of existing parenting measures was created assessing five key parenting constructs that have been identified across multiple theoretical approaches to parenting (nurturance, overprotection, coercive control, behavioral control, and structure). Caregivers of 5- to 13-year-olds were asked to complete the online survey in the Netherlands $(N=835)$, Belgium $(N=435)$ and the United States $(N=241)$. In addition, a questionnaire regarding personality characteristics ('Big Five') of the caregiver was administered. Factor analyses and Item-Response Modeling (IRM) techniques were used for assessing the underlying parenting constructs and for item reduction. Correlation analyses were performed for assessing the relations between general parenting and personality of the caregivers. The reduced questionnaire revealed acceptable fit of our parenting model and acceptable IRM item fit statistics. Caregiver personality was related to the parenting constructs as measured by the CGPQ. The personality traits of extraversion, agreeableness, conscientiousness, and openness to experience were positively associated with parenting constructs (i.e., nurturance, structure, behavioral control) previously found to be related to more positive child health outcomes, whereas the trait of neuroticism was associated with coercive control and a chaotic home environment. Based on expert panel review and cognitive interviews the questionnaire was further modified. The proposed 85 -item questionnaire may facilitate research exploring how parenting influences children's health related behaviors.
\end{abstract}




\section{Introduction}

General parenting has commonly been defined as the approach parents use to raise their child, and are a function of the parent's attitudes, beliefs and behaviors, creating a family emotional climate (Baumrind, 1968, 1971; Darling \& Steinberg, 1993). Parenting is a complex interplay of specific behaviors intended to influence child outcomes, and displayed across many different situations (Darling \& Steinberg, 1993). Parenting has been examined from a variety of theoretical perspectives including psychoanalytic (Orlansky, 1949), operant learning (Gewirtz, 1956; Patterson \& Fleischman, 1979), social learning (Bandura, 1977; Sears, Maccoby, \& Levin, 1957), acceptance-rejection (Rohner, 1986), attachment (Ainsworth, Blehar, Waters, \& Wall, 1978; Bowlby, 1958), self-determination (Grolnick \& Ryan, 1989), and Vygotskian (Wood, Bruner, \& Ross, 1976) theories. In contrast to early investigations that examined the child development consequences of specific parenting practices (e.g., the nature and timing of weaning or toilet training) (Orlansky, 1949), most theoretical approaches (operant and Vygotskian approaches being notable exceptions) have led to studies examining the child development correlates of general, cross-situational variations in general parenting approach-often referred to as parenting styles or dimensions. These studies focused less on what parents do and more on how they do it. Skinner, Johnson, and Snyder (2005), in a review of this literature, showed that independent of theoretical perspective, most researchers have focused on three core dimensions of parenting practices (warmth versus rejection, structure versus chaos, and autonomy support versus coercion). These are the three dimensions we focused on in the development of our instrument, referred to below as parental nurturance, structure, and control.

\section{Toward a Comprehensive Assessment of General Parenting}

Although there is considerable convergence across studies on the child development correlates of parental nurturance and structure (Skinner et al., 2005), the literature on parental control is much less consistent (Barber, 1996; Rollins \& Thomas, 1979). While nurturance and structure are well defined in the parenting literature, multiple forms of control have been identified by several scientists, some inhibiting and others supporting a child's emotional development. Forms of control proposed to inhibit a child's development include parental strictness and excessive involvement or worry (overprotection), and parental dominance or intrusiveness (coercive control). Generally accepted controlling practices supporting a child's development include the application of developmentally appropriate forms of guidance and direction (behavioral control).

Thus, we identified five parenting constructs (i.e., nurturance, overprotection, coercive control, behavioral control, and structure) that describe the major individual differences in general parenting behavior. Each of these constructs will be clarified in the following sections. Figure 1 displays our comprehensive general parenting model. 
Figure 1. Comprehensive General Parenting Model

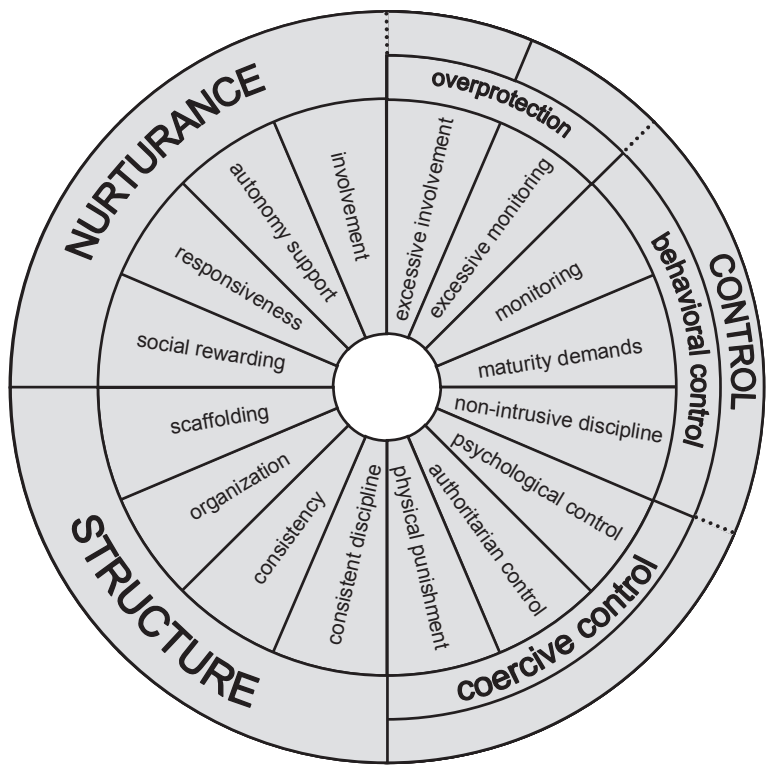

Note: Five-factor parenting model for the development of the Comprehensive General Parenting Questionnaire

\section{Nurturance}

This is one of the parenting constructs most frequently assessed. It is the degree to which parents foster and recognize individuality and self-assertion by being supportive and responsive to their child's needs, showing interest in child activities, spending time with their child, praising their child for good behavior, and expressing affection and care (warmth; Rohner, 1986) toward their child. The literature supports four sub-constructs that encompass nurturance. These include 'responsiveness' (the extent to which parents are aware of their child's feelings, problems, and difficulties, and the way they respond in a supportive and attuned manner), 'autonomy support' (parenting behaviors in which children are promoted to express their feelings and opinions; e.g., Grolnick, Price, Beiswenger, \& Sauck, 2007; Grolnick \& Ryan, 1989), 'social rewarding' (verbally praising their child as a reward for good behavior), and 'involvement' (parents being involved with their child by attending the child's events and activities, and spending time with their child).

\section{Overprotection}

One of the most understudied aspects of parental control is parental overprotection (Power \& Hill, 2008). With a few notable exceptions (e.g., Holmbeck et al., 2002; Kiel \& Buss, 2011), most of what has been written about the negative effects of parental overprotection come from clinical case studies (e.g., Parker, 1983; Sargent, 1983) or from media reports of 'helicopter parents.' Parents who are overprotective, score 
high on 'excessive involvement' (excessive nurturing) and 'excessive monitoring' (strict control). They are believed to negatively impact child development through interfering with the development of children's autonomy. Although it is difficult to describe for a given child what constitutes 'excessive' involvement or monitoring, it is defined here as involvement or monitoring that is excessive given the child's developmental level. Therefore, if a parent shows a level of involvement or monitoring that is more appropriate for a much younger child, it is viewed as excessive. Because this newer construct was not specifically addressed in the Skinner et al. (2005) model, we are including it under the control construct. The sub-construct of 'excessive involvement' is defined as being too involved with their child (parents being overprotective by not letting their child get involved in activities if there is a slight chance to fail, and spending every free minute they have with their children). 'Excessive monitoring' is defined as parents who excessively monitor their child's behavior (characterized by overprotective parental behaviors such as frequently checking where the child is and what the child is doing, more than is considered appropriate for the child's age).

\section{Coercive control}

We refer to coercive control as parents characterized by pressure, intrusion, domination, and discouragement of child independence and individuality. The sub-constructs of this parenting construct are 'authoritarian control' (parents who tend to enforce rules harshly, expect their child to accept their judgments, values, and goals without questioning, and attempt to control their child's emotions at all times; Baumrind 1968, 1971), 'physical punishment' (using corporal punishment as a way of disciplining the child), and 'psychological control' (parental behaviors that are intrusive and manipulative of children's thoughts, feelings, attachments to parents (Barber \& Harmon, 2002, page 15)). Psychological control intrudes into the psychological and emotional development of the child through use of parenting practices such as guilt and anxiety induction, love withdrawal, constraining verbal expressions, and personal attacks on a child (Barber, 1996). It was first defined by Becker in 1964 as negative, love-oriented discipline such as child isolation from the parent and love withdrawal. Schaefer's work $(1965 a, b)$ included psychological control as the presence of parental dominance, intrusiveness, and coercive, autocratic discipline. From the 70 s to the 90s, the construct of psychological control was largely neglected in empirical research on the socialization process, because in these decades the typological approaches to parenting focusing on the 'responsiveness' and 'demandingness' dimensions dominated the socialization literature (Baumrind, 1991; Maccoby \& Martin, 1983). After this period, Steinberg (1990) and Barber (1996, 2002a) re-focused on the construct of psychological control.

\section{Behavioral Control}

This construct could be regarded as parents supervising and managing their child's activities, providing clear expectations for behavior (in this paper referred to as maturity demands), and using disciplinary approaches in a non-intrusive manner. Parents scoring high on behavioral control provide adequate levels of control, they are not too strict or over-controlling, but rather allow their child to have enough space to 
develop independence and autonomy. As Darling and Steinberg formulated in 1993, it refers to the parent's 'willingness to act as a socializing agent.' The identified sub-constructs are as follows: 'monitoring' (supervising their child's activities) and 'maturity demands' (expectations for behavior) (Barber, Olsen \& Shagle, 1994), and 'non-intrusive discipline' (the use of disciplinary approaches when children misbehave that are mainly based on explaining a child's misbehaviors, taking away privileges and correcting the child in a non-intrusive manner).

\section{$\underline{\text { Structure }}$}

It is the degree to which parents organize their child's environment, by helping their child when necessary to gradually achieve a certain goal, and consistently enforcing rules and boundaries. Sub-constructs include 'inconsistent discipline' (reverse coded; parents scoring high on concepts such as non-contingency and inconsistency, acting erratic, unpredictable and undependable, and not following through when disciplining their child), 'consistency' (acting in a predictable manner by providing and explaining clear and consistent guidelines, enforcing those rules, and keeping promises to their child; Slater \& Power, 1987), 'organization' (helping their child to organize regular activities; e.g., Sessa, Avenevoli, Steinberg, \& Morris, 2001; Slater \& Power, 1987), and 'scaffolding' (exposing children to activities that foster the development of new skills and providing just enough structure and assistance to help them solve problems and learn with the ultimate goal of enabling children to perform the task independently; Carr \& Pike, 2012; Wood, Bruner, \& Ross, 1976).

\section{Relationship with Adult Personality}

Parenting is influenced by numerous facets of the caregiver; one of the main determinants is parent personality (Belsky, 1984). Assessment of personality is commonly based on five-factor taxonomy of traits, the so-called 'Big Five', which has proven very useful for conceptualizing and measuring individual differences in personality (e.g., Goldberg, 1990; McCrae \& Costa, 1987; McCrae \& John, 1992). Consensus has been achieved concerning the five-factor personality structure as it has been proven to replicate in diverse samples, across languages and cultures, and across several assessment methods and factor analytic procedures (John \& Srivastava, 1999). The 'Big-Five' factors have been labeled as follows: (1) extraversion, (2) agreeableness, (3) conscientiousness, (4) openness to experience (or intellect, culture), and (5) neuroticism (vs. emotional stability) (Costa \& McCrae, 1992).

Within the parenting literature, a meta-analytic review was previously conducted examining links between the 'Big Five' personality factors and parenting (Prinzie, Stams, Deković, Reijntjes, \& Belsky, 2009). Findings showed that higher levels of extraversion, agreeableness, conscientiousness, and openness to experience and lower levels of neuroticism were related to more parental warmth and behavioral control, whereas only higher levels of agreeableness and lower levels of neuroticism were related to more autonomy support. Neuroticism has been repeatedly found to be associated with less adaptive parenting behaviors. 
In the current study, relations between general parenting and personality characteristics of the caregivers were assessed as a measure of construct validity as child-rearing varies depending on parent personality. Based on previous findings (Prinzie et al., 2009) we expected that caregivers who score high on 'positive' parenting (i.e., nurturance, structure, and behavioral control) would also score high on the more positive related personality traits including agreeableness, extraversion, conscientiousness, openness to experience, and score low on neuroticism.

\section{The Study Rationale}

Despite the large number of general parenting instruments (Holden \& Edwards, 1989; Locke \& Prinz, 2002), considerable disagreement exists about how to best assess parenting. Most of the instruments only assess limited aspects of parenting, and consensually identified questionnaires of high quality measures are lacking (Sleddens, Gerards, Thijs, De Vries, Kremers, 2011). Hence, it is necessary to identify the core constructs of parenting and to elaborate and clarify their defining features. Although Skinner et al. (2005) developed a questionnaire to measure the three core constructs of warmth, control, and structure, the length of their questionnaire limited the number of parenting constructs they could assess. For example, they had limited (or no items) on such well-studied constructs as parental monitoring, organization, consistency in discipline, scaffolding, and overprotection. This study aimed to develop and validate a new 'Comprehensive General Parenting Questionnaire' (CGPQ) to assess the five key constructs of parenting reviewed above. Developing a single parenting questionnaire to assess the major parenting constructs (versus piecing together a large number of individual questionnaires) greatly reduces participant response burden. Moreover, by measuring the major parenting dimensions simultaneously, it will be possible in future studies to examine individual differences in parenting styles that involve simultaneously assessing individuals across multiple parenting dimensions. The ultimate goal is to promote comparability across studies and facilitate research exploring how parenting influences children's health related behaviors.

The mixed methods developmental process of our CGPQ comprises the following four steps: 1) items were identified from existing parenting questionnaires based on our framework including the five constructs of parenting; 2) cognitive interviews and author review informed the modification, deletion and/or replacement of items; 3) advanced statistical analyses including Classical Test Theory, Confirmatory Factor Analyses (CFA) and Item-Response Modeling (IRM) were conducted to test our theoretical five-factor parenting model and to develop fit items using an online survey containing the parenting item bank; and 4) additional author reviews and cognitive interviews were done to review the fit items, determine if any construct was missing or inadequately assessed, assess content validity, and verify wording of the modified items. 


\section{Method}

\section{Scale development}

We searched for validated instruments measuring our defined parenting constructs (see Figure 1), and selected some of the most commonly used instrument in research. An item bank was created by pulling and adapting items from the following existing questionnaires: the 'Parents as Social Context Questionnaire' (Skinner et al., 2005); the 'Ghent Parental Behavior Scale' (Van Leeuwen \& Vermulst, 2004); the 'Child Rearing Practices Report' (Block, 1965; Deković, Janssens, \& Gerris, 1991); the 'Parenting Dimensions Inventory' (Power, 2002; Slater \& Power, 1987); the 'Parental Regulation Scale - youth selfreport: parental expectations for behavior scale and parental monitoring of behavior scale' (Barber, 2002b; Soenens, Vansteenkiste, Luyckx, \& Goossens, 2006); the 'Psychological Control Scale - youth self-report' (Barber, 1996; Soenens et al., 2006) and its adaptations to parent self-reported parenting (Olsen et al., 2002; Soenens, Vansteenkiste, Duriez, \& Goossens, 2006); the 'Parental Authority Questionnaire' (Buri, 1991); the Dutch Parenting Questionnaire ('Nijmeegse Opvoedingsvragenlijst'; Gerris, Van Boxtel, Vermulst, Janssens, Van Zutphen, \& Felling, 1993), and the 'Perceptions of Parents Scales' (Grolnick, Ryan, \& Deci, 1991; Soenens et al., 2007; Vansteenkiste, Zhou, Lens, \& Soenens, 2005). Team meetings were held to ensure face validity of the items, and modifications were made to improve ambiguous items. In case of unavailability of the measures in both Dutch and English, the items of concern were translated by the first author, a Dutch native speaker and fluent speaker of the English language, and cross-checked by the coauthors. All authors approved the final English translations. Cognitive interviewing was conducted on several of these questionnaires (Deković et al., 1991; Skinner et al., 2005; Soenens et al., 2006) with 10 to 20 Dutch parents to ensure that they understood the items and response scales. This pre-test consisted of parents completing the questionnaire, followed by discussion of particular words/phrases to see whether parents understood the items as intended, and discussion of items parents identified as complex. For the interview a pre-defined interview script was used. Minor changes were made in wording. Moreover, based on an in-depth review of existing parenting literature and validated measures, we wrote additional items to provide adequate number of items to cover all sub-constructs of the five different parenting constructs. The resulting questionnaire included 145 items that measured nurturance, overprotection, coercive control, behavioral control, and structure. For all items the same five-point Likert scale was used, ranging from 1 (Strongly disagree) to 5 (Strongly agree).

\section{Additional measures}

In addition to the parenting questions, we collected demographic information (e.g., child gender and age, living situation, parental education level and employment status, see Table 1). Caregiver's personality was measured using a 30-item scale for the 'Big Five' (six for each of the traits) (Gerris, Houtmans, KwaaitaalRoosen, Schipper, Vermulst \& Janssens, 1998). The criterion validity, test-retest reliability and internal consistency of this 30-item scale have been well established in previous studies (Vermulst \& Gerris, 2005). Caregivers were asked to score on a seven-point Likert scale the degree to which the personality 
characteristics were descriptive of themselves. Cronbach's alphas were as follows: extraversion 0.88 , agreeableness 0.85 , conscientiousness 0.88 , openness to experience 0.80 , and neuroticism 0.81 .

\section{Data collection and participants}

The survey was administered as a web-based survey which has more advantages than disadvantages compared with traditional modes of data collection. Advantages include lower proneness to social desirability bias, no missing data when using forced-choice formats, and more rapid return than postal questionnaires (Van Gelder, Bretveld, \& Roeleveld, 2010). Disadvantages include selection bias for those that have access to a computer, and higher non-response rates, although subjects responding to an online survey are comparable to those responding to traditional modes of data collection in terms of demographics (Van Gelder et al., 2010).

\section{The Netherlands}

Data were collected using a random sample of eligible parents (i.e., caregivers of 5- to 13-year-olds) from two Dutch Internet survey panels (Flycatcher Internet Research BV and Thesistools). The companies performed the random selections, ensuring the sample remained representative of the countries. Participants who take part in the Flycatcher panel are financially rewarded for their contribution, e.g. by collecting points for every completed questionnaire in order to be able to receive a gift coupon after a number of questionnaires. Only participants who had completed all parenting items were used for the current study. In total, 517 questionnaires were completed via Flycatcher and 304 via Thesistools. Child mean age was $8.64(S D=2.00)$ years.

\section{Belgium}

Similar procedures were used to generate data from Belgium parents. A Dutch Internet panel, Thesistools, was used for distribution of our online survey to eligible Dutch speaking parents in Belgium. In total, 421 questionnaires were used for analysis. Child mean age was $9.43(S D=1.88)$ years.

\section{$\underline{\text { United States }}$}

In the United States (US), English-speaking parents were informed about the online survey by (a) posting and handing out flyers in the vicinity of the Texas Medical Center, community centers, public libraries, universities, sports centers, and museums throughout Houston, Texas; (b) posting the study on the website of Baylor College of Medicine and the Children's Nutrition Research Center (CNRC); and (c) listing the study in the CNRC's nationally distributed newsletter and recruiting from the CNRC participant database. From all completed entries $(N=241)$, three names from the US sample were randomly selected to receive a $\$ 50$ gift 
card. Only participants who agreed to take part in the raffles had a chance to win one of the gift cards. Child mean age was $9.18(S D=2.26)$ years.

\section{Data analysis}

Based on several expert meetings with some of the leading researchers from the parenting field having extensive experience in questionnaire item development (based on qualitative and advanced statistical methods), 30 items were dropped prior to data analysis from the list of 145 parenting items. These items were dropped because of redundancy of item content or ambiguity. Data reduction procedures (i.e., CFA and IRM) were used to further reduce the list of 115 items on the total sample of parents $(N=1497)$. The use of the total sample provided adequate power to perform the data reduction procedures on the list of 115 items. Table 2 gives an indication of the number of items within each of the five parenting constructs and the corresponding sub-constructs.

A second-order CFA was used to validate the hypothesized five-factor structure (nurturance, overprotection, coercive control, behavioral control, and structure). The second-order model allowed for sub-constructs loading onto the higher order constructs. In the first model we constrained the parenting factors so they did not correlate, whereas in the second model they were allowed to correlate. Given that the data were not severely skewed or kurtosed, parameter estimates were obtained using the maximum likelihood estimation procedure. Items were dropped that did not fit the model (i.e., with factor loadings equal or less than 0.40). The chi-square goodness-of-fit test and three fit indices were used to assess model fit, including the Root Mean Square Error of Approximation (RMSEA), the Comparative Fit Index (CFI), and the Non-Normed Fit Index (NNFI). Criteria of Hu and Bentler (1995) were used to evaluate model fit: RMSEA with a value of $\leq 0.05$ indicating a good fit and an upper value of 0.08 representing a reasonable fit; $\mathrm{CFI}$ and NNFI with a value $>0.95$ indicating a good fit.

Rasch Modeling (Multidimensional Partial Credit Model) was employed to further assess the psychometric properties of the parenting questionnaire and to reduce items, using the ConQuest software $(\mathrm{Wu}$, Adams, \& Haldane, 2007). These analyses were performed on the five parenting constructs separately, allowing us to incorporate the multidimensionality of sub-constructs within parenting constructs. The IRM analyses yield item infit statistics, item parameter difficulty estimates, Wright maps, and reliability indices. Item fit was determined by computing the weighted mean square fit statistics for each item, which indicate whether residuals varied as much as expected given the observed distribution. Items with a weighted infit statistic between 0.75 and 1.33 and/or items with a corresponding weighted $t$-statistic between -2.00 and 2.00 were indicative of a good fit (Adams \& Khoo, 1996). Examination of item fit was the first step in removing items using IRM.

The next step was to identify items with overlapping levels of item average difficulty via the Wright map. In the context of general parenting, item difficulty refers to the level of agreement in performing the parenting practices. Item difficulty is the item's location on the underlying parenting construct, a 'higher' 
location indicating an increment in level of difficulty for the respondent to answer more agreeably to an item. Among items with overlapping levels of difficulty, item removal decisions were based on several meetings with the research group ensuring content validity was not threatened. Item separation reliability (EAP/PV) was calculated for the parenting scales' underlying the parenting constructs. It indicated 'how well the sample of subjects had spread the items along the measure of the test' (Fisher, 1992, page 238). The EAP/PV reliability is analogous to Cronbach's alpha and can be interpreted similarly where the minimum acceptable cut-off level for Cronbach's alpha is 0.50 (Portney \& Watkins, 2000).

Mean factor scores were computed for the five constructs of the CGPQ (see Table 2) and the 'Big Five' personality questionnaire (see Table 3). Correlation coefficients were used to assess associations between the scores for the five parenting constructs and to assess the associations between the scores for the parenting constructs and the 'Big Five' personality constructs, partialling out the effects from child gender and age, parental education level (ranging from 1: lowest level of education, to 3: highest level of education), and parental employment status (dichotomized as 1: employed or 2: non-employed). The strength of the relationship between the variables studied was assessed using correlation effect sizes as suggested by Cohen (1988): small (0.02 - 0.15), medium (0.15 - 0.35), and large (0.35 - 1.0).

\section{Results}

\section{Sample Characteristics}

Characteristics of the study samples are depicted in Table 1. Most often, female caregivers completed the online survey. Child gender was nearly equally divided across the three samples. Most caregivers indicated they lived with the child and spouse (percentages ranging from $77.2 \%$ in the US to $88.6 \%$ in the Netherlands). The US study sample was ethnically diverse. The majority was White (46.5\%), but Hispanics (24.5\%) and African-Americans (19.1\%) were also represented. A minority of the US participants were combined into 'other,' consisting of American Indians, Native Hawaiians, Pacific Islanders, and Asians (10.0\%). A large percentage of participants from the Netherlands, Belgium and the US reported higher levels of education $(47.4 \%, 81.1 \%$, and $58.1 \%$, respectively, indicated having a college degree or higher) and were employed $(87.5 \%, 90.3 \%$, and $83.0 \%$, respectively). Our study populations were roughly representative samples of the Dutch, Belgium and US population. Compared to the general US population, whites were underrepresented in the current study (46.5\%), but our US sample had a demographic distribution (i.e., ethnically diverse sample) similar to the Houston population. Participants with higher levels of education were slightly overrepresented in the current samples, but employment rates were largely similar to the general populations. 
Table 1. Sample Characteristics

\begin{tabular}{|c|c|c|c|c|c|c|c|}
\hline \multirow[t]{2}{*}{ Description } & & \multicolumn{2}{|c|}{$\begin{array}{c}\text { Netherlands } \\
(N=821)\end{array}$} & \multicolumn{2}{|c|}{$\begin{array}{l}\text { Belgium } \\
(N=435)\end{array}$} & \multicolumn{2}{|c|}{$\begin{array}{l}\text { United States } \\
\qquad(N=241)\end{array}$} \\
\hline & & $n$ & $\%$ & $n$ & $\%$ & $n$ & $\%$ \\
\hline \multirow[t]{2}{*}{ Child gender } & Male & 408 & 49.7 & 213 & 49.0 & 128 & 53.1 \\
\hline & Female & 413 & 50.3 & 222 & 51.0 & 113 & 46.9 \\
\hline \multirow[t]{2}{*}{ Relationship to child } & Female caregiver & 519 & 63.2 & 336 & 77.2 & 203 & 84.2 \\
\hline & Male caregiver & 302 & 36.8 & 99 & 22.8 & 38 & 15.8 \\
\hline \multirow[t]{4}{*}{ Race (US only) } & Black or African-American & & & & & 46 & 19.1 \\
\hline & White or Euro-American & & & & & 112 & 46.5 \\
\hline & Hispanic or Latino & & & & & 59 & 24.5 \\
\hline & Other & & & & & 24 & 10.0 \\
\hline \multirow[t]{3}{*}{ Living situation } & Together with child and spouse & 727 & 88.6 & 377 & 86.7 & 186 & 77.2 \\
\hline & Together with child and no spouse & 84 & 10.2 & 56 & 12.9 & 43 & 17.8 \\
\hline & Other & 10 & 1.2 & 2 & 0.5 & 12 & 5.0 \\
\hline \multirow[t]{3}{*}{ Education $^{a}$} & Low & 127 & 15.5 & 21 & 4.8 & 13 & 5.4 \\
\hline & Medium & 305 & 37.1 & 61 & 14.0 & 88 & 36.5 \\
\hline & High & 389 & 47.4 & 353 & 81.1 & 140 & 58.1 \\
\hline \multirow[t]{6}{*}{ Employed: paid job } & Yes & 718 & 87.5 & 393 & 90.3 & 200 & 83.0 \\
\hline & 36 hours or more per week & 290 & 40.4 & 210 & 53.4 & 167 & 83.5 \\
\hline & 20 to 35 hours per week & 275 & 38.3 & 144 & 36.6 & 18 & 9.0 \\
\hline & 12 to 19 hours per week & 105 & 14.6 & 35 & 8.9 & 10 & 5.0 \\
\hline & Less than 12 hours per week & 48 & 6.7 & 4 & 1.0 & 5 & 2.5 \\
\hline & No & 103 & 12.5 & 42 & 9.7 & 41 & 17.0 \\
\hline
\end{tabular}

Note: ${ }^{a}$ Highest education attained, categorized into low level $\left(8^{\text {th }}\right.$ grade or less, attended some high school, technical school graduate), medium level (high school graduate or GED, some college), and high level (college graduate, post graduate study).

\section{Confirmatory Factor Analysis}

CFA revealed a relatively adequate fit of our hypothesized general parenting model $\left(\mathrm{X}^{2}=26606.39, d f=\right.$ $6418, p<0.001$; RMSEA $=0.06, \mathrm{CFI}=0.91, \mathrm{NNFI}=0.91$ ) when the parenting constructs were not allowed to correlate. The fit slightly improved after allowing the parenting constructs to correlate (i.e., $\mathrm{X}^{2}=25434.68$, $d f=6414, p<0.001 ; \mathrm{RMSEA}=0.06, \mathrm{CFI}=0.92, \mathrm{NNFI}=.92)$. Subsequently, 33 items were removed based on the following criteria: magnitude of loadings (e.g., < 0.40 ), contribution to construct coverage, and theoretical considerations. The reduced 82 -item model had a slightly better fit compared to the 115 -item model (parenting constructs not allowed to correlate: $\mathrm{X}^{2}=14013.87, d f=3217, p<0.001$; RMSEA $=0.05$, $\mathrm{CFI}=0.93, \mathrm{NNFI}=0.92$; parenting constructs allowed to correlate: $\mathrm{X}^{2}=12864.61, d f=3213, p<0.001$; RMSEA $=0.05, \mathrm{CFI}=0.93, \mathrm{NNFI}=0.93$ ).

\section{Item-Response Modeling}

IRM analyses on each of the five parenting constructs using multidimensional models indicated that all 82items had acceptable values for both the weighted mean square statistic and $t$ statistic. To further reduce the number of items in the questionnaire, the Wright maps were visually inspected to assess overlapping item coverage across the latent parenting factors. Subsequently, 20 items were removed, until the total number of items per parenting sub-construct was around five based on the following criteria: items with 
overlapping levels of difficulty, contribution to construct coverage, and theoretical considerations. Thereafter, IRM was repeated on the reduced set of items (62 items in total) for each of the five parenting constructs. All items had acceptable values for both the weighted mean square statistic and $t$ statistic (range of infit statistics, $t$ statistic between brackets: nurturance $0.85(-2.0)-1.26(4.9)$, overprotection 0.98 $(-0.5)-1.05(0.9)$, coercive control $0.91(-1.6)-1.21(3.3)$, behavioral control $0.88(-2.8)-1.17(3.7)$, and structure $0.86(-4.4)-1.17(5.0))$. Item difficulty estimates (SE) ranged from - $0.84(0.04)$ to $0.64(0.05)$ for nurturance, from $-1.24(0.02)$ to $1.24(0.02)$ for overprotection, from $-0.96(0.02)$ to 0.77 (0.04) for coercive control, from $-0.58(0.03)$ to $0.77(0.03)$ for behavioral control, and from $-0.56(0.02)$ to $0.67(0.02)$ for structure. Based on the Wright map, the parenting constructs of nurturance, structure, and behavioral control, the items covered a restricted portion of participants (only those scoring low on this factor) in that the upper end of the continuum remained uncovered by items with higher levels of difficulty. The reverse was seen for the other two parenting constructs of coercive control and overprotection. EAP/PV reliability estimates slightly dropped for the several parenting constructs as expected, most likely due to item removal (ranged between 0.52 and 0.86). We refer to Table 2 for an overview of the number of items per parenting sub-construct and the reliability estimates.

Table 2. Comprehensive General Parenting Questionnaire average scores and item separation reliability

\begin{tabular}{|c|c|c|}
\hline Parenting constructs & Mean (SD) & EAP/PV reliability \\
\hline Nurturance (19 items) & $4.46(0.40)$ & 0.86 \\
\hline Responsiveness (6 items) & $4.48(0.47)$ & 0.79 \\
\hline Autonomy support (5 items) & $4.51(0.47)$ & 0.73 \\
\hline Involvement (4 items) & $4.22(0.64)$ & 0.79 \\
\hline Social rewarding (4 items) & $4.63(0.46)$ & 0.75 \\
\hline Overprotection (6 items) & $2.55(0.55)$ & 0.53 \\
\hline Excessive monitoring ( 2 items) & $3.31(0.73)$ & 0.49 \\
\hline Excessive involvement ( 4 items) & $2.17(0.63)$ & 0.52 \\
\hline Coercive control (12 items) & $2.06(0.50)$ & 0.75 \\
\hline Psychological control (5 items) & $1.84(0.66)$ & 0.71 \\
\hline Physical punishment (3 items) & $1.34(0.58)$ & 0.62 \\
\hline Authoritarian control (4 items) & $2.87(0.69)$ & 0.66 \\
\hline Behavioral control (10 items) & $4.00(0.49)$ & 0.69 \\
\hline Monitoring (3 items) & $4.02(0.73)$ & 0.68 \\
\hline Maturity demands (5 items) & $4.31(0.52)$ & 0.75 \\
\hline Non-intrusive discipline ( 2 items) & $3.19(1.00)$ & 0.33 \\
\hline Structure (15 items) & $3.84(0.45)$ & 0.75 \\
\hline Inconsistent discipline (R, 3 items) & $2.90(0.86)$ & 0.73 \\
\hline Consistency (5 items) & $4.47(0.52)$ & 0.69 \\
\hline Organization (3 items) & $3.73(0.89)$ & 0.74 \\
\hline Scaffolding (4 items) & $4.64(0.41)$ & 0.67 \\
\hline
\end{tabular}

Note: Number of questionnaire items $=62$ (following CFA and IRM analyses). 


\section{Correlations Parenting and Caregiver Personality}

Associations between the parenting constructs on the reduced 62-item questionnaire were as follows (see Table 3): nurturance, structure and behavioral control were positively intercorrelated as well as the constructs of overprotection and coercive control, with small to large effect sizes. Additionally, both nurturance and structure were positively related with behavioral control and negatively related with coercive control. The negative relationship with overprotection was only significant for structure, not for nurturance. Behavioral control on the other hand was positively related with overprotection and coercive control (medium effect sizes).

Associations between the five parenting constructs on the reduced 62-item questionnaire and 'Big Five' personality characteristics of the caregivers are also reported in Table 3. Positive correlations (small to large effect sizes) were found for the association between the four features of the 'Big Five' (i.e., extraversion, agreeableness, conscientiousness, and openness to experience) and the three positive parenting constructs (i.e., nurturance, structure, and behavioral control). These personality characteristics tended to be negatively correlated with coercive control and overprotection. However, conscientiousness was positively associated with overprotection and not associated with coercive control, and agreeableness was not associated with overprotection. For the personality characteristic of neuroticism, negative correlations with nurturance and structure were found, whereas positive correlations were found with behavioral control, coercive control and overprotection (small to medium effect sizes). 


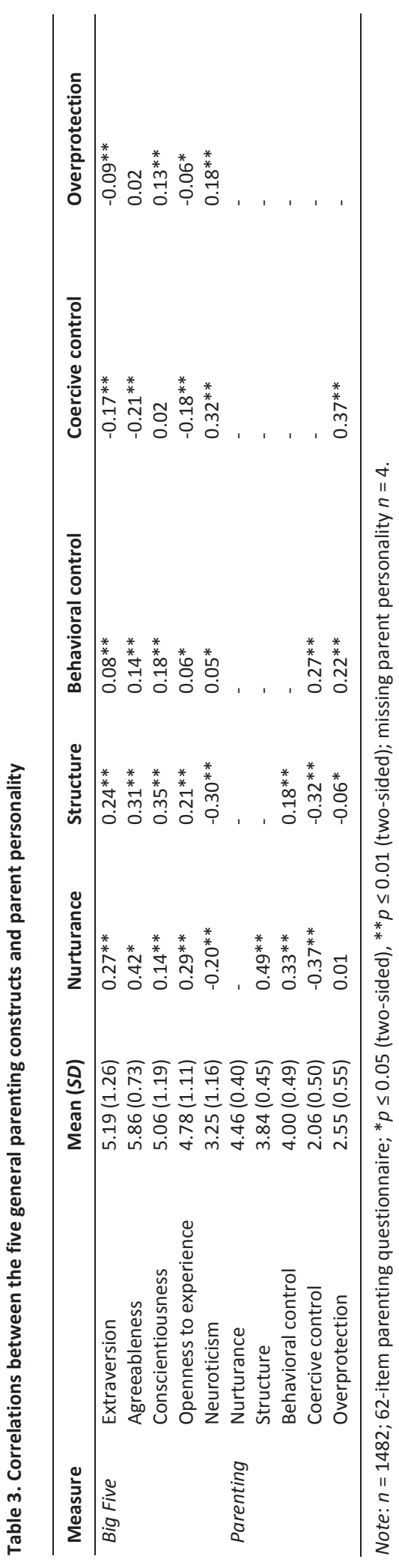




\section{Questionnaire refinements based on quantitative and qualitative analyses}

We started the development of the CGPQ with a 145-item instrument based on our parenting model, populated with existing items from previously developed questionnaires and refinement through expert reviews. Prior to data analysis, 30 items were dropped because of redundancy of item content or ambiguity. Based on the CFA and IRM analyses, 53 additional items were dropped. The questionnaire was reviewed again, resulting in the rewording of some items and 23 additional items were added. We added items to better cover the sub-constructs of (excessive) monitoring and involvement; Hardy, Power and Jaedicke's (1993) modification of the Hetherington and Clingempeel's (1983) 'Parent Assessment of Child Monitoring scale' and the 'Protectiveness scale' developed by Hardy et al. (1993) were used for this purpose. As a result of the expert review, we elected to incorporate an additional sub-construct in the construct of behavioral control, i.e., 'considering child input' (not being too strict to give a child space for personal development). This process resulted in an 85-item questionnaire representing the five parenting constructs and their corresponding sub-constructs each covered by five items.

To ensure that parents could comprehend the wording of the parenting items, the answer options and the instructions, five cognitive interviews were conducted in the Netherlands and the US, respectively. For the US cognitive interviews, caregivers were recruited through the CNRC participant database. Families with eligible 5- to 13-year-old children, who previously indicated an interest in being contacted for studies, were identified and contacted. Baylor College of Medicine's Institutional Review Board approved the study; all caregivers completed informed consent prior to data collection. A fifteen dollar gift card was provided to the caregiver for participation. For the Dutch cognitive interviews, participants also represented a convenience sample, recruited using personal network of the interviewer. The participants received a ten euro gift card for participation. For both countries, only minor changes were made in wording of items. Questionnaire completion time was about 15 minutes. Caregivers reported the instruction, items and answer options of the questionnaire were easy to understand and parents agreed that all aspects of parenting were covered. The current version of the questionnaire that resulted from the mixed-method approach as described above is incorporated in the Supplement of this chapter on page 241-244.

\section{Discussion}

\section{Validation of the CGPQ}

A parenting model, consisting of five constructs of parenting (i.e., nurturance, overprotection, coercive control, behavioral control, and structure) was used as the basis for the development of the CGPQ. CFA supported our five-factor model (moderately fitting) and together with IRM analyses helped us to reduce redundant items. The low reliability (a sample-dependent measure) of the 'overprotection' parenting construct could be due to less number of items assessing this construct and possible heterogeneity of this construct in this sample. 
Different approaches have been developed to conceptualize patterns of parenting, besides the typological approach to parenting. Whereas Maccoby and Martin (1983) described authoritative parents high on two dimensions (responsiveness and demandingness), Steinberg (1990) typified it by high levels on the dimensions of warmth and acceptance, psychological autonomy or democracy, and behavioral control. Grolnick and Pomerantz (2009) tried to adapt the multiple-forms approach to defining parental control, by proposing that 'only parenting characterized by pressure, intrusion and domination should be considered control, whereas parenting frequently labeled control but characterized mainly by guidance should be considered structure' (abstract, page 165, see also Pomerantz \& Grolnick, 2009). However, this approach does not take into account the possibility of having different combinations of parenting and its multidimensionality (Conger, 2009), and all identified facets of the control construct (Grusec, 2009). Skinner et al. (2005) identified three core dimensions in the assessment of parenting, each consisting of two opposing constructs: 'warmth and rejection', 'structure and chaos', and 'autonomy support and coercion', and supported the multidimensionality of these constructs. We suggest using latent class analyses or mixture modeling (Muthen \& Asparouhov, 2006) for future studies using the CGPQ in order to assess the contribution and interaction of all five parenting constructs, which we propose will allow for better differentiation among parenting styles. As such, different combinations of the five parenting constructs may be used to characterize different clusters of parenting. This approach is supported in work of Grusec and Davidov (2010), who imply that processes within each parenting domain are interacting with those in other domains.

\section{Parenting and Personality}

Confirming the findings of the meta-analytic review by Prinzie et al. (2009), but also the recently conducted study of De Haan, Deković and Prinzie (2012), this study showed that parent's personality, in terms of the 'Big Five', was related to general parenting. Parents scoring high on the traits of extraversion, agreeableness, conscientiousness, and openness of experience also scored higher on positive aspects of parenting (i.e., nurturance, structure, behavioral control), as expected. Such parents generally provide supportive, structured and consistent home climates in which their parenting behaviors are expressed. These personality characteristics were generally inversely related to coercive control. Relationships of personality with overprotection were less pronounced. A reason for this might be that this construct was not covered by a wide range of items and reliability was low. Neuroticism (characterized by proneness to frustration, anger, and distress) was indeed associated with low levels of nurturance and coercive forms of control, but also with chaotic home environments and overprotection.

\section{Study Limitations and Strengths}

Some limitations of the present study should be mentioned. First, it is likely that a bias occurred due to potential social desirability in reporting parenting behaviors, in particular as regards coercive forms of parenting. Second, correlations with parent personality were examined using the reduced 62-item questionnaire and not the full 85-item questionnaire as this examination was part of the iterative 
development and validation process. Additionally, other indicators of parenting could have been included to assess construct validity, such as associations with similar parenting dimensions as measured by existing questionnaires using different items; observations of parenting; or reports from other family members. Demonstration of validity would be enhanced with performing a cross-validation, however, our sample was not sufficiently large to allow this. And lastly, caution is needed when generalizing these results as the samples might deviate from the general populations. A strength of our study is that we used a systematic mixed methods approach. We thoroughly searched the literature to develop our comprehensive general parenting model and identified questionnaires measuring each of our five parenting constructs. Based on advanced statistical analyses we assessed fit of our parenting model with a large sample of parents across three different countries and reduced questionnaire length.

\section{Future Directions}

Future work on the precursors and outcomes of parenting can benefit from measures that include all domains of parenting and make use of cluster-analytic approaches. Our questionnaire attempts to give such a comprehensive overview of parenting. Next steps include validation of the 85-item CGPQ and applicability to other target groups (adolescent self-reported parenting, and parent-reported parenting of infants and toddlers). Future directions should include studies that use the CGPQ across other cultural groups (e.g., Eastern cultures) without excluding important parenting constructs, to test for differential item functioning, factorial invariance and identify underlying universal characteristics of parenting that cut across cultures - characteristics that may differ in the way they are expressed in different cultures. Additionally, the contextual influence of parenting moderating the association between more specific parenting practices and children's health outcomes (Darling \& Steinberg, 1993) could be investigated more thoroughly. Also other variables including child temperament, child age, socio-economic status and culture are assumed to interact with parenting style, and should be taken into account in future research efforts. 


\section{Supplement. 85-item Comprehensive General Parenting Questionnaire (caregivers of 5- to 13-year-olds)}

On the following pages you will see statements about parenting. We are interested in your opinion about these statements. Please read all statements carefully.

Sometimes there may be questions you think are not applicable to your family or child. Please try to answer these questions to the best of your ability. At times, there may be questions you might think: 'I would like to act this way, but in reality I am not doing this'. Please answer these questions by indicating what you are actually doing.

How much do you agree or disagree with the following statements?

(strongly disagree, somewhat disagree, neutral, somewhat agree, strongly agree)

\begin{tabular}{cc}
\hline Questionnaire items divided by (sub)scales & Measure \\
\hline Nurturance - Autonomy support &
\end{tabular}

Nurturance - Autonomy support

1 I encourage my child to be curious, to explore, and to question things

CRPR/PDI

2 I trust my child

PSCQ

3 I respect my child's opinion and encourage him to express it

CRPR/PDI

4 I encourage my child to be true to himself

PSCQ

5 I encourage my child to express his opinions even when I do not agree with him

PSCQ

Nurturance - Social rewarding

6 I praise my child when he does something good

DPQ a

7 I say something nice to my child as a reward for good behavior

CPBS a

8 When my child does his best, I praise him/her

GBPS a

9 I tell my child how much I appreciate it when he spontaneously helps me

CPBS a

10 I praise my child when he deserves it

$\mathrm{DPQ}$ a

Nurturance-Responsiveness

11 I know exactly when things are not going very well for my child

DPQ a

12 When my child is sad, I know what is going on with him

$\mathrm{DPQ}$

13 I feel good about the relationship I have with my child

PSCQ

14 My child and I have warm affectionate moments together

CRPR a

15 I know exactly when my child has difficulty with something

$\mathrm{DPQ}$

Nurturance - Involvement

16 I find time to talk with my child

POPS

17 I spend a lot of time with my child

POPS

18 I easily find a way to make time for my child

PASQ a

19 I attend as many of my child's events and activities as possible

New item

20 I find it interesting and educational to be with my child for long periods

CRPR

Structure - Inconsistent Discipline

21 I have a hard time consistently enforcing rules with my child

New item

22 I do not always follow through when I threaten to discipline my child

New item

23 I threaten discipline more often than I actually give it

CRPR a CPBS a

24 When I discipline my child, I sometimes end the punishment early

CPBS a

25 There are times I just do not have energy to make my child behave as he should

PDI 


\section{Questionnaire items divided by (sub)scales}

Measure

\section{Structure - Consistency}

26 When I tell my child I will do something, I do it

PSCQ

27 I use clear and consistent messages when I tell my child to do something

New item

28 I try not to change the rules at home very often

PSCQ a

29 I try not to forget the promises I make to my child

CRPR a

30 I explain the reasons behind our family rules

New item

\section{Structure-Organization}

31 I make sure my child has enough time to get ready for school

New item

32 I help my child to schedule time for household chores

New item

33 I help my child plan his activities for the day/week

New item

34 I teach my child to keep his bedroom clean and orderly

New item

35 I make sure my child is at school on time

New item

\section{Structure - Scaffolding}

When my child faces a difficult problem, I help him break it down into smaller steps

When I talk with my child about his/her problems, I really try to help him

I put time and energy into helping my child, when he asks for it

When my child has difficulties, I help him

When my child has a problem, I help him figure out what to do about it

\section{Behavioral Control - Monitoring}

41 I keep track of my child's activities with friends

PACMS a

I pay attention to where my child is

43 I watch my child to make sure he behaves appropriately

44 I am aware of what my child is doing when he is at home

I am aware of my child's choice of friends, who they are, what they are like

\section{Behavioral Control - Maturity demands}

I expect my child to follow our family rules

PSCQ

47 I have clear expectations for how my child should behave

PRS (E) a

48 I require my child to behave in certain ways

PRS (E)

I make sure that my child understands what I expect of him

PSCQ a

50 I teach my child to follow rules

\section{Behavioral Control - Non-intrusive discipline}

51 When I correct my child's behavior, I explain why

PSCQ

52 When my child goes against a rule I take away a privilege

New item

53 I correct my child when he breaks the rules

New item

54 I correct my child's minor misbehaviors with explanations

New item

55 I would ground my child if he committed serious offenses
New item 
Behavioral Control - Considering child input

56 I want my child to always obey me (reversed coding)

PDI a

57 I place a lot of emphasis on obedience in my child (reversed coding)

PDI a

58 If I give my child too many rules, he will grow up to be a unhappy adult

PDI a

59 I make sure I give my child lots of freedom to make mistakes and learn from them

PDI a

60 I give my child a lot of freedom to make up his own mind

PDI a

Coercive control - Psychological control

When my child does something that is not allowed, I do not talk to him until he says sorry

GPBS

I am less friendly with my child if he does not see things my way

PCS

I make sure my child is aware of how much I sacrifice for him/her

CRPR a

I make my child feel guilty when he does not meet my expectations

Olsen (2002)

page 246

When my child hurts my feelings, I stop talking to him until he pleases me again

PCS

Coercive control-Physical punishment

\section{Coercive control - Authoritarian control}

When I ask my child to do something, I expect it to be done immediately without questions

PAQ

\section{Overprotection - Excessive monitoring}

I am always aware of what my child is doing

I let my child play a lot by himself without my supervision (reversed coding)

When my child has a friend over, I frequently check to see what they are doing

I make sure I know exactly where my child is at all times

I prefer my child play at our house with his friends rather than playing at a friend's house

Overprotection - Excessive involvement 
Note: This questionnaire is copyright protected. This questionnaire is also available in the Dutch and Spanish language. Please contact Ester Sleddens if you are interested in using or adapting the questionnaire for your research. Please note that the scale of 'Considering Child Input' (Behavioral Control) is not previously tested before using CFA and IRM; $\mathrm{a}=$ adapted item.

\section{Abbreviations:}

CRPR: Child Rearing Practices Report

DPQ: Dutch Parenting Questionnaire ('Nijmeegse Opvoedingsvragenlijst')

GPBS: Ghent Parental Behavior Scale

PAQ: Parental Authority Questionnaire

PASMS: Parental Assessment of Child Monitoring Scale

PCS: Psychological Control Scale

PDI: Parenting Dimensions Inventory

PPOS: Perceptions of Parents Scales

PRS (EB): Parental Regulation Scale, parental expectations for behavior scale

PRS (M): Parental Regulation Scale, parental monitoring of behavior scale

PSCQ: Parents as Social Context Questionnaire

$P Q$ : Protectiveness Questionnaire 


\section{CHAPTER 12}

Food parenting practices and child dietary patterns:

Prospective relations and the moderating role of general parenting, child temperament, and child eating style

Ester FC Sleddens

Stef PJ Kremers

Annette Stafleu

Pieter C Dagnelie

Nanne K de Vries

Carel Thijs

In revision 


\begin{abstract}
Research on parenting practices has focused on individual behaviors while largely failing to consider the larger context of their use. This study aims to unravel the conditions under which food parenting practices, general parenting and child characteristics (i.e., temperament and eating style) shape children's dietary behavior. First, we examined the extent to which food parenting practices predict the development of children's dietary behavior (classified as unhealthy: snacking, sugar-sweetened beverage; and healthy: water and fruit intake). Second, we tested the moderating role of both general parenting and child characteristics on this relationship. Within the KOALA Birth Cohort Study, the Netherlands, questionnaire data were collected prospectively at 6 and 8 years $(N=1654)$. Correlations were computed to assess the association between food parenting practices and the potential moderating factors. Linear regression models were fitted to assess whether food parenting practices predict dietary behavior at age 8 , as well as dietary behavior development from age 6 to 8 . Finally, moderation analyses were performed by evaluating interactions with general parenting and child characteristics. Instrumental and emotional feeding, and pressure to eat were found to have undesirable associations with child dietary behavior (increased unhealthy intake and decreased healthy intake), whereas associations were in the desirable direction for covert control and restriction. General parenting and child characteristics moderated several of the associations between food parenting and child dietary behavior. Future research should assess the influence of factors moderating on the food parenting - child dietary behavior/weight relationship for the development of more effective family-based interventions.
\end{abstract}




\section{Introduction}

It is well-known that childhood overweight has a tendency to persist into adulthood (Singh, Mulder, Twisk, Van Mechelen, \& Chinapaw, 2008) and is associated with multiple adverse health outcomes, both in the short term (Daniels, 2009) and longer term (Reilly \& Kelly, 2011).

While it is generally accepted that parenting has important influences on children's dietary intake and ultimately weight status, confusion exists about the exact conditions of its influence. In the feeding domain, previous reviews showed the relationships of parents' use of so-called food parenting practices (i.e., content-specific acts of parenting (Darling \& Steinberg, 1993)) on child eating and weight status (e.g., Clark, Goyder, Bissell, Blank, \& Peters, 2007; Faith, Scanlon, Birch, Francis, \& Sherry, 2004; Ventura \& Birch, 2008; Wardle \& Carnell, 2007). Most of the included studies focus on highly controlling food parenting practices (restricting the type and amount of food and using food as a reward), failing to assess other practices such as encouragement and guidance. These (mainly cross-sectional) studies have yielded contradictory results, and have in common that they do not assess the larger context, e.g., by examining influence of parenting practices in the context of so-called general parenting styles.

General parenting is independent of specific socialization content; it has commonly been defined as the approach parents use to raise their child, and is a function of parent's attitudes, beliefs and behaviors, creating a family emotional climate (Darling \& Steinberg, 1993). In a recent review, Sleddens, Gerards, Thijs, De Vries, and Kremers (2011) found that authoritative forms of parenting (characterized by parental warmth and guidance) were associated with more positive weight-related outcomes than more permissive or coercive forms of parenting. This was confirmed in some recent observational studies (Fuemmeler et al., 2012; Johnson, Welk, Saint-Maurice, \& Ihmels, 2012; Rodenburg, Kremers, Oenema, \& Van de Mheen, 2011; Topham et al., 2011).

Studies assessing both general parenting and food parenting practices are scarce (e.g., Blissett \& Haycraft, 2008; Blissett, Meyer, \& Haycraft, 2011; Hennessy, Hughes, Goldberg, Hyatt, \& Economos, 2010; MusherEizenman \& Holub, 2006; Taylor, Wilson, Slater, \& Mohr, 2011; Van der Horst, Kremers, Ferreira, Singh, Oenema, \& Brug, 2007; Vereecken, Rovner, \& Maes, 2010; Hubbs-Tait, Kennedy, Page, Topham, \& Harrist, 2008), and only few test whether general parenting influences the impact of food parenting practices (Hennessy et al., 2010b; Musher-Eizenman \& Holub, 2006; Taylor et al., 2011; Van der Horst et al., 2007; Vereecken et al., 2010). Two of these studies found stronger protective effects for restrictive food parenting on child dietary intake in the context of certain types of authoritative parenting (MusherEizenman \& Holub, 2006; Van der Horst et al., 2007).

Besides the contextual role of general parenting in altering the effectiveness of food parenting practices, there are other moderators. Some studies have reported on associations between food parenting and child temperament and/or child eating styles (Blissett et al., 2011; Gregory, Paxton, \& Brozovic, 2010; Haycraft \& Blissett, 2011; Horn, Galloway, Webb, \& Gagnon, 2011; Hughes \& Shewchuk, 2012; Jansen et al., 2012; 
Vereecken et al., 2010). Other studies examined interaction effects between these variables, indicating that controlling practices induce relations between these child characteristics and health behavior (Anzman \& Birch, 2009; Gubbels et al., 2009; Gubbels et al., 2011). Restriction was related to higher weight status among children with low levels of inhibitory control (Anzman \& Birch, 2009), soft drink intake among children with high levels of overactivity (Gubbels et al., 2009), and increases in energy intake among children with a hungry eating style (Gubbels et al., 2011). Monitoring was found to have desirable effects on food intake of children with a non-deviant eating style (i.e., non-picky and non-hungry) and stimulation to eat healthy was effective for children with a deviant eating style (Gubbels et al., 2011).

The current study is a first attempt to unravel the conditions under which both global dimensions of parenting and child characteristics shape children's dietary intake applying a longitudinal study design (see Figure 1). The first objective of this study was to examine the extent to which food parenting practices predict the development of child dietary behavior. The second objective was to assess the moderating role of general parenting, child temperament, and child eating style on the relationship between food parenting practices and children's dietary behavior. 


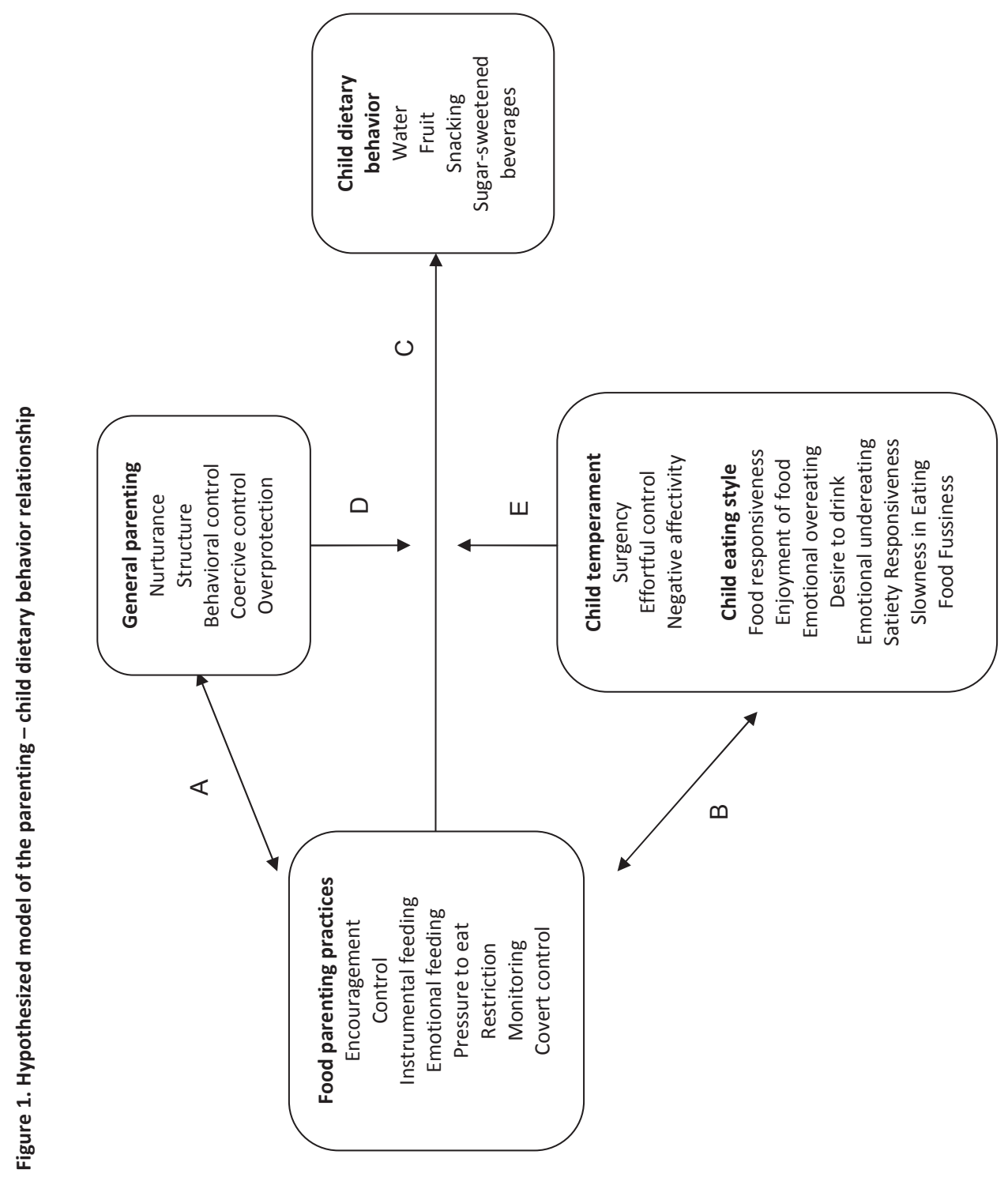




\section{Method}

\section{Respondents and procedure}

Data were collected within the KOALA Birth Cohort Study in the Netherlands. The study design has been previously described in detail (Kummeling et al., 2005). Briefly, from 2000 onwards, healthy pregnant women with a conventional lifestyle $(N=2343)$ were recruited from an existing cohort study on pregnancyrelated pelvic girdle pain. In addition, pregnant women with an alternative lifestyle $(N=491)$ with regard to dietary habits (e.g., preferring organic foods), vaccination programs, and/or antibiotics use were recruited through several 'alternative' circles like anthroposophical physician offices and midwives, and organic food shops. All participants $(N=2834)$ were enrolled between 14 and 18 weeks of gestation and completed questionnaire during pregnancy and regularly after birth. Informed consent was signed by all parents, and ethical approval was obtained from the Maastricht University/University Hospital Maastricht medical ethics committee.

\section{Measures}

When the children were around 6 to 7 years old (mean age $6.61(S D=0.65)$ years), parents completed a questionnaire regarding their food parenting practices, their child's temperament, eating style and dietary intake. A total of 1828 questionnaires (76\%) were returned. After removing the second born of twins $(n=$ 18), removing cases who did not complete any of the food parenting practices scales $(n=1)$ and cases who did not complete any of the dietary items $(n=156), 1654$ cases remained for the analyses. At age 7 to 8 (mean age $8.60(S D=0.66)$ years), another questionnaire was administered to parents of the KOALA study, assessing general parenting and children's dietary intake. A total of 1853 questionnaires (79\%) were returned. Of those, 1657 cases also had completed the previous measurement, but only 1654 cases remained for the analyses (see above).

\section{Food parenting practices}

Different validated instruments were used to measure food parenting practices at the child's age of 6 to 7 : the Child Feeding Questionnaire (CFQ; Birch, Fisher, Grimm-Thomas, Markey, Sawyer, \& Johnson, 2001), the Parental Feeding Style Questionnaire (PFSQ; Wardle, Sanderson, Guthrie, \& Rapoport, 2002), and parental covert control over eating (Ogden, Reynolds, \& Smith, 2006). Mean scores were computed for each subscale providing at least $60 \%$ of the items were completed.

The measures of restriction, pressure to eat and monitoring were based on the CFQ (Birch et al., 2001): (1) 'restriction' ( 2 items, Pearson's $r=0.31$ ), the extent to which parents restrict their child's access to food ('I have to be sure that my child does not eat too many unhealthy foods' and 'I have to be sure that my child does not drink too many sugar-sweetened beverages'), (2) 'pressure to eat' (4 items, Cronbach's alpha = 0.53; e.g., 'my child should always eat all of the food on his/her plate), and (3) 'monitoring' (4 items, 
Cronbach's alpha $=0.75$; e.g., 'how much do you keep track of the snacks/sweets that your child eats'). Three additional items, not originally in the CFQ, asked parents to report on monitoring of their child's sugar-sweetened beverage and fruit intake, and the amount of foods their child consumes during breakfast

(e.g., 'how much do you keep track of the sugar-sweetened beverage/fruit that your eat consumes', 'how much do you keep track of the foods that your child consumes during breakfast'). The response format consisted of a five-point Likert scale from 0 (Never) to 5 (Always).

The Dutch validated translation of the PFSQ (Sleddens, Kremers, De Vries, \& Thijs, 2010) was used to assess the following four subscales: 'instrumental feeding' ( 4 items, Cronbach's alpha $=0.65$ ), 'emotional feeding' (5 items, Cronbach's alpha $=0.82)$, 'control over eating' (10 items, Cronbach's alpha $=0.72$, and 'encouragement to eat' ( 8 items, Cronbach's alpha $=0.75$ ). Parents were asked to rate their feeding behavior on a five-point Likert scale from 0 (Never) to 5 (Always).

Parental covert control has been defined as forms of control related to eating that cannot be detected by the child. It was assessed using 3 items adapted from the 5 -item covert control scale developed by Ogden et al. (2006). The items, with a five-point Likert scale ranging from 1 (Totally disagree) to 5 (Totally agree), were: 'I try not to eat unhealthy food when my child is around', 'I avoid buying unhealthy foods at grocery stores', and 'I try not to buy foods that I would like because I do not want my child to have them' (Cronbach's alpha $=0.65$ ).

\section{General parenting}

The Comprehensive General Parenting Questionnaire (CGPQ) is a recently developed and validated parental-reported measure to assess the five key constructs of general parenting: nurturance, structure, behavioral control, coercive control and overprotection (Sleddens, O'Connor et al., in revision). The first three constructs can be regarded as the more 'positive aspects' of parenting, or so-called authoritative behaviors (Sleddens, O'Connor et al., in revision). Parents have to indicate on a five-point Likert scale how much they agree with statements regarding parenting. The Cronbach's alpha for each of the five higher order constructs in our sample were $0.82,0.76,0.74,0.74$ and 0.69 , respectively. Mean scores were computed for each subscale providing at least $60 \%$ of the items were completed.

\section{Child temperament}

The validated 36-item form of the Children's Behavior Questionnaire (CBQ; Putnam \& Rothbart, 2006) was used to assess three broad dimensions of temperament: surgency/extraversion (SR; tendency to perform impulsive and active behavior, Cronbach's alpha $=0.78$ ), negative affectivity (NA; predisposition to experience negative affective states, Cronbach's alpha $=0.74)$, and effortful control (EC; ability to control attentional processes and behavior, Cronbach's alpha $=0.73$ ). Parents were asked to report on a sevenpoint Likert scale ranging from 1 (Extremely untrue of your child) to 7 (Extremely true of your child) how well each statement describes their child's reaction to a given situation within the past 6 months. Moreover, a 
'not applicable' answer option was provided to be used when parents could not answer because they had not seen their child in the particular situation described. For our study, we used a Dutch translation by Majdandžić and Van den Boom (2007) which has proven to have factorial resemblance with the original study from the United States and adequate reliability in a sample of Dutch 5- to 8-year-olds (Sleddens, Kremers, Candel, De Vries, \& Thijs, 2011).

\section{Child eating style}

The Dutch validated translation (Wardle, Guthrie, Sanderson, \& Rapoport, 2001b) of the Children's Eating Behavior Questionnaire (Sleddens, Kremers, \& Thijs, 2008) was used to assess the food approach subscales of 'food responsiveness' ( 5 items, Cronbach's alpha $=0.71$ ), 'enjoyment of food' ( 4 items, Cronbach's alpha $=0.83$ ), 'emotional overeating' ( 4 items, Cronbach's alpha $=0.71$ ), and 'desire to drink' ( 3 items, Cronbach's alpha $=0.80$ ), and the food avoidant subscales of 'satiety responsiveness' ( 5 items, Cronbach's alpha $=$ 0.79), 'slowness in eating' ( 4 items, Cronbach's alpha $=0.83$ ), 'emotional undereating' (4 items, Cronbach's alpha $=0.76$ ), and 'food fussiness' ( 6 items, Cronbach's alpha $=0.91$ ). Parents were asked to rate their child's eating behavior on a five-point Likert scale from 0 (Never) to 5 (Always). Mean scores were computed for each subscale providing at least $60 \%$ of the items were completed.

\section{Child dietary behavior}

Dietary behaviors of children were assessed using several items from a validated Food Frequency Questionnaire (FFQ) designed to accurately assess energy intake of Dutch children aged 2 to 12 (Brants, Stafleu, Ter Doest, Hulshof, \& Thijs, 2006; Dutman et al., 2010). For all of the eating and drinking variables the following response categories were used: never, less than 1 day a week, 1 day a week, 2 to 3 days a week, 4 to 5 days a week, and 6 to 7 days a week.

Children's snacking frequency of several sugar-sweetened and energy-dense food products (between meals) was assessed with 4 items derived from this FFQ. Parents were asked to indicate how many days a week (normal week) their children consumed the following snacks in between meals: (1) fried snacks, (2) potato crisps, salted biscuits, and peanuts, (3) cake or large biscuits, and (4) pie, pastry, candy bars, and chocolates. A single score was calculated for the number of snacking occasions (between meals) in days a week, by adding reported frequency (in days a week) of the different snacks.

Both children's sugar-sweetened soft drink consumption and water consumption were assessed with two items. Parents were asked to indicate on how many days a week their child consumed these drinks. Additionally, parents were asked to indicate the number of glasses their child consumed of these drinks on such a day. Frequency and amount of soft drinks and water consumed were multiplied to obtain an average score of glasses soft drinks and water consumed a week. 
Fruit consumption was assessed by asking parents to indicate how many days a week (normal week) their child consumed fruit. Additionally, parents were asked to indicate the number of servings their child consumed on such a day, corresponding with earlier validation studies (Bogers, Van Assema, Kester, Westerterp, \& Dagnelie, 2004). One apple or one pear counted as one serving, two mandarins counted as one serving, and one bunch of grapes counted as one serving as well. Multiplying frequency with the reported usual amount computed an average score for the number of fruit servings consumed per week.

\section{Parental background characteristics}

For educational level, seven categories were distinguished which were recoded into three levels (low, medium, and high), in line with international classification systems (Eurostat, 2007). Country of birth was recoded into 'Netherlands' versus 'other country'. In addition, maternal age at birth of the child and recruitment group (conventional versus alternative lifestyle) was used in the current analyses.

\section{Data analyses}

Partial Rank correlations were computed for a general assessment of the correlations between food parenting practices and the potential moderating factors (i.e., general parenting, child temperament, and child eating style), adjusted for several covariates. Thereafter, separate linear regression analyses were performed to examine the relationship between food parenting practices and child dietary behavior (i.e., snacking, sugar-sweetened beverage intake, fruit intake, and water consumption). For each of the four dietrelated outcomes the analyses were performed twice using different dependent variables, one predicting dietary behavior at age 8 , the other predicting dietary behavior development from age 6 to age 8 (adjusting dietary behavior at age 8 for dietary behavior at age 6). All food parenting practices were entered simultaneously, correcting for potential confounding by the other variables. Finally, in order to examine whether several parental and child characteristics moderated the association between food parenting practices and children's dietary behavior, we calculated interaction terms between the food parenting variables and general parenting, child temperament, and child eating style. The interaction terms were added to three different regression models in the fourth step using a forward entering procedure (three different models for the interactions of food parenting with each of the potential moderators). The threshold for including interaction terms in the stepwise regression was set at $p<0.10$. For each of the three models, we added all main effects of the potential moderating variables together in the third step. The resulting interaction terms were entered in a separate regression with only one interaction term to check whether the interaction remained significant $(p<0.10)$. If so, stratified linear regression analyses were performed, in order to examine the association between food parenting practices and child dietary behavior in the different strata of the moderator variables (i.e., general parenting, child temperament, and child eating style). For each of the moderators, three groups were created based on half a standard deviation from the mean score ( $<-0.5 S D,-0.5 S D$ to $0.5 S D$, and >0.5 SD from the mean), to obtain roughly similar group sizes. All analyses were adjusted for the influence of several potential confounders: recruitment group (conventional versus alternative lifestyle), educational level (low, medium, and high; 
highest educational level attained within a family), country of birth (Netherlands versus other), maternal age at birth, and gender of the child.

\section{Results}

Of the 1654 children eligible for the current study $51.3 \%$ were boys and $48.7 \%$ were girls. The majority of the mothers $(96.8 \%)$ and fathers $(95.8 \%)$ were born in the Netherlands. Moreover, most of the families were characterized by a conventional lifestyle (81.3\%) compared to an alternative lifestyle (18.7\%). Educational level was high for $66.6 \%$ of the families, medium for $28.7 \%$, and low for $4.8 \%$. Average maternal age at the time of their child birth was $32.31(S D=3.72)$ years.

The mean number of snacking occasions per week was $1.23(S D=0.71)$ days at age 8. Children's mean intake of sugar-sweetened beverage and water was $2.20(S D=4.26)$ and $9.75(S D=8.60)$ glasses per week, respectively. The mean number of servings of fruit per week was $7.75(S D=4.32)$. 


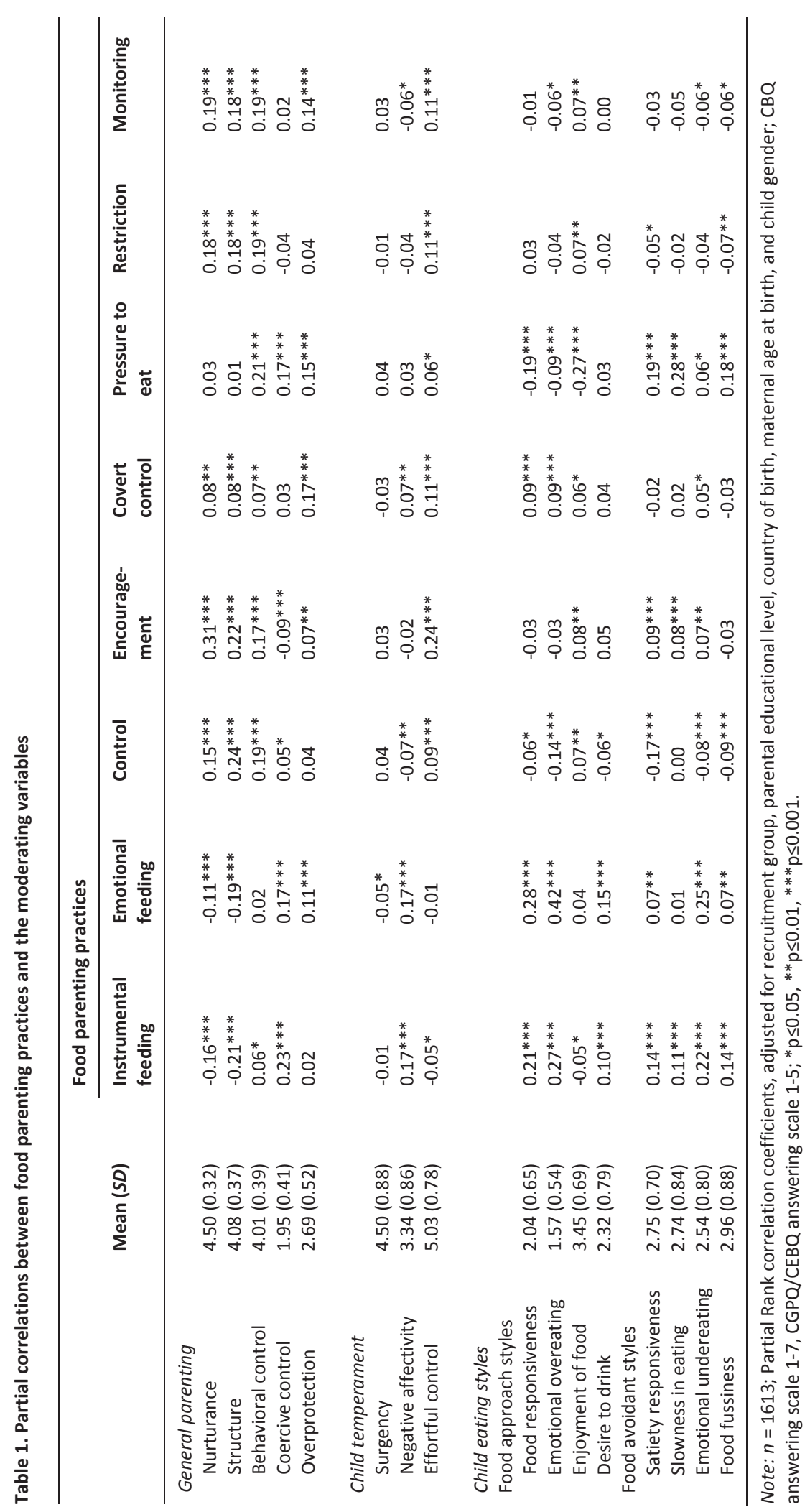




\section{Associations between food parenting and the potential moderators ( $A$ and $B$ in Figure 1 )}

General parenting practices correlated with most of the food parenting practices, although modestly at most ( $r=0.31$ or lower, Table 1). Nurturing and structuring parents applied less instrumental and emotional food parenting practices and more encouragement and controlling practices. Encouraging and controlling practices were also more often used by parents scoring high on behavioral control. Coercive control was positively related to instrumental and emotional feeding, and overprotection was related to emotional feeding. Overprotective parents more often indicated to keep foods out of reach of their children (covert control). Pressure to eat was positively related to the controlling general parenting constructs (i.e., behavioral control, coercive control, and overprotection).

Among the child temperament characteristics, surgency did not correlate with any of the food parenting practices ( $r$ not exceeding 0.05 , Table 1). Parents of children scoring high on negative affectivity more often applied instrumental feeding (rewarding their child with foods) as well as emotional feeding (giving foods when their child is distressed). The child's effortful control was positively correlated with parental encouragement, covert control, restriction, and monitoring.

Among the child's eating styles, the strongest correlation was shown between emotional eating and emotional feeding, which is not suprising given that both are parent reported ( $r=0.42$, Table 1). Pressure to eat was negatively correlated with the food approach eating styles of the $C E B Q$, and positively with the food avoidant eating styles. Most of the eating styles were related to parental instrumental and emotional feeding ( $r>0.20$ for food responsiveness, emotional overeating, and emotional undereating).

\section{Associations between food parenting and child dietary behavior (C in Figure 1)}

The strength of associations was generally low as indicated by the size of the standardized regression coefficients between -0.16 and 0.12 (see Table 2). The associations between food parenting practices and children's dietary behaviors at age 8 were mostly attenuated by controlling for baseline dietary behavior at age 6 (compare models 1 and 2, Table 2). Instrumental feeding at age 6 was related to less fruit intake at age 8. This association was also present with fruit intake development from age 6 to 8 as an outcome. Emotional feeding was positively related to child snacking, both cross-sectionally and longitudinally. Encouragement was related to increases in healthy eating (i.e., fruit intake and water consumption). Pressure to eat was positively associated with unhealthy behaviors (i.e., snacking and consumption of sugar-sweetened beverages) and negatively with water consumption. The results for the controlling food parenting practices (e.g., PFSQ control, CFQ restriction, and covert control) were mixed. Control as measured by the PFSQ was not associated with any of the child dietary outcomes. Parental restriction had a desirable effect on the consumption of sugar-sweetened drinks and water (leading to a lower and higher intake, respectively). Parents that kept unhealthy foods out of reach from their children (covert control) were more likely to have children eating healthily (i.e., less snacking and sugar-sweetened beverage intake, and more fruit and water intake). 


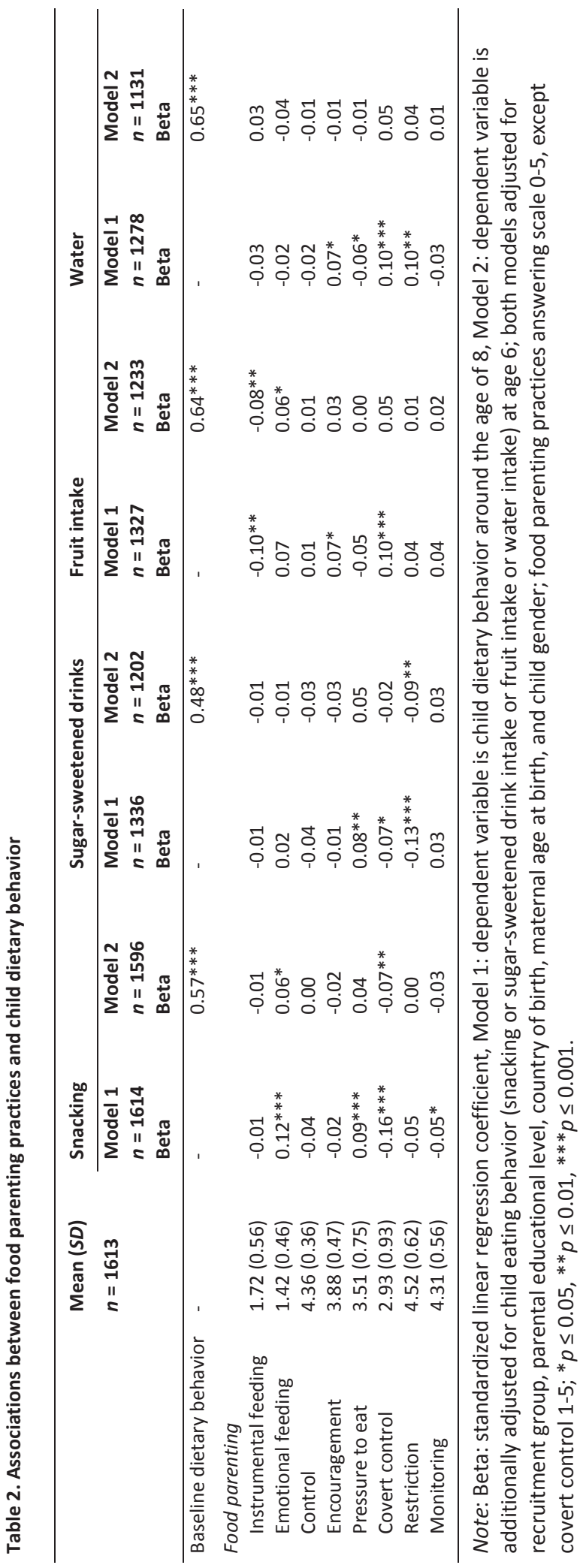




\section{Factors moderating the food parenting-child dietary behavior relationship ( $D$ and E in Figure 1)}

In Supplement 1 on page $273-277$ we provide all significant interaction terms between the moderating variables and food parenting practices in explaining child dietary behavior at age 8 and development from age 6 to 8, and the results of the stratified analyses. In Figure 2, we graphically display these stratified analysis only for associations between the food parenting practice and the outcome that were statistically significant in one or more of the strata of the moderator variable. The red bars in the figures represent associations between food parenting practices and undesirable child dietary behavior (i.e., increased unhealthy intake/decreased healthy intake), while the green bars represent associations between food parenting practices and desirable child dietary behavior (i.e., increased healthy intake/decreased unhealthy intake). In Table 3 an overview of these significant associations is also given, categorized by type of food parenting practice and moderating variable. Below, the results are organized below by each of the hypothesized moderators (i.e., general parenting, child temperament, and child eating style).

\section{General parenting}

In total, 15 interactions were found between food parenting practices and general parenting in predicting child dietary behavior. Generally, we found that the parenting practices of encouragement (Figure 2.4), covert control (Figure 2.5), and restriction (Figure 2.7) had more desirable effects when parents scored higher on positive (i.e., behavioral control, nurturance) and lower on negative (i.e., coercive control, overprotection) general parenting constructs, respectively. Instrumental and emotional feeding (Figure 2.1/2.2) had stronger relations with undesirable child dietary behavior in unfavorable home environments (i.e., less behavioral control and nurturance, higher levels of overprotection). When parents scored intermediate on behavioral control, instrumental feeding was related to lower sugar-sweetened beverage intake, whereas when parents scored high on behavioral control, instrumental feeding was related to higher sugar-sweetened beverage intake (Figure 2.1b). Other exceptions were as follows. The relation between instrumental feeding and water consumption was stronger (and negative) among children of parents that scored high on nurturance (Figure 2.1d), and the relation between emotional feeding and fruit intake was stronger (and positive) among children of parents that scored high on behavioral control (Figure 2.2c). Additionally, pressure to eat indicated to have an undesirable effect on child dietary behavior among parents with positive parenting characteristics; it was significantly related to child snacking among parents scoring high on structure (Figure 2.6a), and it was significantly related to child intake of sugar-sweetened beverage among parents scoring intermediate and high on behavioral control (Figure 2.6b).

\section{Child temperament}

In total, 7 interactions were found between food parenting practices and child temperament in predicting child dietary behavior. No interactions with child temperament were found for (covert) control, pressure to eat, and instrumental feeding. Generally, the relations between food parenting practices and child dietary behavior were stronger for children that score low on negative affect and/or high on effortful control. For this group of children, emotional feeding was related to higher intake of snacks (Figure 2.2a), 
encouragement was related to lower intake of snacks (Figure 2.4a) and higher intake of water (Figure 2.4d), and monitoring was related to lower intake of snacks (Figure 2.8a). Monitoring was significantly related to less snacking consumption among children scoring high on surgency (Figure 2.8a), and restriction was negatively related to sugar-sweetened beverage intake among children scoring low on effortful control (Figure 2.7b).

\section{Child eating style}

In total, 22 interactions were found between food parenting practices and child eating style in predicting child dietary behavior. Generally, the parenting practices of restriction, (covert) control, and monitoring had more desirable effects when children displayed healthy weigh-promoting eating styles (scoring low on the food approach and/or high on the food avoidance scales). Instrumental feeding had a detrimental impact on dietary behavior when children displayed overweight-inducing eating styles (scoring high on the food approach and/or low on the food avoidance scales). In addition, some food parenting practices had more desirable effects when children scored high on overweight-inducing eating styles; covert control showed stronger positive associations with fruit intake (Figure 2.5c), and encouragement showed stronger negative associations with snacking (Figure 2.4a). There were some exceptions. For restriction, children with low scores on satiety responsiveness and intermediate scores on desire to drink showed stronger positive associations with water intake (Figure $2.7 \mathrm{~d}$ ), and children with intermediate and high scores on desire to drink showed stronger negative associations with sugar-sweetened beverage intake (Figure 2.7b). Finally, the relationship between pressure to eat and child sugar-sweetened drink intake was highest for children scoring high on both overweight-inducing eating styles (i.e., desire to drink) and healthy weightpromoting eating styles (i.e., satiety responsiveness and food fussiness) (Figure 2.6b). 


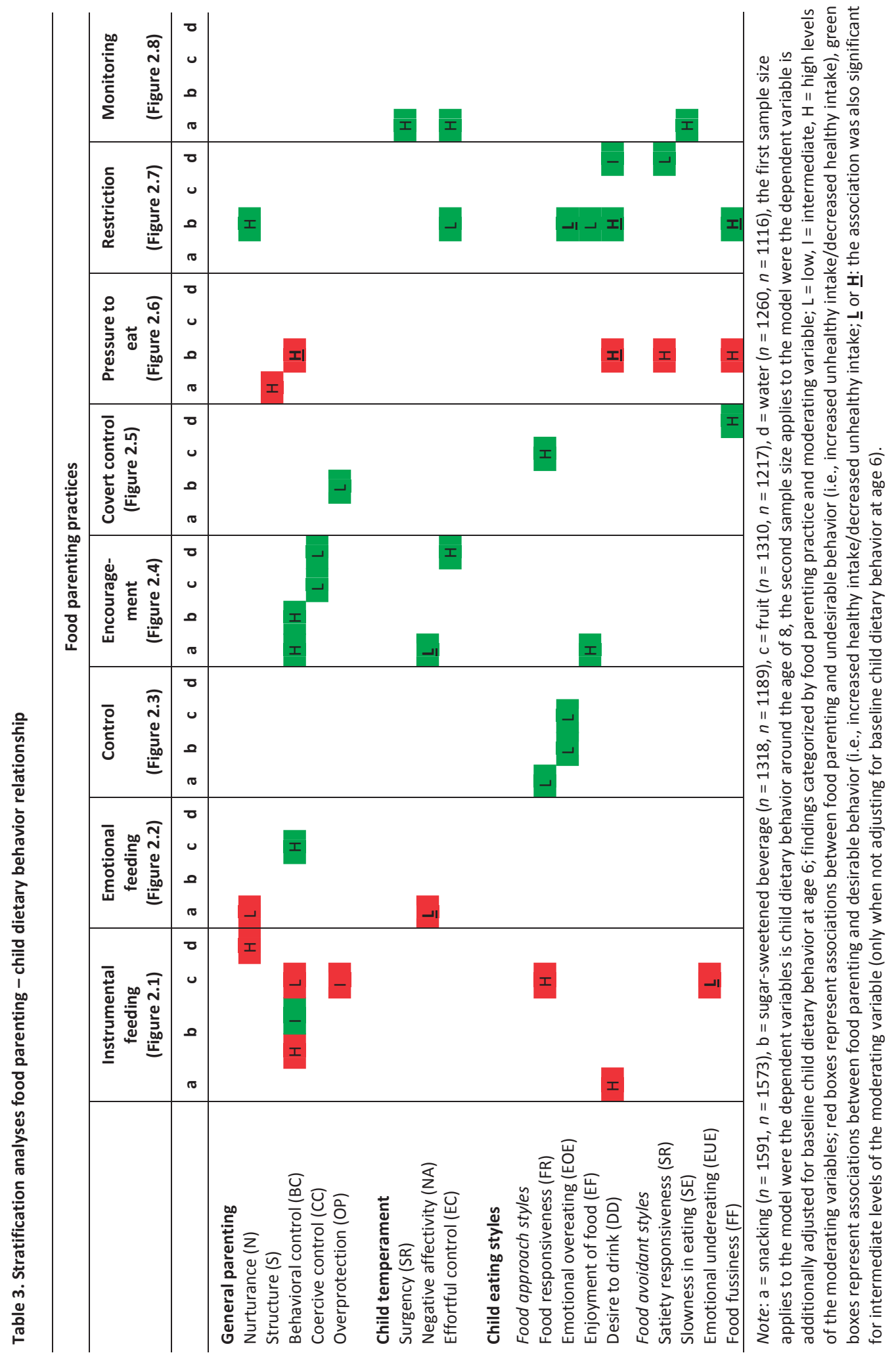



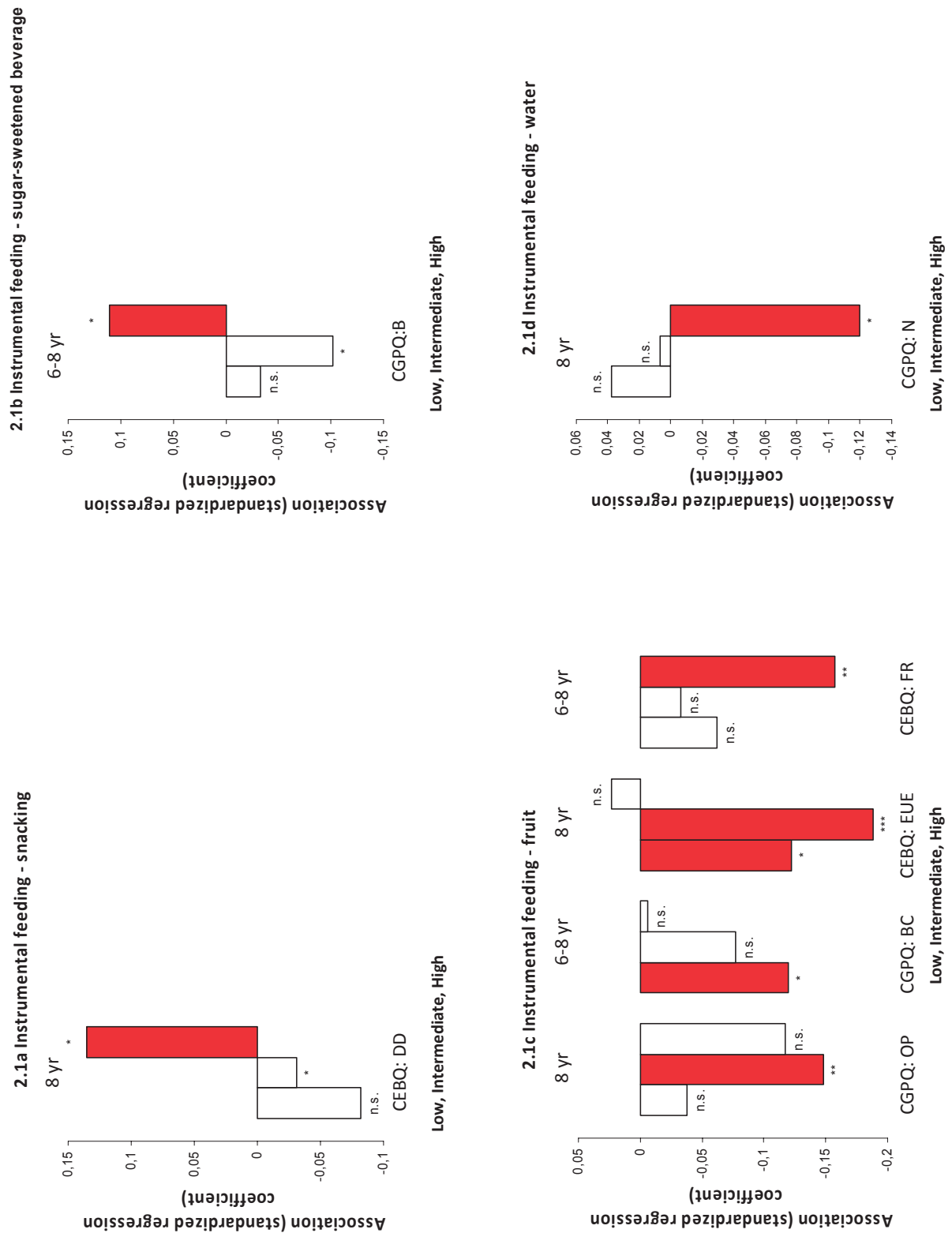

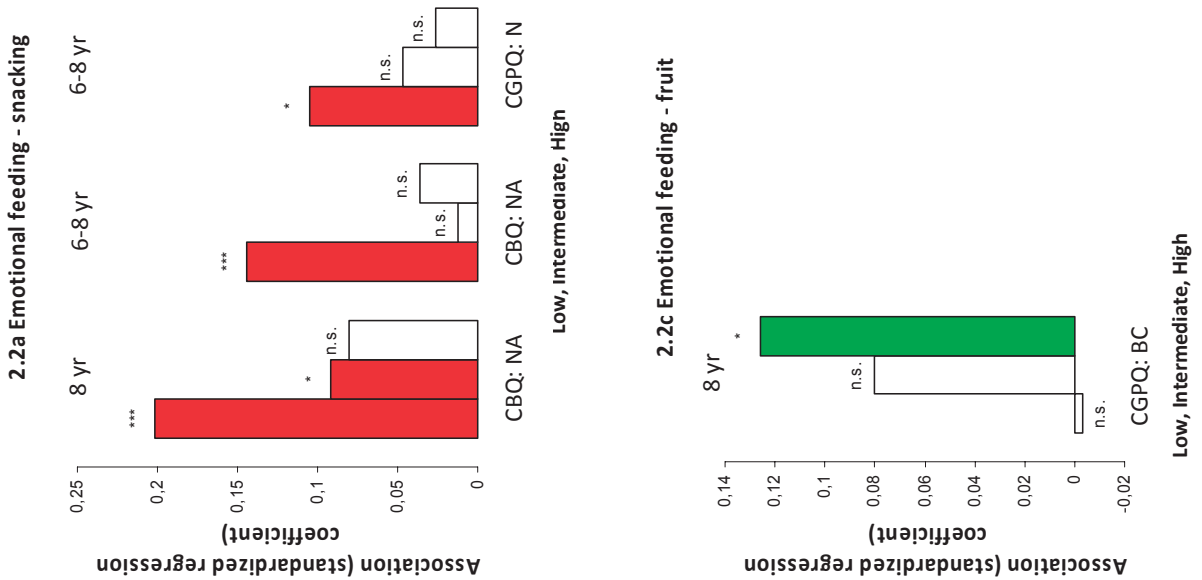

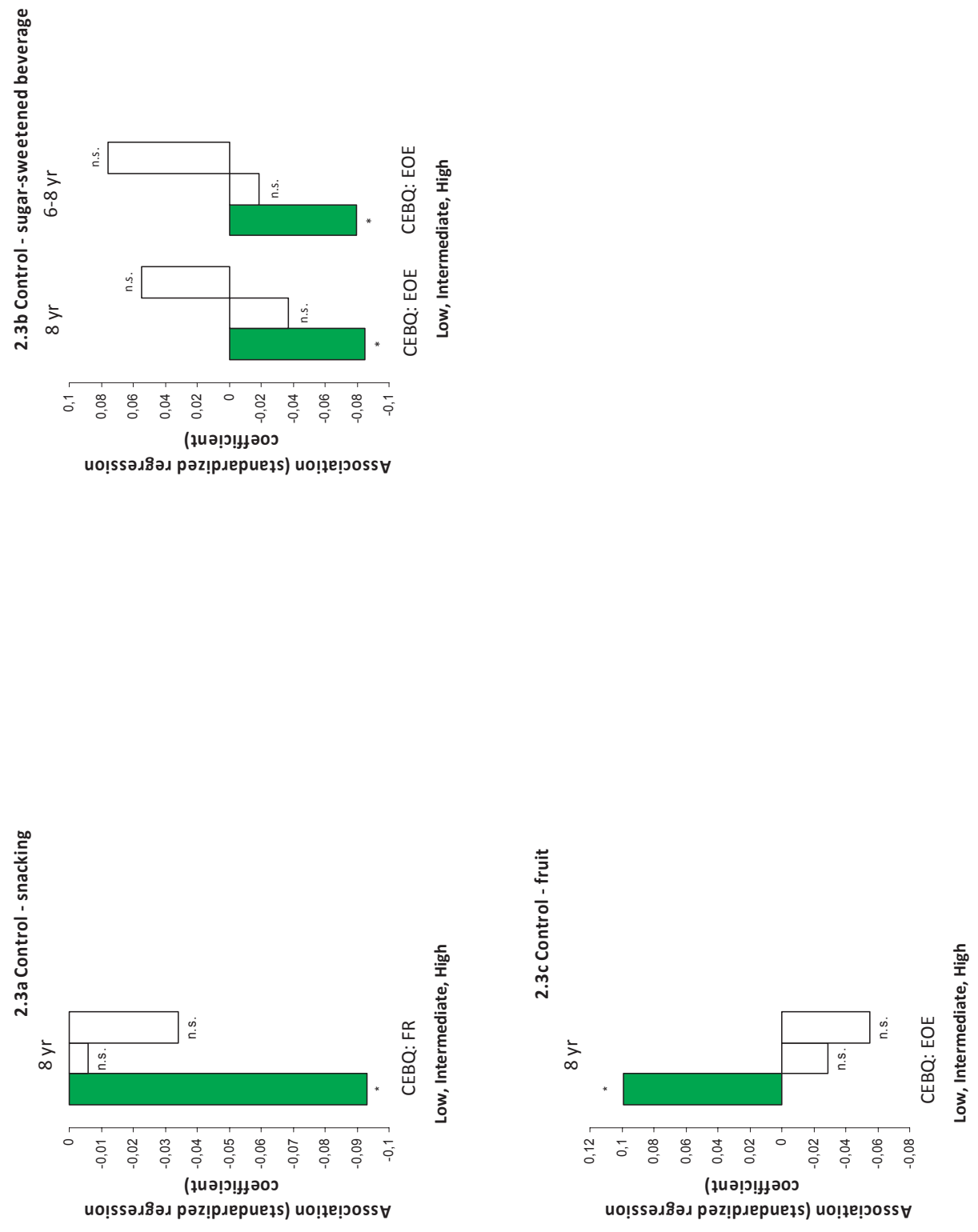


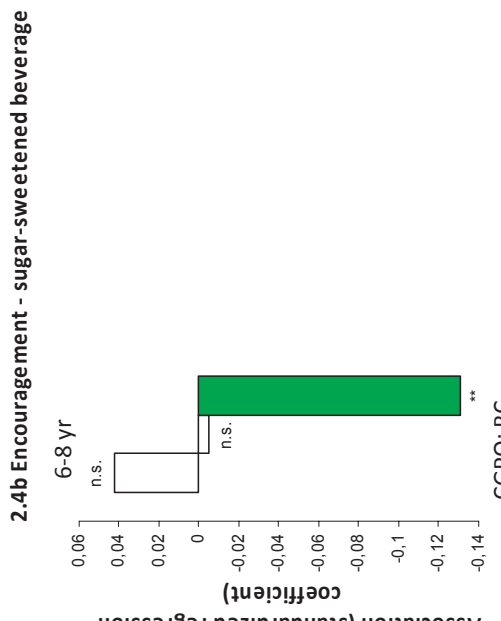

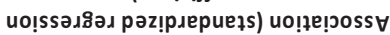

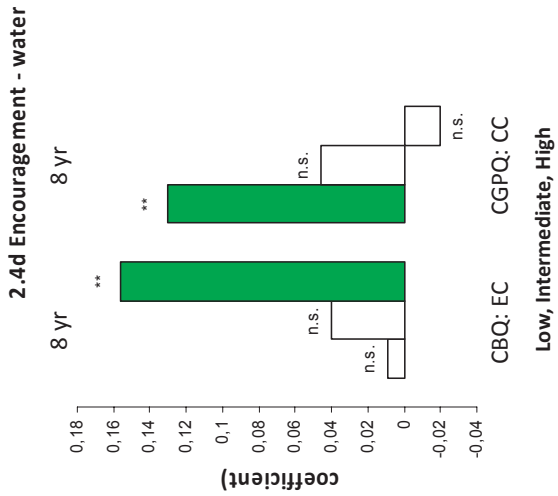

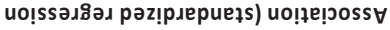

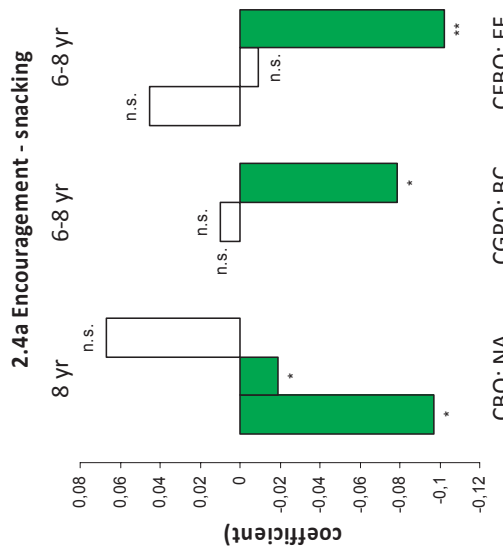

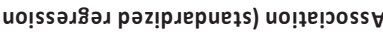

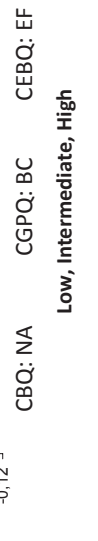

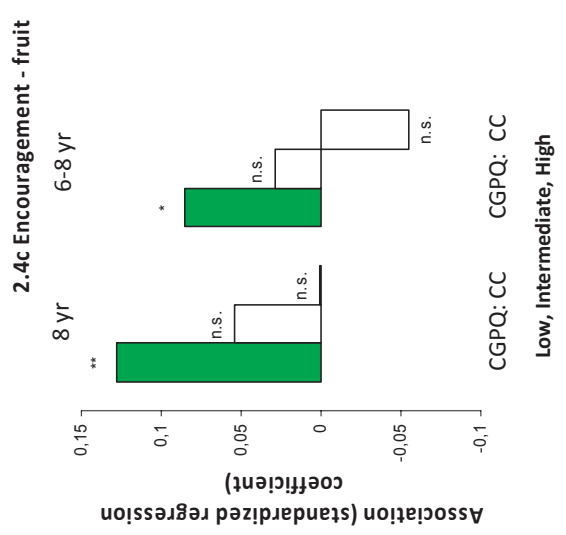



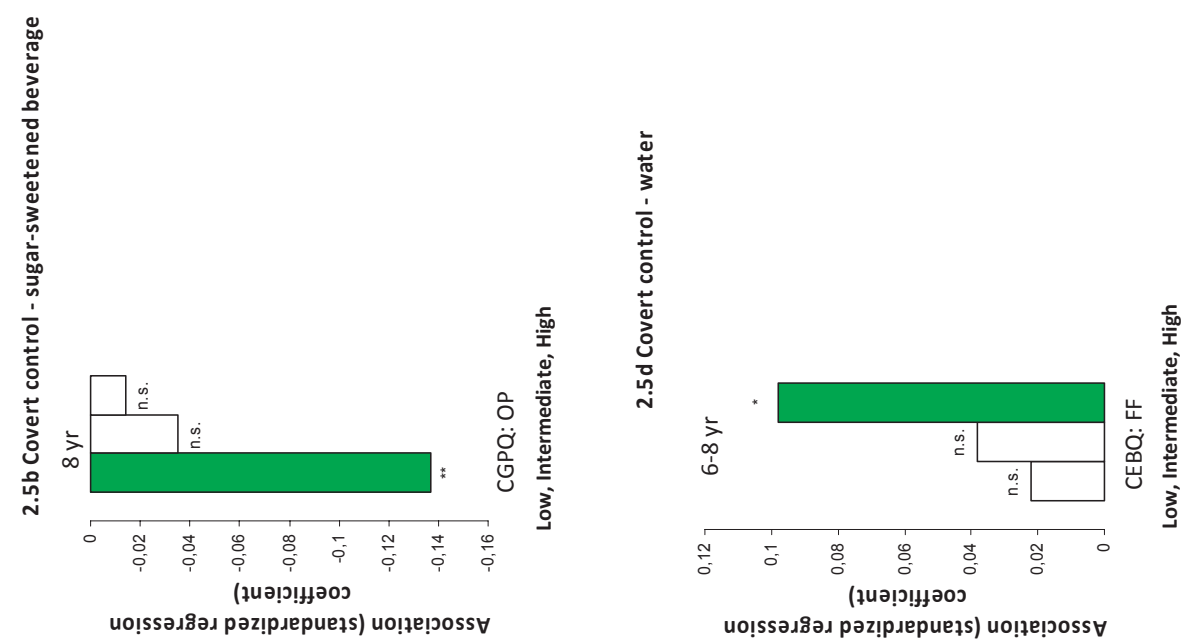

일

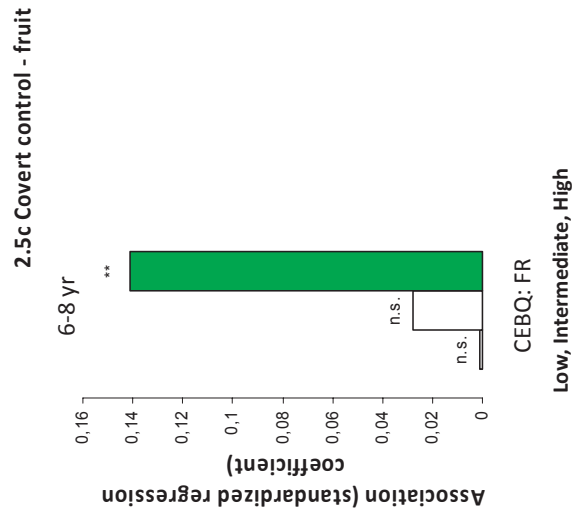



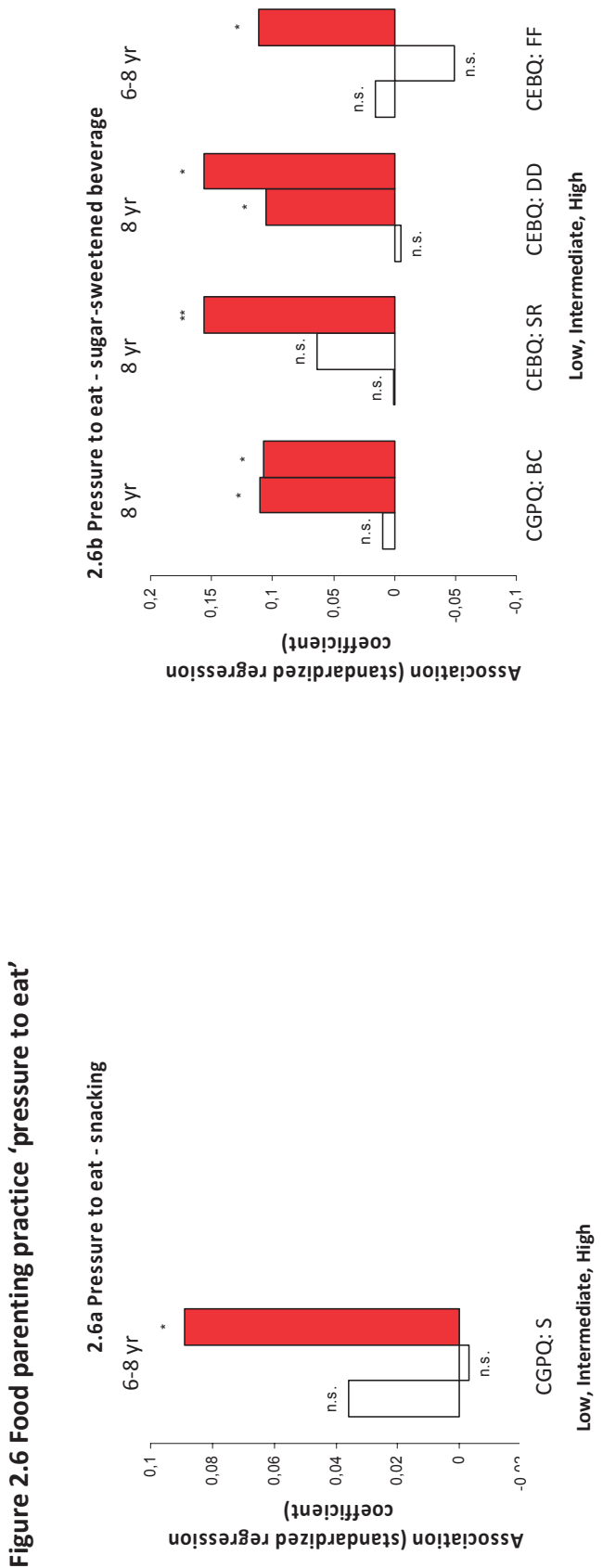

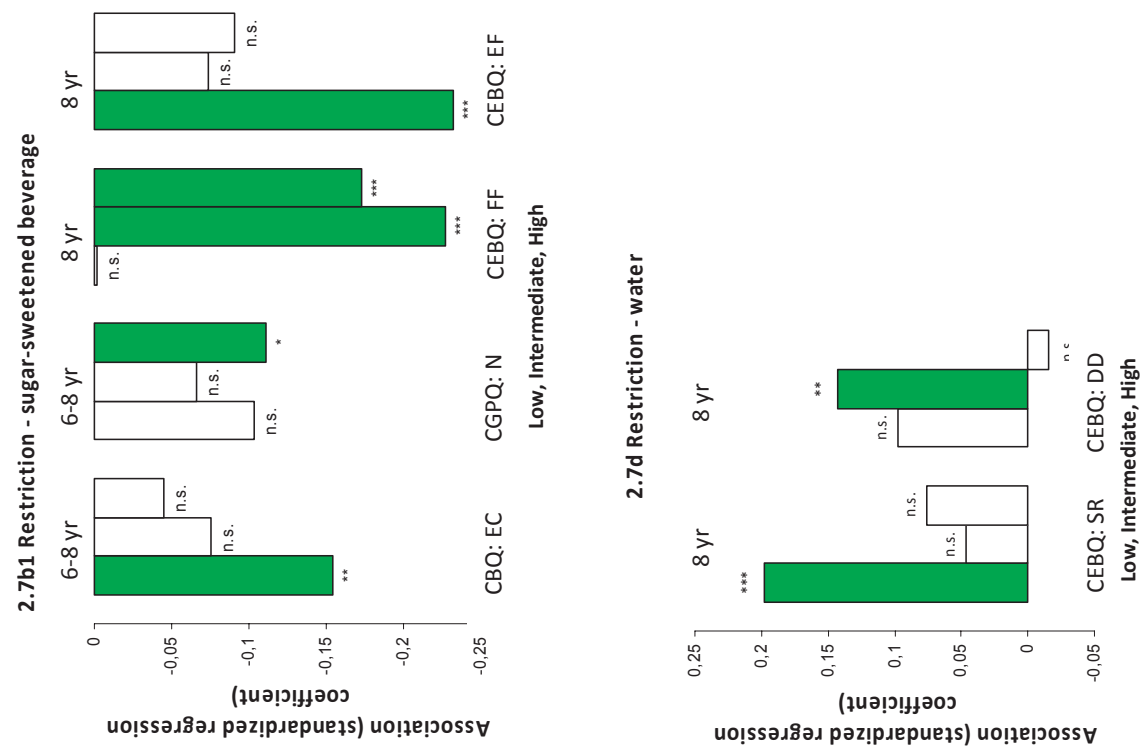

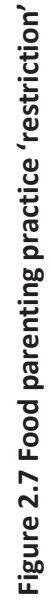

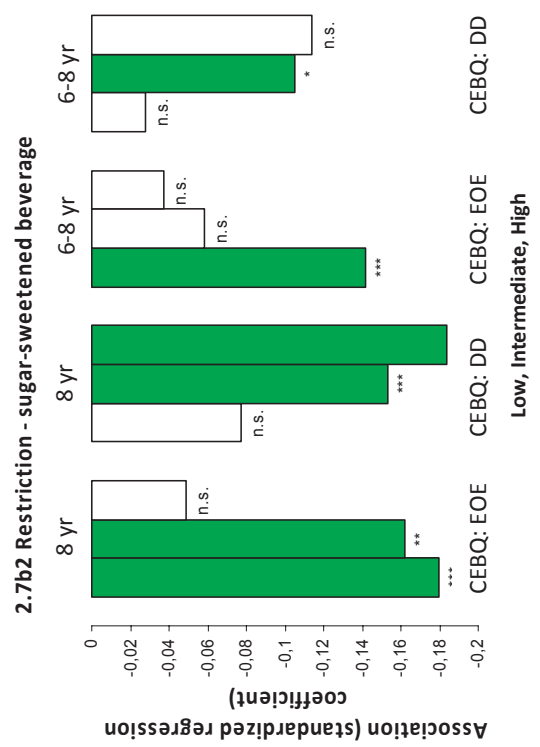




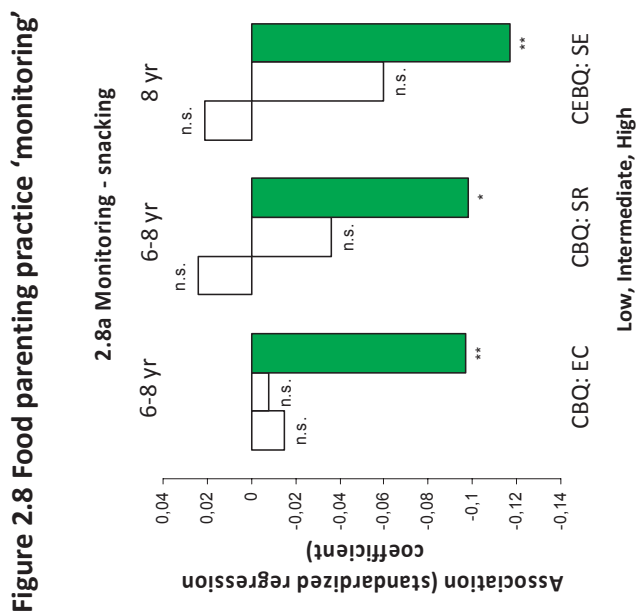




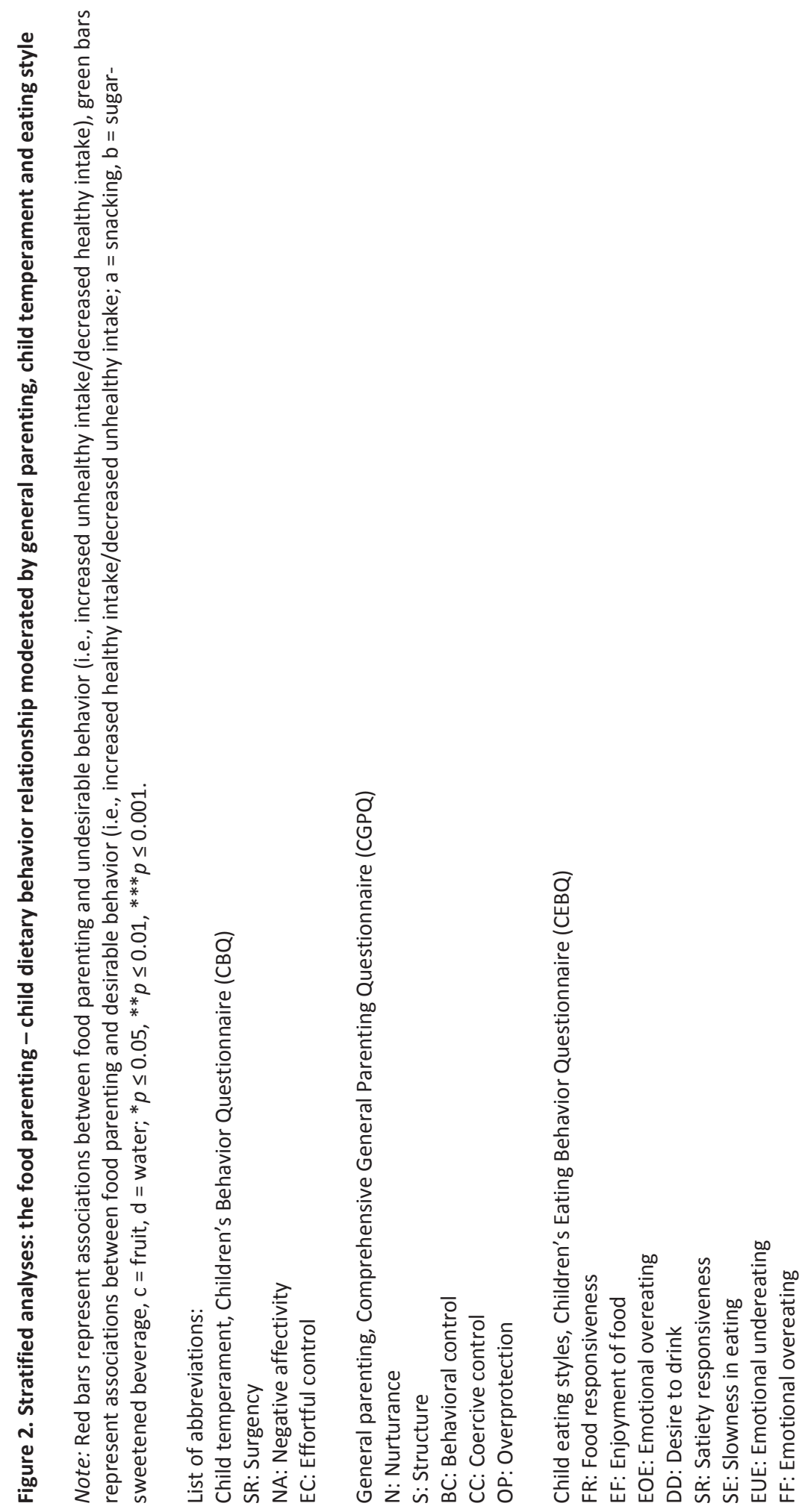




\section{Discussion}

Many studies on food parenting fell short to consider the larger family context. The present study provides evidence for effective and ineffective food parenting practices, as well as for child- and parent-related contexts that induce their impact. The main effects could be summarized as follows: instrumental and emotional feeding practices, and pressure to eat were found to have detrimental associations with child dietary behavior, whereas covert control, encouragement and restriction were found to have favorable associations with child dietary behavior. These associations were stronger for some sub-groups of the moderating variables, which will be discussed later in this section.

The more 'positive' constructs of general parenting (i.e., nurturance and structure) (Sleddens, O'Connor et al., in revision) were associated with lower use of parental instrumental and emotional practices. Reversed associations were found for the more firmly controlling parents. Thus, those parents tend to give their child more foods in response to good behavior and to reduce stress in their child. The other scales, except pressure to eat, were positively related to the authoritative aspects of parenting. Vereecken et al. (2010) also found that the more positive child-centered food practices, including reasoning and praising, were related to more positive parent-child interactions. Most studies relating parenting style to food parenting have been performed with the CFQ (e.g., Blissett \& Haycraft, 2008; Blissett et al., 2011; Hennessy et al., 2010b; Hubbs-Tait et al., 2008). Our study found that pressure to eat, also assessed by the CFQ, was positively associated with controlling parenting styles, which is partially supported by previous studies (Hennessy et al., 2010b; Hubbs-Tait et al., 2008).

In our study, there was a positive association between child negative affectivity and parental use of both instrumental and emotional feeding. Few studies have been performed regarding this topic, but a recently published study demonstrates the existence of a relationship between difficult temperament and parents' use of food to soothe their infant (McMeekin, Jansen, Mallan, Nicholson, Magarey, \& Daniles, 2013). These food parenting practices have been found to impede self-regulation of eating by impairing satiety responses thereby leading to eating for reasons other than hunger (DiSantis, Hodges, Johnson, \& Fisher, 2011). Other positive relations between child temperament and food parenting were found for effortful control and encouraging and controlling practices, similarly to the findings in the study of Hughes et al. (2012).

The relationships between food parenting practices and child eating style were plausible, especially the associations between instrumental and emotional feeding and children's overweight-inducing eating styles, as well as the positive associations of these practices with healthy weight-promoting eating styles. Note that parenting is the result of bidirectional relationships, as child eating styles could either follow or elicit food parenting practices. The relationships between pressure to eat and the food approach and avoidant subscales of the CEBQ were also (partially) found in previous studies (Haycraft \& Blissett, 2011; Jansen et al., 2012; Gregory et al., 2010). The associations of restriction and monitoring with snacking and sugarsweetened beverage intake were less pronounced compared to other studies (Haycraft \& Blissett, 2011; 
Jansen et al., 2012; Gregory et al., 2010; Gubbels et al., 2011] which found stronger associations with deviant eating styles.

We confirmed some of the results of previous studies in which non-directive child-centered food practices were related to consuming healthier diets (Murashima, Hoerr, Hughes, \& Kaplowitz, 2012; Vereecken et al., 2010), and parental reward of food was related to unhealthy food (Kröller \& Warschburger, 2009; Sleddens et al., 2010) and soft drink intake (Kröller \& Warschburger, 2009). We also found some contradictory findings (Faith, Scanlon et al., 2004; Ventura \& Birch, 2008). Parental restriction was negatively related to sugar-sweetened beverage intake and positively related to water intake, in contrast to previous studies (e.g., Jansen E, Mulkens, \& Jansen A, 2007) that showed that restriction can lead to increases in calorie intake and liking for the restricted food. Coercing children to eat was associated with unhealthy dietary behavior. Higher levels of pressure in child feeding could have detrimental effects on children's development of healthy dietary behavior, as children are focused away from internal cues to hunger and satiety (Francis et al., 2001), leading to a decrease in preference and intake of the healthy foods and subsequent increases in consumption of unhealthy foods.

Moderating effects (i.e., general parenting, child temperament, and child eating style) on the food parenting - child dietary behavior relationship were found. For children who were reared in a positive parenting context, restriction, encouragement and covert control were found to work better. For children who grew up in a less positive parenting context, instrumental and emotional feeding worked more detrimental. For children low on negative affectivity and/or high on effortful control, relations between food parenting practices and child dietary behavior were stronger; encouragement and monitoring had a desirable association with child dietary behavior and emotional feeding an undesirable association. For children with a healthy weight-promoting eating style, restriction, monitoring and (covert) control had more desirable effects. For children with an overweight-inducing eating style, instrumental feeding had more undesirable effects. Some conflicting findings were found. The one concept standing out most was pressure to eat, which was related to unhealthy dietary behavior for parents scoring high on authoritative aspects of parenting and for children scoring high on fussy eating. Pressure to eat often occurs when parents feel that their child is eating insufficient amounts of food or in response to their child's underweight (Francis et al., 2001). Secondary analyses confirmed this assumption, as we found that parents of children who are underweight scored significantly higher on pressure to eat compared to parents of children who are overweight or obese (data not reported). Low weight status has been proven to be related with more food avoidant behaviors (Jansen et al., 2012; Sleddens et al., 2008). As a result, parents could apply coercing food parenting practices trying to increase their child's weight, and probably also increasing their child's unhealthy dietary behaviors (i.e., snacking and sugar-sweetend beverage intake).

This study benefitted from a longitudinal design, with measures on child dietary behavior repeated at age 6 and 8. The included variables (food parenting practices, general parenting, child temperament, and child eating) were measured with validated instruments in the Dutch context (Bogers et al., 2004; Brants et al., 2006; Dutman et al., 2010; Gubbels et al., 2011; Sleddens et al., 2008/2010; Sleddens, Kremers et al., 2011; 
Sleddens, O'Connor et al., in revision). Moderation analysis was possible thanks to a large sample size and sufficient diversity within the study. We confirmed the operation of higher order moderation processes, implying that parenting and child factors at higher, more distal, levels alter the impact of food parenting practices at more proximal levels. This study is unique as we defined these processes prior to analyses. We recommend future studies to include the theory-based examination of possible moderation effects, and ensure sufficient study size to do so; or examine specific hypotheses in smaller scale studies with careful selection of the contextual situation.

There were also some limitations that need special attention. Highly educated parents and parents with an 'alternative' lifestyle were overrepresented in our sample, partially due to the choice of recruitment methods (Gubbels et al., 2009). We therefore adjusted all analyses for highest education level attained and recruitment channel. We did not correct for multiple testing due to the explorative nature of the study. In spite of the longitudinal analysis, causality is difficult to establish, since part of the associations (and interactions) may be modified by parental adaptations to unwanted behavior. Snacking and intake of sugarsweetened drinks were studied as unhealthy behaviors, and fruit and water intake as healthy behaviors. Of course, other behaviors such as breakfast and vegetable consumption are important as well to determine children's dietary behavior. Finally, any choice of single food groups as healthy or unhealthy is deemed to be debatable due to complexities such as substitution (e.g., between fruit, natural fruit drinks, sweetened fruit drinks and soft drinks, tea, water and milk drinks), and the ambiguities of relations with specific health indicators such as nutritional imbalances, dental health, and overweight development. Further studies are needed with specific health outcomes to evaluate whether moderation by contextual factors as shown in our study with health behaviors as outcome also translate to health outcomes such as overweight development.

In conclusion, our results show that food parenting practices are important determinants in explaining child dietary behavior, and that general parenting behaviors as well as child characteristics moderate this association. Future research efforts should continue to focus on testing the influence of factors impacting on the food parenting - child dietary behavior and/or weight relationship in order to gain insights into relevant contextual factors that need to be taken into account in designing interventions. 


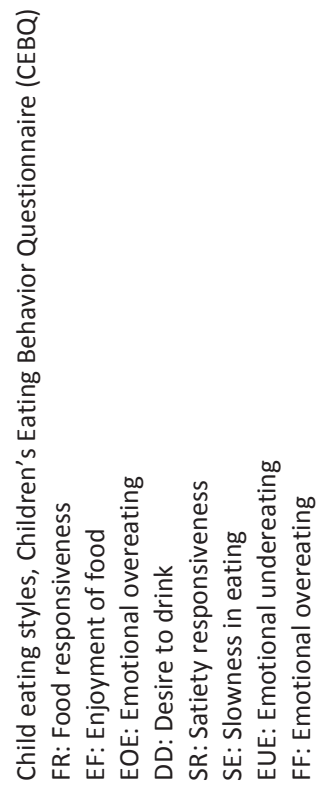




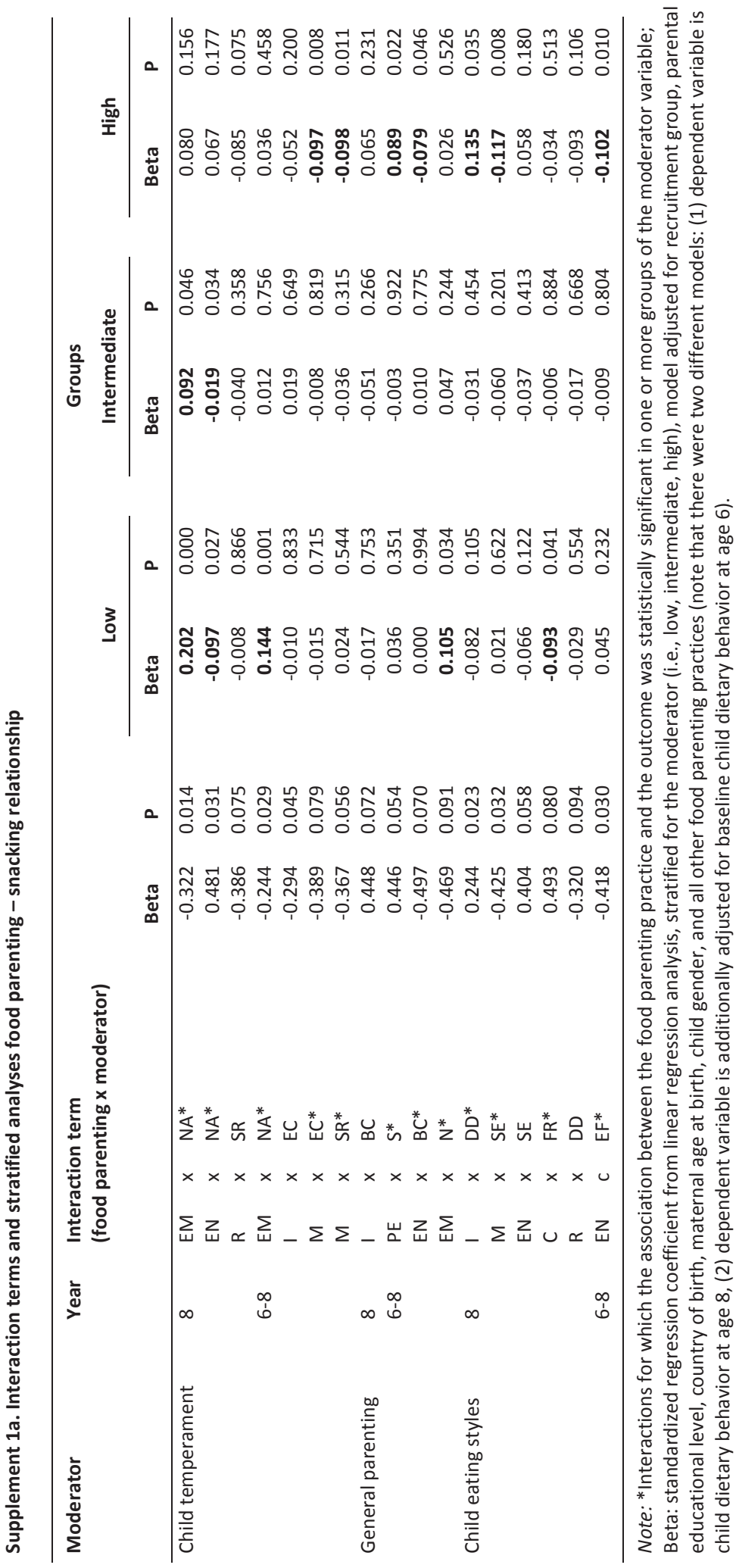




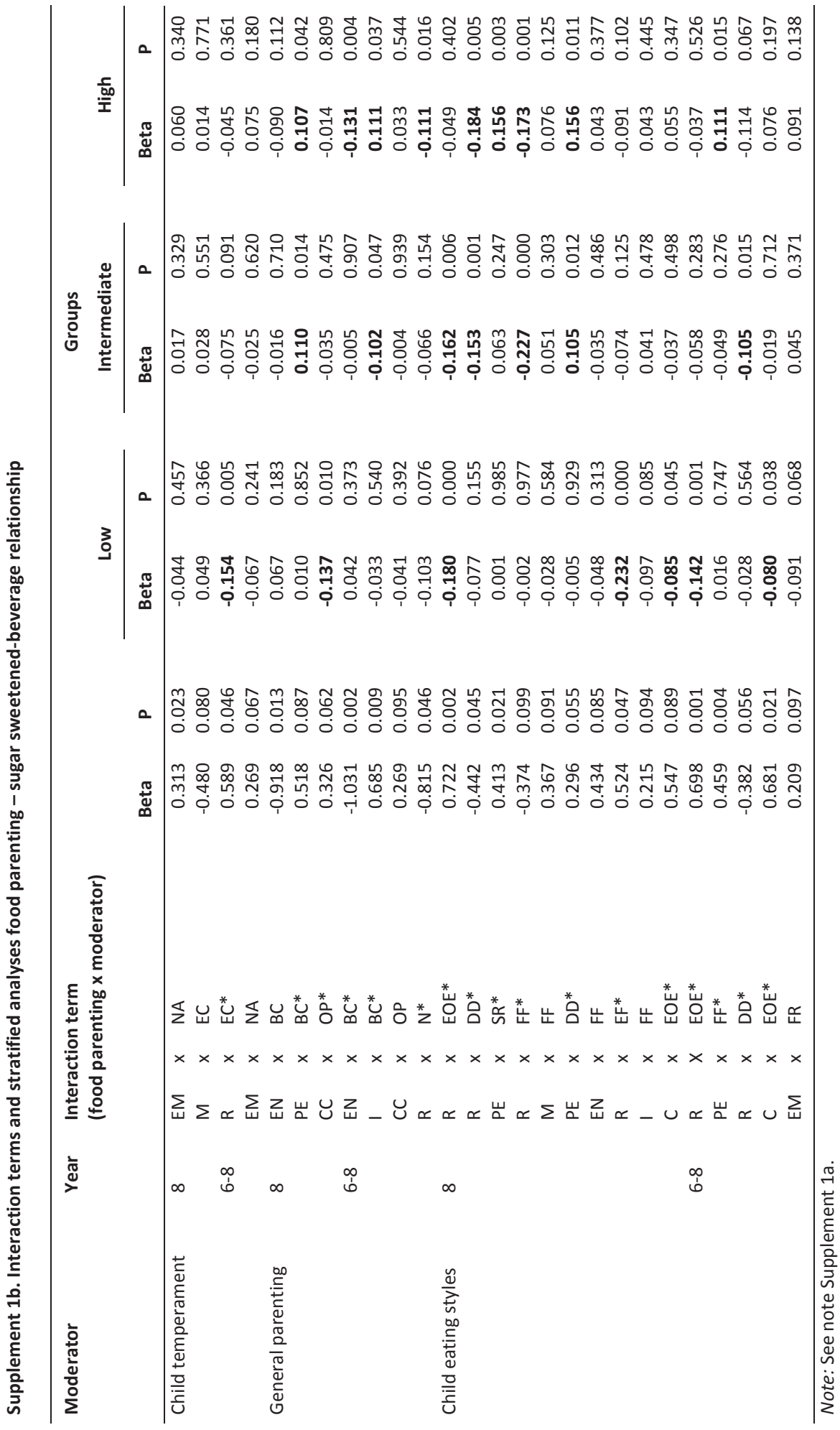




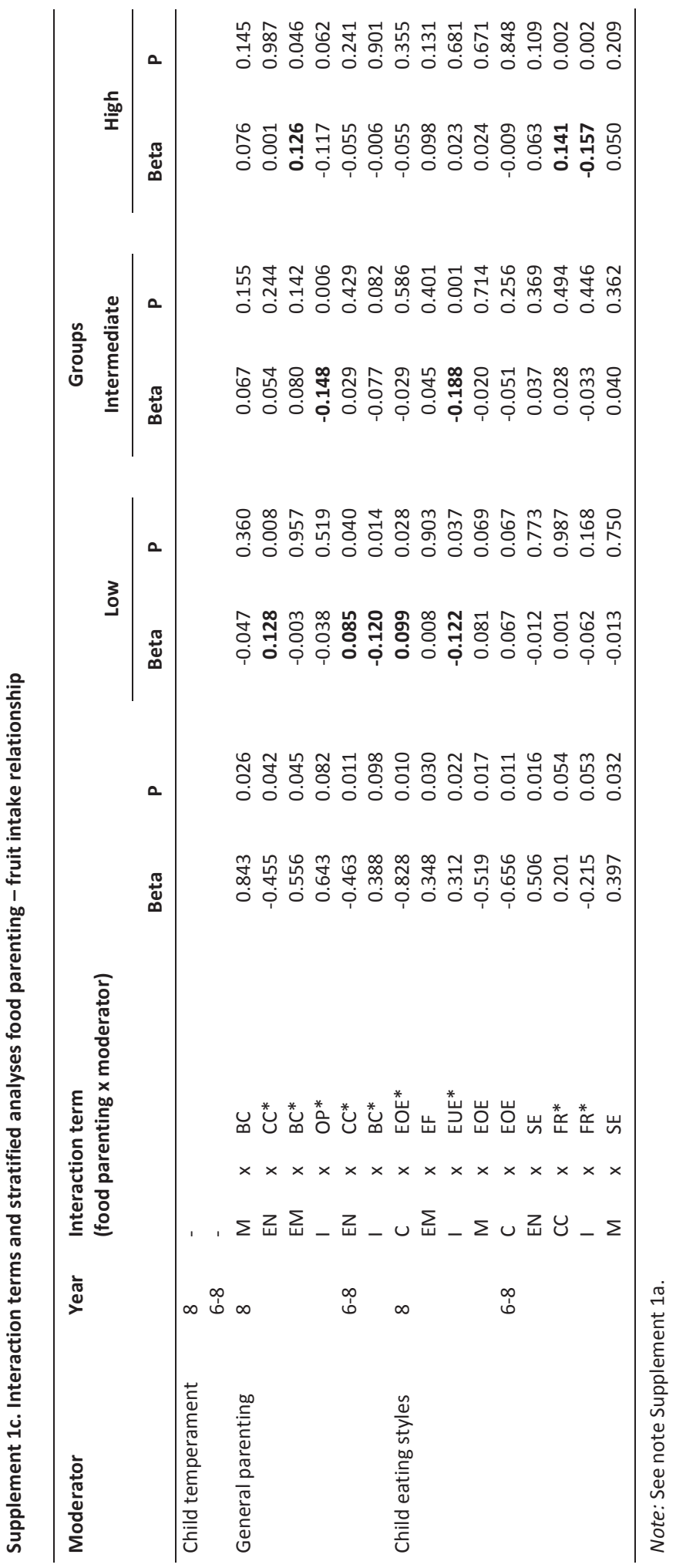




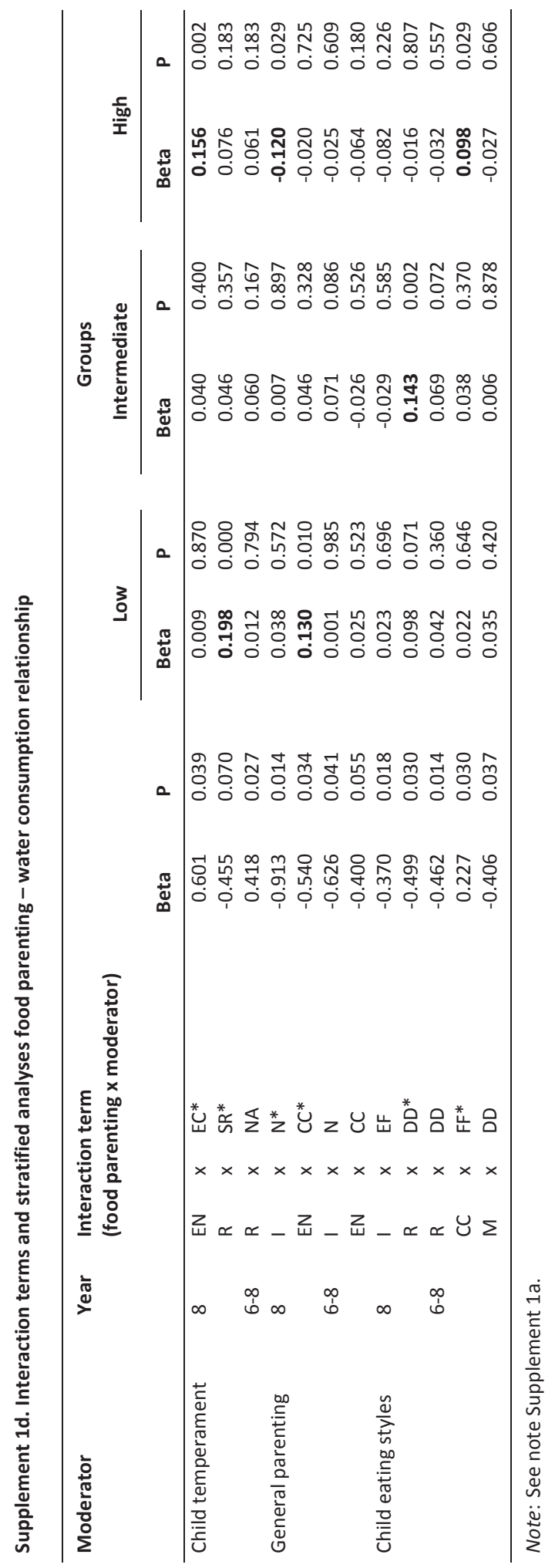


CHAPTER 13

General discussion 


\section{Introduction}

Childhood overweight and obesity is a dominant concern because of its dramatic increase, its persistence into adulthood, its association with a host of negative health outcomes and its burden on healthcare systems. Parents are utterly important in influencing children's health behaviors and subsequently their weight status.

The aim of this dissertation was three-fold. First, three reviews were conducted about the role of parents in influencing their children's energy balance-related behaviors (EBRBs). Second, questionnaires were developed and/or validated related to child temperament, eating and feeding styles, and general parenting. Third, interrelationships between parenting variables and their contribution to children's EBRBS were examined in the ongoing KOALA Birth Cohort Study, using the validated instruments.

This final chapter is divided into several parts. The main findings of each study are summarized, followed by a general discussion. This discussion focuses on methodological issues and implications of the findings for research, practice and theory, followed by a general conclusion and directions for future research.

\section{Main findings}

\section{PART 1: Parenting reviews}

Chapter 2 summarizes existing observational studies about the relationships between general parenting and child EBRBs and weight status. In total, 36 studies were included. Findings suggest that children raised in authoritative homes ate more healthily, were more physically active and had lower Body Mass Index (BMI) levels, compared to children who were raised with other styles (authoritarian, permissive, neglectful). Findings of the few moderation studies indicate that general parenting has a differential impact on children's weight-related outcomes, depending on child and parental characteristics. Discrepancies in study results were found due to the broad diversity of parenting measures (some measuring similar and some very different constructs and some were completed by parents or children), the diverse demographical contexts, and different methods used to assign parents to styles.

Observational studies increasingly emphasize the impact of general parenting on the development of childhood overweight and obesity. Chapter 3 provides an overview of existing interventions addressing general parenting in order to prevent or treat childhood obesity. Seven studies were eligible for inclusion. The studies described four different general parenting programs promoting authoritative parenting, which were supplemented with lifestyle components (i.e., nutrition and physical activity). All studies showed significant small to moderate intervention effects on at least one weight-related outcome measure. These interventions provide evidence that the promotion of authoritative parenting is an effective strategy for the prevention and management of childhood overweight and obesity. 
Chapter 4 reviews existing questionnaires of parenting practices in regard to physical activity, their psychometric performance and correlation with children's physical activity levels. Eleven unique PA parenting questionnaires were identified, and 46 studies that used these instruments were included. Findings highlight the tremendous variation in the conceptualization and measurement of physical activity parenting, common use of non-validated instruments, and lack of comprehensive measures. The development of theory-based physical activity parenting measures should be prioritized to guide the study of the parental role in promoting child physical activity as well as the design of family-based physical activity interventions.

\section{PART 2: Instrument development}

The studies below described the development and/or validation of child temperament, eating and feeding styles, and general parenting questionnaires.

\section{Child temperament}

Chapter 5 focuses on validating several forms of the Children's Behavior Questionnaire (CBQ; 195 items and 36 items) using factor analytic procedures to see whether the three-factor temperament structure as previously identified in the United States (US) could be replicated in a Dutch sample. Cross-cultural comparisons of temperament structure were also performed. In total, 353 parents of 6-8 year olds completed the instrument. The original factor structure of the different CBQ forms was generally replicated and represented the three broad dimensions of temperament. Results demonstrated a relatively high degree of factor similarity of the Dutch sample with other cultures. The findings provide evidence for applicability of the $\mathrm{CBQ}$ in Western Europe as a promising instrument to comprehensively assess reactive and self-regulative temperamental dimensions in young children.

Chapter 6 describes the development and validation of a one-item temperament scale, with three vignettes addressing the three global traits of temperament. This one-item scale was developed to assist in tailoring interventions. The one-item measure was tested against the 36-item CBQ. Both questionnaires were completed by 237 caregivers of 3- to 5 -year-olds in the US. Additionally, the psychometric properties of the 36-item measure were assessed. Classical test theory analysis demonstrated adequate internal consistency and factor analysis confirmed a three-factor structure. Potential improvements to the measure were identified using item response modeling. The three response categories (one-item temperament scale) correlated with the temperament factors of the 36 -item scale, as expected. The one-item temperament scale may be applicable for clinical use.

Chapter 7 describes the validation of a 3-item temperament measure and 13-item impulsivity scale. First, the one-item temperament measure described in chapter 6 was adapted to keep intact the multidimensionality of the scale. For each of the three vignettes, parents were asked to select how much it applied to their child. Again, this measure was tested against the 36-item CBQ. Additionally, a child-report 
13-item impulsivity questionnaire was tested for its applicability. Psychometrics of the CBQ and the impulsivity scale were examined, which were considered good in terms of internal consistency and factorial structure. The three temperament items correlated with the averages scores on the corresponding $\mathrm{CBQ}$ factors. Furthermore, surgency was highly related to impulsivity. Findings provide evidence for the applicability of the impulsivity and temperament measures for observational research.

\section{Eating and feeding styles}

Chapter 8 describes the translation and validation of the Children's Eating Behavior Questionnaire (CEBQ) in a Dutch sample of 135 6- to 7-year-olds. The CEBQ is a parent-report measure designed to assess variation in eating style among children, and initially developed in the United Kingdom. Factor analyses were performed and relationships between child eating style and BMI were investigated. Results generally confirmed the theoretical factor structure, with acceptable internal reliability and between-subscale correlations. Linear regression analyses revealed that BMI z-scores were positively associated with the 'food approach' (food responsiveness, enjoyment of food, emotional overeating) and negatively with 'food avoidant' subscales (satiety responsiveness, slowness in eating, emotional undereating, and food fussiness). The results support the use of the CEBQ as a psychometrically sound tool for assessing eating behaviors in Dutch children and the study demonstrates its applicability in overweight-related studies.

Chapter 9 describes the translation and validation of the Parental Feeding Style Questionnaire in a Dutch sample of 135 6- to 7-year-olds. Psychometric evaluations, including factor analyses, were performed. Additionally, associations between parental feeding styles and dietary intake behaviors of both the parent and the child were assessed. Results indicated considerable similarity of factor structure, internal reliability and between-subscale correlations with the original British study. The parental feeding dimensions of instrumental feeding' (i.e., using food as a reward) and 'emotional feeding' (i.e., feeding in response to children's emotional distress) were positively related to children's snacking behavior. The feeding style 'encouragement to eat' was negatively associated with children's snacking behavior. Various feeding styles were found to be related to parental dietary behaviors (e.g., 'encouragement to eat' positively with fruit intake and breakfast consumption). Findings indicate the importance of acknowledging parental feeding styles in future research efforts as well as in the development of family-based interventions promoting healthy eating habits among children.

\section{General parenting}

Chapter 10 summarizes a range of issues and offered numerous directions for future research regarding the assessment of parenting. These included: 1) general versus domain specific parenting styles and practices; definitions were provided and comparisons were made; 2) novel approaches to parenting measurement; recommendations were made regarding the development of rapid assessment parenting tools using advanced psychometric methods, use of multiple measuring tools to collect complementary information, and use of cluster analytic approaches for assigning parents to styles; 3 ) the role of ethnicity and culture; 
future research should try to identify underlying universal characteristics of parenting that operate across cultures; 4) assessing bidirectional influences; the interactions between styles and practices may be complex; 5) broadening assessments beyond the immediate family; a child's development and behavior is influenced by all of the influences in his environment; and 6) designing effective interventions. Thus, this chapter outlined general existing concepts, nomenclature, core constructs, measurement issues and challenges, emerging technology, new and evolving perspectives on parenting, and design issues for interventions. Collaboration within the burgeoning parenting research community is a top priority.

Chapter 11 describes the development of the 'Comprehensive General Parenting Questionnaire' (CGPQ), designed for use in this dissertation. Despite the large number of general parenting instruments, considerable disagreement exists about how to best assess parenting. Most of the instruments only assess limited aspects of parenting, and consensually identified questionnaires of high quality measures are lacking. Hence, it is necessary to identify the core constructs of parenting and to elaborate and clarify their defining features. Developing a single parenting questionnaire to assess the major parenting constructs (versus piecing together a large number of individual questionnaires) greatly reduces participant response burden. Moreover, by measuring the major parenting dimensions simultaneously, it will be possible in future studies to examine individual differences in parenting styles that involve simultaneously assessing individuals across multiple parenting dimensions. The ultimate goal is to promote comparability across studies and facilitate research exploring how parenting influences children's health-related behaviors. The questionnaire is comprehensive as it measures the five key aspects of parenting: nurturance, structure, behavioral control, coercive control, and overprotection. The survey was administered to large samples of parents of 5- to 13-year-old children in the Netherlands, Belgium and the US.

A mixed methods approach was used for the development of the CGPQ comprising the following four steps: (1) Items were identified from existing parenting questionnaires based on our framework including the five constructs of parenting. (2) Cognitive interviews and author review informed the modification, deletion and/or replacement of items. (3) Advanced statistical analyses including classical test theory, confirmatory factor analyses and item-response modeling were conducted to test our theoretical fivefactor parenting model and to develop fit items using an online survey containing the parenting item bank. (4) Finally, additional author reviews and cognitive interviews were done to review the fit of the items, determine if any construct was missing or inadequately assessed, assess content validity, and verify wording of the modified items.

Additionally, a questionnaire regarding personality characteristics ('Big Five') of the caregiver was administered. The reduced 62-item questionnaire revealed acceptable fit of our parenting model and acceptable item-response modeling item fit statistics. Caregiver personality was related to the parenting constructs as measured by the CGPQ. The personality traits of extraversion, agreeableness, conscientiousness, and openness to experience were positively associated with parenting constructs (i.e., nurturance, structure, behavioral control) previously found to be related to more positive child health outcomes, whereas the trait of neuroticism was associated with coercive control and a chaotic home 
environment. Based on expert panel review and cognitive interviews the CGPQ was further modified. The proposed 85-item scale may facilitate research exploring how parenting influences children's health behaviors.

\section{PART 3: Parenting - child eating behavior relationship}

Research on parenting practices has focused on individual behaviors while largely failing to consider the larger context of their use. Chapter 12 aims to examine the extent to which food parenting practices predict the development of child eating behavior. Additionally, the study tested the moderating role of both general parenting and child characteristics on the relationship between food parenting practices and children's eating patterns. For this purpose, we used data from the KOALA Birth Cohort Study. Associations between food parenting and child eating were present. Instrumental and emotional feeding, and pressure to eat were found to have detrimental associations with child eating behavior, whereas covert control, encouragement and restriction were found to have favorable associations. Although the strength of relations differed depending on the context, all findings (strength of association between food parenting and child eating behavior) of the stratified analyses were in the same direction. Two examples, for children who were reared in a positive parenting context, restriction, encouragement and covert control worked better than for children who were reared in a less positive parenting context (e.g., coercive and overprotective home environments). For children who grew up in a less positive parenting context, emotional and instrumental feeding worked more detrimental. Moreover, some food parenting practices did not interact with other factors in explaining child eating behavior. These might be robust for contextual factors and suitable to target to parents when using more general types of interventions where tailoring to an individual is not possible. Thus, parents have a large influence on their children's eating behavior. Food parenting practices are important correlates of children's eating behavior, and it is important to take into account the parents' parenting style and temperament and eating behavior of children.

\section{Methodological issues}

In this paragraph various characteristics (i.e., age and ethnicity) of the study populations will be discussed, followed by a more in-depth description of the KOALA Birth Cohort Study. Finally, issues related to selfreported questionnaire data and findings related to the validation of some questionnaires used for this dissertation will be discussed.

\section{Study populations}

For the current dissertation various samples were used which differed in age and ethnicity. We used national and international samples (i.e., US and Belgium). The study samples described in chapter 5 , 8, and 9 were recruited by teachers of third graders (6- to 7-year-olds) in Dutch primary schools. Children received 
a questionnaire to give to their parents for completion. Only parents holding Dutch nationality were requested to complete the survey. For the validation of the 'Comprehensive General Parenting Questionnaire' (chapter 11) we used large samples of parents of 5- to 13-year-olds from the Netherlands, Belgium and the US. The participants for this study were recruited through internet panels (i.e., the Netherlands and Belgium) and through (a) posting and handing out flyers; (b) posting the study on websites; and (c) listing the study in newsletters and recruiting from participant databases (i.e., Houston, Texas, US). For the validation of the one-item temperament scale in the US (chapter 6), English-speaking caregivers of 3- to 5-year-olds were recruited in the vicinity of the Texas Medical Center in Houston (similar procedure as the recruitment procedure for the US sample just described). This was an ethnically diverse sample (about $40 \%$ were Whites, $25 \%$ were Hispanics and $25 \%$ were African-Americans). For the validation of the 3-item temperament scale (chapter 7) a subsample was used from the KOALA Birth Cohort Study when children were around the age of 6 to 7. A larger sample from this longitudinal study was used when evaluating the parenting - child eating behavior model (chapter 12). Although longitudinal studies have more advantages above cross-sectional studies, for questionnaire validation studies the latter type of study design is appropriate to use. With regard to ethnicity, we are aware that when validating an existing questionnaire for another cultural group this should be done with caution. Some questionnaire items developed for a particular country might not be suitable for other cultures. It is important to perform cognitive interviews with the target group for pretesting purposes to check whether they comprehend the questionnaire. Additionally, the factorial structure of the questionnaire should be checked for equivalence between cultures. For instance, we found that the factor structure of the CBQ was roughly similar to different US study samples, but slight differences were found; the subscale of shyness loading more on the temperamental trait of Negative Affectivity than reversed coded on the trait of Surgency (see chapter 5). Especially for comparability purposes it is recommended to develop and validate questionnaires that can be used across cultural groups without excluding important cultural constructs (see chapter 10). Thus, the various samples used for the current dissertation varied with regard to demographics and study design.

\section{The KOALA Birth Cohort Study}

This cohort, as the only longitudinal study of the current dissertation, deserves some attention. Although the KOALA study has more special features, this paragraph is restricted to issues with regard to recruitment, study design, and drop-out rate. Recruitment of the study participants of the KOALA study started already in the year 2000. More than 2800 women and their offspring have been followed from pregnancy onwards. The children born from the pregnancies have been followed with repeated questionnaires and home visits at many different time points. An advantage of longitudinal studies compared to the cross-sectional studies is that they are unique in following a certain population over time. However, one should be cautious in drawing inferences regarding causality. Experimental studies (e.g., randomized controlled trials) are strictly needed to show causation; to test the effect of changes of a particular variable in the environment. The KOALA study has some other interesting features. Pregnant women with different lifestyle characteristics were recruited through so-called 'alternative' lifestyle channels including anthroposophist physician offices and midwives, and organic food shops (Kummeling et 
al., 2005). Many of these women had specific opinions about dietary habits (e.g., preferring biological/organic foods), vaccination schemes and/or antibiotic use. Women with a conventional lifestyle were recruited from an existing cohort on pregnancy-related pelvic girdle pain. Recruiting both pregnant women with a conventional and 'alternative' lifestyle was done in the first place to increase contrast of interesting determinants for allergy and asthma. In the current dissertation, the analyses were corrected for recruitment channel, because an 'alternative' diet (e.g., organic) and 'alternative' viewpoint on child rearing might have consequences for the study findings. Another feature of the KOALA cohort which needs some attention is the drop-out rate. This is a problem in all birth cohort studies. Only $65 \%$ of the participants completed the questionnaire around their child's age of 8 . This attrition rate is comparable to that of similar cohort studies (Environmental Health Risks in European Birth Cohorts (ENRIECO), 2012).

\section{Measurement}

The data that were used for the current dissertation were collected through questionnaires using paperand-pencil (chapter 5, 7, 8, 9, and 12) and online (chapter 6 and 11) administration formats. For the development of the general parenting questionnaire (chapter 11) we additionally used cognitive interviewing to test whether parents understood the questionnaire items and expert review to further optimalize the items. Some differences in responses between the paper-and-pencil and the online administration methods have been reported (Whitehead, 2011), whereas others only found equivalences between the two methods (Bishop et al., 2010). Advantages of web-based survey include lower proneness to social desirability bias, no missing data when using forced-choice formats, and more rapid return than postal questionnaires (Van Gelder, Bretveld, \& Roeleveld, 2010), but also lower levels of missing data when no forced-choice format was used (Kongsved, Basnov, Holm-Christensen, \& Hjollund, 2007). Disadvantages of this mode of data collection include selection bias for those that have access to a computer (Van Gelder et al., 2010), and higher non-response rates (Kongsved et al., 2007; Van Gelder et al., 2010), although subjects responding to an online survey have been found to be comparable to those responding to traditional modes of data collection in terms of demographics (Van Gelder et al., 2010). It is important to note that we consistently administered only paper-and-pencil or online surveys for each of the conducted studies, to reduce changes of finding different underlying factor structures for instance. Previous research found mixed results; some found a similar factor structure across the different administration modes (e.g., Cole, Bedeian, \& Field, 2006), whereas other found factorial validities to be prone to changes when administered online (Buchanan, Johnson, \& Goldberg, 2005).

In the current dissertation we rely on parent-reported data; mainly the mothers completed the questionnaires. Self-reported data, especially relating to their own general parenting behaviors, may be prone to social desirability bias and could potentially pose a threat to construct validity. Social desirability refers to a tendency by respondents to portray an overly positive image of their true selves (Uziel, 2010). Although social desirability scales have been developed to identify and statistically correct for a respondent's biased answering style, these scales are far from ideal (Jo, 2000; Leite, \& Beretvas, 2005; Uziel, 2010). As Paulhus and Vazire (2007) have said, 'the way to correct for socially desirable response bias 
in self-reports is probably not by statistically controlling for results on another self-report measure'. Little support has been found that social desirability corrections can adjust for attenuated associations between the self-report and external criteria (Jo, 2000; Leite \& Beretvas, 2005; Uziel, 2010). A small correlation between responses to social desirability scales and a self-report measure does not necessarily mean that there is no social desirability bias in that scale, nor does correcting for the presence of a correlation mean that the problem is resolved as correction could results in misleading results or unnecessary reduction of power (Leite \& Beretvas, 2005). Implicit measures (Fazio \& Olson, 2003) of parenting constructs may be worthwhile to develop, as these have the potential to solve issues of social desirability and capture impulsive influences related to parenting (Mâsse \& Watts, submitted).

In this dissertation, first steps were taken to validate some of the well-respected and often used comprehensive questionnaires for child temperament (Children's Behavior Questionnaire (CBQ); Rothbart, Ahadi, Hershey, \& Fisher, 2001; Sleddens, Kremers, Candel, De Vries, \& Thijs, 2011; see chapter 5) and child eating style (Children's Eating Behavior Questionnaire (CEBQ); Sleddens, Kremers, \& Thijs, 2008; Wardle, Guthrie, Sanderson, \& Rapoport, 2001b; see chapter 8). Although, the Child Feeding Questionnaire (Birch, Fisher, Grimm-Thomas, Markey, Sawyer, \& Johnson, 2001) is the most frequently used food parenting practices measure worldwide during the last decade (Vaughn, Tabak, Bryant, \& Ward, submitted), we felt this questionnaire mainly focussed on highly controlling food parenting practices (e.g., restriction, pressure to eat). As the instrument was already previously administered to participants of the KOALA study (Gubbels, Kremers, Goldbohm, Stafleu, \& Thijs, 2012), the assessment of food parenting was broadened in the current dissertation by including the Parental Feeding Style Questionnaire (PFSQ; see chapter 9; Sleddens, Kremers, De Vries, \& Thijs, 2010; Wardle, Sanderson, Guthrie, Rapoport, \& Plomin, 2002), because besides assessing controlling food practices this measure also assesses the more positive encouraging practices.

Additionally, we developed very brief temperament measures (see chapter 6 and 7) for use in applied studies. First, a one-item scale was developed to assist in tailoring interventions (Sleddens, Hughes et al., 2012a). An example of such an intervention is 'Kiddio: Food Fight', a smart phone application (app) game prototype to help parents of preschool children use effective parenting practices related to eating vegetables (Baranowski et al., 2012; Beltran et al., 20212). The goal of the game was for players (parents) to persuade the character of the game (Kiddio, a 3- to 5-year-old child) to taste a vegetable. To create a child character that would behave similarly to the game player's preschool child, the player needed to specify their child's temperament. Since employing the 36-item CBQ form to assess the child's temperament on three dimensions would detract from immersive game play, a one-item temperament measure was developed (Sleddens, Hughes et al., 2012a). By using this rapid assessment method to identify the most dominant temperament characteristic, tailoring of games is feasible. Our one-item scale was not intended to replace the multi-dimensional scales for assessing child temperament for observational studies. In order to keep intact the multi-dimensional approach, Sleddens, Kremers, De Vries, and Thijs (2012) developed a 3-item temperament measure based on the traits of Surgency, Negative Affectivity, and Effortful Control as measured by the $\mathrm{CBQ}$. 
We also decided to develop a new and comprehensive general parenting questionnaire for use in multiple countries, which we called the 'Comprehensive General Parenting Questionnaire' (CGPQ; see chapter 11), as a single parenting questionnaire to measure all major parenting constructs was absent. For the development of this questionnaire we followed a mixed methods approach, including the development of a conceptual model for general parenting, creating an item bank, and using cognitive interviewing, expert review, and advanced statistical analyses (i.e., Confirmatory Factor Analysis and Item Response Modeling). The questionnaire was validated against a parent personality measure. Further validations of this instrument are ongoing using different data including correlations with independently assessed and parental reports of child outcomes (e.g., BMI, behavior problems, temperament) and independent ratings of the parents' practices. Although observations of parent-child interactions would be ideal to assess the instruments' construct validity, these were not conducted (yet) due to time and financial constraints. The questionnaire is currently also translated into the Spanish, Indonesian and Norwegian languages, adapted to other age groups (parents of 1- to 4-year-olds, and parents of 13-to 15-year-olds and adolescents aged 13 to 15 ) and applied in different countries worldwide (e.g., the Netherlands, Belgium, US, Indonesia, Mexico, and Norway). Thus, validation of the CGPQ and further refinement is still ongoing. With enough research, we may be able to identify underlying universal characteristics of parenting that operate across cultures, but may be expressed in different ways (chapter 10). Further research with the CGPQ should focus on assessing factorial invariance of the instrument across cultures.

\section{Statistical analyses}

In the current dissertation we used several advanced psychometric statistical procedures to validate questionnaires using Confirmatory Factor Analyses and Item Response Modeling beyond more classical test theory approaches (Wilson, Allen, \& Li, 2006a/b). Although we collected large amounts of data from three different countries for the development of the CGPQ (i.e., Netherlands $N=821$, Belgium $N=435$, and US $N$ =241), we lacked statistical power to conduct these analyses on the sub-groups. Only the use of the total sample provided adequate power to perform the data reduction procedures on the list of 115 items.

Statistical procedures to evaluate our hypothesized parenting model such as Structural Equation Modeling were not used, but may be used in the future. This analysis technique has many advantages above multiple regression (Chin, 1998; Kline, 1998; Musil, Jones, \& Warner, 1998), including testing models with multiple dependent variables, using Confirmatory Factor Analysis to reduce measurement error, testing the overall models, ability to model mediating and moderating variables at once, and ability to model error terms. Fortunately, our sample was large enough $(N=1654)$ to evaluate whether the relationship between food parenting practices and child eating behavior was moderated by several contextual factors (i.e., general parenting, child temperament, and child eating style). This study provided evidence for effective and ineffective food parenting practices, as well as for child- and parent-related contexts that moderate their impact. However, we did not adjust for multiple testing as our study was explorative in nature. A drawback of these additive regression models is that they usually are underpowered when multiple higher order 
interactions are incorporated. Regression trunk modeling could solve this problem. This is a multiple regression model with main effects and a parsimonious number of higher order interaction effects (Dusseldorp, Conversano, \& Van Os, 2010). The model is especially appropriate for prediction problems with multiple predictors and a combination of linear main effects and interaction effects. We recommend future studies with power problems and/or lack of exact a priori hypotheses about the number of interaction effects to use regression trunk modeling as a suitable solution to overcome problems related to multiple testing.

\section{Implications for research}

In this paragraph, the research implications concerning parenting measurement and parent-child interactions will be described.

\section{Valid measurement}

The development of well-validated generally respected parenting measures requires consideration of many aspects. Most importantly the choice of a measurement method depends on the research question and the resources available (see chapter 10, Power et al., submitted). For instance, within the KOALA study we were interested in asking the total sample about the broad spectrum of general parenting. Using questionnaires was the best method for this purpose. Of course, multiple measuring tools are preferable (observations, child reports, etc.), but this was simply not feasible for the studies included in this dissertation. There may be researchers who do not include parenting assessments in their studies because instruments with good reliability and validity take too long to complete. Advanced psychometric methods including Confirmatory Factor Analyses and Item Response Modeling should be applied to develop short forms and rapid assessment parenting tools. These new tools could be used for multiple purposes, such as adding a short set of questions to large national surveys where parenting is not the main focus, developing screening instruments for identifying high-risk participants for targeted interventions, assessing parenting in clinical settings such as pediatric and primary care medical practices, and evaluating parenting-related interventions (see chapter 10, Power et al., submitted). However, for the development of a parenting instrument one should consider using other methods to assist in this process. For instance, video-taped observations during mealtime in the home setting to monitor food parenting practices of parents and compare these findings to self-report data, and collecting data from other sources (e.g., spouses). All these additional data collection methods can cross-validate the data collected with the self-report parenting measure. Attention should also be paid to develop and validate parenting instruments that can be used across cultural groups without excluding important cultural constructs. There are several strategies to consider for improving the measurement of parenting constructs (Morsbach \& Prinz, 2006). Mâsse \& Watts (submitted) recently discussed whether the solution to improve self-report parenting measures lies in '1) improving the question asked (Morsbach \& Prinz, 2006); 2) improving the methods used to correct for social desirability; 3 ) changing our measurement paradigm to assess implicit parenting behaviors; 4) 
changing how self-report is collected by taking advantage of ecological momentary assessment methods; 5) using better psychometric methods, such as item banking and computerized adaptive testing; and 6) considering alternative data collection methods such as portable technologies, gaming, and virtual reality simulation'.

Much more research is needed to further optimize the current parenting tools, which lack to reflect the full dynamics and complexity of the parental role in shaping their children's EBRB, especially parenting related to food (Vaughn et al., submitted), physical activity (Sleddens, Kremers et al., 2012), and screen media (Jago, Edwards, \& Sebire, submitted). There is no agreement as to which dimensions should be assessed by these measures, how a specific dimension should be operationalized, and which items to include. And there is no consensus about exact definitions of the major parenting constructs in this field (e.g., particularly the distinction between 'style', 'dimensions', and 'practices') (Jansen, Daniels, and Nicholson, 2012). Recent statements emphasize the need for the development, validation, and use of better parenting measures (Baranowski et al., submitted; Faith et al., 2012; Jansen, Daniels, \& Nicholson, 2012). During a conference of leading investigators and practitioners in Houston, US from May 20-22, 2012, there was agreement about the following issues to measurement of parenting (cf Baranowski et al., submitted, summary manuscript): 'A fundamental reconsideration was needed of the foundational knowledge of parenting in regard to EBRB. More qualitative and observational research is needed since key dimensions of parenting may yet be discovered. Investigators need to adhere to consistent definitions to enhance consistency of findings, and to better understand when and why the inconsistencies occurred. Since it seems unlikely that the dimensions or mechanisms of influence of parenting on different child behaviors will differ substantially at the conceptual level, more transdisciplinary research is needed among these investigators so the research on physical activity and screen media parenting practices can benefit from the advances in general parenting and food parenting practices. New methods are needed to minimize the likely socially desirable responses to existing methods, relieve respondent burden, and better understand the functioning of scales and items. Attendees rightly identified inadequate attention to the role of the child (e.g., temperament and other characteristics) in the selection or use of types of parenting. The extent to which different measures are needed for different genders, ages of children or different cultures (e.g., across ethnic groups, countries) need serious attention.'

To develop 'better' parenting tools it is important 1) to clarify what is being understood by the different dimensions; 2) to identify existing items belonging to these dimensions; 3 ) to classify the items into the appropriate dimension ('binning' the items); 4) to reduce the pool of items ('winnowing') by eliminating redundancy, rewrite double-barreled items, and modify poorly worded items; 5 ) to supplement the pool of items whenever a dimension is found to be under-represented by existing items; 6) to standardize the items; and 7) to review the items by experts. Sophisticated methods including the use of an Item Response Modeling study need to be used to develop an item bank, rare to date in the behavioral sciences (Mâsse, Wilson, Baranowski, \& Nebeling, 2006), and use of Computerized Adaptive Testing (Forrest et al., 2012), a dynamic method that can reflect the full range of parenting practices while maintaining the reliability of scores with fewer items. Using IRM item banking by using Computerized Adaptive Testing has the potential 
to: (a) create measures that can be used across a wide range of studies and populations; (b) allow the flexibility of selecting which items are included in a given study while maintaining the ability to compare results across studies; and (c) reduce participant burden (Mâsse \& Watts, submitted).

\section{Parenting in perspective}

Parenting does not occur in isolation, it is the result of bi-directional relationships between parent and child, influenced by interactions with the environment. Parents are utterly important, more so than other environments, in influencing especially young children's behaviors. Child outcomes are likely to be influenced by the combined parenting practices of mothers and fathers. Most studies focused on only assessing parenting of mothers (Sleddens, Gerards, Thijs, De Vries, \& Kremers, 2011), the ones also assessing paternal practices did not assess the interaction between those two. However, there was one exception (see chapter 2, Sleddens, Gerards et al., 2011). In the study of Berg, Wall, Bauer, and NeumarkSztainer (2010), the co-occurence of an authoritarian mother and a neglectful father was associated with higher BMI in adolescent sons, but there was no protective effect of authoritative parenting in general. Additionally, incongruent parenting practices were associated with higher BMIs in adolescents (i.e., when mothers modeled and encouraged healthful eating and physical activity, but fathers did not, adolescents had higher BMIs). Thus, differences between mothers and fathers in parenting have important consequences for their children's health outcomes. Further studies should focus on assessing the influences of incongruent parenting practices.

Longitudinal studies about the influence of parenting on child EBRBs and overweight development are scarce (Fuemmeler et al., 2012), and only few of them asessing more complex mechanims of parental influence. Although most of the conducted cohort studies in this respect tend to show more favorable outcomes for children who are raised in authoritative homes (i.e., healthier diet and activity patterns, and lower BMI) (see chapter 2, Sleddens, Gerards et al., 2011), more research is needed to further support these findings. A recent study of Fuemmeler et al. (2012) reported on associations between parenting style and transitions in BMI from adolescence to young adulthood (over 11 years). Future studies should go beyond assessing associations between parenting and child weight-related outcomes, and also assess other factors which potentially can alter the parenting - child weight relationship such as child temperament and eating style. Using valid measurement, assessing more complex mechanisms, and following-up parents and children for long time periods would be a challenge to progress in this area. Our study described in chapter 12 was a first attempt to assess the influence of parents on children's eating behavior from age 6 to 8 , and assessing factors impacting on this association, using several validated questionnaires, although only short periods of assessment ( 2 years) were included.

The broader home environment is an aspect which was outside the scope of this dissertation. Future research efforts should aim to also include the broader context, e.g., quality of a child's environment, parental stress, attachment (Pritchett, Kemp, Wilson, Minnis, Bryce, \& Gillberg, 2011; Stenhammer et al., 2010; Strauss \& Knight, 1999; Trombini, Baldaro, Bertaccini, Mattei, Montebarocci, \& Rossi, 2003; Walker \& 
Kirby, 2010), but also influence of other caregivers including grandparents, and siblings (or even birth order of the child). Additionally, looking at other environmental influences outside the immediate home environment would be informative; e.g., neighborhood and/or school environment (e.g., Safron, Cislak, Gaspar, \& Luszczynska, 2011; Williams, Wyatt, Hurst, \& Williams, 2012) and day care (Gubbels, Kremers, Stafleu, Dagnelie, De Vries, \& Thijs, 2010; Gubbels et al., 2011).

\section{Implications for practice}

Practical implications discussed will relate to the development of family-based intervention programs for childhood overweight and obesity.

\section{Contextual intervention efforts}

Programs aimed to change children's EBRB to prevent or reduce childhood overweight may benefit from being tailored to family characteristics, including general parenting behaviors, child temperament and child eating styles (see chapter 12, Sleddens, Kremers, Stafleu, Dagnelie, De Vries, \& Thijs, in revision). The operation of higher order moderation processes was confirmed, implying that parenting and child factors at higher, more distal, levels alter the impact of parenting practices (i.e., food parenting practices) at more proximal levels. However, the food parenting - child eating relationship was not reversed when taking into account these moderating factors. Food parenting practices were also found to not interact with other factors in explaining child eating behavior. These might be robust for contextual factors and suitable to target to parents when using more general types of interventions where tailoring to an individual is not possible. For instance, parents should be encouraged to use covert controlling practices (e.g., not bringing unhealthy foods in the home). It is too early to give specific advice about what approach to use, invidualized or general types of interventions. This individual approach is especially recommended when adverse effects are present depending on the moderator.

Whereas a few years ago only few intervention studies were found targeting general parenting, some in combination with lifestyle components (Gerards, Sleddens, Dagnelie, De Vries, \& Kremers, 2011), this number is fortunately growing (e.g., Brotman et al., 2012; Gerards et al., 2012; Golley, Magarey, \& Daniels, 2011; Magarey et al., 2011; Moens \& Braet, 2012; Robertson, Thorogood, Inglis, Grainger, \& StewartBrown, 2012; Shelton, LeGros, Norton, Stanton-Cook, Morgan, \& Masterman, 2007; Ward et al., 2011; West, Sanders, Cleghorn, \& Davies, 2010). Most of them showed positive intervention effects on children's weight-related outcomes (Brotman et al., 2012; Golley et al., 2011; West et al., 2012; Moens \& Braet, 2012). Some of them also reported positive effects on general parenting (parents reported applying more authoritative forms of parenting after participating in the intervention) (Magarey et al., 2011; West et al., 2012), but not all (Moens \& Braet, 2012; Shelton et al., 2007). An example of such a family-based intervention program for childhood obesity is the Lifestyle Triple P - Positive Parenting Program (West et al., 2010). This program is based on the general Triple P, developed by Sanders and colleagues (Sanders, 
2012; Sanders, Markie-Dadds, \& Turner, 2003; Sanders, Turner \& Markie-Dadds, 2002). General Triple P contains five levels, ranging from the use of universal media to disseminate positive parenting information to an intensive individually-tailored program which includes home visits. Lifestyle Triple focuses on Level 4, moderate to high intensity consisting of group sessions, and is tailored to the concerns of parents of overweight and obese children (West et al., 2010). The program aims to reduce children's risk of chronic weight problems by increasing parents' skills and confidence in managing children's weight-related behavior (West et al., 2010). The findings so far are promising; the short-term findings support the efficacy of the program (West et al., 2010). Lifestyle Triple P is translated and adapted to the Dutch situation and currently being evaluated (Gerards et al., 2012). F

It remains a difficult question whether to change parenting practices or parenting styles or both (chapter 10 , Power et al., submitted) to improve child health; current research does not fully answer this question. One might argue that changes in general parenting have a large public health effect (Gerards et al., 2011), potentially changing the impact of a broad range of specific parenting practices on child behavior. Therefore, targeting parenting styles might be more effective (Wilson et al., 2012), i.e., having a broader impact on child developmental outcomes, than changing parenting practices alone. On the other hand, it might be easier to change specific parenting practices, which are potentially more amendable to change and more proximal indicators of child behavior. Probably it is best trying to change both parenting styles and practices when intervening with parents. Previous studies also showed that interventions combining general parenting components with lifestyle components (i.e., parenting practices with regard to healthy eating and activity) may lead to better results than interventions focusing exclusively on general parenting (Gerards et al., 2011). Future research should further explore the relationships between specific and general parenting practices on child behavior and health.

Interventions to prevent or reduce childhood overweight and obesity should not only be focused on learning parents to use effective parenting practices. They should also take into account their child's temperamental traits (see chapter 12). An example of an intervention tailored to a child's most dominant temperament trait is 'Kiddio: Food Fight', a smart phone app game prototype to help parents of preschool children use effective vegetable parenting practices (Baranowski et al., 2012; Beltran et al., 2012). The game is intended to learn parents' skills for effective vegetable parenting. Extensive formative research has been conducted, including: specifying effective and ineffective vegetable parenting practices; testing these parenting practices in a family-based behavior change intervention; assessing the feasibility of a smart phone app with parents; testing alternative story lines with parents; testing a one-item measure of child temperament (see chapter 6, Sleddens, Hughes et al., 2012a/b) for use in the game to create a child character (Kiddio) that would behave similarly to the game player's child; creating one episode of Kiddio to get the 3- to 5-year-old to taste a vegetable at home; and alpha testing the prototype with prospective users (Baranowski et al., 2012).

There are also intervention programs that take into account a child's eating style. An example is the NOURISH randomized controlled trial to encourage positive food parenting practices and food preferences 
in early childhood with the goal of preventing childhood obesity (Daniels et al., 2009; Daniels, Mallan, Battistutta, Nicholson, Perry, \& Magarey, 2012). Parents were given guidance on the 'when, what and how' of food parenting (Daniels et al., 2012), including managing the child's eating behaviors and eating styles such as food refusal and fussy eating. Short-term results of this study are promising with reducing anthropometric indicators of obesity risk (Daniels et al., 2012). To our knowledge, interventions to teach parents effective food parenting practices to prevent or reduce childhood obesity taking into account their child's eating style are rare.

\section{Implications for theory}

In this paragraph implications for theory related to EBRB parenting are discussed, thereby acknowledging the importance of interactions between types and levels of parental influence.

\section{From an isolated approach to an interactionist, dynamic ecological approach}

The current dissertation was based on an ecological view of environmental influences on behavior (Friedman \& Wachs, 1999), in which different types and levels of environment are interrelated with factors at the individual level (Sallis \& Owen, 2002; Wachs, 1992/1999). As Wachs (1992) outlined in his structural model of the environment, environmental factors are categorized in a hierarchical, multilevel, and multidimensional manner. A change at one level can affect all other levels (Spence \& Lee, 2003). For years, researchers have tended to rely on static, context-free generalizations about determinants of EBRBs. Environmental and personal factors interact in explaining and predicting EBRBs, giving us more information than only studying potential determinants of EBRB as isolated factors (Kremers, 2010). But those moderation studies are rare in the study of environmental influences on EBRBs (Kremers, De Bruijn, Droomers, Van Lenthe, \& Brug, 2007). During the last few years, the call for the inclusion of interaction variables in models (Kremers et al., 2007) has led to interesting findings in the EBRB determinant research domain. Whereas one used to search for person-related moderators of parenting influences (Kremers et al., 2007), this may not sufficiently reflect the complexity of the impact of parenting (Kremers et al., submitted). Parenting does not occur in isolation. Distal processes can modify the pattern of relationships between environment and individual behavior occurring at levels that are more proximal. This theory was supported in the present dissertation, as general parenting was found to modify the association between some food parenting practices and child eating patterns (see chapter 12). General parenting forms the context in which proximal parenting processes operate. In addition, several child characteristics (i.e., temperament and eating style) also interacted with food parenting practices in predicting child eating behavior. This is in line with the Environmental Research framework for weight Gain prevention (EnRGframework; Kremers, De Bruijn, Visscher, Van Mechelen, De Vries, \& Brug, 2006), which presents a dualprocess view on the environment - behavior relationship. In this framework, environmental influences are hypothesized to influence behavior both directly and indirectly. The latter pathway is hypothesized to be moderated by specific personal and behavior factors, including personality. In our study (chapter 12), for 
instance, the food parenting practice of 'encouragement' found to be negatively associated with snacking consumption, but only for children scoring low on negative affect. Children scoring high on negative affect may not be responsive to parental encouragement strategies to get their child to eat healthy; however, these strategies work in a detrimental manner for these children. We advocate always including the theorybased examination of possible moderation effects.

\section{Conclusions and future directions}

The studies presented in this dissertation focused on: 1) reviewing evidence regarding the importance of general parenting in the development of children's EBRB; 2) reviewing the literature regarding important parenting (i.e., general parenting and parenting related to food and physical activity) and child (i.e., child temperament and eating style) measures and validating some of these measures; and 3) examining the parenting - child EBRB relationship. Parents are key to the development of their child's EBRB, and should be targets in interventions programs to prevent or reduce childhood overweight. However, there continue to be inconsistencies in the measurement of parenting and the current literature is lacking regarding the exact role of parents. We found that programs aimed to change children's EBRB to prevent or reduce childhood overweight may benefit from focusing on parents. Practices which were related to positive child eating behavior (increased healthy eating and decreased unhealthy eating) were covert control and restriction. Practices related to negative child eating behavior outcomes were instrumental and emotional feeding, and pressure to eat. We did not find that the association was reversed when taking into account the moderating factors of general parenting behaviors, child temperament and child eating styles (see chapter 12, Sleddens, Kremers et al., in revision). In short, the effects (desirable or undesirable) of these practices were robust for contextual factors. Nevertheless, we confirmed the operation of higher order moderation processes, implying that parenting and child factors at higher, more distal, levels alter the impact of parenting practices (i.e., food parenting practices) at more proximal levels (see our model presented in chapter 1 on page 11). Thus, we found food parenting practices to be important determinants in explaining child eating behavior, and general parenting behaviors as well as child characteristics have been found to induce or reduce this association, but not reversing the association. Future research efforts should continue to focus on testing the influence of factors impacting on the parenting - child EBRB relationship in order to gain insights into relevant contextual factors that need to be taken into account in designing interventions. Thus, theory-based ecological research approaches need to be used acknowledging the dynamic interplay of types and levels of parental influence on child EBRB using longitudinal research designs. As our understanding of familial influences on children's obesogenic behavior and weight status improves, health promoters can develop more effective childhood overweight programs intervening on parenting. 
REFERENCES 
Adams RJ, \& Khoo ST. (1996). Quest-Interactive test analysis system. Victoria, Australia: The Australian Council for Education Research.

Adkins S, Sherwood NE, Story M, \& Davis M. (2004). Physical activity among African-American girls: The role of parents and the home environment. Obesity Research, 12(S), 38-45.

Agras WS, Hammer LD, McNicholas F, \& Kraemer HC. (2004). Risk factors for childhood overweight: A prospective study from birth to 9.5 years. Journal of Pediatrics, 145, 20-25.

Ahadi SA, Rothbart MK, \& Ye R. (1993). Children's temperament in the US and China: Similarities and differences. European Journal of Personality, 7, 359-378.

Ainsworth MDS, Blehar MC, Waters E, \& Wall S. (1978). Patterns of attachment: A psychological study of the strange situation. Hillsdale, NJ: Lawrence Erlbaum.

Ajzen I, \& Fishbein M. (1977). Attitude-behavior relations: A theoretical analysis and review of empirical research. Psychological Bulletin, 84, 888-918.

Anderson CB. (2004). Athletic identity and its relation to exercise behavior: Scale development and initial validation. Journal of Sport and Exercise Psychology, 26, 39-56.

Anderson CB, \& Coleman KJ. (2008). Adaptation and validation of the athletic identity questionnaireadolescent for use with children. Journal of Physical Activity and Health, 5, 539-558.

Anderson CB, Mâsse LC, \& Hergenroeder AC. (2007). Factorial and construct validity of the athletic identity questionnaire for adolescents. Medicine and Science in Sport and Exercise, 39, 59-69.

Anderson CB, Mâsse LC, Zhang H, Coleman KJ, \& Chang S. (2009). Contribution of athletic identity to child and adolescent physical activity. American Journal of Preventive Medicine, 37, 220-226.

Anderson CB, Mâsse LC, Zhang H, Coleman KJ, \& Chang S. (2011). Ethnic, gender, and BMI differences in athletic identity in children and adolescents. Journal of Physical Activity and Health, 8 , 200-209.

Anzman SL, \& Birch LL. (2009). Low inhibitory control and restrictive feeding practices predict weight outcomes. Journal of Pediatrics, 155, 651-656.

Aragona J, Cassady J, \& Drabman RS. (1975). Treating overweight children through parental training and contingency contracting. Journal of Applied Behavioral Analysis, 8, 269-278.

Archer LA, Rosenbaum PL, \& Streiner DL. (1991). The Children's Eating Behavior Inventory: Reliability and validity results. Journal of Pediatric Psychology, 16, 629-642.

Arnold DS, O'Leary SG, Wolff LS, \& Acker MM. (1993). The Parenting Scale: A measure of dysfunctional parenting in discipline situations. Psychological Assessment, 5, 137-144.

Arredondo EM, Elder JP, Ayala GX, Campbell N, Baquero B, \& Duerksen S. (2006). Is parenting style related to children's healthy eating and physical activity in Latino families? Health Education Research, 21, 862-871.

Ashcroft J, Semmler C, Carnell S, Van Jaarsveld CHM, \& Wardle J. (2007). Continuity and stability of eating behaviour traits in children. European Journal of Clinical Nutrition, 62, 985-990.

Asendorpf JB, Denissen JJA, \& Van Aken MAG. (2008). Inhibited and aggressive preschool children at 23 years of age: Personality and social transitions into adulthood. Developmental Psychology, 44, 997-1011. 
Babbitt RL, Edlen-Nezien L, Manikam R, Summers J, \& Murphy CM. (1995). Assessment of eating and weight-related problems in children and special populations: Measures, theory, and research. In DB Allison (Ed.), Handbook of assessment methods for eating behaviours and weight-related problems (pp. 431-492). Thousand Oaks, CA: Sage Publications.

Baldwin AL. (1948). Socialization and the parent-child relationship. Child Development, 19, 127-136.

Bandura A. (1971). Social learning theory of aggression. In JF Knutson (Ed.), Control of aggression: Implications from basic research (pp. 201-250). Chicago, IL: Adline Publishing Co.

Bandura A. (1977). Social learning theory. Englewood Cliffs, NJ: Prentice-Hall.

Baranowski T, O’Connor T, Hughes S, Beltran A, Baranowski J, Nicklas T, Sleddens E, Thompson D, Lu A, \& Buday R. (2012). Smart phone video game simulation of parent-child interaction: Learning skills for effective vegetable parenting. In S Arnab, I Dunwell, \& K Debattista (Eds.), Serious games for healthcare: Applications and implications (pp. 247-264). Hershey, PA: IGI Global.

Baranowski T, O’Connor T, Hughes S, Sleddens E, Beltran A, Frankel L, Mendoza J, \& Baranowski J. (submitted). Houston...We have a problem! Measuring parenting: Overview of a conference.

Barber BK. (1996). Parental psychological control: Revisiting a neglectful construct. Child Development, 67, 3296-3319.

Barber BK. (2002a). Reintroducing parental psychological control. In BK Barber (Ed.), Intrusive parenting: How psychological control affects children and adolescents (pp. 3-13). Washington, DC: American Psychological Association.

Barber BK. (2002b). Regulation as a multicultural concept and construct for adolescent health and development. Unpublished manuscript.

Barber BK, \& Harmon EL. (2002). Violating the self: Parental psychological control of children and adolescents. In BK Barber (Ed.), Intrusive parenting: How psychological control affects children and adolescents (pp. 15-52). Washington, DC: American Psychological Association.

Barber BK, Olsen JE, \& Shagle SC. (1994). Associations between parental psychological and behavioral control and youth internalized and externalized behaviors. Child Development, 65, 11201136.

Barkeling B, Ekman S, \& Rössner S. (1992). Eating behaviour in obese and normal weight 11-year-old children. International Journal of Obesity and Related Metabolic Disorders, 16, 355-360.

Barlow J, \& Stewart-Brown S. (2006). Understanding parenting programmes: Parents' views. Primary Health Care Research and Development, 2, 117-130.

Barrett P. (2005). Pbs: Shortform [Computer software]. Retrieved from http://www.pbarrett.net/ shortform.htm

Baumrind D. (1965). Parental control and parental love. Children, 12, 230-234.

Baumrind D. (1967). Child care practices anteceding three patterns of preschool behavior. Genetic Psychology Monographs, 75, 43-88.

Baumrind D. (1968). Authoritarian vs. authoritative parental control. Adolescence, 3, 255-272.

Baumrind D. (1971). Current patterns of parental authority. Developmental Psychology Monographs, 4, 1-103. 
Baumrind D. (1972). An exploratory study of socialization effects on black children: Some blackwhite comparisons. Child Development, 43, 261-267.

Baumrind D. (1978). Parental disciplinary patterns and social competence in children. Youth and Society, 9, 239-276.

Baumrind D. (1991). The influence of parenting style on adolescent competence and substance use. Journal of Early Adolescence, 11, 56-95.

Beck S, \& Terry K. (1985). A comparison of obese and normal-weight families' psychological characteristics. American Journal of Family Therapy, 13, 55-59.

Becker WC. (1964). Consequences of different kinds of parental discipline. In ML Hoffman, \& LW Hoffman (Eds.), Review of child development research (pp. 169-208). New York, NY: Russell Sage Foundation.

Beets MW, Cardinal BJ, \& Brandon LA. (2010). Parental social support and the physical activityrelated behaviors of youth: A review. Health Education Behavior, 37, 621-644.

Beets MW, Pitetti KH, \& Forlaw L. (2007). The role of self-efficacy and referent specific social support in promoting rural adolescent girls' physical activity. American Journal of Health Behavior, 31, 227-237.

Beets MW, Vogel R, Forlaw L, Pitetti KH, \& Cardinal BJ. (2006). Social support and youth physical activity: The role of provider and type. American Journal of Health Behavior, 30, 278-289.

Bell RQ, \& Chapman M. (1986). Child effects in studies using experimental or brief longitudinal approaches to socialization. Developmental Psychology, 22, 595-603.

Belsky J. (1984). The determinants of parenting: A process model. Child Development, 55, 83-96. Beltran A, O'Connor TM, Hughes SO, Baranowski J, Nicklas TA, Thompson D, \& Baranowski T. (2012). Alpha test of a video game to train parents in effective vegetable parenting. Games for Health Journal: Research Development and Clinical Applications, 1, 219-222.

Berge JM, Wall M, Bauer KW, \& Neumark-Sztainer D. (2010). Parenting Characteristics in the Home Environment and Adolescent Overweight: A Latent Class Analysis. Obesity, 18, 818-825.

Berge JM, Wall M, Loth K, \& Neumark-Sztainer D. (2010). Parenting style as a predictor of adolescent weight and weight-related behaviors. Journal of Adolescent Health, 46, 331-338.

Berge JM, Wall M, Neumark-Sztainer D, \& Bauer K. (2010). Parenting characteristics in the home environment and adolescent obesity: A latent class analysis. Obesity, 18, 818-825.

Biddle SJH, Atkin AJ, Cavill N, \& Foster C. (2011). Correlates of physical activity in youth: A review of quantitative systematic reviews. International Review of Sport and Exercise Psychology, 4, 25-49.

Biddle SJH, Gorely T, Marshall SJ, Murdey I, \& Cameron N. (2003). Physical activity and sedentary behaviours in youth: Issues and controversies. Journal of the Royal Society of the Promotion of Health, 124, 29-33.

Biddle SJH, Pearson N, Ross GM, \& Braithwaite R. (2010). Tracking of sedentary behaviours of young people: A systematic review. Preventive Medicine, 51, 345-351.

Biddle SJH, Whitehead SH, O'Donovan TM, \& Nevill ME. (2005). Correlates of participation in physical activity for adolescent girls: A systematic review of recent literature. Journal of Physical Activity and Health, 2, 423-434. 
Birch LL, Birch D, Marlin D, \& Kramer L. (1982). Effects of instrumental eating on children's food preferences. Appetite, 3, 125-134.

Birch LL, \& Fisher JO. (2000). Mothers' child-feeding practices influence daughters' eating and weight. American Journal of Clinical Nutrition, 71, 1054-1061.

Birch LL, Fisher JO, \& Davison KK. (2003). Learning to overeat: Maternal use of restrictive feeding practices promotes girls' eating in the absence of hunger. American Journal of Clinical Nutrition, 78, 215-220.

Birch LL, Fisher JO, Grimm-Thomas K, Markey CN, Sawyer R, \& Johnson SL. (2001). Confirmatory factor analysis of the Child Feeding Questionnaire: A measure of parental attitudes, beliefs and practices about child feeding and obesity proneness. Appetite, 36, 201-210.

Birch LL, Marlin DW, \& Rotter J. (1984). Eating as the 'means' activity in a contingency: Effects on young children's food preference. Child Development, 55, 431-439.

Birch LL, Zimmerman S, \& Hind H. (1980). The influence of social-affective context on the development of children's food preferences. Child Development, 52, 856-861.

Bishop FL, Lewis G, Harris S, McKay N, Prentice P, Thiel H, \& Lewith GT. (2010). A within-subjects trial to test the equivalence of online and paper outcome measures: The Roland Morris disability questionnaire. Musculosketelal Disororders, 11:113.

Blissett J, \& Haycraft E. (2008). Are parenting style and controlling feeding practices related? Appetite, 50, 477-485.

Block JH. (1965). The child-rearing practices report. Berkely: University of California, Institute of Child Development.

Block JH. (1981). The Child-Rearing Practices Report (CRPR): A set of Q items for the description of parental socialization attitudes and values. Unpublished manuscript, Institute of Human Development, University of California, Berkeley.

Blissett J, \& Haycraft E. (2008). Are parenting style and controlling feeding practices related? Appetite, 50, 477-485.

Blissett J, Meyer C, \& Haycraft E. (2011). The role of parenting in the relationship between childhood eating problems and broader behaviour problems. Child: Care, Health and Development, 37, 642648.

Bluford DAA, Sherry B, \& Scanlon KS. (2007). Interventions to prevent or treat obesity in preschool children: A review of evaluated programs. Obesity, 15, 1356-1372.

Bogers RP, Van Assema P, Kester ADM, Westerterp KR, \& Dagnelie PC. (2004). Reproducibility, validity, and responsiveness to change of a short questionnaire for measuring fruit and vegetable intake. American Journal of Epidemiology, 159, 900-909.

Boone-Heinonen J, Gordon-Larsen P, \& Adair LS. (2008). Obesogenic clusters: Multidimensional adolescent obesity-related behaviors in the U.S. Annals of Behavioral Medicine, 36, 217-230.

Bowlby J. (1958). The nature of the child's tie to his mother. International Journal of Psychoanalysis, 39, 350-373. 
Bradley RH, \& Corwyn RF. (2008). Infant temperament, parenting, and externalizing behavior in first grade: A test of the differential susceptibility hypothesis. Journal of Child Psychology and Psychiatry, 49, 124-131.

Braet C, \& Van Strien T. (1997). Assessment of emotional, externally induced and restrained eating behaviour in nine to twelve-year-old obese and non-obese children. Behaviour Research and Therapy, 35, 863-873.

Brann LS, \& Skinner JD. (2005). More controlling child-feeding practices are found among parents of boys with an average body mass index compared with parents of boys with a high body mass index. Journal of the American Dietetic Association, 105, 1411-1416.

Brants H, Stafleu A, Ter Doest D, Hulshof K, \& Thijs C. (2006). Ontwikkeling van een voedselfrequentievragenlijst: Energie-inneming van kinderen van 2 tot en met 12 jaar [Development of a food frequency questionnaire: Energy intake of children 2-12 years of age]. Voeding Nu, 2, 25-28.

Brockman R, Jago R, Fox KR, Thompson JL, Cartwright K, \& Page AS. (2009). 'Get off the sofa and go and play': Family and socioeconomic influences on the physical activity of 10-11 year old children. BMC Public Health, 9:253.

Bronfenbrenner U. (1977). Toward an experimental ecology of human development. American Psychologist, 32, 513-531.

Brotman LM, Dawson-McClure S, Huang K, Theise R, Kamboukos D, Wang J, Petkova E, \& Ogedegbe G. (2012). Early childhood family intervention and long-term obesity prevention among high-risk minority youth. Pediatrics, 129, e621-e628.

Brown KA, Ogden J, Vogele C, \& Gibson EL. (2008). The role of parental control practices in explaining children's diet and BMI. Appetite, 50, 252-259.

Bruch H. (1973). Eating disorders: Obesity, anorexia nervosa, and the person within. New York, NY: Basic Books.

Brustad RJ. (1993). Who will go out and play? Parental and psychological influences on children's attraction to physical activity. Pediatric Exercise Science, 5, 210-223.

Buchanan T, Johnson JA, \& Goldberg LR. (2005). Implementing a five-factor personality inventory for use on the Internet. European Journal of Psychological Assessment, 21, 115-127.

Buri JR. (1991). Parental authority questionnaire. Journal of Personality Assessment, 57, 110-119.

Burton P, Smit HJ, \& Lightowler HJ. (2007). The influence of restrained and external eating patterns on overeating. Appetite, 49, 191-197.

Campbell KJ, Crawford DA, Salmon J, Carver A, Garnett SP, \& Baur LA. (2007). Associations between the home food environment and obesity-promoting eating behaviors in adolescence. Obesity, 15, 719-730.

Campbell KJ, \& Hesketh KD. (2007). Strategies which aim to positively impact on weight, physical activity, diet and sedentary behaviours in children from zero to five years. A systematic review of the literature. Obesity Reviews, 8, 327-338.

Carnell S, Haworth CMA, Plomin R, \& Wardle J. (2008). Genetic influence on appetite in children. International Journal of Obesity, 32, 1468-1473. 
Carnell S, \& Wardle J. (2007). Measuring behavioural susceptibility to obesity: Validation of the Child Eating Behaviour Questionnaire. Appetite, 48, 104-113.

Carnell S, \& Wardle J. (2008). Appetite and adiposity in children: Evidence for a behavioral susceptibility theory of obesity. American Journal of Clinical Nutrition, 88, 22-29.

Carr A, \& Pike A. (2012). Maternal scaffolding behaviour: Links with parenting style and maternal education. Developmental Psychology, 48, 543-551.

Carruth BR, \& Skinner JD. (2000). Revisiting the picky eater phenomenon: Neophobic behaviors of young children. Journal of the American College of Nutrition, 19, 771-780.

Carruth BR, Skinner J, Houck K, Moran J, Coletta F, \& Ott D. (1998). The phenomenon of 'picky eater': A behavioral marker in eating patterns of toddlers. Journal of the American College of Nutrition, 17, 180-186.

Caspi A, Harrington H, Milne B, Amell JW, Theodore RF, \& Moffitt TE. (2003). Children's behavioral styles at age 3 are linked to their adult personality traits at age 26. Journal of Personality, 71, 495514.

Caspi A, Roberts BW, \& Shiner RL. (2005). Personality development: Stability and change. Annual Review of Psychology, 56, 453-484.

Cecil JE, Palmer CAN, Wrieden W, Murrie I, Bolton-Smith C, Watt P, Wallis DJ, \& Hetherington MM. (2005). Energy intakes of children after preloads: Adjustment, not compensation. American Journal of Clinical Nutrition, 82, 302-308.

Centraal Bureau voor de Statistiek [Statistics Netherlands]. (2010). Statline CBS Databank: Education level working population. Retrieved from http://www.cbs.nl/en-GB/menu/themas/ onderwijs/cijfers/default.htm

Cervera MD, \& Méndez RM. (2006). Temperament and ecological context among Yucatec Mayan children. International Journal of Behavioral Development, 30, 326-337.

Chao RK. (1994). Beyond parental control and authoritarian parenting style: Understanding Chinese parenting through the cultural notion of training. Child Development, 65, 1111-1119.

Chen JL, \& Kennedy C. (2004). Family functioning, parenting style, and Chinese children's weight status. Journal of Family Nursing, 10, 262-279.

Chen JL, \& Kennedy C. (2005). Factors associated with obesity in Chinese-American children. Pediatric Nursing, 31, 110-115.

Chen JL, Kennedy C, Yeh CH, \& Kools S. (2005). Risk factors for childhood obesity in elementary school-age Taiwanese children. Progress in Cardiovascular Nursing, 20, 96-103.

Chen JL, Unnithan V, Kennedy C, \& Yeh CH. (2008). Correlates of physical fitness and activity in Taiwanese children. Internaitional Nursing Review, 55, 81-88.

Chin WW. (1998). Commentary: Issues and opinion on structural equation modeling. Management Information Systems Quarterly, 22, 7-16.

Clark HR, Goyder E, Bissell P, Blank L, \& Peters J. (2007). How do parents' child-feeding behaviours influence child weight? Implications for childhood obesity policy. Journal of Public Health, 29, 132141. 
Cleland V, Timperio A, Salmon J, Hume C, Baur LA, \& Crawford D. (2010). Predictors of time spent outdoors among children: 5-year longitudinal findings. Journal of Epidemiology and Community Health, 64, 400-406.

Cleland VJ, Timperio A, Salmon J, Hume C, Telford A, \& Crawford D. (2011). A longitudinal study of the family environment and physical activity among youth. American Journal of Health Promotion, 25, 159-167.

Cohen J. (1983). The cost of dichotomization. Applied Psychological Measurement, 7, 249-253.

Cohen J. (1988). Statistical power analysis for the behavioral sciences $\left(2^{\text {nd }}\right.$ edition). Hillsdale, NJ: Lawrence Erlbaum Associates, Inc.

Cohen J. (1992). A power primer. Psychological Bulletin, 112, 155-159.

Cole TJ, Flegal KM, Nicholls D, \& Jackson AA. (2007). Body mass index cut offs to define thinness in children and adolescents: International survey. British Medical Journal, 335:194.

Cole MS, Bedeian AG, \& Field HS. (2006). The measurement equivalence of web-based and paperand-pencil measures of transformational leadership: A multinational test. Organizational Research Methods, 9, 339-368.

Cole TJ, Bellizzi MC, Flegal KM, \& Dietz WH. (2000). Establishing a standard definition for child overweight and obesity worldwide: International survey. British Medical Journal, 320:1240.

Conger RD. (2009). Commentary on Grolnick and Pomerantz, 'Issues and challenges in studying parental control: Toward a new conceptualization'. Child Development Perspectives, 3, 173 175.

Corder K, Van Sluijs EMF, Goodyer I, Ridgway CL, Steele RM, Bamber D, Dunn V, Griffin SJ, \& Ekelund U. (2011). Physical activity awareness of British adolescents. Archives of Pediatric and Adolescent Medicine, 165, 603-609.

Corder K, Van Sluijs EMF, McMinn AM, Ekelund U, Cassidy A, \& Griffin SJ. (2010). Perception versus reality: Awareness of physical activity levels of British children. American Journal of Preventive Medicine, 38, 1-8.

Costa PT, \& McCrae RR. (1992). Revised NEO Personality Inventory (NEO-PI-R) and NEO Five Factor Inventory (NEO_FFI) professional manual. Odessa, FL: PAR.

Craigie AM, Lake AA, Kelly SA, Adamson AJ, \& Mathers JC. (2011). Tracking of obesity-related behaviours from childhood to adulthood: A systematic review. Maturitas, 70, 266-284.

Crawford D, Cleland V, Timperio A, Salmon J, Andrianopoulos N, Roberts R, Giles-Corti B, Baur L, \& Ball K. (2010). The longitudinal influence of home and neighbourhood environments on children's body mass index and physical activity over 5 years: The CLAN study. International Journal of Obesity, 34, 1177-1187.

Croake JW, \& Hinkle DE. (1991). Attitudes toward child rearing scale (ATCRS). In, The ETS Test Collection Catalog. Phoenix, AZ: Oryx Press.

Daniels SR. (2009). Complications of obesity in children and adolescents. International Journal of Obesity, 33(S1), 60-65. 
Daniels LA, Magarey A, Battistutta D, Nicholson JM, Farrell A, Davidson G, \& Cleghorn G. (2009). The NOURISH randomised control trial: Positive feeding practices and food preferences in early childhood - a primary prevention program for childhood obesity. BMC Public Health, 9:387.

Daniels LA, Mallan KM, Battistutta D, Nicholson JM, Perry R, \& Magarey A. (2012). Evaluation of an intervention to promote protective infant feeding practices to prevent childhood obesity: Outcomes of the NOURISH RCT at 14 months of age and 6 months post the first of two intervention modules. International Journal of Obesity, 36, 1292-1298.

Darling N, \& Steinberg L. (1993). Parenting style as context: An integrative model. Psychological Bulletin, $113,487-496$.

Davison KK. (2004). Activity-related support from parents, peers, and siblings and adolescents' physical activity: Are there gender differences? (2004). Journal of Physical Activity and Health, 1, 363-376.

Davison KK, Li K, Baskin ML, Cox T, \& Affuso O. (2011). Measuring parental support for children's physical activity in white and African American parents: The Activity Support Scale for Multiple Groups (ACTS-MG). Preventive Medicine, 52, 39-43.

Davison KK, \& Birch LL. (2001). Childhood overweight: A contextual model and recommendations for future research. Obesity Reviews, 2, 159-171.

Davison KK, Cutting TM, \& Birch LL. (2003). Parents' activity-related parenting practices predict girls' physical activity. Medicine and Science in Sports and Exercise, 35, 1589-1595.

Davison KK, \& Deane GD. (2010). The consequence of encouraging girls to be active for weight loss. Social Science and Medicine, 70, 518-525.

Davison KK, Downs DS, \& Birch LL. (2006). Pathways linking perceived athletic competence and parental support at age 9 years to girls' physical activity at age 11 years. Research Quarterly for Exercise and Sport, 77, 23-31.

Davison KK, \& Jago R. (2009). Change in parent and peer support across ages 9 to 15 yr and adolescent girls' physical activity. Medicine and Science in Sports and Exercise, 41, 1816-1825.

Davison K, \& Schmalz DL. (2006). Youth at risk of physical inactivity may benefit more from activityrelated support than youth not at risk. International Journal of Behavioral Nutrition and Physical Activity, 3:5.

De Bourdeaudhuij I, Te Velde SJ, Maes L, Pérez-Rodrigo C, De Almeida MD, \& Brug J. (2009). General parenting styles are not strongly associated with fruit and vegetable intake and socialenvironmental correlates among 11-year-old children in four countries in Europe. Public Health Nutrition, 12, 259-266.

De Haan AD, Deković M, \& Prinzie P. (2012). Longitudinal impact of parental and adolescent personality on parenting. Journal of Personality and Social Psychology, 102, 189-199.

Deković M, Janssens JMAM, \& Gerris JRM. (1991). Factor structure and construct validity of the Block child rearing practices report (CRPR). Psychological Assessment, 3, 182-187.

De Lauzon-Guillain B, Musher-Eizenman D, Leporc E, Holub S, \& Charles MA. (2009). Parental feeding practices in the United States and in France: Relationships with child's characteristics and parent's eating behavior. Journal of the American Dietetic Association, 109, 1064-1069. 
Den Exter Blokland EAW, Engels RCME, \& Finkenauer C. (2001). Parenting-styles, self-control and male juvenile delinquency: The mediating role of self-control. In M Martinez (Ed.), Prevention and control of aggression and the impact on its victims (pp. 201-207). Dordrecht/New York: Kluwer/Plenum Press.

De Onis M, Blössner M, \& Borghi E. (2010). Global prevalence and trends of overweight and obesity among preschool children. American Journal of Clinical Nutrition, 92, 1257-1264.

De Vet E, De Ridder DTD, \& De Wit JBF. (2011). Environmental correlates of physical activity and dietary behaviours among young people: A systematic review of reviews. Obesity Reviews, 12, e130-e142.

DiSantis KI, Hodges EA, Johnson SL, \& Fisher JO. (2011). The role of responsive feeding in overweight during infancy and toddlerhood: A systematic review. International Journal of Obesity, 35, 480-492.

Dishman RK, Sallis JF, \& Orenstein DR. (1985). The determinants of physical activity and exercise. Public Health Reports, 100, 158-171.

Doak CM, Visscher TLS, Renders CM, \& Seidell JC. (2006). The prevention of overweight and obesity in children and adolescents: A review of interventions and programmes. Obesity Reviews, 7, 111-136.

Dovey TM, Staples PA, Gibson EL, \& Halford JCG. (2008) Food neophobia and 'picky/fussy' eating in children: A review. Appetite, 50, 181-193.

Drucker RR, Hammer LD, Agras WS, \& Bryson S. (1999). Can mothers influence their child's behavior? Journal of Developmental and Behavioral Pediatrics, 20, 88-92.

Dubois L, Farmer A, Girard M, Peterson K, \& Tatone-Tokuda F. (2007). Problem eating behaviors related to social factors and body weight in preschool children: A longitudinal study. International Journal of Behavioral Nutrition and Physical Activity, 4:9.

Dubois L, Girard M, Potvin Kent M, Farmer A, \& Tatone-Tokuda F. (2008). Breakfast skipping is associated with differences in meal patterns, macronutrient intakes and overweight among preschool children. Public Health Nutrition, 12, 19-28.

Duncan SC, Duncan TE, \& Strycker LA. (2005). Sources and types of social support in youth physical activity. Health Psychology, 24, 3-10.

Dusseldorp E, Conversano C, \& Van Os BJ. (2010). Combining an additive and tree-based regression model simultaneously: STIMA. Journal of Computational and Graphical Statistics, 19, 514-530.

Dutman AE, Stafleu A, Kruizinga A, Brants HAM, Westerterp KR, Kistemaker C, Meuling WJA, \& Goldbohm RA. (2010). Validation of an FFQ and options for data processing using the doubly labelled water method in children. Public Health Nutrition, 14, 410-417.

Dzewaltowski DA, \& Ryan GJ. (2002). Comparing the relationships between different types of selfefficacy and physical activity in youth. Health Education and Behavior, 29, 491-504.

Edwardson CL, \& Gorely T. (2010). Parental influences on different types and intensities of physical activity in youth: A systematic review. Psychology of Sport and Exercise, 11, 522-535.

Edwardson CL, \& Gorely T. (2010). Activity-related parenting practices and children's objectively measured physical activity. Pediatric Exercise Science, 22, 105-113. 
Eggers K, De Nil LF, \& Van den Bergh BRH. (2009). Factorial temperament structure in stuttering, voice disordered, and normal speaking children. Journal of Speech, Language, and Hearing Research, 52, 1610-1622.

Eggers K, De Nil LF, \& Van den Bergh BRH. (2010). Temperament dimensions in stuttering and typically developing children. Journal of Fluency Disorders, 35, 355-372.

Elfhag K, Tholin S, \& Rasmussen F. (2008). Consumption of fruit, vegetables, sweets and soft drinks are associated with psychological dimensions of eating behaviour in parents and their 12-year-old children. Public Health Nutrition, 11, 914-923.

Enten RS, \& Golan M. (2008). Parenting styles and weight-related symptoms and behaviors with recommendations for practice. Nutrition Reviews, 66, 65-75.

Environmental Health Risks in European Birth Cohorts (ENRIECO). (2012). Retrieved from http://www.enrieco.org.

Epstein LH, Gordy CC, Raynor HA, Beddome M, Kilanoswski CK, \& Paluch R. (2001). Increasing fruit and vegetable intake and decreasing fat and sugar intake in families at risk for childhood obesity. Obesity Research, 9, 171-178.

Epstein LH, McCurley J, Wing RR, \& Valoski A. (1990). Five-year follow-up of family-based behavioural treatments for childhood obesity. Journal of Consulting and Clinical Psychology, 58, 661-664.

Epstein LH, McKenzie SJ, Valoski A, Klein KR, \& Wing RR. (1994). Effects of mastery criteria and contingent reinforcement for family-based child weight control. Addictive Behaviors, 19, 135-145.

Epstein LH, Paluch RA, \& Raynor HA. (2001). Sex differences in obese children and siblings in family-based obesity treatment. Obesity Research, 9, 746-753.

Epstein LH, Valoski A, Wing RR, \& McCurley J. (1994). Ten-year outcomes of behavioral family-based treatment for childhood obesity. Health Psychology, 13, 373-383.

Epstein LH, Valoski A, Wing RR, \& McCurley J. (1990). Ten-year follow-up of behavioral, family-based treatment for obese children. Journal of the American Medical Association, 264, 2519-2523.

Epstein LH, Wing RR, Koeske R, \& Valoski A. (1985). A comparison of lifestyle exercise, aerobic exercise, and calisthenics on weight loss in obese children. Behavior Therapy, 16, 345-356.

Eurostat. Task Force on Core Social Variables. Final Report 2007. Luxembourg: European Communitees.

Fagot BI, \& Leve LD. (1998). Teacher ratings of externalizing behavior at school entry for boys and girls: Similar early predictors and different correlates. Journal of Child Psychology \& Allied Disciplines, 39, 555-566.

Faith MS, Berkowitz RI, Stallings VA, Kerns J, Storey M, \& Stunkard AJ. (2004). Parental feeding attitudes and styles and child body mass index: Prospective analysis of a gene-environment interaction. Pediatrics, 114, e429-e436.

Faith MS, Scanlon KS, Birch LL, Francis LA, \& Sherry B. (2004). Parent-child feeding strategies and their relationships to child eating and weight status. Obesity Research, 12, 1711-1722. 
Faith MS, Van Horn L, Appel LJ, Burke LE, Carson JAS, Franch HA, Jakicic JM, Kral TVE, Odoms-Young A, Wansink B, \& Wylie-Rosett J. (2012). Evaluating parents and adult caregivers as 'agents of change' for treating obese children: Evidence for parent behavior change strategies and research gaps: A scientific statement from the American Heart Association. Circulation, 125, 1186-1207.

Fazio RH, \& Olson MA. (2003). Implicit measures in social cognition research: their meaning and use. Annual Review of Psychology, 54, 297-327.

Ferreira I, Van der Horst K, Wendel-Vos W, Kremers S, Van Lenthe FJ, \& Brug J. (2006). Environmental correlates of physical activity in youth - A review and update. Obesity Reviews, 8 , 129-154.

Fisher PA. (1993). Temperament goodness of fit and psychosocial adjustment in children (Unpublished doctoral dissertation). University of Oregon, Eugene.

Fisher WP. (1992). Reliability statistics. Rasch Measurement Transactions, 6:3.

Fisher JO, \& Birch LL. (1999a). Restricting access to foods and children's eating. Appetite, 32, 405419.

Fisher JO, \& Birch LL. (1999b). Restricting access to palatable foods affects children's behavioral response, food selection, and intake. American Journal of Clinical Nutrition, 69, 1264-1272.

Fisher A, Saxton J, Hill C, Webber L, Purslow L, \& Wardle J. (2010). Psychosocial correlates of objectively measured physical activity in children. European Journal of Public Health, 21, 145-150.

Fisher AB, Schaefer BA, Watkins MW, Worrell FC, \& Hall TE. (2006). The factor structure of the fear survey Schedule for children-II in Trinidadian children and adolescents. Anxiety Disorders, 20, $740-$ 759.

Flodmark CE, Ohlsson T, Rydén O, \& Sveger T. (1993). Prevention of progression to severe obesity in a group of obese schoolchildren treated with family therapy. Pediatrics, 91, 880-884.

Foltz JL, Cook SR, Szilagyi PG, Auinger P, Stewart PA, Bucher S, \& Baldwin CD. (2011). US adolescent nutrition, exercise, and screen time baseline levels prior to national recommendations. Clinical Pediatrics, 50, 424-433.

Forrest CB, Bevans K, Tucker C, Riley AW, Ravens-Sieberer U, Gardner W, \& Pajer K. (2012). Commentary: The Patient-Reported Outcome Measurement Information System (PROMIS ${ }^{\circledR}$ ) for children and youth: Application to pediatric psychology. Journal of Pediatric Psychology, 37, 614621.

Francis LA, Hofer SM, \& Birch LL. (2001). Predictors of maternal child-feeding style: Maternal and child characteristics. Appetite, 37, 231-243.

Freedman DS, Khan LK, Serdula MK, Dietz WH, Srinivasan SR, \& Berenson GS. (2005). The relation of childhood BMI to adult adiposity: The Bogalusa Heart Study. Pediatrics, 115, 22-27.

Friedman SL, \& Wachs TD. (1999). Measuring environment across the life span: Emerging methods and concepts. Washington, DC: American Psychological Association.

Fuemmeler BF, Yang C, Costanzo P, Hoyle RH, Siegler IC, Williams RB, \& Østbye T. (2012). Parenting styles and body mass index trajectories from adolescence to adulthood. Health Psychology, 31, 441449. 
Gable S, \& Lutz S. (2000). Household, parent, and child contributions to childhood obesity. Family Relations, 49, 293-300.

Gartstein MA, Knyazev GG, \& Slobodskaya HR. (2005). Cross-cultural differences in the structure of infant temperament: United States of America (U.S.) and Russia. Infant Behavior and Development, 28, 54-61.

Gartstein MA, \& Rothbart MK. (2003). Studying infant temperament via the Revised Infant Behavior Questionnaire. Infant Behavior and Development, 26, 64-86.

Gattshall ML, Shoup JA, Marshall JA, Crane LA, \& Estabrooks PA. (2008). Validation of a survey instrument to assess home environments for physical activity and healthy eating in overweight children. International Journal of Behavioral Nutrition and Physical Activity, 5:3.

Gerards SMPL, Dagnelie PC, Jansen MWJ, Van der Goot LOHM, De Vries NK, Sanders MR, \& Kremers SPJ. (2012). Lifestyle triple P: A parenting intervention for childhood obesity. BMC Public Health, $12: 267$

Gerards SMPL, Sleddens EFC, Dagnelie PC, De Vries NK, \& Kremers SPJ. (2011). Interventions addressing general parenting to prevent or treat childhood obesity. International Journal of Pediatric Obesity, 6, e28-e45.

Gerris JRM, Houtmans MJM, Kwaaitaal-Roosen EMG, Schipper JC, Vermulst AA, \& Janssens JMAM. (1998). Parents, adolescents and Young adults in Dutch families: A longitudinal study. Nijmegen: Institute of Family Studies, University of Nijmegen.

Gerris JRM, Van Boxtel DAAM, Vermulst AA, Janssens JMAM, Van Zutphen RAH, \& Felling AJA. (1993). Parenting in Dutch families. Nijmegen, the Netherlands: University of Nijmegen, Institute of Family Studies.

Gewirtz JL. (1956). A program of research on the dimensions and antecedents of emotional dependence. Child Development, 27, 205-221.

Gibson LY, Byrne SM, Davis EA, Blair E, Jacoby P, \& Zubrick SR. (2007). The role of family and maternal factors in childhood obesity. Medical Journal of Australis, 186, 591-595.

Golan M. (2006). Parents as agents of change in childhood obesity - from research to practice. International Journal of Pediatric Obesity, 1, 66-76.

Golan M, \& Crow S. (2004). Parents are key players in the prevention and treatment of weight-related problems. Nutrition Reviews, 62, 39-50.

Golan M, Fainaru M, \& Weizman A. (1998). Role of behaviour modification in the treatment of childhood obesity with the parents as the exclusive agents of change. International Journal of Obesity, 22, 1217-1224.

Golan M, Kaufman V, \& Shahar DR. (2006). Childhood obesity treatment: Targeting parents exclusively v. parents and children. British Journal of Nutrition, 95, 1008-1015.

Golan M, \& Weizman A. (2001). Familial approach to the treatment of childhood obesity: Conceptual model. Journal of Nutrition Education, 33, 102-107.

Golan M, Weizman A, Apter A, \& Fainaru M. (1998). Parents as the exclusive agents of change in the treatment of childhood obesity. American Journal of Clinical Nutrition, 67, 1130-1135. 
Goldberg LR. (1990). An alternative 'description of personality': The Big-Five factor structure.

Personality processes and individual differences, 59, 1216-1229.

Goldsmith HH, Buss KA, \& Lemery KS. (1997). Toddler and childhood temperament: Expanded content, stronger genetic evidence, new evidence for the importance of environment. Developmental Psychology, 33, 891-905.

Golley RK. (2005). Family-focused management of overweight in pre-pubertal children - a randomised controlled trial. The healthy eating and lifestyle through positive parenting (HELPP) study. School of Medicine, Flinders University.

Golley RK, Magarey AM, Baur LA, Steinbeck KS, \& Daniels LA. (2007). Twelve-month effectiveness of a parent-led, family-focused weight-management program for prepubertal children: A randomized, controlled trial. Pediatrics, 119, 517-525.

Golley RK, Magarey AM, \& Daniels LA. (2011). Children's food and activity patterns following a sixmonth child weight management program. International Journal of Pediatric Obesity, 6, 409-414.

Golley RK, Perry RA, Magarey A, \& Daniels L. (2007). Family-focused weight management program for fiveto nine-year-olds incorporating parenting skills training with healthy lifestyle information to support behaviour modification. Nutrition and Dietetics, 64, 144-150.

Goodyer IM, Ashby L, Altham PM, Vize C, \& Cooper PJ. (1993). Temperament and major depression in 11- to 16-year-olds. Journal of Child Psychology and Psychiatry, 34, 1409-1423.

Gordon R. (1987). An operational definition of disease prevention. In JA Sternberg, MM Silverman, \& MD Rockville (Eds.), Preventing Mental Disorders (pp. 20-26). U.S. Department of Health and Human Services.

Gottlieb NH, \& Baker JA. (1986). The relative influence of health beliefs, parental and peer behaviors and exercise program participation on smoking, alcohol use, and physical activity. Social Science and Medicine, 22, 915-927.

Gregory JE, Paxton SJ, \& Brozovic AM. (2010). Maternal feeding practices, child eating behaviour and body mass index in preschool-aged children: A prospective analysis. International Journal of Behavioral Nutrition and Physical Activity, 7:55.

Grolnick WS, \& Pomerantz EM. (2009). Issues and challenges in studying parental control: Toward a new conceptualization. Child Development Perspectives, 3, 165-170.

Grolnick WS, Price CE, Beiswenger KL, \& Sauck CC. (2007). Evaluative pressure in mothers: Effects of situation, maternal, and child characteristics on autonomy supportive versus controlling behavior. Developmental Psychology, 43, 991-1002.

Grolnick WS, \& Ryan RM. (1989). Parent styles associated with children's self-regulation and competence in school. Journal of Educational Psychology, 81, 143-154.

Grolnick WS, Ryan RM, \& Deci E. (1991). Inner resources for school achievement: Motivational mediators of children's perceptions of their parents. Journal of Educational Psychology, 83, 508517.

Grolnick WS, \& Slowiaczek ML. (1994). Parents' involvement in children's schooling: A multi-dimensional conceptualization and motivational model. Child Development, 65, 237-252. 
Grusec JE. (2009). Commentary on the proposed abandonment of multiple forms of control. Child Development Perspectives, 3, 171-172.

Grusec JE, \& Davidov M. (2010). Integrating different perspectives on socialization theory and research: A domain-specific approach. Child Development, 81, 687-709.

Gubbels JS. (2010). Influence of micro-environments on pre-school children's energy balance-related behaviors and weight status. Dissertation.

Gubbels JS, Kremers SPJ, Goldbohm RA, Stafleu A, \& Thijs C. (2012). Energy balance-related behavioural patterns in 5-year-old children and the longitudinal association with weight status development in early childhood. Public Health Nutrition, 15, 1402-1410.

Gubbels JS, Kremers SPJ, Stafleu A, Dagnelie PC, De Vries NK, \& Thijs C. (2010). Child-care environment and dietary intake of 2- and 3-year-old children. Journal of Human Nutrition and Dietetics, 23, 97-101.

Gubbels JS, Kremers SPJ, Stafleu A, Dagnelie PC, Goldbohm RA, De Vries NK, \& Thijs C. (2009). Dietrelated restrictive parenting practices: Impact on dietary intake of 2-year-old children and interactions with child characteristics. Appetite, 52, 423-429.

Gubbels JS, Kremers SPJ, Stafleu A, De Vries SI, Goldbohm RA, Dagnelie PC, De Vries NK, Van Buuren S, \& Thijs C. (2011). Association between parenting practices and children's dietary intake, activity behavior and development of body mass index: The KOALA Birth Cohort Study. International Journal of Behavioral Nutrition and Physical Activity, 8:18.

Gubbels JS, Kremers SPJ, Van Kann DH, Stafleu A, Candel MJJM, Dagnelie PC, Thijs C, De Vries NK. (2011). Interaction between physical environment, social environment, and child characteristics in determining physical activity at child care. Health Psychology, 30, 84-90.

Gupta N, Goel K, Skah P, \& Misra A. (2012). Childhood obesity in developing countries: Epidemiology, determinants, and prevention. Endocrine Reviews, 33, 48-70.

Gustafson SL, \& Rhodes RE. (2006). Parental correlates of physical activity in children and early adolescents. Sports Medicine, 36, 79-97.

Hallal PC, Victora CG, Azevedo MR, \& Wells JCK. (2006). Adolescent physical activity and health: A systematic review. Sports Medicine, 36, 1019-1030.

Hardy DF, Power TG, \& Jaedicke S. (1993). Examining the relation of parenting to children's coping with everyday stress. Child Development, 64, 1829-1841.

Harrison F, \& Jones AP. (2012). A framework for understanding school based physical environmental influences on childhood obesity. Health \& Place, 18, 639-648.

Harvey-Berino J, \& Rourke J. (2003). Obesity prevention in preschool native-american children: A pilot study using home visiting. Obesity Research, 11, 606-611.

Haycraft EL, \& Blissett JM. (2008). Maternal and paternal controlling feeding practices: Reliability and relationships with BMI. Obesity, 16, 1552-1558.

Healey DM, Brodzinsky LK, Bernstein M, Rabinovitz B, \& Halperin JM. (2010). Moderating effects of neurocognitive abilities on the relationship between temperament and global functioning. Child Neuropsychology, 16, 20-31. 
Heesch KC, Mâsse LC, \& Dunn AL. (2006). Using Rasch modeling to re-evaluate three scales related to physical activity: Enjoyment, perceived benefits and perceived barriers. Health Education Research, 21 (S1), i58-i72.

Heitmann BL, Westerterp KR, Loos RJF, Sørensen TIA, O’Dea K, Mc Lean P, Jensen TK, Eisenmann J, Speakman JR, Simpson SJ, Reed DR, \& Westerterp-Plantenga MS. (2012). Obesity: Lessons from evolution and the environment. Obesity Reviews, 13, 910-922.

Heitzler CD, Lytle LA, Erickson DJ, Barr-Anderson D, Sirard JR, \& Story M. (2010). Evaluating a model of youth physical activity. American Journal of Health Behavior, 34, 593-606.

Heitzler CD, Martin SL, Duke J, \& Huhman M. (2006). Correlates of physical activity in a national sample of children aged 9-13 years. Preventive Medicine, 42, 254-260.

Hejazi S. (2007). Temperament, parenting, and the development of childhood obesity. The University of British Columbia.

Haycraft E, \& Blissett J. (2011). Predictors of paternal and maternal controlling feeding practices with 2- to 5-year-old children. Journal of Nutrition Education and Behavior, 44, 390-397.

Hennessy E, Hughes SO, Goldberg JP, Hyatt RR, \& Economos CD. (2010a). Parent-child interactions and objectively measured child physical activity: A cross-sectional study. International Journal of Behavioral Nutrition and Physical Activity, 7:71.

Hennessy E, Hughes SO, Goldberg JP, Hyatt RR, \& Economos CD. (2010b). Parent behavior and child weight status among a diverse group of underserved rural families. Appetite, 54, 369-377.

Hetherington EM, \& Clingempeel G. (1983). The molecular family coding system. Unpublished manuscript, University of Virginia.

Hinkley T, Crawford D, Salmon J, Okely AD, \& Hesketh K. (2008). Preschool children and physical activity: A review of correlates. American Journal of Preventive Medicine, 34, 435-441.

Ho DYF. (1986). Chinese patterns of socialization: A critical review. In MH Bond (Ed.), The psychology of the Chinese people (pp. 1-37). New York, NY: Oxford University Press.

Holden GW, \& Edwards LA. (1989). Parental attitudes toward child rearing: Instruments, issues and implication. Pyschological Bulletin, 106, 29-58.

Hollenstein T, \& Lewis M. (2006). A state space analysis of emotion and flexibility in parent-child interaction. Emotion, 6, 656-662.

Holmbeck GN, Johnson SZ, Wills KE, McKernon W, Rose B, Erklin S, \& Kemper T. (2002). Observed and perceived parental overprotection in relation to psychosocial adjustment in preadolescents with a physical disability: The meditational role of behavioural autonomy. Journal of Consulting and Clinical Psychology, 70, 96-110.

Horn MG, Galloway AT, Webb RM, \& Gagnon SG. (2011). The role of child temperament in parental child feeding practices and attitudes using a sibling design. Appetite, 57, 510-516.

Horne PJ, Tapper K, Lowe CF, Hardman CA, Jackson MC, \& Woolner J. (2004). Increasing children's fruit and vegetable consumption: A peer-modelling and rewards-based intervention. European Journal of Clinical Nutrition, 58, 1649-1660.

Hu L, \& Bentler PM. (1995). Evaluating model fit. In RH Hoyle (Ed.), Structural equation modeling: concepts, issues, and applications (pp. 76-99). Thousand Oaks, CA: Sage Publication. 
Huaidong DU, \& Feskens E. (2010). Dietary determinants of obesity. Acta Cardiologica, 65, 377-386. Huang JS, Becerra K, Golnari G. Fernandez S, Opalach A, \& Andres del Valle A. (2009). Digital facial image modification, body image, and parental support for dietary and physical activity behaviors. Journal of Pediatrics, 154, 74-78.

Hubbs-Tait L, Kennedy TS, Page MC, Topham GL, \& Harrist AW. (2008). Parental feeding practices predict authoritative, authoritarian, and permissive parenting styles. Journal of the American Dietetic Association, 108, 1154-1161.

Hughes SO, Patrick H, Power TG, Fisher JO, Anderson CB, \& Nicklas TA. (2007). The impact of child care providers' feeding on children's food consumption. Journal of Developmental and Behavioral Pediatrics, 28, 100-107.

Hughes SO, Power TG, Fisher JO, Mueller S, \& Nicklas TA. (2005). Revisiting a neglected construct: Parenting styles in a child-feeding context. Appetite, 44, 83-92.

Hughes SO, \& Shewchuk RM. (2012). Child temperament, parent emotions, and perceptions of the child's feeding experience. International Journal of Behavioral Nutrition and Physical Activity, 9:64.

Hughes SO, Shewchuk RM, Baskin ML, Nicklas TA, \& Qu H. (2008). Indulgent feeding style and children's weight status in preschool. Journal of Developmental and Behavioral Pediatrics, 29, 403-410.

Humenikova L, \& Gates GE. (2008). Social and physical environmental factors and child overweight in a sample of American and Czech school-aged children: A pilot study. Journal of Nutrition Education and Behavior, 40, 251-257.

Hunt C. (2003). The parenting puzzle: How to get the best out of family life. Oxford, UK: Family Links. Hurley KM, Cross MB, \& Hughes SO. (2011). A systematic review of responsive feeding and child obesity in high-income countries. Journal of Nutrition, 141, 495-501.

Israel AC, Stolmaker L, \& Andrian CA. (1985). The effects of traning parents in general child management skills on a behavioral weight loss program for children. Behavior Therapy, 16, 169-180.

Israel AC, Guile CA, Baker JE, \& Silverman WK. (1994). An evaluation of enhanced self-regulation training in the treatment of childhood obesity. Journal of Pediatric Psychology, 19, 737-749.

levers-Landis CE, Burant C, Drotar D, Morgan L, Trapl ES, \& Kwoh K. (2003). Social support, knowledge, and self-efficacy as correlates of osteoporosis preventive behaviors among preadolescent females. Journal of Pediatric Psychology, 28, 335-345.

Jackson C, Bee-Gates DJ, \& Henriksen L. (1994). Authoritative parenting, child competencies, and initiation of cigarette smoking. Health Education Quarterly, 21, 103-116.

Jago R, Davison KK, Brockman R, Page AS, Thompson JL, \& Fox KR. (2011). Parenting styles, parenting practices, and physical activity in 10- to 11-year olds. Preventive Medicine, 52, 44 47.

Jago R, Edwards MJ, Sebire SJ. (submitted). General and specific approaches to media parenting: A systematic review of current measures, associations with screen-viewing and measurement implications. 
Jago R, Fox KR, Page AS, Brockman R, \& Thompson JL. (2009). Development of scales to assess children's perceptions of friend and parental influences on physical activity. International Journal of Behavioral Nutrition and Physical Activity, 6:67.

James J, \& Kerr D. (2005). Prevention of childhood obesity by reducing soft drinks. International Journal of Obesity, 29 (S2), 54-57.

James J, Thomas P, Cavan D, \& Kerr D. (2004). Preventing childhood obesity by reducing consumption of carbonated drinks: Cluster randomised controlled trial. British Medical Journal, 328, 1237-1239.

Janicke DM, Sallinen BJ, Perri MG, Lutes LD, Silverstein JH, Huerta MG, \& Guion LA. (2008). Sensible treatment of obesity in rural youth (STORY): Design and methods. Contemporary Clinical Trials, 29, 270-280.

Jansen E, Daniels LA, \& Nicholson JM. (2012). The dynamics of parenting and early feeding constructs and controversies: A viewpoint. Early Child Development and Care, 182, 967-981.

Janssen I, Katzmarzyk PT, Boyce WF, Vereecken C, Mulvihill C, Roberts C, Currie C, Pickett W, \& the Health Behaviours in School-Aged Children Obesity Working Group. (2005). Comparison of overweight and obesity prevalence in school-aged youth from 34 countries and their relationships with physical activity and dietary patterns. Obesity Reviews, 6, 123-132.

Jansen E, Mulkens S, \& Jansen A. (2007). Do not eat the red food! Prohibition of snacks leads to their relatively higher consumption in children. Appetite, 49, 572-577.

Jansen PW, Roza SJ, Jaddoe VWV, Mackenbach JD, Raat H, Hofman A, Verhulst FC, \& Tiemeier H. (2012). Children's eating behavior, feeding practices of parents and weight problems in early childhood: results from the population-based Generation R Study. International Journal of Behavioral Nutrition and Physical Activity, 9:130.

Jebb SA. (2005). Dietary strategies for the prevention of obesity. Proceedings of the Nutrition Society, 64, 217-27.

Jo M-S. (2000). Controlling social-desirability bias via method factors of direct and indirect questioning in structural equation models. Psychology \& Marketing, 17, 137-148.

John OP, \& Srivastava S. (1999). The Big Five trait taxonomy: History, measurement, and theoretical perspectives. In LA Pervin, \& OP John (Eds.), Handbook of personality: Theory and research $\left(2^{\text {nd }}\right.$ edition, pp. 102-138). New York, NY: Guilford Press.

Johnson R, Welk G, Saint-Maurice PF, \& Ihmels M. (2012). Parenting styles and home obesogenic environments. International Journal of Environmental Research and Public Health, 9, 14111426.

Joyce JL, \& Zimmer-Gembeck MJ. (2009). Parent feeding restriction and child weight. The mediating role of child disinhibited eating and the moderating role of the parenting context. Appetite, 52, 726-734.

Kahan D. (2005). Jewish day-schooled adolescents' perceptions of parental and environmental support of physical activity. Research Quarterly for Exercise and Sport, 76, 243-250.

Kalarchian MA, Levine MD, Arslanian SA, Ewing LJ, Houck PR, Cheng Y, Ringham RM, Sheets CA, \& Marcus MD. (2009). Family-based treatment of severe pediatric obesity: Randomized, controlled trial. Pediatrics, 124, 1060-1068. 
Kaplan HI, \& Kaplan HS. (1957). The psychosomatic concept of obesity. Journal of Nervous and Mental Disease, 125 , 181-201.

Kiel EJ, \& Buss KA. (2011). Prospective relations among fearful temperament, protective parenting, and social withdrawal: The role of maternal accuracy in a moderated mediation framework. Journal of Abnormal Child Psychology, 39, 953-966.

Kim MJ. (2006). Parenting style and older children's and young adolescents' dietary intake and nutritional status. Texas A\&M University.

Kim MJ, Mclntosh WA, Anding J, Kubena KS, Reed DB, \& Moon GS. (2008). Perceived parenting behaviours predict young adolescents' nutritional intake and body fatness. Maternal and Child Nutrition, 4, 287-303.

Kimiecik JC, \& Horn TS. (1998). Parental beliefs and children's moderate-to-vigorous physical activity. Research Quarterly for Exercise and Sport, 69, 163-175.

Kimiecik JC, Horn TS, \& Shurin CS. (1996). Relationships among children's beliefs, perceptions of their parents' beliefs, and their moderate-to-vigorous physical activity. Research Quarterly for Exercise and Sport, 67, 324-336.

King AC, Parkinson KN, Adamson AJ, Murray L, Besson H, Reilly JJ, Basterfield, \& the Gateshead Millennium Study Core Team. (2011). Correlates of objectively measured physical activity and sedentary behaviour in English children. European Journal of Public Health, 21, 424-431.

Kirby J, Levin KA, \& Inchley J. (2011). Parental and peer influences on physical activity among Scottish adolescents: A longitudinal study. Journal of Physical Activity and Health, 8, 785-793.

Kitzmann KM, \& Beech BM. (2006). Family-based interventions for pediatric obesity: Methodological and conceptual challenges from family psychology. Journal of Family Psychology, 20, 175-189.

Kitzmann KM, Dalton WT, \& Buscemi J. (2008). Beyond parenting practices: Family context and the treatment of pediatric obesity. Family Relations, 57, 13-23.

Klesges RC, Eck LH, Hanson CL, Haddock CK, \& Klesges LM. (1990). Effects of obesity, social interactions, and physical environment on physical activity in preschoolers. Health Psychology, 9, 435-449.

Kline RB. (1998). Principles and Practice of Structural Equation Modeling. New York, NY: The Guilford Press.

Kochanska G, Aksan N, \& Joy ME. (2007). Children's fearfulness as a moderator of parenting in early socialization: Two longitudinal studies. Developmental Psychology, 43, 222-237.

Kochanska G, Aksan N, Knaack A, \& Rhines HM. (2004). Maternal parenting and children's conscience: Early security as a moderator. Child Development, 75, 1229-1242.

Kochanska G, De Vet K, Goldman M, Murray K, \& Putnam SP. (1994). Maternal reports of conscience development and temperament in young children. Child Development, 65, 852-868.

Kochanska G, Murray K, Jacques TY, Koenig AL, \& Vandegeest KA. (1996). Inhibitory control in young children and its role in emerging internalization. Child Development, 67, 490-507.

Kohnstamm GA. (1989). Temperament in childhood: Cross-cultural and sex differences. In GA Kohnstamm, JE Bates, \& MK Rothbart (Eds.), Temperament in childhood (pp. 483-508). Chichester, England: Wiley. 
Koivisto UK, Fellenius J, \& Sjödén PO. (1994). Relations between parental mealtime practices and children's food intake. Appetite, 22, 245-257.

Kongsved SM, Basnov M, Holm-Christensen K, Hjollund NH. (2007). Response rate and completeness of questionnaires: A randomized study of internet versus paper-and-pencil versions. Journal of Medical Internet Research, 9:e25.

Kremers SPJ. (2010). Theory and practice in the study of influences on energy balance-related behaviors. Patient Education and Counseling, 79, 291-298.

Kremers SPJ, Brug J, De Vries H, \& Engels RCME. (2003). Parenting style and adolescent fruit consumption. Appetite, 41, 43-50.

Kremers SPJ, De Bruijn G-J, Droomers M, Van Lenthe F, Brug J. (2007). Moderators of environmental intervention effects on diet and activity in youth. American Journal of Preventive Medicine, 32, 163172.

Kremers SPJ, De Bruijn G-J, Visscher TLS, Van Mechelen W, De Vries NK, \& Brug J. (2006).

Environmental influences on energy balance-related behaviors: A dual-process view. International Journal of Behavioral Nutrition and Physical Activity, 3:9.

Kremers SPJ, Sleddens EFC, Gerards SMPL, Gubbels JS, Rodenburg G, Gevers D, \& Van Assema P. (submitted). General and food-specific parenting: Measures and interplay.

Kremers SPJ, Visscher TLS, Seidell JC, Van Mechelen W, \& Brug J. (2005). Cognitive determinants of energy balance-related behaviours: Measurement issues. Sports Medicine, 35, 923-933.

Kröller K, \& Warschburger P. (2009). Maternal feeding strategies and child's food intake: Considering weight and demographic influences using structural equation modeling. International Journal of Behavioral Nutrition and Physical Activity, 6:78.

Kummeling I, Thijs C, Penders J, Snijders BEP, Stelma F, Reimerink J, Koopmans M, Dagnelie PC, Huber M, Jansen MC, De Bie R, \& Van den Brandt PA. (2005). Etiology of atopy in infancy: The KOALA Birth Cohort Study. Pediatric Allergy and Immunology, 16, 679-684.

Kusanagi E. (1993). A psychometric examination of the Children's Behavior Questionnaire. In Annual Report of the Research and Clinical Center for Child Development, 1991-1992. Sapporo, Japan: Hokkaido University, Faculty of Education.

Lamborn SD, Mounts NS, Steinberg L, \& Dornbusch SM. (1991). Patterns of competence and adjustment among adolescents from authoritative, authoritarian, indulgent, and neglectful families. Child Development, 62, 1049-1065.

Landsford JE, Deater-Deckard K, Dodge KA, Bates JE, \& Pettit GS. (2004). Ethnic differences in the link between physical discipline and later adolescent externalizing behaviors. Journal of Child Psychology and Psychiatry, 45, 801-812.

Larios SE, Ayala GX, Arredondo EM, Baquero B, \& Elder JP. (2009). Development and validation of a scale to measure Latino parenting strategies related to children's obesigenic behaviors. The parenting strategies for eating and activity scale (PEAS). Appetite, 52, 166-172.

LeCuyer EA, Swanson DP, Cople R, \& Kitzman H. (2011). Effect of African- and European-American maternal attitudes and limit-setting strategies on children's self-regulation. Research in Nursing and Health, 34, 468-482. 
Leite WL, \& Beretvas N. (2005). Validation of scores on the Marlowe-Crowne social desirability scale and the balanced inventory of desirable responding. Educational and Psychological Measurement, 65, 140-154.

Levy P. (1967). The correction for spurious correlation in the evaluation of short-form tests. Journal of Clinical Psychology, 23, 84-86.

Liem DG, Mars M, \& De Graaf C. (2004). Sweet preferences and sugar consumption of 4- and 5-yearold children: Role of parents. Appetite, 43, 235-245.

Li S, Treuth MS, \& Wang Y. (2010). How active are American adolescents and have they become less active? Obesity Reviews, 11, 847-862.

Lindahl KM, Clements M, \& Markman H. (1997). Predicting marital and parent functioning in dyads and triads: A longitudinal investigation of marital process. Journal of Family Psychology, 11, 139151.

Lindhout IE, Markus MT, Hoogendijk THG, \& Boer F. (2009). Temperament and parental child-rearing style: Unique contributions to clinical anxiety disorders in childhood. European Child and Adolescent Psychiatry, 18, 439-446.

Lipsey MW. (1990). Design sensitivity: Statistical power for experimental research. Newbury Park, CA: Sage Publications.

Lissau I, \& Sørensen TIA. (1994). Parental neglect during childhood and increased risk of obesity in young adulthood. Lancet, 343, 324-327.

Lobstein T, Baur L \& Uauy F. For the IASO International Obesity TaskForce. (2004). Obesity in children and young people: A crisis in public health. Obesity Reviews, 5 (S1), 4-85.

Lock K, Pomerleau J, Causer L, Altmann DR, \& McKee M. (2005). The global burden of disease attributable to low consumption of fruit and vegetables: Implications for the global strategy on diet. Bulletin of the World Health Organization, 83, 100-108.

Locke LM, \& Prinz RJ. (2002). Measurement of parental discipline and nurturance. Clinical Psychology Review, 22, 895-929.

Lohaus A, Vierhaus M, \& Ball J. (2009). Parenting Styles and Health-Related Behavior in Childhood and Early Adolescence. Journal of Early Adolescence, 29, 449-475.

Lollis S, \& Kuczynski L. (1997). Beyond one hand clapping: Seeing bidirectionality in parent-child relations. Journal of Social and Personal Relationships, 14, 441-461.

Loprinzi PD, \& Trost SG. (2010). Parental influences on physical activity behavior in preschool children. Preventive Medicine, 50, 129-133.

Lowe CF, Horne PJ, Tapper K, Bowdery M, \& Egerton C. (2004). Effects of a peer modelling and rewards-based intervention to increase fruit and vegetable consumption in children. European Journal of Clinical Nutrition, 58, 510-522.

Ludrosky JM. (2005). Childhood obesity: Multifactoral determinants of child weight and eating behaviors. Miami University.

Ludwig DS, Peterson KE, \& Gortmaker SL. (2001). Relation between consumption of sugarsweetened drinks and childhood obesity: A prospective, observational analysis. Lancet, 357, 505508. 
Lytle LA, Varnell S, Murray DM, Story M, Perry C, Birnbaum AS, \& Kubik MY. (2003). Predicting adolescents' intake of fruits and vegetables. Journal of Nutrition Education and Behavior, 35, 170-175.

Maccoby EE, \& Martin JA. (1983). Socialization in the context of the family: parent-child interaction. In EM Hetherington (Ed.), PH Mussen (Series Ed.), Handbook of child psychology, Vol. 4: Socialization, personality, and social development (pp. 1-101). New York, NY: Wiley.

Magarey AM, Daniels LA, Boulton TJ, \& Cockington RA. (2003). Predicting obesity in early adulthood from childhood and parental obesity. International Journal of Obesity, 27, 505-513.

Magarey AM, Perry RA, Baur LA, Steinbeck KS, Sawyer M, Hills AP, Wilson G, Lee A, \& Daniels LA. (2011). A parent-led family-focused treatment program for overweight children aged 5 to 9 years: The PEACH RCT. Pediatrics, 127, 214-222.

Majdandžić M, \& Van den Boom DC. (2007). Multimethod longitudinal assessment of temperament in early childhood. Journal of Personality, 75, 121-168.

Majdandžić M, Van den Boom DC, \& Heesbeen DGM. (2008). Peas in a pod: Biases in the measurement of sibling temperament? Developmental Psychology, 44, 1354-1368.

Malina RM, \& Little BB. (2008). Physical activity: The present in the context of the past. American Journal of Human Biology, 20, 373-391.

Marshall SJ, Biddle SJH, Gorely T, Cameron N, \& Murdey I. (2004). Relationships between media use, body fatness and physical activity in children and youth: A meta-analysis. International Journal of Obesity and Related Metabolic Disorders, 28, 1238-1246.

Martin GC, Wertheim EH, Prior M, Smart D, Sanson A, \& Oberklaid F. (2000). A longitudinal study of the role of childhood temperament in the later development of eating concerns. International Journal of Eating Disorders, 27, 150-162.

Mâsse LC, \& Tremblay RE. (1997). Behavior of boys in kindergarten and the onset of substance use during adolescence. Archives of General Psychiatry, 54, 62-68.

Mâsse LC, \& Watts AW. (submitted). Stimulating innovations in the measurement of parenting constructs.

Mâsse LC, Wilson M, Baranowski T, \& Nebeling L. (2006). Improving psychometric methods in health education and health behavior research. Health Education Research, 21 (S1), i1-i3.

Matheson DM, Robinson TN, Varady A, \& Killen JD. (2006). Do Mexican-American mothers' food-related parenting practices influence their children's weight and dietary intake? Journal of the American Dietetic Assocation, 106, 1861-5.

McAdams DP, \& Bradley DO. (2010). Personality development: Continuity and change over the life course. Annual Review of Psychology, 61, 517-542.

McCrae RR, \& Costa PT. (1987). Validation of the five-factor model of personality across instruments and observers. Journal of Personality and Social Psychology, 52, 81-90.

McCrae RR, \& John OP. (1992). An introduction to the five-factor model and its applications. Journal of Personality, 60, 175-215.

McGuire MT, Hannan PJ, Neumark-Sztainer D, Cossrow NHF, \& Story M. (2002). Parental correlates of physical activity in a racially/ethnically diverse adolescent sample. Journal of Adolescent Health, 30, 253-261. 
McMeekin S, Jansen E, Mallan K, Nicholson J, Magarey A, \& Daniels L. (2013). Associations between infant temperament and early feeding practices. A cross-sectional study of Australian mother-infant dyads from the NOURISH randomized controlled trial. Appetite, 60, 239-245.

McMinn A, Van Sluijs EM, Wedderkopp N, Froberg K, \& Griffin SJ. (2008). Sociocultural correlates of physical activity in children and adolescents: Findings from the Danish arm of the European Youth Heart Study. Pediatric Exercise Science, 20, 319-332.

Mendelson BK, White DR, \& Schliecker E. (1995). Adolescents' weight, sex, and family functioning. International Journal of Eating Disorders, 17, 73-79.

Miller KS, Vannatta K, Compas BE, Vasey M, McGoron KD, Salley CG, \& Gerhardt CA. (2009). The role of coping and temperament in the adjustment of children with cancer. Journal of Pediatric Psychology, 34, 1135-1143.

Milyavskaya M, Gingras I, Mageau GA, Koestner R, Gagno H, Fang J, \& Bioché J. (2009). Balance across contexts: Importance of balanced need satisfaction across various life domains. Personality and Social Psychology Bulletin, 35, 1031-1045.

Mitchell S, Brennan L, Hayes L, \& Miles CL. (2009). Maternal psychosocial predictors of controlling parental feeding styles and practices. Appetite, 53, 384-389.

Mitchell J, Skouteris H, McCabe M, Ricciardelli LA, Milgrom J, Baur LA, Fuller-Tyszkiewicz M, \& Dwyer G. (2011). Physical activity in young children: A systematic review of parental influences. Early Child Development and Care, 182, 1411-1437.

Moens E, \& Braet C. (2012). Training parents of overweight children in parenting skills: A 12-month evaluation. Behavioural and Cognitive Psychotherapy, 40, 1-18.

Moens E, Braet C, \& Soetens B. (2007). Observation of family functioning at mealtime: A comparison between families of children with and without overweight. Journal of Pediatric Psychology, 32, 52-63.

Montgomery C, Jackson DM, Kelly LA, \& Reilly JJ. (2006). Parental feeding style, energy intake and weight status in young Scottish children. British Journal of Nutrition, 96, 1149-1153.

Moore SN, Tappen K, \& Murphy S. (2007). Feeding strategies used by mothers of 3-5-year-old children. Appetite, 49, 704-707.

Morsbach SK, \& Prinz RJ. (2006). Understanding and improving the validity of self-report of parenting. Clinical Child and Family Psychology Review, 9, 1-21.

Mullis F. (1999). Active parenting: An evaluation of two Adlerian parent education programs. Journal of Individual Psychology, 55, 225-232.

Murashima M, Hoerr SL, Hughes SO, \& Kaplowitz SA. (2012). Feeding behaviors of low-income mothers: Directive control relates to a lower BMI in children, and a nondirective control relates to a healthier diet in preschoolers. American Journal of Clinical Nutrition, 95, 1031-1037.

Musher-Eizenman DR, De Lauzon-Guillain B, Holub SC, Leporc E, \& Charles MA. (2009). Child and parent characteristics related to parental feeding practices. A cross-cultural examination in the US and France. Appetite, 52, 89-95. 
Musher-Eizenman DR, \& Holub SC. (2006). Children's eating in the absence of hunger: The role of restrictive feeding practices. In RK Flamenbaum (Ed.), Childhood obesity and health research (pp. 135-156). New York, NY: Nova Science Publishers.

Musil CM, Jones SL, \& Warner CD. Structural equation modeling and its relationship to multiple regression and factor analysis. Research in Nursing and Health, 21, 271-281.

Must A, \& Strauss RS. (1999). Risks and consequences of childhood and adolescent obesity. International Journal of Obesity and Related Metabolic Disorders, 23 (S2), 2-11.

Mustillo S, Worthman C, Erkanli A, Keeler G, Angold A, \& Costello EJ. (2003). Obesity and psychiatric disorder: Developmental trajectories. Pediatrics, 111, 851-859.

Muthen B, \& Asparouhov T. (2006). Item response mixture modeling: Application to tobacco dependence criteria. Addictive Beahviors, 31, 1050-1066.

Netherlands Organization for Applied Scientific Research, TNO: Growth monitor (based on results of Dutch Fourth Growth Study, 1997). Retrieved from http://www.tno.nl/content.cfm?\& context=markten\&content $=$ case\&laag1=189\&item_id=469\&Taal=2

Neumark-Sztainer DR, Friend SE, Flattum CF, Hannan PJ, Story MT, Bauer KW, Feldman SB, \& Petrich CA. (2010). New moves - Preventing weight-related problems in adolescent girls: A grouprandomized study. American Journal of Preventive Medicine, 39, 421-432.

Newman K, Harrison L, Dashiff C, \& Davies S. (2008). Relationships between parenting styles and risk behaviors in adolescent health: An integrative literature review. Revista Latino-Americana de Enfermagem, 16, 142-150.

Newman J, \& Taylor A. (1992). Effect of a means-end contingency on young children's food preferences. Journal of Experimental Child Psychology, 64, 200-216.

Nickelson J, Alfonso ML, McDermott RJ, Bumpus EC, Bryant CA, \& Baldwin JA. (2011). Characteristics of 'tween' participants and non-participants in the VERB summer scorecard physical activity promotion program. Health Education Research, 26, 225-238.

Nowicka P, \& Flodmarkt CE. (2008). Family in pediatric obesity management: A literature review. International Journal of Pediatric Obestiy, 3 (S1), 44-50.

Nowicka P, Hoglund P, Pietrobelli A, Lissau I, \& Flodmark CE. (2008). Family weight school treatment: 1-year results in obese adolescents. International Journal of Pediatric Obesity, 3, 141-147.

Nowicka P, Pietrobelli A, \& Flodmark CE. (2007). Low-intensity family therapy intervention is useful in a clinical setting to treat obese and extremely obese children. International Journal of Pediatric Obesity, 2, 211-217.

Nunnally JC. (1978). Psychometric theory ( $2^{\text {nd }}$ edition). New York, NY: McGraw-Hill.

Nunnally JC, \& Bernstein IH. (1994). Psychometric theory ( $2^{\text {nd }}$ edition). New York, NY: McGraw-Hill.

Nygaard E, Smith L, \& Torgersen AM. (2002). Temperament in children with Down syndrome and in prematurely born children. Scandinavian Journal of Psychology, 43, 61-71.

O'Connor BP. (2000). SPSS and SAS programs for determining the number of components using parallel analysis and Velicer's MAP test. Behavior Research Methods, Instrumentation, and Computers, 32, 396-402. 
O'Connor TM, Hilmers A, Watson K, Baranowski T, \& Giardino AP. (2011). Feasibility of an obesity intervention for paediatric primary care targeting parenting and children: Helping HAND. Child: Care, Health and Development, 39, 141-149.

O’Connor TM, Hughes SO, Watson KB, Baranowski T, Nicklas TA, Fisher JO, Beltran A, Baranowski JC, Qu H, \& Shewchuk RM. (2010). Parenting practices are associated with fruit and vegetable consumption in pre-school children. Public Health Nutrition, 13, 91-101.

Ogden CL, Carroll MD, Kit BK, \& Flegal KM. (2012). Prevalence of obesity and trends in Body Mass Index among US children and adolescents, 1999-2010. Journal of the American Medical Association, 307, 483-490.

Ogden J, Reynolds R, \& Smith A. (2006). Expanding the concept of parental control: A role for overt and covert control in children's snacking behaviour? Appetite, 47, 100-106.

Olsen SF, Yang C, Hart CH, Robinson CC, Wu P, Nelson DA, Nelson LJ, Jin S, \& Wo J. (2002). Maternal psychological control and preschool children's behavioral outcomes in China, Russia, and the United Sates. In BK Barber (Ed.), Intrusive parenting: How psychological control affects children and adolescents (pp. 235-262). Washington, DC: American Psychological Association.

Olvera N, \& Power TG. (2010). Brief report: Parenting styles and obesity in Mexican American children: A longitudinal study. Journal of Pediatric Psychology, 35, 243-249.

Orlansky H. (1949). Infant care and personality. Psychological Bulletin, 46, 1-48.

Ommundsen Y, Klasson-Heggeb $\varnothing$ L, \& Anderssen S. (2006). Psycho-social and environmental correlates of location-specific physical activity among 9- and 15- year-old Norwegian boys and girls: The European Youth Heart Study. International Journal of Behavioral Nutrition and Physical Activity, $3: 32$.

Ommundsen Y, Page A, Ku PW, \& Cooper AR. (2008). Cross-cultural, age and gender validation of a computerized questionnaire measuring personal, social and environmental associations with children's physical activity: The European Youth Heart Study. International Journal of Behavioral Nutrition and Physical Activity, 5:29.

Ogden J, Reynolds R, \& Smith A. (2006). Expanding the concept of parental control: a role for overt and covert control in children's snacking behaviour? Appetite, 47, 100-106.

Ortega RM, Requejo AM, López-Sobaler AM, Quintas ME, Andrés P, Redondo MR, Navia B, LópezBonilla MD, \& Rivas T. (1998). Difference in breakfast habits of overweight/obese and normal weight schoolchildren. International Journal for Vitamin and Nutrition Research, 68, 125-132.

Oude Luttikhuis H, Baur L, Jansen H, Shrewsbury VA, O’Malley C, Stolk RP, \& Summerbell CD. (2009). Interventions for treating obesity in children. Cochrane Database of Systematic Reviews, 1, article no. CD001872.

Paez S, Maloney A, Kelsey K, Wiesen C, \& Rosenberg A. (2009). Parental and environmental factors associated with physical activity among children participating in an active video game. Pediatric Physical Therapy, 21, 245-253.

Parikh T, \& Stratton G. (2011). Influence of intensity of physical activity on adiposity and cardiorespiratory fitness in 5-18 year olds. Sports Medicine, 41, 477-488. 
Parker G. (1983). Parental overprotection: A risk factor in psychosocial development. New York: Grune \& Stratton.

Parker G, Tupling H, \& Brown LB. (1979). A parental bonding instrument. British Journal of Medical Psychology, 52, 1-10.

Patrick H, \& Nicklas TA. (2005). A review of family and social determinants of children's eating patterns and diet quality. Journal of the American College of Nutrition, 24, 83-92.

Patrick H, Nicklas TA, Hughes SO, \& Morales M. (2005). The benefits of authoritative feeding style: Caregiver feeding styles and children's food consumption patterns. Appetite, 44, 243-249.

Patterson GR. (1976). Living with children: New methods for parents and teachers (Revised). Champaign, IL: Research Press.

Patterson GR. (1982). Coercive family process. Eugene, OR: Castalia Press.

Patterson GR, \& Fleischman MJ. (1979). Maintenance of treatment effects: Some considerations concerning family systems and follow-up data. Behavior Therapy, 10, 168-185.

Patterson GR, \& Guillion ME. (1968). Living with children: New methods for parents and teachers. Champaign, IL: Research Press.

Paulhus DL, \& Vazire S. (2007). The self-report method. In RW Robins, RC Fraley, \& R Krueger (Eds.), Handbook of research methods in personality psychology (pp. 224-239). New York, NY: Guilford Press.

Pearson N, Atkin AJ, Biddle SJ, Gorely T, \& Edwardson C. (2010). Parenting styles, family structure and adolescent dietary behaviour. Public Health Nutrition, 13, 1245-1253.

Pearson N, \& Biddle SJH. (2011). Sedentary behavior and dietary intake in children, adolescents, and Adults: A systematic review. American Journal of Preventive Medicine, 41, 178-188.

Pérez-Escamilla R, Obbagy JE, Altman JM, Essery EV, McGrane MM, Ping Wong Y, Spahn JM, \& Williams CL. (2012). Dietary energy density and body weight in adults and children: A systematic review. Journal of the Academy of Nutrition and Dietetics, 112, 671-684.

Pomerantz EM, \& Grolnick WS. (2009). Toward a clear and inclusive conceptualization of parental control: Reply to the commentaries. Child Development Perspectives, 3, 176-177.

Portney LG, \& Watkins MP. (2000). Foundations of clinical research: Applications to practice $\left(2^{\text {nd }}\right.$ edition). Upper Saddle Rive, NJ: Prentice Hall Health.

Power TG. (1993). Parenting Dimensions Inventory (PDI): A research manual. Unpublished manuscript, University of Houston, Houston, TX.

Power TG. (2002). Parenting dimensions inventory - Short version (PDI-S): A research manual. Unpublished manuscript, Washington State University.

Power TG, Sleddens EFC, Berge J, Connell L, Govig B, Hennessy E, Ligget L, Odoms-Young A, Mallan K, Santa Maria D, \& George SM. (submitted). Issues in the measurement of parenting style.

Powers SW, Chamberlin LA, Van Schaick KB, Sherman SN, \& Whitaker RC. (2006). Maternal feeding strategies, child eating behaviors, and child BMI in low-income African-American preschoolers. Obesity, 14, 2026-2033.

Power TG, \& Hill LG. (2008). Maternal protectiveness and child adjustment: A multidimensional study. Parenting: Science and Practice, 8, 187-212. 
Power TG, Kobayashi-Winata H, \& Kelley ML. (1992). Childrearing patterns in Japan and the United States: A cluster analytic study. International Journal of Behavioral Development, 15, 185-205.

Prinzie P, Stams G-JJM, Deković M, Reijntjes AHA, \& Belsky J. (2009). The relations between parents' Big Five personality factors and parenting: A meta-analytic review. Journal of Personality and Social Psychology, 2, 351-362.

Pritchett R, Kemp J, Wilson P, Minnis H, Bryce G, \& Gillberg C. (2011). Quick, simple measures of family relationships for use in clinical practice and research. A systematic review. Family Practice, 28, 172-187.

Prochaska JJ, Rodgers MW, \& Sallis JF. (2002). Association of parent and peer support with adolescent physical activity. Research Quarterly of Exercise and Sport, 73, 206-210.

Pugliese J, \& Tinsley B. (2007). Parental socialization of child and adolescent physical activity: A meta-analysis. Journal of Family Psychology, 21, 331-343.

Pulkkinen L. (1982). Self-control and continuity from childhood to late adolescence. In BP Baltes, \& OG Brim (Eds.), Life-span development and behavior (pp. 63-105). Orlando, FL: Academic Press.

Putnam SP. (2011). Stability and instability of childhood traits: Implications for personality development of animals. Developmental Psychobiology, 53, 510-520.

Putnam SP, \& Rothbart MK. (2006). Development of short and very short forms of the Children's Behavior Questionnaire. Journal of Personality Assessment, 87, 102-112.

Putnam SP, Rothbart MK, \& Gartstein MA. (2008). Homotypic and heterotypic continuity of finegrained temperament during infancy, toddlerhood, and early childhood. Infant and Child Development, 17, 387-405.

Putnam SP, Sanson AV, \& Rothbart MK. (2002). Child temperament and parenting. In MH Bornstein (Ed.), Handbook of parenting: Vol. 1. (pp. 255-277). Mahwah, New Jersey: Lawrence Erlbaum.

Rampersaud GC, Pereira MA, Girard BL, Adams J, \& Metzl JD. (2005). Breakfast habits, nutritional status, body weight, and academic performance in children and adolescents. Journal of the American Dietetic Association, 105, 743-760.

Raudsepp L. (2006). The relationship between socio-economic status, parental support and adolescent physical activity. Acta Paediatrica, 95, 93-98.

Reau NR, Senturia YD, Lebailly SA, \& Christoffel KK. (1996). Infant and toddler feeding patterns and problems: Normative data and a new direction. Pediatric Practice Research Group. Journal of Developmental and Behavioral Pediatrics, 17, 149-153.

Reilly JJ, \& Kelly J. (2011). Long-term impact of overweight and obesity in childhood and adolescence on morbidity and premature mortality in adulthood: Systematic review. International Journal of Obesity, 35, 891-898.

Reineke PR. (2008). The relationship between parenting style and child weight. New York University.

Rhee K. (2008). Childhood overweight and the relationship between parent behaviors, parenting style, and family functioning. Annals of the American Academy of Political and Social Science, 615, 11-37. 
Reitman D, Rhode PC, Hupp SDA, \& Altobello C. (2002). Development and validation of the parental authority Questionnaire-Revised. Journal of Psychopathology and Behavioral Assessment, 24, 119127.

Rhee KE, Lumeng JC, Appugliese DP, Kaciroti N, \& Bradley RH. (2006). Parenting styles and overweight status in first grade. Pediatrics, 117, 2047-2054.

Riddoch CJ, Leary SD, Ness AR, Blair SN, Deere K, Mattocks C, Griffiths A, Davey Smith G, \& Tilling K. (2009). Prospective associations between objective measures of physical activity and fat mass in 1214 year old children: The Avon Longitudinal Study of Parents and Children (ALSPAC). BMJ, 339:b4544.

Robertson W, Friede T, Blissett J, Rudolf MCJ, Wallis M, \& Stewart-Brown S. (2008). Pilot of 'Families for Health': Community-based family intervention for obesity. Archives of Disease in Childhood, 93, 921-928.

Robertson W, Thorogood M, Inglis N, Grainger C, \& Stewart-Brown S. (2012). Two-year follow-up of the 'families for health' programme for the treatment of childhood obesity. Child: Care, Health and Development, 38, 229-236.

Robinson TN, Kiernan M, Matheson DM, \& Haydel KF. (2001). Is parental control over children's eating associated with childhood obesity? Results from a population-based sample of third graders. Obesity Research, 9, 306-312.

Robinson CC, Mandleco B, Olsen SF, \& Hart CH. (1995). Authoritative, authoritarian, and permissive parenting practices: Development of a new issue. Psychological Reports, 77, 819-830.

Robinson CC, Mandleco B, Olsen SF, \& Hart CH. (2001). The parenting styles and dimensions questionnaire (PSDQ). In BF Perlmutter (Ed.), Handbook of family measurement techniques (pp. 319-321). Thousand Oaks, CA: Sage.

Rodenburg G, Kremers SPJ, Oenema A, \& Van de Mheen D. (2011). Psychological control by parents is associated with a higher child weight. International Journal of Pediatric Obesity, 6, 442-449.

Rohner RP. (1986). The warmth dimension: Foundations of parental acceptance-rejection theory. Newbury Park, CA: Sage Publications.

Rollins BC, \& Thomas DL. (1979). Parental support, power, and control techniques in the socialization of children. In WR Burre, R Hill, FI Nye, \& IL Reiss (Eds.), Contemporary theories about the family: Research-based theories (pp. 317-364). New York, NY: Free Press.

Rosenkranz RR, \& Dzewaltowski DA. (2008). Model of the home food environment pertaining to childhood obesity. Nutrition Reviews, 66, 123-140.

Rothbart MK, Ahadi SA, \& Evans DE. (2000). Temperament and personality: Origins and outcomes. Journal of Personality and Social Psychology, 78, 122-135.

Rothbart MK, Ahadi SA, \& Hershey KL. (1994). Temperament and social behavior in childhood. Merrill-Palmer Quarterly, 40, 21-39.

Rothbart MK, Ahadi SA, Hershey KL, \& Fisher P. (2001). Investigations of temperament at 3 to 7 years: The Children's Behavior Questionnaire. Child Development, 72, 1394-1408. 
Rothbart MK, \& Bates JE. (1998). Temperament. In W Damon (Series Ed.) \& N Eisenberg (Vol. Ed.), Handbook of child psychology: Vol. 3. Social, emotional and personality development $\left(5^{\text {th }}\right.$ edition, pp. 105-176). New York, NY: Wiley.

Ruxton CHS, \& Kirk TR. (1997). Breakfast: A review of associations with measures of dietary intake, physiology and biochemistry. British Journal of Nutrition, 78, 199-213.

Ryan RM, \& Deci EL. (2000). Self-determination theory and the facilitation of intrinsic motivation, social development, and well-being. American Psychologist, 55, 68-78.

Rydell AM, Dahl M, \& Sundelin C. (1995). Characteristics of school children who are choosy eaters. Journal of Genetic Psychology, 156, 217-229.

Safron M, Cislak A, Gaspar T, \& Luszczynska A. (2011). Micro-environmental characteristics related to body weight, diet, and physical activity of children and adolescents: A systematic umbrella review. International Journal of Environmental Health Research, 21, 317-330.

Sallis JF, Alcaraz JE, McKenzie TL, Hovell MF, Kolody B, \& Nader PR. (1992). Parental behavior in relation to physical activity and fitness in 9-year-old children. American Journal of Diseases of Children, 146, 1383-1388.

Sallis JF, Grossman RM, Pinski RB, Patterson TL, \& Nader PR. (1987). The development of scales to measure social support for diet and exercise behaviors. Preventive Medicine, 16, 825-836.

Sallis JF, Hovell MF, Hofstetter CR, Faucher P, Elder JP, Blanchard J, Caspersen CJ, Powell KE, \& Christenson GM. (1989). A multivariate study of determinants of vigorous exercise in a community sample. Preventive Medicine, 18, 20-34.

Sallis JF, Nader PR, Broyles SL, Berry CC, Elder JP, McKenzie TL, \& Nelson JA. (1993). Correlates of physical activity at home in Mexican-American and Anglo-American preschool children. Health Psychology, 12, 390-398.

Sallis JF, \& Owen N. (2002). Ecological models of health behavior. In K Glanz, BK Reimer, \& FM Lewis (Eds.), Health Behavior and Health Education. Theory, Research, and Practice ( $3^{\text {rd }}$ edition, pp. 462484). San Francisco, CA: Jossey-Bass.

Sallis JF, Prochaska JJ, \& Taylor WC. (2000). A review of correlates of physical activity of children and adolescents. Medicine and Science in Sports and Exercise, 32, 963-975.

Sallis JF, Wendell CT, Dowda M, Freedson PS, \& Pate RR. (2002). Correlates of vigorous physical activity for children in grades 1 through 12: Comparing parent-reported and objectively measured physical activity. Pediatric Exercise Science, 14, 30-44.

Salmon J, Timperio A, Telford A, Carver A, \& Crawford D. (2005). Association of family environment with children's television viewing and with low level of physical activity. Obesity Research, 13, 19391951.

Sameroff AJ. (1975). Transactional models in early social relations. Human Devevelopment, 18, 6579.

Sanders MR. (2012). Development, evaluation, and multinational dissemination of the Triple PPostive Parenting Program. Annual Review of Clinical Psychology, 8, 345-379. 
Sanders MR, Markie-Dadds C, \& Turner KMT. (2003). Theoretical, scientific, and clinical foundations of the Triple P-Positive Parenting Program: A population approach to the promotion of parenting competence. Parenting research and practice monograph, 1, 1-24.

Sanders MR, Turner KMT, \& Markie-Dadds C. (2002). The development and dissemination of the Triple PPositive Parenting Program: A multilevel, evidence-based system of parenting and family support. Preventive Science, 3, 173-189.

Sargent J. (1983). The sick child: Family complications. Developmental and Behavioral Pediatrics, 4, 50-56.

Saucier G. (1994). Mini-markers: A brief version of Goldberg's unipolar Big-Five markers. Journal of Personality Assessment, 63, 506-516.

Saunders KL. (2007). Preventing obesity in pre-school children: A literature review. Journal of Public Health, 29, 368-375.

Scaglioni S, Salvioni M, \& Galimberti C. (2008). Influence of parental attitudes in the development of children eating behaviour. British Journal of Nutrition, 99(S1), 22-25.

Schaefer ES. (1959). A circumflex model for maternal behavior. Journal of Abnormal Social Psychology, 59, 226-235.

Schaffer R. (1977). Mothering. Cambridge, MA: Harvard University Press.

Schmitz KH, Lytle LA, Phillips GA, Murray DM, Birnbaum AS, \& Kubik MY. (2002). Psychosocial correlates of physical activity and sedentary leisure habits in young adolescents: The Teens Eating for Energy and Nutrition at School study. Preventive Medicine, 34, 266-278.

Schokker DF, Visscher TLS, Nooyens AC.J, Van Baak MA, \& Seidell JC. (2006). Prevalence of overweight and obesity in the Netherlands. Obesity Reviews, 8, 101-107.

Scholtens S, Brunekreef B, Visscher TLS, Smit HA, Kerkhof M, De Jongste JC, Gerritsen J, \& Wijga AH. (2007). Reported versus measured body weight and height of 4-year-old children and the prevalence of overweight. European Journal of Public Health, 17, 369-374.

Schönbeck Y, Talma H, Van Dommelen P, Bakker B, Buitendijk SE, HiraSing RA, \& Van Buuren S. (2011). Increase in prevalence of overweight in Dutch children and adolescents: A comparison of nationwide growth studies in 1980, 1997 and 2009. PLoS one, 6, e27608.

Schaben JA, Welk GJ, Joens-Matre R, \& Hensley L. (2006). The predictive utility of the children's physical activity correlates (CPAC) scale across multiple grade levels. Journal of Physical Activity and Health, 3, 59-69.

Schaefer ES. (1965a). A configurational analysis of children's reports of parent behavior. Journal of Consulting Psychology, 29, 552-557.

Schaefer ES. (1965b). Children's reports of parental behavior: An inventory. Child Development, 36, 413-424.

Sears RR, Maccoby EE, \& Levin H. (1957). Patterns of childrearing. Stanford: Stanford University Press.

Sears RR. (1951). A theoretical framework for personality and social behavior. American Psychologist, 6, 476-82. 
Sessa FM, Avenevoli S, Steinberg L, \& Morris AS. (2001). Correspondence among informants on parenting: Preschool children, mothers, and observers. Journal of Family Psychology, 15, 5368.

Sha MM, \& Pan Y. (2009). The use of vignettes in evaluating multilingual questionnaires. Presented at the American Association for Public Opinion Research, May 14-17.

Shelton D, Le Gros K, Norton L, Stanton-Cook S, Morgan J, \& Masterman P. (2007). Randomised controlled trial: A parent-based group education programme for overweight children. Journal of Paediatrics and Child Health, 43, 799-805.

Simonds J, \& Rothbart MK. (2004). The Temperament in Middle Childhood Questionnaire (TMCQ): A computerized self-report measure of temperament for ages 7-10. Poster session presented at Occasional Temperament Conference, Athens, GA, October.

Singh AS, Mulder C, Twisk JWR, Van Mechelen W, \& Chinapaw MJM. (2008). Tracking of childhood overweight into adulthood: A systematic review of the literature. Obesity Reviews, 9, 474-488.

Skinner BF. (1953). Science and human behavior. New York, NY: MacMillan.

Skinner BF. (1965). Science and human behaviour. New York, NY: Free Press.

Skinner E, Johnson S, \& Snyder T. (2005). Six dimensions of parenting: A motivational model. Parenting: Science and Practice, 5, 175-235.

Slater MA, \& Power TG. (1987). Multidimensional assessment of parenting in single-parent families. In JP Vincent (Ed.), Advances in family intervention, assessment, and theory (pp. 197-228). Greenwich, CN: JAI Press.

Sleddens EFC, Gerards SMPL, De Vries NK, Thijs C \& Kremers SPJ. (2011). General parenting, childhood overweight and obesity-inducing behaviors: A review. International Journal of Pediatric Obesity, 6, e12-e27.

Sleddens EFC, Hughes SO, O’Connor TM, Beltran A, Baranowski JC, Nicklas TA, \& Baranowski T. (2012a). The Children's Behavior Questionnaire very short scale: Psychometric properties and development of a one-item temperament scale. Psychological Reports, 110, 197-217.

Sleddens EFC, Hughes SO, O’Connor TM, Beltran A, Baranowski JC, Nicklas TA, \& Baranowski T. (2012b). Reply to commentary on Putnam's promise of, problems with, and potential refinement of the 'Extremely Short Form of the Children's Behavior Questionnaire". Psychological Reports, 111, 621-623.

Sleddens EFC, Kremers SPJ, Candel MJJM, De Vries NK, \& Thijs C. (2011). Validating the Children's Behavior questionnaire in Dutch children: Psychometric properties and a cross-cultural comparison of factor structures. Psychological Assessment, 23, 417-426.

Sleddens EFC, Kremers SPJ, De Vries NK, \& Thijs C. (2010). Relationship between parental feeding styles and eating behaviours of Dutch children aged 6 to 7. Appetite, 54, 30-36.

Sleddens EFC, Kremers SPJ, De Vries NK, \& Thijs C. (2012). Measuring child temperament: Validation of a 3-item temperament scale and 13-item impulsivity scale. European Journal of Developmental Psychology, Epub 30 May. 
Sleddens EFC, Kremers SPJ, Hughes SO, Cross MB, Thijs C, De Vries NK, O'Connor TM. (2012).

Physical activity parenting: A systematic review of questionnaires and their associations with child activity levels. Obesity Reviews, 13, 1015-1033.

Sleddens EFC, Kremers SPJ, Stafleu A, Dagnelie PC, De Vries NK, \& Thijs C. (in revision). Food parenting practices and child eating patterns: Prospective relations and the moderating role of general parenting, child temperament, and child eating style.

Sleddens EFC, Kremers SPJ, \& Thijs C. (2008). The Children's Eating Behaviour Questionnaire: Factorial validity and association with Body Mass Index in Dutch children aged 6 to 7. International Journal of Behavioral Nutrition and Physical Activity, 5:49.

Sleddens EFC, O'Connor TM, Watson KB, Hughes SO, Power TG, Thijs C, De Vries NK, \& Kremers SPJ. (in revision). Development of the Comprehensive General Parenting Questionnaire for caregivers of 5 to 13 year olds.

Soenens B, Vansteenkiste M, Duriez B, \& Goossens L. (2006). In search of the sources of psychologically controlling parenting: The role of parental separation anxiety and parental maladpative perfectionism. Journal of Research on Adolescence, 16, 539-559.

Soenens B, Vansteenkiste M, Lens W, Luyckx K, Goossnes L, Beyers W, \& Ryan RM. (2007). Conceptualizing parental autonomy support: Adolescent perceptions of promotion of independence versus promotion of volitional functioning. Developmental Psychology, 43, 633-646.

Soenens B, Vansteenkiste M, Luyckx K, \& Goossens L. (2006). Parenting and adolescent problem behavior: An integrated model with adolescent self-disclosure and perceived parental knowledge as intervening variables. Developmental Psychology, 42, 305-318.

Spence JC, \& Lee RE. (2003). Toward a comprehensive model of physical activity. Psychology of Sport and Exercise, 4, 7-24.

Spera C. (2005). A review of the relationship among parenting practices, parenting styles, and adolescent school achievement. Educational Psychology Review, 17, 125-146.

Spoth R, Redmond C, \& Shin C. (1998). Direct and indirect latent-variable parenting outcomes of two universal family-focused preventive interventions: Extending a public health-oriented research base. Journal of Consulting and Clinical Psychology, 66, 385-399.

Spruijt-Metz D, Lindquist CH, Birch LL, Fisher JO, \& Goran MI. (2002). Relations between mothers' child-feeding practices and children's adiposity. American Journal of Clinical Nutrition, 75, 581-586.

Stark LJ, Spear S, Boles R, Kuhl E, Ratcliff M, Scharf C, Bolling C, \& Rausch J. (2010). A pilot randomized controlled trial of a clinic and home-based behavioral intervention to decrease obesity in preschoolers. Obesity, 19, 134-141.

Stice E, Shaw H, \& Marti CN. (2006). A meta-analytic review of obesity prevention programs for children and adolescents: The skinny on interventions that work. Psychological Bulletin, 132, 667-691.

Stein RI, Epstein LH, Raynor HA, Kilanowski CK, \& Paluch RA. (2005). The influence of parenting change on pediatric weight control. Obesity Research, 13, 1749-1755.

Steinberg L. (1990). Autonomy, conflict, and harmony in the family relationship. In SS Feldman, \& GR Elliott (Eds.), At the threshold: The developing adolescent (pp. 255-276). Cambridge, MA: Harvard University Press. 
Steinberg L, Elmen JD, \& Mounts NS. (1989). Authoritative parenting, psychosocial maturity, and academic success among adolescents. Child Development, 60, 1424-1436.

Stenhammar C, Olsson GM, Bahmanyar S, Hulting A-L, Wettergren B, Edlund B, \& Montgomery SM. (2010). Family stress and BMI in young children. Acta Paediatrica, 99, 1205-1212.

Strauss RS. (2000). Childhood obesity and self-esteem. Pediatrics, 105, e15.

Strauss RS, \& Knight J. (1999). Influence of the home environment on the development of obesity in children. Pediatrics, 103, e85.

Summerbell CD, Waters E, Edmunds LD, Kelly S, Brown T, \& Campbell KJ. (2005). Interventions for preventing obesity in children. Cochrane Database of Systematic Reviews, 3, article no. CD001871.

Swithers SE, \& Davidson TL. (2008). A role for sweet taste: Calorie predictive relations in energy regulation by rats. Behavioral neuroscience, 122, 161-173.

Tamis-LeMonda CS, Briggs RD, McClowry SG, \& Snow DL. (2008). Challenges to the study of African American Parenting: Conceptualization, sampling, research approaches, measurement, and design. Parenting: Science and Practice, 8, 319-58.

Taylor WC, Baranowski T, \& Sallis JF. (1994). Family determinants of childhood physical activity: A social-Cognitive model. In RK Dishman (ed.), Advances in exercise adherence (pp. 319-342). Champaign, IL: Human Kinetics.

Taylor WC, Sallis JF, Dowda M, Freedson PS, Eason K, \& Pate RR. (2002). Activity patterns and correlates among youth: Differences by weight status. Pediatric Exercise Science, 14, 418431.

Taylor A, Wilson C, Slater A, \& Mohr P. (2011). Parent- and child-reported parenting. Associations with child weight-related outcomes. Appetite, 57, 700-706.

Telama R. (2009). Tracking of physical activity from childhood to adulthood: A review. Obesity Facts, 3, 187-195.

Te Velde SJ, Van Nassau F, Uijtdewilligen L, Van Stralen MM, Cardon G, De Craemer M, Manios Y, Brug J, \& Chinapaw MJM. On behalf of the ToyBox-study Group. (2012). Energy balance-related behaviours associated with overweight and obesity in preschool children: A systematic review of prospective studies. Obesity Reviews, 13(S1), 56-74.

Thompson ME. (2010). Parental feeding and childhood obesity in preschool-age children: Recent findings from the literature. Issues in Comprehensive Pediatric Nursing, 33, 205-267.

Tiggemann M, \& Lowes J. (2002). Predictors of maternal control over children's eating behaviour. Appetite, 39, 1-7.

Timperio A, Salmon J, Ball K, Baur LA, Telford A, Jackson M, Salmon L, \& Crawford D. (2008). Family physical activity and sedentary environments and weight change in children. International Journal of Pediatric Obesity, 3, 160-167.

Tohill BC, Seymour J, Serdula M, Kettel-Khan L, \& Rolls BJ. (2004). What epidemiologic studies tell us about the relationship between fruit and vegetable consumption and body weight. Nutrition Reviews, 62, 365-374. 
Topham GL, Hubbs-Tait L, Rutledge JM, Page MC, Kennedy TS, Shriver LH, \& Harrist AW. (2011).

Parenting styles, parental response to child emotion, and family emotional responsiveness are related to child emotional eating. Appetite, 56, 261-264.

Topham GL, Page MC, Hubbs-Tait L, Rutledge JM, Kennedy TS, Shriver L, \& Harrist AW. (2010). Maternal depression and socio-economic status moderate the parenting style/child obesity association. Public Health Nutrition, 13, 1237-1244.

Trombini E, Baldaro B, Bertaccini R, Mattei C, Montebarocci O, \& Rossi N. (2003). Maternal attitudes and attachment styles in mothers of obese children. Perceptual and Motor Skills, 97, 613-620.

Trost SG, \& Loprinzi PD. (2011). Parental influences on physical activity in children and adolescents: A brief review. American Journal of Lifestyle Medicine, 5, 171-181.

Trost SG, Sallis JF, Pate RR, Freedson PS, Taylor WC, \& Dowda M. (2003). Evaluating a model of parental influence on youth physical activity. American Journal of Preventive Medicine, 25, 277-282.

Tumanova V, Zebrowski PM, Throneburg RN, \& Kulak Kayikci ME. (2011). Articulation rate and its relationship to disfluency type, duration, and temperament in preschool children who stutter. Journal of Communication Disorders, 44, 116-129.

Turner HM, Rose KS, \& Cooper MJ. (2005). Schema and parental bonding in overweight and nonoverweight female adolescents. International Journal of Obesity, 29, 381-387.

Utter J, Scragg R, Schaaf D, Fitzgerald E, \& Wilson N. (2007). Correlates of body mass index among a nationally representative sample of New Zealand children. International Journal of Pediatric Obesity, 2, 104-113.

Uziel L. (2010). Rethinking social desirability scales: From impression management to interpersonally oriented self-control. Perspectives on psychological Science, 5:243.

Van den Bergh BRH, \& Ackx M. (2003). Een Nederlandse versie van Rothbarts ‘Children's Behavior Questionnaire: Interne consistentie en driefactoren model van de subschalen [A Dutch version of Rothbart's Children's Behavior Questionnaire: Internal consistency and three-factor model of the scales]. Kind en Adolescent, 24, 77-84.

Van den Hurk K, Van Dommelen P, De Wilde JA, Verkerk PH, Van Buuren S, \& HiraSing RA. (2006). Prevalentie van overgewicht en obesitas bij jeugdigen 4-15 jaar in de periode 2002-2004 [Prevalence of overweight and obesity in children aged 4-15 from 2002-2004]. Leiden: TNO Kwaliteit van Leven, TNO-rapport.

Van den Hurk K, Van Dommelen P, Van Buuren S, Verkerk PH, \& HiraSing RA. (2007). Prevalence of overweight and obesity in the Netherlands in 2003 compared to 1980 and 1997. Archives of Disease in Childhood, 92, 992-995.

Van der Horst K, Chin A. Paw MJ, Twisk JWR, \& Van Mechelen W. (2007). A brief review on correlates of physical activity and sedentariness in youth. Medicine and Science of Sports and Exercise, 39, 1241-1250.

Van der Horst K, Kremers SPJ, Ferreira I, Singh A, Oenema A, \& Brug J. (2007). Perceived parenting style and practices and the consumption of sugar-sweetened beverages by adolescents. Health Education Research, 22, 295-304. 
Van der Horst K, Oenema A, Ferreira I, Wendel-Vos W, Giskes K, Van Lenthe F, \& Brug. (2007). A systematic review of environmental correlates of obesity-related dietary behaviors in youth. Health Education Research, 22, 203-226.

Van Gelder MMHJ, Bretveld RW, \& Roeleveld N. (2010). Web-based questionnaires: The future in epidemiology? American Journal of Epidemiology, 172, 1292-1298.

Van Leeuwen KG, \& Vermulst AA. (2004). Some psychometric properties of the Ghent Parental Behavior Scale. European Journal of Psychological Assessment, 20, 283-298.

Vansteenkiste M, Zhou M, Lens W, \& Soenens B. (2005). Experiences of autonomy and control among Chinese learners: Vitalizing or immobilizing? Journal of Educational Psychology, 97, 468-483.

Van Strien T, Frijters JER, Bergers GPA, \& Defares PB. (1986). The Dutch Eating Behavior Questionnaire (DEBQ) for assessment of restrained, emotional, and external eating behavior. International Journal of Eating Disorders, 5, 295-315.

Van Strien T, \& Oosterveld P. (2008). The Children's DEBQ for assessment of restrained, emotional, and external eating in 7- to 12-year-old children. International Journal of Eating Disorders, $41,72-81$

Van Strien T, \& Ouwens MA. (2007). Effects of distress, alexithymia and impulsivity on eating. Eating Behaviors, 8, 251-257.

Van Zeijl J, Mesman J, Stolk MN, Alink LR, Van Ijzendoorn MH, Bakermans-Kranenburg MJ, Juffer F, \& Koot HM. (2007). Differential susceptibility to discipline: The moderating effect of child temperament on the association between maternal discipline and early childhood externalizing problems. Journal of Family Psychology, 21, 626-636.

Vartanian LR, Schwartz MB, \& Brownell KD. (2007). Effects of soft drink consumption on nutrition and health: A systematic review and meta-analysis. American Journal of Public Health, 97, 667-675.

Vaughn AE, Tabak RG, Bryant MJ, \& Ward DS. (submitted). Measuring parent food practices: A systematic review of existing measures and examination of instruments.

Ventura AK, \& Birch LL. (2008). Does parenting affect children's eating and weight status? International Journal of Behavioral Nutrition and Physical Activity, 5:15.

Vereecken CA, Keukelier E, \& Maes L. (2004). Influence of mother's educational level on food parenting practices and food habits of young children. Appetite, 43, 93-103.

Vereecken C, Legiest E, De Bourdeaudhuij I, \& Maes L. (2009). Associations between general parenting styles and specific food-related parenting practices and children's food consumption. American Journal of Health Promotion, 23, 233-240.

Vereecken C, Rovner A, \& Maes L. (2010). Associations of parenting styles, parental feeding practices and child characteristics with young children's fruit and vegetable consumption. Appetite, 55, 58996.

Vermulst AA, \& Gerris JRM. (2005). Quick Big Five persoonlijkheidstest [The Quick Big Five personality test]. Leeuwarden, NL: LDC Publishers.

Viana V, Sinde S, \& Saxton JC. (2008). Children's Eating Behaviour Questionnaire: Associations with $\mathrm{BMI}$ in Portuguese children. British Journal of Nutrition, 100, 445-450. 
Wachs TD. (1992). The nature of nurture. Newbury Park, CA: Sage.

Wachs TD. (1999). Celebrating complexity: Conceptualization and assessment of the environment. In SL Friedman, \& TD Wachs (Eds.), Measuring environment across the life span: Emerging methods and concepts (pp. 357-392). Washington, DC: American Psychological Association.

Wake M, Nicholson JM, Hardy P, \& Smith K. (2007). Preschooler obesity and parenting styles of mothers and fathers: Australian national population study. Pediatrics, 120, e1520-e1527.

Walker LO, Kirby RS. (2010). Conceptual and measurement issues in early parenting practices research: An epidemiologic perspective. Maternal and Child Health, 14, 958-970.

Wang Y, \& Lobstein T. (2006). Worldwide trends in childhood overweight and obesity. International Journal of Pediatric Obesity, 1, 11-25.

Ward TM, Gay C, Alkon A, Anders TF, \& Lee KA. (2008). Nocturnal sleep and daytime nap behaviors in relation to salivary cortisol levels and temperament in preschool-age children attending child care. Biological Research for Nursing, 9, 244-253.

Ward DS, Vaughn AE, Bangdiwala KI, Campbell M, Jones DJ, Panter AT, \& Stevens J. (2011).

Integrating a family-focused approach into child obesity prevention: Rationale and design for the my parenting SOS study randomized control trial. BMC Public Health, 11:431.

Wardle J, \& Carnell S. (2006). Parental feeding practices and children's weight. Acta Paediatrica, 96, 5-11.

Wardle J, Carnell S, Haworth CMA, Farooqi IS, O'Rahilly S, \& Plomin R. (2008). Obesity-associated genetic variation in FTO is associated with diminished satiety. Journal of Clinical Endocrinology and Metabolism, 93, 3640-3643.

Wardle J, Guthrie C, Sanderson S, \& Plomin R. (2001a). Food and activity preferences in children of lean and obese parents. International Journal of Obesity, 25, 971-977.

Wardle J, Guthrie CA, Sanderson S, \& Rapoport L. (2001b). Development of the Children's Eating Behaviour Questionnaire. Journal of Child Psychology and Psychiatry, 42, 963-970.

Wardle J, Sanderson S, Guthrie CA, Rapoport L, \& Plomin R. (2002). Parental feeding style and the inter-generational transmission of obesity risk. Obesity Research, 10, 453-462.

Watkins MW. (2000). Monte Carlo PCA for parallel analysis [Computer software]. State College, PA: Educational and Psychological Associates.

Watkins MW. (2005). Coefficient of Congruence [Computer software]. State College, PA: Educational and Psychological Associates.

Watkins MW. (2006). Determining parallel analysis criteria. Journal of Modern Applied Statistical Methods, 5, 344-346.

Waylen A, Stallard N, \& Stewart-Brown S. (2008). Parenting and health in mid-childhood: A longitudinal study. European Journal of Public Health, 18, 300-305.

Welk GJ, Wood K, \& Morss G. (2003). Parental influences on physical activity in children: An exploration of potential mechanisms. Pediatric Exercise Science, 15, 19-33.

West F. (2007). The Lifestyle Triple P project: Exploring the link between parenting and childhood obesity. University of Queensland. 
West F, Sanders MR, Cleghorn GJ, \& Davies PSW. (2010). Randomised clinical trial of a family-based lifestyle intervention for childhood obesity involving parents as the exclusive agents of change. Behaviour Research and Therapy, 48, 1170-1179.

Whitaker RC, Wright JA, Pepe MS, Seidel KD, \& Dietz WH. (1997). Predicting obesity in young adulthood from childhood and parental obesity. New England Journal of Medicine, 337, 869-873.

Whitehead L. (2011). Methodological issues in Internet-mediated research: A randomized comparison of internet versus mailed questionnaires. Journal of Medical Internet Research, 4, 13, e109.

Williams SL, \& Mummery WK. (2011). Links between adolescent physical activity, body mass index, and adolescent and parent characteristics. Health Education Behavior, 38, 510-520.

Williams AJ, Wyatt KM, Hurst AJ, \& Williams CA. (2012). A systematic review of associations between the primary school built environment and childhood overweight and obesity. Health \& Place, 18, 504-514.

Wilson M, Allen DD, \& Li JC. (2006a). Improving measurement in health education and health behavior research using item response modeling: Introducing item response modeling. Health Education Research, 21 (S1), i4-i18.

Wilson M, Allen DD, \& Li JC. (2006b). Improving measurement in health education and health behavior research using item response modeling: Comparison with the classical test theory approach. Health Education Research, 21 (S1), i9-i32.

Wilson P, Rush R, Hussey S, Puckering C, Sim F, Allely CS, Doku P, McConnachie A, \& Gillberg C. (2012). How evidence-based is an 'evidence-based parenting program'? A PRISMA systematic review and meta-analysis of Triple P. BMC Medicine, 10:130.

Windle M, Iwawaki S, \& Lerner RM. (1988). Cross-cultural comparability of temperament among Japanese and American preschool children. International Journal of Psychology, 23, 547-567.

Windle M, \& Lerner RM. (1986). Reassessing the dimensions of temperamental individuality across the life span: The Revised Dimensions of Temperament Survey (DOTS-R). Journal of Adolescent Research, 1, 213-230.

Withrow D, \& Alter DA. (2011). The economic burden of obesity worldwide: A systematic review of the direct costs of obesity. Obesity Reviews, 12, 131-141.

Wolman J, Skelly E, Kolotourou M, Lawson M, \& Sacher P. (2008). Tackling toddler obesity through a pilot community-based family intervention. Community Practitioner, 81, 28-31.

Wong SL, \& Leatherdale ST. (2009). Association between sedentary behavior, physical activity, and obesity: Inactivity among active kids. Preventing Chronic Disease, 6, A26.

Wood D, Bruner JS, \& Ross G. (1976). The role of tutoring in problem solving. Journal of Child Psychology and Psychiatry, 17, 89-100.

World Health Organization. (2000). Obesity: preventing and managing the global epidemic. Report of a WHO consultation. Geneva: Switzerland (WHO Technical Report Series, No. 894).

World Health Organization. (2006). Obesity and overweight. Geneva: Switzerland (WHO Fact Sheet, No. 311). 
Wu M, Adams R, \& Haldane S. (2007). Conquest. Victoria, Australia: The Australian Council for Educational Research.

Wu T, Dixon WE, Dalton WT, Tudiver F, \& Liu X. (2010). Joint effects of child temperament and maternal sensitivity on the development of childhood obesity. Maternal and Child Health Journal, 15, 469-477.

Wudy SA, Hagemann S, Dempfle A, Ringler G, Blum WF, Berthold LD, Alzen G, Gortner L, \& Hebebrand J. (2005). Children with idiopathic short stature are poor eaters and have decreased Body Mass Index. Pediatrics, 116, e52-e57.

Young KM, Northern JJ, Lister KM, Drummond JA, \& O'Brien WH. (2007). A meta-analysis of familybehavioral weight-loss treatments for children. Clinical Psychology Review, 27, 240-249.

Zabinski MF, Saelens BE, Stein RI, Hayden-Wade HA, \& Wilfley DE. (2003). Overweight children's barriers to and support for physical activity. Obesity Research, 11, 238-246.

Zecevic CA, Tremblay L, Lovsin T, \& Michel L. (2010). Parental influence on young children's physical activity. International Journal of Pediatrics. Epub July 7.

Zeller MH, Boles RE, \& Reiter-Purtill J. (2008). The additive and interactive effects of parenting style and temperament in obese youth seeking treatment. International Journal of Obesity, 32, 1474-1480.

Zeller MH, Reiter-Purtill J, Modi AC, Gutzwiller J, Vannatta K, Davies WH. (2007). Controlled study of critical parent and family factors in the obesigenic environment. Obesity, 15, 126-136. 
SUMMARY 
Worldwide, childhood overweight and obesity are a major concern because of its dramatic increase and prevalence, its persistence into adulthood and its association with a host of negative health outcomes. Parents are pivotal to developing obesogenic behaviors in children (overconsumption of calories, low levels of physical activity, and high levels of sedentary behavior).

Childhood overweight has elicited broad research interest in the question how families influence child energy balance-related behaviors (EBRBs). Accurate data on intervening processes by which parenting impact child's weight are lacking. Progress in research on specific parenting practices related to food and physical activity (so-called food and activity-related parenting) is severely hampered by the lack of common measures of consensually defined constructs that have been validated in multiple samples around the world. Research on food and activity-related parenting practices has looked at individual dimensions while largely failing to consider the context of their use. Alternatively, research focusing on general parenting alone is limited by a vague understanding of the processes or mechanisms through which global dimensions of parenting shape development specifically in the eating and activity domain. The majority of studies fail to assess parenting context as one of the moderating factors. Another issue inhibiting progress in this research is the broad diversity of parenting measures, some measuring similar and some very different constructs, with no common set of consensually identified priority items. These problems are compounded by poor theoretical development, and limited consideration of diverse demographical contexts. Finally, much of the research has been cross-sectional.

Although extensive parenting research has been conducted around the world, conflicting findings have been reported. Therefore, intervention programs are based on incomplete knowledge regarding important and modifiable causes of childhood overweight. This is an important problem because without such information, we are unable to comprehensively understand the role of parents and make public health and preventive recommendations to educate parents and children to reduce childhood overweight. Hence, interventions have had disappointing effects.

Parenting is the central theme of this dissertation. The aim is to evaluate complex parenting - child weight relationships. We know parents play a key role in the development of children's EBRB, but accurate data on intervening processes by which parenting impact these behaviors are lacking. With this dissertation, we hope to get more insights in this issue to fight childhood overweight.

This dissertation consists of three parts to increase our knowledge about the role of parents. It is important to summarize existing knowledge about this subject. Therefore, in the first part, three reviews were conducted on the role of parents in influencing their children's EBRBs. In the second part, we developed and/or validated questionnaires measuring parenting and child behavior (child temperament, feeding and eating styles, and general parenting). And finally, parental influences on children's EBRBs are evaluated using the validated instruments that were administered to participants of the ongoing KOALA Birth Cohort Study. This knowledge could be applied to improve existing family-based overweight intervention programs. 


\section{PART 1: Parenting reviews}

First of all, we were interested in the research findings with regard to the influence of general parenting styles and practices on children's overweight-related behavior. Therefore, we decided to conduct a literature review. Chapter 2 summarizes existing observational studies about the relationships between general parenting and child EBRBs and weight status. In total, 36 studies were included meeting the inclusion criteria, and 21 different parenting instruments were used to measure general parenting. Findings suggest that children raised in authoritative homes (characterized by a family context of expressing warmth and emotional support, together with using clear, bidirectional communication) ate more healthily, were more physically active and had lower Body Mass Index (BMI) levels, compared to children who were raised with other styles (authoritarian, permissive, neglectful). Findings of the few moderation studies indicate that general parenting has a differential impact on children's weight-related outcomes, depending on child and parental characteristics. Discrepancies in study results were found due to the broad diversity of parenting measures (some measuring similar and some very different constructs and some were completed by parents or children), the diverse demographical contexts, and different methods used to assign parents to styles. It is concluded that general parenting is important, but the exact mechanisms of the influence of parents in influencing weight-related behaviors of their children remains unclear.

We were interested in assessing the effectiveness of current intervention programs including general parenting for the prevention and treatment of childhood overweight. Chapter 3 provides an overview of existing interventions addressing general parenting in order to prevent or treat childhood obesity. Seven studies were eligible for inclusion. The studies described four different general parenting programs, which were supplemented with lifestyle components (i.e., nutrition and physical activity). All studies showed significant small to moderate intervention effects on at least one weight-related outcome measure. This review shows that despite the emerging observational evidence for the role of parenting in children's weight-related outcomes, few interventions have been developed that address general parenting in the prevention of childhood obesity. These interventions provide evidence that the promotion of authoritative parenting is an effective strategy for the prevention and management of childhood overweight and obesity.

A third literature review which we conducted was based on parenting practices in regard to physical activity. We were interested in the developmental and validation processes of existing questionnaires assessing these practices. Chapter 4 reviews existing questionnaires of parenting practices in regard to physical activity (e.g., parental support and facilitation of enrolment in physical activity classes), their psychometric performance and correlation with children's physical activity levels. Eleven unique PA parenting questionnaires were identified, and 46 studies that used these instruments were included. Findings highlight the tremendous variation in the conceptualization and measurement of physical activity parenting, common use of non-validated instruments, and lack of comprehensive measures. The development of theory-based physical activity parenting measures should be prioritized to guide the study of the parental role in promoting child physical activity as well as the design of family-based physical activity interventions. 


\section{PART 2: Measurement}

Based on the findings of the literature reviews described in chapter 2 to 4 , accurate data on the influence of parents on children's EBRBs are lacking partially due to the broad diversity of measures which are often poorly validated. Therefore, we decided to develop and/or validate questionnaires related to child temperament, feeding and eating styles, and general parenting. These instruments were administered to participants of the KOALA Birth Cohort Study to examine the hypothesized parenting model.

\section{Child temperament}

In our hypothesized parenting model, visualized in the introduction (chapter 1), we describe temperament as one of the moderating factors in the relation between parenting practices and children's EBRBs. An indepth validation study of the comprehensive Children's Behavior Questionnaire (CBQ) was absent in the Netherlands. In the following three chapters we describe the validation of diverse forms of the CBQ.

Chapter 5 focuses on validating several forms of the CBQ (195 items and 36 items), developed in the United States (US). Three global traits of temperament are measured including 'extraversion', 'negative affectivity', and 'effortful control'. In total, 353 Dutch parents of 6- to 8-year-olds completed the instrument. Following factor analytic procedures, the three-factor temperament structure as previously identified in the US could be replicated in the Dutch sample. Cross-cultural comparisons of temperament structure were also performed. Results demonstrated a relatively high degree of factor similarity of the Dutch sample with other cultures. The findings provide evidence for applicability of the CBQ in the Netherlands as a promising instrument to comprehensively assess reactive and self-regulative temperamental dimensions in young children.

Chapter 6 describes the development and validation of a one-item temperament scale, with three vignettes addressing the three global traits of temperament. This one-item scale was developed to assist in tailoring interventions. Parents were asked to choose the temperament trait (vignette) most applicable to their child. The one-item measure was tested against the 36-item CBQ. Both questionnaires were completed by 237 caregivers of 3- to 5-year-olds in the US. Additionally, the psychometric properties of the 36-item measure were assessed. Classical test theory analysis demonstrated adequate internal consistency and factor analysis confirmed a three-factor structure. Potential improvements to the measure were identified using item response modeling. The findings with regard to the validation of the one-item temperament scale show that the three response categories correlated with the temperament factors of the 36-item scale, as expected. The one-item temperament scale may be applicable for clinical use.

Chapter 7 describes the validation of a 3-item temperament measure and child-report 13-item impulsivity scale. Impulsivity is one of the characteristics of extraversion, which was previously found to be correlated with childhood overweight. First, the one-item temperament measure described in chapter 6 was adapted. For every vignette, parents are asked to select how much it applied to their child on a scale ranging from (Extremely untrue of your child) to (Extremely true of your child), thereby keeping intact the multi- 
dimensionality of the scale. Again, this measure was tested against the 36 -item CBQ. In total, 229 Dutch parents and their 6- to 7-year-old children completed the temperament measures and 13-item impulsivity scale, respectively. Psychometrics of the $\mathrm{CBQ}$ and the impulsivity scale were examined, which were considered good in terms of internal consistency and factorial structure. The findings with regard to the validation of the 3-item temperament measure show that the three items correlated with the averages scores on the corresponding $\mathrm{CBQ}$ factors. Furthermore, surgency was highly related to impulsivity. Findings provide evidence for the applicability of the impulsivity and temperament measures for observational research.

\section{Eating and feeding styles}

Eating behavior of children is one of the other potential moderating factors in the relationship between food parenting practices and children's overweight-related behavior. A questionnaire comprehensively assessing (variation in) children's eating style was developed in the United Kingdom (UK) and only available in English. Chapter 8 describes the translation and validation of the Children's Eating Behavior Questionnaire (CEBQ) in a Dutch sample of 135 parents of 6- to 7-year-olds. Factor analyses were performed and relationships between child eating style and BMI were investigated. Results generally confirmed the theoretical factor structure, with acceptable internal reliability and between-subscale correlations. Linear regression analyses revealed that BMI z-scores were positively associated with the 'food approach' subscales (food responsiveness, enjoyment of food, emotional overeating) and negatively with 'food avoidant' subscales (satiety responsiveness, slowness in eating, emotional undereating, and food fussiness). The results support the use of the CEBQ as a psychometrically sound tool for assessing eating behaviors and the study demonstrates its applicability in overweight-related studies.

Dutch questionnaires assessing food parenting practices are also scarce. Therefore, we decided to translate a food parenting questionnaire from the UK, in addition to the already used Child Feeding Questionnaire within the KOALA birth cohort study. Chapter 9 describes the translation and validation of the Parental Feeding Style Questionnaire in a Dutch sample of 135 parents of 6- to 7-year-olds. Psychometric evaluations, including factor analyses, were performed to assess whether the four factors underlying this questionnaire ('food control', 'instrumental feeding', 'emotional feeding', 'encouragement to eat') were replicated in the Dutch sample. Additionally, associations between parental feeding styles and dietary intake behaviors of both the parent and the child were assessed to predict eating behavior. Results indicated considerable similarity of factor structure, internal reliability and between-subscale correlations with the original British study. The food parenting practices 'instrumental feeding' (i.e. using food as a reward) and 'emotional feeding' (i.e. feeding in response to children's emotional distress) were positively related to children's snacking behavior. The food parenting practice 'encouragement to eat' was negatively associated with children's snacking behavior. Various feeding styles were found to be related to parental dietary behaviors. Findings indicate the importance of acknowledging food parenting practices in future research efforts as well as in the development of family-based interventions promoting healthy eating habits among children. 


\section{$\underline{\text { General parenting }}$}

General parenting is another important factor besides the more specific parenting practices. We think that the relationships between parenting practices and children's EBRBs are influenced by the general parenting context within a family. Parenting is difficult to measure and operationalize. Chapter $\mathbf{1 0}$ summarizes the discussions from a working group of experts in the childhood overweight field. This working group, led by Thomas Power and the author of this dissertation, on general parenting measurement took place at the pre-conference meeting to the 'International Society of Behavioral Nutrition and Physical Activity' annual meeting, 'Measuring parenting: Current status and consensus reports'. The group discussed a range of issues and offered numerous directions for future research regarding the assessment of parenting. These included: 1) discussing issues related to differences in conceptualizations with regard to general versus domain specific parenting styles and practices and its influence on children's overweight-related behaviors; 2) suggestions for novel approaches to parenting measurement; 3 ) the development of measurement instruments that can be used across cultural groups and necessity to identify underlying universal characteristics of parenting that operate across cultures; 4) identifying bi-directional influences between parents and children and interaction with other family members; 5) broadening assessments beyond the immediate family; and 6) designing effective interventions at all levels. Collaboration within the burgeoning parenting research community is a top priority to develop qualified instruments.

Despite the large number of general parenting instruments (see chapter 2) most of them only assess limited aspects of parenting. Considerable disagreement exists about how to best assess parenting (chapter 10). Therefore, we decided to develop a general parenting questionnaire. Chapter 11 describes the development of the 'Comprehensive General Parenting Questionnaire' (CGPQ), developed for use in this PhD project. First, we identified the core constructs of parenting (i.e., 'nurturance', 'structure', 'behavioral control', 'coercive control', and 'overprotection'), and clarified their defining features. The ultimate goal is to facilitate research exploring how parenting influences children's health related behaviors.

A mixed methods approach was used for the development of the CGPQ comprising the following four steps: 1) items were identified from existing parenting questionnaires based on our framework including the five constructs of parenting; 2) cognitive interviews and author review informed the modification, deletion and/or replacement of items; 3) advanced statistical analyses including confirmatory factor analyses and item-response modeling were conducted to test our theoretical five-factor parenting model and to develop fit items; and 4) additional author reviews and cognitive interviews were done to review the fit items, determine if any construct was missing or inadequately assessed, assess content validity, and verify wording of the modified items.

The survey was administered to large samples of parents of 5- to 13-year-olds in the Netherlands, Belgium and the US. In total, 1497 caregivers completed the questionnaire. Additionally, a questionnaire regarding personality characteristics ('Big Five') of the caregiver was administered. The reduced 62-item questionnaire revealed acceptable fit of our parenting model and acceptable item-response modeling item fit statistics. Caregiver personality was related to the parenting constructs as measured by the CGPQ. The 
personality traits of extraversion, agreeableness, conscientiousness, and openness to experience were positively associated with parenting constructs (i.e., nurturance, structure, behavioral control) previously found to be related to more positive child health outcomes, whereas the trait of neuroticism was associated with coercive control and a chaotic home environment. Based on expert panel review and cognitive interviews the questionnaire was further modified to include 85 items. This proposed 85 -item scale may facilitate research exploring how parenting influences children's health behaviors.

\section{PART 3: Parenting - child eating behavior relationship}

In this last part, we partially tested our hypothesized parenting model. Research on parenting practices has focused on individual behaviors while largely failing to consider the larger context of their use. Chapter 12 aims to examine the extent to which food parenting practices predict the development of child eating behavior. Additionally, the study tested the moderating role of both general parenting and child characteristics on the relationship between food parenting practices and children's eating patterns. For this purpose, we used data from the KOALA Birth Cohort Study. Associations between food parenting and child eating were present. Instrumental and emotional feeding, and pressure to eat were found to have detrimental associations with child eating behavior, whereas covert control, encouragement and restriction were found to have favorable associations. These findings are partially confimed in chapter 9. Although the strength of relations differed depending on the context, all findings (strength of association between food parenting and child eating behavior) of the stratified analyses were in the same direction. Two examples, for children who were reared in a positive parenting context, restriction, encouragement and covert control worked better than for children who were reared in a less positive parenting context (e.g., coercive and overprotective home environments). For children who grew up in a less positive parenting context, emotional and instrumental feeding worked more detrimental. Moreover, some food parenting practices did not interact with other factors in explaining child eating behavior. These might be robust for contextual factors and suitable to target to parents when using more general types of interventions where tailoring to an individual is not possible. Thus, parents have a large influence on their children's eating behavior. Food parenting practices are important correlates of children's eating behavior, and it is important to take into account the parents' parenting style and temperament and eating behavior of children.

\section{General discussion}

Chapter 13 discusses the methodological issues and the scientific, practical and theoretical implications of the studies described in this dissertation. It was concluded that the role of parents is pivotal in the development of their child's eating and activity behaviors. Several parent and child characteristics have been proven to impact the relationship between parenting practices and child overweight-inducing behaviors. With regard to assessment of parenting, there is a need for further development and validation of questionnaires, especially assessing food parenting practices and physical activity parenting practices. Efforts were made to clarify as to which constructs should be assessed and how a specific construct should be operationalized. Input from different sources is needed to progress in this area. The conference 
described in chapter 10 partially contributed to this. It is important to assess parenting practices using theory-based ecological research approaches acknowledging the dynamic interplay of types and levels of parental influence on child EBRB using longitudinal research designs. As our understanding of familial influences on children's obesogenic behavior and weight status improves, health promoters can develop more effective family-based interventions. 
SAMENVATTING 
Overgewicht en obesitas bij kinderen is wereldwijd een groot probleem. Dit komt doordat overgewicht vaker voorkomt en zowel gedurende de kindertijd en de volwassen leeftijd is gerelateerd aan een slechte gezondheid. Ouders spelen een belangrijke rol in de ontwikkeling van overgewichtgerelateerd gedrag van hun kinderen (hoge calorie-inname, weinig beweging, en veel sedentair gedrag).

Overgewicht bij kinderen krijgt veel aandacht. Wetenschappers zijn geïnteresseerd in de vraag hoe ouders energiebalans-gerelateerd gedrag van hun kind beïnvloeden. Er zijn echter weinig gegevens beschikbaar over de mate waarin ouders het gewicht van kinderen kunnen beïnvloeden. Voortgang in het onderzoek naar specifieke ouderschapspraktijken op het gebied van voeding en beweging (zogenaamde voedings- en beweegpraktijken) is ernstig verstoord door gebrek aan inhoudelijke en valide vragenlijsten die getest zijn in verschillende steekproeven wereldwijd. Daarnaast is het onderzoek naar ouderschapspraktijken beperkt, omdat de meeste studies de context van deze praktijken niet onderzoeken. Onderzoek dat zich juist richt op de context van deze specifieke praktijken, zoals algemene opvoeding (het emotionele klimaat in de thuisomgeving), is tevens beperkt. $\mathrm{Er}$ is namelijk niet precies bekend hoe algemene opvoeding energiebalans-gerelateerd gedrag van kinderen beïnvloedt en er is een tekortkoming van veel onderzoeken om de opvoedingscontext als een van de modererende factoren mee te nemen. Er zijn daarnaast nog andere tekortkomingen aan te wijzen in de studies naar opvoeding en overgewicht bij kinderen. Voortgang wordt belemmerd door een veelvoud aan opvoedingsvragenlijsten, die telkens weer gebruik maken van verschillende constructen. Als deze constructen wel hetzelfde zijn, dan worden ze vaak weer anders geoperationaliseerd. Deze problemen worden verder gecompliceerd door een slechte theoretische onderbouwing en veelvoud aan demografische contexten waardoor vergelijkbaarheid tussen studies wordt gecompliceerd. Ook maken bestaande studies vaak gebruik van dwarsdoorsnedeonderzoek.

Hoewel er wereldwijd veel onderzoek is verricht naar opvoeding, zijn er bevindingen die elkaar tegenspreken. De huidige interventieprogramma's zijn daarom veelal gebaseerd op onvolledige informatie over belangrijke en veranderbare oorzaken van overgewicht bij kinderen, waardoor ze veelal teleurstellende effecten hebben. Dit is een belangrijk probleem, want zonder deze informatie is het niet mogelijk om precies te achterhalen wat de rol is van ouders. Hierdoor is het lastig om interventieprogramma's te ontwikkelen voor ouders en kinderen om overgewicht te beperken.

Opvoeding is het centrale thema van dit proefschrift. Het doel is om de complexe relatie tussen opvoeding en gewichtsstatus van kinderen te onderzoeken. We weten dat ouders een belangrijke rol spelen in de ontwikkeling van overgewichtgerelateerd gedrag in hun kinderen, maar er zijn weinig gegevens beschikbaar over de mate waarin ouders dit gedrag beïnvloeden. We hopen met dit proefschrift hier meer inzicht in te krijgen zodat uiteindelijk de stijging van overgewicht en obesitas onder kinderen een halt toegeroepen kan worden.

Dit proefschrift bestaat uit drie delen om onze kennis over de rol van ouders te vergroten. Allereerst is het van belang om te weten wat er al bekend is over dit onderwerp. Daarom hebben we in het eerste deel van dit proefschrift drie literatuurstudies uitgevoerd over de rol van ouders in het beïnvloeden van 
energiebalans-gerelateerd gedrag van hun kinderen. In het tweede deel hebben we vragenlijsten die opvoeding en gedrag van kinderen meten ontwikkeld en/of gevalideerd (temperament, eetstijl, voedingspraktijken en algemene opvoeding). Als laatste hebben we gekeken naar de invloed van ouders op energiebalans-gerelateerd gedrag van hun kinderen waarbij gebruik is gemaakt van deze gevalideerde meetinstrumenten. De instrumenten zijn afgenomen bij deelnemers van de lopende grootschalige KOALA ('Kind, Ouders en gezondheid, Aandacht voor Leefstijl en Aanleg') cohortstudie. De gegevens van deze metingen kunnen worden gebruikt voor het verbeteren van bestaande interventieprogramma's voor ouders met kinderen die overgewicht hebben.

\section{DEEL 1: Literatuurstudies op het gebied van opvoeding}

Allereerst waren we geïnteresseerd naar de bevindingen van eerder onderzoek over de relatie tussen algemene opvoedingsstijlen- en praktijken van ouders en overgewichtgerelateerd gedrag bij kinderen. We hebben daarom besloten om een literatuurstudie uit te voeren. In hoofdstuk $\mathbf{2}$ vatten we de bevindingen van observationele studies samen die dit onderzoeken. In totaal werden 36 studies gevonden die aan de inclusiecriteria voldeden. Er werden in totaal 21 verschillende vragenlijsten gebruikt om algemene opvoeding te meten. De bevindingen laten zien dat kinderen die zijn opgegroeid in autoritatieve gezinnen (gekenmerkt door opvoedingscontext waarin warmte en emotionele steun centraal staan, samen met het gebruik van duidelijke, tweerichtingscommunicatie) over het algemeen gezond aten, vaker fysiek actief waren en een lagere Body Mass Index rapporteerden, in vergelijking met kinderen die zijn opgegroeid in gezinnen met andere opvoedingsstijlen (autoritaire, permissieve en verwaarlozende opvoeding). De uitkomsten van de enkele moderatiestudies laten zien dat de invloed van algemene opvoeding op gewichtsgerelateerd gedrag van kinderen afhankelijk is van bepaalde kenmerken van het kind en de ouder. De verscheidenheid in resultaten kan worden verklaard op grond van de diversiteit aan opvoedingsvragenlijsten (sommige meten dezelfde en andere meten verschillende constructen, sommige zijn ingevuld door ouders en andere door kinderen), de diversiteit in demografische contexten en de verschillende methodes, die gebruikt zijn om ouders in de opvoedingsstijlen in te delen. Er kan worden geconcludeerd dat algemene opvoeding een factor is die aan gewichtsgerelateerd gedrag bij kinderen is gerelateerd. De mate waarin dit effect zich voordoet, dient nog verder te worden onderzocht.

We hebben vervolgens de effectiviteit van bestaande interventies die zich richten op algemene opvoedingsstijl- en praktijken voor preventie en behandeling van overgewicht bij kinderen onderzocht. Hoofdstuk 3 geeft een overzicht van bestaande interventieprogramma's die zich richten op dit onderwerp. Zeven studies zijn geschikt bevonden voor inclusie. Deze studies beschrijven in totaal vier verschillende programma's gericht op algemene opvoeding en die zijn aangevuld met op leefstijlgerichte componenten (zoals voeding en beweging). Alle studies laten kleine en matige significante effecten zien op tenminste één gewichtsgerelateerde uitkomstmaat. Deze literatuurstudie laat zien dat er maar weinig interventies zijn ontwikkeld die zich richten op algemene opvoeding in de preventie en behandeling van overgewicht bij kinderen. Dit ondanks de toenemende overtuiging in empirisch onderzoek van het belang van opvoeding in de ontwikkeling van gewichtsgerelateerde uitkomsten van hun kinderen (hoofdstuk 2). De weinige 
interventies die er wél zijn benadrukken de effectiviteit van het bevorderen van een autoritatieve opvoedingsstijl voor de preventie en aanpak van overgewicht en obesitas bij kinderen.

Een derde literatuurstudie die we hebben uitgevoerd, richt zich op beweegpraktijken van ouders. We waren geïnteresseerd in het ontwikkelings- en validatieproces van vragenlijsten die deze ouderschapspraktijken meten. Hoofdstuk 4 geeft een overzicht van deze beweegpraktijkenvragenlijsten (zoals steun van ouders om te bewegen en bevordering van lidmaatschap van sportclubs). Daarnaast worden de psychometrische eigenschappen van deze vragenlijsten beschreven en de correlatie hiervan met het beweeggedrag van kinderen. In totaal hebben we 11 unieke beweegpraktijkenvragenlijsten geïdentificeerd en 46 studies zijn opgenomen die deze vragenlijsten hebben gebruikt. Er blijkt een grote variatie te zijn in conceptualisatie van constructen die beweegpraktijken meten, er wordt veelvuldig gebruik gemaakt van niet-gevalideerde vragenlijsten en er is een schaarste aan uitgebreide vragenlijsten die meerdere constructen meten. De ontwikkeling van op theorie gebaseerde beweegpraktijkenvragenlijsten moet worden bevorderd om dit te voorkomen. Het is belangrijk dat de rol van ouders in de bevordering van fysieke activiteit van hun kinderen in toekomstig onderzoek beter onderzocht wordt. Alleen dan kunnen er betere interventies voor ouders worden ontwikkeld om beweeggedrag bij kinderen te bevorderen.

\section{DEEL 2: Meetinstrumenten}

De literatuurstudies in de hoofdstukken 2 tot 4 laten zien dat er nog veel onduidelijk is over de rol van ouders in het beïnvloeden van energiebalans-gerelateerd gedrag van kinderen. Dit komt mede door gebruik van een veelvoud aan vragenlijsten die bovendien vaak slecht zijn gevalideerd. Omdat de huidige resultaten niet afdoende zijn en veel vragenlijsten niet beschikbaar zijn in het Nederlands, hebben we besloten een aantal vragenlijsten te ontwikkelen en/of te valideren. Deze vragenlijsten hebben betrekking op de volgende onderwerpen: 'temperament van kinderen', 'voedingspraktijken van ouders en eetstijlen van kinderen' en 'algemene opvoeding'. Deze vragenlijsten zijn uiteindelijk afgenomen bij deelnemers van de KOALA cohortstudie om te kijken wat de invloed is van opvoeding op energiebalans-gerelateerd gedrag van kinderen.

\section{Temperament van het kind}

In ons voorgestelde model over de rol van opvoeding, gevisualiseerd in de introductie (hoofdstuk 1), is temperament van het kind beschreven als een van de modererende factoren in de relatie tussen ouderschapspraktijken en energiebalans-gerelateerd gedrag van kinderen. Een uitgebreide validatiestudie van de veelomvattende temperamentsvragenlijst voor kinderen was afwezig in Nederland. De komende drie hoofdstukken beschrijven de validatie van diverse varianten van deze temperamentsvragenlijst.

Hoofdstuk 5 richt zich op de validatie van de temperamentsvragenlijst voor kinderen (in twee varianten van respectievelijk 195 vragen en 36 vragen), ontwikkeld in de Verenigde Staten. De drie belangrijkste 
temperamentskenmerken die worden onderscheiden in deze vragenlijst zijn 'extraversie', 'negatieve affectiviteit', en 'zelf-controle'. In totaal hebben 353 ouders van Nederlandse kinderen in de leeftijd van 6 tot 8 jaar de vragenlijst ingevuld. Het drie-factorenmodel dat in de Verenigde Staten resulteerde uit het onderzoek bleek ook van toepassing te zijn op de Nederlandse steekproef. Daarnaast zijn de resultaten van de temperamentsvragenlijst ook op basis van land met elkaar vergeleken. Er werd een hoge mate van gelijkheid in factorstructuur van de Nederlandse steekproef met andere culturen vastgesteld. Deze bevindingen laten zien dat de temperamentsvragenlijst in Nederland in ieder geval toepasbaar is, als een instrument om de drie belangrijkste temperamentskenmerken in jonge kinderen in kaart te brengen.

Hoofdstuk 6 beschrijft de ontwikkeling en de validatie van een zeer korte temperamentsvragenlijst in de Verenigde Staten, met drie vignettes welke de algemene temperamentskenmerken omvatten. Deze vragenlijst is ontwikkeld voor gebruik in interventiestudies. Ouders dienen te kiezen welk temperamentskenmerk (vignette) het meest van toepassing is op hun kind en krijgen hierbij de mogelijkheid om te kiezen tussen drie alternatieven (de algemene temperamentskenmerken). De lijst is afgezet tegen de temperamentsvragenlijst van 36 vragen. Beide vragenlijsten werden ingevuld door 237 opvoeders van 3- tot 5-jarige kinderen in de Verenigde Staten. Ook de psychometrische eigenschappen van de 36 vragen lange temperamentsvragenlijst werden onderzocht. Analyses gebaseerd op de klassieke testtheorie laten zien dat de interne consistentie hiervan afdoende was en een factoranalyse bevestigde de validiteit van de drie-factorenstructuur. Potentiële verbeteringen voor de 36 vragen lange vragenlijst werden geïdentificeerd met behulp van item-responsemodellen. Hierbij wordt de kwaliteit van een vragenlijst onderzocht door elk van de factoren individueel te beschouwen. De resultaten met betrekking tot de validatie van de zeer korte temperamentsvragenlijst laten zien dat de drie temperamentskenmerken correleerden met de temperamentsfactoren van de 36 vragen lange vragenlijst zoals verwacht. Er zijn mogelijkheden om de zeer korte temperamentsvragenlijst toe te passen voor klinische doeleinden.

Hoofdstuk 7 beschrijft de validatie van een temperamentsvragenlijst van 3 vragen en een impulsiviteitvragenlijst van 13 vragen. De impulsiviteitsvragen werden door kinderen beantwoord. Impulsiviteit is een van de onderdelen van extraversie, waarvan voorheen gebleken is dat er een positieve correlatie is met overgewicht bij kinderen. Hiervoor werd de zeer korte temperamentsvragenlijst uit hoofdstuk 6 aangepast. In de zeer korte temperamentsvragenlijst werd ouders gevraagd te kiezen welk van de drie temperamentskenmerken (vignette) het meest van toepassing is op hun kind. Ouders moeten in de aangepaste versie voor elk temperamentskenmerk (vignette) aangegeven in hoeverre dat van toepassing is op hun kind om zo de multi-dimensionaliteit van de schaal te waarborgen. Hiervoor werd een vijfpuntsschaal gebruikt van 1 (Helemaal niet van toepassing) tot 5 (Volledig van toepassing). Ook deze aangepaste lijst is afgezet tegen de temperamentsvragenlijst van 36 vragen. In totaal hebben 229 ouders en hun 6- tot 7-jarige kinderen respectievelijk de temperamentsvragenlijsten en de impulsiviteitsvragenlijst ingevuld. De psychometrische eigenschappen van de 36 vragen lange temperamentsvragenlijst en de impulsiviteitvragenlijst werden onderzocht. De interne consistentie en factorstructuur waren ook hier afdoende; de betrouwbaarheid en validiteit van de drie-factorenstructuur werd bevestigd voor de temperamentsvragenlijst van 36 vragen en de betrouwbaarheid en validiteit van de onderliggende een- 
factorstructuur van de impulsiviteitsvragenlijst werd bevestigd. De resultaten met betrekking tot de validatie van de temperamentsvragenlijst van drie vragen laten zien dat de drie vragen correleerden met de gemiddelde scores van de drie temperamentsfactoren. Bovendien bleek extraversie hoog gecorreleerd met impulsiviteit. De bevindingen laten zien dat beide vragenlijsten toepasbaar zijn voor gebruik in observationeel onderzoek.

\section{Voedingspraktijken en eetstijlen}

Naast temperament kan ook eetgedrag van het kind als een van de modererende factoren worden gezien op de relatie tussen voedingspraktijken van ouders en overgewicht bij kinderen. Een vragenlijst die uitgebreid de (variatie in) eetstijl van kinderen meet, was ontwikkeld in het Verenigd Koninkrijk en niet beschikbaar in het Nederlands. Hoofdstuk 8 beschrijft daarom de vertaling en de validatie van de eetgedragsvragenlijst voor kinderen ('Children's Eating Behavior Questionnaire') in een Nederlandse steekproef van 135 ouders met kinderen in de leeftijd van 6 tot 7 jaar. We hebben factoranalyses uitgevoerd en correlaties tussen eetstijl en Body Mass Index onderzocht. De factorstructuur was vrijwel gelijk aan de structuur die in het Verenigd Koninkrijk is gevonden, met acceptabele interne consistenties en correlaties tussen de verschillende schalen van de vragenlijst. Lineaire regressie analyses lieten zien dat de gestandaardiseerde Body Mass Index scores positief geassocieerd zijn met de 'voedingsbevorderende' schalen ('voedselresponsiviteit', 'genieten van voedsel', 'emotioneel overeten') en negatief met de 'voedingsvermijdende' schalen ('voedingsinname minderen na verzadiging', 'trager eten', 'emotioneel ondereten', 'kieskeurig ten aanzien van voedsel') van de eetgedragsvragenlijst. De eetgedragsvragenlijst is dus betrouwbaar en valide om eetgedrag te meten. De vragenlijst is toepasbaar in overgewichtgerelateerde studies om kinderen te identificeren die een hoger risico lopen om overgewicht te ontwikkelen.

Net zoals vragenlijsten die eetstijlen bij kinderen meten zijn ook vragenlijsten die ouderschapspraktijken meten ten aanzien van voeding schaars in de Nederlandse taal. We hebben als goede aanvulling op de 'Child Feeding Questionnaire' (voorheen opgenomen binnen de KOALA studie) een voedingspraktijkenvragenlijst vertaald vanuit het Engels. In hoofdstuk 9 wordt de validatie van de voedingspraktijkenvragenlijst ('Parental Feeding Style Questionnaire') in een Nederlandse steekproef van 135 ouders met kinderen in de leeftijd van 6 tot 7 jaar beschreven. Psychometrische evaluaties waaronder factoranalyses zijn uitgevoerd om te onderzoeken of de vier voedingspraktijken die deze lijst meet ('controle over voeding', 'instrumenteel voeden', 'emotioneel voeden', 'aanmoedigen om te eten') ook kunnen worden herkend in een Nederlandse steekproef. Bovendien zijn de correlaties tussen voedingspraktijken en voedingsgedrag van zowel de ouder als het kind onderzocht om te bestuderen in welke mate voedingsgedrag voorspeld kan worden. De factorstructuur, interne consistentie en correlaties tussen de verschillende schalen vertoonden ook hier grote overeenkomsten met de oorspronkelijke studie uit het Verenigd Koninkrijk. 'Instrumenteel voeden' (ouders die voeding gebruiken als beloning) en 'emotioneel voeden' (het geven van voeding als reactie op emotionele stress van hun kind) waren positief gecorreleerd met snackgedrag van kinderen. De voedingspraktijk 'aanmoedigen om te eten' was negatief geassocieerd 
met snackgedrag van kinderen. Verschillende voedingspraktijken waren gerelateerd met voedingsgedrag van ouders. De bevindingen laten zien dat het belangrijk is om voedingspraktijken te meten in toekomstige studies en mee te nemen in de ontwikkeling van interventieprogramma's voor ouders om gezonde eetgewoontes van kinderen te bevorderen.

\section{Algemene opvoeding}

Naast ouderschapspraktijken is ook algemene opvoeding belangrijk. Wij gaan toetsen of de relatie tussen ouderschapspraktijken en energiebalans-gerelateerd gedrag van kinderen beïnvloed wordt door de algemene opvoedingscontext binnen een gezin. Algemene opvoeding blijkt lastig meet- en operationaliseerbaar. Dit leidt tot veel discussie onder wetenschappers. Hoofdstuk $\mathbf{1 0}$ vat de discussie samen van een expertpanel van onderzoekers op het gebied van overgewicht bij kinderen. Dit expertpanel heeft plaatsgevonden tijdens een congres voorafgaand aan de jaarlijkse bijeenkomst van de 'International Society of Behavioral Nutrition and Physical Activity' onder leiding van Thomas Power en de auteur van dit proefschrift. De bijeenkomst had als titel: 'Measuring parenting: Current status and consensus reports'. De werkgroep besprak een aantal punten over het meten van algemene opvoeding en kwam bovendien met een aantal aanbevelingen waar toekomstig onderzoek zich op kan richten. De belangrijkste discussiepunten zijn: 1) onduidelijkheden oplossen over de verschillen tussen algemene versus domein specifieke opvoedingsstijlen- en praktijken en hun invloed op overgewichtgerelateerd gedrag van kinderen; 2) suggesties geven over nieuwe benaderingen om opvoeding te meten; 3) de ontwikkeling van meetinstrumenten die inzetbaar zijn voor meerdere culturele groepen en noodzaak om universele kenmerken van opvoeding te ontdekken voor verschillende culturen; 4) het belang van het meten van wederkerige invloeden tussen ouder en kind en interactie met andere gezinsleden; 5) belang van het meten van opvoeding buiten de directe familieleden (zoals door leraren en vrienden); en 6) de ontwikkeling van effectieve interventies op verschillende niveaus en intensiteiten, rekening houdend met de doelgroep. Samenwerking met de groeiende onderzoeksgemeenschap op het gebied van opvoeding heeft prioriteit om geschikte meetinstrumenten te ontwikkelen.

Zoals we hebben geconstateerd in hoofdstuk 2 worden er veel verschillende algemene opvoedingsvragenlijsten gebruikt, maar richten de meeste van deze lijsten zich op een beperkt aantal aspecten van opvoeding. Er is veel onduidelijkheid over hoe je opvoeding het beste kunt meten (zie hoofdstuk 10). Daarom hebben we een algemene opvoedingsvragenlijst ('Comprehensive General Parenting Questionnaire') ontwikkeld voor gebruik in dit promotieproject. Hoofdstuk 11 beschrijft de ontwikkeling van deze vragenlijst. Allereerst hebben we de belangrijkste constructen van opvoeding geïdentificeerd en beschreven ('zorgzaamheid', 'structuur', 'gedragscontrole', 'strikte controle (dwang)' en 'overprotectie'), evenals de onderliggende kenmerken. Het ultieme doel is om onderzoek te bevorderen naar de invloed van ouders op gezondheidsgedrag van kinderen.

Een stapsgewijze validatie met diverse technieken ('mixed methods approach') is toegepast bij de ontwikkeling van de algemene opvoedingsvragenlijst. Deze bestaat uit vier stappen: 1 ) De identificatie van 
vragen uit bestaande opvoedingsvragenlijsten gebaseerd op een raamwerk van vijf opvoedingsconstructen; 2) Het houden van cognitieve interviews en onderlinge discussie tussen de auteurs om informatie te krijgen over het eventueel aanpassen, weglaten of vervangen van vragen; 3) Het uitvoeren van geavanceerde statistische analyses (confirmatorische factoranalyses en item-responsemodellen) om het vijf-factoren opvoedingsmodel te testen en onderzoek te doen naar geschikte vragen; en 4) Het uitvoeren van additionele cognitieve interviews en onderlinge discussie tussen de auteurs om de aangepaste vragen te beoordelen op relevantie voor de constructen, op toetsing van de inhoudsvaliditeit en op woordgebruik.

Vervolgens is de ontwikkelde lijst afgenomen bij grote groepen opvoeders van 5- tot 13-jarige kinderen in Nederland, België en de Verenigde Staten. In totaal hebben 1497 opvoeders de vragenlijst ingevuld. Bovendien is er ook een vragenlijst over persoonlijkheidskenmerken (zogenaamde 'Big Five') afgenomen bij de opvoeders. De gereduceerde opvoedingsvragenlijst met 62 vragen laat een acceptabele overeenkomst zien met ons vijf-factoren opvoedingsmodel. Persoonlijkheid van de opvoeder hing samen met de vijf opvoedingsconstructen in onze vragenlijst. De persoonlijkheidstrekken 'extraversie', 'meegaandheid, 'zorgvuldigheid', en 'openheid voor ervaringen' waren positief geassocieerd met de opvoedingsconstructen zorgzaamheid, structuur bieden en gedragscontrole. Deze opvoedingsconstructen zijn gerelateerd aan positieve gezondheidsuitkomsten van kinderen. De persoonlijkheidstrek 'neuroticisme' was geassocieerd met dwangcontrole en een chaotische thuisomgeving. De opvoedingsvragenlijst is verder aangepast naar aanleiding van cognitieve interviews en bespreking van de vragenlijst met een expertpanel, waardoor deze nu 85 vragen bevat. De voorgestelde opvoedingsvragenlijst draagt bij aan onderzoek dat tot doel heeft om de rol van ouders te bestuderen in de ontwikkeling van gezondheidsgedrag bij kinderen.

\section{DEEL 3: Opvoeding - eetgedrag kind relatie}

In dit laatste deel zijn we gestart met het toetsen van ons model van de relatie tussen opvoeding en overgewicht bij kinderen. Eerder onderzoek over ouderschapspraktijken richt zich grotendeels op deze individuele gedragingen; de context waarbinnen deze ouderschapspraktijken worden uitgevoerd wordt vaak niet meegenomen (zie hoofdstuk 2). Hoofdstuk 12 heeft als doel om te bepalen welke voedingspraktijken van ouders de ontwikkeling van het eetgedrag van hun kinderen voorspellen. Bovendien heeft de studie tot doel de modererende invloed van zowel algemene opvoeding als de kindkenmerken (temperament en eetstijl) te testen op de relatie tussen voedingspraktijken en eetgedrag van kinderen. Om dit te onderzoeken is gebruik gemaakt van gegevens die zijn verzameld binnen de grootschalige KOALA cohortstudie. $\mathrm{Er}$ is een aantal verbanden gevonden tussen voedingspraktijken van de ouders en het eetgedrag van hun kinderen. Instrumenteel en emotioneel voeden en druk die ouders uitoefenen om hun kinderen voedingsproducten te laten consumeren leidden tot meer ongezond eetgedrag en minder gezond eetgedrag, zoals deels bevestigd in hoofdstuk 9. Kinderen eten gezonder als ouders ervoor zorgen dat ongezonde voedingsproducten niet in huis worden gehaald, ouders hun kinderen aanmoedigen een grote variëteit aan voedingsproducten te consumeren en ouders strikte voedingsregels toepassen. Ondanks het feit dat de sterkte van de correlatie afhing van de context, lieten alle bevindingen van de gestratificeerde analyses dezelfde trend zien. Twee voorbeelden: voor kinderen die zijn opgegroeid in een positieve 
opvoedingscontext heeft restrictie, aanmoedigen en het niet in huis halen van ongezonde producten betere effecten op voedingsgedrag dan voor kinderen die zijn opgegroeid in een andere opvoedingscontext (dwangmatige en overprotectieve thuisomgeving). Voor kinderen die zijn opgegroeid in een mindere positieve opvoedingscontext heeft emotioneel en instrumenteel voeden negatievere effecten op voedingsgedrag. Bovendien is gebleken dat sommige voedingspraktijken niet interacteren met andere factoren om eetgedrag van kinderen te verklaren. Deze factoren zijn misschien robuust voor variatie in contextuele factoren en geschikt voor gebruik in meer algemene interventies. Ouders hebben een aanzienlijke invloed op het eetgedrag van hun kinderen. Om ervoor te zorgen dat kinderen gezonder gaan eten, is het belangrijk te letten op de voedingspraktijken die ouders hanteren. Daarnaast is het van belang om rekening te houden met algemene opvoedingspraktijken en temperament en eetstijl van het kind.

\section{Algemene discussie}

In hoofdstuk 13 worden conclusies voor de wetenschap en de praktijk getrokken uit het onderzoek dat dit proefschrift wordt gerapporteerd. De rol van ouders blijkt uiterst belangrijk in de ontwikkeling van eet- en beweeggedrag van kinderen. Verschillende ouder- en kindfactoren beïnvloeden de relatie tussen specifieke ouderschapspraktijken en overgewichtgerelateerd gedrag van kinderen. Om opvoedingspraktijken nog beter te kunnen meten, is het noodzakelijk om specifieke vragenlijsten verder te ontwikkelen en te valideren. Dit geldt met name voor ouderschapspraktijken met betrekking tot voeding en beweging. De bestaande voedings- en beweegpraktijkenvragenlijsten zijn vaak slecht gevalideerd. In dit onderzoek hebben we getracht duidelijkheid te brengen over welke constructen er gemeten moeten worden en hoe deze constructen geoperationaliseerd moeten worden. Input van verschillende wetenschappers wereldwijd is nodig om voortgang te boeken in dit onderzoeksveld. Het congres beschreven in hoofdstuk 10 heeft hier een bijdrage aan geleverd. Het is belangrijk dat dynamische interacties tussen verschillende typen en niveaus van opvoeding (algemene opvoeding en ouderschapspraktijken) op energiebalans-gerelateerd gedrag van kinderen worden onderzocht. Hierbij dient gebruik te worden gemaakt van longitudinale onderzoeksontwerpen. Als we beter weten hoe ouders overgewichtgerelateerd gedrag en gewichtsstatus van hun kind beïnvloeden, dan kunnen gezondheidsbevorderaars effectievere interventieprogramma's ontwikkelen. 


\section{DANKWOORD ACKNOWLEDGMENT}


Overgewicht en obesitas hebben al vanaf mijn bachelorstudie mijn interesse omdat de gevolgen enorm kunnen zijn. Tijdens mijn bachelorstudie heb ik me in dit onderwerp verder verdiept door middel van een stage en wilde hier graag tijdens mijn masterstudie mee verder gaan. Ik kwam in contact met Stef Kremers en Carel Thijs die zich deels bezig hielden met dit onderwerp. Carel was tevens hoofdonderzoeker van de KOALA-studie. Dit geboortecohort gaf me de mogelijkheid om de rol van de ouders in de ontwikkeling van energiebalans-gerelateerd gedrag van hun kind te onderzoeken. Na het binnenhalen van een subsidie vanuit de Hartstichting ben ik mijn onderzoek gestart bij de Vakgroep Gezondheidsbevordering.

Graag wil ik iedereen bedanken die heeft bijgedragen aan het tot stand komen van dit proefschrift.

Allereerst mijn promotor Nanne de Vries en co-promotoren Stef Kremers en Carel Thijs, voor hun vertrouwen in mij, hun prettige begeleiding en inspirerende discussies. Ik ben jullie dankbaar voor het steunen van mijn initiatieven. Stef, jij stond achter mijn ideeën; heel erg bedankt daarvoor. Jouw positiviteit waardeer ik enorm. Carel, door onze inspirende discussies werd ik gestimuleerd om kritisch mijn onderzoek te beoordelen. Ook wil ik de leden van de leescommissie bedanken voor hun deelname en tijdinvestering in het beoordelen van mijn proefschrift. Daarnaast zou ik graag collega's en oud collega's van de vakgroep Gezondheidsbevordering bedanken voor alle gezellige momenten, praktische hulp en inhoudelijke discussies.

Dank ook aan alle deelnemers van mijn onderzoek. Zonder hun interesse en deelname was het niet mogelijk geweest dit proefschrift te schrijven. In het bijzonder wil ik de deelnemers van de KOALA-studie bedanken. Zij hebben steeds met enthousiasme de vragenlijsten ingevuld en diverse kinderen hebben meegedaan aan de fitheidstesten. Aan de dataverzameling en logistieke planning hebben verschillende assistenten hun bijdrage geleverd; met name wil ik Ine Bonekamp en Teun Remmers daarvoor bedanken.

Special thanks go to the employees of the Department of Pediatrics, Baylor College of Medicine from the USDA/ARS Children's Nutrition Research Center in Houston, Texas, United States. You have made my work visit a very special and inspiring one. In particular, I want to thank Tom Baranowski, Teresia O'Connor, and Sheryl Hughes. I was lucky having a chance to work with the godfather of the ISBNPA and some of the most well-known scientists in the 'parenting - childhood overweight' field. I am happy to say: 'Houston, I have landed'. Hopefully, our cooperation will continue for a long time. Teresia, special thanks to you; you are a great person to work with, and many thanks for all your help and hospitality during my stay. I enjoyed spending time with you and your family. Kathy Watson, thank you for giving me statistical advice regarding the advanced statistical analyses presented in this dissertation. Thomas Power, thank you for providing me with the opportunity to visit your Department of Human Development at Washington State University. Your help as a parenting expert is highly appreciated.

Linda en Floortje, wat fijn dat jullie mijn paranimfen zijn. Bedankt voor jullie steun en goede advies. Familie en vrienden, bedankt voor jullie betrokkenheid en interesse in de voortgang van mijn onderzoek. 
CURRICULUM VITAE 
Ester Francisca Catharina Sleddens was born on June 9, 1985 in Eindhoven, the Netherlands. After completing high school in 2003 (Were Di College, Valkenswaard), she began her studies in Health Sciences at the Faculty of Health, Medicine and Life Sciences of Maastricht University, the Netherlands. She attained her Bachelor degree (specializations: Health Promotion and Mental Health) and Research Master degree (specializations: Social Sciences and Epidemiology) in Health Sciences at Maastricht University. She started her scientific career at the Department of Health Promotion of Maastricht University, the Netherlands in September, 2008. Besides research on parenting, child temperament and childhood overweight, she is responsible for various educational activities at the Faculty of Health, Medicine and Life Sciences of Maastricht University. While studying for her Bachelor's degree, she was an exchange student at Central Michigan University, Mount Pleasant, MI, United States (Spring 2006). She was a visiting researcher at the USDA/ARS funded Children's Nutrition Research Center, Department of Pediatrics, Baylor College of Medicine, in Houston, TX, United States (November 2010 to April 2011, and May 2012), for which she received a travel grant from the Dutch Heart Foundation, and at the Department of Human Development of Washington State University, in Pullman, WA, United States (May 2012). She co-organized a pre-conference to the annual meeting of the International Society of Behavioral Nutrition and Physical Activity entitled 'Parenting measurement: Current status and consensus reports', held in Houston, TX, United States, from May 20-22, 2012. Currently, she is involved in several international research projects related to parenting and childhood overweight. She obtained a one-year Kootstra Talent Fellowship from Maastricht University Medical Centert. In 2013, she started working as a postdoctoral researcher at the Department of Health Promotion of Maastricht University. 
PUBLICATION LIST 
Sleddens EFC, Kremers SPJ, \& Thijs C. (2008). The Children's Eating Behaviour Questionnaire: Factorial validity and association with Body Mass Index in Dutch children aged 6 to 7. International Journal of Behavioral Nutrition and Physical Activity, 5:49.

Sleddens EFC, Kremers SPJ, De Vries NK, \& Thijs C. (2010). Relationship between parental feeding styles and eating behaviours of Dutch children aged 6 to 7. Appetite, 54, 30-36.

Stafleu A, Goldbohm S, Van Dommelen P, Van den Bosch L, Dutman E, Gubbels JS, Sleddens EFC, \& Thijs C. (2009). Regels en opvoedpraktijken van ouders ten aanzien van eten - het KOALA geboortecohort. Voeding $\mathrm{Nu}, 10$ (okt), 18-21.

Sleddens EFC, Kremers SPJ, Nanne NK, \& Thijs C. (2010). De vertaling en validatie van twee Engelse voedingsvragenlijsten: Bruikbaarheid in Nederland. Voeding Nu, 1/2 (dec/jan), 25-28.

Sleddens EFC, Kremers SPJ, Candel MJJM, De Vries NK, \& Thijs C. (2011). Validating the Children's Behavior Questionnaire in Dutch children: Psychometric properties and a cross-cultural comparison of factor structures. Psychological Assessment, 23, 417-426.

Sleddens EFC, Gerards SMPL, Thijs C, De Vries NK, \& Kremers SPJ. (2011). General parenting, childhood overweight and obesity-inducing behaviors: A review. International Journal of Pediatric Obesity, 6, e12-27.

Gerards SMPL, Sleddens EFC, Dagnelie PC, De Vries NK, \& Kremers SPJ. (2011). Interventions addressing general parenting to prevent or treat childhood obesity. International Journal of Pediatric Obesity, 6, e2845.

Sleddens EFC, Hughes SO, O'Connor TM, Beltran A, Baranowski JC, Nicklas TA, \& Baranowski T. (2012). The Children's Behavior Questionnaire very short scale: Psychometric properties and development of a oneitem temperament scale. Psychological Reports, 110, 197-217.

Baranowski T, O'Connor T, Hughes S, Beltran A, Baranowski J, Nicklas T, Sleddens E, Thompson D, Lu A, \& Buday R. (2012). Smart phone video game simulation of parent-child interaction: Learning skills for effective vegetable parenting. In S Arnab, I Dunwell, \& K Debattista (Eds.), Serious games for healthcare: Applications and implications (pp. 247-264). Hershey, PA: IGI Global.

Sleddens EFC, Kremers SPJ, De Vries NK, \& Thijs C. (2012). Measuring child temperament: Validation of a 3item temperament scale and 13-item impulsivity scale. European Journal of Developmental Psychology, Epub 30 May. 
Sleddens EFC, Kremers SPJ, Hughes SO, Cross MB, Thijs C, De Vries NK, \& O'Connor TM. (2012). Physical activity parenting: A systematic review of questionnaires and their associations with child activity levels. Obesity Reviews, 13, 1015-1033.

Sleddens EFC, Hughes SO, O'Connor TM, Beltran A, Baranowski JC, Nicklas TA, \& Baranowski T. (2012). Reply to commentary on Putnam's promise of, problems with, and potential refinement of the 'Extremely Short Form of the Children's Behavior Questionnaire'. Psychological Reports, 111, 621-623.

Mercken L, Sleddens EFC, De Vries H, \& Steglich C. (2013). Choosing adolescent smokers as friends: The role of parenting and parental smoking. Journal of Adolescence, Epub 21 Jan.

Sleddens EFC, O'Connor TM, Watson KB, Hughes SO, Power TG, Thijs C, De Vries NK, \& Kremers SPJ. (in revision). Development of the Comprehensive Parenting Questionnaire for caregivers of 5 to 13 year olds.

Baranowski T, O'Connor TM, Hughes SO, Sleddens EFC, Beltran A, Frankel L, Mendoza J, \& Baranowski J. (submitted). Houston... We have a problem! Measuring parenting: Overview of a conference.

Kremers SPJ, Sleddens EFC, Gerards SMPL, Gubbels JS, Rodenburg G, Gevers D, \& Van Assema P. (submitted). General and food-specific parenting: Measures and interplay.

Power TG, Sleddens EFC, Berge J, Connell L, Govig B, Hennessy E, Ligget L, Odoms-Young A, Mallan K, Santa Maria D, \& George SM. (submitted). Issues in the measurement of parenting style.

Remmers T, Sleddens EFC, Thijs C, \& Kremers SPJ. (submitted). Correlations between physical activity enjoyment, physical activity behavior, and physical fitness in 7 to 10 year old Dutch children: The KOALA Birth Cohort Study.

Sleddens EFC, Kremers SPJ, Stafleu A, Dagnelie PC, De Vries NK, \& Thijs C. (in revision). Food parenting practices and child dietary patterns: Prospective relations and the moderating role of general parenting, child temperament, and child eating style.

Remmers T, Sleddens EFC, Gubbels JS, De Vries SI, Mommers M, Penders J, Kremers SPJ, \& Thijs C. (submitted). Relationships between physical activity and the development of BMI in children. 